

WILSON COPEPOD LIBRARY Smithsonian Institution invertebrate Zoology (Crustacea) 

WILSON COPEPOD LIBRARY

Smithsonian Institution

Invertebrate Zoology

(Crustacea) 



\subsection{4.

TRAVAUX

DU

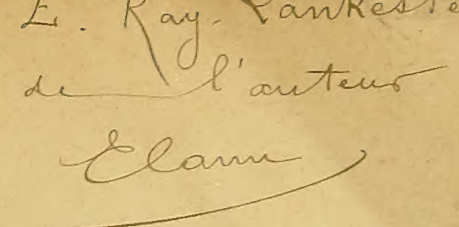

WILSON COPEPOD LIBRARY Smithsonian Institution Invertebrate Zoology

(Crustacea)

\section{LABORATOIRE DE ZOOLOGIE MARITIME}

DE

\section{WIMEREUX-AMBLETEUSE \\ (PAS-DE-C ALAIS )}

Tоме VI

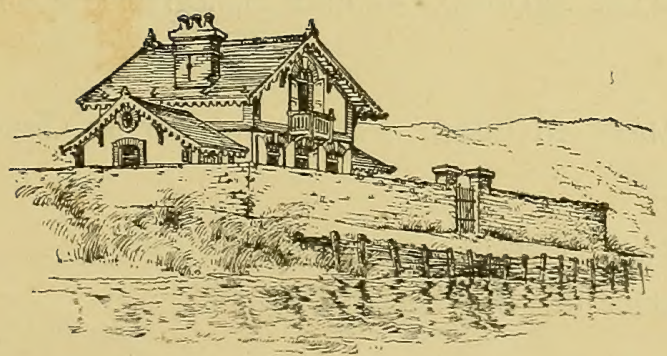

L I L L E, IMPRIMERIE L. DANEL.

I 892 . 


\title{
TRAVA UX
}

\author{
$\mathrm{DU}$ \\ LABORATOIRE DE ZOOLOGIE MARITIME \\ DE
}

WIMEREUX-AMBLETEUSE.

Tome I. Jules Barrois. - Recherches sur l'embryologie des Bryozoaires.

Tome II. Paul Hallez. - Contributions à l'histoire naturelle des Turbellariés.

Tome III. Romain Moniez. - Essai monographique sur les Cysticerques.

Tome IV. Romain Moniez. - Mémoires sur les Cestodes.

Tome V. Alfred Giard et Jules Bonnier. - Contributions à l'étude des Bopyriens. 
LES

\section{COPÉPODES DU BOULONNAIS}

MORPHOLOGIE, EMBRYOLOGIE, TAXONOMIE,

PAR

\section{EUGENe CANU, Docteur ès-sciences,}

Naturaliste à la Station aquicole de Boulogne-sur-Mer. 
348470

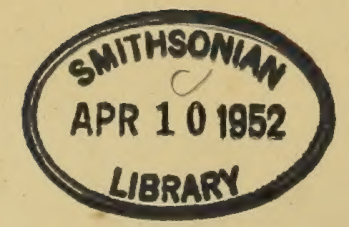




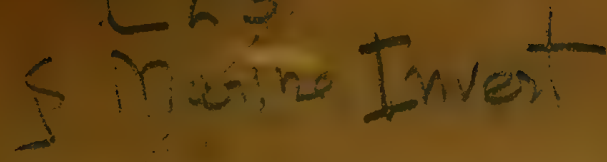

TRAVAUX DU LABORATOIRE DE ZOOLOGIE MARITIME DE WIMEREUX-AMBLETEUSE (PAS-DE-CALAIS) TOME VI.

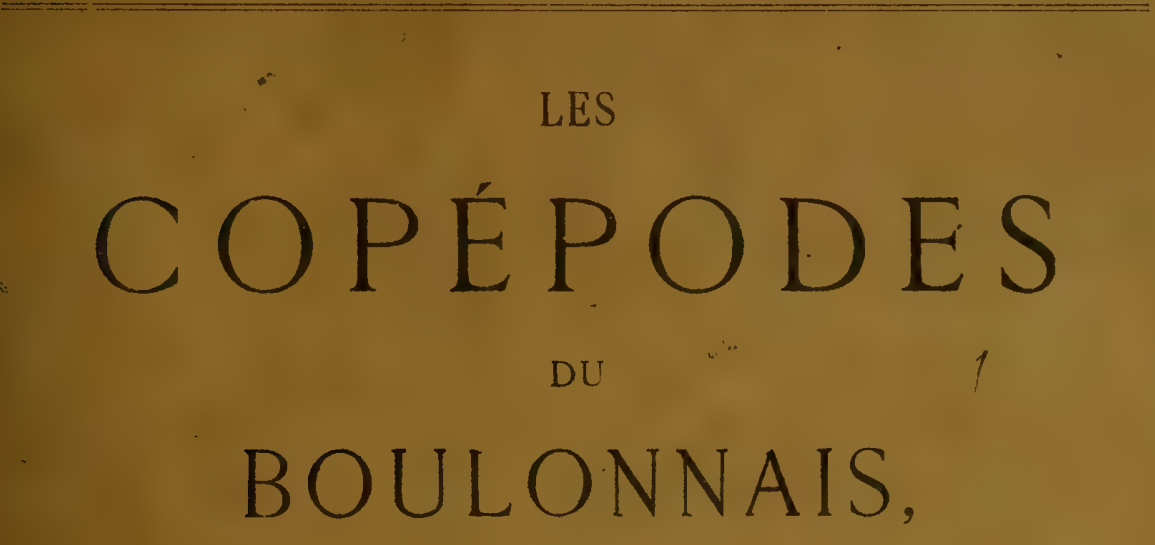

MORPHOLOGIE, EMBRYOLOGIE, TAXONOMIE,

PAR

\section{EUGÉNE CANU,}

MATURALISTE A LA STATION AQUICOLE DE. BOULOGNF-SUR-MER.

LI LLE,

IMPRIMERIE L; DANEL

1892. 
Publications de la station zoologique de wimereuX-Ambletelse,

SOUS LA DIRECTION DE

ALFRED GIARD,

PROFESSEUR A LA SORBONNE。

I.

BULLETIN SCIENTIFIQUE

DE LAA FRANCE ET DE LA BELGIQUE.

Vingt-quatrième annće '(189a).

Le Bullelin scicntifique parait par livraisons datées du jour de leur publication. Chaque volum: frand in $-8^{\circ}$ contient 500 pages environ et de 15 a 30 planches hors texte.

Sans négliger aucune des parties des sciences hiologiques, la direction s'attache surtout a publier des travaux ayant trait à l'Évolution (ontogénie et phylogénie) des êtres vivants. L.es recherches relatives a l'éthologie et à la distribution gréographique dans leurs rapports avec la théorie de la Descendance occupent aussi une large place dans le Bulletin.

Entin, ce recucil peut être consideré comme le Journal de la Station naritime de // 'imcreux-tmblclcuse (Pas-de-Calais), londée et dirigée depuis $187^{3}$ par le Protesseur A. Guard.

Les tomes I, II, III, IV. VIII, IX, Xet XI sont epuisés. Quelques exemplaires des tomes V, VI et VII sont encore en vente au prix de 15 fr le vol.; les tomes XII a XVI au prix de 10 fr.; les tomes XVII et XVIII au prix de 20 fr., et à partir du tonre XIX au prix de 30 fr. le volume.

Le tirage étant limité, ces prix seront rapidement augmentés.

\section{PRIX DE L'ABONNEMENT A UN VOLUME:}

\section{Pour Paris ............................. \\ Pour les Départements et l'Etranger $\ldots \ldots$.}

L'abonnement est payable après la livraison du premier fascicule de chaque volume, et sera continué, sauf avis contraire et par écrit.

Adresser tout ce qui concerne la rédaction et l'administration à

MM. AlFRED GIARD, 14, rue Stanislas,
Jules BONNIER, 75, rue Madame, 
A Monsieur

\section{le professeur Alfred Giard,}

En témoignage de mon affection

et de ma reconnaissance.

Eug. Canu. 



\title{
AVANT-PROPOS.
}

\begin{abstract}
II n'y a que ceux qui se sont longtemps et fortement occupés de la determination des espèces, et qui ont consulté de riches collections, qui peuvent savoir jusqu'à quel point les especces, parmi les corps vivants, se fondent les unes dans les autres, et qui ont pu se convaincre que dans les parties où nous voyons des espèces isolées, cela n'est ainsi que parce qu'il nous en manque d'autres qui en sont plus voisines et que nous n'avons pas encore recueillies.

Lamarck, Philosophie zoologique. Première partie, chapitre III.
\end{abstract}

Les débuts de ce travail remontent à l'année 1885. Depuis cette époque, j’ai recueilli et étudié les Copépodes marins du Boulonnais.

Mes recherches ont été effectuées principalement au Laboratoire de Zoologie maritime de Wimereux, et poursuivies, dans l'intervalle des séjours à la mer, à l'Institut zoologique de Lille jusqu'en $\mathbf{1 8 8 7}$, et depuis lors au Laboratoire zoologique de l'École normale supérieure de Paris. Grâce à l'obligeance de M. le Prof. GIARD, directeur de ces laboratoires, j'y ai trouvé la plus large hospitalité et toutes les ressources nécessaires à mes travaux.

Une méthode rigoureuse était indispensable dans l'étude d'un groupe zooloyique aussi varié et étendu que celui des Copépodes. Aussi me suis-je efforcé d'éclairer la 
zoologie de ces Crustacés en suivant les règles fixées dès 1876 par le Prof. GIARD (I). Appliquant la méthode de superposition embryogénique, j'ai tenté d'établir les rapports exacts de ces animaux par l'étude de leur morphologie aux divers stades de leur existence; j'ai eu grand soin d'utiliser également les renseignements fournis par l'éthologie, si importante comme agent évolutif.

Devant l'accumulation des matériaux recueillis pendant cinq années de récoltes, j'ai dû limiter ce premier mémoire aux résultats fournis par les formes libres et semi-parasites qui présentent un intérêt spécial aux différents points de vue de la biologie.

Néanmoins, si j'ai tenté quelques discussions d'ordre général, je crois m'excuser en rappelant que ces spéculations sont appuyées sur l'étude consciencieuse de plus de 80 espèces observées à l'état vivant et fréquemment suivies dans leurs métamorphoses (2).

Diverses préoccupations ont amené la subdivision de mon travail en plusieurs parties :

Dans l'introduction, à l'historique résumé de notre connaissance des formes étudiées, j’ai adjoint une nomenclature destinée à faciliter la compréhension du texte descriptif aux naturalistes spécificateurs peu familiarisés avec la terminologie morphologique.

Dans la première partie, j’ai passé en revue la morphologie de ces espèces considérées dans leur évolution toute entière, et non pas seulement à l'état adulte.

Dans la deuxième partie sont décrites les métamorphoses d'une série de Copépodes appartenant à une même famille commensale des Tuniciers.

(1) Giard. Les faux principes biologiques et leurs conséquences en taxonomie, "Conclusions "; Rezue scientifique, 2e série, 5e année ( 18 mars 1876 ).

(2) Voir pages 277 et 278 la liste des formes étudiées dans le présent travail. 
La troisième partie contient l'exposé des particularités éthologiques des Copépodes libres et semi-parasites, avec la recherche des influences qu'elles exercent sur leur histoire naturelle.

La quatrième partie a trait à la phylogénie des Copépodes, examinés dans leurs relations entre eux et avec les autres Crustacés.

Enfin, la cinquième comprend l'étude taxonomique de Copépodes libres et semiparasites recueillis pour la plupart dans le Boulonnais, la description des formes nouvelles ou peu connues, augmentée de nombreux détails anatomiques qui mont paru insuffisants pour prendre place dans la partie morphologique.

Pour la description systématique, je me suis efforcé de disséquer et de figurer dans leurs moindres détails les appendices caractéristiques de ces petits Crustacés, étude aujourd'hui indispensable, quoiqu'elle soit souvent longue et difficile.

C'est avec une vive satisfaction que je puis témoigner publiquement ma reconnaissance envers les naturalistes qui m'ont aidé dans la préparation de mon travail :

A MM. Alfred Giard, Fondateur et Directeur, et Jules Bonnier, DirecteurAdjoint du Laboratoire de Wimereux, qui m’ont amicalement prodigué durant mes recherches leurs précieux conseils et le concours le plus éclairé;

A M. et $\mathrm{M}^{\mathrm{me}}$ Pierre Bonnier, qui m'ont obligeamment facilité la préparation des planches définitives et la lecture des mémoires publiés en langues slaves ;

A mon ami M. Alfred Bétencourt, poursa collaboration active et dévouée dans la recherche et la préparation du matériel;

A M. le $D^{r}$ H.-E. Sauvage, Directeur de la Station aquicole de Boulognesur-Mer,qui m'a communiqué, de la plus aimable façon, la collection de Copépodes formée par lui dans cet établissement.

Parmi les carcinologistes qui m'ont, à diverses reprises, fait connaltre leur avis 
sur des points discutables, ou communiqué de précieux échantillons, je désire remercier tout spécialement :

MM. le D W. Giesbrecht, le Prof. G. O. Sars, le Rév. A. M. Norman, le $D^{r}$ G. St. Brady et Isaac C. Thompson.

Durant trois années consécutives, j’ai obtenu du Conseil Municipal de Paris une subvention destinée à faciliter mes recherches au bord de la mer. Qu'il me soit permis d'en témoigner ici toute ma gratitude.

Boulogne-sur-Mer, le ${ }_{1}^{\text {er }}$ Décenıbre 1891.

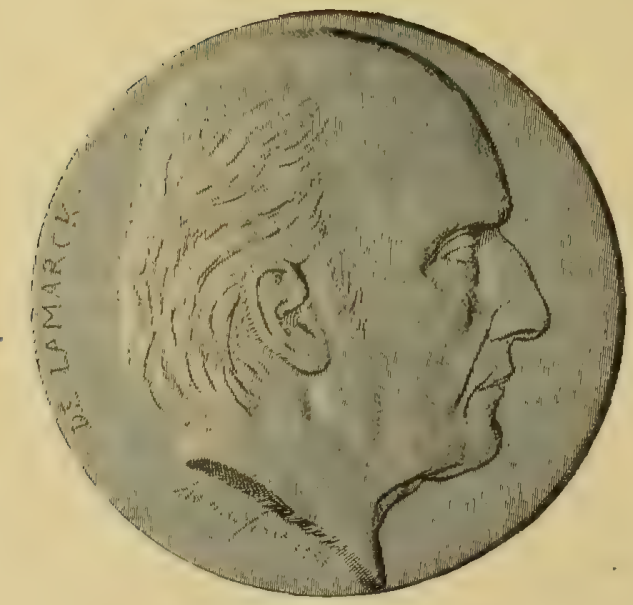




\section{TABLE DES MATIĖRES.}

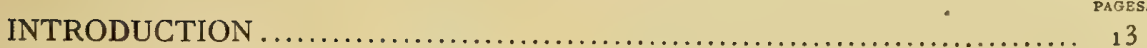

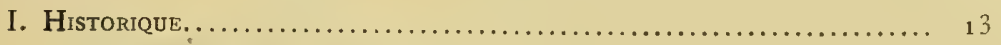

II. Nomenclature d'un copépode typtQue....................... 19

1. État adulte .................................. 19

2. Métamorphose................................... 23

Premiére partie. - MORPHOLOGIE................................... 24

I. Forme générale........................................ 24

1. Céphalothorax.................................... 26

2. Abdomen .......................................... 30

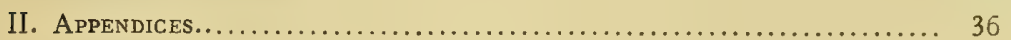

1. Antennules .................................... 36

2. Antennes..................................... 38

3. Pièces buccales .................................. 39

4. Premières maxilles............................... 56

5. Secondes maxilles................................ 58

6. Pattes thoraciques............................... 60

Deuxieme, partie. - EMBRYOGÉNIE DES ASCIDICOLID无............... 64

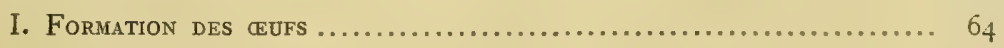

II. MÉtamorphoses..........................................

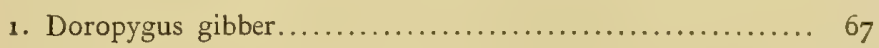

2. Bonnierilla longipes............................. 77

3. Notodelphys agilis .............................. $7^{8}$

4. Notodelphys allmani ............................ 81

5. Doroixys uncinata ............................... 83

6. Enterocola fulgens................................ 85

7. Aplostoma brevicauda ........................... 88

III. Conclusions............................................ g1

1. Condensation du développement .................... g1

2 Mésoderme secondaire.............................. 93 
Troisieme partie - ÉTHOLOGIF

I. Milieux cosmiques..................................... 97

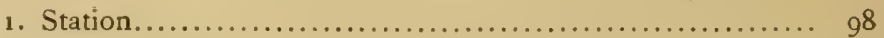

2. Latitude ..................................... 102

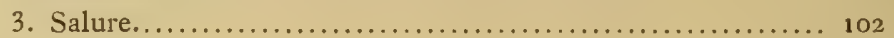

II. Milieux Bjologiques...................................... 104

1. Espèces libres ................................... 104

- 2. Espèces parasites.................................. 104

III. Caractères sexuels secondatres; Progenèse..................... 110

IV. Commensaux et ennemis des copépodes ......................... 120

Quatriéme partie. - PHYLOGÉNIE................................... 124

I. RAPPORTS DES COPÉPODES ENTRE EUX........................ 124

1. Le Copépode le moins spécialisé ........................ 124

2. Classification phylogénétique des Copépodes .............. 127

II. Rapports des copépodes avec les aUtres CRustacés............. 134

Cinquième partie. - DESCRIPTION SYSTÉMATIQUE..................... 138

I. Monoporodelphya........................................... 143

a. - Harpacticidæ ................................ 143

b. - Calanidæ............................... 168

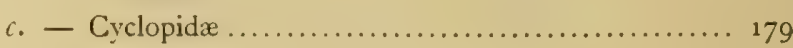

d. - Ascidicolid $x \ldots \ldots \ldots \ldots \ldots \ldots \ldots \ldots \ldots \ldots \ldots \ldots, 186$

II. Diporodelphya ......................................... 224

1. - Monochila ...................................... 225

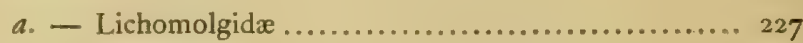

b. - Ergasilidæ.............................. 244

2. - Auliostoma.................................... 247

a. - Hersiliidæ .................................... 247

b. - Cancerilla et Caligidium....................... 254

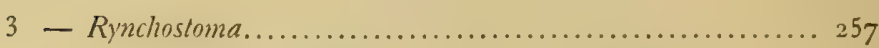

a. - Ascomyzontidæ.......................... 257

Index analytique des descriptions............................. 273

Liste synoptique des espèces étudiées ......................... 277

Table des figures intercalées dans le texte $\ldots . \ldots \ldots \ldots \ldots \ldots \ldots \ldots \ldots . . . \ldots 279$

Littérature ..................................................... $28_{1}$ 


\section{INTRODUCTION.}

I.

\section{HISTORIQUE.}

Si quelques espèces de Copépodes parasites des Poissons ont attiré, par leur grande taille, l'attention des premiers naturalistes, et ont été remarquées par ARISTOTE, il n'en est pas de même des formes moins dégradées de Copépodes libres ou semi-parasites, qui font l'objet de ce travail.

\section{LES PREMIERES DESCRIPTIONS.}

La découverte du premier Copépode normal, attribuée jusqu'à ce jour à STEPHAN BLANKAART (10) (1) pour une mauvaise figure de Cyclops ? publiée à Amsterdam en 1688, revient de droit à FranCESCO REDI (173).

-Dans quelques lignes écrites en 1684 , - et dont je dois la connaissance à M. le Professeur GIARD, - le célèbre naturaliste italien fournit sur un Copépode ascidicole tous les renseignements nécessaires, accompagnés d'un dessin suffisant pour nous faire reconnaitre un Doropygus $(\mathbf{2})$.

Voici d'ailleurs la traduction du texte même de REDI : "A l'intérieur du gros " trou qui est la bouche, on voit une large et longue cavité dans laquelle se " tiennent des animalcules. Dans toutes les Mentules ou "pincii " que j'ai observées " durant plusieurs années consécutives, dans les mois de janvier, février, mars, " avril èt juillet, ces mèmes animalcules furent toujours trouvés sans aucune " exception. Lorsqu'ils sont vivants, ils sont de couleur noirâtre, ou gris bruns

(1) Les chiffres gras, ici entre parenthèses, correspondent aux numéros des ouvrages cités dans la littérature, pages 28:-292.

(2) Le mémoire de RBDI renferme encore bien des observations remarquahles pour l'époque, et on a déjà reconnu les Daphnies dans d'autres animalcules aquatiques figurés par cet auteur. 
" tachetés de noir ; mais lorsqu'ils sont cuits dans l'eau, ils deviennent de couleur " rouge vif. Ils ne sont pas plus gros qu'un petit grain de blé, ni durs, ni revêtus

" d'une carapace; au contraire, ils sont tendres, et lorsqu'on les serre dans les " doigts, ils crèvent subitement et se désagrègent sans aucune résistance. Parfois,

" dans certains " pincii ", je n'en ai trouvé que cinq ; dans d'autres, six, et jusque "sept, huit, mème neuf, mais pas plus de douze. Ils demeurent vivants lorsque " la Mentule a été détachée du rocher et est morte defuis deux jours, voire mème " depuis trois jours. Leur figure, plus grande que nature et vue sous le microscope, " est représentée au $n^{0} 7$ de la planche XXI, où est également figuré, sous le $n^{0} 6$,

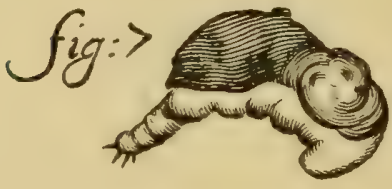

Fig. I. - Doropygien de la Mentule, d'après REDI (loc. cit., PI. XXI, fig. 7).

"le " pincio " de mer, de grandeur naturelle.

"Ces vermicules sont-ils nés dans la cavité " de la Mentule? Ou bien étant d'abord " dans la mer, se retirent-ils et se cachent-ils " spontanément en cet endroit, comme dans " une grotte? Attirés au contraire par la "Mentule, y viennent-ils pour être tenus " comme dans un vivier ou conservés " comme nourriture en cas de nécessité? Je ne saurais ni ne voudrais me " prononcer maintenant sur cette question".

Bien que le travail de REDI ne nous laisse aucun doute sur la nature de l'animal qu'il a observé, c'est à Leeuwenhoek (132) qu'est dû le premier document véritablement zorlogrique sur ces animaux microscopiques. Le micrographe hollandais écrivit, en 1699, à propos d'un Cyclops, une description accompagnée de figures où il constate le premier la dissemblance de l'embryon et de la mère.

\section{LES PREMIERS ESSAIS TAXONOMIQUES.}

Depuis le commencement du dix-huitième siècle, il a été publié sur le groupe entier des Copépodes une multitude de mémoires descriptifs ou anatomiques, dont la substance a été résumée d'une façon complète dans divers traités devenus classiques ( $\mathbf{I})$.

Renvoyant le lecteur à ces sources pour les détails de la révision historique, je m'attacherai surtout à indiquer l'évolution graduelle de nos connaissances avec les innovations importantes tentées aux diverses époques, et j'insisterai plus spéciale-

(1) Consulter spécialement Milne Edwards (149), Baird (9), Gerstecksr (83), Desmiarets (77), Claus (51). 
ment sur les travaux récents d'une réelle importance. On trouvera, d'ailleurs, dans le relevé de la littérature et dans le texte descriptif, l'indication et la discussion des mémoires d'importance secondaire relatifs aux sujets traités ici.

La première période a été - comme de coutume - consacrée exclusivement à l'énumération des formes observées par les divers naturalistes, décrites d'ailleurs sans détails anatomiques par suite des faibles moyens d'investigation de l'époque. Ces descriptions n'en restent pas moins utilisables pour les naturalistes exercés et patients, qui savent suppléer aux insuffisances systématiques et morphologiques par leur expérience et l'emploi judicieux des renseignements éthologiques.

Au dix-huitième siècle, c'est à Copenhague que florissait la Zoologie descriptive : GUNNer (102) et STR $\phi$ M(193) s'occupèrent de quelques espèces de Copépodes libres marins. O.-F. Mueller (152), le véritable fondateur de la microcarcinologie, créa le groupe des Entomostracés; mais il eut le tort de méconnaitre les résultats embryogéniques acquis par les recherches de Leeuwenhoek et de De GeER ( $\mathbf{7} \mathbf{4})$, puisqu'il plaça les embryons des Copépodes dans un genre spécial nommé par lui Nauplius.

Au temps de O.-F. Mueller, il n'existait point encore de classification naturelle des Crustacés, et la voie ouverte par Fabricius (r9) dans l'etude des caractères fournis par les appendices de ces animaux n'avait pas été suffisamment suivie pour qu'on puisse fixer nettement les limites des différentes familles.

C'est en $\mathbf{1} 796$ que Latreille (12r) voulut former, parmi les Insectes, une famille des Crustacés, d’après leurs caractères organiques. Il fut suivi plus tard par CUvier (72), qui sépara (après un essai de BRISSON, 1765) les Crustacés des Insectes. Ces arrangements ont subi diverses améliorations de la part de LATrellle, Cuvier, LeACH (130), jusqu'en 1827 , époque à laquelle Latreille (128) publia lui-mème une nouvelle classification naturelle des Crustacés en familles d'après l'organisation de la bouche. Desmarets (rar), Milne Edwards $(\mathbf{1 4 8}, \mathbf{1 4 9})$, Claus $(\mathbf{4 4}, \mathbf{5 1})$, et GERST ÆCKER (83) ont suffisamment insisté sur ces points, pour me dispenser d'y revenir.

\section{LES RECHERCHES ANATOMIQUES.}

A la fin du dix-huitième siècle, l'anatomie des Crustacés avait ainsi accompli d'importants progrès, dont la plus grande part revenait encore aux Crustacés supèrieurs. En ce qui concerne spécialement les Copépodes, les travaux de zoologie descriptive et anatomique ne commencent qu'avec le dix-neuvième siècle. 
C'est alors que RAmDohr (168) et JURINE père (115) nous instruisent soigneusement des particularités les plus importantes de l'histoire naturelle des Cyclopes. Le mémoire du dernier de ces auteurs, sur les " Monocles ", conserve encore une importance considérable. En réalité, nous devons à l'attention portée par les naturalistes de cette époque vers les variations embryonnaires, le plus grand progrès réalisé dans la connaissance de tous les Crustacés ; car il ne pouvait être question des véritables rapports des nombreuses formes de Crustacés qu'après la découverte définitive faite par Alex. von Nordmann (154) sur l'embryon des Lernéens et sur ses métamorphoses. La synthèse de cette série d'études anatomiques se trouve incontestablement dans le Traité classique de H. Milne Edwards (149), malgré les imperfections de ce livre.

L'impulsion fut grande à partir de cette époque. Parmi les nombreux naturalistes qui étudièrent alors les Copépodes, on doit citer: BurmeISTER (26), RathKe (171, 17z), KR $\varnothing$ Yer (122-5), von Siebold (187), Pickering et Dana (160), Philippi (159), Goodsir (94, 95), Baird (9), Lilljeborg (136), Lubbock (140-4), et surtout Dana (73) dont l'œuvre splendide sur les Crustacés de l'exploration américaine reste encore l'un des plus beaux monuments de la science zoologique.

Le nombre des espèces connues était déjà.considérable; mais il devait prendre encore, durant la seconde moitié du dix-neuvième siècle, un développement nouveau dans l'appoint fourni par les formes, si intéressantes, parasites des Invertébrés. Nicothoe, Staurosoma, Notodelphys, Doridicola ont inauguré une série de découvertes que les zoologistes n’ont point vu décroitre encore, malgré les efforts soutenus de chercheurs tels que : Audouin et Milne-Edwards (3), Will (21:), Allman $(\mathbf{1}, \mathbf{2})$, Leydig (135), Costa (71), Bruzelius (23), Leuckart (133), van Beneden (11-13), Thorell(204), Hesse (110), Boeck (14), M. Sars (183-5), Keferstein (116), Hancock et Norman (104), von Nordmann (155), Krøyer (125), Claparède (38, 39), Claus (55), Brady (20), Buchholz (24), Giesbrecht (90), Aurivillius (4-7), etc.

\section{LES ÉTUDES MORPHOLOGIQUES.}

A côté de tous ces travaux, dont la plupart doivent être considérés comme ayant participé exclusivement au dénombrement des formes de Copépodes, il convient de mentionner tout particulièrement les études de ZENKER (214), qui ont véritablement inauguré, pour les Crustacés inférieurs, la méthode analytique et comparative. Le “System der Crustaceen" et la "Critik der Erichson'schen 
Gliedmassentheorie" contiennent les règles d'où procèdent les morphologistes modernes, tels que Huxley, Claus, Boas, etc.

En ce qui concerne la taxonomie des parasites, ZeNKER (214, p. 112) eut l'honneur de rejeter le groupe purement artificiel et physiologique des Crustacés "suceurs" de MiLne-EDWARDS, et d'établir sur de véritables bases zoologiques la parenté des Siphonostomes et Lernéens avec les Cyclopides ou Copépodes libres. S'il existe parmi les Crustacés suceurs des ressemblances dans l'organisation de la bouche, c'est pour ZENKER un effet de la convergence produite par le parasitisme dans diverses familles de Crustacés; les Siphonostomes et Lernéens, si semblables aux Cyclopides dans tous leurs caractères morphologiques de l'adulte et des jeunes, ne sont en somme que des Copépodes suceurs. Abordant d'autre part (ibid., p. I 18) les discussions plus élevées de la morphologie, l'auteur établit l'homologie des appendices d'après leur position relative et leurs rapports anatomiques, la forme et la fonction ne fournissant que des renseignements insuffisants et souvent trompeurs (I). Il considère les appendices de la région prébuccale, innervés par les ganglions supra-œesophagiens, comme homologues entre eux et comme différents des appendices post-buccaux, innervés par la chaîne ganglionnaire ventrale.

En continuant la méthode de Zenker, Claus (40 à ro) a su conquérir une renommée indiscutable dans l'étude minutieuse des Crustacés. Les divers mémoires publiés par cet auteur, durant trente années consécutives, sur les Copépodes les plus variés, le placeraient, seuls, au premier rang des zoologistes. Chacun des points importants de l'organisation de ces animaux a attiré l'attention de Claus, et j'examinerai en détail, dans les pages qui vont suivre, les diverses opinions qu'il a émises.

Dans toutes ses recherches, Claus a donné la prépondérance aux caractères morphologiques; il a vigoureusement soutenu $(50,55,66)$ ses idées contre THORELL (204) et plus récemment encore contre KOSSMANn (118-120): l'anatomie des appendices, et spécialement des pièces buccales, est toujours invoquée par lui dans la fixation des rapports des Crustacés, parfois peut-être au mépris des réserves de ZENKER lui-même. Rarement Claus s'est écarté de cette méthode trop rigoureusement objective, en recherchant certains arrangements taxonomiques discutables; il a notamment attribué des origines multiples aux trompes-suçoirs ou siphons des Copépodes siphonostomes $(\mathbf{6 3})$ et rattaché, par de vagues liens

(1) «MILnE-EdWARDS.... bildet aus den «saugenden Crustaceen » eine eigne Unterklasse. Aber kann die " Gestalt der Mundorgane hier von so grosser systematischer Wichtigkeit sein, hier, wo durch die parasitische \$. Lebensweise eine bestimmte 2weckmässige Form verlangt und hervorgebracht wird? Wären die Thiere nun " auch verwandt oder nicht, so würden sich doch ihre Mundbildungen ähnlich sehen ". 
de parenté, les Notodelphyidés et les Lichomolgidés (66). La classification des Copépodes établie dans son livre fondamental $(\mathbf{5 0 , 5 1})$ et conservée dans ses traités élémentaires, porte la marque de cette tendance.

A la suite de Claus, un grand nombre de naturalistes se sont efforcés de faire connaitre complètement les Copépodes des différentes familles : les descriptions ont acquis, depuis lors, des qualités d'exactitude et de méthode remarquables, tant par l'emploi des moyens d'observation les plus perfectionnés que par l'effet de préoccupations théoriques toujours croissantes.

Les Copépodes libres marins ont occupé, entre autres: $\operatorname{BoECK}(\mathbf{1 5}, \mathbf{1} \boldsymbol{7})$, Brady (20), Norman (157), G. O. Sars $(\mathbf{1 8 1}, \mathbf{1 8 2})$, Lilljeborg $(\mathbf{1 3 6}, \mathbf{1 3}$ ) Mebius 150,151 , P. O. C. Aurivillius 8, S. A. Poppe 161-166, Haller (103), Giesbrecht $(\mathbf{8 8}, \mathbf{8 9}, \mathbf{9 1}, \mathbf{9 2 , 9 3})$, G. M. Thomson (202, z03), Sparre SChneider (192), CAR (36, 37), I.C. Thompson (194-201), CANu $(\mathbf{2 8}, \mathbf{2 9}, \mathbf{3 2}, \mathbf{3 3})$, Gilbert C. Bourne $(\mathbf{1 8}, \mathbf{1 9})$, etc.

Les formes semi-parasites furent l'objet des études de bon nombre de chercheurs, tels que: Buchiolz (24), Kerschner (117), Brady (20), Giesbrecht (90), C. W. S. Aurivillius (4-7), Della $\operatorname{Valle}(\mathbf{7 5}, \mathbf{7 6})$, Canu $(\mathbf{2 7}, \mathbf{3 0}, \mathbf{3 1}, \mathbf{3 4}, \mathbf{3 5})$, Schimkevitsch (188), Salensky (180), Hansen (105), Giard et Bonnier (87), Claparède $(\mathbf{3 8}, \mathbf{3 9})$, Kossmann $(\mathbf{1 1 8}, \mathbf{1 1 9})$, A. Valle (zor), Ridley (17ry), Wright (213), Raffaele è Monticelli (167), Rossoll 178), Giard (84-86), Grube (100), Mac Intosh (145), Kurz (126), Levinsen (134), Greffe (96), LIST (139), etc.

Quand leurs auteurs sont peu soucieux des données générales de la biologie moderne, ces mémoires diffèrent uniquement des anciens par les détails plus approfondis des observations micrographiques. Dans quelques-uns d'entre eux, on a cependant tenté d'èlucider les points les plus difficiles de la morphologie des Copépodes (ex.: HARTOG, 106) ou soigneusement recherché les véritables rapports zoologiques de ces êtres. C'est ainsi que GiesBreCHT (89, p. 97) a critiqué la méthode taxonomique courante et repoussé les subdivisions des Gnathostomes et des Parasites, établies sur les particularités d'organes trop visiblement influencés par les conditions secondaires de l'existence. Pour cet auteur, la classification fondamentale doit reposer sur des caractères plus fermes (I)

(1) «... so scheint es mir nothwendig, hiezu solche Merkmale zu wählen, welche an sich einer grösseren "Variabilität fähig, thatsachlich eine solche doch nicht erlangt haben. Schon darum halte ich es für unrichtig, " die Form der so unendlich variirten Mundtheile zum Haupt-Eintheilungs-Princip sämmtlicher Copepoden

" zu wählen; ganz abgesehen davon, dass sich die Eintheilung der Halb-und Ganz-Parasiten an die der Frei-

"lebenden wird anschliessen müssen " (loc. cit., p. 97). 
et l'essai tenté par lui en I88 I (1), recevra pleine confirmation en ce qui concerne les Copépodes pélagiques (communication in litt.).

Dès I886, j'avais réclamé (ar, p. 3 I2) pour l'étude des parasites des Synascidies, l'application de la méthode de la zoologie générale (2); c'est encore la mème tendance que j'ai suivie, autant que possible, dans la préparation de ce travail, d'après les principes enseignés par le Prof. Giard (3) qui en a fait lui-même l'application à quelques Copépodes (4).

\section{I.}

\section{NOMENCLATURE D'UN COPÉPODE TYPIQUE.}

\section{ETAT ADULTE.}

Le corps de tout Copépode se compose d'un tronc et d'appendices reliés à celui-ci par des articulations.

$\mathrm{I}^{\circ}$. - Le tronc est le plus souvent divisé par des articulations transversales en un certain nombre d'anneaux successifs de forme variable.

Dans la règle, à la plupart des anneaux primitifs correspond une paire d'appendices. Tout anneau primitif, avec une seule paire d'appendices, porte le nom de somite.

Quand plusieurs paires d'appendices s'attachent à la face ventrale d'un seul anneau, montrant ainsi que celui-ci est formé de plusieurs somites, cet anneau n'est qu'un segment, dénomination physiologique appliquée, par extension, au somite demeuré simple (5).

(1) Loc. cit. p. 163.

(2) "Celle qui, à l'étude comparative des formes adultes, allie la connaissance de l'embryon dans toutes 》 les modifications qu'il subit jusqu'à l'état parfait de développement, et qui, en outre, s'éclaire des nom" breux renseignements fournis par l'éthologie."

(3) Consulter spécialement Giard. Les faux principes biologiques, Reoue scicntifique, 1876; - Giard et BONNIER. Contribution à l'étude des Bopyriens, p. 222 et suiv.

(4) $85 ; 87$, p. 352.

(5) Il faut néanmoins distinguer, dans une description morphologique, entre les deux appellations. Le céphalon, composé au moins de cinq somites, ne forme qu’un seul segment. Le thorax, également composé de cinq somites, ne compte souvent que quatre segments. 
Dans un somite, il y a :

A. une face dorsale - tergum —, convexe et plus ou moins bombée ;

B. une face ventrale - scutum - convexe, mais plus aplatie que le tergum, et portant latéralement les appendices.

C. deux pleura, formées par la face dorsale repliée vers la face ventrale, en débordant mème souvent au-dessus de celle-ci sous forme de lames infléchies vers la ligne médiane.

Malgré la grande variabilité de la forme générale du corps, on peut toujours reconnaître aussi :

a. l'extrémité antérieure arrondie, entière, pourvue, plus ou moins latéralement, de deux paires d'appendices, parfois très saillants, extrèmement réduits dans d'autres cas ;

b. l'extrémité postérieure, sans appendices, mais fendue en son milieu et partagée ainsi en deux pièces plus ou moins divergentes constituant la furca.

Dans la moitié postérieure du corps, on trouve ventralement $(\delta)$ ou latéralement $\left(\sigma^{\top}\right.$ et $q$ ) ou mème latéro-dorsalement $(q)$, les deux orifices génitaux. La limite articulaire antérieure du segment portant ces orifices sépare les deux grandes régions du corps :

A. l'antérieure, constituant le céphalothorax; elle est plus élargie et porte des appendices saillants visibles sur la face ventrale ;

B. la postérieure, formant l'abdomen; elle est dépourvue d'appendices saillants et généralement plus effilée que la précédente. Cette région est souvent très réduite dans les parasites.

A. - Le céphalothorax se compose à son tour de deux régions : céphalon et thorax.

a. Le céphalon est constitué par les cinq premiers somites appendiculés. II est toujours compris dans le premier segment du corps, mais il n'y est pas toujours seul, par suite de sa soudure fréquente avec le premier somite thoracique (I).

(1) Soudure reconnaissable à la présence des premières pattes thoraciques sur le premier segment.

Suivant l'exemple donné par la plupart des zoologistes qui ont étudié les Copépodes, j'emploie pour indiquer le nombre des segments ou des articles d'une région déterminée l'épithète formée par le chiffre correspondant suivi de la terminaison convenable: ex. 2-, 3-, 4- segmenté, 2-, 3-, 4- articulé. Cette terminologie me semble préférable pour la brièveté et la netteté des descriptions. 
b. Le thorax comprend les cinq derniers somites appendiculés. Le quatrième et le cinquième se soudent ensemble dans quelques cas assez rares.

B. - L'abdomen est assez variable dans le nombre de ses segments. Il ne présente point d'appendices véritablement articulés, comparables à ceux du céphalothorax. Le dernier somite, ou furca, se compose de deux pièces séparées suivant l'axe longitudinal. L'avant-dernier somite, qui porte l'anus, présente parfois, dans une partie de sa longueur, la même modification, tandis que les autres restent entiers. Le premier somite abdominal porte, dans les deux sexes, les ouvertures génitales.

\section{$2^{\circ}$ - Les appendices sont de deux sortes :}

A. les appendices simples de la première paire, ou antennules, toujours reconnaissables à leur situation, leur structure ou leurs organes sensoriels.

B. les appendices biramés des paires suivantes, se rencontrant jusqu'à l'extrémité du céphalothorax.

Ils se présentent sous l'aspect des appendices primitifs des Crustacés, avec leur région basilaire, ou protopodite, biarticulée et portant deux rames. Le premier article du protopodite est le coxopodite; le second, le basipodite. La rame interne, toujours conservée, est l'endopodite; la rame externe, souvent réduite (rame accessoire de quelques auteurs) et parfois même atrophiée, est l'exopodite.

Les quatre premières paires d'appendices biramés appartiennent au segment céphalique. Elles portent, d'avant en arrière, les noms de :
a. Antennes;
b. Mandibules;
c. Premières maxilles;
d. Secondes maxilles.

Ces dernières ont, chez l'adulte, une apparence trompeuse : les deux rames de chaque seconde maxille sont séparées dès la base d'insertion de l'appendice; chacune d'elles semble ainsi former une appendice spécial. La rame externe, ou seconde maxille externe, un peu extérieure par rapport à l'autre, correspond au premier maxillipède ou maxillipède externe des auteurs; la seconde maxille int'sn', plus rapprochée de la ligne médiane, est le deuxième maxillipède ou maxillipède interne. 
La première maxille montre encore des pièces accessoires dont la plus importante est bien un lobe externe du basipodite : l'épipodite.

Les mandibules et maxilles, placées sur les côtés et en arrière de la bouche, sont souvent désignées sous les noms collectifs de pièces buccales, d'appendices buccaux, etc.

La bouche est protégée en avant par une saillie ou repli du tégument: la lèvre supérieure. Le bord postérieur de l'ouverture buccale ou lèvre inférieure, est protégé fréquemment par deux saillies symétriques disposées entre les pièces buccales, un peu en avant des premières maxilles, et qui sont appelées paragnathes. La dépression comprise entre la lèvre supérieure et les paragnathes, loge l'extrémité du coxopodite mandibulaire : c'est l'atrium buccal, au fond duquel est la véritable ouverture buccale. Les extrémités saillantes de la lèvre et des paragnathes peuvent se souder par coalescence et fermer imparfaitement l'atrium buccal, en formant une trompe ou un siphon qui prolonge le tube digestif.

Les dernières paires d'appendices biramés, au nombre de cinq au maximum, sont les appendices thoraciques. Ils présentent la forme typique, avec protopodite (coxo-, basi-podite), endopodite et exopodite. Leurs lobes, aplatis et pourvus de soies ou de crochets, sont disposés pour la locomotion, de sorte qu'on les désigne fréquemment sous les noms de pattes, pattes thoraciques, pattes natatoires. La variabilité de ces appendices est grande, en vertu d'adaptations diverses; la cinquième paire, servant à la réunion des sexes, diffère beaucoup dans le mâle et dans la femelle, ainsi qu'avec l'espèce. On constate aussi parfois l'absence d'une ou de plusieurs paires.

La position - très variable d'ailleurs, suivant les genres - des ouvertures génitales femelles, règle la nature des sacs ovigères :

a. Il n'y a qu'un sac ovigère ventral, quand ces orifices sont ventraux et très rapprochés.

b. Il y a deux sacs, quand les orifices, écartés, sont latéraux ou latérodorsaux.

$c$. Un genre n'a qu'un seul sac ovigère dorsal.

Certaines formes, de différentes familles, développent, par un reploiement de leur tégument, une cavité incubatrice dans laquelle se développent les embryons jusqu'à l'éclosion. 


\section{MÉTAMORPHOSE.}

Au möment de l'éclosion, l'embryon des Copépodes montre, avec un tronc non segmenté, les trois premières paires d'appendices: antennules, antennes et mandibules. Il est alors au stade nauplien; on l'appelle larve nauplius. On le reconnaît déjà comme Copépode aux deux soles furcales divergentes de l'extrémité postérieure.

Dès qu'apparaissent, à la surface yentrale du tronc non segmenté et au-delà des mandibules, deux replis symétriques saillants, porteurs d'une soie raide, l'embryon, ainsi pourvu des ébauches des premières maxilles, entre dans le stade métanauplien : c'est une larve métanauplius.

Plus tard, le tronc se segmente, la furca apparaît, le nombre des appendices augmente, leur nature définitive s'affirme et on y distingue : mandibules, maxilles, pattes natatoires. L'embryon entre alors dans les stades-copépodes.

Les premiers d'entre eux se distinguent comme stades-cyclopoides; divers caractères importants permettant de reconnaître morphologiquement les espèces adultes ne sont pas encore apparus : par exemple la nature définitive des ornements de la furca et des appendices (soies, épines, denticules).

Après les stades cyclopoïdes, le genre, l'espèce mème de ces embryons peuvent être discernés, et les stades-copépodes sont plus spécialement des stadescyclops, des stades-tachidius, des stades-temora, des stades-calanus, des stadesenterocola, etc. 


\section{PREMIERE PARTIE.}

\section{MORPHOLOGIE.}

Les Copépodes normaux, - que j'appelle ainsi, parce qu'ils sont indubitablement ceux qui, au cours du développement phylogénétique du groupe, ont subi le moins d'influences perturbatrices et adaptatives, - se reconnaissent à la division toujours nette de leur corps en segments et à la présence d'appendices rappelant la structure embryonnaire, dans l'organisation et dans le fonctionnement.

En réalité le Copépode actuel le plus normal sera le plus voisin des Crustacés ancêtres des Copépodes. Il présentera une organisation relativement peu spécialisée en ce qui concerne l'adaptation aux conditions d'existence. Dans l'étude comparative des particularités morphologiques, tous les éléments constitutifs des diverses parties du tronc et des appendices apparaitront à l'état moyen dans les types normaux; dans les formes spécialisées, on les rencontrera çà et là avec le développement que comporte un fonctionnement particulier.

\section{FORME GENÉRALE.}

Elle varie :

I. - Dans les Copépodes libres, avec le développement relatif des régions antérieure et postérieure :

Dans les formes constituant la famille des Harpacticidés (Pl. IV, V), les segments sont, comme on le sait, presqu'identiques dans toute la longueur; c'est évidemment là un caractère primitif commun à tous les membres de cette famille. 
Chez les Cyclopidés (PI. III, IV) et les Calanidés (PI. I, II), la région antérieure ou céphalothoracique présente, dans le sens latéral, un développement bien plus grand que la région postérieure ou abdominale; à ce titre, les Copépodes de forme cyclopoïde ou calanoïde se distinguent facilement des espèces harpacticoïdes.

Remarquons encore que, le plus souvent, les Calanidés se servent couramment du fonctionnement de l'abdomen comme moyen de locomotion.

Lorsque le Calanide plane, soutenu presque verticalement dans l'eau par l'action de ses longues antennules, qui servent de balancier, et des autres appendices céphaliques qui vibrent comme de petites nageoires, l'abdomen replié dorsalement dans la position habituelle est vigoureusement ramené vers la face ventrale par de puissants muscles extenseurs; dans ce mouvement, la furca et les soies qui la terminent agissent comme une palette natatoire et projettent le corps de l'animal dans diverses directions.

Dans les Cyclopidés, ce rôle de la furca est beaucoup moins répandu et moins fréquent, ce qui tend à séparer, avec les particularités des deux premiers appendices, la forme cyclopoïde de la forme calanoïde.

2. - Dans les Copépodes semi-parasites, d'après l'espèce et le sexe :

$\mathrm{I}^{0}$ Chez les Lichomolgidés (Pl. XXII à XXV) et les Ascomyzontidés (P1. XXVI à XXIX), la forme cyclopoïde est encore conservée dans les mâles et les femelles; elle se retrouve aussi, avec quelques déformations peu importantes, dans les femelles de Thersites (Ergasilidés) et dans les Hersiliidés.

$2^{0}{ }^{0}$ Dans les Ascidicolidés (P1. VI, VII, VIII, XX), le dimorphisme sexuel est très accentué, et les femelles, en vue de l'incubation des œufs (Notodelphys, Doropygus) ou d'un parasitisme plus complet (Enterocola, Aplostoma, Enteropsis), sont plus déformées que les mâles.

Quand la forme varie dans de telles proportions, peut-elle prétendre, suivant l'opinion (I) de Kossmann (1 18, page 2 du tiré à part), à une importance morphologique de premier ordre ?

Dans un groupe zoologique, les particularites les plus importantes ont une origine ancienne. Elles sont héritées d'ancêtres communs à tous les animaux du groupe, et ceux-ci les ont conservées presqu'intactes au cours du développement phylogénétique. Dans les formes actuellement vivantes, l’embryogénie seule peut

(1) "Meiner Meinung nach muss man, soweit die Unkenntniss der Entwickelung ein wissenschaftliches "System unmöglich macht, den ganzen Habitus, d. h. Körperform und Grad der Degeneration als Haupt-

" karachteristikum betrachten ". 
permettre de distinguer, par la date d'apparition plus ou moins précoce de ces particularités, l'importance relative qu'il est possible de leur attribuer, par application de la loi de répétition de la phylogénie par l'ontogénie.

Comment s'établit donc la forme générale du corps ? Et dans quelles conditions apparaissent les segments de la carapace chitineuse de l'adulte?

Dans le nauplius et le metanauplius (PI. VII, X, XII, XIII, XV, XIX, XXI, $\mathrm{XXII}, \mathrm{XXIII}$ ), le tronc est recouvert d'une mince chitine continue sans segmentation, et ses muscles fonctionnels sont les muscles extrinsèques des appendices : ce sont les " muscles primaires" d'URBanowicz (206), comme le montrent les dessins publiés par cet auteur (1).

Sous l'enveloppe métanauplienne, à mesure que se réalisent la disposition définitive du mésoderme secondaire et la métamérisation du corps en somites, apparaissent, sous forme d'étranglements dans la paroi chitineuse, les replis séparant les divers segments de la première larve cyclopoïde. Mais ce n'est pas encore dans les larves cyclopoïdes que la forme définitive est acquise.

L'aspect général de l'adulte, en relation avec la disposition des somites et dépendant de modifications adaptatives, synthétise en réalité une foule d'actions morphologiques et physiologiques d'importance très inégale, qu'on devra préciser pour utiliser convenablement ce caractère.

\section{CÉPHALOTHORAX.}

Dans tous les Copépodes, dès la première apparition des segments, les cinq somites céphaliques sont unis en une seule division : le céphalon. Cette disposition apparaissant avec une généralité parfaite et à une époque très précoce dù développement, nous en pouvons conclure qu'elle est acquise d'ancètres éloignés et qu'elle est d'une grande importance taxonomique.

L'union du premier somite thoracique avec le céphalon, très fréquente dans ies espèces libres et semi-parasites, peut s'observer ou non dans des formes pourtant très voisines:

Le genre Paracalanus la présente (Pl. I, fig. I et 3), tandis que le genre Calanus en est privé (Pl. I, fig. I 3 et 14 ).

(1) Le peu de renseignements que nous possédons sur le développement des Copépodes ne permet pas de juger actuellement de la valeur taxonomique des premières formes embryonnaires. Quelques caractères similaires laissent supposer qu'on pourrait ultérieurement, avec une connaissance plus approfondie de l'embryologie, différencier les larves des Calanidés, des Harpacticidés, etc. ; mais dans cette distinction, les détails d'organisation l'emportent encore sur la forme générale. 
La femelle adulte du genre Clausia (PI. II, fig. I) ne porte aucune trace de limite céphalothoracique, tandis que la jeune femelle (fig. 4), le mâle adulte (fig. 2) ou jeune (fig. 5 et 7 ) montrent, sur la face dorsale, une séparation incomplète entre le céphalon et le premier somite thoracique.

Dans les Harpactidés, le premier somite thoracique est soudé au céphalon (Pl. IV et V);

Chez Cyclops, le premier somite est soudé au céphalon (PI. III, fig. I ; Pl. IV, fig. I), mais il est libre chez Cyclopina et Oithona.

Dans tous les Hersiliidés, le premier somite thoracique est soudé au céphalon ; de mème chez les Ascomyzontidés.

Chez les diverses espèces de Lichomolgidés que j'ai pu étudier, ce somite est tantôt libre (PI. XXII, XXIII et XXV), et tantôt uni au céphalon (PI. XXIV).

Cette énumération suffira pour établir le peu d'importance d'un caractère, réparti aussi irrégulièrement dans des famillesparfaitement naturelles et basées sur une foule d'autres particularités de morphologie, d'embryologie et d'éthologie. Mais il convient de remarquer cependant la précocité de cette soudure, que l'on trouve déjà effectuée au premier stade cyclopoïde. L'inconstance du caractère nous empêche de le considérer comme acquis depuis longtemps par voie d'hérédité; dans la formation de cette soudure, il existe donc une condensation embryogénique, une hétérochronie remarquable du développement qui peut s'expliquer seulement par une adaptation.

Dans la soudure, d'ailleurs assez rare, des derniers somites thoraciques, il n'y a pas la même précocité :

J'ai montré en effet (p. 235 et suiv.) que, dans le genre Isias - où le premier somite thoracique est indépendant du céphalon, tandis que les $4^{\mathrm{e}}$ et $5^{\mathrm{e}}$ somites du thorax forment un seul segment avec deux paires d'appendices, - cette soudure est très tardive et s'accomplit seulement à la dernière mue.

Le genre Paracalanus fournit un autre exemple de cette soudure tardive, et j'ai pu suivre la disposition des $4^{\mathrm{e}}$ et $5^{\mathrm{e}}$ somites thoraciques :

I. dans la femelle (PI. I, fig, 3) et le mâle (PI. I, fig. I) adultes, où les deux somites sont soudés et où on ne reconnất la trace de leur limite que par une légère dépression marginale de la carapace ;

2. dans le jeune mâle avant la dernière mue, où la limite, moins nette que celle des autres segments, est encore très sensible à la face dorsale (Pl. I, fig. 2) et à la face ventrale (PI. 1, fig. 5). 
De mème, dans le genre Clausia (Pl. II), la jeune femelle (fig. 4) et le jeune mâle (fig. 7) présentent les $4^{p}$ et $5^{\mathrm{e}}$ somites thoraciques libres, tandis que chez les adultes mâle (fig. 2) et femelle (fig. I) ces somites s'unissent en un seul segment.

Dans les parasites, la soudure des $4^{\mathrm{e}}$ et $5^{\mathrm{e}}$ somites du thorax est plus répandue. Elle manque tout à fait chez les semi-parasites cyclopiformes des deux sexes; mais, dans les esfèces plus profondément modifiées, présentant un dimorphisme sexuel plus accentué, elle est générale dans le sexe femelle et ne peut être considérée que comme une modification liée à la sexualité.

Chez les Ascidicolidés mâles, dans les cas extrêmes, la forme du thorax est relativement peu différente de la normale : les somites sont libres et ne subissent point de modification profonde (Pl. XI, fig. 2 ; PI. VIII, fig. 13). Chez les femelles le $5^{\mathrm{e}}$ somite thoracique se soude plus ou moins complètement au $4^{\mathrm{e}}$, tandis que le $\mathrm{I}^{\mathrm{er}}$ reste indépendant du céphalon.

Dans les Notodelphys, Doropygus, Notopterophorus, Goniodelphys, Gunenotophorus, Bonnierilla, Doroixys et Buprorus, la face dorsale des téguments thoraciques subit une modification pour constituer une cavité incubatrice dans laquelle les œufs sont logés après la ponte, jusqu'à l'éclosion des larves naupliennes. Signalée par Allman (1), étudiée soigneusement par THORELL (204), KERSCHNER(117) et Giesbrecht (90), cette cavité est constituée par une duplicature du tégument qui forme voute, et dont le bord libre vient s'accoler lateralement et dorsalement sur l'abdomen, là où se trouve conservée l'ouverture de la chambre incubatrice par laquelle sortent les embryons (voir Pl. VI, fig. 3 et 4 ; PI. VIII, fig. 1 et I 2 ; Pl. X, fig. I ; Pl. XI, fig. I et I4) (1).

L'évolution de cette cavité prouve l'exactitude de cette interprétation; et je ne puis que confirmer les descriptions de KERSCHNER ( $l$. cit.) vérifiées et corrigées par GIESBRECHT (l. cit.).

La première ébauche du repli incubateur s'effectue très hâtivement dans les plus jeunes formes de développement des femelles, dès le stade antépénultième. Elle se présente extérieurement comme un repli saillant, détaché du segment d'origine et s'étendant peu à peu au-dessus des segments suivants (Pl. VI, fig. 3), jusqu'au delà du $5^{\mathrm{e}}$ somite thoracique. Au fur et à mesure du développement, les bords latéraux du repli progressent (PI. VI, fig. 4) et se soudent à la paroi latérale des segments thoraciques de sorte qu'il ne reste plus qu'un orifice dorsal,

(1) La compréhension de cette cavité incubatrice a été, pour différents auteurs : CLaus (45), BuchHolz (24), BRADY (20, part. I), le sujet d'erreurs relevées avec beaucoup de raison par Kerschner (117, p. 4 et 5 ) et sur lesquelles je ne reviendrai pas. 
assez réduit, mettant en communication la cavité et l'extérieur, au niveau du $\mathrm{I}^{\text {er }}$ segment abdominal (PI. XIV, fig. I4). Le somite thoracique donnant naissance au repli de la cavité incubatrice est tantôt le $4^{e}$ (Doropygus: P1. VI, fig. 3, 4 ; Notodelphys, Doroixys: Pl. XIV, fig. I), tantôt le $2^{\mathrm{e}}$ (Bonnierilla: Pl. X, fig. I ; Gunenotophorus).

Les deux ailes symétriques qui protègent les deux sacs ovigères externes dans Ascidicola et Enterocola, ainsi que les courtes saillies arrondies en situation analogue chez Aplostoma et Enteropsis, ne rappellent en rien la cavité incubatrice des autres ascidicoles; ces genres font partie d'une série de formes non moins intéressantes qui portent, après la ponte, des sacs ovigères externes.

Chez Botryllophilus femelle, le thorax est gonflé et boursoufflé par le développement des œufs (Pl. XVI, fig. I et 2); ses deux derniers somites sont soudés l'un à lautre. Près de lui sont d'autres genres fortement modifiés dans le sexe femelle par une dépendance plus étroite vis-à-vis de l'hôte nourricier, et qui tendent vers l'aspect vermiforme des animaux abrités dans une cavité restreinte. En effet, les segments thoraciques, dont le $4^{\mathrm{e}}$ est double, se montrent moins netlement distincts les uns des autres; ils présentent un développement égal et sont presque cylindriques (Enteropsis, Enterocola: Pl. XVIII, fig. I et 2, Pl. XX, fig. I; Aplostoma : Pl. XX, fig. 5). Dans cette dernière série d'Ascidicolidés, le dimorphisme sexuel atteint ses limites extrèmes; l'étude suivie de son apparition ontogénétique, que j'ai pu faire dans le genre Enterocola, ajoute un intérèt tout nouveau à la question déjà si intéressante dela sexualité des Copépodes parasites.

Quand on suit le développement de l'embryon chez Enterocola fulgens (PI. XIX), on voit succéder au stade nauplius (Pl. XIX, fig. I, 2) deux stades cyclopoïdes successifs, pendant lesquels l'embryon, pourvu d'appendices natatoires parfaitement organisés, est très mobile et nage avec la plus grande vivacité (PI. XIX, fig. 3, 4, 7; pl. XVIII, fig. 3).

Dans le sexe mâle, les appendices natatoires ainsi que la forme cyclopoïde normale se conservent au delà de cette période; le céphalothorax est composé chez l'adulte (PI. XIX, fig. 13) de six segments indépendants légèrement aplatis.

Dans le sexe femelle au contraire, l'embryon se présente (PI. XIX, fig. 8) sous l'aspect vermiforme après le deuxième stade cyclopoïde. La régression parasitaire du céphalothorax est ici parfaitement manifeste et précoce, et cette précocitê trouve encore son explication dans un phénomène des plus curieux de condensation embryogénique, sous l'influence des conditions éthologiques.

Les mèmes différences existent dans les autres Copépodes ascidicoles voisins 
de Enterocola. Et le genre Aplostoma en fournit encore un exemple (PI. XX, fig. 5: ?: fig. 13: 万!

C. W. S. Aurivillius a décrit (5) deux genres distincts d'une même famille nouvelle des Enteropsidés (loc. cit., p. 236):

I. le genre Enteropsis (p. 237-242), d'après des exemplaires femelles trouvés dans Molgula ampulloides;

2. le genre Haligryps (p. 242-246) d'après des exemplaires mâles trouvés dans la même Molgule.

Ces deux genres sont en réalité les deux sexes d'un mème animal, voisin d'Aplostoma et présentant les mèmes particularités de dimorphisme sexuel. La femelle d'Enteropsis sphinx (loc. cit., pl. VIII, fig. 17-19) est un Copépode vermiforme, à thorax cylindrique. Le màle (loc. cit., pl. IX, fig. I et 2) est un Copépode de forme normale, ressemblant beaucoup, par l'aspect extérieur, aux mâles des autres ascidicoles, Notodelphys ou Enterocola par exemple.

En résumé, dans la famille de Copépodes ascidicoles (Notodelphyidés + Enteropsidés), nous voyons des variations considérables dans la structure des somites thoraciques, variations qui ont poussé tous les auteurs à séparer, jusqu'à ce jour, dans des familles distinctes, les membres de ce groupe parfaitement naturel. Ici encore, la forme des mâles a conservé le caractère primitif et m'a permis de rectifier cette erreur.

Une autre famille bien naturelle de Copépodes semi-parasites, dont les membres vivent sur des Annélides polychètes, pourrait fournir encore des résultats de mème ordre, si son étude était conduite avec plus de méthode et basée sur un nombre de types suffisant. Notre connaissance actuelle de ces Copépodes suffit d'ailleurs amplement à démontrer l'importance très secondaire des replis incubateurs dans l'établissement des caractères taxonomiques, puisque chez ces êtres tellement distincts des. Notodelphyidés (auct.), il se rencontre des Gastrodelphys GrÆFFE (96) avec cavité incubatrice, à côté de types vermiformes comme Nereicola KefERstein (116), ou caligiformes tels que Eunicicola KURz (126) et Chelonidium HESSE (110, 1889), porteurs de sacs ovigères externes.

\section{ABDOMEN.}

L'abdomen des Copépodes libres et semi-parasites est, al plus haut degré, sujet à d'importantes modifirations. 
Cette région, qui doit, à l'encontre des interprétations de divers auteurs, s'étendre du I I $^{\mathrm{e}}$ somite à l'extrémité du corps, est réellement dépourvue d'appendices homologues aux pièces buccales et aux pattes thoraciques. Avec les particularités constitutionnelles des appendices, ceci constitue la meilleure caractéristique de l'ordre des Copépodes.

La séparation des régions, dans les divers groupes de Crustacés, a une importance considérable, comme Claus l'a reconnu depuis longtemps: nulle part elle n'est plus nette que chez les Copépodes, dans lesquels la région céphalothoracique a acquis phylogénétiquement un régime particulier facile à saisir dans l'étude embryologique, tandis que l'abdomen héritait de tout autres propriétés.

Les prétendues "pattes abdominales rudimentaires" des Copépodes ne méritent point ce nom au même titre que les appendices thoraciques. Dans un assez grand nombre de formes, sur-les bords des orifices génitaux, il existe un très léger repli lamellaire porteur de deux soies raides: c'est, chez le màle, la valvule génitale recouvrant l'orifice externe des sacs à spermatophores, et chez la femelle, le bord supérieur de l'ouverture génitale. L'insertion de ces deux soies peut s'effectuer à l'extrémité de petites saillies tuberculaires que Della Valle (75, fig. I 3, 14) prend pour l'endopodite et l'exopodite rudimentaires, déduction morphologique si hasardée qu'il semble inutile de la réfuter ici.

GIESBRECHT (89, p. 97) a fait remarquer que ces "pattes rudimentaires " de l'abdomen manquent chezila plupart des Copépodes nageant en haute mer, qu'il a nommés pour ce fait “Gymnopléodes ", tandis qu'elles existent chez les autres espèces, “ Podopléodes", vivant au fond de l'eau parmi les algues.

Ces replis - ou pléopodes, si l'on veut employer le terme utilisé par GIESBRECHT, - apparaissent d'une manière spéciale comparativement aux pattes thoraciques.

Dans l'évolution de l'embryon, lorsque se forment les replis appendiculaires qui, après la mue métanauplienne, fournissent les pattes natatoires de la première larve cyclopoïde, on constate très nettement (PI. VII, X, XII, XIII, XIX) les formations suivantes :

$I^{0}$ l'épaississement de la paroi ectodermique au point d'apparition de chaque appendice ;

$2^{0}$ l'apparition d'un repli à la partie externe de cet épaississement, pour la région extérieure de l'appendice ;

$3^{\circ}$ la séparation d'un groupe de cellules exodermiques à l'intérieur de chaque appendice, four constituer la paire de ganglions du somite correspondant.

De plus, dans les divers stades-copépodes, lorsqu'apparait un nouveau somite 
thoracique, les mèmes règles président à la formation des véritables pattes (P1. VII, $\mathrm{X}, \mathrm{XV}, \mathrm{XIX)}$ : l'épaississement exodermique et la prolifération de l'exoderme caractérisent les åppendices.

ZenKer (214, p. I 20) a d'ailleurs soutenu, depuis longtemps, que “dans la " détermination des appendices homologues, nous devons abandonner toute consi" dération sur l'aspect et la fonction, et les supposer reportés à leur condition "primitive de moignon pédiculiforme".

Rien de semblable à l'apparition d'un tel appendice, si rudimentaire qu'il soit, ne se rencontre sur l'abdomen des formes mâles ou femelles. L'existence des deux épines, parfois sétiformes, qui ornent le bord supérieur de l'ouverture génitale des " Podopléodes", ne me paraît nullement suffisante pour admettre l'existence de pattes abdominales que l'on se verrait forcé de considérer comme étant à peine ébauchées par suite d'un arrêt de développement ou devenues rudimentaires par régression. Les étonnantes considérations phylogénétiques qui se déduisent tout naturellement de semblables suppositions seront d'ailleurs examinées dans un autre chapitre.

Le somite non appendiculé abdominal a, comme division du corps, la mème valeur que le somite thoracique. La segmentation mésodermique, qui est la cause première de la formation des segments abdominaux, est, en effet, dans ses lignes essentielles, semblable à celle qui produit les divisions du thorax. Dans la larve cyclopoïde comme dans les derniers stades copépodes, on voit apparaittre les deux derniers somites thoraciques ou les somites abdominaux (Pl. IX, fig. 6, 7, 8), à l'avant du segment préfurcal ; la seule différence réside dans la formation ultérieure des appendices, quand il s'agit d'un somite thoracique.

L'abdomen est séparé du thorax dès le $2^{\mathrm{e}}$ stade cyclopoïde ; à cet âge il est intégralement compris dans le segment préfurcal ou anal. Dans celui-ci apparaissent successivement, d'avant en arrière ( $\mathbf{I}$ ), les segments abdominaux en nombre d'ailleurs très variable :

$I^{0}$ Suivant le sexe

Dans le màle, il existe presque toujours un segment de plus que chez la femelle. De cette disposition, la plupart des auteurs ont conclu que les deux premiers somites abdominaux, libres chez le mâle, étaient soudés en un seul segment chez la

(1) Exception faite pour le $4^{\mathrm{e}}$ sọmite abdominal de Doropygus, qui apparait en sens inverse dans l'avantdernier anneau du jeune mâle ou de la jeune femelle, à l'avant-dernier stade du développement (Pl. VI, fig. 3 et 4 ). 
femelle. Au cours du développement on ne voit pas s'opérer cette soudure, comme pour les $4^{\mathrm{e}}$ et $5^{\mathrm{e}}$ somites thoraciques de Clausia. Isias et Paracalanus. Mais il existe quelques formes rares dont les deux sexes présentent exactement le mème nombre de segments abdominaux : Doropygus (PI. VI, fig. 2 et 4; PI. VIII, fig. I2 et I 3), Modiolicola (P1. XXV, fig. I4 et I5). Pour cette raison, j'admettrai qu'une condensation embryogénique affecte, dans la plupart des femelles de Copépodes, la formation ontogénétique des segments abdominaux et supprime la séparation des deux premiers somites dans l'embryon, pour éviter leur soudure consécutive au cours de l'évolution.

Le nombre des somites abdominaux, à l'exclusion de la furca, est donc typiquement de 5 chez le mâle comme chez la femelle. La disposition primitive est conservée dans le sexe màle, mais il n'en est pas de même chez les femelles où il varie :

$2^{0}$ Suivant les espèces.

Chez les Calanidés, il existe 4 (Clausia, Pl. II, fig. I) ou plus généralement 3 segments (PI. II, fig. 17 et I9). Dans la plupart des autres Copépodes libres, l'abdomen de la femelle compte 4 segments, et il en est de mème pour les semiparasites cyclopiformes.

Chez Modiolicola et les Notodelphyidae (auct.), nous savons que l'abdomen de la femelle est 5 -segmenté ; mais, dans les autres Copépodes ascidicoles, le nombre des segments se réduit à mesure que le parasitisme devient plus intime : Enterocola femelle présente encore 4 segments abdominaux (PI. XVIII, fig. 2; Pl. XIX, fig. I2), tandis que Aplostoma femelle n'en possède que trois.

La furca n'est que le dernier somite abdominal, divisé suivant l'axe longitudinal pour former deux pièces distinctes. Cette séparation s'étend souvent sur le segment anal, qui est alors profondément incisé postérieurement et porte les pièces furcales à l'extrémité de deux lobes saillants parfois très écartés dans le sens latéral.

L'apparition des pièces furcales a lieu dès la mue métanauplienne (PI. VII, fig. 3 et 4 ; Pl. X, fig. 5; Pl. XII, fig. 4, 5, 6, etc.) et mème parfois, dans les embryogénies condensées, dès la mue nauplienne (Enterocola, Aplostoma: PI. XXI, fig. 6). Elles constituent un somite simple; les quelques trabécules qui traversent leur cavité dans le premier stade cyclopoïde ne suffisent point pour y distinguer, comme l'a fait Urbanowicz ( 206 , Pl. III, fig. 23, S XI et S XII), deux somites différents. Et, puisqu'aucun somite n'entre en régression (I) dans les Copépodes, rien ne force à admettre que les ancêtres de ces Crustacés possédaient

(1) Ce qui existerait chez Branchipus, d'après Claus: 62, p. 9. 
un abdomen plus richement segmenté que celui de leurs descendants actuels. D'ailleurs, la formation du somite furcal est très précoce. Elle débute chez l'embryon encore emprisonné dans l'enveloppe de l'œuf; et le nauplius, avant son éclosion, montre déjà l'ébauche des deux soies furcales naupliennes (Pl. XV, fig. I, $f$ ). Au premier stade cyclopoïde, lorsque la furca a acquis sa disposition caractéristique, les deux soies terminales du nauplius sont remplacées par d'autres soies, d'une manière constante pour beaucoup d'espèces (Pl. VII, fig 5; Pl. X, fig. 6 et 7 ; etc.) :

a. La soie furcale nauplienne (PI. VII, fig. 4; PI. X, fig. 5) est remplacée par une petite soie dorsale généralement plumeuse;

b. Il existe sur le bord externe une soie divergente, dite soie marginale;

c. Les soies terminales sont au nombre de quatre : la plus longue, avec sa base élargie, est la soie terminale interne; les trois externes sont plus petites. Au deuxième stade cyclopoïde, la disposition des 4 soies terminales a changé et pris l'aspect définitif de l'adulte (PI. VIII, fig. 6 et 7 ; PI. X, fig. 8; PI. XII, fig. 8); la plus longue d'entre elles n'est plus latérale interne : c'est la plus interne des deux médianes, les deux latérales étant alors les plus petites.

La section transversale de l'abdomen est le plus souvent circulaire ou elliptique, comme dans toutes les especes cyclopiformes, dans la majorité des Harpacticidés, dans les Calanidés. C'est le cas général dans le sexe mâle. Le tégument ne présente alors aucune des dispositions constantes dans le céphalothorax, et il n'existe plus de bouclier dorsal ni de replis pleuraux. La chose n'est point générale cependant, et chez certains Harpacticidés où les segments de l'abdomen s'aplatissent, on retrouve assez nettement les diverses parties des somites thoraciques; c'est le cas dans les Peltidies (Alteutha, etc.) et dans les femelles de Thalestris, Dactylopus, etc. pour les deux ou trois premiers somites de l'abdomen.

Le genre Amymone Claus mérite une mention spéciale pour l'aplatissement lateral du céphalothorax et de l'abdomen.

Le segment préfurcal porte toujours l'ouverture anale sur la face dorsale. Mais l'anus subit fréquemment, dans sa position, des variations assez grandes, liées au clivage longitudinal du segment qui le porte (PI. III, fig. I; PI. IV, fig. 20; Pl. V, fig. 24 ; etc.). C'est ainsi que dans plusieurs Harpacticidés (Ectinosoma, Dactylopus, etc.) où le segment anal est presque entièrement séparé en deux saillies latérales sur lesquelles s'attachent les pièces furcales, l'ouverture anale est 
reportée jusqu'à la limite des segments préfurcal et antépénultième. Dans les espèces de Doropygus du groupe de Doropygus pulex, le segment anal est aussi très profondément incisé. De même dans les Copépodes pélagiques du groupe des Calanidés, parmi lesquels les mâles de Calanus finmarchicus (Pl. I, fig. I 3 et 15) et les femelles de Acartia discaudata (Pl. III, fig. 17), montrent avec la division du segment préfurcal en deux lobes saillants, un élargissement notable de l'extrémité postérieure.

Chez les espèces rampantes ou fouisseuses, la furca perd, par adaptation, sa conformation habituelle: elle se trouve transformée en organe d'adhésion par sa forme spéciale et l'apparition de crochets terminaux (Doropygus: Pl. VI, fig. 2, 3, 4; Gunenotophorus: PI. XI, fig. I2; Botryllophilus: PI. XVI, fig. I, 2, 4; Aplostoma: Pl. XX, fig. Io).

Ailleurs elle est terminée par un nombre plus restreint de soies épineuses bien résistantes, destinées à faciliter la marche du Copépode dans la vase ( $N$ annopus: Pl. IV, fig. 6, Io). Nous retrouvons alors l'aspect primitif des soies furcales, en ce sens que la plus grosse des soies terminales est la plus interne. Claus avance (51, p. I 3) que dans des cas analogues, ce sont les deux soies terminales externes qui disparaissent; on se trouverait par conséquent en présence d'une structure normalement particulière au stade de développement qui suit immédiatement la mue du nauplius. Je ne puis admettre cette opinion; dans le cas spécial de $N$ annopus, les deux soies terminales interne et externe ont disparu et c'est la plus grande des soies furcales externes du $2^{e}$ stade cyclopoïde ( $\mathrm{I}$ ), la soie médiane interne par conséquent, qui forme la grande soie rigide terminale de l'adulte. Les divers passages graduels présentés par beaucoup d'autres espèces limicoles des genres Laophonte (PI. V, fig. 24), Cletodes, etc., ne laissent aucun doute sur ce point.

Les modifications de la furca résultant d'adaptations spéciales apparaissent donc assez tardivement, après le $2^{\mathrm{e}}$ stade cyclopoïde qui présente la condition primitive encore générale chez les types actuels les moins modifiés du groupe des Copépodes.

De ce qui est exposé dans les pages précédentes, il ressort bien que les influences sol's lesquelles l'aspect, c'est-à-dire la forme générale du corps s'établit dans le Copépode, n'exercent leur action qu'à une époque tardive du développement ontogénétique. Dans la suite, je montrerai par divers exemples que d'autres caractères d'organisation sont acquis d'une manière beaucoup plus précoce. En vertu de la loi de répétition de la phylogénie par l'ontogénie, je suis donc autorisé à n'accorder

(1) Et non du ter stade cyclopoïde, comme le dit Claus. 
à la forme générale qu'une importance morphologique et taxonomique secondaire, par rapport aux caractères d'apparition plus précoce.

\section{APPENDICES.}

Les appendices vraiment dignes de ce nom apparaissent dans tous les Crustacés, par paires, sur la face ventrale, sous forme de bourrelets exodermiques qui s'accroissent et se relient au tronc par une articulation qu'actionnent des muscles extrinsèques. Ainsi compris, et quelle que soit leur forme, ils sont plus ou moins rigoureusement homologues, comme le croyait déjà ZENKER.

Comme je l'ai montré plus haut, le segment génital des Copépodes ne porte point d'appendices véritables ; il diffère nettement en cela des segments correspondants de beaucoup d'autres Crustacés où les orifices génitaux sont placés à la base de pattes souvent modifiées sensiblement en vue de la copulation (1).

Dans les Copépodes, le nombre des appendices du céphalothorax est au maxinum de 10 paires.

\section{ANTENNULE.}

C'est toujours un appendice simple, dépourvu de la rame accessoire d'apparition tardive que l'on voit si souvent dans les Crustacés supérieurs. Ce caractère de simplicité de l'antennule, parfaitement reconnu par Claus (5\%, p. 24; 61, p. 6), la sépare nettement des autres appendices qui sont biramés. Toutefois, il ne me parait pas suffisant pour admettre l'existence, chez les Crustacès, de deux sortes d'appendices morphologiquement distincts, qui seraient : l'antennule simple d'une part; les autres appendices biramés d'autre part. Le mode d'origine des antennules et des deux autres paires de membres du nauplius étant exactement le mème dans l'embryon, toute discussion sur ce point est, à mon avis, écartée; et il me décide à considérer l'antennule comme un organe métamérique de même ordre que les appendices suivants.

Voulant demeurer strictement sur le te̊rrain des faits observés, je ne m'arrêterai pas à discuter, après Ray LANkester (Quart. Journ. Micr. Sc., vol. XXI, I88I) et Claus $(\mathbf{6 1}, \mathbf{6 2})$, la situation primordiale qu'occupait l'antennule chez

(1) C'est le cas des Branchipus, des Malacostracés, exception faite de beaucoup d'isopodes chélifères, de Priapion Fraissei GD et B., dont les malles portent un pénis médian de formation secondaire. 
les ancètres des Crustacés. Dans le nauplius des Copépodes (PI. XV, fig. I), l'antennule apparaît latéro-ventralement, un peu en avant de l'ouverture buccale et sur le côté du capuchon formant la lèvre supérieure. Topographiquement, elle est donc à ce stade, un appendice préstomial. Je me rallie, four cette raison, à l'opinion de Claus, tout en n'attachant, pour mon compte, qu'une importance assez faible à cette question. Rien ne prouve, en effet, que la position de la bouche n'ait subi, au cours du développement phylogénétique du groupe, un déplacement antéro-postérieur; d'autre part aucune observation ne vient appuyer la supposition d'après laquelle l'antennule aurait été primitivement métastomiale et ultérieurement reportée par un phénomène de céphalisation vers l'extrémité antérieure, comme il est démontré pour l'antenne des Branchiopodes ( $\mathbf{I}$ ).

Quoi qu'il en soit, l'antennule est soumise à de sérieuses modifications chez les Copépodes qui nous occupent :

Dans la plupart des formes pélagiques, elle joue dans la natation le rôle de balancier, et assure l'équilibre de l'animal placé verticalement au sein du liquide. C'est alors une longue suite d'articles (jusqu'à 24 et 25) garnis de soies, pouvant s'étendre transversalement (Pl. I, fig. I) ou se rabattre sur les côtés du corps (Pl. I, fig. I 3).

Ces longues antennules, si richement articulées, sont, sans nul doute, une acquisition adaptative en vue de l'existence pélagique. Le type normal réside certainement dans l'antennule moins complexe des Harpacticidés (Pl. V, fig. I, 2, 3) vivant à l'abri des algues sur le fond de la mer : c'est également la disposition que l'on rencontre dans l'embryon.

Diverses espèces parasites subissent, d'autre part, des réductions curieuses que j'ai pu suivre de très près dans un ascidicole fortement dégradé, Enterocola fulgens. Le premier embryon cyclopoïde possède des antennules sétifères 4-articulées (Pl. XIX, fig. 3, 4) du type normal. Au second stade cyclopoïde, elles sont plus allongées et comptent 5 articles garnis de longues soies et de bàtonnets sensoriels (PI. XIX, fig. 7). Lorsque cet embryon prépare la mue qui doit fournir le premier stade enterocolien de la femelle, l'antennule diminue considérablement en longueur et se trouve réduite à deux articles garnis de quelques courtes épines (Pl. XIX, fig. 8, 9, Io). Au contraire, chez le mâle (Pl. XIX, fig. I3) le nombre des articles augmente et le caractère d'appendice en balancier du Copépode semipélagique persiste. Enterocola fulgens fournit donc un exemple frappant de la faculté d'adaptation qui caractérise les antennules, et il permet de comprendre facilement le passage de l'antennule normale et pauci-articulée des jeunes, vers

(1) P. PelseneER. Observations on the Nervous System of Apus, Quar/. Jourm. Micr. Sci., vol. XXV, 1885. 
l'antennule réduite des parasites ou vers l'antennule hypertrophiée des grands nageurs.

Je n'insisterai pas longuement sur les détails de variation des antennules, tant chez les femelles que chez les mâles ; leur étude trouvera place dans la description systématique.

Toutefois, il convient de noter dès à présent le rôle sensoriel de ces appendices, dans les deux sexes. Chez les Copépodes, on connaît depuis les premières recherches de Claus (46), l'existence sur l'antennule d'organes spéciaux répandus dans tout le groupe des Crustacés. Ce sont des sortes de soies cylindriques, légèrement renflées, hyalines, revêtues d'une cuticule très mince, et qui se trouvent en rapport avec des prolongements du système nerveux. Ces filaments sensoriels, bâtonnets réfringents ou soies tactiles, sont disposés en divers points de l'appendice, plus développés et plus nombreux dans le sexe mâle. Leur disposition constante dans une espèce déterminée est spécialement intéressante au point de vue systématique, car on peut constater dans des formes très voisines des différences considérables. C'est ainsi que le mâle de Caligidium vagabundum Claus porte un bouquet de longs filaments réfringents vers la base de l'antennule, tandis que Cancerilla tubulata, seul Copépode connu qui soit vraiment parent de Caligidium, en est dépourvu dans les deux sexes (Pl. XXIX, fig. 5 et 6). D'autre part, parmi les Ascomyzontidés, le mâle de Dyspontius striatus est le seul, à notre connaissance, qui présente une semblable richesse de filaments sensoriels (Pl. XXVIII, fig. 2).

L'adaptation très fréquente de l'antennule des mâles, en vue de la réunion des sexes, est de règle dans plusieurs familles. L'une des antennules (1), ou les deux (2) sont modifiées très tardivement en appareils préhensiles qui retiennent la femelle pour la copulation. Plusieurs articles déjà formés suivant le mode commun aux deux sexes. peuvent se souder en un seul ; certains d'entre eux s'hypertrophient pour loger de puissants muscles qui replient l'extrémité distale sur la région basilaire jusqu’à former une pince géniculée.

\section{ANTENNE.}

Elle conserve sa forme primitive biramée avec la fonction natatoire, chez un grand nombre de Copépodes nageurs : c'est le cas général chez les Calanidés, où

(1) Chez beaucoup de Calanidés, à l'exception de Calanus (PI. I, fig. 13), Paracalanus (Pl. I, fig. 1), Clausia (Pl. II, fig. 2) et des gentes voisins.

(2) Chez les Cyclopidés (Pl. IV, fig. 2), Harpacticidés (Pl. V, fig. 4, 5), Nolodelphys, Agnathainer (PI. XVII, fig. 2 et 12), Ascomyzontidés, etc. 
l'exopodite, fort développé et richement articulé, joue un rôle considérable dans les mouvements de l'animal. La grande majorité des Harpacticidés présentent la mème organisation fondamentale, bien que l'exopodite soit souvent très réduit (Pl. IV, fig. 9) et rarement absent (P1. V, fig. 7) : il est rare (Longipedia, Sunaristes) qu'il présente dans cette famille, un développement suffisant pour être comparable à celui des Calanidés.

Dans les espèces où l'exofodite antennaire disparaît par suite d'une adaptation à la préhension ou à l'adhésion, la constitution primitive de l'appendice, toujours bien nette dans les larves naupliennes, se retrouve encore au premier stade cyclopoïde, comme j'ai pu le constater chez divers Copépodes et notamment dans Notodelphys (P1. XIII, fig. 6), Enterocola (P1. XIX, fig. 4), Aplostoma (P1. XXI, fig. 8, 9).

La soudure des divers articles du protopodite et de l'endopodite (qui transforme ces régions articulées en une pièce unique plus solide) donne lieu à diverses modifications de l'appendice. La plus considérable réduit l'antenne à 2 articles (PI. IV, fig. 9 ; Pl. XXI, fig. 8,9). La régression parasitaire agissant sur l'embryon femelle d'Enterocola, ne laisse plus de l'antenne 3-articulée du jeune cyclopoïde qu'un petit moignon sans articles (Pl. XIX, fig. 8, 9, Io).

\section{PIECES BUCCALES.}

La morphologie de la région buccale des Copépodes des diverses familles nous est surtout connue par les recherches méthodiques de Claus. Plus que toute autre, la région buccale semble être soumise à des adaptations profondes et nombreuses, et certains auteurs, trompés par les variations multiples qu'elle présente, ont refusé à l'organisation des organes masticateurs toute l'importance taxonomique et morphologique que d'autres lui prodiguent, en revanche, d'une manière trop exclusive.

La bouche, placée sur la face ventrale au niveau de la $3^{\mathrm{e}}$ paire d'appendices. est, en général, une fente transversale, au fond de l'atrium buccal plus ou moins déprimé en entonnoir.

Dans la plupart des mémoires, le nom de bouche est confusément donné à tort à l'atrium buccal considéré dans son ensemble sans souci de sa forme. Mais la bouche véritable n'est que le point précis où le pharynx débouche à la surface du tégument ventral.

Lorsque la cuticule, déprimée autour de l'ouverture buccale, y produit une concavité notable et sensible, la bouche n'est pas rigoureusement dans le plan de 
la surface ventrale: elle rentre, si l'on peut s'exprimer ainsi, dans le corps de l'animal (Pl. XXX, fig. 9).

Elle devient au contraire saillante, lorsque le tégument qui borde exactement l'orifice pharyngien, se prolonge au-dessus du plan ventral et qu'il entraine dans son mouvement le tube œsophagien (PI. XVII, fig. 4, 5 et 13).

La bouche ne subit elle-même aucune modification lorsque les formations tégumentaires accessoires qui l'abritent produisent, par un développement centrifuge particulier, un allongement excentrique de l'atrium buccal, comme c'est le cas dans la plupart des Copépodes dits siphonostomes.

Toutes ces formations, bien différentes dans leurs caractères, ont été jusqu'ici confondues sous les divers noms de trompe, suçoir ou siphon. Claus a seul tenté d'établir, pour certaines d'entre elles, une distinction qui ne me parait pas heureuse (63, p. I5) et j'y reviendrai plus loin.

$I^{0}$. - Dans les espèces libres et dans beaucoup de semi-parasites, - vivant de matières divisées par leurs organes masticateurs et amenées à l'orifice buccal par les organes de préhension ou par le courant d'eau que produisent les appendices, - l'atrium buccal est délimité :

A. antérieurement, par la lèvre supérieure :

B. postérieurement, par les paragnathes;

C. latéralement, par les mandibules.

La lèvre supérieure se présente alors comme une saillie convexe en avant de la bouche; son bord postérieur vient affleurer au bord antérieur de l'ouverture buccale (Pl. V, fig. 8; Pl. XXX, fig. 9). Cette saillie forme donc un bourrelet qui recouvre en partie l'atrium buccal et qui peut présenter divers ornements, soies et épines, intéressants dans leurs détails (Pl. XXX, fig. 9; PI. III, fig. 16). Ainsi comprise, la levre supérieure apparait très hâtivement au cours du développement, en mème temps que s'accroissent, dans l'œuf, les appendices naupliens (PI. XV, fig. I). Chez le nauplius, elle présente déjà sensiblement la même disposition que dans l'adulte (Pl. VII, fig. 3; PI. XII, fig. 3, 4, 5, 6, etc.), avec l'aspect d'un capuchon recouvrant jusqu'à la bouche l'œsophage recourbé.

En arrière de l'atrium buccal, se trouvent à droite et à gauche de la ligne médiane, au niveau du $4^{\mathrm{e}}$ somite ou de la $\mathrm{I}^{\mathrm{re}}$ maxille, deux pieces saillantes, symétriques, auxquelles Claus a donné le nom de paragnathes. Cet auteur les a depuis longtemps signalées chez les Calanidés (51, p. 25) et chez Clausidium 
(55, p. 330), en les consıdérant comme la lèvre inférieure. Il a récemment déduit la valeur morphologique des paragnathes de Malacostracés, d'une intéressante disposition découverte chez Euphausia. Les paragnathes de ce Schizopode sont

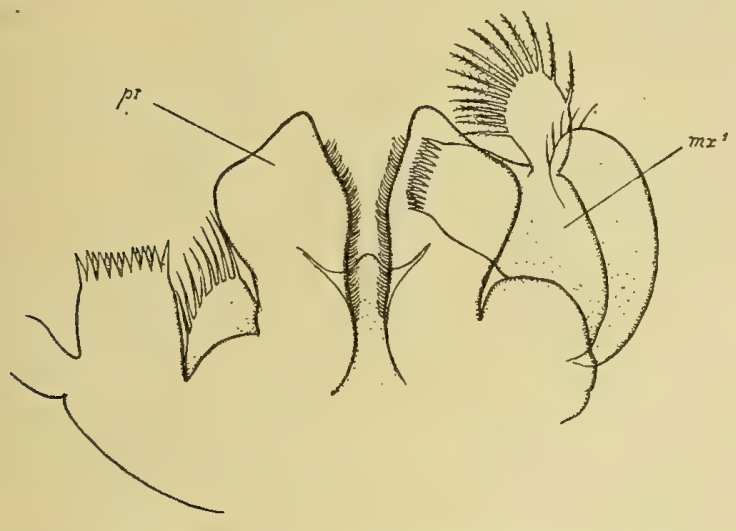

Fig. 2. - Premières maxilles et paragnathes d'Euphausia, d'après Claus: loc. cit., Taf. 1, Fig. 4. $m x$, première maxille; pr, paragnathe. particulièrement développés; ce sont de grands lobes ciliés sur le bord interne et réunis à la base par un septum transversal en forme de lèvre. Chacun des deux lobes est en relation continue avec la première maxille du. còté correspondant et paraît en être un lobe inférieur, saillant vers la ligne médiane.

Lesparagnathes des autres Malacostracés ne seraient d'après Claus que les lobes basilaires - devenus auto-

nomes - de la $\mathrm{I}^{\mathrm{re}}$ maxille. Claus doute d'ailleurs (loc. cit., p. I4) que les paragnathes des Entomostracés (Ostracodes, Halocypris et Copépodes) soient véritablement homologues de ceux des Malacostracés. Quoi qu'il en soit, on sait qu'ils ne sont nullement équivalents à une paire d'appendices, puisque dans aucun groupe ils ne reçoivent les nerfs d'une paire ganglionnaire spéciale.

Dans les Copépodes, les paragnathes sont bien développés, non seulement chez les Calanidés, mais encore chez les Harpacticidés (PI. V, fig. 8) et principalement dans la famille des Hersiliidés, où j'ai pu les étudier dans tous leurs rapports (31).

Chez Giardella callianassa $\mathrm{CANU}$, on remarque à la face ventrale ( $\mathrm{Pl}$. XXX, fig. 9) une série d'épaississements chitineux limitant plus ou moins complètement les cadres articulaires dans lesquels s'insèrent les appendices buccaux. Le cadre antérieur, placé sur les côtés de l'atrium buccal et de la lèvre supérieure, loge, à droite et à gauche, la mandibule et la première maxille. La mandibule est appuyée sur la branche du cadre d'insertion qui s'avance vers l'extrémité antérieure latéralement à la lèvre supérieure, et la maxille sur la branche latérale divergente. La crète intermédiaire réunissant ces deux branches, sépare presque entièrement du cadre mandibulo-maxillaire une autre cavité dans laquelle s'insère le paranathe. Ce dernier, dans Giardella callianassa, est une pièce massive, fortement 
arquée, qui forme voùte au-dessus de la mandibule, en dirigeant son extrémité distale vers la lèvre supérieure. Le bout du paragnathe est dentelé, tandis que sa face postérieure est recouverte de longs poils raides. L'articulation du paragnathe s'effectue sur la crète chitineuse intermédiaire dont je viens de parler, ainsi que sur une petite saillie chitineuse qu'envoie vers l'intérieur le second cadre articulaire, celui de la $2^{\mathrm{e}}$ maxille externe.

Entre les deux paragnathes se trouve la lèvre inférieure, abritée sous leur dôme et suivie d'une saillie complexe, denticulée dans la ligne médiane, garnie de poils sur les bords. Cette saillie à base élargie réunit entre elles les crêtes articulaires postérieures des paragnathes. Mais il n'existe nullement ici l'union indiquée par Claus entre les paragnathes et les $I^{\text {re's }}$ maxilles, sans qu'il ait pourtant décrit la disposition exacte de leurs crètes articulaires. Au contraire, le paragnathe est très éloigné de cet appendice et il se présente déjà, dans les stades les plus jeunes (PI. XXX, fig. IO), comme une pièce parfaitement indépendante. Les paragnathes paraissent donc des saillies secondaires du tégument ventral, développées à l'intérieur de la double rangée des appendices buccaux sans aucune intervention de ces derniers.

A cet emploi de la disposition des crêtes chitineuses articulaires, on pourrait cependant objecter le cas des secondes maxilles interne et externe, qui constituent une seule paire d'appendices et s'appuient de chaque côté sur deux crêtes articulaires distinctes. Je remarquerai d'abord qu'il n'est pas demontré que les deux branches postérieures des cadres d'insertion ne puissent dériver de la ramification secondaire d'une branche unique; et je comparerai la disposition des crêtes articulaires dans les deux cas extrèmes:

a. d'un développement considérable du paragnathe chez Giardella;

b. de l'absence complète de paragnathes dans les Lichomolgidés, particulièrement chez Modiolicola insignis (P1. XXV, fig. 18).

Dans les deux cas, la disposition des crètes chitineuses est la méme. Je crois être en droit d'en conclure que, chez Giardella, il n'y a pas de crète articulaire surajoutée pour séparer, comme paragnathe, un lobe basilaire de la maxille,

En somme, dans Giardella comme dans tous les Hersiliidés semi-parasites, la région buccale est constituée par un vaste atrium dans lequel entrent les extrémités des mandibules qui viennent faire saillie entre la lèvre supérieure et les paragnathes. Ces derniers limitent, en arrière de la lèvre supérieure, un champ presque triangulaire que vient fermer postérieurement la saillie qui fait suite à la lèvre inférieure. Par le mouvement des paragnathes, cette cavité prébuccale peut 
s'appliquer assez étroitement à la surface de l'hôte qui abrite le Copépode (Clausidium); elle recueille probablement une partie de la nourriture. Les mandibules mancuvrent à l'intérieur de la cavité sus-mentionnée, et il n'existe guère de différence entre la disposition que je viens de décrire et le " disque-suçoir "signalé par Claus (55, p. 342) chez Nicothoe astaci Aud. ET M. Edw.

Dans ce dernier, la cohésion des éléments constitutifs de l'atrium buccal est plus grande. Nous y retrouvons encore, dans une cavité assez restreinte limitée par la lèvre supérieure et les paragnathes $(=$ la lèvre inférieure avec deux lobes réniformes de Claus), deux mandibules en stylet dépourvues de palpes et presque entièrement abritées dans un suçoir à la formation duquel la véritable lèvre inférieure est totalement étrangère. Nicothoe astaci est le dernier terme de la série des Hersiliidés dégradés de plus en plus par l'effet du parasitisme croissant : c'est le type "siphonostome " de cette famille, et son disque-suçoir se range dans les trompes-siphons de Claus (63, p. 15 ).

$2^{0}$ Pour toute une série de Copépodes siphonostomes - que l'on a jusqu'ici rangés pêle-mèle dans les classifications zoologiques, en vertu de la caractéristique fournie par leur trompe-siphon, c'est-à-dire pour leur aptitude au parasitisme le plus varié - , on reste incapable de fixer nettement les relations des types actuels avec les formes gnathostomes qui leur ont donné naissance. Sans parler des nombreuses espèces parasites des Poissons que j'ai dû étudier comparativement à ce point de vue, je m'occuperai seulement des formes semi-parasites, vivant sur les Invertébrés.

A. - Dans les curieux Copépodes qui appartiennent aux deux genres Cancerilla Dalyell et Caligidium Claus, les mandibules sont encore abritées dans une sorte de siphon très court, constitué - autant qu'il est permis d'en juger sur l'étude exclusive des adultes - par la lèvre supérieure et le tégument ventral consécutif a la véritable bouche.

L'observation des mandibules dans leur position naturelle est rendue très difficile chez Cancerilla tubulata. L'aplatissement de la région buccale, sous une pression trop considérable du couvre-objet, suffit pour provoquer artificiellement la sortie de la mandibule hors de l'atrium buccal; le gonflement des organes internes sous l'action dissolvante de la potasse caustique bouillante produit fréquemment le mème effet.

J'ai reproduit (PI. XXIX, fig. 6) l'aspect des pièces buccales de Canierilla tubulata mâle, éclaircies par la potasse caustique: on peut constater ainsi la fosition des mandibules au-dessous de la paroi postérieure du siphon. Ce dernier 
forme, à la face ventrale du Copépode, une saillie tronc-conique qui loge les mandibules ordinairement obliques et dirigées vers le sommet, tandis que dans mon dessin elles retombent vers le fond de l'atrium buccal par suite de la disparition des muscles qui les maintiennent.

Les mandibules (PI. XXIX, fig. Io) sont de courtes pièces légèrement arquées, terminées par un pinceau de poils rigides; elles sont réduites au coxopodite et manquent du fouet extérieur assez répandu chez les Ascomyzontidés.

Claus a décrit une disposition tout à fait semblable dans le mâle de Caligidium vagabundum, et il émet, à ce sujet, les considérations suivantes: “ D'après les " remarquables particularités des appendices

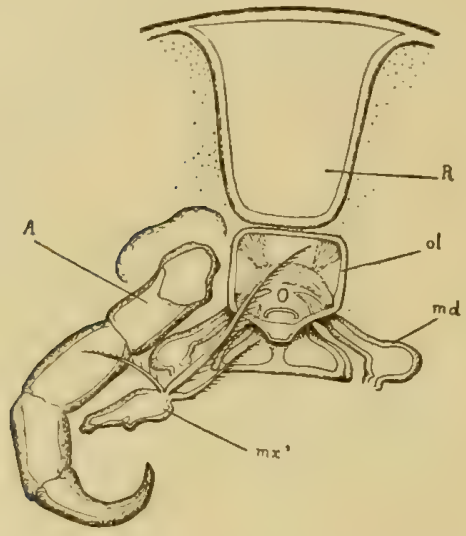

Fig. 3. - Région buccale de Caligidium vagabundum d'après Claus: loc, cit. Taf. 1, Fig. 3. $A$, antenne; ol, lèvre supérieure; $m d$, mandibule; $m x x^{1}$, première maxille; $R$, rostre frontal.
" et en particulier des pièces buccales, on " pourrait réclamer pour notre genre (Cali" gidium! la création d'une famille spéciale. "Pourtant, avant de se prononcer sur ce " point, il est nécessaire d'attendre la décou"verte de la femelle et des formes voisines" (66, p. 39).

La structure du siphon et de la mandibule ne paraît pas suffisamment différente, dans sa morphologie, des parties homologues des autres siphonostomes pour faire triompher cette opinion prématurée, qui trouve cependant un sérieux appui dans la disposition des appendices thoraciques chez les deux sexes.

Le siphon de Cancerilla et de-Caligidium reproduit sensiblement l'aspect que l'on connait dans certains Copépodes semi-parasites (Nereicola, Gastrodelphys), dont il ne saurait être rapproché dans aucune classification naturelle.

B. - Dans les Ascomyzontidés (PI. XXVI à XXIX), le siphon est de forme conique, il se relie à la face ventrale par sa base élargie. L'extrémité distale est percée d'un orifice que l'on a souvent considéré comme l'ouverture buccale, alors qu'il représente seulement l'entrée de l'atrium buccal. Je prendrai comme type le genre Asterocheres BOECK dans lequel le siphon, relativement court, ne dépasse pas la base des secondes maxilles.

Dans Asterocheres lilljeborgi BoEck, lorsque le siphon est rabattu contre la face ventrale, dans la position naturelle, et qu'il dirige son extrémité libre vers le 
thorax (PI. XXVII, fig. 3), la lèvre supérieure, vue de face, recouvre la portion distale du stylet mandibulaire. Ce dernier pénètre dans le cône siphonien par une échancrure située entre les crêtes articulaires de sa paroi supérieure et de sa paroi inférieure; il se prolonge, en se courbant légèrement vers l'extérieur, jusqu'au bout du tube siphonien, et son extrémité dentée se trouve à l'entrée de l'atrium buccal (PI. XXVIII, fig. 4). Les stylets mandibulaires d' $A$. lilljeborgi peuvent donc jouer le rôle d'un appareil térébrant, de mème que les mandibules des Hersiliidés et en particulier de Nicothoe astaci.

Quelle signification morphologique faut-il attribuer à la paroi postérieure du siphon?

Elle se rattache à la face ventrale, par une série de crètes articulaires placées au niveau de la première maxille et dans une situation absolument identique à celle des paragnathes chez les Copépodes gnathostomes. Dans la région -médiane du siphon, la paroi postérieure se soude intimement à la paroi antérieure, mais il persiste, dans les épaississements chitineux, des indications très nettes d'une séparation primitive que l'on retrouve d'ailleurs vers l'extrémité distale. Celle-ci se compose, en effet, de deux lobes, supérieur et inférieur, entre lesquels se logent les stylets mandibulaires.

La disposition indiquée ci-dessus pour Asterocheres lilljeborgi se retrouve très nettement encore chez $A$. renaudi Canu (PI. XXVI, fig. I4) et Dermatomyzon elegans Claus.

L'absence totale d'une dépendance du pharynx à l'intérieur du siphon nous oblige à considérer la face postérieure de la trompe des Ascomyzontidés comme une formation tégumentaire externe comparable aux paragnathes, dans leurs ròle et position.

La bouche véritable des Ascomyzontidés est placée dans la portion élargie du siphon, au sommet d'une saillie indépendante : c'est un orifice plus ou moins arrondi et étroit (PI. XXIX, fig. 3), vers lequel des dépressions dans la paroi de la trompe conduisent les matières nutritives aspirées par les mouvements du tube digestif.

La même structure fondamentale se retrouve dans toutes les espèces d'Ascomyzontidés que j'ai pu étudier; mais il existe de sérieuses différences dans le développement des diverses parties. Ainsi chez Artotrogus normani BRADY ET RoB. et Dyspontius striatus THORELL, le siphon, très développé, s'ètend jusqu'au milieu du thorax; les lobes terminaux sont également très allongés et laissent passer l'extrémité denticulée des mandibules. Chez _. Icontiophorus siululus BRADY ET ROB., le siphon présente une forme plus spéciảlisée : il se termine par un long 
tube capillaire qui s'étend jusqu'à l'extrémité postérieure du corps, entre les piècęs furcales (Pl. XXIX, fig. I). Les stylets mandibulaires sont moins allongés et terminés, à la base du tube capillaire, par un long poil grêle et effilé, incapable de jouer aucun rôle dans la recherche de la nourriture.

En réalité, le siphon des Ascomyzontidés n'est point une simple lèvre supérieure enroulée en tube, mais bien une formation comparable en tous points au siphon des Caliges et des Lernæopodes. On ne peut le considerer, ainsi que l'a fait autrefois Claus (63, p. 15), comme un "tube épipharyngien" dans lequel le coxopodite mandibulaire n’aurait pu trouver place.

$3^{0}$ - La famille des Ascidicolidés est certainement la plus intéressante par les modifications qu'y subissent les diverses pièces buccales. L'étude approfondie que j'ai pu faire de la structure et du développement de ces organes, dans les formes les plus diverses de cette famille, m'a fourni plusieurs résultats entièrement. nouveaux et des plus importants four la morphologie des Crustacés.

A. Dans les types commensaux, qui constituent le groupe des Notodelphyidés de THORELl, l'organisation de la région buccale est assez semblable à celle que j'ai décrite plus haut chez les Copépodes gnathostomes.

Chez Notodelphys allmani THorell, la mandibule - dont le coxopodite mandibulaire, fortement denté à son extrémité interne, vient s'abriter au-dessous de la lèvre supérieure, - est suivie de la $\mathrm{I}^{\mathrm{re}}$ maxille, très apte à la mastication par le développement des épines internes de son protopodite. Auprès de la $\mathrm{I}^{\mathrm{re}}$ maxille et vers l'intérieur, se trouve une petite saillie recourbée du tégument ventral, séparée des appendices par des crètes articulaires et qui se montre en tous points semblable aux paragnathes des gnathostomes; elle présente mème un développement plus grand que chez les Calanidés. Mais il existe, sur la face ventrale, entre les paragnathes de Notodelphys, un écartement plus considérable que chez les Hersiliidés, et l'atrium buccal est moins fermé chez Notodelphys que dans ces derniers.

La même disposition des organes buccaux se retrouve chez Doropygus (PI. VI) et les autres Notodelphyidés, notamment chez Doroixys ou les paragnathes bien développés sont plus grands que la seconde maxille interne (Pl. XIV, fig. 7).

B. - Dans le genre Agnathaner Canu les propriétés masticatrices de la mandibule ont tout à fait disparu par suite de la réduction considérable qui atteint le coxopodite de cet appendice.

Dans le mâle d'Agnathaner typicus Canu la bouche se trouve à l'extrémité postérieure d'une sorte de bulbe saillant (Pl. XVIII, fig. 4 et 5), à l'intérieur 
duquel on reconnait l'œsophage avec sa paroi chitineuse plissée. Ce dernier, assez étroit auprès de la bouche, se renfle aussitôt en une sorte de vésicule au delà de laquelle il se rétrécit de nouveau pour traverser le collier nerveux œsophagien et aboutir dans l'estomac. Les muscles élévateurs et abaisseurs de l'œsophage, qui s'insèrent en divers points sur la paroi extérieure, augmentent par leur contraction la cavité de cette vésicule et font jouer à cet œesophage renflé le rôle d'une pompe aspirante, ainsi que LiST l'a déjà décrit chez Gastrodelphys clausi GrefFEE (96, p. 97).

La bouche est un orifice transversal de forme plus ou moins elliptique, qui fait suite à la paroi supérieure convexe du bulbe buccal; cette paroi supérieure est véritablement homologue de la lèvre supérieure des autres Copépodes. La paroi inférieure du bulbe est à peu près verticale ; par sa position au niveau des mandibules et son contact immédiat avec l'orifice buccal, elle correspond à la lèvre inférieure. Agnathaner est donc le premier Copépode dans lequel soit signalée une trompe buccale fonctionnant comme un siphon aspirateur, véritablement formée par les deux lèvres sans intervention des paragnathes ou de replis tégumentaires analogues (I).

Le tégument ventral ne présente, d'ailleurs, dans Agnathaner typicus, aucun indice de paragnathes à la place habituellement occupée par ces organes dans le premier somite maxillaire.

La mandibule montre, dans ce Copépode, les caractères essentiels d'une mandibule de Notodelphys, par le basipodite, l'exopodite et l'endopodite richement garnis de soies plumeuses (PI. XVII, fig. 6). Le coxopodite se compose d'une région articulaire élargie sur laquelle s'insère la portion terminale, et d'une lame masticatrice réduite à un moignon effilé vers l'extrémité et confusement dentelé ; il est disposé sur le còté du bulbe buccal (PI. XVII, fig. 4 et 5 ) et n’atteint pas à l'extrémité. Il ne peut donc être d'aucune utilité dans la préparation de la nourriture.

Tous les appendịces buccaux montrent cette régression de leurs appareils masticateurs qui est la conséquence logique du développement de l'appareil aspirateur. C'est ainsi que la première nıaxille (PI. XVII, fig. 7) diffère considérablement de celle de Notodelphys, Doropygus, etc. par la réduction des épines du protopodite, et que les deuxièmes maxilles sont réduites à de simples moignons épineux ou sétifères (Pl. XVII, fig. 8 et 9).

On retrouve exactement la mème disposition des organes buccaux dans une

(1) La formation de la trompe épipharyngienne signalée par CLAUS, chez la femelle de Lernatiscus nemaloxys CLAUS, n'est pas encore clairement expliquée et ne semble pas se rattacher rigoureusement à l'appareil ici décrit. 
seconde espèce du mẻme genre: Agnathaner minutus Canu (P1. XVII, fig. I3). Quant à l'organisation de la bouche chez la femelle des Agnathaner, elle reste inconnue.

C. - Dans le genre Enterocola VAN BEN., la région buccale est plus difficile à comprendre, car elle diffère notablement dans les deux sexes. Je n'ai pu arriver à la connaissance complète de l'organisation de ces Copépodes qu'avec l'aide de l'étude embryologique.

a. Au premier stade cyclopoïde, l'embryon de Enterocola fulgens VAN BEN. possède les trois paires habituelles d'appendices buccaux (PI. XIX, fig. 4 et 5): les mandibules, les I $^{\text {res }}$ et $2^{\text {es }}$ maxilles.

La bouche est assez semblable à ce que nous connaissons chez Agnathaner: c'est un arifice transversal placé au sommet d'un bulbe buccal composé de la lèvre supérieure convexe et d'une lèvre inférieure légèrement saillante.

La mandibule (PI. XIX, fig. 5, md) est un appendice biramé, très réduit, formé d'un protopodite, d'un exopodite et d'un endopodite nettement reconnaissables mais constitués par une cuticule mince et plissée. Sous cette forme, dépourvue de soies et d'épines, la mandibule est évidemment incapable de remplir son rôle dans la préparation de la nourriture. L'aspect même de son tégument, comparable à celui de l'exopodite antennaire en voie de dégradation $(\mathrm{Pl}$. XIX, fig. $4, A)$, indique bien que l'on a affaire à un appendice en régression, ce que démontrent d'ailleurs sa taille infime comparée à celle du $3^{\mathrm{e}}$ appendice nauplien (PI. XIX, fig. I, $m d$ ) et le sort quelle subit dans la suite du développement.

La I $^{\text {re }}$ maxille (PI. XIX, fig. 5, $m x^{4}$ ) est une pièce bilobée dont la lame interne est déjà très solidement construite et dont la lame externe, palpiforme, porte deux courtes épines.

La $2^{\mathrm{e}}$ maxille est une simple pièce non biramée, composée de deux articles : l'un basilaire, plus large; l'autre terminal, formant griffe.

En résumé, dans cet embryon, les deux paires postérieures sont seules destinées à la mastication.

b. Au second stade cyclopoïde (PI. XIX, fig. 7), la même disposition s'accentue davantage. La mandibule a subi le sort de l'exopodite antennaire et n'est plus qu'un petit moignon à peine visible ; elle a presque complètement disparu.

Les maxilles présentent à peu près la même forme que dans le stade précédent et le bulbe buccal persiste également dans son organisation primitive. 
c. Si nous passons directement du second stade cyclopoïde au mâle adulte, semi-parasite et bon nageur, nous retrouvons les mèmes pièces essentielles, avec quelques faibles modifications (PI. XVIII, fig. 4 et 5). La bouche $(b)$ est encore placée au sommet d'un petit bulbe buccal. Sur les côtés de la face ventrale, et en arrière de la bouche, se retrouvent deux paires d'appendices : les premières $\left(m x^{1}\right)$ et les secondes maxilles $\left(m x^{2}\right)$. Mais aucun de ces organes n'est devenu véritablement masticateur; ils se rapprochent ainsi d'Agnathaner.

La première maxille $\left(m x^{1}\right)$ se compose d'un article basilaire, à peu près cylindrique et très court, sans prolongement en forme de lobe vers l'intérieur; d'un article terminal élargi, palpiforme, avec six grosses soies richement barbelées. La deuxième maxilie $\left(m x^{2}\right)$ est également composée de deux articles dont le deuxième est très court et terminé par une seule soie barbelée.

Si nous comparons cette disposition à celle qui a été décrite précédemment pour le genre Agnathaner, nous trouvons que les différences résident surtout dans la disparition "d'une partie des organes masticateurs, incapables de remplir leur rôle habituel dans ce dernier. Le mâle d'Enterocola fulgens représente donc un degré plus élevé de la spécialisation qui atteint les membres de la famille des Ascidicolidés et qui les a conduits précédemment des Notodelphyidés vers Agnathaner.

d. Chez la femelle, après le second stade cyclopoïde, l'embryon commence la vie parasitaire et entre Jans le premier stade entérocolien. Les deux paires de maxilles sont alors organisées pour la mastication; leurs griffes sont plus solides et plus aiguës, mais on reconnaît encore dans chacun de ces appendices les deux articles basilaire et terminal dont il a été question plus haut.

e. Dans la femelle adulte, les appendices se conservent avec leur organisation fondamentale, mais ils sont encore mieux appropriés à la fonction masticatrice. J'insisterai sur quelques détails mal compris ou mal interprétés par d'autres auteurs, et je prendrai comme type Enterocola betencourti Canu, espèce qui ne diffère nullement de sa congénère au point de vue des pièces buccales.

Le bulbe buccal est ici moins saillant que dans l'embryon. La paroi chitineuse de la lèvre supérieure est fortement épaissie; la bouche (PI. XVIII, fig. ${ }_{13}, b$ ) est cachée au-dessous de son bord postérieur (Pl. XVIII, fig. 14, ls). L'œsophage chitineux et élargi aboutit à la bouche; comme dans Agnathaner, il est à mème d'aspirer les matières nutritives préparées par l'action des pièces buccales. Cette aspiration est facilitée par l'existence d'une sorte de cuvette entre 
les premières maxilles et la saillie chitineuse médiane qui sépare les secondes maxilles (PI. XVIII, fig. I4).

La première maxille $\left(m x^{1}\right)$ compte deux articles. L'article basilaire est une pièce solide, à parois chitineuses épaisses; son extrémité interne fait saillie dans la cuvette métastomiale et présente deux lobes inégaux ; le bord antérieur porte, en son milieu, une grosse épine rigide barbelée, dirigée vers la bouche. Le second article est une lame aplatie, aux parois moins épaisses, légèrement échinée vers l'extrémité; il est bordé par une série de six grosses épines coniques à surface échinée.

La seconde maxille $\left(m x^{2}\right)$ se compose également de deux articles. L'article basilaire, renflé et solide, fournit, vers son extrémité distale, une saillie recourbée et recouverte d'épines qui se dirige vers l'intérieur. Le second article est une puissante mâchoire bidentée; il porte, sur la face externe, une petite soie hyaline implantée dans une zone transparente polygonale.

Les deux maxilles s'appuient sur deux puissantes crêtes chitineuses articulaires, dont la réfringence spéciale les distingue au milieu des nombreux épaississements du tégument céphalique (Pl. XVIII, fig. 13). Ces crêtes chitineuses suffiraient à démontrer que les appendices buccaux sont réduits à deux paires chez la femelle de Enterocola, si l'organogénie ne le faisait voir d'une façon plus péremptoire.

Aucun des auteurs qui ont étudié soigneusement l'organisation de Enterocola n’a pu comprendre l'intérêt de cette disposition. Claus (55, p. 352) désigne comme "larges mandibules coniques "l'article basilaire des premières maxilles, et comme " petites maxilles palpiformes " le $2^{\mathrm{e}}$ article palmé. Il fait suivre ces appendices de "deux paires de pattes crochues très saillantes qui sont les maxillipèdes " et qui correspondent probablement à l'apophyse interne du $\mathrm{I}^{\mathrm{er}}$ article et au $2^{\mathrm{e}}$ article des secondes maxilles. Dans une description accompagnée de nombreuses figures capables d'inspirer confiance au lecteur, Della VAlle (76, p. 246) n’est pas plus heureux lorsqu'il interprète ces parties, car il considère le $2^{\mathrm{e}}$ article de la $\mathrm{I}^{\mathrm{re}}$ maxille comme la portion distale, élargie, de la mandibule et comme sa portion basilaire, une partie du I $^{\text {r }}$ article avec la grosse soie barbelée interne. La maxille serait, pour lui, composée uniquement de l'article basilaire avec la soie palpiforme. Dans la $2^{\mathrm{e}}$ maxille, les articles sont encore artificiellement séparés, pour constituer deux paires de maxillipèdes.

En résumé, dans le genre Enterocola se trouve réalisée la caractéristique imposée par THORELL à ses Copepoda Pacilostomata, caractéristique démontrée 
inapplicable aux Copépodes (Corycæidæ, Sapphirinidæ, Lichomolgidæ, Ergasilidæ, etc.) pour lesquels fut établi ce sous-ordre. L'influence de l'adaptation étant trop évidente dans l'établissement de ces caractères, il me semble inutile d'insister sur leur valeur taxonomique très restreinte au point de vue de la séparation des grandes familles de Copépodes.

D. - Chez Aplostoma brevicauda, je rencontre une tout autre disposition des pièces buccales qui doit encore trouver son explication dans l'étude organogénique.

a. Après le stade nauplius (PI. XXI, fig. 6), l'embryon d'Aplostoma prend aussitôt la forme du premier stade cyclopoïde. La bouche (PI. XXI, fig. Io, b) est à la partie antérieure d'un bulbe buccal terminé par une longue épine $(b)$ recourbée vers l'avant. De chaque côté du bulbe buccal, un petit appendice représente la mandibule rudimentaire (fig. IO, $m x^{1}$ ); il se compose (fig. II) d'une portion basilaire biarticulée supportant un endopodite très petit terminé par deux soies, et un exopodite réduit à une saillie bilobée très faible.

La première maxille manque complètement; elle n'apparait même jamais à l'extérieur du corps, puisque le stade metanauplius est supprimé dans le développement condensé.

La seconde maxille (PI. XXI, fig. 8, 9 et Io, $m x^{2}$ ) est un appendice 3-articulé dont le dernier article recourbé porte une griffe terminale.

Chez Aplostoma brevicauda, la régression des appendices buccaux s'effectue par conséquent suivant un mode différent de celui qu'elle affecte dans Enterocola fulgens et elle atteint profondément les deux premières paires masticatrices, respectant seulement la troisième paire, adaptée à la fixation dans les jeunes.

b. Dans la femelle adulte, la bouche est encore située sur le bulbe buccal fortement convexe (PI. XX, fig. 8, ls $+l i$ ). Les mandibules aussi réduites que chez l'embryon, sont encore constituées de mème que je l'ai décrit ci-dessus ( $\mathrm{Pl} . \mathrm{XX}$, fig. 9). La $2^{\theta}$ maxille, 3 -articulée, est fortement organisée et peut servir à la fixation et à la reptation (Pl. XX, fig. $\left.8, m x^{2}\right)$.

$c$. Dans un Copépode mâle, que je rapporte pour ses deux pièces buccales au genre Aplostoma, la bouche (PI. XX, fig. I6) est également située sur un bulbe buccal saillant. La mandibule est une suite de trois articles portant une grosse soie barbelée en son milieu et deux petites soies recourbées, à son extrémité. La seconde maxille est un puissant appareil préhensile, 4-articulé et terminé par une forte griffe. 
E. - Dans le genre Enteropsis Auriviluıus, gràce à l'étude attentive d'une espèce nouvelle parasite de Diazona hebridica ForBes, j'ai pu comparer plus utilement entre elles les descriptions relatives aux deux autres espèces de ce genre.

a. Très voisine de Aplostoma par la forme générale du corps, la femelle adulte de Enteropsis s'en rapproche encore notablement par la morphologie de la région buccale, mal interprétée jusqu'à ce jour.

La bouche d'Enteropsis pilosus Canu est située sur un bulbe buccal, comme chez Aplostoma. Le tégument ventral est fortement saillant au-dessous de la lèvre inférieure; il est couvert, en ce point, de poils raides et triangulaires. Sur les côtés

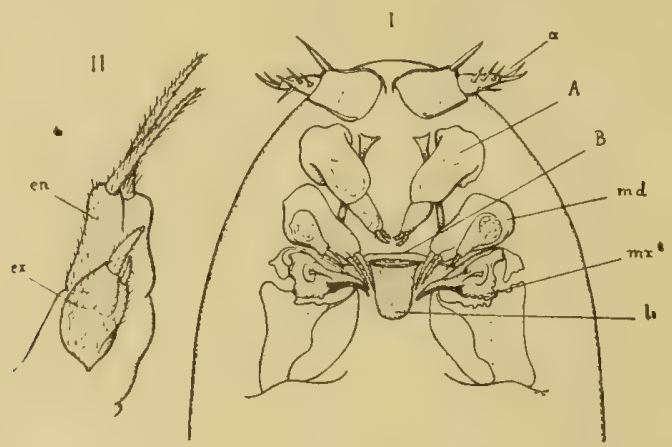

Fig. 4. - Femelle d'Enteropsis pilosus Canu.

I. - Céphalon vu par la face ventrale. $a$, antennule; $A$, antenue ; $m d$, mandibule ; $m x$, seconde maxille; $B$, bouche; $l i$, lèvre inférieure.

II. - Mandibule isolée, plus fortement grossie ; en, endopodite ; ex, exopodite.

du bulbe et en avant de l'orifice buccal s'insère une première paire d'appendices : les mandibules. Au-dessous se trouve une seconde paire : les secondes maxilles. Comme chez Aplostoma, la première maxille fait défaut.

La mandibule se compose d'une région basilaire aux contours irréguliers, indiquant d'une manière très confuse la division en deux articles que nous avons constatée chez Aplostoma. Cette région basilaire est immédiatement continuée par un endopodite bilobé à son extrémite distale et terminé par deux longs stylets cylindriques obliquement ảirigés vers la lèvre inférieure. Sur la face externe de la base des mandibules, vient se fixer un petit exopodite, terminé par deux courts stylets. Tout cet appareil - base, rames et stylets - est recouvert, vers l'extérieur, de nombreux poils raides et triangulaires, semblables à ceux de la saillie ventrale métastomiale. 
Les secondes maxilles sont des appendices simples, bi-articulés, dont la base

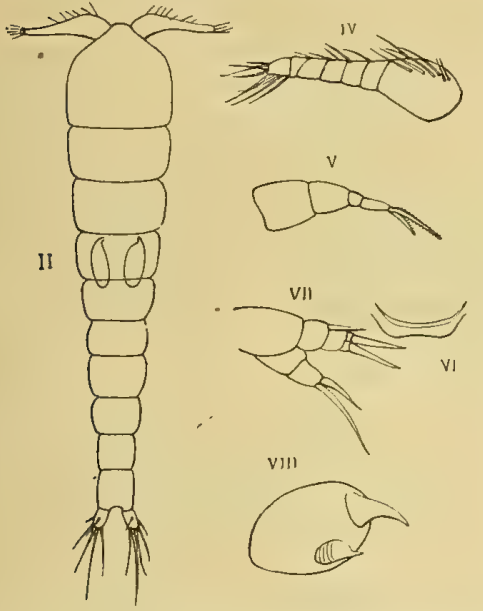

Fig. 5. - Mâle adulte d'Enteropsis sphinx Aur (- Haligryps teres AUR). Reproduction des figures données par C. W. S. Aurivillius (loc. cit., Tafl. 9).

II. - Mâle vu par la face dorsale.

IV. - Antennule.

V. - Antenne.

VI. - Bouche.

VII. - Mandibule.

VIII. - Seconde maxille.

élargie et solide est complètement inerme et dont l'extrémité est formée par une forte griffe uncinée portant une soie délicate fixée au centre d'une zone claire et transparente (tubercule à paroi mince ?).

En réalité, la seule différence importante qui sépare les pièces buccales d'Enteropsis de celles d'Aplostoma consiste dans la solide organisation de la seconde maxille exclusivement adaptée à la fixation. Le rapprochement apparent qui existe entre les mandibules et les $2^{\mathrm{es}}$ maxilles, résulte simplement de cette adaptation et n'infirme en rien les conclusions morphologiques énoncées ici.

Dans la description originale du genre Enteropsis, Auriviluius (5, p. 237, 238 ) signale l'existence de maxilles 2-ou 3-articulées, fixées à la base des mandibules; dans ma notice préliminaire (27, p. 367 ), tout en faisant quelques réserves sur l'exactitude de cette interprétation, j'avais suivi la mème nomenclature et séparé, comme mandibule, l'exopodite, et comme maxille, l'endopodite du véritable appendice
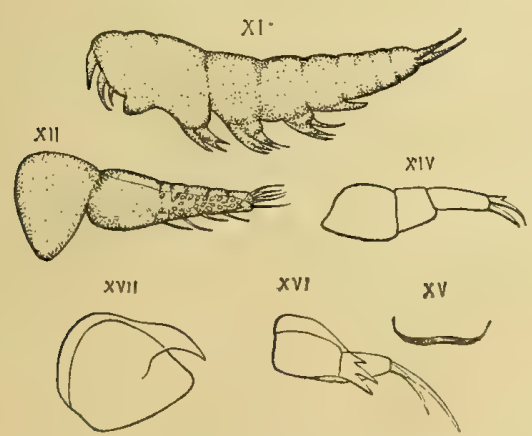

Fig. 6. - Jeune mâle d'Enteropsis sphinx AUR ( $=$ Haligryps aculeatus AUR). Reproduction des figures données par C. W. S. Aurivillius (loc. cil., Tafl. 9).

XI. - L'animal vu de profil.

XIV. - Antenne.

XV. - Bouche.

XVI. - Mandibult.

XVII. - Seconde maxille. mandibulaire. Plus récemment, SchimkEVITSCH a décrit une nouvelle espèce d'Enteropsis pour laquelle il laisse indéterminée l'existence de la mandibule, sans s'étendre particulièrement sur la constitution biramée de. la maxille.

b. Il est vraiment regrettable que je n'aie pu étendre au genre Enteropsis l'étude embryologique des appendices céphaliques, afin de confirmer entièrement les résultats fondés sur l'étude comparative des adultes. SCHIMkEvitsch (1) a négligé, dans ses recherches embryologiques sur Enteropsis dubius Schimk., les détails de la métamorphose. En conséquence nous ne possédons 
de renseignements que sur le mâle jeune et sur le mâle adulte d'Enteropsis sphinx, décrits par Aurivillius comme deux espèces du genre Haligryps Aur. Et nous y retrouvons la mème disposition morphologique que dans la femelle, si l'on en juge d'après les descriptions publiées par le naturaliste suédois.

D'après ce qui précède, il me semble bien établi que le genre Enteropsis est très voisin du genre Aplostoma, plutòt que du genre Enterocola. Mais si l'on choisit comme terme de comparaison l'état de régression des pièces buccales dans les deux genres, on est forcé de conclure que Enteropsis, avec sa mandibule plus développée, est moins dégradé et moins élevé dans la série morphologique que Aplostoma.

En comparant entre elles les diverses dispositions des pièces buccales dans les types d'Ascidicolidés ici décrits, nous voyons qu'il existe tous les passages entre les formes gnathostomes les plus élevées et les formes parasites les plus profondément modifiées.

Le genre Agnathaner établit l'union des deux types gnathostome et suceur; il vient démontrer l'inexactitude des opinions émises jusqu'à ce jour sur les relations morphologiques et taxonomiques des Copépodes commensaux et parasites des Tuniciers.

$4^{0}$. - Parmi les Copépodes semi-parasites, il est un groupe de formes où l'organisation buccale diffère notablement de tout ce qui nous a occupé plus haut. La première maxille, très réduite, semble n'être chez eux qu'une dépendance palpiforme de la mandibule; cette apparence est assez nette pour qu'une classe spéciale ait été créée pour ces animaux dans le groupe des Copépodes, sous prétexte qu'une paire d'appendices buccaux, les mandibules, faisaient défaut. Le groupe des Copépodes pœcilostomes de ThORELL a été vivement combattu par CLAUS, avec beaucoup de raison; il ne semble plus actuellement accepté par aucun zoologiste.

J'ai pu étudier attentivement plusieurs espèces de ce groupe appartenant aux deux familles des Lichomolgidés et des Ergasilidés : je me rallie entièrement aux conclusions morphologiques de Claus $(43,49,51,55,66)$, contre l'opinion de THORELL (204)

A. La lèvre supérieure des Lichomolgidés forme, à la face inférieure du céphalon, une saillie fortement convexe dont le contour antérieur est à peu près semicirculaire. Du côté postérieur cette lèvre est profondément incisée en son milieu, elle forme deux ailes qui divergent latéralement et recouvrent en partie les premiers 
appendices buccaux ou mandibules (PI. XXII, fig. 3 et fig. I7; Pl. XXIV, fig. 18 ; Pl. XXV, fig. 3). Sous la lèvre supérieure se trouve une grande et large cavité en entonnoir, à l'intérieur de laquelle pénètrent les portions distales effilées et falciformes des pièces buccales (PI. XXII, fig. I7; P1. XXIV, fig. 18; Pl. XXV, fig. 3). L'ouverture de cette cavité n'est pas la véritable bouche, que l'on trouve reportée au fond de l'entonnoir, au point où aboutit l'extrémité des pièces buccales; la lèvre supérieure recouvre donc, au-dessus de la face ventrale, une sorte d'atrium (ou propharynx) qui conduit à l'orifice buccal. Les secondes maxilles et parfois même la saillie interne du prolongement falciforme mandibulaire, viennent affleurer à l'entrée de ce propharynx pour remplir un ròle masticateur ; mais les matières nutritives ainsi divisées sont conduites sous la lèvre supérieure, jusqu'à la bouche, au travers d'une sorte de canal constitué par les prolongements falciformes ou barbelés des mandibules et des secondes maxilles. Ainsi les appendices buccaux sont légèrement détournés de leur rôle habituel; mais leur valeur morphologique n'a nullement varié comme le croyait THORELL.

La première maxille ( $\mathrm{Pl}$. XXII-XXV) est une courte pièce, presque cylindrique, terminée par une, deux ou trois soies; elle vient s'insérer au-dessous de la portion basilaire élargie de la mandibule, mais elle reste totalement indépendante de cette dernière, comme le prouvent parfaitement les crètes articulaires spéciales sur lesquelles s'appuient les deux appendices. Comme je l'ai déjà fait observer précédemment, il n'existe, chez les Lichomolgidés, aucune trace d'une disposition rappelant, mème de très loin, la formation des paragnathes.

b. Chez les Ergasilidés, la disposition est la mème; toutefois la lèvre supérieure n'est point, à beaucoup près, aussi développée que dans les précédents.

Dans la femelle de Thersites gasterostei PAgenstecher, la mandibule falciforme, fortement dentée sur le bord interne, sert visiblement à la mastication; sa base, très large, recouvre presque complètement la première maxille réduite à un moignon portant deux fines soies (PI. XXIII, fig. 14, 15 et 16). L'organisation fondamentale ressemble à ce qui existe chez les Lichomolgidés; mais le rôle des pièces est plus nettement broyeur.

Les types intermédiaires, rattachant graduellement ces animaux singuliers aux Copépodes gnathostomes, restent encore inconnus, et les études embryoligiques ne sont pas encore assez avancées pour nous renseigner sur les affinités phylogénétiques fondamentales des Corycoeidés, Lichomolgidés, Ergasilides, Chondracanthidés, évidemment apparentés entre eux. 


\section{Conclusions.}

De l'étude qui précede, il ressort clairement que la région buccale, avec les lèvres, paragnathes et mandibules est douée d'une variabilité considérable dans un méme ensemble de Copépodes voisins. La formation des appareils si curieux qui ont reçu les noms de siphon et de trompe, a pu s'effectuer, aux dépens des Copépodes gnathostomes primitifs et ancestraux, de diverses façons différentes qu'il importait de fixer. L'existence de ces formations, dans deux Copépodes siphonostomes, n'indique nullement la parenté immédiate de ces ètres. Giardella et Nicothoe, qui sont : le premier, un gnathostome, et le second, un siphonostome, sont plus voisins que Nicothoe et Nereicola, tous deux pourvus d'une courte trompe presque discoïdale.

Les siphonostomes ne constituent donc point un groupe naturel; et, pour déduire de la structure des appareils buccaux de l'adulte toutes les conséquences qu'elle comporte, il est nécessaire de saisir, dans le développement de l'être, le mode qui a présidé à leur constitution et d'établir, par l'étude comparative des formes voisines, les diverses étapes phylogénétiques qu'ont dû traverser les Copépodes gnathostomes ancestraux avant de se transformer en siphonostomes. En un mot, l'étude de la morphologie évolutive des siphons est indispensable. Elle n'avait point été entreprise jusqu'à ce jour. J'ai montré, le premier, à propos des femelles d'Ascidicolidés, les intéressants résultats que l'on peut en tirer pour la connaissance des formes parasites.

\section{PREMIERE MAXILLE.}

Cet appendice apparaît dans l'embryon dès le stade métanauplius. I1 se montre alors, le plus souvent, à l'état d'un bourrelet peu différencié portant vers l'extérieur une longue épine ou soie légèrement infléchie (PI. XII, fig. 3, 4, 6, etc., $\left.m x^{1}\right)$. Dans ce bourrelet se constitue l'appendice presque définitif de l'adulte, qui présente normalement tous les caractères d'un appendice biramé adapté à la mastication.

La ${ }^{\text {re }}$ maxille normale se rencontre, à mon avis, dans le curieux Longipedia coronata. Elle y possède, en effet, dans un état moyen de complication, toutes les 
parties essentielles de cet appendice considéré dans l'ensemble des Copépodes. La région basilaire, ou coxopodite, est pourvue, à son bord interne, d'une saillie armée de solides épines barbelées qui servent à diviser la nourriture; dans la région

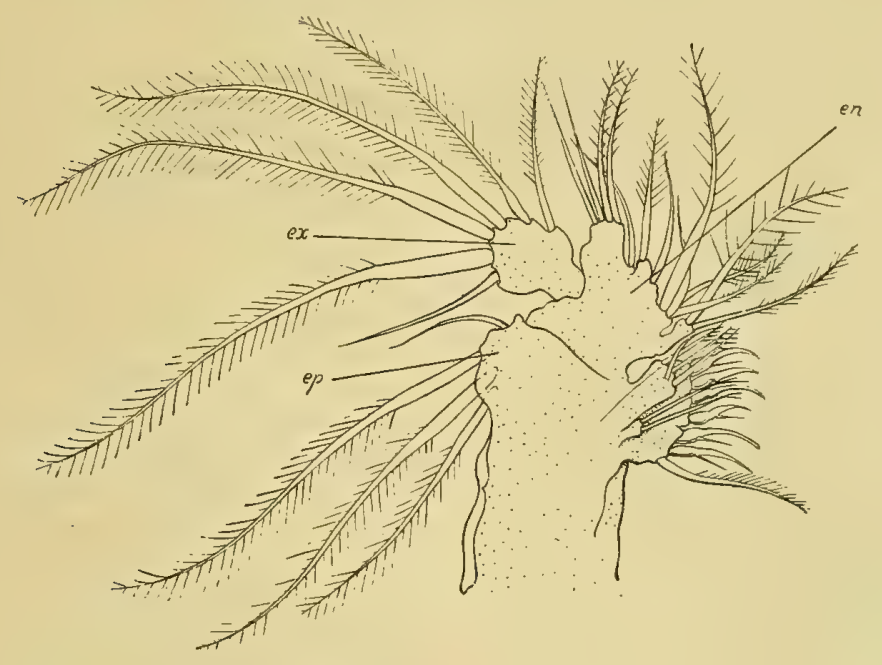

Fig. 7. - Première maxille de Longifedia coronata, d'après GIEsBrecht, Taf. IX, fig. 11. $e n$, endopodite; $e x$, exopodite ; $p$, épipodite.

distale de ce coxopodite, se trouvent quelques lobes saillants armés de soies barbelées, prolongements digitiformes accessoires qui appartiennent encore (comme l'indique l'anatomie comparée) au $\mathrm{I}^{\mathrm{er}}$ article primordial du protopodite. Vers la face externe, une autre saillie du coxopodite, portant trois grandes soies plumeuses et une petite soie lisse, constitue l'épipodite signalé déjà sous ce nom par Claus chez les Calanidés. Sur le coxopodite s'articule une pièce impaire (deuxième article du protopodide, ou basipodite) armée de soies vers l'intérieur et supportant deux lobes plus ou moins nettement articulés à leur base : l'endopodite et l'exopodite.

Dans presque tous les groupes de Copépodes, on retrouve, avec plus ou moins de développement, les divers détails de structure décrits ici pour la première maxille de Longipedia.

Chez les Calanidés, les deux rames terminales sont bien développées et nettement distinctes. De plus, l'épipodite est véritablement hypertrophié, il se frésente (Pl. II, fig. II, ep) sous forme d'un lobe presque distinct, portant jusqu'à dix 
longues soies richement barbelées. Une variation intéressante se rencontre dans le mâle de Clausia elongata, dont l'épipodite tombe avec la dernière mue et fait complètement défaut chez l'adulte.

Chez les Notodelphys, Doropygus, Agnathaner, etc., l'épipodite existe à l'état assez rudimentaire et porte seulement deux soies barbelées : l'une très grosse et postérieure, l'autre très petite.

Dans la plupart des Harpacticidés, les rames terminales sont plus ou moins

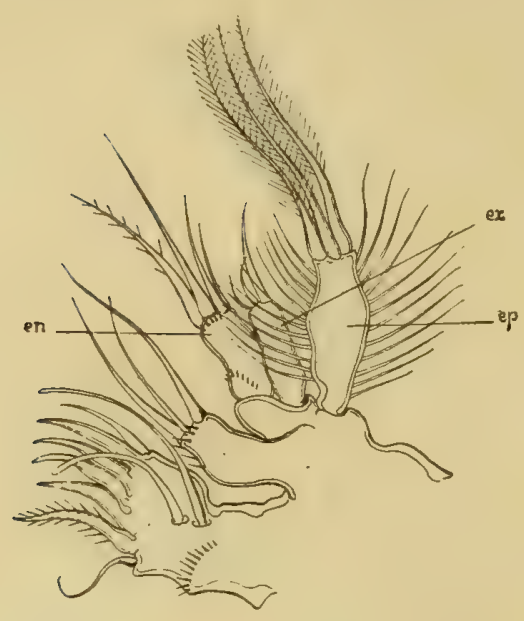

Fig. 8. - Première maxille de Zaus spinosus. en, endopodite; $e x$, exopodite; ep, épipodite. réduites et varient beaucoup sans présenter d'intérêt morphologique spécial ; l'épipodite fait défaut.

Chez les Peltidiens, l'épipodite existe presque toujours à un état de développement relativement considérable; c'est alors un lobe articulé à sa base et terminé par deux grosses et longues soies très richement barbelées. Dans certains cas, il pourrait être comparé au fouet de la cavité branchiale des Crustacés décapodes, qui est lui-même l'épipodite du premier maxillipède.

Dans les Cyclopidés, le genre Cyclops présente une première maxille très spécialisée en vue de la mastication et dans laquelle on ne reconnait plus qu'avec peine l'endopodite et l'exopodite (PI. III, fig. 3, 13 et 14). .

Chez les Copépodes commensaux ou parasites, la première maxille est le plus souvent très réduite (Lichomolyidés), parfois même absente (Enterocola, Enteropsis); je ne reviendrai pas sur ce sujet déjà traité plus haut.

\section{SECONDE MAXILLE.}

Claus a depuis longtemps démontré (XLVII, p.59), à l'aide de l'embryogénie, que cet appendice est, chez les Copépodes, très souvent séparé dans toute sa longueur en deux parties correspondant: l'interne, à l'endopodite, l'externe, à l'exopodite. Sans insister spécialement sur ce point encore discuté par quelques auteurs, je me rallie à cette interprétation, en vertu du mode de développement de la seconde maxille. 
Dans le métanauplius, lorsqu'avant la mue qui fournira le premier embryon cyclopoïde, apparaissent sous la cuticule les divers appendices des $4^{\mathrm{e}}, 5^{\mathrm{e}}$ et $6^{\mathrm{e}}$ paires, tous les replis appendiculaires qui leur donnent naissance subissent exactement les mêmes transformations. Dans la première paire - qui fournira les secondes maxilles - ils sont comme dans les deux autres (les deux premières pattes thoraciques) simples au début, puis bilobés, nettement divisés enfin en deux portions toujours accolées côte à côte, comme l'exopodite et l'endopodite d'un mème appendice (PI. VII, fig. 3; Pl. X, fig. 2, 3; Pl. XII, fig. 3, 4; Pl. XIII, fig. 2, 3; Pl. XV, fig. 3). C'est à la fin de la période métanauplienne que ces appendices perdent peu à peu la ressemblance parfaite qui les unit aux pattes thoraciques (Pl. XII, fig. 6).

Je n'insisterai pas ici sur les variations des deux lobes de la seconde maxille dans la série des Copépodes ( $\mathrm{r}$ ) et je signalerai seulement le cas où l'un des lobes semble disparaitre, afin d'attirer l'attention sur le mode de développement des secondes maxilles simples. J'ai pu l'étudier chez Enterocola fulgens VAN BeN. et Aplostoma brevicauda Canu. Chez tous deux, l'embryogénie est condensée et le stade métanauplius supprimé. Sous l'enveloppe nauplienne, on voit apparaitre comme un repli le bourrelet d'abord simple qui donne naissance à la seconde maxille. Jusqu'alors, il n'est rien de spécial dans cette dernière. Mais dans la suite du développement, alors que les pattes thoraciques sont déjà biramées, la seconde maxille reste simple (PI. XIX, fig. I, $m x^{2}$ ); elle tend ainsi peu à peu vers sa conformation définitive (Pl: XXI, fig. 6).

Il n'est point douteux pour moi qu'il s'agit encore ici d'un phénomène de condensation embryogénique. Dans le cours du développement phylogénétique des Copépodes à secondes maxilles simples — qu'il s'agisse des Ascidicolidés ou de toute autre famille - , les formes ancestrales ont subi une régression graduelle de l'une des rames de leur seconde maxille, tendant ainsi à devenir simple (le cas existe encore pour Doropygus psyllus, D. pulex, Gunenotophorus globularis, Doroixys uncinata; voir P1. VIII, IX, XI, XIV). Dans la série des descendants successifs, la simplicité, acquise d'abord pour l'adulte, s'est peu à peu reportée sur les stades embryonnaires jusqu'à l'époque d'origine dans le stade nauplien. Ne trouvons-nous pas, d'ailleurs, la mème condensation - moins accentuée, il est vrai et par suite plus facile à saisir —, dans la simplification de l'antenne de Notodelphys et de tant d'autres? Chez Notodelphys allmani, l'exopodite n'existe plus qu'aux stades nauplius, métanauplius et premier cyclopoïde (PI. XIII, fig. $\left.6^{\prime}\right)$.

(1) La seconde maxille la plus primitive se rencontre aussi chez Longipedia coronuta. 
Dans les Ergasilidés et Myicola WrICHT (Lichomolgidés), la seconde maxille s'est simplifiée aussi par un effet de l'hérédité limitée à un seul sexe ; elle est simple dans les femelles et double dans les mâles. Il faut d'ailleurs remarquer que chez ces animaux, la seconde maxille interne a été conservée dans le mâle seul en vue du rôle spécial qu'elle y remplit pour l'union des sexeschez tous les Copépodes voisins. Dans les formes faisant partie d'un groupe où ce rôle copulateur n'est point dévolu aux secondes maxilles internes du mâle, la concurrence sexuelle n'intervenant pas pour conserver cet organe, la suppression s'établit dans les deux sexes. Nous en avons des exemples chez les Ascidicolidés.

\section{PATTES THORACIQUES.}

J'ai décrit précédemment, à propos des prétendus pléopodes et de la naissance de la seconde maxille, le mode d'apparition de ces appendices (I).

Dès le premier stade cyclopoïde, les pattes thoraciques existantes (les deux premières paires) présentent déjà la constitution typique et fondamentale du groupe des Copépodes, caractère principal sur lequel Milne Edwards (148) établit ce groupe. Ce sont des appendices biramés (PI. VII, fig. 5; Pl. X, fig. 6, 7 ; PI. XV, fig. 5; Pl. XIX, fig. 4 et 5), aplatis en lame dans le sens antéropostérieur ; le protopodite y est 2-articulé et les rames sont également articulées et armées de soies et d'épines. Un repli tégumentaire s'étend entre les deux pattes d'une même paire, les soutient et accouple leurs mouvements : c'est le "Bauchwirbelkorper" de ZENKER, le " coupler" de HARTOG. Avec les progrès du développement, ces pattes natatoires se modifient peu à peu: le nombre des articles s'accroit, les soies et les épines augmentent ou se transforment suivant les adaptations spéciales auxquelles elles sont soumises.

Dans les Copépodes nageurs, libres ou semi-parasites, les pattes conservent l'aspect embryonnaire et servent à la natation durant toute la vie. Elles sont le mieux caractérisées dans les formes pélagiques, bien qu'elles montrent toujours sensiblement les mèmes farties essentielles. D'après les travaux descriptifs approfondis des vingt-cinq dernières années, on sait que la patte natatoire typique du Copépode est un appendice avec les coxopodite et basipodite libres et munis : le premier d'une soie interne, et le second d'une soie externe (et parfois d'une épine 
interne dans la première paire). Les deux rames sont 3-articulées, pourvues de soies barbelées à leur bord interne; l'exopodite porte à son bord externe des épines en lame de poignard, c'est-à-dire formées d'un axe solide tt épais avec des bords graduellemerit amincis et denticulés.

Les nombreuses variations des diverses parties seront décrites en détail dans le dernier chapitre (systématiq̨ue) de ce travail. Je signalerai seulement une disfosition curieuse qui se présente dans les formes glissant sur les surfaces lisses, dans les formes caligoïdes comme Artotrogus et Scutellidium. Les deux rames y sont terminées à leur extrémité par des épines solides et rigides, tandis que dans les types libres une seule - l'exopodite - présente cette disposition.

Chez les Copépodes parasites, la locomotion ne s'effectue plus du tout de la même façon et se transforme en reptation. Les pattes natatoires de l'embryon deviennent alors des appareils adhésifs par la disparition plus ou moins complète des soies et l'apparition de crochets terminaux ; mais elles demeurent toujours bâties sur le mème type d'un appendice simple, réduit, 2-articulé et encore embryonnaire, ou bien d'un appendice biramé et peu développé (1).

Les réductions des pattes natatoires dans un seul sexe sont fréquentes chez les parasites dimorphes, comme Enterocola (PI. XVIII et XIX). Cette réduction apparait mẻme dans ce dernier d'une façon très précoce après le deuxième stade cyclopoïde, et cette précocité 'est due à une condensation remarquable du développement limitée au sexe femelle.

Chez Cancerilla tubulata femelle, les pattes thoraciques des deux premières paires sont réduites à un rôle préhensile (PI. XXIV, fig. I 2, I3), tandis qu'elles sont natatoires chez le mâle (fig. 6). Dans ce curieux Copépode, ainsi que dans Caligidium, la $4^{\mathrm{e}}$ paire de pattes thoraciques manque presque totalement chez l'adulte, tandis que la $5^{\mathrm{e}}$ persiste.

La cinquième paire de pattes thoraciques est formée d'organes parfaitement homodynames des autres appendices du thorax, comme le démontrent:

a. une origine ontogénétique concordante;

b. des ressemblances parfaites qui les unissent aux diverses époques de leur évolution. En effet, dans la plupart des Copépodes, la $5^{\mathrm{e}}$ paire de pattes est très

(1) D'étonnantes formes d'appendices simples, multiarticulés, ressemblant à des pattes d'lsopodes ou de Myriapodes, ont été décrites par HESSE. Mais on ne peut attacher aucune importance morphologique à ces descriptions erronées. 
peu développée far rapport aux précédentes. Elle se compose (Pl. III, fig. 7, 9 , I7 : PI. IV, fig. 5, I9; Pl. V, fig. 22 et 31 ; etc. ) :

$\alpha$. d'une lame simple, soudée vers la base avec le $5^{\mathrm{e}}$ somite thoracique et pourvue d'une soie flexible à son bord externe ;

३. d'un article terminal plus ou moins fourni de soies et d'épines.

Dans cet état, la $5^{\mathrm{e}}$ patte est comparable aux premiers rudiments libres des appendices thoraciques : elle est à l'état embryonnaire.

Il existe cependant des cas fréquents, dans le groupe des Calanidés, où la $5^{\circ}$ patte frésente un développement considérable, comparable en tous points. (Calanus, Centropages, etc.) à celui des appendices antérieurs. Mais cette disposition n'est nullement générale, puisque, à côté du genre Calanus ainsi constitué (P1. I, fig. I 8 , 19), vient se ranger le genre très voisin Paracalanus, où la $5^{\mathrm{e}}$ patte est très réduite (Pl. I, fig. 6) par rapport aux I $^{\text {re }}$ et $3^{\mathrm{e}}$ (Pl. I, fig. 9 et Io). De plus, les mâles de Clausia elongata possèdent des $5^{\text {es }}$ pattes à l'état adulte ou jeune (P1. II, fig. $5,6,7,8$ ) tandis que la femelle en est dépourvue (PI. II, fig. I et 4).

Deux interprétations sont possibles, en ce qui concerne la conception morphologique de cette cinquième paire de pattes.

$\mathbf{I}^{0}$ Elle serait formée d'apfendices thoraciques ayant présenté, dans les ancêtres des Copépodes, un développement parfait (comme chez Calanus) au même titre que les 4 fremières paires; et elle aurait subi, dans l'évolution du groupe, une régression à peine indiquée chez Calanus, Centropages, etc., mais presque complète chez les Cyclops, Asterocheres, etc. C'est l'opinion de Claus, qui insistait encore récemment (61, p. 105) sur la filiation des Copépodes aux Protostracés (= Urphyllopoden, 5r) (1).

A cette théorie, j'objecterai que : si les derniers segments ont subi une semblable régression d'arrière en avant, il est logique d'admettre qu'elle s'est établie peu à feu, atteignant successivement les diverses pattes abdominales pour s'étendre ensuite aux 4 dernières pattes thoraciques, qui l'auraient subie à un égal degré dans un type déterminé. Dans ce cas, comment comprendre cette disposition étonnante des Calanidés " gymnopléodes "? Leurs $6^{\text {es }}$ pattes thoraciques (les pléopodes d'après GIESBRECHT, 89) seraient entièrement disparues, tandis que leurs $5^{\text {es }}$ pattes restaient mieux conservées que dans les " podopléodes ", où Fersistent constamment les $6^{\text {es }}$ pattes.

(1) Pourvus d'un céphalon à 5 somites, d'un thorax à 8 somites avec pattes natatoires, d'un abdomen à 6 somites avec pléopodes biramés, et d'un telson (61, p. 11 à 13). 
$2^{0}$ La seule opinion acceptable dans l'état actuel de la science est celle où la $5^{\mathrm{e}}$ patte thoracique constitue le dernier appendice apparu phylogénétiquement dans les ancêtres des Copépodes actuels, et fixé par hérédité à l'état relativement embryonnaire dans tous les “Gymnopléodes ". Les développements exagérés présentés par ces appendices dans quelques Copépodes pélagiques, supérieurement adaptés à la natation en haute mer, ne sont que des hypertrophies secondairement acquises en vue de perfectionner les organes locomoteurs, de même que, sous l'influence de la concurrence sexuelle, s'établissaient d'autres hypertrophies en organes préhensiles chez les mêmes animaux. 


\section{DEUXIEME PARTIE.}

\section{EMBRYOLOGIE DES ASCIDICOLIDÆ.}

\section{FORMATION DES GUFS.}

Les recherches approfondies de GieSBRECHT (90, p. 328-339) sur l'ovogenèse de Notopterophorus ont donné à cette question un intérêt spécial; pour les formes très variées d'Ascidicolidés que j'ai pu étudier, j'ai recherché dans quelle mesure se justifiaient les descriptions des auteurs, sur ce point particulier.

L'appareil génital femelle des Ascidicolidés est batti sur le type habituel parmi les Copépodes; il se compose de :

a. un ovaire médian et dorsal, situé à la limite du céphalon êt du $\mathbf{I}^{\text {er }}$ somite thoracique,

b. deux oviductes latéraux aboutissant à la face latéro-dorsale du $\mathbf{I}^{\mathrm{er}}$ somite abdominal, dans deux atriums génitaux débouchant à l'extérieur par les ouvertures génitales,

C. un appareil séminifère, comprenant lui-même un pore de fécondation, unique et ventral, placé dans le $\mathrm{I}^{\mathrm{er}}$ somite abdominal au fond d'une petite dépression des téguments, et duquel part un court canal séminifère bientôt bifurqué pour fournir deux canalicules divergents qui remontent le long des faces latérales jusqu'aux réceptacles séminaux. Ces derniers déversent le sperme dans l'atrium génital par l'intermédiaire d'un court conduit.

Kerschner (117, p. 9 et 10) a signalé, le premier, l'existence de l'ovaire 
impair chez Notodelphys, Doropygus, etc, et indiqué le mode de développement des ovules par chapelets entrelacés au sein des oviductes.

Mais c'est à GIESBRECHT qu'on doit la découverte certaine de l'origine cellulaire des ovules dans le genre Notopterophorus, comme celle de la nature épitheliale des parois oviducales à l'état jeune.

I. - Chez Notopterophorus, GiesBrecht a étudié le mode de formation de la première génération d'ovules et constaté que : dans l'oviducte et sa ramification aveugle dorsale, composés ainsi que l'ovaire d'une masse cellulaire pleine, se forment aux dépens de l'épithélium de la paroi des files de cellules ovulaires dans lesquelles apparaissent les œufs. J'ai pu vérifier ces observations chez la femelle de $N$.gibber Thorell, à l'avant-dernier stade du développement (PI. VI, fig. 3) et dans le cours ultérieur de la période reproductrice.

Dans Notopterophorus, les ovules de première génération sont fournis par l'épithélium de tout l'appareil femelle; ceux des générations ultérieures sont formés dans les petites taches germinatrices des parois du tube ovarien (90, p. 334 et 335 ).

2. - Il en est tout autrement dans un genre très voisin : Doroixys KERSCHNER, et les réserves faites à ce sujet par GIESBRECHT $(90, p$. 336) sur les généralisations de KERSCHNER sont parfaitement justifiées.

Dans une femelle âgée de Doroixys uncinata Kerschner, peu de temps après la ponte, l'ovaire est formé par une masse de petites cellules polygonales nucléées (PI. XI, fig. I3, ov). Les tubes aveugle et latéral de l'oviducte sont vides et formés d'une paroi mince, parsemée çà et là de groupes de noyaux (Pl. XI, fig. I3; PI. $\mathrm{XIV}$, fig. I4, ovd). Au sortir de l'ovaire, et dans la portion simple de l'oviducte, se trouvent enchevêtrés de nombreux chapelets d'ovules tous semblables, dérivant de l'épithélium ovarien, lequel se présente ainsi comme le centre de production des ovules de seconde génération.

A mesure que les embryons de la chambre incubatrice avancent dans leur développement, et dès qu'ils montrent les premiers rudiments des appendices naupliens, les ovules se différencient (P1. XI, fig. I4) :

en cellules très grosses, chargées de vitellus nutritif, avec gros noyau nucléolé $(m)$, et qui vont fournir les œufs de la prochaine ponte;

en cellules plus petites, polygonales, avec noyau et nucléole, et qui sont de taille assez variable : petites à l'extrémité des chapelets, très grosses vers le milieu de ceux-ci. Mais les cordons ovulaires de la région simple de l'oviducte sont encore, à ce stade, pour leur position et leur taille, en relation intime avec les dernières cellules de l'ovaire. 
Rien dans cette disposition n'indique la participation des parois de l'oviducte à la formation des ovules de nouvelle génération.

3. - Chez Botryllophilus macropus, l'oviducte est simple sur toute sa longueur, et les œufs apparaissent dans sa région antérieure toute entière, sa partie postérieure servant de glande agglutinante pour la formation du sac ovigère externe. Je n'ai pas vu, dans cette espèce, même chez des femelles venant immédiatement de subir la dernière mue (PI. XVI. fig. 2), la constitution de chapelets ovulaires, et je rapporte, pour cette raison, le développement des ovules de Botryllophilus au type que je vais décrire pour Enterocola.

4. - Chez Enterocola fulgens, dans une femelle adulte en voie de former la première ponte, l'appareil génital (Pl. XVIII, fig. I2) se compose des mêmes parties essentielles que dans Botryllophilus macropus. La portion antérieure de l'oviducte est germigène; la postérieure, d'une structure toute différente, constitue, par l'épithélium secréteur de sa paroi, une glande agglutinante pour la préparation des sacs ovigères $(\mathbf{I})$.

Dans la région germinative de l'oviducte, les cellules ovulaires restent petites du còté interne et dorsal. Elles grandissent beaucoup et se chargent de vitellus dans la portion extérieure. Lne semblable disposition excentrique des œufs mùrs a été décrite par Claus (62, p. 82-85, pl. XI1, fig. I3 et 14) chez Branchipus, où elle est reliée à une formation de cordons ou de chapelets ovulaires beaucoup moins nets que chez Notopterophorus. En conséquence cet auteur a considéré les cordons ovulaires comme formés par des clivages plus ou moins réguliers de la masse germinative, au cours du développement des œufs. Je n'ai point vu de chapelets ovulaires chez Enterocola, ni mème chez Aplostoma, bien qu'ils aient été signalés par Schimkevitsch (188, p. 81, Pl. V, fig. 55) chez Enteropsis.

Dans tous les cas, la maturation des ovules s'effectue dans l'oviducte, et même dans ses deux branches chez les Notodelphyidés. Les œufs mùrs sont conduits au moment de la ponte dans l'atrium génital (Pl. XIV, fig. 14; Pl. XVI, fig. 2 ; Pl. XVIII, fig. 2 et 12 ), au côté interne et ventral duquel arrivent les spermatozoïdes maintenus dans les réceptacles séminaux. La compression des réceptacles sous la pression des œufs mûrs, alors nus et capables de changer leur forme, est la cause effective de l'expulsion des spermatozoïdes et par suite de la fécondation des œufs avant la ponte. Je n’ai pas pu déterminer avec certitude si la

(1) La structure est identique chez Aplostoma brevicauda CANU. 
membrane de l'œuf est formée par une secrétion de l'œuf lui-même (I) après la ponte, ou bien si elle dérive de la secrétion agglutinante des oviductes terminaux.

Après la ponte, dans les genres Notodelphys, Doropygus, Doroixys, Bonnierilla et Gunenotophorus, les œufs sont abrités dans la cavité incubatrice : ils sont disposés au début en deux paquets distincts, correspondant à droite et à gauche aux ouvertures génitales. Plus tard, ils se touchent étroitement et se compriment latéralement jusqu'à devenir plus ou moins polyédriques.

Chez Botryllophilus macropus, les œufs sont pondus à l'extérieur et forment un seul sac. Par suite du rapprochement des ouvertures génitales sur la face dorsale, les œufs pondus à droite ou à gauche se touchent sur la ligne médiane, et la fusion de la secrétion agglutinante qui les enveloppe à leur sortie est seule cause (comme chez les Harpacticidés à un seul sac ovigère) de la disposition constatée.

Chez Ascidicola rosea, Enterocola fulgens, Enterocola betencourti, Aplostoma brevicauda, Enteropsis pilosus, les œufs pondus sont réunis dans deux sacs extérieurs latéraux, abrités sous la $5^{\mathrm{e}}$ patte thoracique.

\section{MÉTAMORPHOSE.}

\section{DOROPYGUS GIBBER ThORELl.}

$\mathrm{I}^{0}$. - A l'éclosion, le nauplius mesure 0,23 mm. de longueur (P1. VII, fig. I). Son corps, de forme elliptique, ramassée et trapue, présente les trois paires de membres habituels, avec les deux soies furcales des larves de Copépodes.

Les antennules sont biarticulées. Le premier article, cylindrique, porte une soie longue et délicaie au côté interne de son bord distal. Le deuxième article, renflé dans sa région proximale et légèrement aminci vers l'autre extrémité, se termine par deux soies dont l'interne est la plus courte. A ce stade, on distingue nettement, avec les muscles qui font mouvoir les articles de l'antennule, de grandes cellules nucléées qui se segmentent rapidement pour fournir les matériaux nécessaires à la transformation ultérieure de l'appendice.

Les antennes sont biramées, et leur base compte deux articles. Le premier article est court et totalement dépourvu de l'épine masticatrice habituelle aux

(1) Comme c'est le cas pour Calanus (Celochilus), d'après Grobren (99). 
larves de Copépodes. Le deuxième article est beaucoup plus long; il porte deux rames insérées :

a. l'exopodite, biarticulé et muni de cinq soies terminales et latérales, sur l'extrémité distale;

b. l'endopodite, monoarticulé et muni de deux soies terminales, sur une saillie latérale, dans la moitié proximale.

Les mandibules sont biramées, avec une base 2-articulée dépourvue d'épine masticatrice. L'exopodite 3-articulé s'insère à l'extrémité et porte quatre soies latérales. L'endopodite très court, 2-articulé, porte deux petites soies terminales et une latérale.

Dans les pattes biramées du nauplius, on prendrait aisément l'exopodite pour l'endopodite. En effet, pendant que s'effectue l'élévation des appendices vers la partie antérieure, il se produit également un léger mouvement de rotation, qui tend a amener la rame interne vers l'extérieur, en faisant pivoter l'appendice entier sur son cadre d'articulation avec le tronc.

Les deux soies furcales, insérées à la face ventrale et tout près de l'extrémité postérieure (Pl. VII, fig. 2), sont composées d'une portion basilaire cylindrique, à paroi chitineuse épaisse, lisse et très réfringente, et d'une portion terminale à paroi mince, barbelée, renflée vers la base et effilée vers l'extrémité.

Vu par la face dorsale, le corps du nauplius montre deux régions bien distinctes :

a. Le bouclier dorsal qui le recouvre presqu'entièrement. Dans le quart antérieur, le contour latéral forme un angle obtus au-dessus des antennules et des antennes. Dans la région médiane et au-dessus des mandibules; les côtés du bouclier sont parallèles et très légèrement ondulés. Dans le tiers postérieur, le corps est renflé; il s'arrondit assez irrégulièrement vers l'extrémité. Les bords postérieurs du bouclier apparaissent très nettement au-dessus des soies furcales divergentes.

b. L'abdomen, que l'on peut ainsi appeler, puisqu'il fournira très prochainement la furca et qu'il porte les deux soies furcales. C'est une petite saillie hémisphérique de la face ventrale du nauplius, que l'on voit déborder au-dessous du bord postérieur du bouclier dans une position convenable de l'embryon.

Quand on observe le nauplius par la face ventrale, la distinction de ces deux régions différentes n'est plus possible, et les contours du corps se continuent régulièrement d'avant en arrière. 


\section{$-69-$}

L'organisation intérieure du nauplius, étudiée sur l'embryon vivant en évitant l'aplatissement causé par la pression du couvre-objet, montre les détails suivants :

L'exoderme est constitué, dans la région antérieure du bouclier, par de grandes cellules nucléées, bien visibles sur les bords, et dans la région postérieure, par de petites cellules polyédriques. A la face ventrale, l'exoderme de la moitié postérieure est également formé de cellules polyédriques en voie de multiplication rapide.

La partie antérieure de la face ventrale est occupée par les insertions des appendices et par la lèvre supérieure. Cette dernière est une saillie convexe, à peu près hémisphérique; le bord postérieur en est légèrement infléchi dans son milieu et porte la petite dépression, à contour très net, qui forme la bouche.

Dans la région antérieure, se trouve l'œil nauplien, composé à l'éclosion de deux corps réfringents plongés chacun dans la concavité d'un double croissant vivement coloré en rouge carmin : à cet âge, le nauplius ne présente nullement l'indication de la troisième partie qui viendra ultérieurement compléter l'œil de l'adulte.

Au-dessus et en avant de l'œil nauplien, existe une grosse glande unicellulaire remplie de globules hyalins et qui se prolonge vers la face ventrale jusqu'à la pointe extrème du bouclier ; c'est la glande frontale.

En arrière de l'œil, se trouvent disposées symétriquement deux masses cellulaires à contour nettement délimité vers la ligne médiane, mais confondu latéralement avec la paroi du corps : ces deux masses sont formées de petites cellules claires et nucléées, en voie de multiplication. Ce sont les deux lobes dorsaux du cerveau, qui se détachent de l'exoderme à cette époque et qui correspondent à ce que Grobben (99) a considéré chez Calanus (Cetochilus) comme le cerveau secondaire.

L'endoderme est fortement chargé de vitellus nutritif. Il forme une grosse masse 'pleine, colorée en vert jaunàtre, à surface mamelonnée; il occupe presque tout l'intérieur du nauplius dans les deux tiers postérieurs. Il est composé d'un petit nombre de très grosses cellules aux contours polyédriques par compression, bourrées de gouttelettes graisseuses colorées avec diverses intensités. A sa surface dorsale, dans la région antérieure, sont dispersés de petits globules graisseux de couleur jaune.

- Sur la face ventrale et spécialement dans la partie postérieure, la surface de la masse endodermique se montre toute différente : les globules graisseux à contours nets et bien arrondis sont remplacés par de petites granulations très serrées, qui marquent dans ce tissu une modification notable, rendue sensible par les 
changements de la coloration de plus en plus claire et virant au bleu. C'est en ce point que frend d'abord naissance, aux dépens de l'endoderme solide du nauplius, la paroi intestinale définitivement constituée dans les stades cyclopoïdes.

A la partie antérieure de l'endoderme, au-dessous du cerveau secondaire, se trouvent amassés, en nombre plus considérable, de petites sphérules peu colorées et jaunâtres, qui sont en ce point situées à la place de l'œsophage auquel elles vont donner naissance.

De mème, à la partie postérieure, un amas de granulations vivement teintées de terre de sienne s'étend ventralement et dorsalement dans la masse endodermique; il forme le rudiment de l'intestin terminal.

Au-dessous de la lèvre supérieure, on voit, accolés de chaque côté, deux amas de très fines granulations jaune-paille, pyriformes, avec le pédicule tourné vers l'extérieur et dirigé vers la base de la mandibule. Je n'ai pu saisir les relations certaines de ces organes avec le tube digestif ni avec l'exterieur. Par leur situation sur la face ventrale entre les deux premières paires de pattes biramées et dans la région moyenne de l'animal, par leur structure, ces organes excréteurs se rapprochent des corps signalés dans l'embryon des Rhizocéphales " reins primitifs " de GIARD; “amas résiduels" de DELAGE); j'ai pu m’en convaincre, à Wimereux, par l'observation des nauplius de Sacculina et de Peltogaster. Comme je n'ai trouvé dans les divers stades naupliens aucune trace certaine des glandes antennales, on pourrait peut-ètre leur rapporter ces organes glandulaires pyriformes et adopter ainsi la dénomination appliquée par GIARD aux formations homologues des Sacculines. Pour admettre avec confiance cette interprétation, il serait nécessaire d'expliquer le déplacement de ces organes vers la face ventrale et de saisir leurs relations avec la deuxième paire d'appendices naupliens.

Le mesoderme du nauplius sortant de la cavité incubatrice de la femelle, immédiatement après l'éclosion, se compose de deux parties essentielles :

a. Les organes mésodermiques fonctionnels, constitués avant l'éclosion durant la formation des trois premières paires d'appendices, aux dépens du mésoderme primaire issu des cellules endodermiques de la gastrula voisines du prostome.

Dans cette catégorie, viennent se ranger les trois paires de muscles doubles qui mettent les affendices en mouvement. De chaque appendice partent en effet deux gros faisceaux musculaires striés, qui cheminent còte à còte au-dessus de la masse endodermique et viennent s'attacher à la face interne du bouclier dorsal auprès de la ligne médiane, dans la moitié postérieure du corps. Ce sont les muscles 
dorsaux des trois premières paires d'appendices que URBANOWICZ a séparés, chez Cyclops, sous la dénomination de “ muscles primitifs ".

b. Les origines du mésoderme secondaire, fournissant par leur prolifération les matériaux mésodermiques pour les somites nouveaux et apparaissant dans la partie postérieure du nauplius.

Elles sont constituées dans le nauplius récemment éclos, par une ou deux grosses cellules médianes accolées à la partie postérieure de l'endoderme, au-dessus des rudiments de l'intestin terminal (Pl, XII, fig. I, c pm). Très peu de temps après l'éclosion, les deux cellules impaires se sont divisées en quatre cellules au protoplasme clair avec leur noyau bien visible, et qui forment sur les côtés de l'endoderme deux bandes latérales de deux cellules chacune (PI. VII, fig. 2, $m s^{2}$ ). En raison de cette disposition, je crois devoir considérer comme les cellules polaires du mésoderme secondaire ces six premières cellules. Je leur applique cette dénomination par une légère modification de la nomenclature établie par HATSCHEK (I). Par leur origine impaire, les cellules polaires du nauplius de Doropygus s'écartent en effet de la définition donnée par cet auteur, puisqu'il appelle ainsi les cellules paires posterieures des bandes mésodermiques dérivant elles-mèmes des cellules mésodermiques primitives ( U Urmesodermzellen"). Je crois, en conséquence de mes observations sur Doropygus et sur d'autres Copépodes voisins, devoir conclure à une distinction plus nette entre le mésoderme primitif de la première larve et les formations mésodermiques dont il est question ici. Je considère ces dernières comme le résultat d'une nouvelle poussée cellulaire, issue de l'endoderme ainsi qu'un mésoderme de nouvelle formation; et je base cette interprétation sur leur situation primitive (PI. VII, fig. I, cpm) autani que sur leur destinée ultérieure (P1. VII, fig. 2, $m s^{2}$ ).

Le nauplius subit, après l'éclosion, plusieurs mues qui n'ont aucune conséquence morphologique - puisque sa forme extérieure n'est point modifiée mais qui présentent une certaine importance au point de vue de l'accroissement de taille et de l'évolution des organes internes. Comme je l'ai dit plus haut, peu de temps après l'éclosion, le mésoderme secondaire se dispose en deux baudes latérales dans lesquelles j'ai compté jusqu'à quatre grosses cellules disposées par files simples. Plus tard, cette disposition n'est plus reconnaissable, par suite de la multiplication rapide des éléments mésodermiques qui envahissent les diverses - parties des somites de nouvelle formation. L'endoderme perd de plus en plus la 
coloration jaunâtre et terne qu'il possède au début, pour prendre un ton plus clair intermédiaire entre le vert et le bleu.

Pendant toutes ces transformations, l'exoderme de la face ventrale s'est fortement épaissi et a proliféré dans des proportions considérables. Sous la cuticule nauplienne se sont formés successivement, d'avant en arrière, des bourrelets saillants, ébauches des replis appendiculaires qui fourniront les diverses paires d'appendices postérieures aux mandibules. Il apparait dans le nauplius quatre paires de ces bourrelets, pour les deux maxilles et les teux premières pattes thoraciques.

A l'intérieur, il se constitue également sous forme de masses séparées qui représentent les ganglions, des amas cellulaires d'origine exodermique, qui font face aux replis appendiculaires et appartiennent au système nerveux ventral.

$2^{\circ}$ Au sortir de l'enveloppe nauplienne, dont il est expulsé à la première mue morphologique, le métanauplius mesure $0,32 \mathrm{~mm}$. (PI. VII, fig. 3). Son corps est de forme vaguement elliptique, avec l'extrémité antérieure pointue et légèrement infléchie sur la face ventrale et l'extrémité postérieure presque tronc-conique. Le gonflement rapide de la région postérieure et en particulier de l'abdomen, provoqué par le grand développement des tissus de la larve, fait disparaître la limite postérieure du bouclier dorsal; la cuticule métanauplienne est alors continue.

Les trois premières paires d'appendices ressemblent presque complètement à celles du nauplius; les antennules présentent déjà à l'intérieur, l'ébauche des cinq articles développés dans le premier stade cyclopoïde.

La première paire d'appendices postmandibulaires n'est plus, comme les suivantes, un simple bourrelet recouvert par la cuticule. C'est un repli extérieur, de forme sensiblement triangulaire, avec l'indication de trois régions distinctes dont la dernière porte une longue soie lisse divergente. Cet état de la première maxille faisant saillie à l'extérieur caractérise très nettement la larve métanauplienne.

Les repiis appendiculaires de la seconde maxille, d'abord simples, se divisent de chaque côté en deux lobes séparés entièrement, par une scission complète, et qui formeront la seconde maxille externe et la seconde maxille interne (Pl. VII, fig. $\left.3, m x^{2}\right)$. Durant tout le stade métanauplien, la situation réciproque des deux lobes démontre, sans discussion possible, que les appendices qui en dérivent constituent une seule paire de membres, homodyname de la première paire de pattes thoraciques.

Les replis appendiculaires des pattes thoraciques se développent sous la cuticule métanauplienne, au nombre de trois paires. Les deux premières sont beaucoup 
plus avancées; dès le début de la période métanauplienne, elles forment, de chaque côté de la face ventrale, des lames basilaires aplaties, articulées avec le tronc, auxquelles s'attachent les deux rames de l'appendice avec leurs soies courtes ( $\mathrm{Pl}$. VII, fig. $3, p^{1}, p^{2}$ ). La troisième paire n'apparait, dans ses premiers rudiments, qu'au stade métanauplius; elle reste bien en retard des précédentes, quoiqu'elle montre distinctement, vers la fin de cet état larvaire, une région basilaire et deux rames.

L'apparition des troisièmes pattes thoraciques et celle du troisième élément visuel (bâtonnet réfringent et cupule colorée qui l'entoure) qui vient compléter l'œil tripartite du Copépode adulte, sont deux particularités remarquables de l'embryon métanauplien.

Les changements intérieurs qui précèdent la mue métanauplienne sont des plus importants : à ce stade, on reconnait encore, dans tous leurs rapports primitifs, les muscles dorsaux du nauplius (PI. VII, fig. 4). Les lobes dorsaux du cerveau sont alors complètement séparés de l'exoderme et forment deux amas cellulaires presqu'accolés sur la ligne médiane, en arrière de l'œil. L'endoderme est réduit dans des proportions considérables, il a pris une teinte plus claire et occupe un peu moins de la moitié de la largeur du tronc; les subdivisions du canal digestif en intestin antérieur, moyen et postérieur sont devenues plus nettes que précédemment. Le mésoderme a complètement évolué pour constituer les organes mésodermiques secondaires; il se trouve dispersé dans toute la cavité du corps, sous forme de tissu conjonctif ou de faisceaux musculaires en développement, et il est impossible de distinguer avec certitude et d'une façon toujours constante, la présence de cavités cœlomatiques définies comme celles décrites par URBANOWICZ (206) chez Cyclops. Le mésoderme secondaire a pris alors l'aspect purement physiologique d'un mésenchyme dont les intervalles irréguliers forment le cœlome.

Il s'est néanmoins établi des divisions du tronc parfaitement nettes, peu de temps après la mue qui délivrera le premier embryon cyclofoïde, alors que la paroi des segments de ce dernier se sépare çà et là de la cuticule métanauplienne (Pl. VII, fig. 4). La première division correspond au céphalon; l'exoderme y est encore constitué par d'assez grosses cellules chitinogènes nucléées, bien visibles sur les bords de la carapace. De chaque côté du corps, un peu au-dessous de la première maxille, on aperçoit une tache claire, au contour polygonal, présentant çà et là des apparences de quelques (2-3) noyaux : c'est un sac à paroi mince, rempli de liquide hyalin; il représente la glande du test rudimentaire. Elle est située dans la partie postérieure du céphalon, dans le somite de la seconde maxille et n'avait pas encore été observée chez Doropygus ou dans les genres voisins. 
A la suite du céphalon viennent encore cinq divisions du tronc, dont l'avantdernière est la plus courte et la dernière la plus longue. C'est dans celles-ci que se trouve la portion terminale de l'intestin. L'extrémité du tronc est occupée par deux pièces cylindriques à extrémité distale arrondie, qui se terminent chacune par quatre petites épines et qui portent dorsalement les deux soies furcales divergentes : c'est la furca (PI. VII, fig. $4, f$ ).

$3^{0}$ La première larve cyclopoïde, débarrassée de la dépouille métanauplienne, mesure $0,46 \mathrm{~mm}$. (PI. VII, fig. 5). Tous les segments, ébauchés dans le métanauplius, ont pris leur liberté; le corps se compose ainsi d'un céphalon, de cinq segments et de la furca. L'antennule compte cinq articles armés de soies au bord antérieur et à l'extrémité; elle est pourvue de trois bâtonnets réfringents sensoriels, sur le dernier et l'antépénultième articles. L'antenne est encore biramée : l'exopodite réduit, 3-articulé, se termine par uné épine recourbée en griffe et quelques soies. Je n'insiste pas sur les pièces buccales, déjà fort semblables dans leur morphologie aux appendices de l'adulte.

Les deux premières paires de pattes thoraciques, propres à la natation, comprennent un protopodite 2-articulé et deux rames également 2-articulées, pourvues d'épines et de soies sur leurs bords. Le caractère le plus saillant de ces pattes natatoires du premier embryon cyclopoïde. réside dans la longueur considérable - égale à celle de l'appendice entier - de l'épine terminale de l'exopodite. La troisième paire de pattes thoraciques, malgré la base et les deux rames sétifères reconnaissables, est encore emprisonnée au-dessous de la cuticule et incapable de fonctionner. Le quatrième segment thoracique, qui est libre, ne porte pas encore de bourrelet appendiculaire bien net. Le segment qui \e suit est de beaucoup plus long; il présente sur sa face dorsale l'ouverture anale, et porte à son extrémité les deux pièces furcales. Ce segment furcal ou anal, simple après la mue, se divise vers la fin de la période du $\mathrm{I}^{\mathrm{er}}$ cyclopoïde, en deux segments réunis par la cuticule de la larve. Les pièces furcales donnent insertion à différentes soies, dont l'une est marginale, une autre dorsale, et les quatre dernières terminales. Parmi celles-ci, l'interne est très développée et très longue, tandis que les trois autres sont à peine indiquées comme rudiments de soies en voie de formation (Pl. VII, fig. 5, $f$ ).

Le tube digestif est déjà assez complètement organisé dans la première larve cyclopoïde : l'intestin moyen est encore rempli d'une masse compacte de globules vitellins colorés en bleu-verdatre : mais l'intestin postérieur est pourvu de parois cellulaires nettes, avec les noyaux de leurs cellules disposés en file simple à la 
face dorsale du canal (i). Le système nerveux ventral forme une masse homogène, légèrement renflée au niveau des segments; il ne s'étend pas au-delà du troisième somite post-céphalique.

La sortie de l'embryon s'effectue, dans toutes les mues naupliennes et cyclopoïdes, par une fente établie suivant le bord antérieur du céphalon.

$4^{0} \mathrm{La}$ deuxième larve cyclopoide mesure $0,5 \mathrm{I} \mathrm{mm}$. Elle est composée (PI. VII, fig. 6) de sept segments et de la furca. Les antennules sont 6-articulées, par la subdivision de l'article terminal des antennules de la larve précédente. Les antennes sont 3-articulées et dépourvues d'exopodite; elles sont préhensiles et ressemblent presqu'exactement aux antennes de l'adulte. De même pour les pièces buccales.

A ce stade, les somites thoraciques sont séparés. Les trois premiers portent chacun une paire de pattes biramées, avec l'endopodite et l'exopodite 2-articulés dans les premières $\left(p^{1}\right.$ et $\left.p^{2}\right)$, simple dans la dernière $\left(p^{3}\right)$. Les deux rames de chaque patte thoracique sont propres à la natation; elles portent, sur leurs bords, des épines en poignard à bords denticulés et des soies barbelées. Un des caractères les plus nets de ces pattes, comparées aux mêmes appendices du stade précédent, consiste dans la forme définitive, persistant chez l'adulte, de ces épines en poignard et particulièrement de la grande épine terminale de l'exopodite, qui est ici plus courte, plus solide et denticulée seulement vers l'extérieur.

Le quatrième segment thoracique porte sous la cuticule ventrale un épaississement exodermique, rudiment de la quatrième paire de pattes. Le cinquième est encore absolument cylindrique. Les soies terminales de la furca sont profondément modifiées; la plus longue est actuellement la plus interne des deux médianes, la plus externe étant encore plus longue que les deux autres.

Les organes internes de la première larve cyclopoïde tendent à prendre leur constitution définitive. Le tube digestif est alors un canal à paroi épithéliale très nette, dans laquelle on ne trouve plus que quelques amas très réduits de granulations deutoplasmiques verdâtres. Le système nerveux s'est définitivement constitué en une masse cérébrale, un collier œsophagien et une chaîne ventrale. Les glandes du test sont encore très visibles. Le mésoderme a pris l'aspect d'un tissu conjonctif. Dans les rames des pattes natatoires, comme dans la furca, se trouvent de grosses cellules glandulaires, à contenu globulaire et réfringent.

Sous cette forme les embryons de Doropygus nagent très vivement dans les vases où ils sont conservés, sans se diriger plus particulièrement vers les points éclairés

(1) Comme l'a déjà constaté Grobaen chez Calamus (Cotochilus), et Cr.aus chez les Phronimidés (Amphipodes). 
comme c'est le cas des embryons plus jeunes et surtout des nauplius et des métanauplius. Au contraire, la deuxième larve cyclopoïde se tient de préférence en repos sur le fond, mais elle est encore très capable de fuir rapidement au milieu du liquide quand on cherche à la saisir au moyen d'une pipette. Le développement considérable des faisceaux musculaires destinés à mettre en mouvement les pattes natatoires explique suffisamment cette mobilité, qui tend à disparaittre dans le cours du développement.

$5^{0}$ En effet, sur le fond des bocaux où j'élevais ces embryons, j'ai toujours trouvé, à la fin de la période d'élevage, des larves moins agiles qui offrent un aspect tout différent. Ces larves au troisième stade cyclopoïde mesurent de o,47 à o, $5 \mathrm{I} \mathrm{mm}$. de longueur selon le degré de courbure du corps et la contraction des segments. Au lieu de nager parfaitement aplatis et de faire dans le liquide des bonds atteignant parfois jusqu’à 2 centimètres, ces larves se tiennent courbées du còté ventral et nagent à la façon des parasites en appuyant les extrémités du corps sur la surface qu'elles parcourent. Cette démarche, particulière aux formes adultes semi-parasites telles que $N$ otodelphys, Lichomolgus, etc., est en relation avec l'état de régression relative que présentent les muscles moteurs des appendices. Ceux-ci (PI. VII, fig. 7) ne forment plus de faisceaux compacts et richement striés, mais bien de petits faisceaux minces et disjoints, avec de petites stries courtes et serrées comme elles existent dans l'adulte (PI. VI, fig. 4). En résumé, au $3^{\mathbf{e}}$ stade cyclopoïde, la musculature et la démarche de l'embryon indiquent, dès cette époque, l'adaptation de l'animal à la vie parasitaire. C'est au $2^{\mathrm{e}}$ stade cyclopoïde qu'il abandonne la surface de ía mer, où il nageait librement à la façon des Copépodes libres, pour aller séjourner au fond et rechercher l'abri que lui offre son hòte habituel. Une fois établi dans celui-ci, les dégradations de l'organisme se succèdent rapidement; la première affecte la musculature comme je viens de l'exposer.

Le troisième stade cyclopoïde diffère des stades doropygiens qui le suivront, par l'existence des soies furcales. Il possède, ęn outre des appendices thoraciques du stade précédent, une paire de replis appendiculaires saillants et terminés par une soie barbelée (Pl. VII, fig. 10) qui formeront la $4^{e}$ paire de pattes.

Le tube digestif présente sensiblement les caractères du stade précédent, avec sa paroi cellulaire parsemée d'amas deutoplasmiques résiduels. Le cerveau est définitivement constitué, et il ne reste plus des lobes dorsaux du cerveau secondaire que deux faibles saillies latérales du ganglion supra-œsophagien (Pl. VII, fig. 7). La chaine ventrale s'étend au-dessous du tube digestif jusqu'au $3^{\mathrm{e}}$ somite thoracique. Cette chaîne présente encore trois renflements ganglionnaires séparés : le premier, plus considérable, correspond aux appendices buccaux et à la première 
paire de pattes ; les deux derniers, particulièrement situés dans les deux premiers somites thoraciques, correspondent aux deux dernières pattes natatoires. La chaine ventrale subit une sorte d'arrèt de développement dans le cours de l'évolution ultérieure, puisque chez l'adulte elle ne s'étend pas au-delà du I ${ }^{\text {er }}$ somite thoracique que dépassent seuls les nerfs destinés aux pattes et à l'abdomen.

Dans le tissu conjonctif se sont développées de grandes cellules à protoplasma granuleux et à petit noyau nucléolé (Pl. VII, fig. 9, cm) qui fourniront plus tard les amas connus, chez l'adulte, sous le nom de corps graisseux.

Les organes génitaux, en voie de formation, sont encore constitués par de grandes cellules claires, à gros noyau, réunies au nombre de 3 ou 4, au-dessus du tube digestif, dans la ligne médiane et à la limite du céphalon et du premier somite thoracique (PI. VII, fig. 7 et $9, r g$ ).

$6^{0}$ Dans la cavité branchiale des Ascidies (Molgules et Phallusies), on trouve tous les stades jeunes ayant la forme doropygienne. Le premier est seul intéressant pour nous, parce qu'il relie les deux périodes de développement : en liberté et à l'état de parasite.

La forme du premier stade doropygien est celle du $3^{\mathrm{e}}$ stade cyclopoïde auquel il succède immédiatement. Cet embryon possède 4 somites thoraciques appendiculés en outre du céphalon et de la furca, un somite thoracique sans appendice articule et le segment préfurcal. Les soies de la furca ont fait place aux crochets et aux épines de l'animal adulte.

\section{BONNIERILLA LONGIPES Kerschner.}

Le nauplius est de forme plus ramassée et mesure $0,27 \mathrm{~mm}$. de longueur. Peu de temps après l'éclosion, apparaissent nettement sur la face ventrale les rudiments des appendices post-mandibulaires qui se developpent ici beaucoup plus rapidement et suivant un mode plus condensé que chez Doropygus gibber. Je n'insisterai pas spécialement sur les caractères communs aux embryons de ces deux formes et je signalerai seulement les différences importantes.

La masse endodermique est colorée en vert ; sa partie antérieure s'accole aux granulations jaunes qui fournissent la région antérieure du tube digestif, et sa partie postérieure disparait dans une tache pigmentaire rouge orangée qui s'étend autour des tissus mésodermiques.

Dans le métanauplius, qui mesure 0,27 à $0,28 \mathrm{~mm}$., apparaissent les diverses particularités déjà signalées pour Doropygus, concernant la formation des somites et 
appendices, et l'évolution des organes intérieurs, du cerveau secondaire, des glandes du test, du tube digestif et de la $3^{\mathrm{e}}$ partie de l'œil de l'adulte. Les modifications que subit la masse endodermique pour aboutir à la constitution du canal digestif sont de mème ordre; elles sont suffisamment nettes dans mes dessins pour qu'il soit superflu d'insister sur leur description (PI. X, fig. 3, 4, 5).

La première larve cyclopoïde ( $\mathrm{Pl}$. X, fig. 7) mesure à l'éclosion $0,45 \mathrm{~mm}$. de long. Elle montre encore les caractères distinctifs de ce stade dans la division du corps en 7 segments et une furca, dans les antennules 5 -articulées, dans les longues épines terminales de l'exopodite des pattes natatoires, dans la $3^{\mathrm{e}}$ patte thoracique encore recouverte par le tégument extérieur mais déjà biramée. Le tube digestif y est complètement organisé en canal digestif, avec des restes de deutoplasme dans sa partie antérieure et une paroi cellulaire bien nette, à noyaux dorsaux, dans sa partie terminale.

La deuxième larve cyclopoïde est le dernier stade embryonnaire que j'aie pu obtenir. Elle atteint $0,47 \mathrm{~mm}$. de longueur et se distingue par sa forme générale (PI. X, fig. 8), ses 8 segments et sa furca, ses 3 paires de pattes natatoires avec épines terminales courtes et denticulées vers l'extérieur. Les antennules sont composées de 6 articles. dont le premier porte, antérieurement et vers son extrémité, une pointe losangique qui correspond à l'épine analogue en mème situation chez l'adulte.

En résumé, dans la première période libre du développement, l'embryologie de Bonnierilla longipes concorde, dans tous ses caractères, avec celle de Doropygus gibber, et vient confirmer les diagnoses que j'ai établies pour les divers stades embryonnaires.

\section{NOTODELPHYS AGILIS THORELl.}

Le nauplius (PI. XII, fig. I) mesure $0,33 \mathrm{~mm}$. Son corps effilé, presqu'ellipsoîdal, montre nettement sur la face dorsale les deux parties du bouclier et de l'abdomen. Les appendices sont constitués sujvant le type commun aux espèces précédemment décrites; ils sont très puissamment organisés et le nauplius de Notodelphys est particulièrement agile.

L'exoderme se présente dans toute l'étendue du bouclier dorsal, sous forme d'une couche chitinogène cellulaire très nette. Les cellules exodermiques sont plus petites et plus serrées sur les cỏtés de l'œil, là où les lobes dorsaux du cerveau se détachent de l'exoderme. Le cerveau secondaire forme encore une paire de masses cellulaires déjà libres vers la ligne médiane, mais attachées à l'exoderme sur 
les côtés. Dans la partie profonde de ces masses, on distingue un contour polygonal (généralement pentagonal) trés nettement accusé, et qui présente toutes les apparences d'un orifice béant dans la masse du cerveau secondaire; je crois devoir considérer cette figure polygonale comme produite par les limites du contact entre les lobes secondaires du cerveau et le ganglion cérébral primitif. En effet dans le cours du développement, lorsque les lobes secondaires se fusionnent complètement avec le ganglion primaire, cette apparence, si nette au début, s'efface; elle disparait chez les larves cyclopoïdes.

La constitution de l'œil nauplien, au début de la vie larvaire, présente un grand intérêt. Directement placé au-dessous de l'exoderme, en avant du ganglion cérébral primitif, cet œil bipartite comprend :

$I^{0}$ deux cupules vivement teintées de carmin, accolées l'une à l'autre sur la ligne médiane par leur côté convexe ;

$2^{0}$ deux parties réfringentes occupant très exactement la dépression concave de ces cupules et montrant chacune, dans leur masse, deux corps polyédriques de réfringence plus considérable, qui indiquent la pluralité primitive des bâtonnets réfringents dans les parties en apparence plus simples de l'œil des Copépodes (I).

En outre des éléments pigmentaires vraiment fonctionnels décrits ici, il existe comme dans la plupart des Copépodes ascidicoles, des taches pigmentaires d'apparence variable qui s'étendent autour de l'œil, en!re les lobes secnndaires dorsaux et autour du ganglion primaire du cerveau ; elles composent une figure assez complexe, colorée en rouge carmin moins vif. Cet amas pigmentaire se découpe de plus en plus au cours du développement; il a complètement disparu dans l'adulte.

La masse endodermique du nauplius venant d'éclore (Pl. XIII, fig. I) est colorée en vert dans la plus grande partie de son étendue, avec une tache superficielle d'un bleu violet dans sa région antérieure, spécialement dans l'espace en V demeuré libre entre les muscles dorsaux de l'antennule. A l'extrémité postérieure se trouve une petite enclave d'un brun jaunâtre située au point d'origine de l'intestin terminal. A l'avant et sur les côtés antérieurs de la région violette, un grand nombre de sphérules jaunes distinguent les origines de l'intestin antérieur.

Les changements de coloration des organes digestifs marchent de pair avec les transformations qui s'y opèrent, comme on peut en juger par la suite du développement.

- (i) J'ai pu également constater, sur des coupes, la pluralité de ces éléments dans les formes de copulation de Lernea branchialis; on connaît des dispositions analogues chez des Calanidés (Claus (5i), Grenazher). 
En effet, dès le début de la période métanauplienne (PI. XII, fig. 3), la portion violette s'étend de plus en plus vers la région postérieure demeurée verte, pour occuper enfin, peu de temps avant la mue qui fournira la première larve cyclopoïde, toute la masse endodermique (Pl. II, fig. 4). Plus tardivement encore et lorsque le métanauplius est sur le point de muer et montre bien différenciés sous sa cuticule tous les segments du corps de la larve cyclopoïde (PI. XII, fig. 5), les matériaux deutoplasmiques, qui oblitèrent le canal digestif en voie de formation, ont pris une jolie teinte violette qu'ils conservent encore au début de la première période cyclopoïde (PI. XII, fig. 7). Et pourtant, à ce dernier stade, le canal digestif est déjà complètement organisé dans ses trois régions distinctes; l'état définitif est alors promptement acquis, par résorption graduelle des globules graisseux colorés auxquels est dû cet aspect de l'organe.

Dans la $i^{\text {re }}$ larve cyclopoïde plus âgée. comme dans la $2^{\mathrm{e}}$ larve cyclopoïde, il reste très peu de deutoplasme. Les parois du tube digestif sont nettement cellulaires; dans l'intestin terminal, elles montrent avec une grande netteté cette localisation des noyaux sur la face dorsale (PI. XII, fig. 8 , i) que j’ai déjà signalée dans Doropygus et Bomierilla.

Le mésoderme secondaire apparait dans Notodelphys agilis avec tous les caractères que j'ai indiqués précédemment chez Doropygus gibber. Au stade nauplius, immédiatement après l'éclosion, une seule grande cellule polaire mésodermique se trouve au-dessus de la tache brun-jaunâtre de l'intestin postérieur, en contact immédiat avec l'endoderme chargé de deutoplasme (PI. XII, fig. I, cpm). Cette cellule est constituée par un protoplasme hyalin et présente un gros noyau nucléolé. Elle se trouve plus tard accompagnée d'une ou parfois de deux autres cellules, également impaires, qui forment ensuite par leur division deux files symétriques de deux ou trois cellules paires : celles-ci sont les bandes mésodermiques secondaires, placées sur les cótés latéraux de la masse endodermique. Ainsi se trouvent entièrement confirmées la description et les conclusions fournies plus haut au sujet de Doropygus gibber.

Le métanauplius mesure aux diverses époques de son existence, de 0,34 à 0,37 $\mathrm{mm}$. de longueur. Ses caractères essentiels concordent (Pl. XII, fig. 3, 4, 5,6) avec ceux que j'ai décrits pour l'embryon correspondant de Doropygus. J'insisterai seulement ici sur la destinée des replis appendiculaires de la seconde maxille. Naissant avec tous les caractères d'une seule paire d'appendices, les secondes maxilles internes et externes se séparent bientòt comme deux appendices distincts jusqu'à leur base d'insertion (PI. XII, fig. $3, m x^{2}$ ), et elles évoluent alors séparément pour acquérir d'une façon indépendante leurs caractères définitifs. Dans le 
métanauplius, très peu de temps avant la mue qui fournira le cyclopoïde, ces appendices ont acquis tous les caractères différentiels, mais ils conservent encore leur situation primitive de pièces nées côte à côte sur la même ligne transversale et par conséquent aux dépens d'une mème et unique paire d'appendices (P̉. XII, fig. 6, $m x^{2} e$ et $\left.m x^{2} i\right)$.

La première larve cyclopoïde (PI. XII, fig. 7) présente dans ses antennules 5-articulées, ses antennes, les longues épines terminales de ses deux paires de pattes natatoires, et sa grosse soie furcale, tous les caractères distinctifs déjà relevés dans le mème embryon pour d'autres espèces. Elle mesure $0,55 \mathrm{~mm}$. de longueur.

La deuxième larve cyclopoïde (P1. XII, fig. 8) a des antennules 7-articulées, des antennes sans exopodite, trois paires de pattes natatoires avec courtes épines terminales denticulées seulement à l'extérieur, et sur Ja furca, deux soies terminales internes plus grandes que les terminales externes. Elle mesure o,6o mm. de long.

Le mode de vie moins particulier des Notodelphys adultes enlève aux stades ultérieurs, semi-parasites dans les Molgules, l'intérèt spécial qu'ils présentent chez Doropygus.

\section{NOTODELPHYS ALLMANI THORELL.}

Le développement de cette espèce concorde dans tous ses détails morphologiques avec ce qui a été dit précédemment pour Notodelphys agilis. Ainsi qu'on peut facilement s'en convaincre par la comparaison des embryons figurés Pl. XII et P1. XIII, les colorations seules diffèrent.

Les embryons ont aux différents stades, dans Notodelphys allmani, la mème longueur que dans $N$. agilis, mais ils sont plus élargis, plus trapus, spécialement durant les périodes nauplienne et métanauplienne.

Dans le nauplius récemment éclos (PI. XIII, fig. I), la masse endodermique, très foncée, est d'une couleur vert-olive un peu jaunâtre avec de grosses sphérules deutoplasmiques de coloration moins vive. A sa partie postérieure se détachent les cellules polaires mésodermiques impaires. A sa partie antérieure et sur la face ventrale des lobes cérébraux secondaires, se dessine une large tache de pigment orangé qui étend ses ramifications digitées vers la base des appendices naupliens en pénétrant même dans le basipodite de la $2^{\mathrm{e}}$ paire.

A la partie postérieure et au point d'origine de l'intestin terminal, se trouve un amas de 6 à 8 globules huileux d'un rouge cerise, qui entourent à peu près la cellule polaire mésodermique (PI. XIII, fig. I). 
Sur les còtés de la masse endodermique, dans le tiers postérieur de la larve, se trouve à droite et à gauche, au-dessous de l'exoderme, une série de granulations formant une trainée pigmentée en rouge (PI. XIII, fig. $1 e$ ).

Au-dessus du point d'insertion de la mandibule, de chaque côté du tronc, existent encore deux amas linéaires de globules d'un beau rouge carmin (PI. XIII, fig. $\mathbf{I}, d)$. Par leur situation, ces divers amas pigmentaires se trouvent reliés au tissu mésodermique et ils peuvent, par leur sort ultérieur au cours du déve'oppement, renseigner très utilement sur les transformations de ce tissu.

Peu de temps après l'éclosion, le nauplius présente encore sensiblement la mème constitution fondamentale : toutefois, on peut constater, sur la face ventrale, la transformation rapide de l'endoderme qui prépare celle du tube digestif. Les parties antérieure et latérales de la masse deutoplasmique prennent, au-dessus du fond vert-olive des cellules non transformées, une jolie teinte verte (Pl. XII, fig. 2) qui va s'étendre de plus en plus, jusqu'à envahir tout l'endoderme (Pl. XIII, fig. 3 et 4). Ces changements de couleur, marchant de pair avec la constitution graduelle de la paroi digestive, s'accentuent encore dans le métanauplius où la région antérieure de l'endoderme devient violette (PI. XIII, fig. 3, 4), teinte que prend d'ailleurs tout le tube digestir dans la première larve cyclopoïde (Pl. XIII, fig. 5).

En mème temps que le tube digestif se constitue dans les divers stades successifs, les taches pigmentaires laiérales $(e)$ changent de forme et de position. Placées sur les còtés du corps de la larve nauplienne (PI. XIII, fig. I et 2), les lignes de granulations pigmentaires $e$ sont reportées vers la face dorsale, par suite du développement rapide et considérable des tissus de la face ventrale qui prolifèrent activement pour constituer les appendices et les divers ganglions ventraux de la larve cyclopoïde. En même temps que cette transformation, s'effectue le développement du mésoderme secondaire, lequel est déjà très avancé chez le nauplius représenté dans la fig. 2 de la Planche XIII. Cette prolifération du mésoderme a pour conséquence l'allongement du grand axe de l'embryon dont les divers somites s'emboitent successivement les uns dans les autres en restant emprisonnés sous la cuticule métanauplienne. C'est ainsi qu'au moment de l'apparition des étranglements latéraux qui préludent à l'établissement des somites, on voit, dans le métanauplius, les taches latérales situées sur la face dorsale se rapprocher de la ligne médiane et s'allonger suivant l'axe de l'embryon, de manière à former deux bandes pigmentaires rouges avec des élargissements assez considérables vers l'avant (PI. XIII, fig. $\left.4, e, e^{\prime}, e^{\prime \prime}, e^{\prime \prime \prime}\right)$. Je ferai remarquer que ces taches plus larges des bandes pigmentaires sont formées successivement d'avant en arrière, dans le mème ordre que les somites. 
Dans le premier stade cyclopoïde, ces bandes se sont allongées dans des proportions considérables. Au lieu d'être continues, comme dans le métanauplius, elles se sont segmentées en un certain nombre de taches disposées avec assez d'ordre dans les diverses régions successives, et on rencontre les taches $e^{\prime}, e^{\prime \prime}, e^{\prime \prime \prime}$, respectivement dans les $\mathrm{I}^{\mathrm{er}}, 2^{\mathrm{e}}$ et $3^{\mathrm{e}}$ somites thoraciques.

Les autres groupes de globules rouges ne se modifient guère jusqu'à leur disparition, comme on peut s'en convaincre par les dessins où j'ai représenté divers stades embryonnaires. Ils ne sont plus visibles dans les larves cyclopoïdes.

Je signalerai seulement, dans les deux premières larves cyclopoïdes de Notodelphys allmani, la grande neteté avec laquelle on peut observer la disposition curieuse des noyaux de l'intestin terminal, à la face dorsale de l'organe.

Tous les autres caractères du développement concordent dans les deux espèces de Notodelphys que j'ai considérées ici. Mais je ferai observer que, dès le $\mathrm{I}^{\mathrm{er}}$ stade cyclopoïde, il est possible d'après la coloration des amas pigmentaires mésodermiques, du segment præfurcal et du céphalon ainsi que d'après la forme des pièces furcales, de distinguer entre les larves de ces deux espèces.

\section{DOROIXYS UNCINATA KeRsChner.}

Dans l'embryon nauplien, encore renfermé dans la membrane de l'œuf et mesurant avant l'éclosion $0,17 \mathrm{~mm}$. de longueur sur $0,12 \mathrm{~mm}$. de largeur, on peut constaler avec une grande netteté, chez Doroixys uncinata, diverses particularités des plus intéressantes.

Lorsque l'œil nauplien est en voie de formation (Pl. XV, fig. 1, $\propto$ ), et que les 3 paires d'appendices se présentent déjà avec l'ébauche de leurs caractères différentiels $(a, A, m d)$, l'endoderme (end) forme une grosse masse pleine, d'une jolie teinte terre de Sienne mèlée de jaune, et composée de grosses cellules polyédriques par pression réciproque.

L'exoderme (exd $)$ est épaissi sur la face ventrale et composé d'assez grandes cellules nucléées, transparentes. A la partie antérieure du corps, au-dessous de l'œil, un premier épaississement exodermique fournit le ganglion cérébral primitif (cer). A la partie postérieure, une petite dépression de l'exoderme loge, de chaque côté, un rudiment en forme de bouton (f) qui correspond à la soie furcale du nauplius après l'éclosion.

A la face ventrale de la masse endodermique, entre l'endoderme et l'exoderme, se trouve une large bande de granulations fines, jaunàtres, qui passent insensiblement à l'endoderme deutoplasmique et qui représentent le premier indice 
des modifications cellulaires qui fourniront le tube digestif de la larve cyclopoïde aux dépens du vitellus nutritif endodermique de l'embryon moins âgé. A la partie postérieure de cette bande endodermique, et au-dessous de l'intestin, se rencontrent deux grosses cellules qui paraitront reportées vers l'arrière à l'éclosion; elles représentent les cellules polaires mésodermiques $(\mathrm{cpm})$, car à cette époque, le mésoderme primitif a évolué depuis longtemps en vue de constituer les appendices naupliens et les muscles - encore non différenciés - qui les manœuvrent.

Dans le nauplius qui vient d'éclore, la longueur atteint $0,20 \mathrm{~mm}$. Le corps est presqu'absolument ellipsoïdal, et présente, en dehors des caractères communs aux nauplius des genres déjà examinés plus haut, les particularités suivantes.

Par une condensation curieuse des premiers phénomènes de la métamorphose, l'ébauche des pattes thoraciques est, chez Doroixys, plus avancée que chez les Copépodes voisins dont il a été question précédemment. L'exoderme de la face ventrale parait donc fortement épaissi et le stade métanauplius ne tarde pas à sortir de la cuticule nauplienne.

L'embryon métanauplien mesure $0,21 \mathrm{~mm}$. de long et présente, sur sa face ventrale (PI. XV, fig. 3); tous les rudiments des appendices ordinaires avec les caractères essentiels que nous connaissons ailleurs. Sur la face dorsale, apparaissent aussi, vers la fin de cette période de la vie larvaire, les divers segments du stade suivant.

La première larve cyclopoïde ( $\mathrm{Pl}$. XV, fig. 5) mesure 0,34 à $0,36 \mathrm{~mm}$., suivant l'extension que le grand prolongement ou rostre frontal $(f r)$ peut prendre en avant.

Ici, comme chez Doropygus, Bonnierilla et Notodelphys, la caractéristique de ce stade se trouve dans les antennes, les épines terminales de l'exopodite des pattes natatoires fonctionnant alors au nombre de deux paires, la soie terminale interne des pièces furcales.

Bientòt apparaissent sous la cuticule les segments et le $4^{e}$ appendice qui existent dans la $2^{\mathrm{e}}$ larve cyclopoïde. Celle-ci a la même taille: $0,34 \mathrm{~mm}$. Ses caractères restent les mèmes que dans les autres genres.

Au moment de la mue qui doit terminer sa période d'existence, la $2^{\mathrm{e}}$ larve cyclopoïde, jusque-là très agile, tombe au fond de l'eau et ne se livre qu'à des mouvements très limités. Il en sort une larve d'aspect tout différent, de même taille, inapte à la natation, privée de soie furcale, avec tous les caractères d'un Copépode dégradé pour le parasitisme (PI. XV, fig.9). Cet embryon compte, entre le céphalon et la furca, 7 segments dont les 3 premiers portent des pattes natatoires, à soies barbelées, réduites $\left(p^{1}, p^{2}, p^{3}\right)$, et une quatrième paire, encore à l'état de repli 
appendiculaire sous-cutané $\left(p^{4}\right)$. En somme, du deuxième embryon cyclopoïde, sort immédiatement et sans interposition d'une période semi-parasitaire (comme nous l'avons vu chez Doropygus), la première larve parasite, qui ne présente pas encore, sur les côtés postérieurs du thorax, les deux épines recourbées et pointues de la forme adulte.

Ces épines existent d'ailleurs dans l'avant-dernier stade de développement de la femelle, mesurant $0,70 \mathrm{~mm}$., où se trouvent aussi les cinq paires de pattes thoraciques (Pl. XV, fig. II).

Dans la première larve parasite, le système nerveux ventral comporte (Pl. XV, fig. Io) un premier renflement ganglionnaire innervant les pièces buccales et fournissant les nerfs de la $\mathrm{I}^{\mathrm{re}}$ paire de pattes $\left(p^{\prime}\right)$. Un deuxième renflement fournit les nerfs de la deuxième paire, et un $3^{\mathrm{e}}$ ceux de la troisième paire. Au delà de cette dernière masse un gros cordon nerveux se dirige encore vers l'abdomen de la larve, mais je n'ai pu le suivre en détail jusque-là. Chez l'adulte, le système nerveux ventral est, comme chez Doropygus, fortement réduit, car il ne dépasse guère le $\mathrm{I}^{\mathrm{er}}$ somite thoracique.

\section{ENTEROCOLA FULlgens van Beneden.}

A l'éclosion, le nauplius (Pl. XIX, fig. I) mesure $0,27 \mathrm{~mm}$. De forme très peu allongée, ovalaire, il présente, avec les 3 paires d'appendices ordinaires, des replis appendiculaires déjà très avancés dans leur développement et qui formeront les maxilles et pattes natatoires. La seconde maxille constitue un lobe simple, sans trace de segmentation en $2^{\mathrm{e}}$ maxille interne et $2^{\mathrm{e}}$ maxille externe. Les matériaux deutoplasmiques rouges, si abondants et d'une si vive couleur dans le nauplius encore enfermé dans la membrane de l'œuf, ont diminué considérablement; le tube digestif est déjà presque formé et d'une teinte rose délicate avec quelques gros globules graisseux plus vifs. Les rudiments de l'intestin anterieur et postérieur sont jaunâtres. L'œil est primitivement bipartite, mais il devient bientòt tripartite par adjonction de l'élément ventral.

Sous l'enveloppe nauplienne, et sans qu'il soit constitué un embryon métanauplien, apparaissent bientòt les somites de la première larve cyclopoïde (PI. XIX, fig. 2).

Au premier stade cyclopoïde (PI. XIX, fig. 3, 4), le corps compte, en outre du céphalon et de la furca, 5 segments; les antennules sont 4 -articulées; l'antenne est biramée avec l'exopodite en voie de régression; il existe deux paires de pattes thoraciques biramées, avec une grande épine terminale sur l'exopodite, et une autre 
paire de pattes biramées ne pouvant pas encore servir à la natation. La soie furcale terminale la plus grande est la plus interne. Le prolongement frontal est très allongé et atteint la région buccale qu'il recouvre en partie; son extrémité distale est élargie en spatule. Le tube digestif en voie de formation présente deux parois bien nettes contenant encore un assez grand nombre de globules deutoplasmiques rougeâtres.

Les pièces buccales de l'embryon (Pl. XIX, fig 5) sont alors des plus intéressantes. Sur le còté du bulbe buccal saillant, les mandibules en vole de régression sont de petits appendices à cuticule plissée et presque fripée, avec une base et deux rames où l'on ne peut distinguer nettement une véritable articulation. Les premières maxilles, situées un peu en arrière, sont 2 -articulées: l'article basilaire est palpiforme, terminé par deux courtes soies pointues. La seconde maxille est un appendice simple, comme on pouvait le supposer d'après son mode de développement dans le nauplius. Elle se compose également de deux articles, le premier constituant une base tres solide sur laquelle s'articule le second, recourbé en forme de griffe, aveç une épine solide à son extrémité et une petite soie accessoire vers l'extérieur.

La deuxième larve cyclopoïde (PI. XIX, fig. 7) compte un segment de plus et mesure $0,47 \mathrm{~mm}$. de long. Le prolongement frontal est entré en régression et ne forme plus qu'une saillie plissée en avant du céphalon. Les antennules sont 5-articulées et portent un long bâtonnet sensoriel réfringent avec de longues soies effilées sur le dernier article. Les antennes sont simples, composées de 3 articles cylindriques et allongés: elles se terminent par une griffe recourbée, accompagnée de quelques petites soies. Les pattes thoraciques des $1^{\mathrm{re}}, 2^{\mathrm{e}}$ et $3^{\mathrm{e}}$. paires sont disposées pour la natation, avec une épine terminale courte et denticulée à l'extérieur de leur exopodite. Dans la furca, la soie terminale la plus longue est la médiane interne.

Dans la région buccale, les modifications des appendices sont peu considérables. La mandibule n'est presque plus visible, et on distingue seulement un petit tubercule à son point d'insertion. La première maxille s'allonge, spécialement en ce qui concerne le prolongement interne du premier article. La seconde maxille est une griffe solidement organisée.

Tandis que la $\mathrm{I}^{\mathrm{re}}$ larve cyclopoïde nage très activement à la façon d'un Copépode libre, la $2^{\mathrm{e}}$ présente plutôt ia démarche indécise et sautillante d'un semiparasite. A la fin deson existence, quand elle se prépare à muer, elle tombe même au fond du vase où l'on élève les embryons et ne se meut presque plus. Quand on l'examine à cette époque, on observe à l'intérieur de la cuticule détachée du 
corps de la larve, une autre larve d'aspect tout différent et dans laquelle presque tous les appendices sont tombés en régression.

Avec beaucoup de peine, j'ai pu obtenir cette transformation de la $2^{\text {e larve }}$ cyclopoïde en $\mathrm{I}^{\mathrm{er}}$ stade entérocolien ou $\mathrm{I}^{\text {er }}$ stade femelle, et cela à plusieurs reprises, dans les mois de juin, juillet et août, c'est-à-dire aux époques où les mâles d'Enterocola fulgens sont le plus rares.

La première larve parasite (Pl. XIX, fig 8) mesure environ $0,45 \mathrm{~mm}$. de longueur; c'est un animal d'aspect vermiforme, recourbé sur la face ventrale et composé de 7 segments et d'une furca recouverts d'une cuticule mince et souple qui rend les limites des divisions moins nettes que dans les stades précédents. L'animal est totalement privé des organes natatoires si bien organisés précédemment. Les antennules $(\mathrm{Pl}$. XIX, fig. $8,9,10, a)$ sont très courtes et composées de 2 articles, avec quelques courtes épines sur leur bord antérieur. Les antennes ( $A$ ) sont de simples petits moignons lamellaires, de forme triangulaire. Il n'y a plus de traces des mandibules. Les premières maxilles $\left(m x^{1}\right)$, encore biarticulées, ont acquis par l'apparition de dents sur l'article basilaire la forme d'une pièce masticatrice surmontée d'un lobe foliacé et denticulé qui correspond au second article. Les secondes maxilles $\left(m x^{2}\right)$ toujours 2-articulées, sont plus trapues et forment une griffe très solide. Les pattes thoraciques au nombre de trois paires sont des appendices (Pl. XIX, fig. I7) à base biarticulée et surmontée par deux rames simples que terminent de petites épines en crochets. Elles peuvent servir à la locomotion comme des griffes s'accrochant sur les aspérités de l'hòte dans lequel vit l'animal; sur une surface lisse comme le fond d'un vase de verre, les mouvements sont devenus presqu'impossibles et se réduisent à des flexions alternativement ventrales et dorsales. La quatrième paire de pattes $\left(p^{4}\right)$ est en voie de formation. La furca se compose de deux pièces foliacées, sans soies ni épines; son extrémité amincie en pointe mousse peut aider, dans de faibles limites, à la locomotion. A ce stade, la cuticule chitineuse mince s'est couverte en beaucoup d'endroits, sur la face ventrale, d'une multitude de petits cils disposés en séries linéaires que l'on peut constater le plus aisément sur la lèvre supérieure (Pl. XIX, fig. 10, $l s$ ) ou sur les pattes thoraciques (Pl. XIX, fig. 17, $p^{1}$ ).

Le tube digestif est un canal à paroi mince, glandulaire dans sa région moyenne et qui a conservé une légère teinte rosée des restes d'éléments deutoplasmiques.

Il est ainsi constaté que, chez Enterocola, la femelle subit un mode de développement très condensé, puisqu'elle tombe sous le coup d'une dégradation organique due au parasitisme bien avant la constitution des appareils morphologiques communs à tous les Copépodes et hérités des ancètres du groupe entier. 
Ainsi que beaucoup de Copépodes ascidicoles, elle pénètre dès le deuxième stade cyclopoïde dans l'hôte qui l'abrite et le nourrit, s'adaptant alors, par une seule mue et en bloc, à l'existence parasitaire. L'accomplissement de l'évolution a lieu suivant le mode habituel; je n'insiste donc pas spécialement sur les détails.

Dans le mâle, la métamorphose est toute différente. Le dimorphisme sexuel s'établit par suite au cours du développement embryonnaire. En effet, entre le mâle de Enterocola et sa deuxième larve cyclopoïde, il n'existe aucune différence morphologique essentielle.

L'antennule 7-articulée du mâle (PI. XVIII, fig. 3) dérive de l'antenne 5-articulée de la larve par l'adjonction de deux articles; toutes deux présentent le mème bâtonnet sensoriel allongé sur le dernier article.

Les antennes 3-articulées sont identiques dans les deux formes (PI. XVIII, fig. 4). La disposition de la bouche sur une còne buccal saillant est pareille. II existe seulement deux paires d'appendices buccaux biarticulés, qui sont garnies de soies plumeuses chez le màle (PI. XVIII, fig. 4) où elles ne servent plus à la prèhension ni a la mastication en raison du mode de vie semi-parasitaire ; l'apparition de ces soies concorde avec l'existence plus vagabonde du màle adulte.

Ainsi, tous les détails de la constitution morphologique tendent à réunir les deux formes; les seules différences résultent de l'âge différent de ces êtres.

De plus, la présence du màle dans le mème hôte que la femelle vient appuyer la mème conclusion sur des bases éthologiques indiscutables. Il est donc certain pour moi - bien que l'apparition graduelle des dernières pattes et des derniers segments du mâle n'ait pu ètre suivie sur l'embryon cyclopoïde - que le mâle dérive de cet embryon par un développement graduel et typique de tous ses caractères. Le dimorphisme sexuel d'Enterocola fulgens me parait établi sur des faits positifs, tirés à la fois de la morphologie et de l'éthologie.

\section{APLOSTOMA BREVICAUDA CANU.}

Le nauplius venant d'éclore (Pl. XXI, fig. I) est ellipsoïdal et légèrement effilé aux deux extremités. Les appendices caractéristiques de ce stade sont relativement allongés. Ce nauplius mesure alors o,2 I mm.; il s'allonge un peu, vers la fin de son existence, par suite du développement des appendices du I $^{\text {er }}$ cyclopoïde. La coloration est jaunâtre, avec des taches pigmentaires brunâtres dispersées à la surface de l'endoderme et qui prendront, au cours du développement, une disposition assez régulière dans les divers somites en formation (fig. 2 et 5). 
La formation des appendices post-mandibulaires mérite, dans cette espèce, une attention toute particulière.

A la face ventrale du nauplius sortant de la membrane de l'œuf, on voit (P1. XXI, fig. 1) l'exoderme de la partie postérieure constitué par un petit nombre de grosses cellules arrondies, composées d'un protoplasme clair environnant un gros noyau, et séparées en deux groupes latéraux symétriques par une dépression superficielle médiane. Au-dessous de ces cellules se trouvent, dans la ligne médiane, l'endoderme, et sur les côtés de l'extrémité postérieure de la masse deutoplasmique deux très grandes cellules sphériques, à noyau nucléolé, qui sont les cellules polaires mésodermiques. Vu par la face dorsale (fig. 2), le nauplius montre encore très nettement les cellules polaires, sur les côtés de l'endoderme postérieur.

Quelques heures après l'éclosion, cette disposition a déjà disparu pour faire place, sur la face ventrale, à un exoderme divisé en deux parties symétriques par une dépression médiane, dont les cellules nucléées plus nombreuses, ont un contour polygonal et s'étendent graduellement sur les côtés de la région postérieure. Les cellules polaires mésodermiques se sont multipliées et ont formé deux bandes mésodermiques de plusieurs cellules plus petites, dont les postérieures présentent encore un très gros noyau.

Cette évolution du mésoderme secondaire, que l'on peut saisir très facilement dans les fig. 1, 2, 3 et 4 de la Pl. XXI, présente, ainsi que la prolifération exodermique signalée ici, tous les caractères d'un phénomène évolutif fortement abrégé; il existe à ce sujet, entre Aplostoma et les genres précédemment décrits, Notodelphys et Doropygus, des différences de mème ordre que celles qui séparent l'embryologie des Rhizocéphales de celie des Cirripèdes.

A mesure que l'exoderme se segmente pour constituer les replis appendiculaires des paires post-mandibulaires, le mésoderme secondaire évolue et la disposition réguiière des deux bandes latérales disparaft. On peut déjà, dans la fig. 4, saisir le début de cette transformation dans leur partie antérieure où plusieurs cellules sont accolées dans le sens transversal. Le nauplius s'allonge alors légèrement (Pl. XXI, fig. 5,6); à sa face ventrale apparaissent :

$I^{0}$. la première maxille $\left(m x^{2}\right)$, sous forme d'un bourrelet exodermique très réduit ;

$2^{\circ}$. la seconde maxille $\left(m x^{2}\right)$, plus importante et qui se divise déjà en plusieurs articles; 
$3^{\circ}$. les deux premières pattes thoraciques $\left(p^{1}, p^{2}\right)$ natatoires, et la $3^{\mathrm{e}}$ rudimentaire ;

$4^{\circ}$. les pièces furcales.

Du nauplius sort directement et sans interposition d'un stade 'métanauplien, la première larve cyclopoïde. Longue d'environ $0,34 \mathrm{~mm}$., elle se compose de 6 segments et d'une furca; elle est colorée en jaune, avec des taches pigmentaires ramifiées sur le bord postérieur des somites (PI. XXI, fig. 7).

Les antennules comprennent 4 articles (Pl. XXI, fig. 8, a) pourvus de soies et portent, sur le $2^{\mathrm{e}}$, un gros bâtonnet réfringent $(s)$ étranglé vers le tiers distal de sa longueur, et sur le dernier, un autre bâtonnet simple et plus court $\left\langle s^{1}\right\rangle$.

Les antennes (Pl. XXI, fig. 9, $A$ ) sont composées d'une partie principale biarticuléé et d'un exopodite en voie de disparition.

La bouche est placée à la partie antérieure d'un cône buccal terminé par une épine saillante recourbée vers l'avant.

Les seuls appendices buccaux existants sont: la mandibule (fig. 10, $m x^{1}$ ) en voie de régression, et la seconde maxille $\left(m x^{2}\right)$; la première maxille n'a pas continué son développement au delà de l'état du bourrelet appendiculaire signalé chez le nauplius.

La seconde maxille est une patte préhensile 3-articulée, atteignant un développement considérable et une solide organisation.

Les soies furcales terminales sont très spéciales : la plus grande, placée au bord interne de l'extrémité, présente, sur une base chitineuse épaisse (Pl. XXI, fig. 12) et réfringente, une région irrégulièrement renflée, à paroi mince et hyaline et au bout de laquelle s'attache, dans une petite invagination, une région grèle, très effilée. A côté de cette soie s'en trouve une autre, plus courte, bátie sur le type ordinaire.

De cette évolution, il ressort que Aplostoma est dépourvu de $\mathrm{I}^{\text {re }}$ maxille et qu'il possède une mandibule très rudimentaire; il s'écarte donc complètement, dans l'organisation des pièces buccales, du genre Enterocola.

L'étude de ce premier stade cyclopoïde m'a permis de rapporter au genre Aplostoma un Copépode mâle possédant la mème disposition morphologique aes pièces buccales, avec quelques différences de détail imputables à la transformation ultérieure des rudiments embryonnaires. Dans ce mâle se retrouvent les soies sensorielles spéciales étranglées vers leur tiers distal, signalées dans l'antennule du cyclopoïde (Pl. XX, fig. I $4, x, y)$ et l'antenne 2 -articulée. 


\section{CONCLUSIONS.}

\section{CONDENSATION DU DÉVELOPPEMENT.}

On ne connaissait, jusqu'à ce jour, que très peu de chose sur le développement des Copépodes ascidicoles. Divers auteurs $(1,204,24,84,117,90,4)$ ont observé les nauplius après l'éclosion, mais ils n'ont point suivi le cours des métamorphoses, et il ne me semble pas nécessaire de réfuter les suppositions déduites des insuccès éprouvés dans leurs tentatives (117, F. I3).

Le détail le rnieux connu consiste dans les modifications profondes de la coloration du deutoplasme dans les ovules et les embryons en voie de développement. THORELl et KERSCHNER (117, p. 10) ont suffisamment insisté, sur ce point, mais ils n'ont point prêté une attention suffisante aux différenciations morphologiques qui accompagnent ces changements de couleur, et pour utiliser - comme on l'a tenté en particulier dans le genre Nolodelphys - d'une façon utile les faibles caractères que fournissent ces derniers, il fallait en préciser davantage les diverses particularités.

BuchHolz a déjà fait observer (24, p. I Io) qu'au I $^{\text {er }}$ stade nauplien, les Notodelphyidés doivent à l'accumulation des matériaux deutoplasmiques un retard sensible dans le développement de leurs organes internes en comparaison des nauplius de Copépodes libres.

Récemment SCHIMKEVITSCH (188) a suivi avec beaucouo de soin l'organogénie d'une curieuse espèce d'Ascidicolidé : Enteropsis dubius, mais il n'a pu établir dans tous ses détails la métamorphose des appendices ni les intéressantes particularités zoologiques qui en découlent.

Ainsi qu'on pouvait l'induire de la remarque précitée de BuchHolz, le développement des Copépodes ascidicoles est un développement condensé, ce qui est dû principalement à l'accumulation considérable du deutoplasme dans l'ovule. Bien qu'elles passent pour habiter simplement dans la cavité respiratoire de leurs hòtes (C. Vogt, Giesbrecht : 90), les formes les moins dégradées comme Notodelphys, Doropygus, etc., présentent une véritable embryologie de parasite, si on les compare, à ce sujet, aux Copépodes libres. 
D’après ce que j’en ai pu étudier malgré la difficulté de son étude, la segmentation de l'œuf nous montre déjà l'effet manifeste de cette condensation, si on la compare aux données acquises à la science par GrobBen (99).

Les stades évolutifs qui suivent cette première période du développement sont encore plus instructifs à ce sujet.

Par les observations que j'ai poursuivies sur les embryons naupliens des Ascidicolidés, se trouve réalisé dans une certaine mesure le désir bien juste exprimé par M. le Prof. H. DE. LaCAze-Duthiers, lorsqu'il réclamait des naturalistes les matériaux d'une étude comparative du naupliưs ( $\mathrm{I}$ ).

Pourtant, malgré l'intérêt qui s'attache à de pareilles comparaisons, on ne peut utiliser les descriptions publiées jusqu'à ce jour $(\mathbf{1 1 5}, \mathbf{4 0}, \mathbf{5 1}, \mathbf{1 1 1}, \mathbf{8}, \mathbf{9 9}$, 206) sur la métamorphose des Copépodes libres de diverses familles, pour établir un parallèle étroit entre le développement de ces formes d'une part, et celui des Ascidicolidés d'autre part. Bien des points parmi les plus importants nécessitent des recherches nouvelles et soigneusement conduites pour nous permettre d'atteindre à ce but.

Néanmoins, parmi les Copépodes libres, chez Calanus (99), nous savons qu'il existe plusieurs stades-nauplius bien distincts dans leurs caractères morphologiques, ainsi que plusieursstades-métanauplius. Dans Doropygus, Notodelphys, Bonnierilla et Doroixys, il n'y a qu'un seul de ces stades, par suite d'une première abréviation dans les étapes de la métamorphose, et tous les Ascidicolidés que j'ai observés ne montrent qu'une seule forme nauplienne. Mais il est juste d'observer que les formes plus parasites, comme Enterocola et Aplostoma, montrent une condensation bien plus grande de leur métamorphose, puisqu'elles manquent du stade métanauplius. Il en est probablement de mème chez Enteropsis dubius, dans lequel Schimkevitsch semble (188, p. 89, 90) avoir considéré comme métanauplius, le nauplius sous la cuticule duquel se forment - dans les sacs ovigères! - les rudiments des quatre paires d'appendices post-mandibulaires. La condensation embryogénique mécaniquement imposée par l'accumulation du deutoplasme, nous apparaît donc en relation étroite avec les conditions éthologiques, et commandée par le degré de parasitisme.

(1) H. DE LACaze-Duthiers; Histoire de la Laura Gerardir. Institul de France, Mémoires de $\boldsymbol{V}$ Académie des Sciences, t. XLII, N" 2, pages 134-137.

"Dans la recherche des analogies qui peuvent faire distinguer les différents nauplius, on devra peut-être s'attacher à mieux étudier qu'on ne l'a fait jusqu'ici la disposition des poils, des articles et la forme générale des pattes. Les renseignements qu'on trouve dans les ouvrages laissent quelque peu à désirer sur ces points... Il n'est pas exact de dessiner les appendices pileux comme on le fait habituellement, en donnant au hasard des coups de crayon destinés à représenter ces poils ". 
Quoi qu'il en soit, les embryons des Ascidicolidés sont libres et nageurs depuis le stade nauplius jusqu'au second stade cyclopoïde. Au delà de cette période ils tombent dans le parasitisme, et, dans tous les cas que j'ai pu étudier, c'est au second stade cyclopoïde que l'animal recherche son hôte pour s'y établir. C'est alors que les adaptations au mode spécial d'existence définitif s'établissent aussi, soit graduellement (Doropygus, Notodelphys), soit subitement (Doroixys, Enterocola). Dans ce dernier cas, l'abréviation de la métamorphose est rendue par ce fait beaucoup plus manifeste.

\section{MESODERME SECONDAIRE.}

Dans l'exposé fait plus haut du développement du mésoderme secondaire chez les Copépodes ascidicoles, on reconnaitra encore une condensation.

La formation de bandes mésodermiques, comme celles du nauplius de Doropygus (Pl. VII, fig. I et 2 ) et d'Aplostoma (Pl. XXI, fig. I, 2 et 4 ) a été constatée dans un grand nombre d'animaux; il me semble nécessaire d'insister sur ce point et de comparer l'évolution des organes mésodermiques dans plusieurs cas semblables.

Suivant l'opinion de GIARD, énoncée dès 1879 ( 1 ) et fréquemment reproduite par lui dans divers mémoires ou dans ses cours d'embryogénie, il me semble nécessaire de distinguer morphologiquement deux catégories de mésodermes - en dehors des préoccupations physiologiques, trop fréquentes aujourd'hui, qui tendent à la séparation des mésenchymes et mésoblastes.- D'après leur ordre d'apparition, et pour ne rien préjuger de leur nature morphologique encore peu étudiée, je les appellerai mésoderme primaire et mésoderme secondaire, en les séparant nettement du pseudo-mésoderme des Célentérés et des Orthonectides.

$\mathrm{I}^{0}$ Le premier (mésoderme solide de GIARD) apparaît très tôt dans l'embryon. Il dérive des cellules mésodermiques primordiales ("Urmesodermzellen " de HATSCHEK), parfois nettement reconnaissables dans la blastula, et qui se détachent toujours des cellules endodermiques au point de formation de la bouche primitive de la gastrula.

C'est toujours ce mésoderme primitif qui forme le tissu conjonctif et les organes musculaires des premières larves dans les animaux à métamorphoses, où l'on peut distinguer avec la plus grande netteté la marche des formations mésodermiques successives.

(1) GIARD, Sur l'organisation et la classification des Orthonectidæ, Complis-rindus Acad. Sci. Paris, 22 septembre 1879. 
Il peut lui être adjoint, dans certains cas spéciaux, des formations mésenchymateuses dérivant des feuillets blastodermiques aux environs du point d'origine ou à une certaine distance de ce point; mais ces cellules migratrices ne modifient en rien la morphologie primaire du mésoderme.

$2^{0}$ Dans les animaux à métamorphoses, après le repos momentané que subissent les organes embryonnaires durant la première période larvaire, il s'établit une nouvelle poussée des éléments dans laquelle apparaît le mésoderme secondaire, pour la constitution des organes nouveaux, souvent importants, de la deuxième larve.

Le mésoderme secondaire (mésoderme cavitaire de GIARD) correspond donc exactement aux cellules polaires mésodermiques des embryogénistes de l'école allemande. C'est une nouvelle formation de l'endoderme qui apparaît, dans les cas d'embryogénie dilatée sous forme d'entérocœles (Tuniciers, Brachiopodes, Chætognathes, Amphioxus), et dans les développements condensés sous forme de cordons pleins (PI. VIII, fig. I et 2 ; Pl. XII et XIII, fig. I; Pl. XV, fig. I; P1. XXI, fig. I, 2 et 4) ou de bandes mésodermiques (Copépodes, Annélides). Sans préjudice de formations mésenchymateuses qui peuvent toujours s'ajouter çà et là, le mésoderme secondaire fournit les éléments conjonctifs et musculaires de la nouvelle poussée évolutive qui préside à la constitution de la $2^{\mathrm{e}}$ forme larvaire.

D'après Hatschek (loc. cit.; voir page I7), GrobBEn (99) et bien d'autres, le mésoderme secondaire ne serait qu'une nouvelle prolifération du mésoderme primaire. Ce serait la suite de l'évolution d'ur mésoderme unique dans sa première origine, après le temps d'arrêt éprouvé dans la I ${ }^{\text {re }}$ larve.

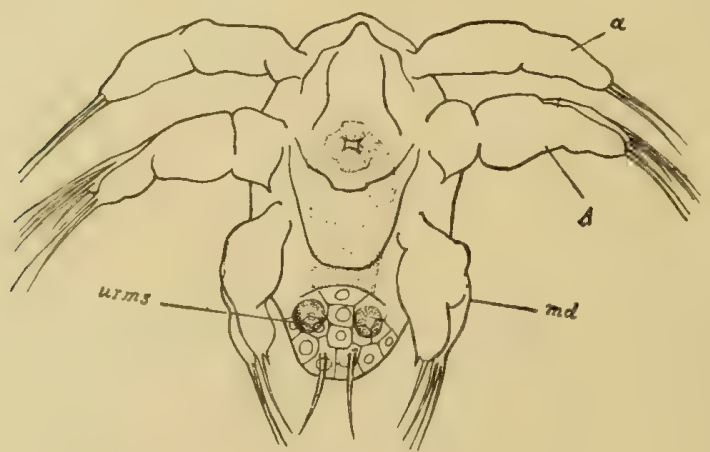

Fig. 9. - Nauplius de Calanus (Cetochilus) à l'éclosion, d'après GrobBen : loc. cit., Fig. 23.

$a$, antennule ; $A$, antenne ; $m d$, mandibule; urms, cellules mésodermiques polaires.

Toutes les observations que j'ai pu faire sur divers Copépodes et Cirripèdes m'empêchent de me rallier à cette interprétation; et je partage absolument, sur ce point, les vues exposées par GIARD et résumées plus haut.

Dans Doropygus gibber (P) VII), Notodelphys agilis (PI. XII), $N$. allmani (PI. XIII), Doroixys uncinata (Pl. XV) et Aplostoma brevicauda (PI. XXI), j'ai vu constamment 
les cellules polaires mésodermiques dans des rapports si étroits avec la partie postérieure de la masse endodermique, qu'il me semble impossible de soutenir une interprétation autre que celle exposée dans les descriptions précédentes.

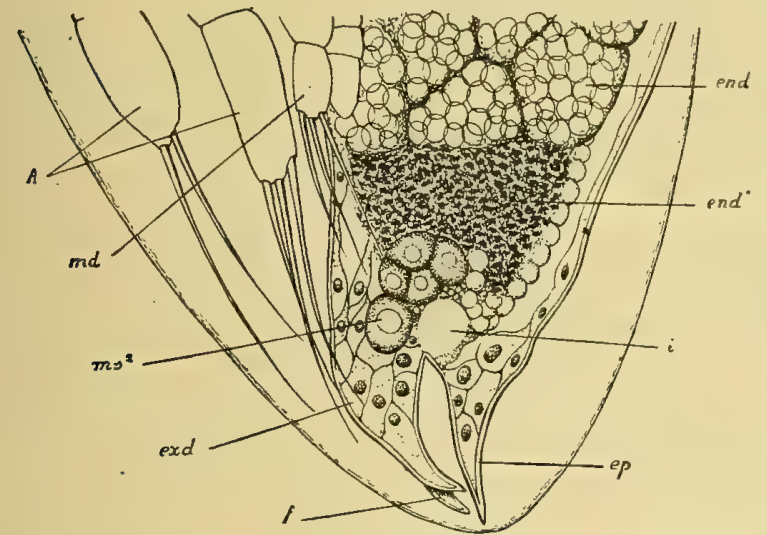

Fig. 10. - Région postérieure d'un nauplius de Balanus balanoĩdes L. peu de temps avant l'éclosion: $A$, antenne; $m d$, mandibule; end, masse endodermique deutoplasmique ; end", renflement intestinal en voie de formation ; $i$, intestin postérieur; exd, exoderme; $m s^{2}$, l'une des bandes mésodermiques secondaires avec sa cellule polaire; $e p$, épine postérieure du bouclier; $f$, fourche terminale de l'abdomen.

Pour les Entomostracés, Grobben (99) a émis une opinion toute différente. Aux cellules mésodermiques primitives, qui auraient persisté durant tout le développement embryonnaire, il rapporte les grosses cellules mésodermiques du nauplius de Calanus (Cetochilus), de Sacculina et de Balanus. Malgré les soins apportés par cet auteur à l'étude embryologique de Calanus, il ne ressort nullement de ses descriptions que les cellules mésodermiques conservent, du stade gastrula au stade nauplius, quelque prédominance sur le reste du mésoderme, ni qu'elles échappent plus que les autres à l'évolution du mésoderme primaire en muscles et tissu conjonctif.

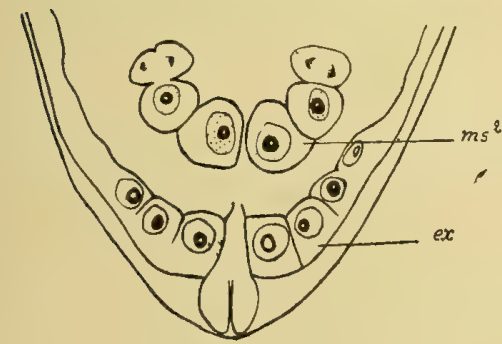

Fig. 11. - Bandes mésodermiques du nauplius de Sacculina (benedeni GIARD) vues de face ; d'après Grobben: loc. cit., Fig. 40.

ex., exoderme ; $m s^{2}$, mésoderme secondaire.

J'ai observé moi-mème, au début de sa formation, le mésoderme secondaire du nauplius de Balanus balanoides L. avant l'éclosion. C'est alors une paire de bandes latérales placées à l'extrémité postérieure de la masse endodermique, et aux dépens de laquelle se constitue le tube digestif. Il est facile de se convaincre que le développement de ces bandes suit le mème mode que dans Aplostoma et les genres voisins. La figure des bandes mésodermiques chez ie nauplius après l'éclosion reproduite par GROBBEN (99, p. 31), ne représente qu'une apparence plus tardive et par suite moins convaincante. 
J'ai également vérifié, chez Sacculina et Peltogaster, la disposition de ces bandes en fer à cheval déjà figurées par GrobBen (99, Pl. IV, fig.40). Longtemps auparavant, GIARD les avait aussi observées, comme le démontrent les dessins déjà anciens et restés inédits qu'il m'a communiqués.

Tout concorde, dans les diverses observations que j'ai pu faire, pour appuyer les conclusions émises précédemment sur la naissance des cellules polaires mésodermiques et du mésoderme secondaire aux dépens de la partie postérieure de l'endoderme, dans le voisinage de l'intestin terminal. 


\section{TROISIEME PARTIE.}

\section{ÉTHOLOGIE.}

L'influence des particularités éthologiques sur les Copépodes parasites a fourni depuis longtemps les exemples les plus nets cités par les naturalistes, qui attachent à ce facteur de modifications une importance pleinement justifiée

Avec GIARD (I), je crois que l'étude d'un groupe d'animaux ne peut ètre détachée de la recherche des diverses manifestations inhérentes à leurs conditions spéciales d'existence; j'ai donc recherché le rôle qui revient aux divers facteurs primaires et secondaires de l'évolution, dans les variations morphologiques constatées par moi chez les Copépodes.

\section{MILIEUX COSMIQUES.}

Les Copépodes libres comptent certainement parmi les animaux qui réagissent avec la plus grande intensité contre l'influence des milieux cosmiques où ils vivent. Par les adaptations nombreuses et variées qui en résultent, il nous est peut-ètre permis de conclure à leur grand état de primitivité, opinion appuyée d'autre part sur de sérieuses bases morphologiques.

(1) Giard, Les facteurs de l'évolution, Reoue scientifique, t. XLIX, 1889, P. 641-648. 


\section{STATION.}

$1^{0}$ Espèces nageuses. - Les Copépodes les plus typiques, les moins adaptés à une existence spéciale, sont, sans nul doute, les formes qui habitent au milieu des algues, y nagent entre deux eaux et se trouvent même parfaitement capables de quitter ces abris où ils trouvent leur nourriture pour des excursions momentanées à la surface de l'eau.

Au premier rang, je puis encore citer Longipedia coronata, que j'ai recueilli en grande quantité dans les pêches au filet fin, dans la zone littorale parmi les algues, aussi bien qu'à la surface où il est toutefois moins abondant.

La même distribution pourrait être signalée en particulier pour un intéressant Peltidien très répandu dans le Boulonnais : Alteutha bopyroídes, que j'ai même recueilli à $\mathbf{2}$ ou $\mathbf{5}$ milles au large, dans la région du Pas-de-Calais comprise entre le Varne et le Colbart.

Mais, dans nos Harpacticidés, l'espèce qui présente au plus haut degré des habitudes pélagiques. est Euterpe acutifrons, commune à la belle saison dans toutes les pêches de surface effectuées au large, accidentelle dans les pêches littorales.

Malgré ces exceptions, le mode d'existence habituel des Harpacticidés reste le moins spécialisé et le plus éclectique; ce fut probablement le régime suivi par les Crustacés, aujourd'hui disparus, qui formèrent la souche de nos animaux.

En conséquence de ces habitudes, les Harpacticidés nageurs ont conservé l'organisation primordiale de leurs ancêtres, sans acquérir, en vue d'une locomotion plus active, les organes perfectionnés ou les autres adaptations inhérentes aux formes pélagiques de tous les groupes d'animaux. Tous les détails de leur anatomie restent dans la note moyenne par rapport aux autres types de Copépodes ayant subi, dans un sens déterminé, l'influence d'un habitat plus restreint.

J'ai pu constater ces faits non seulement dans Longipedia coronata, Euterpe acutifrons, Ectinosoma minutum, Ectinosoma sarsi, Tachidius discipes, Tachidius littoralis, mais aussi dans Amymone sphcrica qui reproduit dans sa démarche et un peu dans sa forme l'aspect de petits Cladocères d'eau douce (Chydorus, Bosmina).

Dans la famille des Harpacticidés eux-mêmes, à côté de ces formes typiques, apparaissent des séries de modifications, souvent plus ou moins importantes, qu'on peut attribuer à l'habitat (I).

(1) Je n'insisterai pas spécialement sur la formation des appareils préhensiles, des griffes et des pinces qui peuvent se présenter pour une foule de raisons difficiles à déterminer quand il s'agit d'animaux de taille aussi réduite que les Copépodes. Les pattes préhensiles de Ia $1^{\text {re }}$ paire, dans les divers genres Laophonle ( $\mathrm{PI} . \mathrm{V}$, fig. 15), Dactylopus, Westwoodia, Thalestris, etc., semblent d'ailleurs indépendantes des influences du milieu. 
$2^{0}$ Espèces adhérentes. - Parmi les Peltidiinés, on rencontre un assez grand nombre de formes dont la plupart rampent en glissant assez rapidement à la surface des roches ou des algues. Dans leur situation habituelle, ces animaux sont très difficiles à découvrir, car ils présentent, en dehors de l'adhérence parfaite qui est occasionnée par leur forme assez semblable à celle des Caliges ou à celle des Isopodes du genre Spharoma, des phénomènes d' “ adaptation protectrice " qui les dissimulent d'une façon complète.

Dans le Boulonnais, j'ai pu recueillir et étudier très aisément Zaus spinosus, petite espèce qui vit en grande abondance à la surface des Algues floridées de la zone à Bugula, et Scutellidium fasciatum, adhérant très fortement et rampant à la surface des frondes de Laminaria saccharina.

J'ai déjà signalé la présence et les aptitudes natatoires de Alteutha bopyroïdes; cantonné à la côte où il se colle aux rochers de Hermelles et particulièrement abondant dans le port en eau profonde de Boulogne, il est accompagné d'une autre espèce beaucoup plus rare et moins vagabonde : Eupelte purpurocincta. Dans ces Harpacticidés, la forme est visiblement modifiée en vue de la reptation à la surface des corps submergés, algues ou rochers. Dans le premier cas, elle tend vers l'aspect des Caliges, et dans le second vers la forme des Sphæromes, qui sont d'ailleurs des Isopodes adaptés au mème genre de vie. Diverses modifications dans le céphalothorax, l'abdomen et les appendices favorisent l'adhérence sur les parois lisses et traduisent nettement l'influence du mode de vie sur l'organisation de ces Copépodes.

$3^{0}$ Espèces limicoles. - Une grande partie des Harpacticidés à forme générale primitive ont abandonné les habitudes de natation précédemment indiquées dans certaines espèces de cette famille. Plus sédentaires, ces formes sont encore très aptes à la locomotion au sein du liquide, mais elles affectionnent plutôt de stationner sur les fonds sablonneux ou vaseux, à l'abri dans les racines de plantes marines. Elles sautent çà et là, par bonds, en conservant constamment leur point d'appui sur le fond de la mer; leur démarche est une reptation sautillante.

Ces formes peu modifiées, fréquentes dans les genres Thalestris, Idya, etc., nous conduisent directement aux espèces limicoles, dans lesquelles les adaptations sont particulièrement profondes et intéressantes.

Le genre Laophonte m'a fourni cinq espèces curieuses, dont les quatre premières sont des formes qui rampent sur le fond de la mer à la surface des fonds de sable ou de vase. La dernière, Laophonte lamellifera, est plus sédentaire et presqu'exclusivement limicole ; de ce fait, elle présente une régression considérable des soies furcales, transformées en épines courtes assurant un point 
d'appui dans la progression à l'intérieur d'une vase trop dense pour permettre la natation. Déjà, dans les espèces moins rampantes, les soies furcales subissent une adaptation curieuse, qui consiste dans un développement considérable de la grande soie médiane interne terminale, transformée en un long stylet ( $\mathrm{Pl}$. V, fig. 24) garni d'épines espacées et non de barbules. Ces dernières eussent subi trop de mutilations, produites par le glissement perpétuel à la surface du sable ou de la vase dans le sautillement particulier qui est le mode de progression de ces espèces.

Dans le genre Laophonte, toutes les fonctions natatoires sont assez bien conservées, même pour les espèces limicoles. Il n'en est pas de même pour une petite série de Copépodes réunis par BRADY dans une sous-famille dont jai pu étudier à nouveau l'espèce type observée jusqu'à ce jour par l'auteur anglais seul. $N$ annopus palustris est un Copépode limicole des régions saumâtres d'estuaires : il est assez fréquent dans le bassin presqu'entièrement comblé du vieux port de Wimereux, et sur les bords de la rivière auprès de l'embouchure. Ses mouvements sont très limités et presqu'exclusivement reptatoires, ce qui concorde parfaitement avec le maintien rigide de cet Harpacticidé et l'ornementation épineuse de ses appendices, presqu'entièrement dépourvus des barbules fines et délicates particulières aux soies propres à la natation (Pl. IV, fig. 6, 2I). Déjà dans les pattes thoraciques de $N$ annopus, nous constatons une tendance à la réduction de la rame interne bi articulée des trois premières paires, allant jusqu'au simple moignon dans la $4^{e}$ paire : ces pattes sont capables de résister à la cohésion de la vase, et, par suite, aptes à remplir leur rôle locomoteur dans ce milieu plutốt que dans l'eau.

La mème tendance rétrograde des pattes thoraciques se continue, d'ailleurs, dans des formes très rapprochées de Nannopus par bien d'autres caractères d'organisation dans les antennules, antennes et pièces buccales; elle ǹous invite à accepter la fondation d'une sous-famille spéciale pour des êtres unis par tant de particularités communes.

Ainsi, dans le genre Platychelipus Brady, les $3^{\mathrm{e}}$ et $4^{\mathrm{e}}$ pattes thoraciques sont bâties sur le type de la $4^{\mathrm{e}}$ patte de Nannopus; chez Huntemannia Poppe, la structure est encore la même pour les $2^{\mathrm{e}}, 3^{\mathrm{e}}$ et $4^{\mathrm{e}}$ paires, bien que l'exopodite n'y compte que deux articles et soit lui-même en régression.

Nannopus, Platychelipus et Huntemannia forment un ensemble de Copépodes limicoles subissant graduellement l'adaptation de plus en plus parfaite à la vie limicole et à la reptation; ils prennent ainsi un grand intérêt dans la famille si homogène, en apparence, des Harpacticidés.

$4^{0}$ Espèces pélagiques. - L'influence des hạbitudes pélagiques que l'on 
rencontre d'une façon générale dans les espèces marines des familles des Calanidés et Cyclopidés, a, pour première conséquence - déjà signalée dans mes descriptions antérieures - la séparation très nette des régions du corps et un commencement de division du travail des appendices, dans la locomotion.

A. - Les trois genres de Cyclopides marins que j'ai recueillis possèdent d'ailleurs des habitudes assez variables. Cyclopina et Oithona sont souvent pêchés avec les Calanidés, à la surface,. où iis vivent en vrais pélagiques; ils se rattachent d'ailleurs au type des Calanides, par les ornements barbelés de leurs appendices céphaliques, comme par le développement de leurs antennules transformées en balanciers d'équilibristes et par l'allongement de leur abdomen. Mais on les rencontre également avec les Harpacticidés, parmi les algues. Quant à Thorellia, il se distingue, avec Cyclops - que je cite ici pour deux espèces saumâtres intéressantes - par des habitudes moins vagabondes, puisque tous deux vivent avec les Harpacticides les moins spécialisés, dans les algues marines des fonds rocheux du littoral. Les Cyclopides non pélagiques peuvent être considérés, vu la disposition simplifiée de leurs divers appendices masticateurs, comme dérivant des espèces péiagiques, et c'est à ce titre que Claus (51) a eu raison de signaler la dégradation qui affecte l'antenne devenue préhensile chez Cyclops. Dans ce sens, il faudrait citer chez Thorellia et Cyclops la disparition plus ou moins complète des portions natatoires (palpes) des mandibules et des deux paires de maxilles.

B. - Dans les Calanidés, Copépodes pélagiques par excellence, la séparation des régions céphalothoracique et abdominale est poussée à son extrème limite. Le céphalothorax est le véritable tronc, et l'abdomen ne forme plus qu'une nageoire caudale extrèmement puissante, capable de mouvements d'une extension et d'une efficacité remarquables. Les Calanidés flottent dans l'eau de mer, avec le céphalothorax placé presque verticalement et leurs longues antennules disposées en $T$ pour servir de balancier dans le maintien de cette position d'équilibre. Les antennes, mandibules er maxilles, pourvues de lames richement garnies de soies barbelées, sont animées d'un mouvement consiant qui a pour effet d'empècher la chute du Copépode au fond du liquide et de l'aider à planer auprès de la surface. L'action des pattes thoraciques ramenées vigoureusement et d'un mouvement commun de haut en bas ou inversement, s'ajoute à l'effet de la nageoire abdominale pour produire les bonds considérables qu'effectue le Calanide quand il est menace d'un danger quelconque. Dans ces déplacements, les antennules grèles et flexibles n'ont qu'un rôle tout à fait passif; elles se replient vers 
l'avant (dans les reculs en arrière), ou sưr les côtés du corps (dans les sauts en avant).

En vue de ce mode tout spécial de locomotion, qui exige un grand espace libre et par conséquent l'éloignement du rivage et l'absence de végétation dans la région habitée, tous les appendices des Calanidés sont hypertrophiés dans le sens de la natation. Les grandes soies barbelées, l'allongement des articles et le développement des rames atteignent dans ces formes un degré considérable; ils s'accordent avec l'apparition d'hypertrophies curieuses dans les organes des sens (Pontellines), dans le développement de pigmentations internes, dans la transparence des tissus, etc., pour éloigner le naturaliste de l'opinion d'après laquelle les Copépodes nageurs les mieux organisés seraient les formes primitives du groupe.

\section{LATITLDE.}

L'influence de la latitude ou du climat sur les Copépodes est encore aujourd'hui presque impossible à déterminer avec un semblant d'exactitude. Les recherches faunistiques et descriptives sur ces petits Crustacés sont en effet tellement insuffisantes qu'on ne peut prétendre à comparer utilement les diverses formes étudiées et signalées, de façon à en établir les variations. En 1866. Claus (53) a cru pouvoir rapporter aux variations d'une mêrne espèce suivant l'habitat chorologique quelques différences insuffisamment observées entre des espèces voisines; mais il a été reconnu par GIESBRECHT (91) que les variétés, établies par Claus, étaient en réalité des espèces bien distinctes, différant entre elles par bien d'autres caractères d'une importance indiscutable.

Après la description de chacune des espèces que j'ai étuđiées, j'ai brièvement résumé la distribution géographique actuellement connue; mais je ne me dissimule, en aucune façon, le peu d'importance que présente semblable compilation, dans l'état actuel de nos connaissances.

\section{SALURE.}

Dans ces dernières années, les études activement conduites, en Allemagne, en Suède, en Norvège, en Suisse et en Italie, sur les habitants des grands lacs plus ou moins éloignés de la mer, et les recherches consciencieuses poursuivies par divers naturalistes, - et notamment à Kiel, sous la direction de MÆeBıs (150), pour la connaissance de la faune de la mer Baltique - ont attribué une plus grande 
valeur à la comparaison des formes d'eau saumâtre et des formes marines; on a tenté, - en s'inspirant des belles recherches devenues classiques de ScHMANKEVITSCH $(189,190)$ sur l'influence modificatrice de la salinité des eaux -, d'attribuer au degré de salure du milieu la plupart des variations qui affectent un genre ou une espèce de Copépode. Mais ces conclusions, hàtivement présentées, ont été renversées dès qu'on les a soumises à un conirôle ou à une critique plus approfondis $(89$, P. I $56 ; 156$, P. II, 20$)$.

J'ai eu l'occasion d'étudier, sur des échantillons provenant de l'embouchure de la Seine, un genre très intéressant de Calanide qui résiste parfaitement à de grandes variations dans le degré de salure de l'eau, et qui se range, par conséquent, avec les formes euryhalines de MÆBius (150) : c'est le genre Temorella Claus, voisin de Temora BAIRD, mais qui s'en distingue par son habitat dans l'eau douce des lacs ou dans l'eau saumâtre des estuaires et des marais salants.

-A l'embouchure de la Seine, dans la baie en facé d'Honfleur, Gadeau DE Kerville a recueilli Temorella affinis POPPE, connu déjà à l'embouchure des grands fleuves de l'Allemagne. C'est la forme type, comme j'ai pu m'en convaincre par l'étude des échantillons recueillis (28); et elle se trouve à Honfleur dans le même habitat que dans l'Ems, l'Eibe, le Weser, le Rhin.

Dans le port mème du Havre, en un point abrité contre les courants puissants, alternativement salés et saumâtres, qui traversent l'embouchure, vit une autre variété (1) de la même espèce supportant, par suite, des conditions spéciales pour l'habitat de ce Copépode. Je la rapporte à Temorella affinis, var. hispida NordQvist (156), pour de légères différences dans la taille des appendices et l'ornementation de la cuticule. Dans la Baltique, qui est un véritable estuaire par le régime hydrographique spécial qu'elle présente (2), Temorella affinis, var. hispida, a été récolté par NORDQVIST dans une station relativement abritée par les récifs d'Åbo (3) contre les courants venant des golfes de Bothnie et de Finlande. Les conditions biologiques de cette station sont en tous points comparables à celles du bassin de l'Eure dans le port du. Havre.

Restant dans le domaine des faits acquis par l'observation, je considère la variété hispida, comme établie — par ségrégation et amixie — sous l'influence d'un ensemble d'actions biologiques et éthologiques confuses, inhérentes à cet habitat spéciál. Mais je ne me crois nullement autorisé à décider, dès maintenant, sans des recherches approfondies et très soigneusement conduites, quelle est l'importance particulière à chacune de ces actions.

(1) Rẹcueillie dans l'estomac de Harengs pèchés dans le port, en février et mars 1891 .

(2) Reclus, Nouvelle géographie universelle, t. IV, p. 33-44.

(3) Reclus, loc, cit., t. IV, le port d'Äbo, Finlande. 
C'est encore avec quelques restrictions que je signalerai ici le cas de Cyclops lubborki Brady. Cette espèce est assez commune à Wimereux dans l'eau saumâtre de l'estuaire; elle manque dans les eaux douces du voisinage. BraDY l'a découverte dans les marais salants du Northumberland et du Durham, et elle a été revue depuis par quelques naturalistes qui ont ignoré l'observation de BrADY. C'est probablement le Cyclops que SCHMANKEviTSCH (189) dénomme C. odessanus, et qu'après lui, sans l'appui de nouvelles expériences, REHBERG et Richard (voir cidessus, page 182) font dériver de $C$. pulchellus $\mathrm{KOCH}$ sous l'influence de la salure. C. pulchellus est très abondant au sommet des falaises, dans des eaux douces peu éloignées de l'estuaire de Wimereux; il serait facilement transporté par plusieurs voies dans les eaux saumâtres où vit $C$. lubbocki, et celui-ci en peut bien dériver après son acclimatation dans ce milieu salin. Je n'ai pas réussi la transformation de C. pulchellus en $C$. lubbocki; néanmoins je ne combattrai pas l'opinion exposée ci-dessus en raison des facilités qui se rencontrent dans la nature pour en favoriser l'accomplissement à longue échéance.

\section{MILIEUX BIOLOGIQUES.}

\section{ESPECES LIBRES.}

Les Copépodes libres sont des animaux plus ou moins carnassiers qui, comme l'a indiqué Claus, se nourrissent aussi bien de débris animaux que de petites proies vivantes (Rotifères, larves d'Annélides), et de végétaux microscopiques (Diatomées, spores d'Ulves, etc.). Parmi les espèces les plus rapaces, je dois citer en première ligne Thalestris longimana CLs., forme robuste et agile, armée de pinces préhensiles puissantes, et que l'on peut très facilement observer en train de dévorer des stades jeunes de Copépodes d'une taille et d'une agilité déjà remarquables.

\section{ESPECES PARASITES.}

L'influence du milieu biologique est plus facile à saisir chez les formes vivant plus ou moins étroitement dans la dépendance d'une autre espèce animale.

Parmi elles on peut distinguer:

$I^{0}$ Des espèces commensales inoffensives, abritées dans la retraite habituelle, voire même à l'intérieur de leur hôte :

$2^{0}$ Des formes semi-parasites qui vivent aux dépens de leur hôte d'une façon temporaire, sont capables de reprendre, pour une période plus ou moins longue, 
l'état d'indépendance et de vivre à la façon des espèces libres, avant de rejoindre leur habitat parasitaire. Ces habitudes sont naturellement reliées à l'organisation, demeurée presque intacte, des appendices natatoires; et, dans les formes semiparasites — avec une dégradation manifeste de la région buccale adaptée à la prise de nourriture aux dépens de l'hôte passager, - on constate un riche développement de soies barbelées natatoires, la délimitation bien nette des régions céphalothoracique et abdominale, en un mot tous les caractères particuliers aux espèces nageuses.

J'ai pu étudier un assez grand nombre d'espèces semi-parasites appartenant aux familles intéressantes des Lichomolgidés (P1. XXII à XXV) et des Ascomyzontidés (P1. XXVI à XXIX) et constater, dans toutes ces formes, les propriétés éthologiques signalées ici avec toutes leurs conséquences.

Dans ces Copépodes, les deux sexes vivent sensiblement de la même façon ; les mâles se rencontrent en compagnie des femelles, aussi bien à l'état de liberté qu'à l'état de parasitisme.

Il existe, en outre, dans ces animaux une différence de taille entre les adultes des deux sexes, différence parfois considérable ct le plus souvent à l'avantage du sexe femelle. Cette particularité qui, comme on le sait, s'accentue davantage avec le degré de parasitisme, semble commandée par le volume des produits sexuels que préparent les êtres de sexe différent.

$3^{0}$ Des parasites immuables dans le sexe femelle, encore mobiles et semi-parasites dans le sexe mâle. Dans cette autre série d'animaux, le dimorphisme sexuel est très accentué, non seulement au point de vue éthologiqué, mais encore au point de vue morphologique.

Chez Enterocola fulgens V. BEN., le mâle désigné comme tel par tous ses caractères de morphologie embryonnaire et définitive, est bien différent de la femelle (PI. XIX, fig. I 3 et I2). C'est un Copépode semi-parasite dans toute l'acception du mot. Ii se rencontre d'ailleurs dans les mêmes cormus de Polyclinum luteum GIARD qui abritent la femelle; mais il sort aisément des Synascidies pour nager vivement dans l'eau, vers les points les plus éclairés, tandis que la femelle, vermiforme et vraiment parasite, reste incapable de mouvements aussi étendus.

Si l'on se reporte à l'embryologie de ces formes, on verra avec la plus grande netteté, que le mâle ne différe à ce point de la femelle, qu'en vertu de sa grande ressemblance avec d'embryon mobile et bon nageur, commun aux deux sexes (Pl. XIX, fig. 7 et I 3). Tandis que la femelle subit une régression manifeste en vue de l'existence parasitaire plus intime qui lui incombe, le màle reste pourvu de tous les attributs du Copépode normal qui existent en rudiments dans l'embryon nageur. 
Le semi-parasitisme nous apparait donc, par rapport au parasitisme, comme une persistance des facultés embryonnaires. A ce titre, dans la comparaison de deux formes parasite et semi-parasite d'espèces différentes, ou de deux formes sexuelles d'une mème espèce, ce mode d'existence constitue un caractère primitif. Par conséquent, nous remarquons, dans ce cas encore, que dans les comparaisons entre formes d'une famille déterminée, l'éthologie conduit aux mêmes résultats que l'étude morphologique et vient appuyer celle-ci dans ses conclusions.

Il est d'ailleurs possible de constater directement, dans les séries un peu complètes de Copépodes parasites et semi-parasites, la marche graduelle des adaptations parasitaires :

A. Hersiliidæe. - Dans cette famille de Copépodes. j’ai rangé diverses formes commensales d'un mème crustacé : Callianassa subterranea. MonTAGU. La Callianasse vit, dans le Boulonnais (I), sur les plages de sable où elle creuse des galeries profondes, ramifiées et tapissées à l'intérieur d'un enduit ochreux qui les limite comme une paroi.

Dans ces galeries vivent, en compagnie de la Callianasse, dans les environs immédiats de ce Crustacé et parfois mème au milieu de ses pattes abdominales, deux espèces de Copépodes, Giardella callianassa et Hersiliodes thompsoni, dont les pièces buccales sont déjà spécialisées et ne servent plus exclusivement à la mastication (PI. XXX, fig. 9).

Sur les Callianasses de la Méditerranée et de l'Adriatique, vit un Copépode voisin, Clausidium (= Hersilia) apodiforme PHILIPPI, recueilli souvent en liberté. Comme Heller (109) l'a démontré, Clausidium est fixé à la surface de la Callianasse, sur laquelle il glisse en conservant son adhérence comme les Caliges glissent sur la peau des Poissons; ce Copépode se réfugie mème dans la cavité branchiale de son hôte. Les trois espèces de Copépodes semi-parasites sur Callianassa subterranea nous présentent par conséquent une gradation dans leurs conditions éthologiques, et il est permis de dire que Clausidium est plus parasite que Giardella ou qu'Hersiliodes thompsoni.

Nicothoe astaci A. ET M.-E., parasite fixé sur les branchies du Homard, représente, à n'en pas douter d'après ses caractères morphologiques, le dernier terme de cette dégradation. Il descend d'un Hersilien mobile et, par l'acquisition d'un degré en plus dans la voie du parasitisme sur les Crustacés décapodes, il est devenu le terme extrême, le parasite définitif, le type "siphonostome" de ce curieux petit groupe de Copépodes.

Tout dàns l'organisation des Hersiliidés - Copépodes actuellement connus

(1) GIARD, Les habitants d'une plage sablonneuse, Bull. scicntif., $2^{\mathrm{e}}$ sér., t.I, 1878, p 35. 
comme parasites des Crustacés décapodes, dont le nombre augmentera sans doute dès qu'on recherchera ces animaux sur les formes voisines de Callianassa et de Homarus - coïncide avec les habitudes particulières à chaque espèce.

Clausidium est bien plus élargi que Giardella ; il est devenu presque discoïdal; ses appendices thoraciques sont pourvus de pelotes chitineuses adhésives qu'on a prises pour des ventouses. Gràce à tous ces perfectionnements, il peut adhérer mieux aux surfaces lisses comme la carapace de son hôte.

Nicothoe, établi dans un endroit abrité, n'a point tous ces accessoires d'adhésion; plus parasite, il présente un développement considérable de la masse du corps par rapport à ses appendices natatoires avec un perfectionnement plus grand de la région buccale.

B. Ascidicolidæ. - Dans cette famille, j’ai réuni toutes les espèces commensales et parasites des Tuniciers, qui formaient autrefois les diverses familles des Notodelphyidés Thorell, des Buproridés Thorell, des Kosmechtridés Della Valle équivalente aux Schizoproctidés Aurivillius, des Enterocolidés Della Valle, des Enteropsidés Aurivillius.

Au point de vue éthologique, ces Copépodes sont, en mème temps que certaines espèces de Lichomolgidés THORELL, d'Ascomyzontidés ThORELl, commensaux ou parasites des Ascidies simples ou composées. Nous trouvons ainsi parmi les Copépodes des Ascidiens, un nouvel exemple de ce fait éthologique très important signalé par GIARD ET Bonnier chez les Bopyriens, à savoir que les diverses espèces d'un même groupe, parasites d'un même hòte, peuvent appartenir à des séries éthologiques toutes différentes, à des sous-familles n'ayant entre elles que des rapports de parenté assez éloignés.

Et pour les Copépodes des Ascidiens, nous savons notamment, d'après les caractères morphologiques, que l'écart existant entre Lichomolgus et Notodelphys est considérable et ne permet nullement d'admettre une filiation réciproque de ces animaux.

Dans les Ascidicolidés, la gradation des effets du parasitisme sur les Copépodes d'une mème série éthologique, commence aux formes gnathostomes perfectionnées, dont le type le plus parfait est Notodelphys, genre sans adaptation à l'existence dégradante d'un parasite. Aussi est-on en droit de considérer ces ètres comme de simples locataires, abrités dans la cavité respiratoire des Ascidies.

Dans les Doropygiens, les facultés de locomotion ne sont nullement perdues. Appropriées à un mode spécial de déplacement, elles consistent à ramper à la surface des parois de la corbeille respiratoire des Tuniciers. Les seules particularités 
de ces êtres sont dans le mode de protection que les femelles réservent aux embryons en voie de développement.

Ainsi, chez tous les Notodelphyidés (= Ascidicolidés, pars), les effets de l'état spécial de dépendance éthologique (et non point biologique) qu'ils subissent, sont en somme très restreints et ont plus d'effet sur les caractères sexuels que sur l'organisation fondamentale.

Mais les manifestations du parasitisme commencent déjà chez des formes visiblement modifiées comme Enterocola. Enteropsis, Aplostoma, et je ne doute pas que l'étude des types si curieux qui forment le genre Ophioseides HesSE ne fournisse aux naturalistes des données nouvelles sur ce groupé.

Agnathaner est le trait d'union entre les formes normales gnathostomes et les types dégradés.

Nous voyons, en comparant l'habitat de Enterocola et Aplostoma avec celui de Notodelphys et Doropygus, etc., que les points d'élection (estomac, tube épicardique) des formes plus dégradées sont profondément situés dans l'ascidiozoïde et indiquent, par suite, un degré de parasitisme plus intime, une plus étroite dépendance. Et de nouveau, nous aboutissons à cette conclusion: que l'éthologie confirme et explique les résultats des recherches morphologiques.

Les Ascidicolidés, qui étaient à l'origine de parfaits gnathostomes nageurs, abrités seulement dans la branchie de leur hôte (Notodelphys), se sont peu à peu adaptés à l'existence cavicole en modifiant leur mode de locomotion dans différents sens (Doropygus, Bonnierilla, Gunenotophorus, d'une part; Ascidicola, d'autre part). Changeant leur genre de vie et devenant de vrais parasitès, ils se sont tranformés en siphonostomes, par les divers stades : Agnathaner, Enterocola, Enteropsis, Aplostoma.

Je dois faire observer encore un fait de nature à appuyer cette compréhension de la biologie des Ascidicolidés. Dans Phallusia, Molgula, Cynthia et Lithonephria qui abritent les commensaux de leur cavité branchiale: Dorogygus, Notodelphys, Bonnierilla,... Botryllophilus, Ascidicola, les glandes génitales fonctionnent parfaitement, et il n'y a pas de castration parasitaire.

Avec Enterocola ou Aplostoma, il en est tout autrement. Dans les ascidiozoïdes pourvus de ces parasites, les produits génitaux sont arrêtés dans leur développement. Les cloaques de Circinalium et de Morchellium ne contiennent pas d'embryons en voie de développement, lorsqu'un Aplostoma est logé dans leur cavité branchiale ou dans leur épicarde. En un mot, les parasites vrais mont seuls présenté les effets de la castration parasitaire. 
C. Nereidicolidæ. - Ne l'ayant pas étudiée personnellement, je ne citerai que brièvement cette famille, établie par Claus (55) et adoptée par KURZ (126). Je ferai observer seulement qu'avec l'habitat sur des animaux du mème groupe, les divers genres: Rhodonicola LevinSEn, Nereicola KefERSTEIn, Eunicicola KURz, Chelonidium HeSSE, Gastrodelphys GR fFFE (= Sabellocheres SARS?)... partagent entre eux une série de caractères qui sont: la disparition graduelle des pattes thoraciques dans le sens postéro-antérieur, la nature des mâles, la morphologie des pièces buccales. Dès à présent je crois pouvoir conclure à l'existence sur les Annélides polychètes, d'une riche série éthologique de formes reliées entre elles par de sérieux rapports de parenté. Elle doit être mentionnée à còté des Hersilidés et des Ascidicolidés. L'étude embryogénique comparative des genres les plus dégradés (comme Herpyllobius ST. ET LUTK.) serait d'un grand secours dans la délimitation définitive des rapports de ces ètres; et c'est le desideratum le plus urgent qui soit à signaler ici à l'attention des naturalistes.

D. Lernæidæ. - A cet exposé des observations éthologiques conduites sur divers groupes semi-parasites, j'ai pu ajouter l'observation personnelle du curieux dimorphisme éthologique qu'ont fait connaitre, chez les Lernéens, les découvertes de Metzger (146) et de Wierzejski (211).

La plus grande partie des Platessa flesus pêchés à la còte dans le Boulonnais, ont les filaments branchiaux garnis d'une foule de petits parasites à divers états de développement, depuis le stade cyclops sortant du nauplius, jusqu'aux adultes prêts à la copulation. Claus (54), sur les échantillons conservés par MetzGer, a suivi les diverses modifications morphologiques de ces êtres, et confirmé l'opinion de ce dernier auteur, d'après laquelle les parasites de la branchie des Flets seraient les jeunes formes de reproduction de Lerncea branchialis L. De mon còté, j'ai vérifié, par comparaison directe des jeınes stades cyclopoïdes au sortir des nauplius de Lerncea branchialis avec les jeunes stades cyclops fixés aux branchies des Flets, l'exactitude de la détermination de Metzger et de Claus. Mais malgré une observation attentive poursuivie durant plusieurs années, jen'ai jamais rencontré sur Platessa flesus, si riche en formes jeunes et en formes de copulation, une seule femelle de Lernée déformée, dans le stade de production des œufs.

Au contraire, chez les Morues qu'on pêche sur les plages du Boulonnais en quantité assez considérable et mème en compagnie des Flets infestés, je n'ai jamais rencontré de jeune Lernée fixée sur les branchies, mais seulement un nombre considérable de femelles múres, avec la déformation caracteristique du stade de production des œufs, sans màle cyclopiforme.

De ces observations qui viennent confirmer à nouveau les données négatives 
anciennes d'un grand nombre de naturalistes, il résulte que Lerncea branchialis femelle, aux deux époques de son existence séparées par la copulation, affectionne deux régimes différents sur deux hôtes également différents (I). Ces deux habitats particuliers sont d'ailleurs séparés par une période de semi-parasitisme, durant laquelle le malle recherche la femelle, et la femelle fécondée recherche le second hôte.

D'après les recherches de WIERZEJSKY sur les jeunes Lernéens des branchies des Céphalopodes (Sepia officinalis, Loligo vulgaris, Eledone moschata), Penella varians ST, et LUTK. présenterait les mèmes habitudes, et passerait la première partie de son existence parasitaire sur les branchies des Seiches et des Calmars, pour se transporter ensuite sur le Dauphin où s'écoule la période de production des œufs

De ces observations dủment acquises sur deux espèces de Lernéens, nous pouvons déduire un mode d'existence spécial à cette famille de parasites, et admettre dans leur évolution complète, pour le sexe femelle, deux périodes de parasitisme avec deux périodes de semi-parasitisme : la première, durant les stades nauplien et cyclopoïde, est suivie d'une periode de parasitisme transitoire; la seconde, au moment de la copulation, est suivie du parasitisme complet et définitif.

Par leur parasitisme embryonnaire, Jes Lernéens ne se distinguent d'ailleurs aucunement d'autres siphonostomes parasites sur les Poissons. Si leurs jeunes sont fixés, n'en est-il pas de même des Caliges (Chalimus), etc.? La seule différence consiste dans le changement d'hôte avant la ponte.

Les femelles de Lernixa et de Penella cherchent-elles une nourriture plus abondante ou un abri plus sûr, afin de produire plus aisément leurs œufs ; ou bien ce changement d'habitat s'est-il établi par sélection naturelle; en vue de la progéniture elle-même? On ne peut faire, sur ce point, que des suppositions purement gratuites, et il me.semble plus prudent d'attendre d'une connaissance plus approfondie de la famille des Lernæidés des documents capables de fixer nos idées à ce sujet.

\section{CARACTERES SEXUELS SECONDAIRES. - PROGENESE.}

L'étude des caractères extérieurs, qui permettent d'arriver - avec la constatation des produits sexuels - à la distinction des sexes, présente, depuis les recherches

(1) Metzger a trouvé, en compagnie de stades de copulation, des Lernées femelles déformées, sur Cyclopterius lumpus. Bien que j'aie observé accidentellement de jeunes Lernées fixées en masse sur les branchies de ce Poisson, je n'en ai jamais obtenu les premiers stades de deformation à côté des jeunes. Accidentellement aussi, j'ai rencontré, sur le Cycloptère, de grandes Lernées fémelles, avec des cufs pondus. 
de Darwin, une importance considérable. Malgré l'attention qu'ont prêtée les naturalistes au problème de l'origine des sexes, cette question demeure l'une des plus obscures de la biologie.

Comme l'a montré Giard (I) l'éthologie joue un rôle prépondérant dans l'établissement du dimorphisme sexuel, et les observations faites à ce sujet, sur les Entonisciens comparés aux diverses espèces dimorphes de Cirripèdes et de Myzostomides (2) ont jeté un jour nouveau sur cette partie interessante de la biologie.

Les Copépodes, dont les conditions d'existence sont si variables, ne pouvaient manquer de montrer, à ce point de vue, d'intéressants phénomènes. Les divergences, depuis longtemps classiques, qui séparent les mâles et les femelles des Copépodes parasites, trouveront peut-ètre d'utiles comparaisons dans les conditions sexuelies que j'ai observées chez les semi-parasites moins dégradés.

Les caractères sexuels secondaires affectent, chez les Copépodes, la plupart des appendices; dans les traités élémentaires de zoologie, sont citées d'importantes variations localisées dans différents types sur les antennules, antennes, maxilles, pattes thoraciques (spécialement de la $5^{\mathrm{e}}$ paire), etc. Mais, en réalité, il existe entre les mâles et les femelles des différences dans presque toutes les parties du corps, avec cette restriction qu'elles se présentent çà et là, dans les diverses familles, plus ou moins accentuées sur telle ou telle partie.

A. Copépodes libres. - Les modifications que subissent leurs appendices pour se transformer en organes préhensiles chez le mâle, sont trop directement reliées à la réunion des sexes pour être mises en question; mais d'autres particularités plus spéciales qui apparaissent dans quelques espèces, restent inexpliquées, et leur mode d'utilisation ou de fonctionnement demeure un problème difficile à résoudre.

Dans les Copépodes pélagiques, que l'on recueille en grande quantité à la surface de la mer et qui vivent en troupes d'une étendue souvent immense, les mâles sont mêlés aux femelles et il ne semble point que le dimorphisme sexuel y affecte l'éthologie, comme c'est le cas pour tant d'autres espèces de Crustacés: pour certains Ostracodes, par exemple, dont les femelles vivent au fond de la mer et les mâles, à la surface, en vrais pélagiques.

Le plus souvent, la transformation de l'antennule droite du màle en pince préhensile, par l'apparition d'une articulation géniculée secondairement acquise, semble assurer seule la réunion des sexes.

(1) Giard, La castration parasitaire et son influence...; Bull, scienr, tome XVIII, 1887 ; page 24.

(2) Giard et Bonmier, Contributions à l'étude des Bopyriens, Trav, du Lab. de Wimerenx, tome V, 1887, page 212-215. 
Dans les Calanidés sans antennule géniculée, d'autres caractères encore inexpliqués, séparent les mâles des femelles.

Chez Calanus finmarchicus et Paracalanus parvus, j'ai pu constater notamment le grand développement des soies terminales et externes des deux derniers articles de la seconde maxille externe chez le mâle (Pl. I, fig. I6 et 7) comparé à la femelle (Pl. I, fig. I7 et 8). De plus, dans la femelle l'abdomen est élargi à la région postérieure et les pièces furcales plus larges sont aussi plus divergentes (Pl. I, fig. I3 et 15) que dans le mâle. Les antennules légèrement épaissies paraissent seulement jouer, chez le mâle, un róle sensoriel plus actif et fonctionner plus efficacement comme balancier d'équilibre (I).

Dans les Euchætinés de Giesbrecht (89, p. 163) se rangent un petit nombre de genres pour lesquels Claus (51, p. 28 et 30 ) signalait déjà une régression des pièces buccales, dans le sexe mâle. J'ai pu réétudier après GiesBRECHT la mème disposition dans Clausia elongata (Lucullus acuspes, GresBr.). Dans la femelle de Clausia elongata, ainsi que dans le jeune mále avant la dernière mue, tous les appendices buccaux présentent les propriétés masticatrices et natatoires habituelles aux Calanides (PI. II, fig. 9, I7, I2). Dans le mâle, avec la dernière mue, les mandibules, premières maxilles et secondes maxilles externes perdent la majeure partie de leurs accessoires masticateurs (PI. II, fig. 12 et 13 ) en même temps que l'épipodite de la $I^{\text {re }}$ maxille disparaît completement.

Il peut être déduit de ce qui précède, que la femelle et le mâle suivent jusqu'au moment de la reproduction, le mème cycle évolutif ; ils possèdent alors les mèmes besoins biologiques, sans distinction de sexe. Dès l'époque de la maturation des produits sexuels, le mâle, dans sa dernière transformation, s'écarte de la femelle, pour prendre, en raison sans doute des exigences de la fonction reproductrice, une toute autre série de propriétés accentuant le dimorphisme sexuel au point de vue éthologique.

En tous cas, par ces faits dûment observés, nous sommes autorisé à dire que dans les Euchætinés, les mâles sont plus spécialisés, plus transformés que les femelles; ils sont par suite plus élevés dans la série morphologique. Mais les caractères extérieurs ne sont pas seuls à distinguer entre elles les formes sexuelles des Copépodes pélagiques.

GiesBRECHT (89) a signalé chez Temora longicornis et Centropages hamatus un développement plus grand de la musculature dans les pattes natatoires, chez le mâle; il ajoute que le fait s'explique par la nécessité pour le mâle de

(i) Ces particularités que j'ai constatées seulement dans les deux espèces que j'ai pu étudier se reproduisent, chez de nombreux Copépodes, comme a bien voulu me le faire savoir le Dr GiesBrECHT. Et il me semble, ainsi qu'à ce naturaliste, parfaitement juste de fonder une sous-famille spéciale comprenant ces Calanides. 
rechercher et poursuivre les femelles au moment de la réunion des sexes. Comme l'a fait remarquer cet auteur, les muscles des pattes thoraciques sont en effet, chez Centropages hamatus mâle (Pl. II, fig. 20), plus développés que chez la femelle (Pl. II, fig. 2I) ; les muscles extrinsèques de l'endopodite $(m)$ sont particulièrement différents.

Mais j'ai rencontré encore un cas particulièrement frappant du dimorphisme sexuel musculaire, dans les deux espèces de Acartia que j'ai recueillies en abondance à Wimereux.

Dans Acartia clausi et $A$. discaudata, les muscles dorsaux forment au-dessous de la carapace céphalique du mâle, un revêtement continu et serré; ils donneñt à la région cervicale un aspect tout à fait différent de celui que présente la femelle (comparer Pl. II, fig. I6 et I7, I 8 et I9). En étudiant cette musculature, on voit que le muscle le plus développé, qui accentue à un si haut degré le dimorphisme musculaire, est le moteur extrinsèque des antennules auquel HarTOG (106, p. 11) a donné le nom de “ double extenseur", et il présente certainement, en raison de sa masse chez le mâle, une puissance presque triple de celle qu'il a chez la femelle.

Ici encore pourrait s'appliquer l'explication fournie par Giesbrecht. Mais ces faits n'ont-ils pas une importance plus grande, si on les examine en détail?

Par ce qui précède, il est démontré que dans les Copépodes pélagiques, les mâles sont mieux musclès et plus vigoureux que les femelles, au moins en ce qui concerne les appendices natatoires. Leur musculature devient même beaucoup plus puissante, dans certains points et chez quelques espèces. Il existerait ainsi, comme dans beaucoup d'autres animaux (I), un perfectionnement acquis ou développé par sélection naturelle qui aurait été fixé dans les descendants mâles par l'hérédité limitée ã ce sexe.

En recherchant les conditions dans lesquelles les facteurs évolutifs ont pu provoquer pareille transformation, DARWIN était arrivé à faire intervenir l'influence du nombre. Il crut d'abord que “ chez la plupart des animaux à caractères sexuels bien développés, le nombre des mâles excédait de beaucoup le nombre des femelles "(2).

(1) Darwin. Descendance de l'homme, trad. franç., p. 229. "Lorsque les deux sexes ont exactement les mêmes habitudes d'existence et que le mâle a les organes des sens et de locomotion plus développés que la femelle, il se peut que ces sens perfectionnés lui servent pour trouver la femelle. Mais dans la grande majorité des cas, ces organes perfectionnés ne servent qu'à procurer à un male une certaine supériorité sur les autres mâles, car les moins privilégiés, si le temps en était laissé, réussiraient tous à s'apparier avec des femelles... La sélection sexuelle a dû évidemment intervenir pour produire les organes auxquels nous faisons allusion, car les malles ont acquis la conformation qu'ils ont aujourd'hui, non parce qu'elle les met à mème de remporter la victoire dans la lutte pour l'existence, mais parce qu'elle leur procure un avantage sur les autres mâles, avantage qu'ils ont transmis à leur postérité mâle seule."

(2) Darwin, Descendance de l'homme, trad. franç., p. 233. 
Et il admet momentanément l'influence de l'hyperpolyandrie comme facteur augmentant dans le sexe màle, flutôt que dans le sexe femelle, la concurrence vitale et amenant, pour le succès des fonctions reproductrices, une hypertrophie organique dont les femelles, moins concurrentes, n'ont nullement besoin.

DARWIN a lui-même abandonné le ròle de l'hyperpolyandrie, car il croyait qu'une grande disproportion numérique entre les sexes serait nécessaire pour fixer dans les descendants les perfectionnements acquis par la concurrence (1).

Nous savons aujourd'hui, par la loi mathémathique établie par DELBCEuF, et maintes fois appliquée par GIARD (2), qu'il n'est nullement besoin d'une aussi notable proportion en faveur des individus variés, pour établir la variation.

Devant ces données nouvelles, je crois devoir reprendre, en faveur des Copépodes pélagiques du Boulonnais, l'intervention de l'hyperpolyandrie, car j'estime malgré les nombreuses causes d'erreur qui peuvent entrer en jeu dans l'établissement d'une pareille opinion, que les mâles adultes y sont un peu plus nombreux que les femelles. Dans ces êtres, la concurrence vitale croissante ressentie par les màles a été l'origine des transformations hypertrophiques qu'ils nous présentent et qui ont été fixées par la sélection sexuelle (3) et l'hérédité limitée.

B. Copépodes parasites et commensaux. - Le véritable dimorphisme sexuel, qui réside simplement dans l'aspect général des animaux, n'apparait qu'au moment où, le parasitisme étant déjà bien accentué, les différentes petites adaptations morphologiques se compliquent de différences éthologiques très grandes dans l'habitat et les facultés de locomotion ou dans le régime nourricier.

Ainsi fut attirée l'attention des auteurs qui ont signalé les divergences considérables qui séparent les formes sexuelles chez les parasites de poissons les plus dégradés.

$1^{0}$ Négligeant actuellement les différences de taille qui ont surtout fait remarquer par les zoologistes les “ mâles nains " des Copépodes parasites, j'examinerai

(1) Loc. cit., p. 233 : "Si les mâles étaient aux femelles, comme 2 est à 1, comme 3 est à 2 , ou mème dans une proportion un peu moindre, la question serait bien simple, car les mâles les plus attrayants laisseraient le plus grand nombre de descendants. Mais après avoir étudié, autant que possible, les proportions numériques des sexes, je ne crois pas qu'on puisse ordinairement constater une grande disproportion numérique. "

(2) Revue Scientifique, t. XIX, 1877 : Delbeuf, p. 669, et A. GIARD, p. 77I. - A. Giard, Les facteurs de l'évolution, Rev. Scient., t. XLIV, 1889, p. 646. - R. BARon, La loi de Delbeufy Bull. Scientif., t. XXII, 1890, p. 123.

(3) DARWIN, loc. cit., p. 233. Dans la plupart des cas, la sélection sexuelle a agi de la maniere suivante: les femelles les plus vigoureuses sont les premières prètes à reproduire, les mâles les plus vigoureux chassent les plus faibles, et de ces couples naissent des rejetons de plus en plus vigoureux et aussi plus nombreux. 
les variations plus restreintes, affectant la morphologie des appendices dans les formes moins dégradées.

$a$. Dans les Lernæopodidés, etc., on trouve certainement de nombreux exemples de mâles dégradés, suivant l'interprétation donnée à cette expression par Giard et Bonnier (loc. cit., p. 2 I 2-2 I 5). Mais dans les Chondracanthidés, Claus (41, p. 17) et $\operatorname{Vogt}(\mathbf{2 1 0}$, p. 92) ont insisté sur les ressemblances que présentent les mâles avec les formes embryonnaires; souvent ces ressemblances se retrouvent dans la forme extérieure du corps aussi bien que dans l'organisation morphologique. Plus récemment, dans la curieuse famille des Philichtyidés, Claus $(63, p$. I I) a constaté une concordance parfaite entre les caractères du mâle et ceux de la jeune femelle.

Chez les parasites vrais, il est donc juste d'admettre que, dans certaines limites, les mâles adultes sont moins transformés que les femelles adultes.

b. Chez Notopterophorus, GIESBRECHT (90) a reconnu de faibles différences entre les antennes, les mandibules, les premières maxilles et les secondes maxilles internes des femelles et des mâles, et j'ai pu vérifier, chez Doropygus gibber, la ressemblance parfaite existant à ce sujet, entre les femelles jeunes et les mâles : ici encure, c'est-à-dire dans les formes les plus spécialisées des Ascidicolidés commensaux, le nombre et le développement des soies plumeuses sur les rames des appendices buccaux, la forme des griffes et des dents des épines masticatrices, (tous caractèrés qui passent à juste titre pour indiquer dans les Copépodes normaux les plus parfaits une métamorphose progressive) atteignent le niveau le plus élevé chez la femelle; après leur entier développement, les mâles semblent, à ce titre, plus jeunes qu'elles. Ainsi donc, chez ces animaux qui présentent apparemment les mèmes conditions d'existence dans les deux sexes, nous observons le dispositif inverse de ce que nous avons constaté chez les Copépodes libres : c'est le sexe femelle qui est plus profondément transformé, mieux organisé que le sexe mâle.

De même que plus haut, cherchons dans quelles conditions les femelles ont pu acquérir un tel avantage sur les mâles.

D'après une longue série d'observations, j'ai toutes raisons de croire à une notable disproportion entre le nombre des mâles et celui des femelles adultes, chez les Doropygiens. Les mâles de ces animaux sont faciles à découvrir, et je puis les distinguer facilement à l'œil nu des jeunes femelles de mème taille. Dans le Boulonnais, je récolte pourtant en nombre considérable les femelles de Doropygiens très abondants sans trouver régulièrement les màles, et il est exceptionnel 
que je puisse découvrir quelques-uns de ces derniers à la suite de recherches cependant persistantes et attentives $(\mathbf{I})$.

Les femelles y sont donc bien plus nombreuses que les màles; et il y a hyperpolygynie chez ces commensaux.

C'est là un point à mettre en parallèle avec l'hyperpolyandrie des Copépodes pélagiques, point d'autant plus curieux qu'il se présente dans des formes sur. lesquelles l'influence de la vie sédentaire ou commensale se traduit morphologiquement dans des limites très restreintes. L'hyperpolygynie dans les Doropygiens vient appuyer d'une façon remarquable la croyance à l'hyperpolyandrie chez les Calanidés et son action sur les formes sexuelles.

$2^{0}$ Dans les cas de dimorphisme sexuel affectant plus spécialement la taille et l'aspect général, les mèmes conclusions ne sauraient être admises d'une façon constante. Les conditions d'existence acquièrent alors une importance prédominante et le mode de nutrition semble être la cause première de ces variations sexuelles.

a. Dans les Hersiliidés, dès que l'adaptation à l'existence parasitaire se montre plus profonde, le dimorphisme sexuel apparaît aussi. Dans Giardella callianasse, le màle est plus grand que la femelle; il vit comme elle en semi-liberté, au voisinage de la Callianasse. Dans Clausidium apodiforme, le mâle, plus petit, est fixé presque constamment sur la femelle et prend l'aspect d'un màle nain, tout en conservant intacte l'organisation morphologique de l'espèce.

N'en est-il pas de même chez Nicothoe; et le mâle de cette espèce, qu'on n'a jamais trouvé sur la femelle, n'est-il pas un semi-parasite, de taille si réduite qu'il ait pu échapper à l'observation?

Le parasitisme croissant semble bien exercer une influence de plus en plus grande sur le dimorphisme sexuel.

b. Chez les Ascidicolidés, nous trouvons les mèmes faits : dans une première série, comprenant les formes logées dans de grandes cavités respiratoires où elles se meuvent et vivent à l'aise, les dégradations sont faibles et le dimorphisme morphologique n'apparait que très légèrement chez les espèces les plus spécialisées (Notopterophorus); - dans la seconde série, composée d'animaux où le sexe femelle est incapable, par habitat et par dégradation, de se mouvoir aussi librement, le dimorphisme biologique est très net.

(1) Deux genres communs, Bonnierilla et Gunenotophorus, sont connus seulement par leurs femelles. 
Je ne reviendrai pas sur les observations relevées plus haut, pour Enterocola, Aplostoma et Enteropsis ( 1 i.

Dans ces ètres, les femelles vermiformes sont tenues pour la vie dans leur habitat spécial, tandis que les mâles sont beaucoup plus mobiles que ceux des Doropygiens. Par comparaison avec ces derniers, on pourrait dire que chez Enterocola, le mâle est devenu plus pélagique et la femelle plus parasite.

Mais il me semble utile de rechercher ici dans quelles conditions a pu s'établir le dimorphisme sexuel si accentué et si spécial de ces formes.

Par le genre Agnathaner, Enterocola et Aplostoma mâles se rattachent à Notodelphys dont le mâle et la fernelle sont bons nageurs et ne diffèrent pas sensiblement. Les mâles d'Enterocola n'ont donc fait que conserver les propriétés éthologiques ancestrales, qui sont aussi celles des embryons cyclopoïdes.

Il est bien certain que dans les ancêtres des Entérocoliens, les femelles étaient moins précocement dégradées qu'elles ne le deviennent actuellement au cours dụ développement ontogénétique. Dans ces formes ancestrales, l'embryon femelle suivait lui-mème, jusqu'à l'apparition de tous les segments et de toutes les pattes natatoires, le développement ordinaire conservé chez le mâle. La régression de la femelle s'effectuait alors, non point après le $2^{\mathrm{e}}$ stade, mais avec la dernière mue. A mesure que le degré de parasitisme s'est accru, le raccourcissement embryogénique subi par la femelle et provoqué par les changements éthologiques s'est accentué davantage, et les condensations successives du développement se sont fixées seulement dans le sexe femelle. par hérédité limitée. Le sexe mâle n'héritait que des modifications communes à toutes les formes semblables et établies dans l'organisation de la bouche par le mode de nutrition. Ainsi s'explique la concordance morphologique parfaite entre les appendices buccaux de l'embryon du mâle et de la femelle.

Dans ces conditions, s'il exista au début de la dégradation parasitaire une légère différenciation morphologique entre les deux sexes des Copépodes entérocoliens ancestraux (différenciation rapprochant les màles adultes des jeunes femelles immatures plutôt que des femelles adultes, comme c'est le cas chez Notopterophorus), le raccourcissement embryogénique dans l'évolution de la femelle eut

(1) J'ai pu étudier dans toutes ses particularités morphologiques un Copépode mâle, très abondant à Wimereux dans Morchellitum argus et Perophora Listeri, dans lesquels il vit en compagnie de Doroixys unciuzta. Je suppose que cette forme est le mâle de Doroixys; mais la preuve morphologique de cette opinion n'est point suffisamment nette. Ce Copépode est très bon nageur et ressemble à Notodclphys; il est pourvu de globules graisseux jaunâtres rappelant exactement ceux du deutoplasme des embryons de Doroixys; mais ses secondes maxilles internes sont un peu différentes de celles de Doroixys femelle. Si mon hypothèse ètait justifiée, Doroixys appartiendrait à la $2^{\circ}$ série des Ascidicolidés. 
pour effet de voiler ( $\mathrm{I}$ ) entièrement l'ontogénie des descendants actuellement connus en lui donnant tous les caractères d'un dimorphisme sexuel précoce.

Le diagramme ci-après résume d'après ces considérations la marche del'évolution phylogénétique des deux sexes chez Enterocola, le mode actuel de différenciation étant indiqué par les traits pleins, et les états ancestraux par les lignes ponctuées.

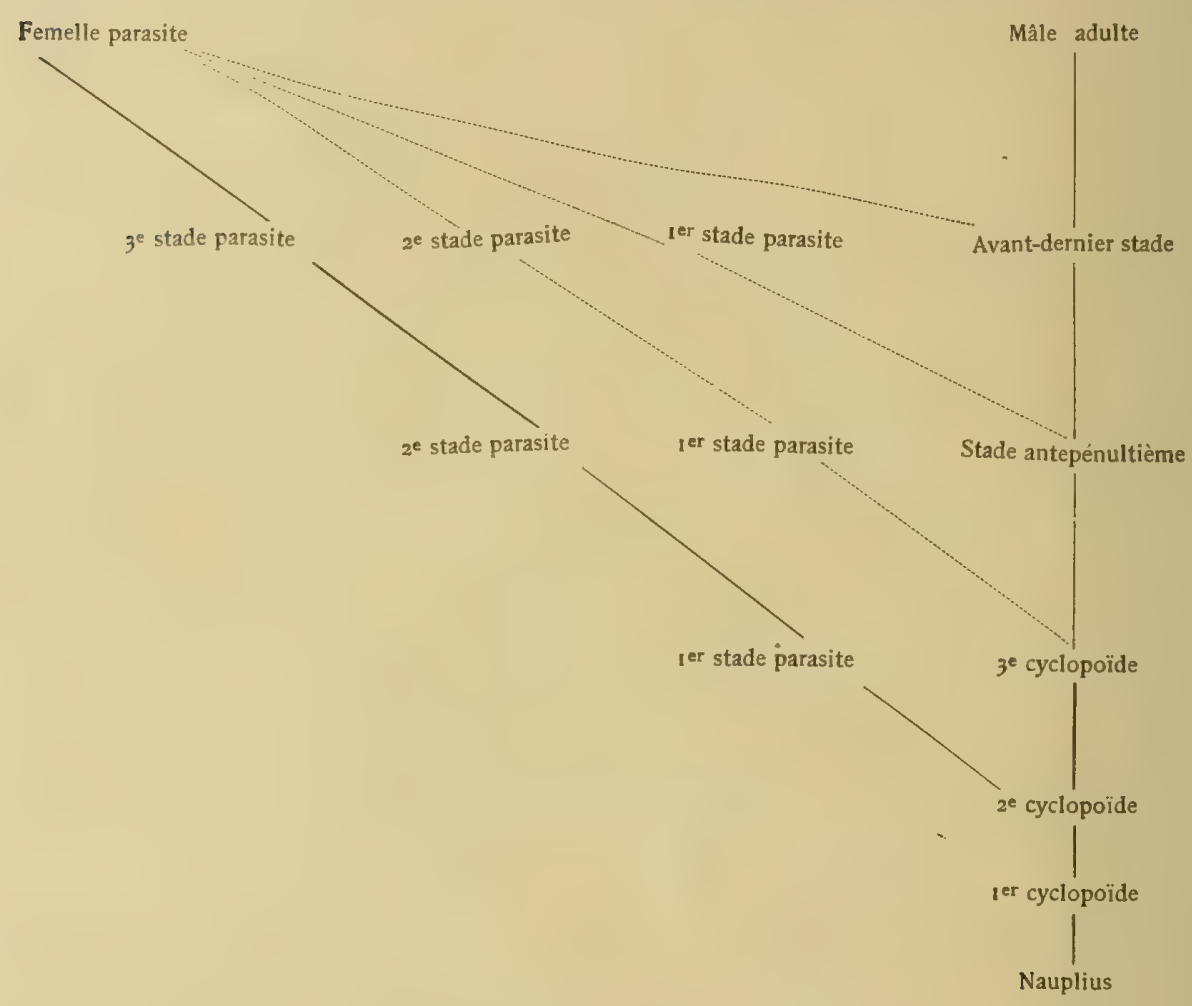

Durant le déveioppement phylogénétique des sexes, à mesure que l'embryon femelle a trouvé, en s'enfonçant plus profondément dans son hôte, un régime plus profitable à la formation des produits gẻnitaux, le dimorphisme biologique est devenu de plus en plus précoce. La sélection naturelle a établi, entre les femelles ainsi privilégiées, une concurrence qui eut pour but l'abréviation croissante de l'existence semi-parasitaire chez leurs embryons.

(1) En faisant disparaître les derniers stades semi-parasites de la femelle et en les remplaçant par des stades dégradés entièrement parasites. 
En ce qui concerne la production du sexe mâle, on pourrait trouver, en faveur $\mathrm{du}$ facteur biologique, d'autres arguments dans l'intervention des saisons. J'ai constaté, en effet, une abondance beaucoup plus grande des mâles d'Enterocola aux époques où la Synascidie qui sert d'hôte nourricier aux deux sexes, est le moins robuste: durant le premier printemps, au sortir de l'hivernage; en septembre, aux abords du même hivernage. Pendant la saison d'été, lorsque Polyclinum prolifère très activement et produit des embryons, Enterocola femelle est très fréquente et donne également beaucoup d'embryons; le mâle est alors très rare.

Il semblerait donc que les embryons fournis par les femelles se transforment plus facilement en femelles parasites lorsque l'Ascidie leur est une proie plus riche, et que, dans le cas inverse, ils forment des mâles.

c. Progenèse. - D'après l'exposé que je viens de faire des particularités sexuelles chez divers Copépodes, il semble naturel d'admettre, dans cette classe, l'existence de phénomènes progénétiques analogues à ceux qu'a signalés GIARD ( I).

Dans les exemples cités plus haut (voir $b, I^{0}$ ) de légères différenciations morphologiques entre les sexes, il est manifeste que la progenèse est acquise dans le sexe mâle; les Doropygiens les plus spécialisés montrent, ainsi que bon nombre de Copépodes parasites des Poissons, des cas de progenèse protandrique.

Chez les Calanides d'autre part, nous avons constaté, d'après les modifications acquises dans les appendices butcaux chez les Euchætinés et dans la musculature chez d'autres Calanidés, un commencement de progenèse protogynique (voir $a$ ).

Mais les Ascidicoles les plus modifiés, comme Enterocola, ne paraissent pas fournir d'exemples précis pour ces comparaisons, à cause des modifications spéciales de la femelle qui donnent à celle-ci, dans le chapitre des différenciations sexuelles chez les animaux, le rang spécial de femelle dégradée, comme certains màles d'Entonisciens se distinguent parmi les autres à titre de mâles dégradés (2).

(1) A. GIARD, La castration parasitaire et son influence sur les caractères sexuels extérieurs du sexe mâle chez les Crustacés Décapodes, Bull. Scientif., t. XVIII, 1887, p. 23. - Giard et Bonnier, Contribution à l'étude des Bopyriens, Trav. du Lab. de Wimereux, t. V, 1887, p. 212: "II y a progene'se lorsque chez un animal la 》 reproduction sexuée s'opère d'une façon plus ou moins précoce, c'est-à-dire lorsque les produits sexués (œufs " ou spermatozoides) se forment et mûrissent avant que l'être n'ait atteint son complet développement......

" Chaque fois qu'il y a progenèse, dans un type déterminé, on constate donc, soit momentanément, soit d'une

" façon définitive, un arrêt de croissauce et de développement : l'animal progenétique a, par suite, l'aspect

1) d'une larve sexuée, lorsqu'on le compare soit à l'autre sexe, soit aux formes voisines qui ne présentent pas le

" phénomène de progenèse. "

(2) Giard Et Bonnier, loc. cit., p. $213-215$. 


\section{COMMENSAUX ET ENNEMIS DES COPEPODES.}

I. Schizomycètes. - A la surface des Copépodes libres ou semi-parasites se développent souvent des végétations de Schizomycètes que l'on peut rapporter avec les auteurs spéciaux au genre Phragmidiothrix. Ces organismes ne sont nuilement parasites de nos animaux. Mais ils trouvent chez eux le mouvement indispensable pour le renouvellement continu du liquide environnant. Ainsi, les soies furcales et appendiculaires de Giardella callianassa et de Hersiliodes thompsoni sont, comme les soies des Callianasses, fréquemment chargées de Phragmidiothrix incrustans GIARD, Schizomycète tapissant également la paroi des galeries de ce Crustacé, où il forme un revètement particulier d'aspect ferrugineux. GIARD a signalé ce commensal des Callianasses ( $\mathbf{r}$ ) et fait remarquer, à juste titre, que le végètal ne recherchait en cet endroit qu'une eau fréquemment renouvelée par les mouvements du Crustacé.

Comme beaucoup d'autres Crustacés (2), les Copépodes libres servent aussi de supports à des Phragmidiothrix que je n'ai pas étudiés plus particulièrement; ils sont fixés aux points les plus découverts : sur les antennules, les soies furcales, la carapace dorsale. les bords des replis pleuraux..., etc. Les mouvements rapides et rarement interrompus des animaux assurent le renouvellement constant du liquide autour d'eux et facilitent les actifs échanges de gaz que réclame le végétal ainsi transporté.

2. Vorticelliens. - Les Copépodes les plus divers, Harpacticidés, Calaniidés, Cyclopidés, etc., servent de support et en même temps de véhicule à des Vorticelliens très divers.

Les Epistylis ne sont pas rares sur les Cyclopides des eaux douces; mais je n'en ai point observé sur les Cyclopidés marins ou d'eau saumâtre que j'ai étudiés.

Temora longicornis, I sias clavipes et Centropages hamatus, si communs dans les pêches de surface effectuées auprès du littoral, portent souvent une belle Vorticelle à la partie antérieure du corps ; je n'ai pu l'étudier assez complètement

(1) Giard, Fragments biologiques; Bull. Scient., tome XX, 1889, page 177.

(2) Consulter spécialement: ENGLER, Ueber die Pilzvegetation des weissen oder todten Grundes in der Kieler Bucht; IV tes Bericht der Comm. Fo wiss. Unt. do deutsch. Meer. in Kiel. VIIl ter-XIter Jahrg. 1877, 2 ter Abth 
pour en donner ici la description. Temorella affinis, de la rade d'Honfleur, porte un Infusoire très voisin sinon identique.

3. Acinétiens. - Tachidius discipes et Tachidius littoralis sont fréquemment couverts d'une quantité considérable de petits Acinétiens, à pédoncule court, à coque granulée et irrégulièrement plissée. Par plusieurs caractères de son organisation, relatifs à la disposition des suçoirs et des ouvertures de la coque, à la position du corps à l'intérieur de l'enveloppe, l'Acinétien des Tachidius se rapproche beaucoup de Acineta fotida Maupas. Mais l'ornementation de la coque rappelle plutôt Acineta cucullus CLAP. ET LACHM. (2), dont elle se distingue pourtant par son irrégularité.

D'après Maupas, Acineta fatida vit à Roscoff et à Alger dans des conditions assez spéciales. Elle devient très abondante dans les aquariums où se trouvent

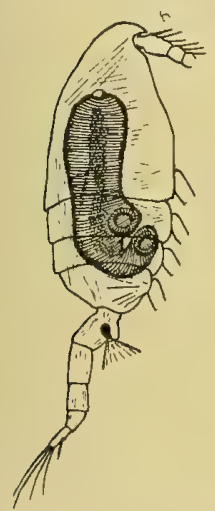

Fig. 12. - Forme jeune d'Apoblemaappendiculatum Diesing dans Clausia elongata BOECK,

d'après Monticelli, loc. cit., Fig. 6. amassées des algues en voie de putréfaction, dans une eau recouverte à la surface d'une couche continue de Bactéries formant une épaisse pellicule.

L'Acinète des Tachidius est très abondant dans l'eau saumâtre, relativement claire et nullement corrompue, de l'embouchure du Wimereux. Par sa fixation sur les Copépodes, par le peu de résistance qu'il offre à la putréfaction, il s'écarte notablement de l'espèce citée plus haut. Je l'appellerai Acineta wimerosa.

Idy a furcata est fréquemment pourvu dans sa région frontale des deux formes, jeune et définitive, du bel Acineta trinacria Gruber, déjà signalé par Claus (51, p. 87, pl. XV, fig. 12) à Messine et étudié par Gruber (3) à Gênes.

4. Apoblema appendiculatum, Rudolphi. Plusieurs fois j'ai trouvé, dans la cavité générale de Paracalanus parvus et Acartia clausi pêchés au large, un jeune Distome coloré en brun-jaunâtre, avec la cuticule ornementée d'annelures transversales saillantes. Je le rapporte à cette espèce, d'après la description récemment publiée par MonTicelli (4). Peut-être est-ce l'animal déjà signalé par

(1) Maupas, Contribution à l'étude des Acinétiens, Arch. de Zool., sér. I. t. IX, 1881, p. 315-323, PI. $\mathrm{XIX}$, fig. 6-32.

(2) Claparède et Lackmann, Études sur les Infusoires et les Rhizopodes, Genève, 1858-1861.

(3) Gruber, Die Protozoen des Hafens von Genua, Nova Acta Leop. Carol, nat. cur. Akad., Bd. XLVI.

(4) Fr. Sav. Monticeilu. Osservazioni intorno ad alcune forme del Gen. Apoblema Dujardin, Alti. $d$. $R$. Accad. Sci. Torimo, vol. XXVI, 1891. 
Claus (51 p. 87, Taf. XXVII, fig. 2), dans la cavité générale du même Copépode, comme un Monostomum asexué. C'est assurément la méme forme qui se montre très abondante dans la baie de Kiel, où elle fut observée par GiesBreCHT (89, p. 163). Je l'ai recueillie parfois à l'état de liberté dans.le produit de mes pêches. Apoblema appendiculatum vit en liberté à la surface de la mer, pénètre dans le corps du Calanide dont il dévore les tissus et termine son développement dans l'intestin des Poissons, en particulier des Clupéides (Willemoes Suhm (I), Mœbius, Giesbrecht, Monticelli).

5. Poissons. - Aux diverses périodes de leur existence, les espèces pélagiques servent de nourriture à de nombreux Poissons. Depuis longtemps, les naturalistes ont signalé la présence de Copépodes dans l'estomac des Clupéides. Déjà O.F. Mueller (152, p. I 16) dit à propos de Temora longicornis: "In mari Finmarchiam alluente reperit immortalis GunNERUs, ego postea in sinu Dröbachiensi. ac in ventriculo Clypea conglomeratos absque instituto examine vidi ".

Par la richesse de leurs réserves graisseuses, souvent si richement colorées, les Copépodes pélagiques constituent une nourriture abondante et profitable, capable d'influer sur la valeur comestible du Poisson; et, malgré l'indifférence apparente avec laquelle les Harengs mangent les diverses espèces de Copépodes qui nagent à la surface des edux où ils se trouvent, on pourrait recueillir dans l'étude attentive de leur alimentation comparée aux variations de la faune pélagique, de précieux renseignements de nature à éclaircir leur biologie (2).

Les jeunes Pleuronectes sont aussi très avides de Copépodes, et peu de temps après avoir abandonné l"existence pélagique, lorsqu'ils vivent sur le fond, Platessa vulgaris et $P$. flesus mesurant I à $2 \mathrm{~cm}$. de long, se nourrissent entre autres choses d'Harpacticidés littoraux.

Les Cirripèdes sont à comparer aux Clupéides comme destructeurs de Copépodes; les Lepas flottant à la surface se nourrissent presqu'uniquement de Calanides pélagiques, qu'ils avalent en nombre considérable, ainsi que j’ai pu le constater maintes fois (3).

(1) Willemoes-Suhm, Biologische Beobachtungen über Meeresthiere: B. Entwickelung eines appendiculate Distomes, Zeitschr. fo wiss. Zool., Bd. XXI.

(2) Les apparitions des bancs de Clupéides, seules profitables aux grandes entreprises de péche, semblent dans certains cas, pour une région déterminée,en relation avec la prédominance saisonnière de certaines formes de Crustacés. C'est ainsi que dans le "Loch Fyne ", d'après G. Brook (Fish. Board f. Scotland, IVth Report, p. 48), le Hareng apparait en même temps que Calanus finmarchicus.

(3) Ce fait avail déjà été reconnu par Leidy (Proc. Acad. Nat. Sci. Philadelphia, 1888, p. 431-432). 
Dans quelques rares occasions, lorsqu'à la suite de journées calmes et chaudes, la vie pélagique s'est multipliée hors de toutes proportions, et qu'elle constitue dans la zone littorale des dépóts considérables de matière organique, d'autres espèces animales se nourrissent aussi de Copépodes; mais ce sont là des cas exceptionnels que j'ai pu constater chez les Actinies (I) et les Pagures.

(1) Mcersus (15i) signale aussi Anomalocera patersoni comme sortant en grand nombre de Actinia plumosa fixés sur les pilotis, à Mandal. 


\title{
QUATRIEME PARTIE.
}

\author{
PHYLOGÉNIE.
}

\section{RAPPORTS DES COPEPOUES ENTRE EUX.}

\section{LE COPEPODE LE MOINS SPÉCIALISÉ.}

Comme je l'ai dit précédemment, Longipedia coronata est, de tous les Copépodes actuellement connus, le plus primitif ou le moins spécialisé :

$\mathrm{I}^{0}$ par la forme relativement indifférenciée des divers somites, dans les parties antérieure et postérieure du corps ;

$2^{0}$ par l'intégrité des appendices céphaliques, qui n'ont subi aucune réduction morphologique, si on les compare au type moyen des Copépodes les mieux organisés, ni aucune hypertrophie d'apparition trop évidemment secondaire ;

$3^{0}$ par son mode de vie, en tous points comparable à l'existence embryonnaire commune à tous les Copépodes, libres ou parasites :

Par tous ces caractères, Longipedia coronata se rapproche, à n'en point douter, de l'ancêtre immédiat commun à tous les Copépodes actuels. Il reste, en outre, 
le type pseudo-ancestral de toute une série de formes assez homogènes : la famille des Harpacticidés, riche en détails variés de l'organisation et pourvue encore d'une partie des caractères primitifs. Je ne prétends pas ainsi que Longipedia soit - leur ancêtre; mais simplement qu'ils dérivent de Copépodes voisins de ce genre, formes encore inconnues ou disparues dans le cours du développement phylogénétique, et que l'on pourrait désigner sous le nom de Protocopepoda.

Ces Protocopépodes sont caractérisés :

a. Par leur corps composé de 15 somites - les cinq derniers manquant d'appendices - et d'une furca ;

b. Par la soudure des 5 premiers somites en un céphalon portant :

$\boldsymbol{x}$, dans la région antérieure, un œil tripartite ;

$\beta$, dans le $2^{\mathrm{e}}$ somite, une paire de glandes antennales de développement moyen (1) ;

$\gamma$, dans le $4^{\mathrm{e}}$ somite, un organe nuchal de développement moyen (2);

$\bar{\delta}$, dans le $5^{\mathrm{e}}$ somite, une paire de glandes du test dans un développement moyen (3).

c. Par la ressemblance parfaite existant entre les divers segments du corps, non divisé en régions ;

d. Par la nature des appendices thoraciques, biramés, accouplés par l'interposition d'une pièce médiane en forme de repli; à rames triarticulées, garnies de soies et d'épines; mais relativement indifférenciés et embryonnaires dans la $5^{\mathrm{e}}$ paire.

(1) Telle qu'on la retrouve passagèrement chez un certain nombre de Copépodes (Cyclopidæ, Calanidæ), à un état spécial de différenciation embryonnaire.

(2) Cet organe glandulaire n'a été signalé chez les Copépodes que par Claus (Lamproglena pulchella, 55, p. 354) et par Grobben (Nauplius de Cyclops serrulatus ; femelle adulte de Ergasilus Sieboldi: 97, p. 60). 11 est homologue de l'organe nuchal des Phyllopodes et Cladocères, des Euphausia, des embryons d'Amphipodes (voir GrobBen, 97, p. 56-61).

J'ai retrouvé cet organe nuchal chez divers Harpacticidés saumâtres et j'ai pu l'étudier soigneusement dans Tachidius discipes et $T$. Littoralis, où il se présente comme une zone ovalaire, claire, toujours visible sur la carapace et recouvrant un revêtement de grosses cellules, au nombre de 6 ou 8. Par sa structure cellulaire, l'organe nuchal de Tachidius se rapproche beaucoup de celui des larves d'Euphausia d'après Grobien : 97, p. 60, pl. VIII, fig. 75 .

(3) Telles qu'on les retrouve aussi passagèrement dans un certain nombre de types (Nolodilphys, Doropygiens) et d'une manière permanente dans beaucoup d'autres. De même que les glandes antennales, ces organes sont hypertrophiés d'une façon secondaire dans les espèces saumâtres (Temorella, Tachidius) et d'eau douce (Diaptomus, Cyclops... etc.) par suite d'un allongement considérable du canal enroulé. 
e. Par la nature des appendices céphaliques, dont :

a. les antennules sont pourvues de bâtonnets sensoriels, réfringents, répartıs indifféremment sur les divers articles;

3. les antennes, biramées, sont des appendices embryonnaires natatoires;

$\%$ les mandibules sont masticatrices avec une extrémité biramée et natatoire;

i. les $I^{\text {res }}$ maxilles sont également masticatrices, avecl'épipodite réduit à une saillie portant 2 ou 3 soies barbelées et la région terminale natatoire et biramée.

$\varepsilon$. les secondes maxilles, fendues de chaque côté en deux pièces présentant encore l'aspect de deux rames d'appendices natatoires (I) avec des soies barbelées et peu d'épines masticatrices (2). Ces deux pièces sont réunies à leur base sur la même crête articulaire, situées à côté l'une de l'autre et non pas disposées obliquement l'une en arrière de l'autre.

f. Par l'absence complète de dimorphisme sexuel dans les segments de l'abdomen et dans les appendices.

8. Par l'existence, dans la région céphalothoracique, d'une chaîne ganglionnaire ventrale, à ganglions à peine fusionnés dans la partie céphalique (3).

b. Par une circulation exclusivement lacunaire (4).

i. Par la parité des conduits genitaux et le fonctionnement des deux orifices externes des oviductes comme ouvertures génitales femelles pour la copulation (5).

(1) Disposition conservée chez Cyclops notamment.

(2) Visible encore chez Longipedia Claus, et Stigmatidium GIESBrECHT.

(3) Puisque certaines espèces pélagiques (Calanus, Euchata) et quelques embryons de Copépodes montrent encore cette division de la chaine ventrale, complètement disparue chez la plupart des adultes.

(4) Le cœur des Calanidés me paraît en effet une acquisition secondaire plutôt que le reste d'une conformation primitive. Claus $(62$, p. 73,74$)$ a décrit, dans le Nauplius de Branchipus, le premier état de la cavité circulatoire. C'est, à mon avis, dans une disposition lacunaire semblable qu'il faut voir la constitution initiale du système circulatoire des Crustacés ancestraux, plutôt que dans un vaisseau dorsal richement segmenté s'étendant dans tous les somites et tombant en régression çà et là pour fournir les appareils plus ou moins simp̨lifiés des diverses familles actuelles d'Arthropodes.

(5) Ce fonctionnement n'est plus conservé intact dans les Copépodes actuels; il a persisté cependant dans les groupes de Crustacès les plus élevés. 


\section{CLASSIFICATION PHYLOGENÉTIQUE DES COPÉPODES.}

Caractère fondamental. - La disposition des ouvertures sexuelles femelles, adjointes durant l'évolution ancestrảle des Copépodes aux orifices des oviductes des Protocopépodes, me semble être le caractère d'organisation capable d'indiquer le premier degré de spécialisation chez ces animaux.

Une partie des descendants directs des Protocopépodes — nous les retrouvons avec quelques variantes dans Longipedia - présentaient une première modification importante dans l'appareil récepteur des spermatozoïdes chez la femelle. Les spermatophores y sont fixés par le mâle à la face ventrale de la femelle, en arrière des orifices externes des oviductes; les spermatozoïdes pénètrent par un seul pore de fécondation (I) dans deux canaux séminifères qui les portent vers les conduits génitaux.

Cette unité de l'ouverture sexuelle a été transmise par les Copépodes primitifs à tout un ensemble de formes actuelles, qui peuvent être opposées, sous la désignation de “COPEPODA MONOPORODELPHYA", aux espèces pourvues du caractère opposé ci-après.

Sous le nom de COPEPODA "DIPORODELPHYA", je réunirai donc les formes qui présentent, chez la femelle, au lieu d'un seul pore ventral de fécondation, deux orifices sexuels distincts, situés sur la face ventrale dans les environs des orifices extérieurs de l'appareil génital et desquels partent deux canaux séminifères aboutissant, avec ou sans interposition d'un réceptacle séminal, dans la cavité terminale des oviductes, où ils amènent les spermatozoïdes pour la fécondation.

Si l'on observe que la très grande majorité des Copépodes diporodelphes sont plus ou moins commensaux ou parasites d'autres animaux, on est tenté d'admettre que la disposition de leur appareil collecteur des spermatozoïdes chez la femelle n'est qu'une conséquence, ou mieux une adaptation secondaire imposée par l'existence parasitaire. On pourrait ainsi conclure à l'ımportance très restreinte de ce caractère organique en matière de classification.

La disposition de l'appareil récepteur chez les Ascidicolida les plus parasites et les plus dégradés, comme Aplostoma et Enterocola, s'oppose absolument à toute opinion de ce genre. Elle nous empêche surtout d'admettre que les Diporodelphes

(1) C'est dans une large dépression du tégument que débouchent parfois les canalícules seminiteres, ou bien dans un canal capillaire assez allongé. 
soient les descendants de Monoporodelphes, dans lesquels l'orifice unique ventral aurait été remplacé par deux orifices latéraux pour faciliter aux mâles l'accès des orifices sexuels femelles dans la copulation.

Une semblable interprétation des faits, toute physiologique, pourrait convaincre ceux qui observeront que beaucoup de. Diporodelphes peu dégradés vivent en semiparasites à la surface du corps de leur hôte, par exemple certains Lichomolgidé et les Hersiliide. Mais comment expliquer ensuite la conservation de l'orifice sexuel impair et ventral chez Notodelphys qui est aussi un semi-parasite, ainsi que chez tous les autres Ascidicolida?

Pour ces Monoporodelphes, il y aurait pourtant grand avantage à se transformer en Diporodelphes!

De mème, l'apparition des orifices femelles pairs et latéraux dans les Copépodes diporodelphes ne pourrait s'expliquer par l'influence des conditions d'existence lorsque ces animaux sont fixés à leur hòte par la région antérieure du corps. Car les màles de Lernceopodide, par exemple, ne rencontrent, à vrai dire, aucun obstacle pour accéder à la face ventrale du segment génital; ils pourraient s'y attacher ausisi facilement que sur les côtés de ce segment. Et dans les Caliges, dont les femelles adhèrent si étroitement à la surface des Poissons qui les portent, les orifices pairs, orifices de diporodelphes, placés à la face ventrale, sont tout aussi difficiles à atteindre qu'un seul orifice, également ventral, de monoporodelphe. A ce point de vue, il n'y a donc aucun progrès réalisé dans l'une ou l'autre catégorie.

Bien plus, dans la distribution des spermatozoïdes après la copulation pour la fécondation bilatérale des oviductes par un seul spermatophore, la position des pores de fécondation (latéraux ou ventral, pairs ou unique) n'a aucune importance. Car, par la présence, chez tout Copépode monoporodelphe, de deux réceptacles séminaux respectivement en relation avec les deux oviductes, par l'existence, chez tout diporodelphe, d'un seul réceptacle séminal également en relation avec les deux oviductes, le liquide spermatique se trouve toujours répandu indifféremment dans les deux oviductes suivant les besoins de l'appareil génital femelle, que la copulation soit médiane, qu'elle soit bilatérale ou unilatérale.

De ces considérations - qui pourraient être étendues encore - il me semble bien résulter clairement :

$I^{0}$ Que la disposition des orifices sexuels femelles échappe, dans les traits généraux, à l'influence perturbatrice des adaptations physiologiques.

$2^{\circ}$ Qu'elle a. par suite, une réelle importance morphologique.

$3^{\circ}$ Que son empioi en taxonomie est bien justifié. 
Tout caractère primordial doit en effet échapper aux influences secondaires qui viennent modifier les premiers stades phylogénétiques d'un groupe. Les pores de fécondation nous semblent bien, par les dispositions qu'ils affectent, satisfaire à ces exigences. Ils présentent donc les qualités essentielles qui manquent à la forme du corps, aux appendices masticatoires et natatoires, etc.

\section{$\mathrm{I}^{0}$ - Les Copépodes monoporodelphes comprennent:}

A. Les Harpacticidés, dont la forme du corps, le rôle à la fois natatoire et masticateur des antennes et des pièces buccales, le rôle natatoire le plus souvent conservé des appendices thoraciques sont, avec l'absence d'hypertrophies spéciales, autant de caractères d'une organisation primitive. Ceci m'invite à les considérer comme une subdivision monophylétique dans laquelle diverses influences secondaires, pour la plupart d'ordre éthologique (voir plus haut), ont amené une longue série de variations peu profondes.

A ce titre, les Harpaticidés, considérés dans leur ensemble, se présentent comme des Copépodes relativement primitifs, si on les compare aux formes voisines que nous allons examiner plus loin.

Parmi les descendants harpacticiformes (opposés aux cyclopiformes: Calanidés et Cyclopidés) des Protocopépodes, ils se distinguent par les caractères suivants :

a. La spécialisation des organes sensoriels de l'antennule. Dans les Harpacticidés, les derniers articles ( fouet) portent seuls un ou deux bâtonnets réfringents.

b. La condensation du système nerveux ventral, réduit à une masse confuse, ramenée vers l'avant du céphalothorax et d'où partent les nerfs.

c. La nature des caractères sexuels secondaires mâles, résidant principalement:

$\alpha$. dans la transformation des deux antennules en organes de préhension;

$\beta$. dans la réduction de la $5^{\mathrm{e}}$ paire de pattes thoraciques.

Dans notre connaissance actuelle des diverses formes d'Harpacticidés, je ne puis trouver de renseignements morphologiques suffisants pour prétendre à établir déjà d'une manière convenable, les rapports phylogénétiques de ces êtres ( $\mathrm{I})$.

Dans le diagramme donné plus loin (p. 133) pour résumer la phylogénie des Copépodes que j'ai étudiés, j’ai dû rapprocher, d'après leur parenté certaine, les sous-familles des Canthocamptinés et des Nannopinés; je les considère comme des formes modifiées (dans leurs pattes thoraciques et leurs organes

(1) Je suis d'ailleurs parfaitement d'accord avec GIESBrecht $(89, p .98)$, pour considérer comme très peu naturels, les rapports établis par Brady (20, vol. I et II) entre les diverses subdivisions de cette famille. 
buccaux) en un sens tout différent des variations éprouvées par les autres sousfamilles.

B. Les Ascidicolidés, bien fius rapprochés des Harpacticidés (par le genre $N$ stodelphys) qu'on ne l'avait cru jusqu'ici, et qui constituent par l'enchaînement morphologique et les caractères éthologiques une sous-famille particulière dont j'ai déjà indiqué les rapports.

En effet, par leurs mandibules, maxilles et maxillipèdes, Notodelphys et Doropygus se rattachent à la souche des Harpacticidés. Dans les formes les plus spécialisées (Enterocola, Aplostoma), la nature des adaptations biologiques masque ces caractères; mais la disposition du pore de fécondation et des canaux séminifères demeure intacte, quel que soit le degré de parasitisme.

C. Les Cyclopidés, qui nous présentent un nombre de formes très bornées dans leur variabilité individuelle, bien qu'elles soient répandues partout.

Leur adaptation à la vie pélagique dans le voisinage du littoral, leur prédominance dans l'eau douce sont tout à fait particulières et ne se rencontrent pour aucune autre division de Copépodes. J'y retrouve tous les caractères (I) d'une branche avortée, d'un " groupe terminus " parmi les formes éminemment nageuses, voire même pélagiques, des Copépodes :

a. "Ils sont très fortement différenciés en vue d'une existence spéciale."

Dans Cyclops et Thorellia, plus particulièrement en ce qui concerne les appendices buccaux. Dans tous les genres, en ce qui concerne les appendices natatoires.

b. "Ils présentent une très faible variabilité."

Les assez nombreuses especes de Cyclops diffèrent par des caractères d'une faible intensité. On ne connaît qu'une espèce bien certaine chez Thorellia, chez Cyclopina et un petit nombre $(\mathbf{9 1}, \mathbf{1 8 9 1})$ chez Oithona.

C. " Ils sont le plus souvent terrestres ou d'eau douce."

Les formes d'eau douce sont bien plus nombreuses que les marines.

d. "Ils ont une embryogénie condensée. "

Mème chez Cyclops, par rapport aux Calanidés.

$e$. "Ils produisent un petit nombre de fœetus à chaque parturition."

Ce qui est vrai spécialement chez Oithona et Cyclopina.

(1) Tels qu'ils ont été indiqués avec précision pal GIARD, Les mammifères ovipares, observations sur une note du Prof. Vinciguerra, Bull. Scicntif., t. XVI1, 1886, p. 416. 
En résumé, les Cyclopidés me semblent dérivés de Copépodes marins adaptés à l'existence pélagique; il en persiste des traces dans les genres Oithona et Cyclopina. Mais après avoir pris, dans leur forme générale, l'aspect des espèces pélagiques sans atteindre à une haute complication adaptative dans leurs appendices natatoires ( $\mathbf{I}$ ), les Cyclopidés pélagiques primitifs ont subi une dégradation (manifeste chez Thorellia et Cyclops, pour les appendices céphaliques masticatoires et préhensiles) en revenant au mode primitif d'existence, dans les algues et sur le fond.

Au début de leur évolution phylogénétique, les Cyclopidés ont subi les mêmes influences et suivi la même voie que les ancêtres des Calanidés, ce qui explique la place qui leur est assignée dans l’arbre phylogénétique établi plus loin.

D. Les Calanidés, qui présentent les caractères les plus nets de l'adaptation pélagique $(2)$.

Je ne reviendrai plus sur ce qui a été dit plus haut à ce sujet.

Les organes des sens sont très développés, mais ils n'atteignent une hypertrophie spéciale que chez les Pontellines, où l'œil tripartite - dissocié en deux ocelles latéro-dorsaux et un ocelle médio-ventral - s'adjoint des cornées réfringentes d'acquisition secondaire.

Par la marche régulière de leur ontogénie, par l'homogénéité de leurs caractères d'organisation et la disposition moins condensée du système nerveux ventral, il semble démontré que les Calanidés se sont phylogénétiquement développés, sans intermédiaire, aux dépens des premiers Copépodes monoporodelphes détachés de la souche. Leur acquisition la plus remarquable a trait à l'apparition d'un cour, qui manque chez tous les autres Copépodes.

2". - Les premiers Copépodes diporodelphes dérivés de la souche conmune à tout le groupe, ont hérité de leurs ancêtres les appareils masticateurs compliqués que l'on voit s'affirmer (Doropygiens) ou disparaitre (Enterocola, Aplostoma) dans une même subdivision des Monoporodelphes.

Chez les Diporodelphya, on peut distinguer, dans l'évolution des pièces buccales et au cours des adaptations parasitaires, trois groupes principaux, sur la morphologie desquels j'ai suffisamment insisté pour qu'il soit inutile d'y revenir.

A. Dansles Monochila, sont réunies toutes les formes diporodelphesquimanquent de paragnathes, et qui possèdent une lèvre supérieure saillante, recouvrant des

(1) Qui, en somme, ne sont pas plus compliqués dans Oithona que dans Longipedia.

(2) Voir Giard, Convergence des types par la vie pélagique, Rov. des Sci. nat. Montpillier, 1. II, 1875, pp. 44-54. 
mandibules réduites, à côté desquelles se voient les màchoires rudimentaires que Thorell considérait comme les palpes des mandibules.

Je n'insisterai pas plus longuement sur ces êtres, dont les rapports avec la souche commune aux Diporodelphya ne seront clairement expliqués qu'après la connaissance parfaite de leur embryogénie et la découverte des types de passage (1).

B. Parmi les Rhynchostoma, viennent se ranger les diverses familles parasites et semi-parasites dont la bouche est surmontée d'une trompe ou d'un siphon solide et capable de servir comme un organe offensif dans la prise de nourriture. Les mandibules en stylets, en même temps que ce siphon, servent à attaquer l'hôte sur lequel vit le Copépode. Les I $^{\text {res }}$ maxilles ne sont point tout à fait rudimentaires; parfois nettement biramées, elles sont aussi réduites parfois à des moignons sessiles.

Par la constitution de leur trompe-siphon, toutes ces formes sont assez particulières pour ne point exiger de plus amples explications, d'autant plus que les espèces traitées dans ce mémoire, ne sont pas, au sujet de leur morphologie, les plus importantes de tous les Rhynchostomes.

C. Parmi les Auliostoma, j'ai réuni toutes les espéces dans lesquelles se constitue autour de la bouche une région antérieure protectrice plutòt qu’offensive dont la délimitation est certainement due à la collaboration des paragnathes et de la lèvre supérieure.

Les mandibules fonctionnent à l'intérieur de cet atrium, sans que celui-ci malgré la ressemblance qu'il affecte parfois avec les siphons précédemment cités - les aide et dirige leurs mouvements. Ces Copépodes semblent être des animaux qui aspirent des matières semi-liquides encore divisées“à l'intérieur par les mandibules, plutôt que des parasites qui attaquent un organe de leur hôte.

J'ai décrit en détail, dans la $2^{\mathrm{e}}$ partie de ce travail, la morphologie et l'évolution de cet organe chez les Hersiliidés; il est donc inutile d'y insister à nouveau (2).

Dans les Nereidicolidés, la même explication pourrait ètre appliquée ; et je ne doute pas que dans des recherches ultérieures, les naturalistes n'observent, parmi ces êtres, une complication morphologique progressive, analogue à celle des Hersiliidés.

(1) De même que les rapports d'Enterocola, Enteropsis et Aplostoma, avec Notodelphys (et par suite avec tous les Gnathostomes) sont établis par la connaissance morphologique d'Agnathaner. La parenté de ces diverses formes a d'ailleurs été déjà discutée par d'autres auteurs; j'aurai l'occasion d'y revenir dans la suite de ce travail.

(2) Dans le groupe des Auliostoma, il est probable qu'il faudra placer encore Caligidiun et Cancerilla d'une part, et les Choniostomatidés (87) d'autre part; mais les renseignements nécessaires manquent pour le faire actuellement. 
$-133-$

Le diagramme suivant indique les rapports phylogénétiques des Copépodes entre eux, tels qu'ils me semblent résulter de l'état actuel de la science (I) :

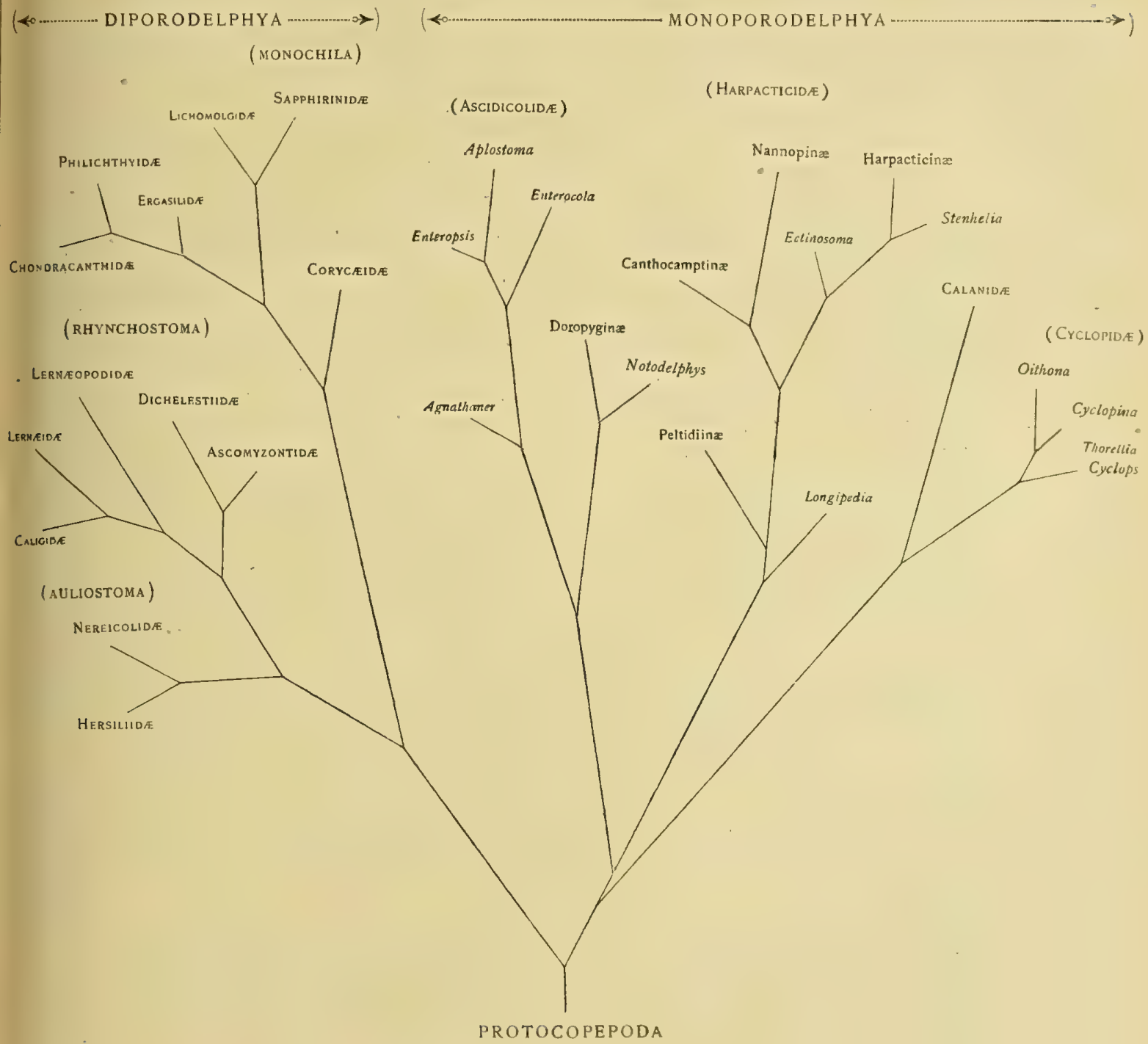

(1) Dans ce tableau, sont indiques seulement les familles ou les genres importants, qui correspondent à un typo bien accusé de différenciation morphologique ; il est fait abstraction des formes peu intéressantes à ce point de vue. - Quand la plupart de s genres sont cités, le nom de la famille qui les comprend êst en parenthèses. 


\section{RAPPORTS DES COPEPODES}

\section{AVEC LES AUTRES CRUSTACES.}

Dans ce qui suit, j'ai utilisé des observations comparatives faites sur divers groupes d'Entomostracés autres que les Copépodes, en mème temps que les résultats publiés par différents auteurs.

Depuis les recherches de Claus sur la phylogénie des Crustacés, il est admis généralement que tous ceux-ci descendent d'animaux hypothétiques, qui ont été successivement appelés par cet auteur "Urphyllopoden " (5'7) ou "Protostraca $"(61)$.

Les "Protostraca" seraient des Crustacés composés d'un grand nombre de segments ainsi distribués :

$\mathbf{I}^{0}$ Un céphalon 5-somité, avec des antennules simples, des antennes, mandibules, premières et secondes maxilles biramées et des paragnathes.

$2^{0}$ Huit somites thoraciques, avec appendices natatoires (comme ceux des Copépodes), et pourvus de branchies.

$3^{0}$ Six somites abdominaux avec appendices biramés.

$4^{0}$ Une pièce terminale aplatie (septième somite abdominal).

En somme, les Protostracés seraient des Crustacés au corps non différencié, ou bien, si l'on peut s'exprimer ainsi, des êtres annélidiformes, quii auraient pour parapodes des appendices biramés peu complexes, distribués dans toute l'étendue du corps.

Parmi les Crustacés actuels ceux qui rappellent le plus exactement ces formes ancestrales, avec quelques différences dans-les pièces buccales et le nombre des segments, sont les Phyllopodes.

Dans les Entomostracés, les Phyllofodes seraient dérivés directement des Protostracés par complication graduelle et régulière des segments, tandis que les autres groupes : Cirripèdes, Copépodes, Ostracodes, etc., seraient des formes peu à peu simplifiées dans leur composition morphologique, et réduites à un nombre parfois très faible de segments et d'appendices. (I)

(1) De même que les Cladocères parmi les Phyllopodes. 
D’après l'opinion récente de Claus (61, pp. 9i-95), les Protostracés dériveraient eux-mêmes d'un être annélidiforme établissant un passage entre les Annélides et les Crustacés par divers caractères de l'organisation des appendices, des organes excréteurs (glandes antennale et coquillière) et de la chaîne ganglionnaire ventrale. Et le Nauplius lui-même, résultant de l'adaptation postérieure et cœnogénétique des premières larves de l'ancêtre annélidiforme à la condition de Crustacé, n'aurait plus qu'une valeur insignifiante dans la phylogénie (I).

Quoi qu'il en soit, en l'absence de faits paléontologiques démontrant l'exactitude de ces vues purement spéculatives, je reste convaincu de la valeur constante qu'il convient d'attribuer à la preuve embryologique, et je persiste à trouver dans les métamorphoses successives des Crustacés, les diverses étapes parcourues par ces animaux au cours de l'évolution phylogénétique. N'est-il point démontré d'ailleurs que les conditions extérieures influent suffisamment sur l'embryogénie, pour falsifier et abréger le cours de la métamorphose? Et le cas de pœcilogonie constaté (2) chez Palcmonetes varians LEACH, n'est-il pas la meilleure preuve expérimentale qu'on puisse exiger pour admettre l'abréviation embryogénique et la suppression chez la plupart des Malacostracés, des premières phases embryonnaires, avec le stade nauplius!

Pour les zoologistes convaincus de l'influence exercée par l'éthologie de l'adulte sur le cours du développement, je suis persuadé que le nauplius restera une forme ancestrale commune à tous les Crustacés, ainsi que le croyait FriTz Mueller (3).

Ainsi que tous les autres Crustacés entomostracés ou malacostracés, les Copépodes dérivent d'un ancêtre nauplien déjà caractérisé comme Crustacé, par un revêtement continu de chitine qui protège le corps entier. Cette chitine, qui commande à l'organisation mécanique de l'animal, est apparue phylogénétiquement dans un ancètre encore plus éloigné et voisin des Gymnotoca GIARD, comme l'a justement pensé Claus (61, p. 95).

(1) L'application la plus curieuse, en méme temps que la moins soutenable, de la théorie de Claus sur l'origine des Crustacés, a été faite récemment par CarL. VoGT, qui trouve dans la larve nauplienne un ancêtre des Crustacés entomostracés, tandis qu'il lui refuse cette propriété à l'égard des Malacostracés. La larve nauplienne des Eubhausia et des Penoens serait donc une forme embryonnaire acquise par adaptation (Recue scientifique, t. XVLVII, 2 mai 1891).

(2) GIARD, Les facteurs de l'évolution, Rev. scientif., t. XLIV, 23 novembre 1889. GIARD, Sur le bourgeonnement des larves d'Astellium spongiformc et sur la Poecilogonie chez les Ascidies composées, Comples rendiks, 2 février 1891. - BoAs, Vidensk. Middel. fia naturh. Foren. i Kjohenhavn, 1880.

(3) F. Mleller, Für Darwin, $\$ 12$. 
Sans vouloir insister plus qu'il ne convient sur ce détail théorique, par suite de la disposition du tube digestif, du système nerveux et des organes segmentaires (glande antennale), je rattache l'ancêtre des Naupliidés aujourd'hui disparus, à des animaux voisins de Dinophilus. Il n'existerait ainsi aucun rapprochement phylogénétique entre les appendices des Crustacés et les parapodes des Annélides; cette opinion est, à mon sens, la plus vraisemblable. Le remplacement du revètement ciliaire continu par de la chitine, dans un Dinophilien peu (2-ou 3-) segmenté, aurait suffi pour la constitution des Naupliidés, desquels. se seraient détachés les divers groupes actuels de Crustacés par un allongement ultérieur et la formation de nouveaux somites homodynames des précédents.

J'ai rejeté précédemment (p. 62) l'opinion tendant à la parenté des Copépodes et des Protostraca de Claus, avec l'hypothèse d'une dégradation de ces derniers pour constituer les premiers. Avec Fritz Mueller (loc. cit.), je retrouve dans l'apparition ontogénétique des somites chez les Copépodes, le mode de formation primitif:

Les Copépodes, Ostracodes et Cirripèdes sont dérivés des Naupliidés par une complicátion graduelle, qui se retrouve encore dans l'ontogénie du corps. Ils se distinguent entre eux dès l'origine, ou mieux dès le stade métanauplius, par des caractères morphologiques précis, qui ont trait aux particularités les plus sensibles par lesquelles sont séparées ces différentes sous-classes.

Pour ces êtres, l’augmentation graduelle du nombre des somites et les caractères morphologiques que prennent ces derniers dès le début de l'évolution embryonnaire sont les bases primitives qui établissent seules les rapports phylogénétiques.

Les Protostraca de Claus n'en restent pas moins des types ancestraux à l'égard des autres familles, et plůs particulièrement des Phyllopodes et des Malacostracés. Mais la plupart des Entomostracés se sont détachés séparément de la souche, bien avant la formation complète d'organismes aussi élevés, alors que les descendants hypothétiques des Naupliidés comptaient 5 (Pentapodostracés), 7 (Heptapodostracés) et I I (Endecapodostracés) somites.

Les Copépodes se sont détachés des Pentapodostracés. En effet, dans toutes leurs larves métanaupliennes, dès que la $5^{\mathbf{e}}$ paire d'appendices se forme, le caractère spécial aux secondes maxilles de ces animaux y apparait. De plus, à partir de cette époque, l'ontogénie affirme de plus en plus leur autonomie, par sa marche spéciale et les caractères particuliers des somites et par les appendices biramés et lamellaires en voie de formation. 
Le diagramme suivant indique les rapports établis ci-dessus entre les divers groupes de Crustacés.

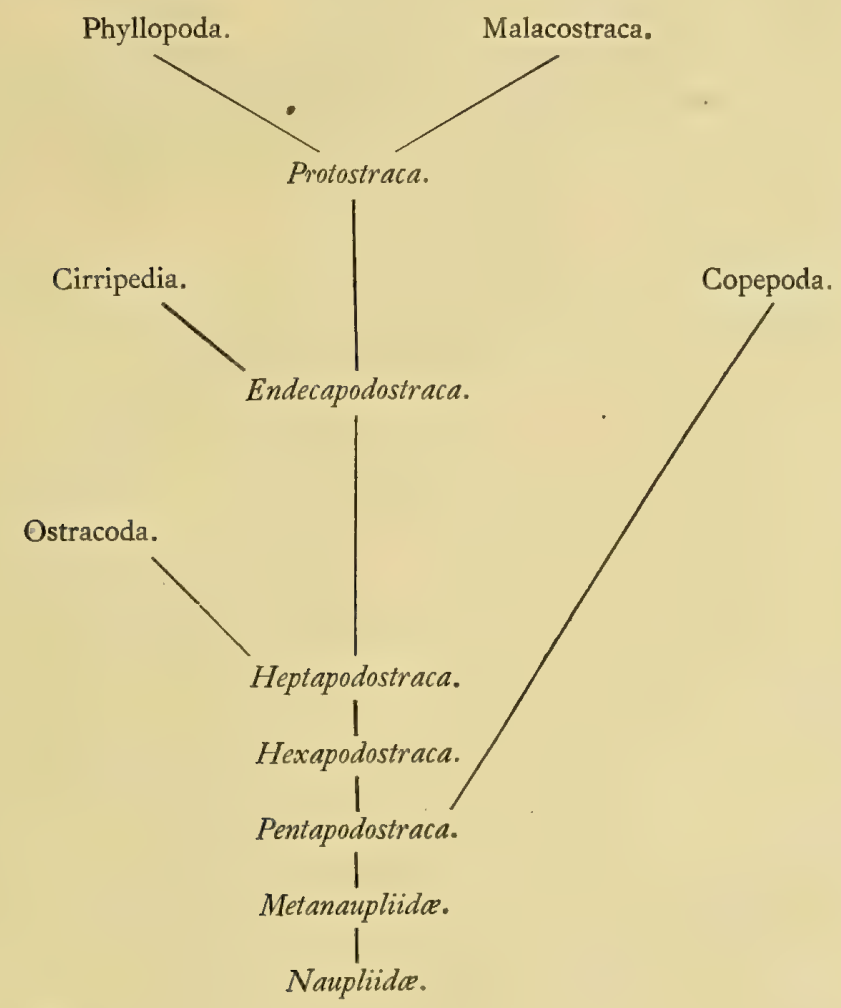




\section{CINQUIEME PARTIE.}

\section{SYSTÉMATIQUE.}

\section{L'ordre des Copépodes comprend tous les Crustacés dont :}

$I^{0}$ Le corps segmenté présente normalement, à l'état adulte, 10 somites appendiculés, 5 somites non appendiculés et une furca composée de deux pièces séparées l'une de l'autre suivant la ligne médiane; et dans lesquels quelques somites peuvent parfois manquer d'appendices, ou bien se fusionner en un seul segment.

$2^{0}$ Les 5 premiers somites constituent un seul segment céphalique, portant d'avant en arrière :

a. Une paire d'antennules simples, avec des organes sensoriels.

b. Une paire d'antennes, le plus souvent biramées, parfois simples. -

c. Une paire de mandibules masticatrices ou perforantes, parfois disparues chez l'adulte, et pourvues d'une région terminale, biramée ou simple, parfois même disparue.

d. Une première paire de maxilles complexe et en forme d'appendices masticatoires et natatoires, ou bien réduite à la seule mastication, ou même à un rudiment insignifinat.

$e$. Une seconde paire de maxilles double, divisées alors en deux pièces accolées ou plus ou moins disjointes, et qui peut parfois demeurer simple par une adaptation secondaire a un rôle préhensile spécial.

$3^{0}$ Les cinq somites post-céphaliques ou thoraciques portent chacun, le plus souvent, une paire de pattes primitivement (dans l'embryon) natatoires et formées :

a. D'un protopodite biarticulé, aplati en une lame transversalement placée sur le corps de l'animal. 
b. D'un exopodite généralement triarticulé, lamellaire et garni d'épines ou de soies barbelées sur ses bords;

c. D'un endopodite généralement triarticulé, avec des soies sur les bords et parfois des épines terminales.

Mais, chez l'adulte, la nature des appendices thoraciques est souvent distraite de sa constitution primitive, en vue d'un rôle spécial à remplir dans l'adhésion ou la reptation. Les pattes thoraciques, lorsqu'elles existent, peuvent être ainsi réduites, dans l'une ou l'autre paire et plus particulièrement dans la dernière. à l'état de lames articulées, avec ou sans épines ni soies.

$4^{0}$ Le système nerveux comprend une masse ganglionnaire supra-œsophagienne assez simple, sans lobes ni subdivisions détachées, et une masse infra-œesophagienne, parfois allongée en chaîne ventrale métamérisée, composée d'un nombre restreint d'amas ganglionnaires le plus souvent confondus, et fournissant un nombre restreint (une dizaine au maximum) de nerfs appendiculaires et abdominaux.

$5^{\circ}$ Les organes visuels sont réduits à un seul œil médian composé de trois parties (ou ommatidies) qui peuvent être disjointes au point de paraître distinctes et de simuler deux yeux latéro-dorsaux et un œil médian.

$6^{0}$ Les organes excréteurs se composent de deux canalicules segmentaires mettant en communication une dépendance de la cavité du corps avec la base des appendices des $2^{\mathrm{e}}$ et $5^{\text {e }}$ somites; ces organes manquent souvent à l'état adulte et se retrouvent seulement alors dans les stades embryonnaires.

$7^{0}$ Le tube digestif droit et simple ne possède pas de glandes distinctement séparées et débouche dans l'avant-dernier somite par un anus dorsal.

$8^{0}$ Les organes génitaux se composent d'une seule (chez le mâle et chez la femelle) ou parfois de deux (chez la femelle) glandes, d'où partent parfois un seul (Calanidés mâles), et plus souvent deux canaux excréteurs aboutissant aux ouvertures génitales situées dans le ${ }_{11^{\mathrm{e}}}$ somite chez les deux sexes.

$9^{0}$ Les métamorphoses compliquées comprennent successivement :

a. Des larves naupliennes pourvues, sur les côtés de l'extrémité postérieure, de deux soies furcales divergentes caractéristiques.

b. Des larves métanaupliennes.

c. Des embryons cyclopoïdes nageurs dont le premier a déjà la forme d'un Copépode peu segmenté et ne comprend que onze somites.

Et dans lesquelles diverses influences secondaires peuvent apporter des abréviations et modifications importantes.

L'ordre des Copépodes fut d'abord établi par Milne-Edwards (en I830: 147), pour une partie des animaux qui le composent actuellement et dans lesquels la 
constitution des appendices natatoires paraissait, aux yeux de ce naturaliste, un caractère taxonomique important.

ZeNKER (214) rapprocha des Copépodes de MiLne-EDWARds les formes parasites qui prèsentent le même caractère.

Le premier, THORELL (204) exagéra l'importance taxonomique des pièces buccales dans ces animaux.

Mais c'est à Claus $(\mathbf{4 9 , 5 1 )}$ qu'est due la découverte de la particularité morphologique importante présentée par la seconde maxille, et la délimitation précise de la catégorie d'animaux qui nous occupent.

Les tableaux suivants résument les classifications les plus importantes qui ont été proposées, pour le groupe des Copépodes, par différents auteurs (I).

\section{ZENKER (214) 1854 .}

1. Malacostraca.

2. Trilobita.

3. Poecilopoda.

4. Aspidostraca... $\left\{\begin{array}{l}a \text { Argulina. } \\ b \text { Branchiopoda. }\end{array}\right.$

5. Entomostraca... $\left\{\begin{array}{l}\text { a Copepoda. } \\ \text { b Siphonostomata } \\ \text { c Lerncooda. }\end{array}\right.$

6. Cirripedia.

7. Ostracoda.

THORELL (204) 1859.

I. Gnathostomata .. $\left\{\begin{array}{l}\text { 1. Calanidæ. } \\ \text { 2. Cyclopidæ......... } \\ \text { 3. Notodelphyidæ. } \\ \text { 4. Buproridæ. }\end{array}\right.$

1. Corycæidæ.

2. Miracidæ.

3. Sapphirinidæ.... \{ $\begin{aligned} & \text { a Sapphirina. } \\ & \text { b Lichomolgus. }\end{aligned}$

II. Poecilostomata.. $\{$ 4. ? Doriclicola.

5. Ergasilidæ.

6. ? Monstrillidæ.

7. Chondracanthidx.

8. Lamippe.

(1) Je renvoie le lecteur aux pages précédentes ( $2^{\mathrm{e}}$ partie ), pour la critique des caractères importants qui y furent utilisés. 


$$
\text { III. Siphonostomata.. }\left\{\begin{array}{l}
\multicolumn{1}{c}{-141-} \\
\text { 1. Ascomyzontidæ. } \\
\text { 2. Nicothoidæ. } \\
\text { 3. Dichelestiidæ. } \\
\text { 4. Caligidæ. } \\
\text { 5. Lernæopodidæ. } \\
\text { 6. Lernæidæ. }
\end{array}\right.
$$

Claus $(\mathbf{4 9}, \mathbf{5 1}, \mathbf{5 5}) 1862-1875$.

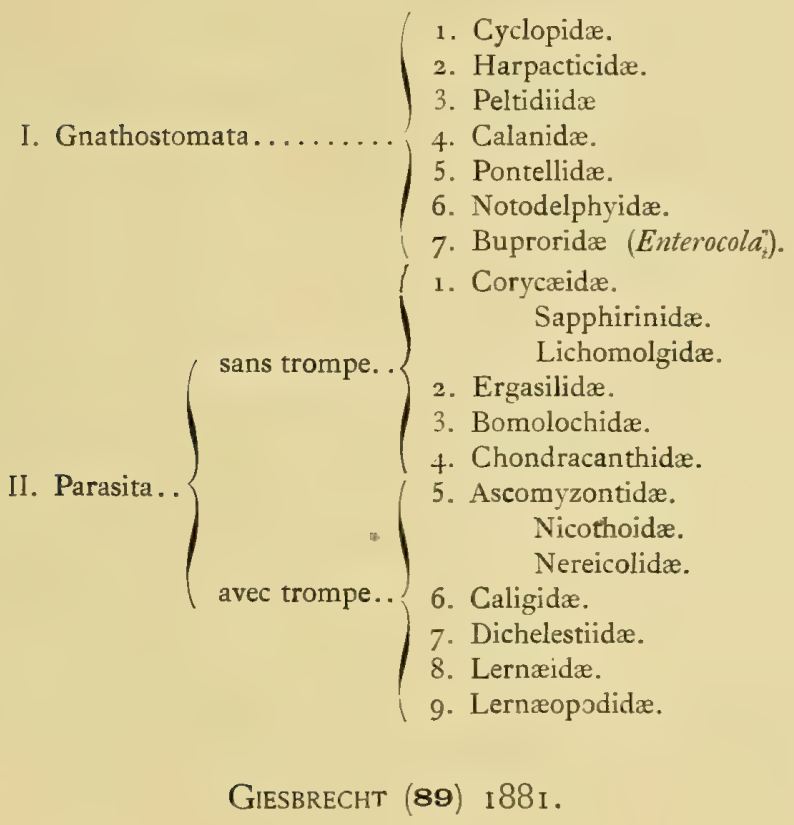

Copépodes libres :
I. Gymnopleoda ......... $\left\{\begin{array}{l}\text { 1. Calanidæ. } \\ \text { 2. Pontellidæ. }\end{array}\right.$
3. ? Misophriidæ.
II. Podopleoda ............ $\left\{\begin{array}{l}\text { 1. Harpacticidæ. } \\ \text { 2. Cyclopidæ. } \\ \text { 3. Corycæidæ. }\end{array}\right.$

La classification phylogénétique, celle qui considère, comme le voulait déjà LAMARCK, les rapports et la série générale des divers degrés d'organisation, l'ordre entre les objets d'une classe, est la seule qui puisse ètre adoptée. 
J'ai tenté dans les pages précédentes d'établir ainsi les relations des familles les plus importantes de Copépodes.

Bien des points de détail nécessitent de nouvelles recherches dans le but d'affermir et de contrôler cet arrangement systématique; mais les rapports naturels des Harpacticidés, des Ascidicolidés et des Hersiliidés s'y trouvent basés de façon plus convenable, sur des particularités morphologiques et embryologiques.

Tableau synoptique des familles étudiées.

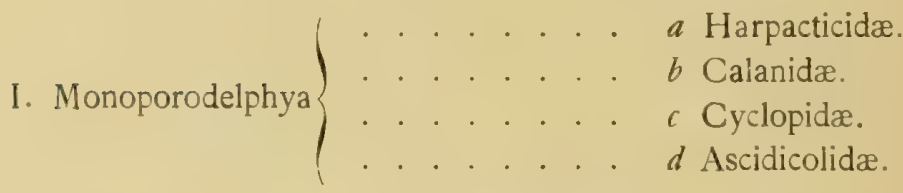

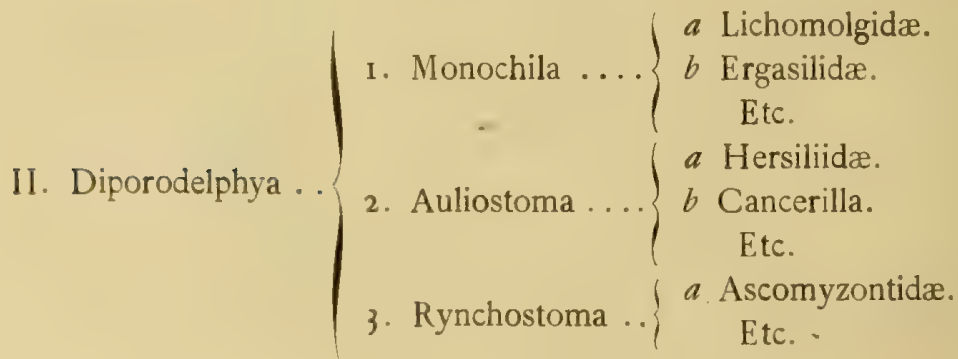




\section{COPEPODA MONOPORODELPHYA.}

Copépodes ayant le corps plus ou moins nettement segmenté et dont la femelle présente un seul orifice sexuel pour la pénétration des spermatozoïdes.

Cette subdivision correspond à peu près aux Gnathostomes de THOReLl (204) et de Claus (49); sa diagnose ainsi modifiée y permet l'introduction, rendue nécessaire par la connaissance que j'ai pu acquérir de leur organisation morphologique et embryonnaire, d'espèces dépourvues de mandibules masticatrices comme Aplostoma brevicauda CANU, Enteropsis pilosus CANU, Enterocola fulgens van Beneden et $E$. betencourti Canu.

L'unité du pore de fécondation est reconnue et démontrée dans les diverses familles de ce sous-ordre par Claus, Giesbrecht, Gruber........ etc. J'ai pu la constater moi-mème dans les types les plus aberrants, même chez ceux qui n'y auraient pu prendre place jusqu'à ce jour, si leurs organes buccaux avaient été connus plus exactement.

\section{HARPACTICIDAE.}

Copépodes monoporodelphes ayant :

$I^{0}$ Le premier somite thoracique soudé avec le céphalon.

$2^{0}$ Les antennules courtes, composées d'un petit nombre d'articles, préhensiles à droite et à gauche chez le mâle.

$3^{0}$ Les antennes 2 - ou 3 -articulées, terminées par des épines recourbées et pourvues le plus souvent d'un exopodite parfois très rudimentaire.

$4^{0}$ Les mandibules très variables, avec la base masticatrice et l'extrémité palpiforme plus ou moins développée et sétifère.

$5^{0}$ Les premières maxilles également variables et masticatrices, avec une région terminale pourvue de soies rarement suffisantes pour la natation. 
$6^{0}$ Les secondes maxilles séparées l'une de l'autre, masticatrices (externes) ou préhensiles (internes).

$7^{0}$ Les appendices thoraciques le plus souvent natatoires, parfois préhensiles par leur terminaison en griffe dans la première paire, réduits dans la cinquième paire à des lames aplaties et sétifères.

$8^{0}$ Le tégument externe composé d'une chitine épaisse et résistante, rarement transparente.

$9^{\circ}$ La circulation exclusivement lacunaire.

$10^{0}$ Les conduits génitaux $q$ pairs et symétriques avec leurs orifices plus ou moins rapprochés l'un de l'autre sur la face ventrale, ce qui explique la disposition presque générale des œufs en une seule masse, après la ponte.

Ainsi délimitée, la famille des Harpacticidés comprend les deux familles des Harpactidés et des Peltididés établies par Claus $(\mathbf{4 9}, \mathbf{5 1})$; elle correspond à la famille des Harpactidés telle que l'ont définie Axel $\operatorname{Boeck}(\mathbf{1 5}, \mathbf{1 7})$ et $\operatorname{Brady}(\mathbf{2 0})$.

Il ne me paraît en effet nullement démontré, même pour un zoologiste attentit aux specialisations anatomo-physiologiques et soucieux des relations phylogénétiques des êtres, que les Peltidiens les plus caractérisés s'éloignent d'un Harpacticidé typique, comme Longipedia, à un degré plus élevé et d'une manière plus fondamentale que certaines formes dûment reconnues apparentées à ce dernier, comme Nannopus ou Huntemannia. C'est pourquoi je considère encore, malgré les récentes remarques de Claus à ce sujet (65, pages I et 2 ), les formes caligoïdes et sphæromoïdes ( $\mathrm{I}$ ) de Peltidiens comme dérivées d'Harpacticidés primitifs ou peu modifiés, par une adaptation à la vie plus ou moins rampante des Caliges et des Sphéromes.

(1) Ressemblant aux crustacés des genres Caligus et Sphacroma. 


\title{
TABLEAU ANALYTIQUE DES SOUS-FAMILLES
}

\author{
ADOPTÉES AVEC LES GENRES ÉTUDIÉS
}

Harpacticidés au corps cylindrique un peu aplati, avec l'exopodite antennal allongé et natatoire, avec la mandibule biramée et la première maxille natatoire pourvue d'un épipodite, avec la seconde maxille interne non préhensile et pourvue de longues soies finement barbelées.

LONGIPEDINE... ... Longipedia.

\begin{abstract}
Harpacticidés avec la mandibule masticatrice, simple ou biramée, la première maxille masticatrice et plus ou moins natatoire, rarement pourvue d'un épipodite, la seconde maxille interne transformée en organe plus ou moins préhensile et dépourvue de longues soies barbelées flexibles.
\end{abstract}

Corps aplati et déprimé avec les appendices thoraciques très écartés l'un de l'autre dans la même paire
Corps cylindrique un peu aplati ou rarement déprimé, avec les appendices thoraciques assez rapprochés l'un de l'autre sur la face ventrale.
Forme légèremeat aplatie ou même déprimée. Mandibule biramée et première maxille masticatoire, toutes deux impropres à la natation.

Forme comprimée latéralement. Mandibule biramée. Appendices thoraciques semblables et tous natatoires.

Mandibule biramée. Pattes de la première paire avec une ou deux rames préhensiles terminées par un crochet recourbé. Pattes natatoires dans les dernières paires.

Mandibule biramée. Patte de la première paire adhésive, sans crochets. Corps aplati.

Caractère masticateur des appendices buccaux bien accusé par la réduction de la région terminale sétifère. Mandibule non biramée. Endopodite des appendices thoraciques fréquemment réduit en longueur et en articulations par rapport à l'exopodite. Espèces nageuses, parfois limicoles.

Mêmes caractères des pièces buccales masticatrices. Endopodite des pattes thoraciques réduit à un simple moignon dans une ou plusieurs paires. Espèces limicoles, rampantes.
Amymonin: ....... Amymone.

(Stenheliinæ Brady)
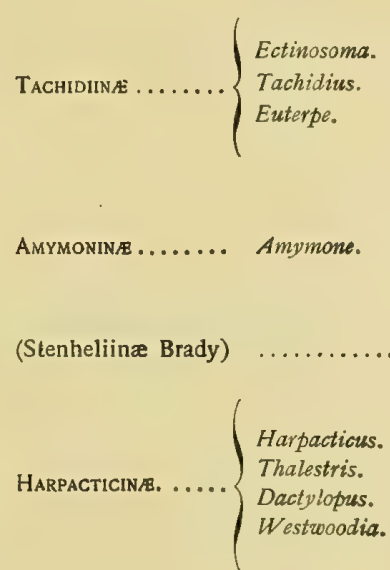

IDYIN/......... Idya.

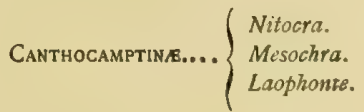

NanNopink ....... Nannopus. 


\section{LONGIPEDIINAE.}

Dans ses caractères principaux, cette sous-famille correspond à celle qu'établit BOECK (15, page 252 , et $\mathbf{1 7}$, page 44 ) sous la mème dénomination.

Je n'y ai pas conservé les trois genres Ectinosoma, Zozime et Bradya introduits far cet auteur, à cause des différences considérables qui les séparent du type de cette subdivision, de Longipedia.

Avec ce dernier vient se grouper un seul genre : Sunaristes Hesse, $110[=$ Longipedina, Mueller; 153], qui comprend lui aussi une seule espèce.

\section{Longipedia Claus.}

\section{I. - Longipedia coronata Claus.}

1863. Longipedia coronala, CLAUS; 51 ; pages I 10 et III; Tafel XIV.

1864. Longipedia coronata, Claus, BOECK ; 17 , pages 252 et 253.

1880. Longipedia coronata, Claus, BraDY, 20, vol. 2 ; pages 5-8; Plates XXXIV et XXXV.

1882. Longipedia coronata, Claus, Giesbrecht, 89, pages 99-104; Taf. I et IV-XII.

Très reconnaissable à la constitution des appendices céphaliques (antenmule épaisse, hérissée d'épines fortement denticulées; exopodite antennaire pluriarticulé, natatoire) et à l'endopodite démesurément allongé de la deuxième paire de pattes. Commune dans la zone littorale à la surface de l'eau et au milieu des algues. Formes jeunes très fréquentes, et peut-être plus nombreuses dans une même pèche que les adultes; ce qui pourrait expliquer les méprises faites par Claus (jeune $\sigma^{\prime}$ décrit pour l'adulte) et Brady (confusion entre les deux sexes, f immatures) et relevées par GiesBRECHT.

Distribution : Mer du Nord, Manche, Océan Atlantique, ......

\section{PELTIDIINAE.}

Dans la sous-famille des Peltidiina, définie plus haut, viennent se ranger les curieuses espèces d'Harpacticidic organisées pour l'adhésion à la surface des algues ou des pierres. L'étude récemment publiée par Claus (65) sur l'organisation 
de nombreux Peltidiens de la Méditerranée me permet d'insister seulement sur quelques particularités importantes.

Première maxille. - Elle diffère assez notablement dans les diverses formes, puisque tantôt elle présente un développement considérable de ses diverses parties constitutives, et spécialement de l'épipodite (voir ci-dessus, page 62), et qu'elle est parfois réduite à un appendice visiblement dégénéré et simplifié (ex. Oniscidium: 65, Tafel V, fig. 6; Tafel VI, fig. 7). Mais l'existence de nombreux lobes bien distincts dans le palpe de la première maxille des formes les plus différentes, comme Alteutha, Eupelte, Scutellidium, Porcellidium et Zaus, prouve bien que dans cette sous-famille, l'organisation primitive de l'appendice est généralement conservée. Et l'homologation du "lobe postérieur " (CLAUS : 65) de la première maxille avec la formation connue chez les Crustacés supérieurs sous le nom d'épipodite ne laisse aucune hésitation. L'épipodite est particulièrement développé dans les espèces des genres Scutellidium Porcellidium et Zaus, tandis qu'il parait subir une régression graduelle dans les genres Alteutha, Eupelte et Oniscidium (I).

AвDomen. - Sa forme, si dissemblable dans les espèces caligoïdes ou sphéromoïdes, ne permet pas d'admettre les subdivisions établies par certains auteurs dans les Peltidiens. En effet, des formes caligoïdes (Scutellidium) et sphéromoïdes (Porcellidium) montrent des rapports zoologiques plus étroits que ne le font certaines espèces sphéromoïdes (Alteutha, Euterpe, Oniscidium) pourtant très voisines à tous égards.

CllL. - Chez les Peltidiens, cet organe présente quelques particularités des plus intéressantes au point de vue comparatif.

Dans les uns (Zaus, Scutellidium), il forme une seule masse compacte et médiane, plongée dans les tissus de l'animal; il ne s'écarte donc point de la disposition habituelle parmi les Copépodes.

Dans les autres, les trois parties constitutives (ommatidies) avec leur cupule pigmentée pluricellulaire et leur lentille réfringente, sont plus (Oniscidium) ou moins (Alteutha, Eupelte) disjointes et disposées en apparence comme un œil médian et deux yeux latéraux. Mais une semblable interprétation ne pourrait être admise au point de vue morphologique (2).

(1) Si l'on en juge d’après les descriptions et les 'figures les plus complètes publiées jusqu'à ce jour, la maxille très réduite dans ce dernier genre, conserverait pourtant un rudiment d'épipodite (65; Taf. V, fig. 6 ; Taf. VI, fig. 7).

(2) Claus (65, page 5) arrive récemment à la mêtne conclusion, aprés avoir soutenu diverses opinions contradictoires sur les modifications analogues des yeux dans les Pontelllens (voir ci-dessous, page 176). 


\section{Zaus Goodsir.}

1845. Zaus, Goodsir, 95.

1863. Zaus, Coodsir; Claus, 51, page 146.

1864. Zaus, Goodsir; Bofck. 15, page 264.

1873. Non Zaus, Goodsir; Kritchaguine, 121, page 396.

\section{Zaus spinosus Claus.}

? 1845. Zaus spinatus; Goodsir, 95.

1863. Zaus spinosus; Claus, 51, page 146: Taf. XXII. fig. 25 ; Taf. XXIII, fig. $1=10$.

1864. Zaus spinatus, Goodsir; Borck, 15, page 264.

1880. Zaus spinatus, Goodsir; BradY, 20, vol. 2 ; page 153.

1889. Zaus spinosus; Claus. 65, page 35.

Copépode mimétique très abondant parmi les petites algues rouges et brunes de la zone des Laminaires. La disposition préhensile des deux rames dans les pattes de la première paire, commune à toutes les formes de ce genre, la cuticule transparente dépourvue de tubercules et d'incrustations calcaires, la largeur du lobe denticulé dans les soies terminales des antennes permettent de le reconnaitre facilement.

Distribution: Mer du Nord, Manche, Océan Atlantique.

\section{Scutellidium Claus.}

1866. Scutellidium; Claus; 53 , page 20.

1868. Aspidiscus; Norman ; 157.

1880. Scutellidium, Cls, BradY; 20, vol. 2 ; page 175.

1884. Scutellidium, Cls; POPPE; 163, page 291.

1889. Scutellidium, Cls ; Claus; 65. page 23.

Comprend les Peltidiens à forme caligoỉde et aplatie, avec la région terminale de la mandibule hypertrophiée par la saillie des lobes antérieur et postérieur, un long épipodite bien reconnaissable sur la première maxille, les deux rames préhensiles dans la première paire de pattes thoraciques. 


\section{Scutellidium tisboïdes Claus.}

1866. Sculellidium tisboïdes; Claus; 53, page 21 ; Taf. IV, fig. 8-15.

1880. Scutellidium tisboïdes, Cls; BradY; 20, vol. 2 ; pages 175-177; PI. LXVIII, fig. 1-10.

1889. Scutellidium tisboïdes, Cls; Claus ; 65, page 23 ; Taf. IX, fig. 5-14.

1890. Scutellidium lisboïdes, Cls; CAR; 37 , page 14.

Commun à la surface des frondes de Laminaires, dans les rochers de la côte.

L'adhérence de ce Copépode à la surface des corps lisses, sur lesquels il glisse facilement à la manière des Caliges, est facilitée non seulement par la forme du céphalothorax, mais aussi par la disposition des appendices. La première paire de pattes natatoires est entièrement adaptée à cet effet; les articles du protopodite, très élargis, portent une saillie chitineuse presque triangulaire formée de stries rayonnantes alternant avec des plis en relief. Cette disposition - qui pourrait être rapprochée des formations analogues constatées par Claus (55, page 333) et Kossmann (118, page I2) sür l'endopodite de Clausidium apodiforme Philippi, et désignées par ces auteurs sous le nom de "ventouses " (Saugscheibe, Saugnäpfchen) - avait échappé jusqu'ici aux naturalistes. De sa présence sur ces deux formes rampantes de familles si différentes, ressort pourtant une réelle importance.

Distribution : Mer du Nord, Manche, Océan Atlantique (îles Scilly, Madère), Méditerranée (Nice, Trieste), Mer Noire.

\section{Alteutha BAIRD.}

1850. Alteutha, BAIRD; 9, page 216.

1863. Alleutha, Brd; Claus; 51, page 141.

1864. Alteutha, Brd; BOECK; 15, page 272.

1880. Peltidium, Philippi ; Brady ; 20, vol. 2 ; page 158.

1885 Peltidium, Philippi ; Poppe; 164, page 191.

1887. Alleutha, Brd; BradY; 22, page 329.

1889. Alteutha, Brd; PoPPE; Abh. Bremen, Bd. IX, page 552.

1889. Alteutha, Brd; Claus; 65, page 6.

\section{Comprend les Peltidiens dont :}

La forme générale du corps, arrondie sur la face dorsale, rappelle les Isopodes (Sphéromes, Armadilles).

Le tégument est incrusté et ornementé de tubercules.

Le rostre frontal est arrondi.

Les antennules comptent 9 articles.

La mandibule supporte une région terminale assez réduite.

La première maxille est pourvue d'une sorte de palpe multilobé. 
La seconde maxille interne est préhensile, avec son premier article très allongé.

Les pattes thoraciques de la première paire ont les deux rames 3-articulées: l'interne natatoire, l'externe terminée en griffe et sans allongement particulier du deuxième article.

La cinquième paire de pattes thoraciques compte deux articles, le basilaire restant relativement court.

\section{Alteutba bopyroides Claus.}

1863. Altcutha bopyroildes; CLaus; 51, page 143; Taf. XXII, fig. 10-17.

1864. Alteutha norvegica; BoECK; 15, page 272.

1880. Peltidium interruptum, Goodsir; BRADY ; 20, vol. 2 ; page 162 ; PI. LXXI, fig. 4-15.

1885. Peltidium conophorum;-Poppe; 164, page 191; Taf. VII, fig. I-9.

1889. Alteutha bopyroides; Claus; 65, page 9; Taf. I. fig. 1-11; Taf. II, fig. 1-8.

Cette espèce est très abondante au milieu des rochers de toute la côte et particulièrement fréquente dans le port en eau profonde de Boulogne.

Par la façon dont elle se roule en boule à la moindre alerte, c'est celle qui rappelle le plus complètement les Isopodes. Elle est colorée en jaune et en brun dans toute sa longueur, et se dissimule parfaitement dans les bocaux au milieu des morceaux de rochers et des plantes. On la découvre aisément lorsqu'elle quitte cet abri pour monter à la surface, où elle reste peu de temps avant de retomber sur le fond.

Distribution : Mer du Nord, Manche, Océan Atlantique, Méditerranée.

\section{Eupelte Claus.}

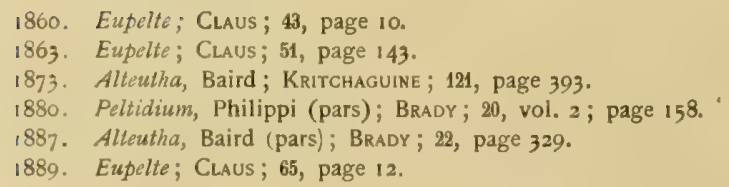

Genre parfaitement distinct du précédent, dont il se rapproche par sa forme (1) d'Isopode et par les appendices céphaliques. Il se distingue nettement par le prolongement frontal saillant et quadrangulaire, par le grand développement des pleura dans les segments thoraciques et abdominaux, par l'organisation de la seconde maxille interne, composée de deux articles basilaires et d'une pince 2-articulée terminale; par la première paire de pattes thoraciques avec l'exopodite 3-articulé, préhensile, dont l'article médian est très allongé; par la cinquième paire de pattes thoraciques 2-articulée, avec l'article basilaire très allongé et saillant.

(B) Plus aplatie que dans Alieutha. 


\section{Eupelte purpurocincta Norman.}

1868. Alteulha purpurocincta; NORMAN ; 157, page 298.

1880. Peltidium depressum, Baird; BRADY ; 20, vol. 2; page 160; Pl. LXXII, fig. 1-5.

1889. Eupelte purpurocincta, Norman ; Claus; 65, page 14, Taf. IIl, fig. $1-8$.

Espèce assez rare dans la zone des Laminaires, au milieu des algues colorées, où elle adhère fortement à la surface des corps submergés. Les deux extrémités du corps sont de couleur jaune-verdâtre, èt les deuxième, troisième et quatrième somites thoraciques forment une bande plus claire de teinte bleuâtre. La surface du tégument est délicatement échinée, le prolongement frontal un peu rétréci vers l'extrémité ; l'endopodite de la première pafte thoracique 3-articulé, la cinquième patte thoracique recouverte de cils courts et serrés vers l'extérieur et terminée par trois épines solides peu effilées; les pièces furcales courtes et peu élargies.

Distribution : Mer du Nord, Manche, Océan Atlantique.

\section{TACHIDIINAE.}

Dans cette série de formes comprenant des Harpacticidés encore peu modifiés par rapport à Longipedia, viennent se grouper naturellement divers genres présentant un certain nombre de caractères primitifs dans leur forme générale, leurs appendices céphaliques, et la similitude des pattes natatoires.

Malgré l'opinion des auteurs, par sa seconde maxille interne un peu préhensile, le genre Ectinosoma se rapproche de Tachidius et Euterpe plutôt que de Longipedia.

\section{Ectinosoma BoEcK.}

1864. Ectinosoma; BOEck; 15, page 254.

1866. Tachidius, Lilljeborg; Claus, 53, page 24.

1872. Ectinosoma; BoEck ; 17, page 44.

1873. Tachidius, Lillj. ; KrITCHÁguine ; 121, page 371.

1880. Ectinosoma, Boeck; Brady; 20, vol. 2 ; page 8.

1882. Ectinosoma, Boeck; Giesbrecht ; 89, page 106.

1885. Ectinosoma, Boeck; РОРPE; 164, page 194.

Les différences génériques avec Tachidius LiLLJ. ne peuvent être mises en doute.

Comme Poppe (164, page 198) l'a indiqué, Tachidius minutus Craus appartient au genre Ectinosoma par tous les caractères du corps et des appendices buccaux $(53,2$ page 4: Taf. 
IV, fig. 1-7). Il en est certainement de même pour Tachidius pygmaeus décrit et figuré plus complètement par Kritchaguine (121; pages 371-373; Pl. XII, fig. 1-9).

La forme du corps presque cylindrique, la constitution normale des quatre paires de pattes thoraciques, le développement particulier des cinquièmes pattes thoraciques élargies et 2-articulées forment - avec les secondes maxilles externes pourvues de deux articles basilaires elliptiques nettement séparés, et les secondes maxilles internes 3-articulées terminées par une longue sjie rigide peu préhensile - la caractéristique de ce genre bien décrit par les auteurs récents (1).

\section{Ectinosoma sarsi, BOECK.}

1872. Ectinosoma Sarsii; BoEck; 17. page 45.

1880. Ectimosona spinipes; Brady; 20, vol. 2, pages 9 et 10.

1885. Ectirosoma Sarsii, Boeck; PoPPE; 164, page 198.

Abondamment répandu dans tous les fonds côtiers légèrement vaseux qui alternent avec les rochers. Recueilli sur toute la còte boulonnaise, fréquemment en compagnie de Longipedia coronata, comme le dit également BRADY (loc. cit., page 10). La taille relativement considérable (de $1 \mathrm{~m} / \mathrm{m}$ à $\mathrm{I}, 2 \mathrm{~m} / \mathrm{m}$ ) pour une espèce de ce genre, la couleur brune, la forme effilée et la rigidité parfaite de son corps, la brièveté des épines échinées qui terminent la cinquième paire de pattes thoraciques - parfaitement indiquée par BoECK dans la description originale (2) caraćtérisent suffisamment cette espèce et confirment la synonymie établie par PoPPE.

Distribution: Mer du Nord, Manche.

(1) On connaît, en effet, très exactement l'organisation des pièces buccales dans quelques espèces étudiées par Giesbrecht (E. gothiceps Gsb.; 89, pp. 106-8) et par Poppe (E. curticorne Boeck; 164, pp. 194-8). Les descriptions de Boeck (15, p. $254 ; 17$, pp. 44 et 45) et de Brady (20, vol, 2, pp. 8-14;21, pp. 99 et too) sont très insuffisantes à ce point de vue.

11 en est de méme des diagnoses rassemblées par DE GUERNE dans sa révision du genre : "Sur les genres Ectinosoma Boeck et Podon LiLLjeborg à propos de deux Entomostracés (Ectinosoma atlanticum G.-Sr.-BradY ET Robertson et Fodolz minutus G.-O. Sars) trouvés à la Corogne dans l'estomac des Sardines, Bull. Soc. Zool. $F r$., tome XII, 1887 \%. Pour l'unique espèce observée, E. allanticum BR. ET RoB., rien n'est ajouté aux descriptions de BRADY sur les appendices buccaux. Mais l'auteur anglais ne les a point décrits particulièrement (21, page 100), parre qu'ils sont trop petits pour qu'il en ait obtenu une préparation suffisante (20, vol. 2, page 14).

C'est dans le mémoire totalement oublié de Krirchaguine (121) que se trouve la première description soignée des organes buccaux d'Ectinosoma, organes dont l'observation est vraiment pleine de difficultés.

(2) « Det femte Fodpar er forlaenget. Grundleddets meget forlaengede indre Rand er paa Enden vaebnet " med tvende. ge ange Torne. Endepladen er omtrent dobbelt saa lang som bred, besat med tvende brede "Torne, der ere meget kortere end denne, og paa Enden af en Lap i den ydre Rand findes der en lignends Torn ». Une description aussi précise, concordant entièrement avec les indications données par Bridi (loc. cill., page io, Pl. XXXVI, fig.9), pouvait écarter les hésitations dont nous fait part ce naturaliste au sujet de cette espèce. 


\section{Ectinosoma minutum, Claus.}

1866. Tachidius minutus; Claus; 53 , page 24, Taf. IV, fig. 1-7.

1873. Tachidius pygmous; Kritchaguine; 121, pages 371-373; PI. XII, fig. 1-9.

1887. Ectinosoma minutum, Claus; PoPPE; 164. page 198.

Espèce très petite $(0,6 \mathrm{~m} / \mathrm{m}$ de long).

Elle se distingue :

$1^{0}$ Par la forme du céphalothorax, bońbé sur la face dorsale.

$2^{0}$ Par les antennules 6 -articulées, assez grandes et pourvues de deux larges filaments sensoriels hyalins.

$3^{0}$ Par l'exopodite 3-articulé des antennes.

$4^{0}$ Par le nombre restreint ( 2 ou 3 ) des soies terminant les deux rames de la mandibule.

$5^{0}$ Par la forme lancéolée et les minces denticulations marginales de l'épine externe insérée sur le prolongement postérieur qu'envoie l'article basilaire de la cinquième patte auprès de la ligne médiane.

$6^{0} \mathrm{Par}$ les pièces furcales très courtes.

Dans la forme du corps et des divers appendices, les exemplaires que j'ai recueillis sur les côtes du Boulonnais concordent assez exactement avec les descriptions données par Claus et KrITCHAGUine. L'organisation de la cinquième paire de pattes est l'argument le plus frappant en faveur de la synonymie acceptée ci-dessus: KrITChaguine (loc. cit., page 373; Planche XII, fig. 9), a particulièrement insisté sur ces ressemblances et sur les caractères de la courte épine terminale de l'article basilaire.

D'après la description de Brady (20, vol. 2 ; page II ; Planche XL, fig. 20), $E$. melaniceps BoECK présenterait dans la disposition des épines de la cinquième paire une certaine ressemblance avec $E$. minutum CLs. L'identité des deux formes paraît très problématique et doit être momentanément rejetée. Du reste, la description de BRADY est fort insuffisante; elle ne nous apprend rien sur l'exopodite antennaire qui, d'après BoECK (15, page 254) serait 2-articulé dans $E$. melaniceps. La courte épine du lobe interne de la cinquième patte thoracique $y$ est dite très courte et lisse. $E$. melaniceps serait aussi de taille plus considérable $(0,84 \mathrm{~m} / \mathrm{m})$ et présenterait réellement à la face dorsale du céphalon une tache foncée que je n'ai pas constatée nettement dans les nombreux exemplaires d' $E$. minutum que j'ai pu examiner.

Abondante avec la précédente sur les fonds vaseux auprès du rivage.

Distribution : Manche, Méditerranée (Nice), Mer Noire. 


\section{Euterpe, Claus.}

1852. Harpacticus, Milne-Edwards (pars); DANA; 73.

1863. Euterpe; Claus; 51 ; page 109.

1880. Euterpe, Claus; BradY; 20, vol, 2 ; page 22.

Genre bien caractérisé : par la seconde maxille interne, avec un article basilaire allongé et beaucoup plus large que les trois suivants, plus grêles et très effilés vers leur extrémité; par la constitution des pattes thoraciques de la première paire, 2-articulées dans chaque branche.

\section{Euterpe acutifrons, DANA.}

1852. Harpacticus acutifrons; DANA ; 73, pp. 1192-1193; PI. LXXXIII, fig. $11 a-b$.

1863. Euterpe gracilis; Claus; 51 , p. 110 ; Taf. XIV, fig. 1-13.

1880. Euterpe gracilis, Claus; BradY; 20, vol. 2, p. 22-24; Pl. XL, fig. $1-16$.

1891. Euterpe acutifrons, Dana; GresBrecht ; 89, vol. VIl; p. 475.

Comme l'a indiqué Giesbrecht, le Copépode mâle étudié par DanA et caractérisé par sa forme générale (presque cyclopoïde, avec prolongement frontal effilé en pointe), par ses antennules préhensiles assez spéciales (I) et par l'extrémité sétiforme (2) de la seconde maxille (3), se confond spécifiquement avec l'espèce européenne signalée par Claus.

Forme pélagique, assez abondante à la surface de la mer, à quelque distance de la côte. Elle était très fréquente parmi les Copépodes que j'ai recueillis en août I 890, au milieu du détroit du Pas-de-Calais durant les sondages faits à bord de I'Ajax pour les études du Pont sur la Manche. De petite taille (I m/m au plus), comme ceux qu'a étudiés DANA, mes échantillons sont incolores, et non pas bruns foncés comme le dit BRADY (loc. cit., page 24) des spécimens recueillis près des côtes d'Irlande à bord du " Porcupine ".

Distribution : Mer du Nord, Manche, Océan Atlantique.

\section{Tachidius, LILLJEBorg.}

1853. Tachidius; LILLJEBORG; 156, page 195.

1864. Tachidius, Lillj.; ВовсK; 15, p. 256.

(1) Voir 73, Plate LXXXI11, fig. 11 a.

(2) Loc. cit., fig. 11 b; et non pas fig. e, comme il est dit dans le texte, page 1193.

(3) DaNa dit avec incertitude : "An extremity of a maxilliped, or perhaps the mandibular palpus......". Mais la figure à laquelle il renvoie ne laisse aucun doute. 
1880. Tachidius, Lillj.; BradY; 20, vol. 2 , page 19

1882. Tachidius, Lillj.; Giesbrecht ; 89, page 108.

1866. Non Tachidius, Lillj. ; Claus; 53, page 24.

Genre suffisamment caractérisé par :

$\mathrm{I}^{0}$ Les antennules courtes et épaisses, garnies de longues soies raides.

$2^{0}$ Les mandibules biramées, à rames simples, presque égales.

$3^{0}$ Les secondes maxilles internes formées de deux articles basilaires cylindriques égaux, d'un troisième article court et rétréci, et d'une longue épine terminale recourbée formant une sörte de pince.

$4^{0}$ Les pattes thoraciques natatoires dans les quatre premières paires.

$5^{\circ}$ La cinquième paire de pattes thoraciques lamellaire, 2-articulée, avec l'article basilaire très court et soudé assez intimement au tronc.

Le genre Tachidius présente un grand intérêt, tant au point de vue des habitudes éthologiques, qu'au point de vue de l'organisation anatomique.

Tachidius discipes GsB. et Tachidius littoralis POPPE vivent dans les eaux plus ou moins saumâtres des estuaires et des marais salants. Dans le Boulonnais, ils sont très répandus à l'embouchure des rivières, au milieu des algues vertes (Enteromorpha intestinalis), en compagnie de Palcemonetes varians LEACH, de $C y$ theridea torosa Jones, et de beaucoup d'autres espèces connues pour leur habitat saumâtre.

Sans compléter, pour les détails, les descriptions des auteurs cités précédemment, j'insisterai sur deux particularités des plus intéressantes que j'ai constatées dans ces Copépodes.

A. La glande du test présente, chez $T$. discipes et $T$. littoralis, un développement relativement considérable. Le canal de cette glande est contourné trois fois, avant d'aboutir à la seconde maxille externe, et ses circonvolutions rappellent beaucoup la disposition connue dans Canthocamptus, ou même dans Cyclops.

Faut-il y voir une confirmation de l'idée émise par GrobBen sur l'influence de la salure dans le développement de cet organe ? En tout cas, le genre Tachidius, franchement adapté à l'existence dans l'eau de mer fortement mélangée d'eau douce, s'éloigne des Harpacticidés marins par le grand développement de sa glande du test pour se rapprocher des Copépodes d'eau douce.

B. L'organe nuchal persiste chez les Tachidius adultes, sous forme d'une plaque d'aspect vaguement glandulaire (voir ci-dessus, page 125). Il apparait très tôt dans le métanauplius, au moment où cet embryon va fournir le premier stade 
cyclopoïde; c'est une sorte de contour elliptique, situé sur la face dorsale au niveau des premières maxilles. Dans l'adulte, la cuticule est en cet endroit moins épaissie que partout ailleurs, ce qui permet de reconnaître plus facilement l'existence de l'organe.

\section{Tacbidius discipes, Giesbrecht.}

1853. Tachidius breoicornis, Müller; LilLjeborg; 136, pages 196-9. Tab. XXII, fig. 12-16; Tab. XXIII, fig. 1,2 et 9 ; Tab. XXVI, fig. 17 et 18.

1864. Tachidius brevicornis, Müller; BoEcK; 15, page 257.

1880. Tachidius brevicornis, Müller; Bradr; 20 , vol. 2 ; pages 20-22; PI. XXXVII, fig. I-16.

1882. Tachiduts discipes; Giesbrecht ; 89, pages 108-111; Taf. II, IV, V, VII-XII.

C'est l'espèce la plus fréquente dans les eaux saumâtres du Boulonnais. Elle peut être distinguée d'après ses antennules légèrement effilées à leur extrémité, d'après son corps ramassé, ses pattes très élargies, spécialement dans la cinquième paire.

Distribution : Baltique, Mer du Nord, Manche, Mer d'Irlande.

\section{Tachidius littoralis, PopPE.}

1881. Tachidius littoralis; POPPE ; 162, pages 149-152; Taf. VI, fig. 1-12.

Moins abondant que le précédent, en compagnie duquel il vit à l'embouchure du Wimereux. Reconnaissable à sa forme moins trapue, à ses antennules très élargies jusqu'à leur extrémité, à l'étroitesse relative des pattes thoraciques.

Distribution : Mer du Nord, Manche, Mer d'Irlande.

AM YMONINAE.

Amymone, Glaus.

1860. Amymone; Claus ; 43, page 11.

1863. Amymone; Claus; 51, page 112.

1864. Amyone, Claus; BoECK; 15, page 255.

1880. Amymone, Claus; Brady; 20, vol. 2, page 28. 


\section{Amymone spharica, Claus.}

1863. Amymone spharica; CLAus; 51, page 114 ; Taf. XX, fig. 1-9.

1864. Amyone sphacrica, Claus; Boeck; 15, page 256.

1880. Amymone spharica, Claus; Brady; 20, vol. 2, pages 28-30; Plate XLIX, fig. I-I1.

Espèce de taille relativement grande $(0,45$ à $0,55 \mathrm{~m} / \mathrm{m})$. de couleur gris-bleuâtre, avec la carapace épaisse et ornée de petits tubercules punctiformes. Abondante dans la zone des Laminaires, dans les divers points rocheux de la côte boulonnaise. Les grandes saillies ventrales, découpées, des bords pleuraux du premier segment abdominal lui donnent un aspect particulier.

Distribution : Mer du Nord, Manche, Méditerranée.

\section{HARPACTICINAE.}

Brièvement définie dans le tableau analytique reproduit précédemment, cette sous-famille présente à côté de variations de détail multiples, certains caractères primitifs assez constants : les espèces qui la composent sont toutes des Copépodes très agiles, carnassiers, vivant au fond des eaux parmi les algues.

\section{Harpacticus, Milne Edwards.}

1840. Arpacticus; Milne-Edwards; 149, vol. 3, page 430.

1850. Arpacticus, M.-Edw.; Balro; 9, page 212.

1853. Harpacticus, M.-Edw. ; LilLjeBorg; 136, page 200.

1863. Harpacticus, M.-Edw. ; Claus ; 51, page 133.

La seconde maxille interne en forme de pince préhensile, les pattes de la première paire préhensiles dans les deux rames, avec l'endopodite court et 2-articulé et l'exopodite allongé et 3-articulé, distinguent à première vue ce genre.

\section{Harpacticus chelifer, O.-F. Mueller.}

1785. Cyclops chelifer; O.-F. Mueller; 152, pages 114 et 115 ; Tab. XIX, fig. 1-3.

..... Harpacticus chelifer, Müller; Milne-Edwards, Claus, Giesbrecht,... etc.

1853. Non Harpacticus chclifer, Müller; LiLlJeborg; 136, page 200; Tab. XXII, fig. 2-11. 
Très abondant parmi les algues de la zone littorale. Se reconnaît entre tous gràce à sa taille restreinte, à sa coloration faible, et à la taille de la seconde maxille interne.

Distribution : Mers arctiques, Mers d'Europe et d'Amérique.

\section{Harpacticus fulvus, Fischer.}

1853. Harpacticus chelifer, Múller; Lilujeborg; 138, page 200; Tab. XXII, fig. 2-II.

1860. Harpacticus fulous; FISCHER; 80.

1880. Harpacticus fultus, Fischer; Bradr; 20, vol, 2, pages 149-152; Plates LXIV, fig. I-11.

Dans les mares d'eau salée exposées au soleil au-dessus du niveau ordinaire de la marée haute. Vit également dans les marais salants de la côte bretonne (Le Pouliguen) ( $\mathrm{I}$ ).

Distribution: Mer du Nord, Baltique, Manche, Océan Atlantique, Ile Kerguelen.

\section{Thalestris Claus.}

1863. Thalestris; Claus; 51 , page 128.

1864. Thalestris, Claus; BoECK; 15, page 264.

1880. Thalestris, Claus; BradY; 20; vol. 2, page 120.

Genre bien caractérisé par l'allongement et la transformation des deux rames des premières pattes thoraciques en puissants organes de préhension, par le développement des secondes maxilles internes en une pince très puissante.

\section{Thalestris longimana, Claus.}

1863. Thalestris longimana; Claus; 51, page 130; Taf. XVIII, fig. Im-11.

1880. Thalestris longimana, Claus; BRADY, 20, vol. 2, p. 136; PI. LX, fig. I-13.

Grande espèce à carapace solide, d'un brun-rougeâtre. Abondante au milieu des algues brunes, dans la zone des Laminaires.

Distribution: Mer du Nord, Manche, Océan Atlantique.

(1) M. TH. Barrois a signalé pour la première fois ce Copépode dans le Boulonnais, dans les conditions éthologiques où Brady l'a recueilli en Angleterre. - Comparer : TH. Barrols, Sur la présence de l'Harpactrcus fulcus, FISCHER, dans le Boulonnais; Reoue biologique du Nord de la France, $2^{\circ}$ Année, 1890, page 288, lignes 10 à 15; et Bradr, 20, vol. 2, pages 151, lignes 6 à 12. 


\section{Thalestris clausi, Norman.}

1868. Thalestris Clausii; NoRman ; 157.

1880. Thalestris Clausii, Norman; BRADY; 20, vol. 2, pages I28-1 30; Pl. LXII, fig. I-I2.

Espèce de taille moyenne, peu colorée, fréquente parmi les Sertularia et les Bugula dans la zone des Laminaires.

Distribution : Mer du Nord, Manche, Océan Atlantique.

\section{Thalestris rufocincta, Norman.}

1880. Thalestris rufocincta, Norman M. S.; BRADY; 20, vol. 2, pages 125 et 126 , PI. LVII, fig. I-9.

Espèce jaunâtre, avec des taches rouges sur la face ventrale et sur les côtés des segments thoraciques dans le milieu du corps.

Très commun dans les rochers de Hermelles de la zone des Laminaires.

Distribution: Merdu Nord, Manche, Océan Atlantique.

\section{Dactylopus, Claus.}

1863. Dactylopus; Claus; 51 , page 124.

1880. Dactylopus, Claus; BRADY; 20, vol. 2; page 105

1882. Dactylopus, Claus; GIESBRECHT; 89, page 122.

Genre différent du précédent par la constitution de l'exopodite dans la première paire de pattes et par l'allongement en griffe de l'extrémité distale dans la seconde maxille interne.

\section{Dactylopus strimi, BAIRD.}

1860. Canthocamptus strómii; BAIRD; 9, page 208; P1. XXVII, fig. 3.

1880. Dactylopus strómii, Baird; BrADY; 20, vol. 2. pages 111-115; P1. LV, fig. 1-13.

Très commun dans la zone des Laminaires, parmi les algues.

Distribution: Mer du Nord, Manche, Océan Atlantique.

\section{Westwoodia, DANA.}

1852. Westwoodia; DANA; 73.

1863. Westrooodia, Dana; Claus, 51 ; page 117. 
Genre bien reconnaissable à la première paire de pattes ayant l'endopodite 3-articulé et préhensile, et l'exopodite composé d'un seul article sétifère.

\section{Westwoodia nobilis, BAIRD.}

1850. Arpacticus nobilis; BaIRD, 9, page 214; Taf. XXVIII, fig. 2.

1863. Westwoodia nobilis, Baird ; CLAus; 51, page 118 ; Taf. XXI, fig. I-9.

Espece très brillamment colorée en vert et en rouge, et reconnaissable même à l'œil nu d'après cette coloration. Commune parmi les Hermelles, dans la zone des Laminaires.

Distribution : Mer du Nord, Manche, Océan Atlantique.

I D Y IN Æ.

Idya, PHILIPPI.

1843. Idya; PhilipPi; 159.

1853. Tisbe; LilljeBORG; 136 , page 191.

1864. Idya, Philippi; Boeck; 15, page 257.

1873. Zaus, Goodsir; Krıtchaguine; 121, page 396.

Son corps élargi et déprimé, au contour cyclopoïde; sa première paire de pattes adhésive, avec la base, l'exopodite et l'endopodite 2-articulés et très larges suffisent pour le caractériser.

$$
\text { Idya furcata, BAIRD. }
$$

1837. Cyclops furcatus; BAlRD.

1850. Canthocamptus furcatus; BAIRD; 9, page 210 ; Tab. XXV et XXX.

1853. Tisbe furcata, Baird; LiLleseBorg; 136, pages 192-195; Tab. XXV, fig. $1-5,11,12$ et 17.

1864. Idya furcata, Baird; BOECK; 15, page 258.

1873. Zaus adoersipes; KrItchaguine; 121, page 396-398, Tab. XIV, fig. 1-5.

Très commun dans la zone littorale, dans le creux des rochers à marée basse. C'est le Copépode qui montre le plus de résistance à la putréfaction : après la mort des animaux contenus dans les aquariums, il apparaît en grand nombre, glissant au-dessous de la pellicule de Bactéries développée à la surface de l'eau de mer conservée pendant quelques jours.

Distribution: Mer du Nord, Baltique, Manche, Océan Atlantique, Méditerranée. 


\section{CANTHOCAMPTIN Æ.}

La tendance accusée dans plusieurs genres de cette sous-famille vers la réduction de l'endopodite dans les pattes natatoires, est, avec les habitudes vagabondes et la puissance masticatrice des mandibules uniramées, des premières et secondes maxilles internes, la caractéristique la plus simple et la plus nette de cette importante série de Copépodes.

\section{Nitocra, Boeck.}

1864. Nitocra; Boeck; 15 , page 274 .

1882. Nitocta, Boeck; GIeSBRErht; 89, page 115.

Genre avec le corps allongé presque cylindrique, le prolongement frontal peu développé, l'exopodite simple dans l'antenne, l'extrémité simple dans la mandibule, toutes les pattes thoraciques 3-articulées dans les deux rames.

\section{Nitocra oligocbceta, GiesBrecht.}

1882. Nitocra oligochoeta; GIESBRECHT ; 89, pages 116 et 117 ; Taf. I, III-XII.

Assez rare dans les algues de la zone littorale.

Distribution: Baltique, Manche.

\section{Mesochra, Boeck.}

1864. Mesochra, BoEck; 15, page 375.

1880. Mesochra, Boeck; BRADY; 20, vol, 2, page 62.

1882. Mesochra, Boeck; Giesbrecht; 89, page 11 .

Genre avec l'endopodite et l'exopodite simples dans l'antenne, l'extrémité de la mandibule uniramée, l'exopodite 3-articulé et l'endopodite 2-articulé dans les quatre paires de pattes natatoires.

\section{Mesocbra lilljeborgi, BOECK.}

1853. Canthocamplus strümii; LILLjEBorG, 136, pages 202-204; Tab. XXIII, fig. 3-8.

1864. Mesochra Lilljeborgii; BOECK; 15, pages 275 et 276. 
1880. Mesochra Lilljeborgii, Boeck; BradY; 20, vol. 2, pages 62-64; PI. XLI, fig. 15-20, Pl. XLVII, fig. 16-21.

1882. Mesochra Lilljeborgii, Boeck ; GiesBrecht ; 89, pages III-115; Taf. I, IV-XII.

Assez rare, parmi les algues, dans la zone littorale.

Distribution : Mer du Nord, Baltique, Manche, Océan Atlantique.

\section{Laophonte, PHILIPPI.}

1840. Laophonte; PhiLıpi, 159, Jahrg Vl.

1863. Cleta; Claus; 51 , page 123.

1864. Laophonte, Philippi ; BOECK; 15 , prges 276 et 277.

Genre bien caractérisé par la forme allongée, presque cylindrique, du céphalothorax; par la faible différence de largeur entre le thorax et l'abdomen; par les antennules composées de 4 à 7 articles; par les antennes 2-articulées avec l'exopodite simple, toujours très réduit et parfois même absent; par la mandibule simple, 2-articulée, par les secondes maxilles externes presque chéliformes, avec l'article terminal très allongé et recourbé; par les pattes natatoires dont l'endopodite est 2-articulé dans les 4 paires et nettement préhensile dans la première.

\section{Laophonte serrata, CLaus.}

1863. Cleta serrata, Claus ; 51, page 124; Taf. XV, fig. 13-20.

1864. Laophonte serrata, Claus, BOECK, 15, page 277.

1880. Laophonte serrata, Claus; Brady ; 20 , vol, 2, pages 71 à 73 , PI. LXXIII, fig. 1-14.

Corps recouvert d'une chitine épaisse; segments bordés postérieurement d'une rangée de denticules triangulaires; pièces furcales cylindriques, aussi longues que le dernier segment; soie furcale médiane rigide et égale à la longueır de l'abdomen. Antennules garnies de grosses saillies épineuses sur les articles basilaires. Exopodite de la première patte thoracique 3-articulé.

Assez commun parmi les algues dans la zone littorale.

Distribution: Mer du Nord, Manche, Océan Atlantique (Iles Britanniques, Madère et Canaries).

\section{Laophonte thoracica, BОЕск.}

1864. Laophonte thoracica; Bosck; 15, page 278.

1880. Laophonte thoracica, Boeck; BRADY; 20, vol. 2, pages 76 et 77 ; Pl. LXXVII, fig. 1-8.

Corps cylindrique au-delà du premier segment renflé; segments garnis d'épines tuberculaires sur le bord postérieur ; pièces furcales plus longues que le dernier segment, avec deux 
fortes soies terminales médianes, l'interne plus longue que l'abdomen. Antennules longues et dépourvues d'épines à leur base; secondes maxilles internes et premières pattes thoraciques très allongées; exopodite de la première patte 3 -articulé; cinquièmes pattes thoraciques longues, avec le second article très étroit.

Commun dans les mares garnies d'algues, entre les rochers.

Distribution : Mer du Nord, Manche.

\section{Laophonte curticaudata, BoEck.}

1864. Laophonte curticaudata; BOECK ; 15 , pages 278 et 279.

1880. Laophonte curticauda, Boeck; BradY; 20, vol. 2, pages 80-82; PI. LXXIII, fig. 15-18 ; PI. LXXVI, fig. $1-9$.

1882. Laophonte curticaudata, Boeck; GIESBRECHT; 89 , page 127.

Espèce plus trapue que la précédente, sans épines sur le bord des segments; pièces furcales de même longueur que le dernier segment. Pattes thoraciques de la première paire solides et peu allongées, avec l'exopodite 3-articulé. Cinquièmes pattes courtes, larges et lamelleuses. Chez le mâle, exopodite des pattes de la troisième paire très élargi et pourvu de très fortes épines sur son bord interne.

Commun sur les fonds sableux et parmi les algues, au milieu des rochers de la zone littorale.

Distribution : Mer du Nord, Baltique, Manche, Océan Atlantique.

\section{Laopbonte lamellifera, Claus.}

1863. Cleta lamellifera; CLaus; 51 , pages 123 et 124 ; Taf. 15 , fig. $2 \mathrm{I}-25$.

1880. Laophonte lamellifera, Cls; BRADY; 20, vol. 2, pages 82-85; Pl. LXXV, fig. 15-23.

Espèce trapue, recouverte d'une carapace solide et ornementée; avec des épines sur le bord des segments. Pièces furcales un peu plus longues que le dernier segment, garnies d'épines moins longues qu'elles, et dépourvues de soies. Pattes de la première paire relativement courtes, avec l'exopodite 3-articulé; de la cinquième paire allongées, solides, avec l'article basilaire pourvu au bord interne d'un prolongement garni de soies presque aussi long que l'article terminal.

Commun dans le sable et la vase, au milieu des algues et dans les creux des rochers, dans la zone des Laminaires et des Fucus.

Distribution : Mer du Nord, Manche, Océan Atlantique, Méditerranée.

\section{Laophonte similis, Claus.}

1866. Cleta similis; Claus; 53 , page 23 ; Taf. V, fig. 13-16.

1880. Laophonte similis, Claus; BradY; 20 , vol. 2 ; pages 78 et 79 ; PI. LXXV, fig. $1-14$. 
1884. Laophonte pilosa ; CAR ; 36, pages 245-247; Taf. XVII, fig. 5-15.

1890. Laophonte similis, Claus; CAR; 37, pages 112 à 113 .

\section{Planche V, Fig. I-24.}

Taille : $q 0,6 \mathrm{~m} / \mathrm{m} ; \sigma^{7} 0,54 \mathrm{~m} / \mathrm{m}$.

Corps (fig. 1 et 2) presque cylindrique, grêle, incurvé sur la face dorsale et même replié dans les spécimens conservés; recouvert de poils très fins sur le bord des segments et sur la face dorsale. Céphalothorax plus long que l'abdomen. Prolongement frontal réduit à une légère saillie arrondie pourvue de deux poils (fig. 6). Pièces furcales (fig. 24) plus courtes que le segment précédent, avec les deux soies terminales médianes bien développées, peu flexibles et garnies d'un petit nombre de barbules rigides; la soie interne étant plus grande et aussi longue que les trois derniers segments abdominaux.

Carapace du segment céphalique montrant trois espaces de chitine plus transparente, dont l'impair, dorsal, correspond à l'organe nuchal (fig. 3).

Antennules assez allongées dans les deux sexes. Chez la ${ }_{\text {}}$, elles comprennent 6 articles (fig. 6 ), dont les $2^{\mathrm{e}}, 3^{\circ}$ et $6^{\theta}$ sont les plus longs, et le $4^{\theta}$, le plus court; le $4^{\theta}$ et le $6^{\mathrm{e}}$ portent chacun un poil sensoriel, et le premier seul est dépourvu de soies. Chez le $\sigma$, elles comptent également 6 articles; le $4^{e}$ est renflé vers l'extérieur et porte : au bord intérieur un gros tubercule pointu, une crête chitineuse denticulée et une longue épine recourbée (fig. 5); puis, au bord supérieur, une saillie avec deux longues soies flexibles et un filament sensoriel; ce $4^{\circ}$ article correspond aux $4^{\circ}$ et $5^{\circ}$ articles de l'antennule . Après lui, viennent encore deux articles disposés en une sorte de griffe et qui portent également des soies et un filament sensoriel; ces derniers correspondent au sixième article antennulaire de la $q$; par une flexion géniculaire, ils viennent s'adapter au bord interne et antérieur du $4^{\circ}$ article pour servir d'organe de préhension dans la copulation.

Antennes 2-articulées, simples (fig:7), l'exopodite paraissant réduit à une simple soie; l'article basilaire présente une légère trace de subdivision. En outre des soies simples, le second article est terminé par des épines uncinées et annelées sur leür bord intérieur a quelque distance de leur extrémité.

Mandibules (fig. 9) solides, avec 4 grosses dents échinées et une petite soie au bord distal du coxopodite, (fig.10) avec un second article simple, portant des traces confuses d'une ramification sous la forme d'un tubercule externe portant trois soies barbelées.

Premières maxilles (fig. 11 et 12 ) avec le coxopodite masticateur pourvu de cinq dents triangulaires, supportant une région terminale quadrilobée, pourvue de soies et qui représente le basipodite et les deux rames encore reconnaissables malgré leur réduction.

Secondes maxilles externes (fig. 13) courtes, avec une portion (article?) basilaire munie de deux tubercules internes portant une épine recourbée finement denticulée et des soies, avec une région terminale pourvue d'une forte épine et de quelques poils grêles.

Secondes maxilles internes (fig. 14) 3-articulées. Article basilaire de forme assez irrégulière, avec des saillies convexes garnies de rangées de denticules et deux soies courtes sur la face antérieure. Article médian long et presque cylindrique. Article terminal en forme de griffe très allongée et recourbée, venant s'accoler au précédent pour constituer une pince puissante. 
Pattes de la première paire relativement courtes (fig. 1 et 2 ). Les deux articles basilaires sont courts (fig. 15), garnis de denticules sur le bord intérieur convexe; le second porte une soie épineuse légèrement barbeiée à son bord externe, et une épine denticulée à la face antérieure de son bord interne. Les deux rames sont 2-articulées; l'externe, plus courte, porte des soies droites à son bord extérieur et deux soies coudées à son extrémité; son bord externe est finement cilié. Le premier article de la rame interne est très allongé, cylindrique, cilié à son bord externe. Le deuxième est très court, cilié à son bord interne et terminé par une forte griffe plus' longue que lui.

Deuxièmes, troisièmes et quatrièmes pattes thoraciques avec la base 2-articulée, l'exopodite 3-articulé et l'endopodite 2-articulé pourvus de soies et d'épines dont le nombre et le développement varient dans les différentes paires (fig. 16,17 et 18 ). Chez le $\sigma$, l'endopodite diffère de celui de la $q$, non pas dans la $2^{\theta}$ paire, comme le dit Brady (loc. cit., page 78 , fig. 10) où il ressemble à celui de la femelle (comparer fig. 19 et fig. 16), mais dans la troisième (fig. 20). il compte alors 3 articles, dont les contours sont assez irréguliers; le second article porte au bord distal une forte épine chitineuse recourbée deux fois en agrafe, et qui sert probablement à la réunion des sexes. L'article terminal porte 4 soies : 2 terminales et 2 internes. En comparant les figures 17 et 20 , on comprendra aisément que les deux derniers articles de l'exopodite du mâle correspondent à l'article terminal de l'exopodite chez la femelle. L'assertion de Brady ( $2^{\theta}$ patte modifiée chez le $\left.\sigma^{\top}\right)$ se trouve réfutée si l'on compare la disposition et la forme des épines de l'exopodite dans la fig. 10 publiée par cet auteur (loc. cit., Pl. LXXV) et dans les fig. 16 et 17 de notre Pl. V.

Cinquièmes pattes thoraciques larges, 2-articulées (fig. 21 et 22). Dans la $q$, l'article basilaire est très développé, surtout vers l'intérieur. Dans le $\sigma$, l'appendice est très réduit , dépourvu de prolongement intérieurau premier article et il présente un nombre moins grand de soies plus courtes.

Lèvre supérieure de l'ouverture génitale mâle (fig. 21) garnie d'une grosse épine denticulée à l'intérieur et d'une soie très fine à l'extérieur.

Ouvertures génitales femelles assez rapprochées l'une de l'autre sur la face ventrale (fig. 23). Elles sont réunies par une rigole transversale, où s'attache le sac ovigère unique. Immédiatement en arrière de cette rigole se trouve le pore de fécondation unique débouchant dans une cavité sphérique assez vaste d'où partent les canalicules séminifères.

Les différences qui existent dans les descriptions de Claus, BradY et CAR entre le nombre des articles de l'antennule, le nombre et la position des soies dans d'autres appendices ont été expliquées par des variations du type. Certaines d'entre elles devront probablement être attribuées à des erreurs d'observation ou à des confusions entre deux espèces voisines. Laophonte similis est, malgré tout, une forme bien caractérisée qui vit à Wimereux dans la région saumâtre de l'estuaire, avec Corophium longicorne, les Tachidius, les Cyclops, Cytheridea torosa,... etc. Je ne l'ai pas recueillie dans la mer, mais elle est signalée par BRADY dans la zone littorale en mème temps que dans les eaux saumâtres.

Distribution: Mer du Nord, Manche, Océan Atlantique, Méditerranée. 


\section{NANNOPIN E.}

Pièces buccales masticatrices; pattes de la première paire préhensiles par l'endopodite, et réduites dans les trois paires suivantes spécialement en ce qui concerne l'endopodite qui devient un simple moignon sétifère dans une ou plusieurs d'entre elles. Habitudes limicoles (1).

\section{Nannopus, Brady.}

1878. Nannopus; BradY; 20, vol, 2, pages 100 et 101.

Corps rigide, légèrement et régulièrement atténué vers l'arrière; exopodite des 4 pattes natatoires 3-articulé; endopodite des 3 premières paires 3-articulé; endopodite de la quatrième paire simple et réduit.

\section{Nannopus palustris, BRADY.}

1878. Nannopus palustris; Brady; 20, vol. 2, pages 101 et 102 ; Pr. LXXVIl, fig. 18-20.

1889. Nannopus palustris, Brady; I. C. THOMPson; 199 , page 188.

\section{Planche IV, Fig. 6-2I.}

Taille : $q, 0,87^{\mathrm{m}} / \mathrm{m} ; \sigma$, inconnue.

Corps (fig. 6 et 7) tout différent de Tachidius (2).

Céphalothorax plus long que l'abdomen; carapace épaisse et peu transparente, salie d'une foule de corps étrangers dans l'animal vivant. Bords des segments garnis d'une rangée continue de dents triangulaires et pointues; bords de la dépression anale également denticulés ( fig. 20). Pièces furcales plus courtes que le dernier segment abdominal, portées sur une saillie latéro-postérieure de ce segment et munies d'un nombre restreint de soies épineuses; soie furcale terminale interne plus grande, renflée à sa base et égale en longueur aux 3 derniers segments abdominaux réunis.

Appendices relativement peu développés (fig. 7).

Antennules plus courtes que le segment céphalique, composées de cinq articles (fig. 8) dont les deux premiers sont plus longs et le quatrième plus court. Au bord antérieur, les $2^{\mathrm{e}}, 3^{\mathrm{e}}, 4^{\mathrm{e}}$ et $5^{\mathrm{e}}$ articles portent de fortes épines recourbées, arrondies à leur extrémité, avec quelques soies flexibles, lisses ou barbelées. Les $3^{\circ}$ et $5^{\circ}$ articles portent chacun un filament sensoriel à leur extrémité distale.

(1) Voir page 100.

(2) "Like Tachidius in general appearance ", dit BradY, qui a donné (loc. cit., PI. LXXVII, fig. 18) un mauvais dessin de l'animal aplati par compression. 
Antenne biramée ( fig. 9); l'exopodite simple, garni de 3 soies grêles et d'une soie renflée et barbelée, est inséré vers le milieu de l'article basilaire cylindrique. Après ce dernier, vient l'endopodite également simple, en forme de lame élargie vers l'extrémité et portant sur le bord 4 grosses épines recourbées et 2 soies plus fines. La face externe de l'endopodite porte plusieurs séries de denticules triangulaires.

Mandibule (fig. 10) simple, avec la base masticatrice solide et élargie, avec l'extrémité simple, irrégulièrement lobée et armée de 4 soies courtes et barbelées.

Première maxille ( fig. 11) avec coxopodite masticateur pourvu de soies barbelées à la face interne et terminé par 4 épines solides et pointues. Région terminale 2-articulée et 3-lobée, ressemblant beaucoup à celle de Laophonte similis, sauf dans le développernent des soies.

Seconde maxille externe (fig. 13) très réduite, 2-articulée (1) avec 3 saillies internes garnies d'épines.

Seconde maxille interne (fig. 14) 3-articulée, ciliée sur les bords, formant une pince courte et peu solide $(\mathbf{2})$.

Pattes natatoires larges et trapues, hérissées d'énormes denticules triangulaires et armées d'épines et de soies épineuses rigides (fig. 15, 16, 17 et 18). Leur base est toujours 2-articulée (3) et leur exopodite toujours 3-articulé. L'endopodite est 2-articulé dans les 3 premières paires et réduit dans la quatrième à un simple moignon peu saillant, porteur d'une soie très longue et d'une autre plus petite (fig. 18) (4).

Cinquièmes pattes 2-articulées (fig. 19). Article basilaire très large, atteignant à peu près le milieu du corps, avec quatre épines denticulées sur la portion intérieure et une soie longue et raide vers l'extérieur.

Second article réduit et foliacé, avec une grosse épine barbelée intérieure, deux soies médianes longues et fines, et deux courtes épines extérieures barbelées.

Ouvertures femelles assez rapprochées l'une de l'autre sur la face ventrale: leur lèvre supérieure porte une soie grêle assez longue; les deux canalicules séminifères débouchent dans une dépression (pore génital) simple, omise dans la figure 21 de la Planche IV.

Cette curieuse espèce est peu commune dans le sable vaseux qui forme le fond de l'estuaire de Wimereux, dans les endroits où poussent abondamment les algues vertes (Enteromorpha intestinalis) et où vivent Palcemonetes varians, Gobius minutus, Mysis vulgaris, ....etc.

Distribution : Côtes de la Mer du Nord, de la Manche et de l'Atlantique.

(1) L'article terminal est en effet incomplètement distinct de l'article médian porteur de la grosse épine.

(2) Cet appendice n'a pas été vu par Brady, qui rappelle que dans Jurinia Claus il est réfuit à quelques soies seulement.

(3) Dans la première paire, le basipodite porte à son bord interne une forte épine non dessinée par BraDY (loc. cit., fig. 19).

(4) C'est évidemment par erreur que BRADY attribue cette organisation aux troisième et quatrième paires de pattes : "In place of the inner branch of the third and fourth pairs is a small tubercle...... n (loc. cil... page 101). 


\section{A L A N I D $Æ$.}

Copépodes monoporodelphes ayant :

$1^{0}$ Tous les caractères d'adaptation à la vie pélagique.

$2^{0}$ Le céphalothorax beaucoup plus développé que l’abdomen, ce dernier armé de longues soies furcales barbelées pour la natation.

$3^{\circ}$ Les antennules très allongées comprenant jusqu’à 23 et 25 articles, symétriques dans les deux sexes ou préhensiles chez le mâle d'un seul côté.

$4^{0}$ Les antennes biramées, bien développées et garnies d'un grand nombre de soies natatoires barbelées.

$5^{0}$ Les mandibules masticatrices avec l'extrémité natatoire.

$6^{0}$ Les premières maxilles généralement pourvues d'un très grand nombre de soies, même sur l'exopodite.

$7^{0}$ Les secondes maxilles internes dépourvues d'épine préhensile et garnies de soies barbelées natatoires.

$8^{0}$ Les appendices thoraciques très développés et natatoires, préhensiles seulement dans la cinquième paire chez le mâle.

$9^{\circ}$ Un cœur avec trois valvules, prolongé parfois d'une artère antérieure courte.

$10^{\circ}$ Les oviductes pairs et symétriques, tandis que le canal déférent est impair et développé seulement à gauche.

II $^{0}$ Les orifices externes des oviductes pairs, mais très rapprochés l'un de l'autre sur la face ventrale, d'où résulte la disposition des œufs pondus en un seul saç, quand ils ne sont pas abandonnés dans l'eau.

Les Calanidés, ainsi délimités, correspondent aux deux familles des Calanida et Pontellide établies par Claus $(\mathbf{4 9}, \mathbf{5 1})$; ils comprennent les deux sous-familles des Calanina et Pontelline de Dana d'une part, de $\operatorname{Boeck}(\mathbf{1 5}, \mathbf{1}$ r) et Brady (20) d'autre part.

Tout en conservant cette dernière classification, j'avais dès 1890 (33, page 470) réclamé la subdivision en sous-familles distinctes du groupement hétérogène et très peu naturel des Calanida, subdivision d'ailleurs tentée déjà par GiEsBRECHT (89, page I63) pour une petite série de formes très voisines les unes des autres qu'il sépara comme sous-famille des Euchcetinx. Les riches collections rapportées par les officiers de la marine italienne ont fourni à GIESBRECHT la plus belle série de Calanidés qui ait été étudiée jusqu’à ce jour (91) et mis à la disposition de cet 
observateur les matériaux nécessaires pour établir sur des bases suffisantes une réforme aussi importante et difficile. Dans l'énumération du petit nombre de Calanidés que j'ai recueillis dans le Boulonnais, j'éviterai toute préoccupation taxonomique, laissant ainsi à un zoologiste plus autorisé le soin d'établir des changements qu'avec lui je regarde comme indispensables (I).

\section{Paracalanus, BoEck.}

1863. Calanus, Leach (pars); Claus; 51, page 172.

1864. Paracalanus; Вовск; 15, page 232.

188\%. Paracalanus, Boeck; Claus; 60, page 14.

\section{Paracalamus parvus, Claus.}

1863. Calanus parvus; Claus ; loc. cit., page 173, Taf. XXVI, fig. 10-14, Taf. XXVII, fig. 1-4 ;

1864. Paracalanus parvus. Claus; BoEck, loc. cit.

\section{Planche I, Fig. I-I 2 .}

Cette espèce est très abondante dans la Manche septentrionale durant toute la saison chaude; on peut l'y recueillir dans les pêches littorales aussi bien qu'au large.

Elle est facile à reconnaître à la soudure du céphalon et du premier somite thoracique, à l'union des quatrième et cinquième somites thoraciques ; dans lesexe femelle, aux pattes de la cinquième paire (Pl. I, fig. 6) 2-articulées et terminées par une longue épine vers l'intérieur et une petite vers l'extérieur; dans le sexe mâle, aux pattes asymétriques de la cinquième paire (fig. II) déjà caractérisées ainsi avant la dernière mue (fig. 5) et aux antennules renflées à la base mais non géniculées (fig. I).

Dans cette espèce, le mâle adulte, après la dernière mue, se distingue de la femelle en particulier par l'écartement en $V$ des pièces furcales (fig. I), par le développement considérable des trois soies externes, richement barbelées, des deux derniers articles de la seconde maxille interne et par la fusion incomplète des troisième, quatrième, cinquième et sixième articles de cette mème maxille (fig. 7).

Distribution : Mer du Nord, Baltique, Manche, Océan Atlantique, Méditerranée, Pacifique, Mer de Chine.

(1) M. le $\mathrm{D}^{\mathrm{r}}$ GiesBrecht a bien voulu me faire connaitre que la classification des Copépodes pélagiques recueillis à bord du "Vettor Pisani " comprendrait une sous-famille nouvelle, dont je lui avais signalé quelques particularités d'après l'étude de deux formes citées plus loin. 


\section{Calamus, LEACH.}

1819. Calanus; LeACH ; 131.

1834. Celochilus; Roussel de VAuzÈMe; 179, page 330.

1863. Cetochilus, R. de Vauz.; Chaus; 51, page 169.

\section{Calamus finmarcbicus, Gunner.}

1765. Monoculus finmarchicus; GUNNER; 102.

1843. Cetochilus septentrionalis; Goodsir; 94.

1863. Cetochilus helgolandicus; Claus; 51, page 171, Taf. XXVI, fig. 2-11.

\section{Planche I, Fig. I3-I9.}

Le plus grand Copépode recueilli dans le détroit du Pas-de-Calais. Il est cantonné au large, à plusieurs milles des côtes. Sa taille, atteignant jusqu'à $4 \mathrm{~m} / \mathrm{m}$ chez la femelle, son aspect général et les caractères des cinquièmes pattes thoraciques, finement denticulées sur le bord interne concave du coxopodite, le rendent facilement reconnaissable $(\mathbf{9 z})$.

Le mâle (Pl. I, fig. 13) se distingue de la femelle (Pl. I, fig. 14) par les antennules, par les cinquièmes pattes thoraciques, par l'écartement des pièces furcales disposées en V (fig. I5), par les trois grandes soies externes barbelées des deux derniers articles de la seconde maxille interne (fig. 16).

Distribution: Mer du Nord, Baltique, Manche, Océan Atlantique, Méditerranée (3ry), Océan Pacifique, Mer de Chine, etc.

\section{Clausia, BOEck.}

1864. Clausia; BoEck; 15, page 233.

1872. Pseudocalanus; BoECK; 17, page 37.

1878. Pseudocalanus, Boeck; Brady; 20, vol. 2, page 44.

1881. Clatusia, Boeck; Claus ; 60, page $16(1)$.

\section{Clausia elongata, BOEcK.}

1864. Clausia elongata; BoEck; 15, page 234.

1872. Pseudocalanus elongatus; BoEcK; 17, page 38.

1881. Clausia elongata, Boeck; Claus; 60, page 16; Taf. III, fig. 11-15.

1882. Lucullus acuspes; Giesbrecht ; 89, pages I60-I67; Taf. II, III, V-XI.

(1) Clausia fut employé d'abord par ClAPARÈDE pour un Copépode parasite décrit d'une manière tellement insuffisante que Claus lui-même croit la description inutilisable et incompréhensible, et qu'il la considère comme destinée à disparaître de la littérature zoologique (60, page 13). 
Espèce commune durant les premiers mois de l'année, plus rare en été.

Elle se reconnait facilement, entre autres caractères, aux antennules et aux cinquièmes pattes thoraciques du mâle (Pl. II, fig, 3), ainsi qu'aux épines délicatement denticulées qui terminent les pattes natatoires.

J'ai signalé précédemment les intéressantes particularités du dimorphisme sexuel de cette formé (page i i2).

A la face interne de la seconde maxille externe du mâle adulte, on retrouve encore, à la base des groupes de petites soies (P1. II, fig. I3), un rudiment de toutes les saillies digitiformes qui supportent dans la femelle et le jeune mâle (PI. II, fig. 12) les longues soies épineuses. Cette disposition est signalée déjà par Claus chez Undina et Euchceta (51, page 30), mais incomplètement décrite et figurée par Giesbrecht (89, page 162, Taf. IX, fig. 18).

Distribution: Mer du Nord, Baltique, Manche, Océan Atlantique, Méditerranée.

\section{Centropages, KR $\phi$ Yer.}

1849. Centropages; KRoYer; 123, page 602.

1853. Ichthyophorba; LILLjEBoRG; 136, page 184 .

1863. Ichthyophorba, Lillj. ; Cuaus ; 51, page 198.

1864. Centropages, Krọyer; Boeck ; 15, page 242.

\section{Centropages bamatus, LILLJEBorg.}

1853. Ichthyophorba hamata; LiLLJEBORG; 136 ; page 18 ; ; Tab. XXI et XXVI.

1863. Ichthyophorba angustata; Claus ; 51, page 199; Taf. XXV.

1864. Centropages hamatus, Lillj.; BoEck; 15, page 244.

L'une des espèces les plus communes en toutes saisons, et particulièrement au printemps. Se distingue aisément par la cinquième patte thoracique biramée dans les deux sexes, préhensile à droite chez le mâle où l'exopodite se termine par une pince à branches assez courtes et effilées; - par l'antennule dépourvue de fortes épines saillantes au bord antérieur des articles dans la région proximale.

Distribution : Mer du Nord, Baltique, Océan Atlantique, Méditerranée.

\section{Centropages typicus, Kropyer.}

1849. Centropages typicus; KR SYer ; 123, page 588; Tab. VI, fig. 22-26.

1863. Ichthyophorba denticornis; Claus; 51, page 199; Taf. XXV, fig. I et 3-9.

1864. Centrobages denticornis, Kroyer; BoEck; 15, page 243. 
Espèce beaucoup plus rare que la précédente, recueillie seulement en été, ạu large. Se distingue par l'épaisseur des deux bras de la pince terminant l'exopodite de la cinquième patte droite chez le mâle et celle de l'épine médiane interne des exopodites de la cinquième paire de pattes chez la femelle, ainsi que par les denticules triangulaires et légèrement recourbés des articles de la région basilaire de l'antennule (28, page 96, Pl. VIII, fig. I et 2; 33, page 324, Pl. XXIII, fig. I).

Distribution : Mer du Nord, Manche, Océan Atlantique, Méditerranée.

\section{Isias, BOECK.}

1864. Isias; BOECK ; 15, page 241.

1878. Isias, Boeck; Brady; 20, vol. I, page 62.

1888. Isias, Boeck; CanU ; 28, page 90.

\section{Isias clavipes, BOECK.}

1864. Isias clavipes; BoEck, 15, page 242.

1878. Isias clavipes, Boeck; BRADY; 20. vol. 1, page 62 ; PI. VII, fig. 3-13.

1888. Isias Bonnieri; CANU ; 29 , page 228 ; PI. XVI-XVIII.

1890. Isias clavipes, Boeck; Canu; 33, page 324.

Espèce très abondante dans les pêches au filet fin exécutées à peu de distance de la còte, depuis mai jusqu'en aoùt. Je ne reviendrai pas sur les descriptions détaillées que j'ai publiées de cette espèce, ni sur la synonymie établie ci-dessus.

Distribution : Mer du Nord, Manche, Océan Atlantique, Méditerranée.

\section{Temora, BAIRD.}

1850. Temora; BaIRD; 9, page 227.

1863. Temora, Baird; Claus; 51, page 197

1881. Temora, Baird; Claus; 59 , pages 482 et suivantes.

1881. Eurytemora; GiesbreCht ; 88 et 89 , pages 149,154 et 168.

1853. Non Temora, Baird; LILLJEBorg; 136.

\section{Temora longicornis, O.-F. Mueller.}

1785. Cyclops longicornis; O.-F. Mueller; 152, pages 115 et 116 ; Tab. XIX, fig. 7-9.

1850. Temora finmarchica, Gunner; BaIRD; 9 , page 228; Tab. XXVIII.

1863. Temora finmarchica, Gunner; Claus; 51, page 195; Taf. XXXIV.

1864. Temora longicornis, Müller; BoECK; 15, page 239 
Forme très nette et très abondante durant la plus grande partie de l'année, aussi bien au large qu'auprès de la côte.

Distribution : Mer du Nord, Baltique, Adriatique (59).

\section{Temorella, Claus.}

1853. Temora, Baird; Lilljeborg; 136, page 177.

1876. Temora, Baird (pars); Høk ; 111.

1878. Temora, Baird (pars); BradY; 20. vol, 1, page 53.

1881. Temorella; Claus; 59 , pages 82 et suivantes,

1881. Eurytemora; GIESBRECHT; 88 et 89, pages 152, 154 et 168.

\section{Temorella affinis, PoPPE.}

1853. Temora velox of; Lilljeborg; 136, page 177 ; Taf. XX, fig. I.

1880. Temora affinis; PopPE; 161, Taf. III.

1881. Temorclla affinis, POPPE; Claus; 59, pages 282 et suivantes; Taf. II, fig. 8-I4.

La forme type de cette espèce vit à l'embouchure de la Seine où elle a été recueillie en abondance par GADEAU DE KeRville (I). La comparaison des échantillons que m'a communiqués ce naturaliste avec les exemplaires de Temorella affinis recueillis par M. le $\mathrm{D}^{\mathrm{r}} \mathrm{H}$.-E. SAUVAGE dans l'estomac de Harengs pèchés dans le port du Havre, m'a convaincu de l'existence de deux variétés distinctes à l'estuaire de la Seine :

$I^{0}$ Dans l'embouchure, exposée aux courants de marée et aux variations de milieu qu'ils entraînent, vit la variété type également observée en mème situation dans les fleuves de l'Allemagne du Nord. Elle se distingue : par sa taille moindre ( $\mathrm{r}, 45 \mathrm{~m} / \mathrm{m}$ de long), par l'allongement moins considérable de la cinquième paire de pattes chez le mâle, et par l'absence de poils courts sur les prolongements aliformes du cinquième somite thoracique.

$2^{0}$ Dans le bassin de l'Eure, abritée contre ces courants, existe une autre variété déjà distinguée par NoRDevist (156, page 53; Taf. V, fig. I, 6, 7, IO; Tafel VI, fig. 4 et 5) sous la dénomination de $T$. affinis, var. hispida, et présentant les caractères cités plus haut comme manquant au type de l'espèce. La variété hispida atteint jusqu'à $\mathrm{I}, 60\left(\sigma^{\prime}\right)$ et I, $87\left(\begin{array}{l}( \\ )\end{array}\right)$ millimètre de longueur, tandis qu'à Ảbo, les exemplaires recueillis par Nordevist ne mesuraient que $1,2\left(\sigma^{\prime}\right)$ à $\mathrm{I}, 38$ (q) millimètres.

(1) La Faune de l'estuaire de la Seine. Extrait de l'Amuaire normand, 1886. 


\section{Acartia, Dana.}

1846. Acartia; Dana.

1852. Acartia; Dana; 73, page 1118.

1853. Dias; Lilljeborg; 136 , page 181

Comme l'ont récemment pensé Brady (21, page 72) et Giesbrecht (91), le genre Liartia est suffisamment caractérisé par DANA pour comprendre le genre Dias plus récemment établi par LILLjEBORG.

Les antennules d'Acartia [Antennæ primæ longæ, rectiusculæ, flexiles, setis quaquaversum insistentibus ornatæ, dextrà maris non geniculante (?) ] correspondent exactement à celles de Dias, et les figures reproduites par DaNA (PI. 79, fig. $2,3,4$ et 5) ne laissent subsistẹr aucun doute sur la synonymie véritable des deux appellations génériques, que vient encore confirmer l'analogie indiquée dans les secondes maxilles d'Acartia et de Pontella (Maxillipedes et pedes antici ferè ac in Pontella).

\section{Acartia clausi, Giesbrecht.}

1863. Dias longiremis, Lillj. (pars); Chaus; 51, page 193.

1866. Dias longiremis, Lillj. ; Claus; 53, page 13, fig. 12.

1889. Acartia clausii; Giesbrecht ; 91.

1890. Acartia clausii, Giesbrecht; Canu ; 33 , page 326 ; P1. XXIV.

Cette espèce est abondante d'avril à septembre dans la zone littorale, à la surface de la mer; elle n'existe pourtant jamais en troupes considérables, comme il arrive pour bien d'autres Calanidés. Sa présence à Nice $(\mathbf{5 3}, \mathbf{9 1})$ pourrait laisser supposer que c'est l'espèce décrite par KRITCHAGuine comme D. Yongiremis, var. spinifer (121, pages 413 et 414, Taf. X, fig. 7-10).

Distribution : Mer du Nord, Manche, Océan Atlantique, Méditerranée.

\section{Acartia discaudata, Giesbrecht.}

188 $\mathrm{t}$. Dias discaudatus; GIESBRECht; 89, pages 148 et 149 ; Taf. III, V, VI, VIII, IX.

1890. Acartia diseaudata, Giesbrecht; Canu; 33, pages 326 et 327; PI. XXIII, fig. 2-6.

Espèce très commune du printemps à l'automne, auprès de la côte, en compagnie de la précédente. Elle se distingue très facilement, dans le sexe femelle, à la taille du spermatophore qu'on trouve fréquemment fixé à l'orifice sexuel et à l'élargissement considérable du dernier segment abdominal et des pièces furcales. ainsi qu'au développement des soies furcales. 
Dès I 873, KRITCHaGuine a décrit et figuré une espèce qui me semble identique (121, pages 4I5-4I9, Taf. X, fig. 2I-22). Par la longueur du spermatophore atteignant l'extrémité de l'abdomen (loc. cit., fig. 22), par l'élargissement des pièces furcales et le renflement basilaire des soies furcales, Dias latisetosus KriTCHAGUine semble bien se confondre avec $A$. discaudata (1). Malgré ces raisons, j'ai conservé le nom donné par Giesbrecht; mais s'il est confirmé par des recherches ultérieures que $A$. discaudata GsB. vit réellement près des rives orientales de la Mer Noire, c'est la désignation spécifique imposée par KRITCHAGUINE qu'il faudra employer, en vertu des droits de la priorité. Cette forme s'appellerait dès lors Acartia latisetosa KRITCHAGUINE.

Distribution : Mer du Nord, Baltique, Manche, Océan Atlantique, Mer Noire (?).

\section{Parapontella, Brady.}

1857. Pontella, Dana; Lubsock; 142.

1878. Parapontella; Brady; 20, vol. 1, page 68.

1890. Parapontella, Brady; Canu; 33, page 320 .

\section{Parapontella brevicornis, LuBBock.}

1857. Pontella breoicornis; LubBock; 142.

1878. Parapontella brevicornis, Lubbock; Brady ; 20, vol. I, pages 69-7I; PI. IX, fig. I-16.

1890. Parapontella brevicornis, Lubbock; Canu; 33, pages 329 et 330; P1. XXV.

Espèce recueillie parfois en abondance dans les pèches au filet fin effectuées en été à la surface, à plusieurs milles du rivage le long des côtes du Boulonnais. Elle était particulièrement fréquente dans le détroit du Pas-de-Calais, entre les bancs du Varne et du Colbart, en août i8go.

Distribution : Mer du Nord, Manche, Océan Atlantique.

\section{Labidocera, Luввоск.}

1853. Labidocera; LuBbock; 140.

1863. Pontella, Dana; Claus; 51, page 207.

(1) D'ailleurs, l'auteur insisle suffisamment sur ce caractère pour lever tous les doutes: " La particularité la plus saillante de cette espèce se voit dans l'arrangement des soies de la furca. Celle-ci contient une petite articulation dans chaque branche; les trois soies marginales ont une conformation normale et se trouvent sur le côté de chaque article. La quatrième soie est extraordinairement élargie, et, dans quelques individus, presque aussi large que la branche elle-mème ». 
1878. Pontella, Dana; Braur; 20, vol. 1, page 72.

1888. Pontella, Dana; Canu; 28, page 99.

1880. Labidocera, Lubbock; GiesBrecht; 89.

1890. Labidocera, Lubbock; Canu; 33, page 330.

\section{Labidocera wollastoni, LuBBock.}

1859. Pontella Wollastoni; Luввоск.

1863. Pontilla helgolandica; Claus; 51, page 208; Taf. XXVI, fig. 1-10, et Taf. XXXVII, fig. 7 .

1878. Pontella Wollastoni, Lubbock; Brady; 20, vol. 1, page 73; PI. X A.

1888. Pontella Wollastoni, Lubbock; Canu; 28, pages 100 et 101 ; Pl. VIII, fig. 4-6.

1889. Labidocera Wollastoni Lubbock; GIESBRECHT; 89.

1890. Labidocera Wollastoni, Lubbock; Canu; 33, page 330.

Espèce de grande taille, parfaitement hyaline. Abondante au large durant l'été depuis 1 886 jusqu'en 1889; rare en 189o. Comme tous les auteurs l'ont observé, cette forme apparait ainsi en grandes troupes, par intermittences.

La transparence de Labidocera wollastoni facilite l'étude de son appareil visuel; à la face dorsale du céphalon, on voit (Fig. 13 ci-contre) deux taches pigmentaires

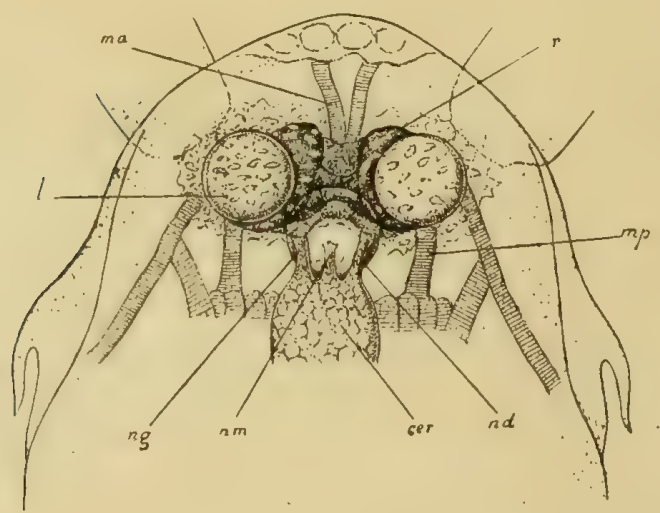

Fig. 13. - Région antérieure du céphalon de Labidocera wollastoni Luввоск, vue par la face dorsale, avec l'œil tripartite.

$c e r$, cerveau ; $n m$, nerf oculaire median; $n g$, nerf oculaire gauche ; $n d$, nerf oculaire droit ; $m p$, muscle postérieur de l'œil ; ma muscle antérieur; $l$, lentilles cornéennes réfringentes ; $\%$, masse pigmentaire. supportant deux lentilles sphériques réfringentes, plus grandes dans le mâle (Pl. II, fig. 14) que dans la femelle (Pl. II, fig, 15). Au-dessous de ces corps, vers la face ventrale et sur la ligne médiane, se trouve un troisième amas pigmentaire au-delà duquel fait saillie vers l'extérieur un renflement cuticulaire réfringent placé entre la lèvre supérieure et le prolongement frontal. Ces trois systèmes (ou ommatidies) semblent diverger d'un point central, et ils arrivent presque à se toucher au sein des tissus; dans d'autres formes voisines, ils sont très intimement accolés l'un à l'autre.

La séparation des trois parties de l'œil a entrainé l'écartement des filets nerveux les unissant au cerveau. On voit, en effet, partir du ganglion supra-œesophagien, à son extrémité antérieure, trois nerfs ( $\mathrm{mm}, \mathrm{nd}, \mathrm{ng}$ dans la Fig. 13) qui viennent se perdre dans les masses pigmentaires sur leur bord postéro-dorsal, un 
peu en arrière du point d'appui des lentilles réfringentes (Voir $n$, dans la Fig. I4,

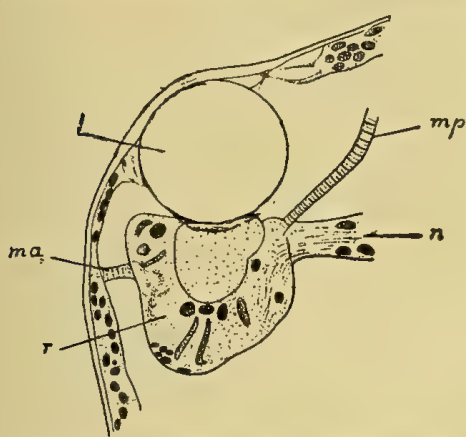

Fig. 14. - Coupe d'une partie de l'œil, chez Pontella, d'après G. H. PARker : The Gompound Eyes in Crustaceans : Bull. Mus. Comp. Zool. Harvard College, Vol. XXI, № 2, Pl. II, Fig. 18.

$l$, lentille cornéenne; $r$, masse pigmentaire; $n$, le nerf oculaire correspondant; $m a$, muscle antérieur ; $m p$, muscle postérieur. ci-contre).

Beaucoup de naturalistes ont tenté une interprétation tout à fait erronée en prétendant retrouver dans cette disposition lac impair et les yeux composés qui se montrent ainsi disposés à l'état adulte dans un grand nombre de Crustacés supérieurs, où ils existent d'ailleurs concurremment au cours des métamorphoses embryonnaires (I).

Il est curieux de remarquer que Claus accepte, à propos des Peltidiens (65, pages 4 et 5) la seule interprétation convenable: disjonction des trois parties constitutives de l'œil impair; mais il en propose une autre pour les Pontelliens (62, pages 42 et 43) considérés seuls : Dans ces derniers,

la partie oculaire antérieure et médiane formerait seule l'œil impair nauplien; les

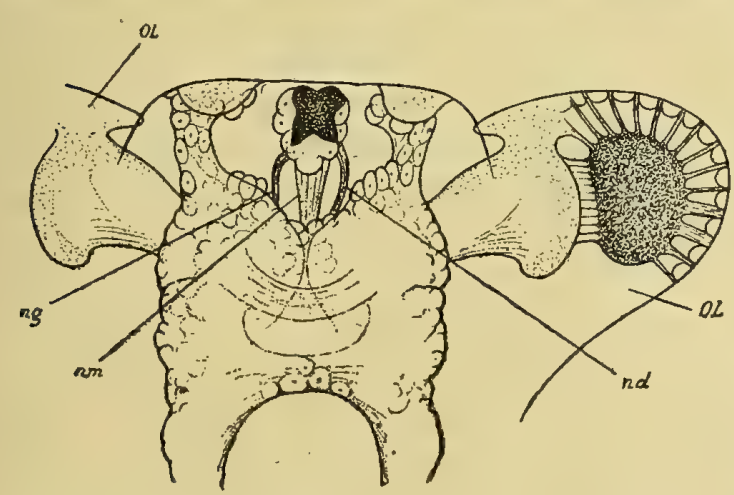

Fig. 15. - Région antérieure du céphalon, chez Branchipus, d'après Claus : loc. cit., Taf. V, Fig. 3

$O L$, yeux latéraux composés; $n m, n g, n d$, les trois nerfs oculaires médian, droit et gauche qui se rendent du cerveau aux trois parties correspondantes de l'œil impair. deux parties latéro-dorsales, avec lentilles réfringentes à la face dorsale du céphalon, seraient les restes, conser¿és par atavisme dans ces seuls Copépodes, des yeux latéraux composés.

Cette opinion ne pourrait êtreacceptée(2). Le nombre, 3 , des filets nerveux qui se rendent du cerveau aux trois ommatidies disjointes, n'est pas même un argument en sa faveur, puisque ces trois nerfs de l'œil médian se

(1) J'ai observé chez l'adulte, dans divers Carides (Palcmon, Palcemonetes, Crangon....), des taches pigmentaires médianes situées à la base du rostre et reliées au cerveau par un nerf en régression. Elles correspondent, à n'en pas douter, à l'œil impair des larves de ces Crustacés. Dans ces animaux, l'œil impair, nauplien, est donc reconnaissable durant toute la vie. - Voir à ce sujet: RoBiwson, on the Nauplius eye persisting...., Quat. Journ. of Micr. sc., vol. XXXIII, pages 283-287, Plate XIV.

(2) Seule, l'embryologie est capable de résoudre cette question d'une manière positive. En effet, les nouvelles 
retrouvent auprès des yeux composés chez Branchipus (voir la fig. Is ci-contre), ainsi que Claus l'a montré lui-même.

Dans les Pontelliens, ainsi que dans tous les autres Copépodes, il n'existe aucune trace de ces yeux composés si répandus dans tous les autres groupes de Crustacés. L'anatomie de cet organe, comparée dans les Peltidiens et les Calanides, le démontre sans aucun doute.

Distribution : Mer du Nord, Manche, Océan Atlantique.

\section{Pontella, Dana.}

1852. Pontella; Dana; 73, page 1131 .

1863. Pontellina, Dana; Claus; 51, pase 210.

1883. Pontella, Dana (pars); Brady; 21, page 87.

1888. Pontellina, Dana; CANu ; 28 , page 101.

\section{Pontella lobiancoi, Giesbrecht.}

1888. Pontellina Lobiancoi, Giesbrecht; Canu ; 28 , pages 101 à $105 ; \mathrm{Pl}$. VIll, fig. 7 et 8 ; Pl. IX, fig. $1-8$.

1889. Pontella Lobiancoi, Gsb. (Canu); Giesbrecht ; 89.

Espèce recueillie en petit nombre pendant le mois d'août I 887 , au large de Boulogne. Elle n'a pas été retrouvée depuis lors dans la même localité.

Distribution: Manche, Océan Atlantique (Gibraltar), Méditerranée (Naples).

\section{Anomalocera, Templeton.}

837. Anomalocera; Templeton; Trans, of Ent. Soc., vol. II, part. I.

1843. Irenotus; GoODSIR; 94.

1849. Pontia, Milne-Edwards (pars); KroYer; 123, pages 501 et 599.

1850. Anomalocera, Templ.; Bairo; 9, page 229.

1863. Irencus, Goodsir; Claus ; 51, page 204.

1878. Anomaloceva, 7 empl.; Brady; 20, vol. 1, page 74.

observations anatomiques de Cuus ne me semblent pas démonstratives (70; pages 23, 24 et 25). Du fait que, dans les Pontelliens, une complication secondaire, affectant simultanément les trois parties de l'œil, donne a ces éléments une apparence multilobée, 3-lobée dans l'inférieur, 4-lobée (Pontellina Cuus) ou 3-lobée (Anomalocera) dans les deux supérieurs, il ne résulte nullement que l'ocelle ventral soit seul homologue de l'œil tripartite des autres Copépodes. On peut en conclure seulement que les trois éléments disjoints de l'œil tripartite ont subi, par une adaption commune, des modifications spéciales aux Pontelliens, modifications toutes différentes de celles qui atteignent l'œil des Coryceides, mais qui n'infirment en rien leur nature d'ocelles composants. 


\section{Anomalocera patersoni, Templeton.}

1837. Anomalocera Patersoni; Templeton; loc. cit.

1843. Irenceus splendidus; GoodsIR; 94.

1849. Pontia Patersoni, Templ.; KRJjYER ; 123. pages 56 t-572.

1850. Anomalocera Patersoni, Templ.; BaIRD; 9, pages 229-232 ; Tab. XXVII, fig. 1 a-l, $2 a-c$.

1863. Irenceus Patersoni, Templ. ; Claus ; 51, pages 206-207; Taf. 11, fig. I; Taf. XXXVII, fig. 1-6.

1878. Anomalocera Patersoni, Templ.; Brady; 20, vol. 1, pages 75-78; Pl. X, fig. 13 et 14 ; Pl. XI, fig. $1-14$.

1889. Anomalocera Patersoni, Templ. ; Giesbrecht ; 89.

La parité des lentilles réfringentes dans chacune des parties latéro-dorsales de l'œil et le grand développement de la partie médiane ventrale, font facilement reconnaître ce Copépode richement coloré, bien caractérisé par d'autres particularités des appendices.

Rare. Recueilli en petit nombre, durant le mois d'août I89o, au milieu du détroit du Pas-de-Calais.

Distribution : Mer du Nord, Manche, Océan Atlantique, Méditerranée.

\section{CY CLOPID}

Copépodes monoporodelphes ayant :

$\mathbf{I}^{0}$ Divers caractères d'adaptation à la vie pélagique.

$2^{0}$ Le céphalothorax plus élargi que l'abdomen; celui-ci terminé par des soies furcales barbelées et ne servant pas spécialement d'organe natatoire.

$3^{0}$ Les antennules assez allongées dans certains cas, mais ne comprenant pas plus de vingt articles, et transformées toutes deux chez le mâle en organes préhensiles.

$4^{0}$ Les antennes simples, sans exopodite, et parfois préhensiles.

$5^{0}$ Les mandibules masticatrices, dont l'extrémité est tantôt bien développée et natatoire, tantôt réduite et impropre à la natation.

$6^{0}$ Les premières maxilles masticatrices, rendues natatoires par la présence de longues soies, barbelées et flexibles sur la région terminale, absentes ou peu nombreuses sur l'épipodite; ou bien les premières maxilles uniquement masticatrices par réduction de la région terminale.

$7^{0}$ Les secondes maxilles masticatrices et préhensiles, semblables dans les deux sexes.

$8^{\circ} \mathrm{La}$ cinquième paire de pattes thoraciques peu développée et semblable dans ies deux sexes.

$9^{0}$ Le tégument chitineux, mince et transparent, sans incrustation de calcaire.

IO ${ }^{0} \mathrm{La}$ circulation lacunaire. 
II ${ }^{0}$ Les conduits génitaux pairs ou symétriques avec orifices bien séparés sur les côtés du segment génital; deux sacs ovigères portés par la femelle après la ponte.

Ainsi délimitée, la famille des Cyclopida a été acceptée par tous les auteurs récents. La position dans ce groupe du genre Oithona — rangé par DANA parmi les Calanidés - est parfaitement justifiée par tous les détails d'organisation. comme l'a reconnu Claus.

\section{Oithona, BAIRD.}

1843. Oithona; BAIRD; Zoologist.

1849. Seribella, Dana; Proc. Amer. Acad. Sc., 11.

1852. Oithona, Baird; Dana, 73, page 1097.

1863. Oithona, Baird; Claus; 51, page 104.

Le corps est élancé, l’abdomen allongé et très grêle. La mandibule porte sur sa base masticatrice, une région terminale biramée, avec l'exopodite pluri-articulé et garni de soies flexibles, et l'endopodite simple armé d'épines solides et recourbées. La seconde maxille est allongée dans ses deux parties.

\section{Oitbona belgolandica, Claus.}

1863. Oithona helgolandica; CLaus; 51, page 105, Taf. XI, fig. 10-12.

1864. Oithona spinifrons; BoEck; 15, page 249.

1878. Oithona spinifrons, Boeck, BRADY; 20, vol. 1, pages 90 et 91, PI.XIV. fig. 1-9, PI. XXIV A, fig. I et 2.

1882. Oithona spinirostris, Claus; GIESBRECHT; 89, pages 139-144.

Assez commun dans les péches au filet fin faites auprès du - littoral et dans le voisinage des rochers de la côte.

Je désigne cette forme sous l'appellation spécifique de helgolandica Claus, à cause de la brièveté des antennules, de sa transparence parfaite, de sa taille inférieure à $\mathrm{I} \mathrm{m} / \mathrm{m}$, qui la séparent d'O. plumifera Dana (= spinirostris CLAuS) avec lequel les auteurs l'ont souvent confondue parsuite de l'insuffisance des descriptions actuelles. La richesse du matériel étudié par GIESBRECHT lui a permis de fixer plus exactement les relations spécifiques de ces étres $(\mathbf{9 1}, \mathbf{1 8 9 1}$, vol. VII, page 475).

Distribution : Mer du Nord, Baltique, Manche, Océan Atlantique.

$$
\text { Cyclopina, Claus. }
$$

1863. Cyclopina; Claus; 51, page 103.

1864. Cyclopina, Claus; BoEck, 15, page 247. 
1872. Cyclopina, Claus; BoEcr; 17 , page $4 \mathrm{I}$.

1878. Cyclopina, Claus; Brady; 20, page 91 .

1882. Cyclopina, Claus; Giesbrecht; 89, page 137.

Le corps est de forme lègèrement ramassée; le palpe mandibulaire est biramé avec l'endopodite et l'exopodite simples et garnis de soies flexibles.

\section{Cyclopina gracilis, Claus.}

1863. Cyclopina gracilis; Claus; 51, page 104, Taf. X, fig. 9-15.

1882. Cyclopina gracilis, Claus; GIESBRECHT, 89, pages 137-139.

Très abondant à la surface de l'eau et au milieu des algues, dans la zone littorale.

Distribution : Mer du Nord, Baltique, Manche, Méditerranée.

\section{Thorellia, BoEck.}

1864. Thorellia; ВOECK, 15, page 249.

1878. Thorellia, Boeck; BRADY; 20, vol. I, page 95.

Le corps cyclopoïde est de forme très ramassée, avec le céphalothorax renflé sur la face dorsale et l'abdomen épais et cylindrique. Les antennules de la femelle sont courtes et comptent 21 articles. L'extrémité mandibulaire est simple et réduite à un tubercule portant un petit nombre de soies rigides. Les deux paires de maxilles sont solides, courtes et préhensiles, la seconde maxille interne étant 4-articulée et terminée par de courts crochets recourbés.

\section{Thorellia brumea, BОЕСK.}

1864. Thorellia brunnea; BoECK; 15, page 250.

1878. Thorellia brunnea, Boeck; BraDY; 20, vol. 1, pages 95-97, Pl. XVI, fig. 1-10.

Cyclopide peu fréquent dans la zone des Laminaires parmi les Hermelles. Je n'ai recueilli qu'un petit nombre d'exemplaires femelles. L'œil n'y est pas rejeté latéralement comme le dit BrADY, qui attribue bien à tort cette opinion à BoECK. Les lentilles réfringentes sont bien écartées sur les côtés (I), mais l'œil n'est point déplacé vers l'un des cótés du corps (2).

Distribution: Mer du Nord, Manche, Océan Atlantique, Nouvelle Zélande.

(1) Comme le dit Вовск : "Lindserne i Oiet....... vaere rykkede ud til Siderne " (loc. ci., page 250).

(2) Ainsi que le prétend BradY: a ........ the eye is not placed in the middle line, but rather to one side........... (loc. cit., pages 96 et 97 ). 


\section{Cyclops, Mueller.}

1776. Cyclops; O-F. Mueller, Zool. Dan. Prodr.

1863. Cjolnps, Müller; Claus, 51, page 96.

Le corps est trapu et ramassé. Les antennules comptent moins de 20 articles (de 6 à 18). Les mandibules sont terminées par un très court tubercule garni de deux ou trois longues soies raides. Les premières maxilles sont courtes, masticatrices et préhensiles. Les secondes maxilles internes portent à̀ leur extrémité des soies rigides en forme d'épines effilées.

Le genre Cyclops fournit dans les eaux saumâtres du littoral un petit nombre d'espèces qui semblent cantonnées dans cet habitat spécial. J'ai recueilli deux espèces habitant exclusivement l'estuaire de Wimereux, où l'influence des marées entretient périodiquement un certain degré de salure par un appoint d'eau de mer. Jamais ces espèces n'ont été rapportées par les nombreuses pêches au filet fin que j'ai exécutées dans l'eau douce des ruisseaux et des mares voisines du vieux port et de la vieille rivière à Wimereux; pour cette raison, j'ai ajouté ces animaux à la liste des formes marines étudiées ici.

\section{Cyclops lubbocki, BRADY.}

1868. Cyclops Luhbockii; BradY.

1875. Cyclops odessanus; Schmankeviтsch, 189, pages 17,67 et 68, Pl. 1, fig. 3, 4 et 6.

1878. Cyclops insignis, Claus; Brady; 20, vol. 1, pages 108 et 109. PI. XXI, fig. I-9.

1880. Cyclops helgolandicus; RehberG, Zool. Anzeiger, Jahrg III, pages 302 et 30 .

1884. Cyclops odessanus, Schmank.; Re HBERG, Inaugural Dissertation Iena, page 3.

1891. Cyclops bicuspidatus, Claus ; Richard, 175, page 230.

Non: Cyclops insignis; Claus, 51, page 101.

$$
\text { PL. 1II, FIG. II-I8. PL. IV, FIG. I-5. }
$$

Taille : $\bar{f}, 1^{\mathrm{n}} / \mathrm{m} ; \sigma, 0,68 \mathrm{~m} / \mathrm{m}$.

Corps de forme élancée ; céphalothorax étroit et allongé, un peu plus grand que l'abdomen (fig. 1 et 2). Prolongement frontal saillant, arrondi à l'extrémité (fig. 3), orné d'écailles à la surface (fig. 16). Lèvre supérieure saillante, élargie dans sa partie postérieure, avec le milieu bordé d'une rangée de dents chitineuses peu pointues (fig. 16).

Autennules moins longues que le céphalothorax. Chez la $q$ (fig. 11), elles comptent 14 articles pourvus de longues soies; les articles 1,8 et 4 sont les plus longs, et le $3^{\mathrm{e}}$ le plus court; le $9^{e}$ article porte seul un poil sensoriel court. Chez le $\sigma$ (fig. 12), elles sont 15 -articulées, géniculées entre les $12^{\mathrm{e}}$ et $13^{\mathrm{e}}$ articles, renflées du $9^{\mathrm{e}}$ au $11^{\mathrm{e}}$, et contournées du $2^{\mathrm{e}}$ au $7^{\mathrm{t}}$.

Antennes (fig. 3) assez courtes, 4 -articulées, avec de longues soies terminales, une rangée de longs poils flexueux au bord interne du $3^{\text {te }}$ article et une longue soie barbelée au bord distal externe du premier.

Mandibules (fig. 4) armées à leur bord masticateur d'un nombre restreint d'épines denticulées fortement saillantes. 
Premières maxilles (fig, 13) courtes et solides, avec l'article basilaire masticateur portant une région terminale biramée en forme de palpe (fig. 14). Article basilaire pourvu : à l'extrémité distale, de quatre épines renflées et peu pointues; sur la face externe, vers le tiers distal. d'une forte épiné triangulaire; vers le milieu, d'une grosse soie légèrement barbelée; et dans l'intervalle, de quelques soies ou épines plus grêles.

Secondes maxilles (fig, 15) internes et externes 4 -articulées, avec des soies rigides et des épines garnies de harbules raides.

Cinquième patte thoracique (fig. 5) grêle, 2-articulée, avec l'article terminal étrost et terminé par une longue soie flexible extérieure et une épine délicate intérieure.

Pièces furcales longues et minces, atteignant à la taille des deux derniers segments abdominaux réunis, sans crête longitudinale visible à la face dorsale et avec une saillie denticulée au tiers proximal de son bord interne.

Eufs pondus par la $q$ en nombre relativement faible et mesurant $0 \mathrm{~m} / \mathrm{m} 06$ de diamètre.

Grâce à la transparence de certains exemplaires, j'ai pu comprendre la disposition des deux troncs nerveux qui se détachent de la corde nerveuse ventrale dans le troisième segment thoracique, à l'intérieur des deux nerfs de la cinquième paire de pattes, pour se diriger vers l'extrémité furcale. Je les ai suivis cheminant côte à côte jusqu'à l'avant-dernier segment abdominal, et j'ai pu observer les courts rameaux qu'ils envoient vers les grosses cellules d'aspect glandulaire régulièrement disposées dans l'hypoderme à la face ventrale de l'abdomen (PI. III, fig. I7). Certains auteurs ont signalé un mode d'innervation semblable pour les mèmes cellules glanduliformes dans les pattes thoraciques, les pièces furcales,... etc. (I)

Dans la furca, l'existence de plusieurs noyaux accolés dans la partie profonde de ces organes glanduliformes, leur donne un aspect particulier (fig. 18) qui se présente aussi parfois dans d'autres points.

Durant plusieurs années consécutives, j'ai trouvé cette espèce très abondante durant les mois de juin et de juillet, dans les points abrités de l'estuaire de Wimereux, sans qu'elle s'étende à une certaine distance de la mer.

A l'encontre de l'opinion dernière soutenue par BRADY, j'attache aux conditions éthologiques une importance assez grande pour la considérer encore comme distincte de $C$. insignis Claus, avec qui elle partage certainement plusieurs de ses caractères. Seule, une étude comparative directe pourrait définitivement fixer les deux formes avec leur synonymie. La taille si réduite (lubbocki $q: 0,9-1 \mathrm{~m} / \mathrm{m}$; insignis, $3-4 \mathrm{~m} / \mathrm{m}$ ) et l'habitat tout différent (puisque $C$. insignis est une espèce rare des eaux douces) sont des variantes trop importantes pour qu'elles soient ainsi négligées.

Distribution : O. de l'Angleterre; Helgoland; N. et O. de la France; Odessa.

(1) Notamment Rehberg (Beiträge zur Naturgeschichte underer Crustaceen; Abhandl. a. d. Naluro. Ver. in Bremen, Bd IX, 1884; Inang. Diss. Iena, 1884) qui a figuré cette disposition chez Cyclops strenuus Fischer. 


\section{Cyclops aquoreus, Fischer.}

1860. Cyclops acquoreus; Fischer ; 80 ; page 054. Taf. XX, fig. 26-29.

1878. Cyclops cequoreus, Fisch.; BradY, 20, vol. 1, page 119, PI. XIX, fig. 8-10, PI. XXI, fig. $10-17$.

1883. Cyclops áquoreus, Fisch.; Tномеson; 202, pages-97 et 98, PI. XI, fig. 16-18.

1888. Cyclops cequoreus, Fis $z h . ;$ Canu; 31, page 425 avec figure, et 33, page 504.

1890. Cyclops aquoreus, Fisch.; Richard et Blanchard; Bull. Soc. Zool. de Fr., 1890, page 138.

PL. III. FIG. I-IO.

Taille : ? $0,61 \mathrm{~m} / \mathrm{m} ; \sigma 0,35 \mathrm{~m} / \mathrm{m}$.

Corps aplati et trapu ; céphalothorax plus long que l'abdomen, avec le contour ovalaire (fig. 1). Prolongement frontal lamellaire, rabattu sur la face ventrale avec l'extrémité légèrement incurvée. Lèvre supérieure peu saillante, ornée, dans le milieu de sa limite postérieure, d'une rangée de dents pointues plus grandes et plus saillantes sur les côtés (fig. 6).

Antennules très courtes. Chez la $q$ (fig. 1), elles sont 6 -articulées avec le quatrième article plus long et le troisième plus court, ornées de longues soies raides, sans filaments sensoriels. Chez le $\sigma$ (fig. 8), elles comptent 12 articles; les articles $7,10,12$ et 1 sont les plus longs; du deuxième au sixième, les antennules sont contournées vers l'intérieur; du septième au dixième elles sont renfées; elles sont coudées entre le dixième et le onzième. Le dernier article est prolongé en une sorte d'épine très épaisse et recourbée, dont la disposition augmente les capacités préhensiles de l'organe. Les antennes du $\sigma$ portent encore de longues soies au bord antérieur; le septième article porte une longue épine pointue, le huitième et le neuvième deux courtes épines recourbées et barbelées, et les quatrième, septième, neuvième. onzième et douzième sont pourvus chacun d'un filament sensoriel particulièrement allongé dans le neuvième et le douzième.

Antenne assez longue, 3-articulée (fig. 2). L'article terminal correspond sans nul doute aux deux derniers articles habituels de l'antenne (dans C $\gamma c l$. hubbocki, par exemple), qui restent soudés dans cette espèce. Brady sépare ces deux articles et figure (loc. cit., fig. 11) une antenne 4-articulée. Malgré son imperfection, le dessin donné par Fischer (loc. cit., fig. 29) est plus exact ; pourtant la base d'insertion de l'appendice sur le tronc y est figurée comme un article, et l'antenne y paraît encore 4 -articulée.

Mandibule (fig. 3, $m d$ ) composée d'une région basilaire (coxopodite) puissante, supportant la portion distale réduite à un petit tubercule garni de trois soies raides et lisses dont la postérieure plus longue n'atteint pas à la taille de l'appendice. La région proximale du coxopodite est large et fortement convexe vers l'intérieur; elle porte une série de petits denticules à la base du tubercule sétifère. La région distale assez longue se compose d'une lame élargie à son extrémité libre (fig. 4) garnie : antérieurement, de cinq dents triangulaires décroissant vers l'arrière; postérieurement, d'une épine courbée et de deux soies dont la plus grande est barbelée.

Première maxille (fig. 3) courte et ramassée, composée d'un coxopodite masticateur et d'une région distale, palpiforme, assez confusémênt biramée.

Seconde maxille (fig. 5) avec ses deux parties nettement tangentes à leur base. — La seconde maxille externe $\left(m x^{2} e\right)$ nettement 3-articulée, avec l'article basilaire très grand et correspon- 
dant sans doute aux deux articles basilaires séparés chez $C$. lubbocki, qui seraient soudés chez $C$. aquorevis; pourtant une trace incomplète de leur séparation, trop nettement accusée par BradY (loc. cit., Pl. XXI, fig. 14), persiste au côté interne de l'appendice; les deux derniers articles sont au contraire bien séparés. Tout l'appendice porte des épines relativement grandes et quelques soies raides. - La seconde maxille interne $\left(m x^{2} i\right)$ compte seulement deux articles; les deux articles basilaires et les deux articles terminaux, si nettement accusés chez $C$. lubbocki, sont ici respectivament soudés; les épines et les soies sont assęz grandes et pauvres en barbules.

Pattes thoraciques des quatre premières paires biramées, avec l'exo- et l'endo-podites 3-articulés. Elles sont très larges, puissantes et garnies au bord externe de fortes épines denticulées.

Pattes thoraciques de la cinquième paire 2-articulées et très développées pour un Cyclops. L'article basilaire, large et saillant sur le côté du cinquième segment (fig. 1), est soudé avec le tronc; il porte à son extrémité une soie relativement courte, peu flexible. L'article terminal est lamellaire, très élargi à son extrémité et légèrement denticulé sur les bords externe et interne; le bord distal est découpé et garni de trois fortes épines et d'une longue soie chez la femelle (fig. 7), tandis que chez le mâle, il porte cinq épines ou soies (fig. 9) (1).

Lèvre supérieure de l'ouverture génitale mâle garnie d'une longue épine interne et de deux soies flexibles externes.

Pièces furcales courtes, égales en longueur au segment précédent; soie furcale médiane interne beaucoup plus longue que les autres et dépassant la taille de l'abdomen.

Eufs pondus en petit nombre dans deux sacs ovigères piriformes, bien arrondis à leur extrémité libre et de couleur verdâtre.

Nauplius très transparent, de forme ramassée, elliptique, avec les appendices peu saillants au-delà des bords du bouclier. La glande antennale relativement peu développée pour un embryon de Cyclops (fig. 10).

Cyclops cequoreus est une espèce très commune de l'estuaire de Wimereux, où elle est cantonnée en compagnie d'une foule d'espèces saumâtres de divers ordres zoologiques. Le mâle est rare, et sa taille réduite en rend la recherche particulièreinent difficile.

Ce Copépode est d'un blanc mat particulier, qui permet de le distinguer au milieu des Tachidius qui vivent en nombre considérable auprès de lui : les gouttelettes huileuses assez communément répandues dans le tissu conjonctif chez la femelle sont, comme le dit FISCHER, souvent teintees de rouge orangé. L'ovaire est légèrement coloré en bleu; les œufs en voie de maturation sont opaques et riches en deutoplasme graisseux.

Distribution: Iles Britanniques, Wimereux. Madère, Nouvelle-Zélande: dans le voisinage de la mer. Algérie: dans les lacs salés de l'intérieur.

(1) Fischer (loc, cit.) indique le dernier article comme pourvu de trois épines seulement. La fig. 27 , dans laquelle il représente la cinquième paire de pattes, me fait croire que la très grosse épine interne s'est produite par la jonction de l'épine interne et de la soie qui l'accompagne, jonction due à un accident de préparation. 


\section{ASCIDICOLIDAE.}

Copépodes monoporodelphes ayant :

$I^{0}$ Des caractères variables d'une adaptation graduelle à l'existence parasitaire dans les Tuniciers.

2" Le céphalothorax plus développé que l'abdomen, tous deux encore nettement segmentés.

$3^{0}$ Les appendices disposés pour la natation ou pour la reptation, de structure très diverse, mais toujours biramés dans les quatre premières paires.

$4^{0}$ Les antennes simples, sans exopodite.

$5^{\circ}$ Les appendices buccaux parfois très développés en vue de la mastication ou de la préhension des aliments, parfois réduits à un rôle fixateur et en nombre incomplet.

$6^{0}$ La circulation essentiellement lacunaire.

$7^{0}$ Les orifices externes des oviductes pairs, latéraux et très souvent dorsaux.

$8^{0}$ Les œufs réunis après la ponte en sacs ovigères externes, ou abrités dans une cavité incubatrice dorsale.

Ayant pu étudier un assez grand nombre de Copépodes ascidicoles, je me suis convaincu aisément de la parenté qui existe entre les espèces commensales ou parasites n'appartenant pas au sous-ordre des Diporodelphes, aux familles des Lichomolgide et des. Ascomy ontide. Les raisons d'ordre morphologique et éthologique, qui appuient cette opinion, ont été largement développées dans les pages qui précèdent; je n'y reviendrai pas. Comme je l'ai montré (pages 60, I08, I 30), les différents naturalistes qui ont créé tant de familles (I) de Copépodes parasites des Tuniciers, n'ont pu, sur un trop faible matériel, se convaincre de l'enchaînement unissant ces formes variées. Mais c'est bien pour les Ascidicolida que semble écrite la phrase de LAMARCK choisie comme épigraphe de ce travail; il n'est point, dans cette famille, d'espèces isolées: toutes s'enchaînent et se relient; mes prédécesseurs ont pensé différemment parce qu'ils ignoraient encore les espèces établissant les relations les plus importantes, les types de passage sans lesquels ne peut être comprise la biologie de ces êtres.

Malgré la diversité des types qui la composent, je crois inutile de subdiviser actuellement cette faible série de Copépodes, où les délimitations génériques suffisent à tous les besoins de la systématique.

(1) Souvent pour une seule espèce très brièvement décrite. 


\section{Notodelphys, Allman.}

1847. Notodelphys, p. p., Allman; 1.

1860. Notodelphys, Allm.; THORELL; 204, pages 26-30.

1869. Notodelphys, Allm.; Виснноц, ; 24, page ir1.

1878. Notodelphys, Allm.; BraDY ; 20, vol. 1, page 125.

Ascidicolidés capables de nager, dans les deux sexes :

$I^{0}$ Avec le corps allongé, le thorax légèrement aplati à peu près aussi long que l'abdomen cylindrique.

$2^{0}$ Avec la cavité incubatrice recouvrant le quatrième et le cinquième somites thoraciques, et aplatie dans le sens dorso-ventral.

$3^{\circ}$ Avec les antennules moins longues que le thorax et composées d'un assez grand nombre d'articles $(q .15 ; \sigma, 12)$, légèrement préhensiles chez le $\sigma$.

$4^{0}$ Avec les antennes longues, 3-articulées, terminées par un crochet fixateur et quelques soies, et pourvues de deux grandes soies géminées au bord distal externe du premier article.

$5^{\circ}$ Avec les mandibules puissantes, composées d'un coxopodite masticateur et d'une extrémité biramée natatoire ayant les deux rames égales entre elles et pourvues de très longues soies barbelées.

$6^{0}$ Avec la première maxille masticatrice à la base et vibratile à l'extrémité, possédant un épipodite sessile réduit à deux soies plumeuses : l'une grosse et longue, l'autre courte et fusiforme.

$7^{0}$ Avec la seconde maxille divisée en deux portions bien développées : l'externe ayant un large article basilaire aplati garni de plusieurs groupes de soies raides finement pectinées, un article médian pourvu d'un fort crochet courbé pour la fixation et une délicate région terminale confusément articulée, pourvue de quelques soies grêles; - l'interne 3-articulée, sétifère, ayant l'article médian pourvu d'une seule grande épine effilée, barbelée ou denticulée.

$8^{0}$ Avec les pattes natatoires larges, aux deux rames 3 -articulées.

$9^{0}$ Avec la cinquième patte lamellaire, 2-articulée, ayant l'article basilaire sessile pourvu d'une petite soie vers l'extérieur, et l'article terminal, presque discoïdal, pourvu à son bord distal d'une courte épine intérieure et d'une courte soie externe.

Le genre $N$ otodelphys contient des formes dont les deux sexes vivent à partir d'une époque précoce de leur existence, dans la cavité branchiale des Ascidies simples ou composées. Ces animaux se meuvent rapidement à la surface de la paroi branchiale, à la façon de semi-parasites; ils peuvent, à la mort de leur hòte, quitter leur abri pour nager librement sur le fond de la mer à la recherche d'un autre hòte. Ils ne sont point pêchés fréquemment en liberté dans la mer, ce qui 
montre bien qu'ils quittent rarement leur abri, et seulement en cas d'urgente nécessité : ce sont des commensaux indépendants plutòt que des semi-parasites.

Trois espèces bien connues des naturalistes sont fréquentes dans le Boulonnais.

\section{Notodelphys agilis, THORELL.}

1860. Notodolphys agilis, THORELL; 201, pages 40 et 41 , Tab. IV et V, No6.

1878. Notodelphys agilis, Th.; BradY; 20, vol. I, PI. XXVI, fig. $1-10$.

1883. Notodelphys agilis, Th. ; Auriviluus ; 4, pages 30-32.

Corps trapu avec céphalothorax relativement large et abdomen épais et plus court ; pièces furcales plus longues que le dernier segment abdominal.

Très fréquent dans le sac branchial de Molgula socialis Alder, de la zone des Laminaires. A été observé par ThORell dans la cavité branchiale de Ascidia (Corella) parallelogramma ALDER; Ascidia (Phallusia) mentula Mueller: Ascidia (Ciona) canina Mueller; Ascidia (Ascidiella) aspersa Mueller; par MceBIus et Høe, dans Ascidia (Phallugia) virginea Mueller ; - par BRAdY, dans Ascidia sordida Alder; par Aurivilluus, dans $P h$. mentula Muell.; $P h$ obliqua ALD.; Ph. patula MUELL., et dans Corella parallelogramma ALDER.

Distribution : Mer du Nord, Manche, Océan Atlantique.

\section{Notodelphys allmani, THORELL.}

1860. Notodilphys allmani, Thorell; 201, pages 3i-35, Tab. I et II, No 1.

1878. Notodelphys allmani, Th.; BRADY; 20, vol. 1, pages 126-129, Pl. XXV, fig. 1-10.

1879. Notodilphys allmani, Th.; KerSCHNER; 117, pages 30 et 31

1883. Notolilphys allmani, Th.; Aurivilulus; 4, pages 28 et 29,77 et 78.

Corps allongé, avec céphalothorax très développé et abdomen épais; pièces furcales un peu plus longues que le segment précédent. Bord postérieur de l'article préfurcal garni de denticules.

Espèce fréquente dans Phallusia fumigata Grube, de la zone des Laminaires. Recueillie par Allman, ..... etc., dans Ascidia communis (?), par Thorell dans Ciona canina Mueller et Phallusia mentula Muell.; par Aurivillius dans Ph. mentula Muell., Ph. obliqua Alder, Ph. virginea Muell., Ph. conchilega Muell., Ciona intestinalis MUELL.; par MÆBIUs dans Ascidia affinis (?).

Distribution : Mer du Nord, Manche, Océan Atlantique, Méditerranée. 


\section{Notodelphys elegans; THORELL.}

1860. Notodelphys elegans; THORELl; 20!, pages 39 et 40, Tab. IV, fig. 5.

Corps effilé, plus petit que da as le précédent; pièces furcales à peine plus longues que le segment précédent, celui-ci dépourvu de denticules à son bord postérieur.

Fréquent dans les exemplaires de Ciona intestinalis Muell. des Roches Bernard. Également recueilli dans ce Tunicier par Thorell, MÆebius et Auriviluius.

Disiribution : Mer du Nord, Manche.

\section{Doropygus, ThORel.L.}

1840. Notopterophorus; O.-G. Costa; 71.

1859. Notopterophorus, Costa ; LeUCKART; 133.

1860. Doropygus; THORell; 204, pages 43-45.

1864. Notopierophorus, Th.; HeSSE; 110.

1866. Doropygus, Th., p. p.; Hesse; 110.

1878. Doropygus, Th. et Notopterophorus, Costa; BRaDY; 20, vol. 1.

1882. Doropygus, Th. ; Giesbrecht ; 90, pages 325 et 326.

1883. Doropygus, Th. ; Auriviluius ; 4 , pages 45 et 57.

Ascidicolidés incapables de nager, dans les deux sexes, avec :

$I^{0}$ Le corps renflé et convexe sur la face dorsale dans la région céphalothoracique, cylindrique dans la région abdominale.

$2^{0}$ La cavité incubatrice recouvrant les quatrième et cinquième somites thoraciques, fortement convexe sur la face dorsale.

$3^{\prime \prime}$ Les antennules à peine égales au céphalon, composées d'un petit nombre ( $\sigma^{7}$ et $q$ : 8 et 9) d'articles et non préhensiles chez le $\sigma^{7}$.

$4^{0}$ Les antennes longues, 3-articulées, préhensiles, et dépourvues de longues soies géminées au bord distal interne du premier article, mais portant un petit tubercule sétifere à la face externe et vers le tiers distal du troisième article.

$5^{\circ}$ Les mandibules masticatrices biramées, aux rames subégales garnies de longues soies barbelées ou plumeuses.

6" La première maxille masticatrice à sa base, vibratile vers l'extrémité et possédant un épipodite sessile réduit à deux soies plumeuses renflées : l'une courte et l'autre très longue.

$7^{0}$ La seconde maxille divisée en deux portions bien développées : l'externe comme dans Notodelphys et l'interne plus ou moins nettement (1-3) articulée; portant des soies épineuses raides ou flexibles, mais point de longue épine rigide et particulièrement préhensile. 
$8^{0}$ Les pattes natatoires larges, aux deux rames le plus souvent 3-articulées, parfois réduites chez le $\sigma^{\nearrow}$ au point de vue des articles et des soies barbelées.

$9^{\circ}$ Les cinquièmes pattes 2-articulées, lamellaires, avec le premier article sessile, pourvu d'une soie grêle à son bord distal externe, et le deuxième article presque rectangulaire, portant à l'extrémité une très courte épine et une petite soie.

$10^{0}$ Les pièces furcales dépourvues de soies plumeuses et portant quelques petites pointes triangulaires ou des épines recourbées.

Etendu à ces limites plus larges par GiesBrecht (90, pages 316-320), le genre Doropygus THORELl comprend un grand nombre de Copépodes ascidicoles de grande taille, bien caractérisés et facilement reconnaissables, que l'on peut distribuer, suivant l'exemple donné par cet auteur, dans les sous-genres $N$ otopterophorus Costa (sens. lat.) et Doropygus Thorell (sens. str.); ils sont représentés tous deux dans le Boulonnais.

\section{S. g. Notopteropborus, Costa (sens. lat.).}

\section{Doropygus ayant :}

I" Le cinquième segment de l'abdomen saillant sur la face ventrale, réduit sur la face dorsale, légèrement entaillé en deux faibles bourrelets supportant les deux pièces furcales.

$2^{11}$ Les pièces furcales courtes et puissantes, terminées par quatre épines en crochet, chitinisées et résistantes.

$3^{0}$ Le deuxième article renflé de la région proximale de l'antennule recouvrant en partie, chez la femelle, le premier article plus grêle de la région distale.

$4^{\prime \prime}$ La seconde maxille interne nettement 3 -articulée, avec de nombreuses soies et des épines sur les divers articles.

Autrefois limitée aux Copépodes ascidicoles qui possèdent au bord postérieur de leurs segments thoraciques des prolongements ou replis aliformes souvent considérables, cette appellation a été étendue par GIESBRECHT aux autres formes dépourvues de ces replis et montrant diverses particularités morphologiques communes, d'une importance taxonomique plus grande.

Les deux catégories de Notopterophorus - avec ou sans prolongements thoraciques dorsaux - sont représentées dans le Boulonnais.

\section{Notopterophorus elongatus, Giesbrecht.}

1882. Notopterophorus elongatus; GIESBRECHT; 90 , pages 327 et 328 . 
Notopterophorus ayant :

$\mathbf{I}^{0}$ Les prolongements dorsaux du thorax sous forme d'ailes ou de bourrelets.

$2^{0}$ La seconde maxille externe avec les soies épineuses du bord interne pourvues de barbules raides et espacées.

$3^{0}$ Les épines terminales de la furca de même taille.

$4^{0}$ L'avant-dernier stade du développement pourvu, dans la femelle, de replis en capuchon sans filaments saillants.

La variété elatus COSTA - avec les appendices du tégument dorsal bien développés, lamellaire, de forme foliacée et relativement arrondie - a été recueillie par M. le D ${ }^{r}$ H.-E. Sauvage dans des exemplaires de Phallusia mentula Mueller provenant d'une bouée mouillée sur le banc du Vergoyer, au large d'Etaples.

Distribution : Mer du Nord, Manche, Méditerranée.

\section{Notopterophorus gibber, THORELL.}

1800. Doropygus gibber; Thorell ; 204, pages 52-55, Tab. VIII, fig. II.

1869. Doropygus gibber, Th.; Bucнноцz ; 24, pages 120-122, Taf. VII, fig. 4.

1879. Doropygus gibber, Th. ; Kerschner ; 117, pages 34 et 35, Taf. Vi, fig. 3-9.

1882. Notopterophorus gitber, Th.; GIESBRECHT; 90, page 328.

1883. Doropygus gibber, Th.; Aurivillius; 4, page 26, Taf. II, fig. 11 et 12.

$$
\text { PL. V, FIG. 25-31 et PL. VI, FIG. I-8. }
$$

Notopterophorus ayant :

$I^{0}$ Les segments thoraciques dépourvus de replis dorsaux et tous semblablement bordés, à leur limite postérieure, d'une saillie tranchante recouvrant légèrement le segment qui les suit.

$2^{0}$ Les épines terminales de la furca de tailles différentes.

En ce qui concerne cette espèce, j'insisterai sur quelques points de détail que j’ai figurés en raison de l'importance spéciale qu'ils présentent.

Taille : $q$ jusqu'à 5 et $6^{\mathrm{m} / \mathrm{m}}$, en rapport avec la taille de l'hôte; ơ de 1 à $1,5^{\mathrm{m}} / \mathrm{m}$.

Céphalothorax épais et renflé sur la face dorsale d'une manière très accentuée.

Abdomen incurvé sur la face ventrale, avec le segment préfurcal profondément incisé et saillant également du côté ventral, où il porte de courts denticules très serrés (Pl. VI, fig. 2 et $4, a 5$ ).

Antennule, $-q(\mathrm{Pl}$. V. fig. 25) : composée de huit articles, dont le deuxième recouvre presque entièrement le troisième vers le bord antérieur; le dernier assez réduit et montrant rarement des traces de subdivision en deux articles, ce qui en porte le nombre à neuf. $\sigma$ (Pl. V, fig. 26) : composée de neuf articles, par suite de la subdivision, plus fréquente mais parfois incomplète, du dernier article de l'antennule femelle; le deuxième ne reccu- 
vrant point le troisième et portant au bord antérieur trois épines tuberculaires légèrement courbes. - Au stade précédant l'état adulte, la conformation est identique dans les deux sexes.

Antenne ( $\mathrm{Pl}$. V, fig. 27) : 3-articulée, préhensile et solide, avec l'article médian plus long que le dernier; celui-ci portant à son extrémité une puissante épine en griffe plus développée chez le mâle.

Mandibule (Pl. V, fig. 28. 万; Pl. VI, fig. 5, ㅇ): biramées avec l'endopodite 2-articulé et l'exopodite simple, avec la forme plus ramassée que présente cet appendice dans le s. g. Notopterophorus comparé au s. g. Doropygus. Le coxopodite masticateur est plus tranchant et ses grosses dents antérieures sont plus saillantes dans le $\sigma$ que dans la + . En résumé, la mandibule du $\sigma^{\text {T }}$ rappelle celle de la femelle avant la dernière ou l'avant-dernière mue, plutôt que la mandibule de la femelle adulte (1).

Première maxille ( $\mathrm{Pl}$. V, fig. 6) biramée, de conformation normale, avec les soies plumeuses effilées plutôt que renflées, conformées suivant la règle dans les g. Notopterophorus.

Seconde maxille externe (PI. VI, fig. 7) bâtie sur le type de ces. g. (2) et comprenant : dans la première portion articulée, une grosse soie hérissée de barbules $(s)$ et une épine recourbée longue et rigide $(e p)$ avec d'autres soies raides, finement barbelées ou plumeuses sur le bord interne; - dans la deuxième portion composée de deux articles, deux grandes soies plumeuses, une petite épine barbelée et un crochet préhensile peu effilé et particulièrement massif à son extrémité sur le premier article; de petites soies sur le second article réduit à un moignon.

Seconde maxille interne (PI. VI, fig. 8) nettement 3-articulée, garnie de soies rigides hérissées de barbules raides, ou bien, vers l'extrémité, de courtes soies plumeuses à barbules flexibles.

Pattes thoraciques des quatre premières paires aux deux rames 3-articulées dans les deux sexes, garnies de soies natatoires barbelées dans la femelle adulte et dans les jeunes mâles, tandis que chez le mâle adulte ces soies tombent en régression dans une (deuxième et troisième paire; Pl. V, fig. 29) ou deux (quatrième paire; Pl. V, fig. 3o) rạmes sans disparaître entièrement (3) pour la plupart.

Pattes thoraciques de la $5^{e}$ paire (Pl. V, fig. 31; Pl. VI, fig. $4, p^{5}$ ) 2-articulées, avec le second article lamellaire, de contour presque rectangulaire pourvu d'une épine intérieure et d'une soie extérieure à son extrémité, et de fines épines en plusieurs ( 7 et 8 ) rangées le long de son bord interne.

A l'encontre de l'opinion récemment soutenue par Gourret (Arch. Biol., t. IX: page 475), les femelles adultes (Pl. VI, fig. 4) et jeunes (Pl. VI, fig. 3) sont munies d'un œil rouge tripartite parfaitement reconnaissable.

Cette espèce est très commune dans un grand nombre d'Ascidies du littoral boulonnais; je l'ai recueillie dans Molgula socialis ALDER, Ciona intestinalis

(1) Comparer Giesbrecht (90), pages 307 et 308.

(2) Voir Giesbrecht, l. c., pages 308 et 309.

(3) Comme l'indique Giesbrecht (1.c., page 311, Taf. XXIV, fig. 16) dans d'autres espèces. 
Muell., Phallusia fumigata Grube, Clavelina lepadiformis Muell., mais elle est plus fréquente dans les deux premières. Elle a été trouvée par d'autres naturalistes dans Ciona intestinalis et dans divers Phallusia.

Distribution : Mer du Nord, Manche, Méditerranée.

\section{S. g. Doropygus, Thorell (sens. str.).}

\section{Doropygus ayant :}

$\mathrm{I}^{0}$ Le cinquième segment de l'abdomen développé également sur les faces dorsale et ventrale, profondément fendu à son extrémité en deux branches tronquées supportant la furca.

$2^{0}$ Les pièces furcales légèrement amincies vers leur extrémité, terminées chacune par des poils grêles, courts et transparents.

$3^{0}$ Le deuxième article antennulaire ne recouvrant pas le troisième.

$4^{0}$ La seconde maxille interne plus ou moins nettement 2-articulée, mais non 3-articulée.

Les espèces rapportées à ce sous-genre sont à juste titre séparées des autres formes du genre par les particularités affectant les antennules, la seconde maxille interne et les pattes thoraciques; elles s'en distinguent plus facilement encore par la constitution de l'abdomen et des pièces furcales. La séparation proposée par Giesbrecht (90, pages 319 et suivantes) est donc en tous points justifiée, comme il ressort de la comparaison des caractères figurés dans les Planches V, VI, VIII et IX.

Par la seconde maxille interne, très nettement 2-articulée $(\mathrm{I})$, Doropygus psyllus THORELl établit le passage au s. g. Notopterophorus (2). Mais la réduction de la région terminale, très sensible chez $D$. pulex TH., s'accentue encore davantage chez les autres types des genres Doroixys Kerschner et Gunenotophorus Costa, où la seconde maxille interne ne compte plus qu'un seul article (3). En somme, $D$. pulex TH. établit le passage du genre Doropygus vers ses voisins Doroixys, Gunenotophorus et aussi Bonnierilla, par la puissance préhensile de l'antenne, par le développement et l'épaississement des portions natatoires des mandibules et des premières maxilles où les soies barbelées deviennent de plus en plus renflées et

(1) Comme le figure d'ailleurs Thoreul (189, Tab. VII, fig. 9 M 3) et malgré l'assertion erronée de Kerschner (10 bis, page 33) qui lui attribue 4 articles.

(2) Comparer PI. VI, fig. 8 et Pl. VIIl, fig. 8.

(3) Comparer PI. IX, fig. 2 ; PI. XI. fig. 7 : Pl. XIV, fig. 7. 
plumeuses, par la ressemblance dans la seconde maxille externe i i et la dégradation des articles terminaux de la seconde maxille interne.

\section{Doropygus psyllus, THORELL.}

1860. Doropygus psyllus; Thorell; 204, pages 49 et 50, Taf. VII, fig. $9 \mathrm{~A}_{1}, \mathrm{~A}_{2}, \mathrm{M}_{3}, \mathrm{P}_{1}, \mathrm{P}_{2}, \mathrm{P}_{5}, \mathrm{~F}_{7}$.

1879. Doropygus psyllus, Th.; KeRschner ; 117, page 33.

1883. Dor. psyllus, Th.; Aurtviluius; 4, pages 24 et 25 . Planche VIII, fig. 1-11.

\section{Planche VIII, Fig. I-II.}

Taille : $7,2 \mathrm{~m} / \mathrm{m}$

Forme ramassée; courbe dorsale du céphalothorax arrondie; limite postéro-dorsale de la cavité incubatrice sphérique.

Pièces furcales courtes et légèrement effilées, avec de très petits poils à son extrémité, représentés à tort comme des épines par Thorell $(l$. c., F7 ).

Antennule (fig. 3) 9-articulée, grêle et légèrement incurvée au-delà du deuxième article.

Antenne (fig. 4) particulièrement allongée et grêle, avec une petite griffe et de petites soies terminales, avec le deuxième article plus court que les deux autres.

Exopodite mandibulaire (fig. 5) très allongé, avec cinq grandes soies plumeuses.

Première maxille (fig. $6^{\circ}$ avec les soies plumeuses plus effilées et plus nombreuses que chez D. pulex (fig. 19). Comme le fait observer Kerschner, c'est l'endopodite (lamina ultima de Thorels) qui porte six soies, et non l'exopodite (lamina extima), qui en porte seulement quatre ainsi que le bord interne du basipodite (lamina media).

Seconde maxille externe (fig. 7) 5-articulée, courte et grêle, avec une forte soie en forme d'épine légèrement infléchie $(e p)$, une soie barbelée et une grosse soie renflée, hérissée de plusieurs séries de barbules $(s)$, sur le tubercule proximal interne du premier article. Épine du deuxième article assez forte vers sa base, légèrement contournée et pointue à son extrémité.

Seconde maxille interne (fig. 8) 2-articulée. L'article distal, correspondant sans doute aux deux derniers articles du même appendice chez Notopterophonus, porte trois soies barbelées, les deux distales plus longues et particulièrement plumeuses.

Première patte thoracique (fig. 9) avec une épine relativement courte et épaisse au bord interne du basipodite.

Deuxième patte thoracique (fig. 10) caractéristique dans la forme des articles de la rame externe et portant des soies barbelées au bord interne de tous les articles, ainsi que l'ont remarqué déjà Kerschner et Aurivillius.

Cinquième patte thoracique (fig. 11) de forme irrégulière, avec deux séries de denticules au bord interne, vers "extrémité. comme dans $D$. pulex.

Doropygus psyllus est une espèce rare qui a été observée en petit nombre

(1) Où l'épine préhensile du deuxième article existe toujours, bien qu'elle soit dans certains cas faible et réduite. Voir GIesbrecht : 90, page 326, note 2. 
d'exemplaires par les seuls auteurs cités plus haut (1). Elle est très rare dans Phallusia virginea Mueller, Ascidie très commune dans les rochers du Boulonnais. Aurivillius l'a recueillie dans le même hòte, et dans Phallusia patula Mueler ; Kerschner l'indique comme habitant Ph. fumigata (Heller!), et Thorell l'a découverte dans Ascidiella aspersa MueLler.

Distribution: Mer du Nord, Manche, Méditerranée.

\section{Doropygus pulex, THORELl.}

1860. Doropygus pulex; Thorell; 201, pages 46-49, Tab. VI, fig. 8.

1869. Doropygus pullus; BuchноLz; 24, pages 116-119, Taf. Vl et VII, fig. 3.

1878. Doropygus pulex, Th.; Brady; 20, vol. 1 , pages 133 et $134, \mathrm{Pl}$. XXVIII, fig. I-12.

1879. Doropygus pulex, Th. ; Kerschner; 117, pages 32 et 33, Taf. VI, fig. 10-12.

1883. Doropygus pulex, Th.; Auriviluius; 4, page 24, Planche VIII, fig. 12-22; Planche IX, fig. 1-3.

\section{Planche VIII, Fig. i i-22 et Planche IX, Fig. $1-3$.}

Taille : $q, 3$ et $4 \mathrm{~m} / \mathrm{m} ; \sigma, 1,5-2 \mathrm{~m} / \mathrm{m}$.

Formẻ assez élancée (fig. 12 et 13 ), spécialement chez le mâle; segments thoraciques distendus par l'énorme développement des cellules conjonctives graisseuses, sur les faces dorsale et pleurale des segments; limite dorsale du thorax irrégulièrement vallonnée, avec la cavité incubatrice prolongée en tronc-de-cône dans son extrémité postérieure. Pièces furcales assez allongées, légèrement amincies ou même effilées, dans la région distale.

Antennule 9-articulée (fig. 14 et 15); antenne 3-articulée (fig. 16), pièces buccales (fig. 1720, fig. 1 et 2) présentant un allongement assez considérable.

Première maxille (fig. 19 et 20 ) garnie d'un nombre assez restreint de soies plumeuses, particulièrement sur l'endopodite.

Seconde maxille externe (fig. 1) comme dans D. psyllus, avec l'épine préhensile du $2^{\mathrm{e}}$ article plus grêle et plus pointue.

Seconde maxille interne (fig. 2) simple plutôt que 2-articulée, mais montrant encore une légèrẻ séparation en deux régions : la distale $\left(=2^{\mathrm{e}}\right.$ article réduit) porte deux soies plumeuses flexibles.

Première patte thoracique (fig. 3) avec le basipodite garni d'une très longue épine à son bord interne, et vers l'extérieur d'une soie à base renflée; avec l'exopodite pourvu extérieurement d'épines en poignard, à bords denticulés.

Deuxième patte thoracique (fig. 21) bien développée, avec le bord externe de l'exopodite

(1) Il existe, dans la description et les dessins publiés par Bradr (20, vol. I, pages 136-138, Plate XXXII) pour $D$. normani BR., des caractères qui ont amené KeRsChner à considérer cette espèce comme une variété de $D$. psyllus. Dans les pattes natatoires et la $I^{\text {ro }}$ maxille, $D$. normani se rapproche en effet, - suivant la description de BradY, de $D$. psy'lltss. Mais la plupart des caractères le rapprochent encore de $D$. pulex. Il me semble done nécessaire d'attendre la confirmation de cette supposition, à la suite de nouvelles recherches, avant de pouvoir se prononcer utilement. 
garni de longues épines sétiformes recourbées, très délicatement denticulées vers leur extrémité. Troisième et quatrième pattes comme la deuxième.

Cinquième patte (fig. 22) 2-articulée, avec le second article irrégulier et deux rangées de denticulẻs faisant saillie sur le bord intérieur, près de l'extrémité.

Doropy gus pulex est commun dans le sac branchial des Cynthiadae (C. morus L.; Styelopsis grossularia VAN BENEDEN; C. lurida Thorell) du Boulonnais, aussi bien dans les rochers de la côte, que sur les bancs plus profonds éloignés du rivage. Cette espèce a été rencontrée par THOREL dans $C$. lurida TH., et dans divers Phallusia et Ciona; par Auriviluus dans Phall. obliqua Alder; Ph. patula Mueller; Ph. virginea Muell.; Ph. conchilega Muell.; Corella parallelogramma Alder, Styela gyrosa Heller, Cynthia loricala KüPPfER, C. rustica Linné; par I. C. Thompson (199) dans Ciona intestinalis, Ascidia plebeja et Ascidiella scabra.

Distribution: Mer du Nord, Manche, Atlantique, Méditerranée.

\section{Bonnierilla, CANU.}

1879. Paryphes; Kerschner, 117, page 27.

1891. Bonnierilla; Canu, 34, page 473.

1835. Non Paryphes; Burmeister.

Ascidicolidés femelles incapables de nager, avec :

$\mathbf{I}^{0}$ Le corps aplati dans le sens transversal et fortement convexe sur la face dorsale dans la région céphalothoracique, presque cylindrique dans la région abdominale.

$2^{0}$ La cavité incubatrice recouvrant les deuxième, troisième, quatrième et cìnquième somites thoraciques, au contour dorsal presque semi-circulaire.

$3^{0}$ Les antennules assez longues, grêles et 8-articulées.

$4^{0}$ Les antennes très allongées, 3-articulées, préhensiles et dépourvues de soies géminées sur le premier article, mais portant à la face externe et vers le tiers distal du troisième article un petit tubercule sétifere.

$5^{0}$ Les mandibules masticatrices, biramées, aux rames subégales garnies de soies barbelées ou plumeuses très longues.

$6^{0}$ La première maxille masticatrice à sa base, vibratile vers son extrémité et possédant un épipodite sessile réduit à deux soies plumeuses renflées, l'une courte, l'autre très longue.

$7^{0} \mathrm{La}$ seconde maxille divisée en deux portions bien développées: l'externe, comme dans Notodelphys et Doropygus; l'interne, 2-articulée, portant des soies plus ou moins raides, mais point d'épine rigide préhensile. 
$8^{0}$ Les pattes natatoires aux deux rames 3-articulées, avec de longues soies barbelées, très allongées dans les deuxième, troisième et quatrième paires, où le bord externe de l'exopodite présente des épines très réduites.

$9^{0}$ Les cinquièmes pattes 2-articulées, lamellaires, avec le premier article sessile pourvu d'une soie grêle à son bord distal externe, avec le second article effilé et terminé par une soie grêle et flexible.

$10^{0}$ Les pièces furcales dépourvues de soies plumeuses et purtant quelques petits poils triangulaires.

Le genre Bonnierilla se distingue nettement des Ascidicolidés voisins par sa forme, par sa cavité incubatrice, par ses pattes thoraciques des deuxième, troisième et quatrième paires. Séparé par KERSCHNER, d'après un unique exemplaire assez mal conservé, sous le nom de Paryphes (I), il se rapproche du genre Gunenotophorus Costa par sa cavité incubatrice développée aux dépens des téguments dorsaux du deuxième somite thoracique; et du sous-genre Doropygus par la structure de ses pièces buccales et furcales.

Une seule espèce est actuellement connue, et elle a été exclusivement rencontrée dans les petites Ascidies du genre Cynthia.

\section{Bomierilla longipes, Kerschner.}

1879. Paryphes longipes; Kerschner; 117, pages 27-30, Taf. III, fig. 10. Taf. IV, fig. I-IO.

7888. Paryphes longipes, Kersch.; Canu, 32, page 505.

1891. Bonnierilla longipes, Kersch. ; Canu, 34, page 473.

$$
\text { PL. X, Fig. I, I } a \text {, I } b ; \text { PL. IX, FIG. 4-I } 3 \text {. }
$$

La femelle adulte est seule connue; le mâle reste inconnu.

Taille : 3 aे $4 \mathrm{~m} / \mathrm{m}$.

Forme générale (Pl. X, fig. 1, $1 a$ et $1 b$ ) assez bien décrite par Kerschner, avec l'aplatissement considérable de la région céphalothoracique dans le sens latéral (fig. $1 b$ ) et le développement particulier du bord postérieur du céphalon en une collerette saillante ornementée, garnie d'une épine à l'angle latéro-postérieur ('fig. 1). Toutefois, à l'encontre de l'opinion de Kerschner, le thorax est très nettement segmenté (fig. 1), et ses divisions sont faciles à reconnaître sur les faces pleurales des segments. Les segments abdominaux sont assez régulièrement cylindriques. Les pièces furcales sont particulièrement incurvées vers la face ventrale (fig. 1). Le prolongement frontal (PI. IX, fig. 4) est saillant, pointu et recourbé ventralement.

Antennule (Pl. IX, fig. 4) 8-articulée, avec les deux premiers articles légèrement renflés. avec une épine saillante en fer de lance au bord antérieur du deuxième article et un filament

(1) Que j'ai dû changer, par suite de l'emploi qui elı avait été fait antérieurement (en 18 85 !) par BurmeISTER, pour un genre d'insecte hémiptère 
sensoriel sur les septième et huitième. En réalité cet appendice diffère aussi bien de l'antennule de Notopterophorus que de celle de Doropygus (1).

Antenne (fig. 5) particulièrement grêle, avec le troisième article très allongé, son tubercule latéral garni de trois soies dont la médiane est plus longue, son épine terminale large et crochue, accompagnée de deux ou trois soies.

Mandibule (fig. 6 ) bien masticatrice, avec cinq longues soies plumeuses à l'extrémité de l'exopodite assez nettement $\mathbf{2}$-articulé, avec l'endopodite $\mathbf{2}$-articulé portant quatre soies barbelées sur le bord interne du premier, sept soies barbelées (2) sur les bords interne et terminal du second article. Le coxopodite masticateur porte au bord intérieur cinq épines plus pointues et plus saillantes, avec une série de nombreux denticules.

Première maxille (fig. 7) avec l'endopodite et l'exopodite subégaux et garnis tous deux de quatre soies plumeuses renflées.

Seconde maxille externe (fig. 8) 4-articulée, avec une soie en forme d'épine (ep), une grosse soie hérissée de plusieurs séries de barbules raides et une petite soie plumeuse placées sur le gros tubercule interne proximal du premier article; avec une épine préhensile recourbée et légèrement efiilée sur le deuxième article; avec des soies grêles sur les autres.

Seconde maxille interne (fig. 9) 2-articulée, avec le second article portant à sa face interne une rangée de poils fins et une petite soie, et à son extrémité distale une grande soie plumeuse.

Première patte thoracique (fig. 10) très aplatie et lamellaire, portant au côté externe de l'exopodite des épines en poignard denticulées et au côté interne des deux rames de très longues soies plumeuses.

Pattes natatoires des trois dernières paires très allongées, avec l'exopodite double de l'endopodite, et pourvues : sur l'endopodite, de neuf (deuxième et troisième paires : fig. 11) ou de sept (quatrième paire; fig. 12) longues soies plumeuses; sur l'exopodite, de très courtes épines rudimentaires au bord externe ou terminal, et des soies plumeuses au bord interne (fig. 11).

Cinquième patte thoracique (fig. 13) insuffisamment décrite par KERSCHNER (loc. cit. page 3o), 2-articulée, avec le second article très allongé, terminé par unè seule soie et pourvu vers l'intérieur de cinq crêtes arquées, garnies de petits poils raides.

Bonnierilla longipes est très commun dans la cavité branchiale de Cynthia lurida THOR., dans les rochers du littoral boulonnais, depuis le cap Gris-Nez jusqu'à la Pointe d'Alprecht. Je l'ai recueilli en plus grande abondance à Audresselles, où il est souvent accompagné dans le mème hóte, de Gunenotophorus globularis CosTA. Doropygus pulex THORELL, et Lichomolgus albens THORELL.

Seul jusqu'à présent, KersChner avait obtenu ce Copépode, en un seul spécimen recueilli dans le sac branchial d'un petit Cynthia sp. de la baie de Muggia.

Distribution: Manche, Adriatique.

(1) Contrairement à ce.que dit KERSCHNER : loc. cit., page 28.

(2) Les trois distales sont beaucoup plus longues et plumeuses. 


\section{Gunenotophorus, Costa.}

1843. Gunenotophonis; O. G. CosTA, 71.

1864. Sphceronotus; Claus, 52 , page 379.

1869. Gunentophorus, Costa; BuchHolz, 24, page 144.

1879. Gunentophorus, Costa ; KerSCHNER, 117, page 190.

1882. Gunenotophorus, Costa; Giesbrecht, 90, page 325.

1883. Gunentophorus, Costa ; Auriviluius, 4, pages 26 et 66.

Ascidicolidés femelles incapables de nager avec :

$I^{0}$ Le corps renflé, convexe sur la face dorsale et presque hémisphérique dans sa région thoracique, cylindrique dans sa région abdominale.

$2^{0}$ La cavité incubatrice recouvrant les deuxième, troisième, quatrième et cinquième somites thoraciques, parfaitement hémisphérique.

$3^{0}$ Les antennules larges et courtes, 7 -articulées.

$4^{0}$ Les antennes fortes et courtes, 3-articulées, préhensiles et dépourvues de soies géminées sur le premier article, mais portant à la face externe du troisième une soie placée sur un léger tubercule.

$5^{\circ}$ Les mandibules masticatrices, biramées, aux rames subégales garnies d'un petit nombre de soies barbelées ou plumeuses.

$6^{0}$ Les premières maxilles de forme très ramassée, masticatrices à leur base, vibratiles vers leur extrémité, et possédant un épipodite sessile réduit à deux soies plumeuses dont l'une est plus longue que l'autre.

$7^{0}$ Les secondes maxilles divisées èn deux portions bien développées : l'externe comme dans Notodelphys, Doropygus et Bonnierilla; l'interne simple et courte, portant quelques soies barbelées grosses et raides, mais point d'épine rigide préhensile.

$8^{0}$ Les pattes natatoires aux deux rames 3 -articulées, pourvues de longues soies barbelées et d'épines marginales dans la première paire, mais sans soies barbelées ni longues épines dans les deuxième, troisième et quatrième.

$9^{0}$ Les cinquièmes pattes thoraciques réduites à un tubercule irrégulier, peu saillant, avec une seule épine courte et pointue.

$10^{\circ}$ Les pièces furcales terminées par quelques petits crochets, sans soies lisses ou barbelées.

La forme du corps, celle de la cavité incubatrice développée, comme dans Bonnierilla, aux dépens du deuxième somite thoracique, contribuent, avec la structure des appendices des quatre derniers segments thoraciques, à caractériser ce genre signalé par quelques naturalistes, généralement dans des Cynthia. 
 \\ Gunenotophorus globularis, Costa.}

1840. Gunenotophorus glohularis; CostA, 71.

1864. Spharonotus thorelli; Claus, 52 , pages 379 et suiv., Taf. XXXVI, fig. 29-34.

1869. Gunentophorus globularis, Costa ; Bucнно1z, 24, pages 144-149, Taf. X, fig. 8.

1879. Gunentophorus globularis, Costa; KERSCHNER, 117, pages 38-40, Taf. II, fig. 3 et Taf. VI, fig. $17-28$.

1885. Gunintophorus globularis, Costa; Auriviluus, 4, pages 26, 28 et 66. Taf. 1V, fig. 13.

1891. Gunenotophorus glomularis, Costa; CANU, 34, page 473.

Planche XI, fig. I-I2.

La seule espèce connue de ce genre; et seulement d'après le sexe femelle.

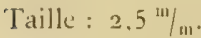

Corps bien segmenté et semblable. sauf dans la forme sphérique et renflée du céphalothorax,

à Bonnierilla longipes. L'abdomen cylindrique, légèrement rétréci vers l'extrémité postérieure (fig. 12) est tran̄sformé en organe de reptation par la légère courbure ventrale de son extrémité et par la puissance adhésive que lui donnent les denticules développés à la surface du dernier segment et des pièces furcales divergentes.

Prolongement frontal et antennule (fig. I) garnis de petits poils raides et serrés sur la face ventrale, lisses sur la face dorsale; bien décrits par Kerschner (loc. cit., page 39), mais figurés par lui d'une manière insuffisante.

Antenne (fig. 2) très résistante par le grand épaississement de sa paroi chitineuse et le développement de crètes de renforcement; l'épine terminale en griffe égale à la moitié du troisième article.

Mandibule biramée, avec le coxopodite masticateur très large portant vers l'intérieur : cinq grosses dents, une série de denticules et une épine pointue accompagnée d'une soie barbelée: avec le basipodite très allongé portant intérieurement deux soies barbeléees; avec les deux rames probablement simples ( 1 ) et portant: l'interne quatre soies barbetées, l'externe cinq soies plumeuses.

Première maxille (fig. 5) avec huit grandes épines sur le lobe masticateur du coxopodite, avec quatre grosses soies plumeuses sur l'exopodite et quatre soies semblables sur l'endopodite soudé au lobe interne saillant du basipodite.

Seconde maxille externe (fig. 6 ) avec la région basilaire large et assez semblable à celle de Bonnierilla longipes; réduite dans sa région terminale à un seul article formé d'une large pièce proximale portant une forte soie plumeuse et une épine recourbée et préhensile, et d'un court prolongement distal armé de trois courtes soies barbelées. Cette région terminale correspond évidemment aux quatre derniers articles des secondes maxilles externes chez Doropygus et Bonnierilla.

Seconde maxille interne (fig. 7) simple, sans indication d'articles, avec sept grosses soies barbelées : cinq marginales plus raides vers l'intérieur et deux terminales flexueuses.

(1) Pourtant, sur l'endopodite persistent les traces d'une segmentation incomplète; toutefois, dans mes exemplaires, la structure diffère bien des descriptions données par KeRSCHNER (loc. cit., page 39) et Auriviluius (loc. cit., pages 27 et 28 ). 
Pattes thoraciques de la première paire (fig. 8) larges, aux contours irréguliers, avec les épines marginales externes de l'exopodite petites et effilées.

Pattes thoraciques des deuxième, troisième et quatrième paires avec l'épipodite plus long, couvert de poils raides et courts, sans soies marginales, mais pourvu de quelqués petites épines rudimentaires sur les bords externe et terminal (fig. 9). L'endopodite est toujours réduit (fig. 9), avec des crêtes saillantes qui donnent à ses deux derniers articles une forme très irrégulière.

Pattes de la cinquième paire soudées intimement avec le corps (fig. 11) et ne formant plus qu'une saillie irrégulière (article basilaire) portant : une épine pointue sur le bord externe, un tubercule saillant (rudiment du deuxième article ?) vers son milieu. Kerschner (loc. cit., page 40; Taf. VI, fig. 27) avait déjà reconnu sa présence sans comprendre exactement sa structure.

Gunenotophorus globularis est assez rare dans la cavité branchiale de Cynthia lurida THORELL; je l'ai recueilli seulement à marée basse, parmi les rochers d'Audresselles. Buchrolz signale ce Copépode dans un Cynthia (C. microcosmus Linné?). Kerschner l'indique aussi comme parasite d'un Cynthia sp. Aurivillius l'a recueilli principalement dans Styela gyrosa Heller, rarement dans Phallusia obliqua Alder et Ph. mentula Mueller.

Distribution: Mer du Nord, Manche, Méditerranée.

\section{Doroixys, Kerschner.}

1879. Dotoixys; Kerschner, 117, page 24.

1883. Doroixys, Kersch.; Della Valle, 76, pages 242 et 244.

1888. Doroixys, Kersch.; CanU, 32, page 505.

1891. Doroixys, Kersch.; Canu, 34, page 472.

Ascidicolidés femelles incapables de nager, avec:

$I^{0}$ Le corps renflé et convexe sur la face dorsale dans la région céphalo-thoracique, avec l'abdomen 4-articulé, court et cylindrique.

$2^{0}$ La cavité incubatrice recouvrant les $4^{\theta}$ et $5^{\theta}$ somites thoraciques, convexe sur la face dorsale.

$3^{\circ}$ Les antennules courtes 7 - et 8 -articulées, sétifères, sans renflement particulier des articles basilaires.

$4^{0}$ Les antennes 3 -articulées, courtes et solides, préhensiles, sans soies barbelées sur le $3^{\text {er }}$ article.

$5^{0}$ Les mandibules biramées, masticatrices, aux rames subégales, ornées de soies plumeuses ou barbelćes.

$6^{0}$ Les premières maxilles masticatrices à leur base, vibratiles à leur extrémité relativement réduite, et possédant un épipodite sessile garni d'une soie barbelée. 
$7^{0}$ Les secondes maxilles externes 4 -articulées, avec une épine préhensile sur le $2^{\mathrm{e}}$ article, et portant, comme dans Doropygus, sur le gros tubercule marginal du $1^{\text {er }}$ article : une soie en forme d'épine, une soie barbelée, une soie hérissée de plusieurs séries de barbules, et une petite soie accessoire; - les secondes maxilles internes très réduites, sans aucune articulation.

$8^{0}$ Les 4 pattes thoraciques biramées, aux 2 rames 3-articulées, sauf pour l'endopodite 2-articulé de la $4^{\theta}$ paire; normalement natatọ̣res dans la $1^{\text {re }}$ paire et transformées dans les trois autres en organes de reptation par l'existence de soies rigides et non barbelées.

$9^{0}$ Les $5^{\text {es }}$ pattes thoraciques sous forme d'un crochet chitineux dépourvu d'articulation.

$10^{\circ}$ Les pièces furcales terminées par quelques petits poils, sans soies barbelées ni crochets.

La forme du corps rappelant Doropygus, la nature et la réduction des appendices buccaux et thoraciques rendent le genre Doroixy's parfaitemeni distinct parmi les autres Ascidicolidés. Le parasitisme dans les Synascidies contribue, avec les particularités morphologiques qu'il présente, à le rendre encore plus intéressant.

Une seule espèce est actuellement connue, et seulement d'après la femelle ( 1 ).

\section{Doroixys uncinata, Kerschner.}

1879. Doroixys uncinata; Kerschner, 117, pages 24-27, Taf. IV, fig. II-13, Taf. V, fig. 1-12.

1882. Doroixys uncinata, Kersch.; Della VAlle, 76, pages 242 et 244, fig. 3.

1888. Doroixys uncinata, Kersch.; Canu, 32, page 505.

1891. Doroixys uncinata, Kersch.; Canu, 34, page 472.

\section{Planche XIV, Fig. I-I4.}

Taille : 1,5 à $2^{113} / \mathrm{m}$.

Corps (fig. i) bien nettement segmenté, avec la cavité incubatrice relativement allongée, au contour ovalaire. L'angle postéro-pleural du céphalon porte une longue épine saillante et repliée, très caractéristique.

Antennule (fig. 2) de forme assez allongée, comptant 7 ou 8 articles par suite de la division accidentelle et souvent douteuse du dernier article.

Antenne (fig. 3) avec la griffe terminale bien recourbée en crochet préhensile, et l'article médian légèrement renfé.

Mandibule (fig. 4) rappelant celle de Notopterophorus par ses deux rames courtes, l'exopodite avec 5 soies plumeuses et l'endopodite 2-articulé avec 6 soies barbelées peu développées.

Première maxille (fig. 5) avec le coxopodite armé de 8 longues épines masticatrices, avec l'endopodite et l'exopodite réduits et pourvus : le premier de 3, le second de 4 grosses soies plumeuses.

Seconde maxille externe (fig. 6) courte et massive.

(1) Au sujet du mâle supposé de cette espèce, voir plus haut: $3^{\circ}$ Partie, Chap. III, $\$$ B; page 117 , note I. 
Seconde maxille interne (fig. $7, m x^{2} \iota$ ) garnie de 5 (et non 6, comme le dit Kerschner: l. c., page 26, Taf. V, fig. 6) soies courtes avec de petites barbules.

Première patte thoracique (fig. 8) toujours contournée vers l'intérieur, avec une courte épine intérieure sur le basipodite, de longues soies barbelées sur l'endopodite, et sur le bord externe de ce dernier des épines effilées et denticulées.

Pattes thoraciques des $2^{\mathrm{e}}, 3^{\ominus}$ et $4^{\mathrm{e}}$ paires armées de courtes épines mousses (fig. 9, 10 et 11 ) au bord externe de l'exopodite, et de longues soies lisses et cylindriques avec l'extrémité arrondie sur les bords interne et distal des deux rames.

Endopodite de la $4^{\mathrm{e}}$ paire 2-articulé et variable: portant à son extrémité tantôt 3 soies visiblements réduites (fig. 12), tantôt 2 soies (fig. 13) ou 2 petits tubercules, comme rudiments des soies tombées en régression.

Cinquième patte (fig. $14, p^{\mathbf{5}}$ ) réduite à une simple pièce chitineuse triangulaire, avec deux petites pointes à l'extrémité et une soie courte et flexible sur une saillie du bord externe.

Pièces furcales (fig. 1) légèrement recourbées vers la face ventrale et un peu plus longues que le segment qui les porte, avec 4 poils terminaux très courts et une petite soie marginale à peine visible.

Doroixys uncinata est très fréquent dans la cavité branchiale de Morchellium argus Milne-Edwards, Circinalium concrescens Giard et Polyclinum luteum Giard, dans tous les rochers littoraux du Boulonnais. Kerschner le signale aussi dans Amarcecium sp.; èt Della Valle dit l'avoir recueilli (I) dans Aplidium cristallinum D. V., A. gibbulosum D. V., Fragarium areolatum GIARD, Diazona violacea SAv., Perophora listeri Wagner, du golfe de Naples. Je l'ai observé également à Concarneau dans diverses Synascidies. C'est probablement l'espèce signalée récemment par PIzon (2) dans Botryllus violaceus M.-EDwards, à SaintWaast la Hougue, avec une série de détails éthologiques des plus extraordinaires. Distribution: Manche, Atlantique, Méditerranée, Adriatique.

\section{Botryllophilus, Hesse.}

1864. Botryllophilus; Hesse, 110, page 345.

1883. Kosmechthrus; Della Valle, 76, pages 248 et 251

1885. Schizoproctus; Auriviluus, 5, pages 247 et 248.

1891. Botryllophilus, Hesse; Canu, 34 , page 473.

Ascidicolidés incapables de nager, dans les deux $(q !, \sigma ! ?)$ sexes, avec :

$I^{0}$ Le corps complètement segmenté, renflé et gibbeux sur la face dorsale dans la région

(1) Loc. cit., page 244. Le dessin (fig. 2) dans lequel cet auteur représente D. uncinala est très imparfait en ce sens que les caractères principaux de ce Copépode y sont omis. Le texte ne signale aucune des particularités caractéristiques.

(2) Le Naturaliste, $13^{\circ}$ année; 15 février 1891. 
thoracique, presque cylindrique et recourbé ventralement dans la région abdominale 4-segmentée.

$2^{0}$ Les œufs pondus à l'extérieur et nullement abrités dans une cavité incubatrice comme chez les précédents.

$3^{\circ}$ Les antennules courtes, élargies dans leur région proximale et comptant un très petit nombre (5-7) d'articles.

$4^{0}$ Les antennes 3 -articulées, avec l'article médian très court et l'article terminal armé de longues épines en poignard nullement préhensiles.

$5^{\circ}$ Les mandibules biramées avec le coxopodite masticateur large et solide, avec l'endopodite 2-articulé bien développé et l'exopodite simple et réduit, garnis tous deux de quelques soies barbelées.

$6^{0}$ Les premières maxilles courtes et massives, avec le coxopodite masticateur et la partie terminale vibratile, pourvues d'un exopodite tuberculaire avec une très petite soie lisse.

$7^{0}$ Les secondes maxilles bien séparées en deux portions: l'externe 1-articulée et pourvue sur le bord interne de plusieurs longues épines rigides; l'interne 3-articulée, avec une griffe terminale uncinée, disposée en pince préhensile par le rabattement du dernier article digitiforme sur le $2^{i}$ plus large et plus solide.

$8^{\circ}$ Les pattes thoraciques des 4 premières paires biramées, courtes, aux rames 2-articulées et garnies d'épines marginales externes ou de soies barbelées marginales internes.

$9^{0}$ Les pattes de la $5^{\circ}$ paire simples, sans articulations, en forme de lames étroites et allongées avec une soie marginale et 2 soies ou épines terminales, attachées chez la femelle adulte aux côtés de la face dorsale dans le $5^{\circ}$ segment thoracique.

$10^{\circ}$ Les pièces furcales courtes et divergentes, dirigées vers la face ventrale, avec une soie marginale et quatre épines uncinées terminales dont l'une est plus longue que les autres.

Ainsi caractérisé, le genre Botryllophilus se rattache nettement, par l'organisation morphologique des appendices, à la série de Copépodes ascidicoles étudiés précédemment. Il en diffère seulement par une dégradation et une spécialisation secondaire de divers appareils, particularités absolument insuffisantes pour justifier la création d'une famille distincte ( $\mathbf{I}$ ) pour ces Copépodes. Ainsi que l'a sommairement indiqué Aurivillius (6, pages 45 et 46), Botryllophilus se rapproche du genre Ascidicola THORELL par les $5^{\text {es }}$ pattes lamelleuses et latéro-dorsales, par les antennules, antennes et mandibules réduites; il s'en écarte d'ailleurs considérablement à bien d'autres points de vue. Dans la série des Ascidicolidés, il occupe au moins une place intermédiaire entre les formes élevées comme Doropygus, Doroixys... etc., et les plus dégradées comme Ascidicola..., etc.

(1) Ce qu'ont tenté à tort Delia Valle et Auriviluius. 
La caractéristique du genre Botryllophilus réside particulièrement :

$\mathrm{I}^{0}$ Dans la structure et la position des $5^{\mathrm{es}}$ pattes thoraciques.

$2^{\circ}$ Dans l'existence presque constante ( $\mathrm{r}$ ) d'un seul sac ovigère de forme rigoureusement sphérique abrité entre les $5^{\text {es }}$ pattes.

La découverte d'une espèce de Botryllophilus voisine du type observé par
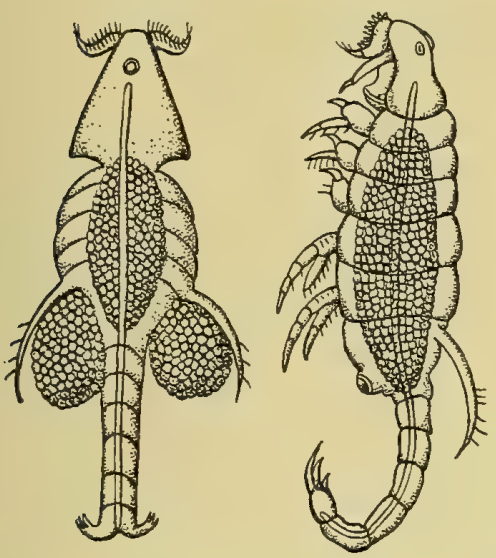

Fig. 16. - Femelle de Botryllophilus ruber Hesse, vue par les faces dorsale et latérale.

D'après Hesse, loc. cit., Pl. XII, Fig. 1 et 2. HeSSE m'a seule permis de fixer la synonymie de ce genre, complètement méconnue par les naturalistes précités. Si Della Valle et Aurivillius n'ont pu reconnaître les relations unissant Botryllophilus Hesse, Kosmechthrus Della Valle et Schizoprocius Auriviluius, la cause en est: d'une part à la nature des espèces (2) qu'ils ont observées; d'autre part à l'insuffisance de leurs observations et de la description publiée sur Botryllophilus. En effet, dans cette dernière, les caractères des $5^{\text {es }}$ pattes et des sacs ovigères $(3)$ peuvent seuls être utilisés avec une certaine confiance. D'autre part, Della Valle n'a fourni pour Kosmechthrus qu'une étude fort imparfaite des appendices buccaux

Enfin, en ce qui concerne Schizoproctus, Auriviluius a commis d'abord (5, pages 247 et 248 ) des erreurs d'observation - ultérieurement corrigées (6, pages 44-46) - bien faites pour motiver des conclusions taxonomiques inexactes.

L'organisation de Botryllophilus que j'ai pu étudier assez complètement sur des spécimens vivants, vient encore appuyer sa parenté avec les autres Ascidicolidés. Au-delà de la bouche, après un court œsophage rétréci, vient (fig. 2) le tube digestif glandulaire sous forme d'une vaste poche cylindrique $(t d)$ qui parcourt toute la région céphalothoracique pour se transformer au niveau du $5^{\mathrm{e}}$ segment en un intestin plus étroit, tubulaire, dirigé vers l'abdomen. Après un renflement

(1) Bien que Hesse indique et figure deux sacs ovigères sphériques, et que le dessin primitivement donné par Aurivilulus (5) laisse supposer l'existence simultanée de deux sacs chez B. (Schizoproctus) inflatus Aur.

(2) Avec les $5^{\text {es }}$ pattes trés courtes, tandis que les formes recueillies par Hesse et par moi ont les $5^{\text {es }}$ pattes très longues.

(3) Loc. cit., page 346: «A la suite du dernier anneau thoracique, on aperçoit de chaque côté une lame plate très longue et très pointue, garnie à son extrémité de poils rigides (fig. 1 et 10). Elle peut se relever presque verticalement et sa forme est incurvée du côté de l'abdomen. En dessous se présentent immédiatement les ouf́s qu'elle semble destinée à protéger et qui, chez ce crustacé, affectent dans leur agglomération une forme sphérique presque régulière." 
assez considérable $(i)$ au niveau des $2^{\mathrm{e}}$ et $3^{\mathrm{e}}$ segments abdominaux, vient le rectum qui débouche par l'anus à la face dorsale du dernier segment.

Le système nerveux est très condensé, comme dans tous les Ascidicolidés. Le cordon nerveux ventral ( $s n$ ) forme une masse compacte ne dépassant guère la limite postérieure du céphalon et envoyant aux quatre paires de pattes et à l'abdomen les paires de filets nerveux qui leur correspondent $\left(n p^{2}, n p^{3}, n p^{4}, n a b d\right)$.

L'œil (oe) tripartite, vivement coloré en rouge-carmin, est très éloigné du cerveau (cer) auquel il se relie par d'assez gros faisceaux nerveux.

L'appareil génital femelle comporte une glande ovarienne et des oviductes producteurs formant dans l'ensemble un $U$ renversé sur les faces dorsale et latérale du tube digestif. Au-delà viennent les oviductes sécréteurs (ovd; qui fournissent la matière agglutinante pour les sacs ovigères. Leurs ouvertures (og) sont reportées sur la face dorsale du $1^{\mathrm{er}}$ segment abdominal, très rapprochées l'une de l'autre (fig. I4, og); leur grand axe est incliné d'environ $45^{\circ}$ sur l'axe longitudinal de l'animal. Le pore de fécondation (fig. 2 et fig. 14, po), ventral et médian, donne accès dans de larges canaux séminifères ( $c s)$, renflés latéralement en poires, prolongés ensuite par un canal plus délicat jusqu'à l'atrium précédant chaque orifice externe des oviductes.

Une observation que j'ai pu faire sur de petits Botryllophilus recueillis dans Polyclinum luteum GIARD tendrait à prouver que, comme l'a pensé Hesse, les males sont dans ce genre très semblables aux femelles, mais plus petits que ces dernières. J'ai figuré : Pl. XVI, fig. 3 et 4 , l'animal entier et son abdomen, et je puis faire observer seulement que ce spécimen n'est ni une jeune femelle caractérisée par l'oviducte rempli d'ovules en voie de formation, ni un mâle complètement adulte. Je crois pouvoir le considérer comme un mâle jeune, encore immature. Sa taille est de $0,7^{\mathrm{m}} / \mathrm{m}$. La $5^{\mathrm{e}}$ patte a, comme dans les jeunes femelles de même taille, la forme et la position ordinaires (Pl. XVI, fig. 4). Dans les femelles plus àgées, la forme de cet appendice se modifie graduellement à mesure qu'il se trouve reporté sur la face dorsale; elle présente avant la dernière mue l'aspect figuré. Pl. XVI, fig. 13, étant plus courte et plus massive qu'à l'état adulte.

\section{Botryllopbilus macropus, CANU.}

1891. Botryllophilus macropus; CANU, 34, p. 473.

\section{Planche XVI, Fig. I-I 4 .}

q. Taille : $1,6 \mathrm{~m} / \mathrm{m}$ environ.

Corps (fig. 1) de forme irrégulière, avec de nombreuses gibbosités que n'ont point signalées les auteurs précités dans les autres espèces du genre. Céphalon très effilé à son extrémité 
antérieure, avec un prolongement frontal grêle et quadrangulaire (fig. 5). Premier segment abdominal déformé par l'apparition de tubercules aux environs du pore de fécondation.

Antennules (fig. 5) 5-articulées, avec de fortes soies raides à base dilatée, spécialement sur les deux articles basilaires élargis.

Antennes (fig. 6) assez longues, coudées vers l'intérieur au niveau de l'article médian très court

Coxopodite mandibulaire (fig. 7) large et tranchant avec 3 grosses épines et une série de petits denticules. Exopodite très réduit (fig. 8, exp) et portant seulement 3 soies. Endopodite (enp) relativepnent considérable, avec 5 grosses soies. Dans le développement relatif de leurs éléments, les mandibules montrent une tendance bien nette à devenir des appendices simples.

Premières maxilles (fig. 9) avec 5 épines masticatrices sur le coxopodite et 7 grosses soies barbelées très courtes sur la portion terminale vibratile.

Secondes maxilles externes (fig. 10) et internes (fig. 11) bien séparées et très développées : les premières, avec 6 grandes épines marginales internes et 3 petites soies accessoires, présentent en outre une grosse soie plumeuse terminale ; les secondes avec le tégument chitineux très épais et résistant, surtout dans la région basilaire, n'ont pas de crêtes denticulées sur la face externe.

Pattes thoraciques des quatre premières paires (fig. 12) presque toujours dirigées vers l'intérieur et repliées sur elles-mêmes; semblables à droite et à gauche dans la même paire (1).

Cinquièmes pattes thoraciques (fig. 1) presque aussi longues que l'abdomen dont elles atteignent le dernier segment, avec une grande épine terminale, une soie terminale et une soie marginale, mais sans poils nombreux à leur bord externe.

Ovaires et œufs récemment pondus de couleur vert cendré. Sac ovigère unique aussi large que le corps, et comprenant de 12 à 15 œufs.

Malgré les quelques suppositions hasardées que l'on pourrait, à la rigueur, introduire d'après la description peu satisfaisante de HESSE, je considère l'espèce du Boulonnais comme distincte des espèces (B. ruber et $B$. virescens) de la rade de Brest. En effet, en l'absence de caractères morphologiques précis dignes d'entrer en comparaison, je trouve que le diamètre ou la couleur des ceufs pondus nous empêchent de considérer ces formes comme sûrement identiques.

Ainsi que je l'ai fait observer précédemment, la taille des cinquièmes pattes sépare également $B$. macropus des deux espèces établies par DElla Valle: $B$. notopus, et par Aurivillius: B. inflatus.

Botryllophilus macropus est assez rare dans la branchie d'un petit Molgulidé vivipare : Lithonephria eugyranda GIARD, très répandu dans la zone des Laminaires et des Bugula, parmi les rochers accessibles à marée basse dans le Boulonnais. B. notopus fut recueilli à Naples dans Distoma pancerii Della VAlle; et

(I) D'après Della VAlle, chez B. notopus D. V., ces pattes seraient dissemblables et asymétriques. 
B. inflatus dans Molgula ampulloides VAN BENEDEN, dans les régions arctiques (Voyage de Nordenskiöld sur la Véga) et sur les côtes septentrionales de Norvège. Distribution: Manche.

\section{Ascidicola, THORELL.}

1860. Ascidicola ; ThOReLL, 204, P. 57.

1863. Coiliacea; HeSsE, 110.

1866. Ascidicola, Th.; HeSsE, 110, p. 68.

1875. Ascidicola, Th.; Claus, 55, p. 350 et 351 .

1878. Ascidicola, Th.; Brady, 20, vol. 1, p. 1/45.

1883. Ascidicola, Th.; Auriviluius, 4, p. 91 et 92

Ascidicolidés femelies ( 1 ) incapables de nager, avec:

$I^{0}$ Le corps cylindrique et vermiforme, complètement segmenté.

$2^{0}$ Les œufs pondus en sacs ovigères externes, sans abri dans une cavité incubatrice close.

$3^{0}$ Les antennules courtes et épaisses dans toute leur longueur, 7 -articulées.

$4^{0}$ Les antennes 3 -articulées, pourvues de crochets pour servir à la reptation, mais sans aucune soie géminée sur le premier article.

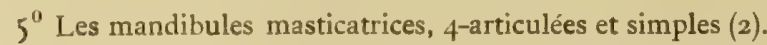

$6^{0}$ Les premières maxilles réduites à un lobe masticateur interne pourvu d'épines et à une région terminale externe simple et pourvue de quelques soies.

$7^{0}$ Les secondes maxilles très réduites, divisées en deux portions : l'externe presque simple sous forme d'une large griffe uncinée avec quelques courtes épines vers l'intérieur et un tubercule sétifere réduit vers la face externe et distale; l'interne sous forme d'un seul article lamellaire avec quelques soies courtes.

$8^{0}$ Les pattes thoraciques des quatre premières paires réduites, avec deux rames 2-articulées incapables d'un rôle natatoire par suite de l'absence des soies plumeuses habituelles ou de leur remplacement par de longues épines lisses, très rigides, servant à ramper.

$9^{0}$ Les pattes thoraciques de la cinquième paire sous forme de deux replis aliformes développés sur la face latéro-dorsale du segment correspondant et recouvrant l'orifice des oviductes de manière à constituer un abri pour les sacs ovigères externes.

$10^{0}$ Les pièces furcales courtes et cylindriques, avec quelques petits poils ou épines à leur extrémité postérieure, mais sans aucune soie barbelée.

(1) Il me paraît très douteux que la forme décrite et figurée par THOReLl comme le mâle d'Ascidicola appartienne à ce sexe ; je crois plutôt qu'elle est une jeune femelle immature.

(2) Par disparition complète de l'exopodite déjà si réduit chez Botryllophilus. 
Complètement séparé des Ascidicolidés précédents par l'aspect cylindrique, presque uniforme, du thorax et de l'abdomen, le genre Ascidicola est à mon avis des plus curieux parce qu'il établit un passage graduel vers les dégradations considérables que subissent les divers organes de ces animaux dans les parasites des Synascidies. En outre de la réduction affectant l'appareil buccal, dans lequel le rôle préhensile ou adhésif vient remplacer les aptitudes masticatrices, les pattes thoraciques de la femelle s'organisent nettement en vue de la reptation, ce qui s'accorde avec le parisitisme plus complet et plus intime de ces animaux.

Dès 1886, j’ai indiqué (ar, p. 319) l'interprétation morphologique qui doit être adoptée pour les lames ou saillies latéro-dorsales et sétifères de ces Copépodes ascidicoles; ce sont les cinquièmes pattes thoraciques qui donnent naissance à ces organes de protection pour les sacs ovigères. On ne peut admettre - comme l'ont fait jusqu'à présent tous les naturalistes — que dans la femelle, ces appendices fassent défaut chez Ascidicola TH., Enterocola van Ben., Enteropsis Auriv., Aplostoma Canu, pas plus qu'on ne l'admet pour Botryllophilus Hesse. L'apparition de ces organes, comparée dans les divers genres, confirme entièrement cette opinion.

\section{Ascidicola rosea, THORELL.}

1860. Ascidicola rosea ; Thorel, 204, p. 59-61, Tab. IX et X, fig. 13.

1863. Coiliacea setigera; HESSE, 110.

1878. Ascidicola rosea, Th.; BRAdY, 20, vol. 1, p. 145 et 146, Plate XXX, fig. I-IO.

1883. Ascidicola rosea, Th.; Auriviluius, 4, p. 106, Taf. VII, fig. 13-22.

C'est la seule espèce connue dans le genre. Bien que je ne l'aie point rencontrée dans le Boulonnais, je l'ai citée pour l'intérêt zoologique qu'elle présente, et pour l'étude que j'en ai pu faire sur des exemplaires récoltés par moi en d'autres points des côtes de France. En effet, elle était très abondante dans l'estomac de grands Ascidiella scabra Mueleer de la baie de Concarneau, où je l'ai trouvée en I886. Je l'ai obtenue également dans le même Tunicier envoyé par la Station zoologique d'Arcachon au laboratoire de zoologie de l'École normale supérieure. Hesse l'a recueillie dans la rade de Brest.

Thorell l'indique dans Ciona intestinalis M., Ciona canina M., Corella parallelogramma Alder, Ascidiella aspersa M.; Auriviluius dans Phallusiaz mentula M., Ph. obliqua ALDer et Ciona intestinalis M.; Norman (fide Brady dans Phal. mentula M. et Ascidia sordida (?).

Distribution: Mer du Nord, Atlantique, Méditerranée. 


\section{Agnatbaner, CANU.}

1891. Agnathaner; CANU, 34, P. 473 et 474.

Ascidicolidés mâles bien organisés pour la natation, avec :

$I^{0}$ Le corps cyclopoide complètement segmenté, comme dans Notodelphys, avec le céphalothorax aplati et ovalaire, l'abdomen cylindrique et étroit.

$2^{0}$ Les antennules légèrement préhensiles des deux côtés et composées d'un nombre assez restreint $(10-13)$ d'articles, avec des soies flexibles, lisses ou barbelées.

$3^{0}$ Les antennes 3 -articulées, terminées par une griffe préhensile et quelques soies, et portant sur le $1^{\text {er }}$ article deux soies géminées comme chez Notodelphys.

$4^{\circ}$ L'ouverture buccale étroite et placée au sommet d'un cône saillant destiné à faciliter le fonctionnement d'un œsophage aspirant les liquides nourriciers.

$5^{\circ}$ Les mandibules biramées, pourvues de longues soies barbelées sur la région terminale semblable à celle de Notodelphys, et présentant dans le coxopodite une réduction considérable de la saillic masticatrice incapable de toute action triturante.

$6^{0}$ Les premières maxilles bien développées dans la région terminale comme chez Notodelphys, etc., réduites dans leur portion masticatrice et possédant parfois un épipodite sessile avec deux soies barbelées inégales.

$7^{0}$ Les secondes maxilles réduites, mais divisées encore en deux portions interne et externe.

$8^{\circ}$ Les pattes des quatre premières paires organisées pour la natation avec les deux rames 3-articulées, richement garnies de soies plumeuses et d'épines à bords denticulés, comme chez Notodelphys.

$9^{\circ}$ Les cinquièmes pattes courtes, lamellaires et 2-articulées, comme chez Notodelphys.

I $\mathrm{O}^{0}$ Les pièces furcales de développement moyen, terminées par de longues soies barbelées en nombre ordinaire.

Ayant longuement insisté dans les pages précédentes sur les déductions morphologiques et taxonomiques qu'entraine l'organisation si intéressante de ces Copépodes, il suffira de rappeler qu'ils sont connus seulement dans le sexe mâle, vivant en semi-parasites dans divers Tuniciers. Néanmoins, ces animaux établissent nettement le passage des Ascidicolidés gnathostomes et supérieurs, aux Ascidicolidés suceurs dépourvus de mâchoires masticatrices. Car dans Agnathaner, les mâchoires des diverses paires persistent avec tous les rudiments d'une organisation normale, sans pouvoir fonctionner utilement en vue de la trituration d'une nourriture solide. Daiss ce genre, apparait encore la disposition buccale et pharyngienne propre aux espèces d'Ascidicolidés suceurs. Par sa forme, par ses aptitudes 
natatoires et l'organisation des appendices locomoteurs, le genre Agnathaner se rapproche beaucoup de $N$ otodelphys, c'est-à-dire des Ascidicolidés ayant conservé la mobilité la plus grande avec le plus grand nombre de caractères primitifs; mais cette ressemblance qu'il partage avec les mâles d'autres Copépodes très dégradés dans le sexe femelle, n'indique nullement une parenté particulièrement étroite entre Agnathaner et Notodelphys.

Une curieuse disposition anatomique, commune aux deux espèces connues dans le genre Agnathaner, consiste dans la présence sur les replis pleuraux du segment céphalique, d'une quantité considérable d'orifices punctiformes garnis d'une petite soie tactile (Pl. XVII, fig. 2 et fig. I2). A ces orifices très nettement circulaires, correspondent de grosses cellules piriformes, comme on les connait en situation analogue dans beaucoup de Copépodes.

\section{Agnatbaner typicus, CANU.}

1891. Agnathaner typicus; Canu, 34, p. 474.

Planche XVII, Fig. i-io.

$\sigma$. Taille : $1,27 \mathrm{~m} / \mathrm{m}^{*}$

Forme (fig. 1) très allongée, avec le céphalon presque semi-circulaire, arrondi dans la région antérieure, le cinquième segment thoracique et l'abdomen étroits et cylindriques. Pièces furcales plus longues que le segment précédent et portant une soie latérale plumeuse et une soie dorsale presque aussi longues qu'elles-mêmes. Soies furcales terminales richement barbelées ou plumeuses, la terminale interne plus longue, à peu près égale à l'abdomen entier. Prolongement frontal (fig. 2) triangulaire et replié sur la face ventrale.

Antennule (fig. 2) ne dépassant point le céphalon en longueur, 13-articulées, les deux derniers articles étant repliés par une articulation géniculée sur le $11^{\mathrm{e}}$, et les $4^{\mathrm{e}}, 5^{\mathrm{e}}, 6^{\mathrm{e}}, 7^{\mathrm{e}}$, $8^{8}, 9^{6}$ et $10^{8}$ disposés pour donner à l'antennule une courbure analogue à celle de cet appendice chez les Cyclopidés mâles. Tous les articles portent à leur bord antérieur des soies flexibles, particulièrement développées et souvent barbelées, et les $10^{\mathrm{e}}$ et $13^{\mathrm{B}}$ sont encore munis de filaments sensoriels très allongés.

Antenne (fig. 3) 3-articulée, avec le dernier article long et supportant une forte épine uncinée avec l'extrémité très effilée.

Mandibule (fig. 6) avec le coxopodite très réduit dans sa région masticatrice, mais saillant et renflé dans sa région articulaire, de sorte que l'extrémité masticatrice réduite n'atteint pas à l'orifice buccal.

Première maxille (fig. 7) avec le coxopodite allongé pourvu d'une très faible saillie masticatrice armée de trois épines assez grêles, avec l'endopodite et l'exopodite garnis de quatre soies plumeuses.

Seconde maxille externe (fig.8) réduite et terminée par une longue épine très effilée et denticulée sur le bord interne. 
Seconde maxille interne (fig. 9) constituée par un faible moignon vaguement 2-articulé et terminé par deux soies en voie de régression.

Cinquième patte thoracique (fig. 10) très courte, avec le premier article large, soudé au tronc et porteur d'une soie à son bord externe; avec le second article large, dirigé vers la ligne médiane et terminé à son extrémité distale par une longue soie flexible extérieure et une très courte épine intérieure accompagnée d'une rangée de fins poils.

J'ai recueilli un petit nombre d'exemplaires mâles de ce Copépode en semiparasite dans la cavité branchiale de Styelopsis grossularia VAN BENEDEN, provenant des rochers d'Audreselles, mais je n'ai pu, malgré mes efforts, en récolter la femelle. Par analogie avec ce que j'ai fait connaître précédemment dans Enterocola, Aplostoma et Enteropsis, je pense que les deux sexes de ce copépode diffèrent notablement dans leurs caractères extérieurs. Néanmoins, dans le Tunicier qui sert d'hôte aux mâles d'Agn. typicus, je n'ai trouvé aucune femelle de Copépode dégradé qui puisse, par sa taille et ses caractères morphologiques ou embryonnaires, être rapprochée du mâle ici décrit.

Distribution : Manche.

\section{Agnathaner minutus, CAnU.}

1891. Agnathaner minutus; CaNu ; 34, page 474.

Planche XVII, Fig. 1 I-I6.

万. - Taille $: 0,8 \mathrm{~m} / \mathrm{m}$.

Forme (fig. 11) cyclopoïde, avec le céphalon étroit dans sa partie antérieure et presque triangulaire. Pièces furcales un peu plus longues que le segment précédènt; soie furcale termmale interne plus longue et égale aux quatre derniers segments abdominaux. Prolongement frontal (fig. 12) pointu, aux bords épaissis, replié sur la face ventrale.

Antennule (fig. 12) 10-articulée, avec le dernier article géniculé sur le $9^{\circ}$, présentant une courbure dans les $3^{\mathrm{e}}, 4^{\mathrm{e}}, 5^{\mathrm{e}}, 6^{\mathrm{e}}, 7^{\mathrm{e}}$ et $8^{\mathrm{\theta}}$ articles, avec de grandes soies flexibles sur le bord antérieur de tous les articles et des filaments sensoriels réfringents sur les $9^{\circ}$ et $10^{\circ}$.

Antenne (fig. 12) 3-articulée, assez grêle, avec une longue griffe terminale et point de soies gémi.lées.

Mandibule ( fig. 13, md ) avec le coxopodile très réduit, le basipodite très long et les deux rames garnies de quatre soies plumeuses dépassant en longueur l'appendice entier.

Première maxille (fig. $13, m x^{1}$ ) avec de courtes pointes saillantes sur le lobe masticateur du coxopodite, avec la région terminale biramée réduite à deux simples lames garnies de soies plumeuses très longues.

Seconde maxille réduite à deux moignons tuberculiformes inermes, l'un externe plus large, l'autre interce très petit. 
Pattes natatoires larges et puissantes, avec de longues épines en pjignard finement denticulées sur les bords, et des soies plumeuses bien développées.

Cinquièmes pattes 2-articulées, avec une seule soie rigide à l'extrémité du second article et une soie barbelée à l'extérieur du premier.

J'ai recueilii seulement quelques exemplaires mâles de ce petit Copépode dans un seul cormus de Circinalium concrescens Giard, et je n'ai pu y découvrir la forme femelle correspondante. Par tous ses caractères, Agn. minutus se distingue nettement du màle des parasites plus fréquents dans le même Tunicier.

Distribution : Manche.

\section{Enterocola, P. J. Van Beneden.}

1860. Enterocola; VAN BENEDEN ; 12, page 151 .

1875. Enterocola, V. Ben.; Claus; 55, page 351.

1883. Enterocola, V. Ben.; Della Valle; 70 , pages 342 et suiv.

1886. Enterocola, V. Ben.; Canu ; 27, page 373.

1891. Enterocola, V. Ben.; Canu; 34, pages 468 et 474.

Non Enterocola, V. Ben.; Norman : 157, et Brady: 20, vol. 1 .

Ascidicolidés dont les mâles sont organisés pour la natation et les femelles pour la reptation. avec :

$I^{0}$ Le corps complètement segmenté, cyclopoïde comme dans Notodelphys pour le $\sigma$, vermiforme ou éruciforme pour la $q$, avec le céphalothorax 6- $\left(\sigma^{7}\right)$ ou 5-( $(q)$. segmenté, un peu plus large que l'abdomen 5- $\left(0^{7}\right)$ ou 4 - ( $(+)$ segmenté.

$2^{0}$ Les œufs pondus par la femelle en deux sacs ovigères externes, sans abri dans une cavité incubatrice.

$3^{0}$ Les antennules constituées : chez le $\sigma^{\top}$ par une suite de huit articles, cylindriques et sétifères; chez la $\&$ par une lame aplatie, très courte, vaguement 3 -articulée.

$4^{0}$ Les antennes : 3 -articulées et préhensiles chez le $\sigma^{\nearrow}$; lamellaires, simples, et sans crochets adhésifs chez la ${ }_{+}$.

$5^{0}$ La bouche placée au sommet d'un cône buccal peu saillant, et complètement dépourvue de mandibules; celles-ci tombées en régression au cours du développement.

$6^{0}$ Les premières maxilles 2-articulées: terminées par six longues soies plumeuses et sans fonctions masticatrices chez le $\sigma^{\nearrow}$; masticatrices, terminées par six courtes épines denticulées sur le deuxième article, avec une grosse épine articulée sur le bord antérieur du premier article chez la + .

$7^{0}$ Les secondes maxilles simples, 2-articulées : terminées par une longue soie plumeuse et sans rôle préhensile ou masticateur chez le o'; préhensiles et masticatrices dans leur région terminale, avec une petite soie appendiculée à l'extrémité distale d second article chez la 9 . 
$8^{0}$ L.es quatre premières pattes thoraciques biramées dans les deux sexes: avec les deux rames natatoires le plus souvent 3 -articulées chez le $\sigma^{\nearrow}$; avec les rames simples, courtes, sans soies natatoires chez la $f$, ou l'exopodite est résistant et unciné et l'endopodite lamelleux avec deux soies glabres.

$9^{\circ}$ Les cinquièmes pattes : petites et 2 -articulées chez le $\sigma$, où elles rappellent $N$ otodelphys; longues, lamellaires, terminées par deux soies et en forme de cuillerons recouvrant les sacs ovigères chez la $q$, où elles rappellent Ascidicola.

$10^{0}$ Les pièces furcales assez courtes et massives : terminées par de longues soies plumeuses atteignant à la longueur de l'abdomen chez le $\sigma$; totalement dépourvues de soies et d'épines terminales chez la $q$.

Comme je l'ai longuement établi dans les parties morphologique et embryologique de ce travail, les auteurs antérieurs : van Beneden, Claus, et surtout Della VALLE, ont méconnu ou mal interprété les particularités si curieuses du genre Enterocola. Je ne puis revenir de nouveau sur les descriptions fournies précédemment à propos des divers organes externes de ces Copépodes, et je renvoie le lecteur aux détails donnés plus haut. Néanmoins, certaines particularités anatomiques ignorées des naturalistes méritent encore d'être signalées.

Quand on observe par la face dorsale un exemplaire femelle d'Enterocola fulgens VAN BEN., s'il est bien vivant et récemment extrait de son hôte, on peut distinguer (PI. XIX, fig. 12) dans le tissu conjonctif de l'animal une grande quantité de cellules graisseuses, de forme irrégulièrement polygonale et qui se détachent au-dessus des oviductes remplis d'œufs mûrs, comme des points brillants plus vivement colorés. A la face dorsale, ces cellules paraissent irrégulièrement disposées dans toute la cavité générale, mais il n'en est pas de même sur la face ventrale. En effet, si l'on observe ventralement un exemplaire femelle d'Ent. fülgens décoloré par un séjour prolongé dans l'eau de mer au dehors de son hôte et par l'action de l'alcool légèrement acidulé, on reconnaît (fig. 2) la présence de grandes cellules conjonctives graisseuses disposées à droite et à gauche, en amas importants près de la base des pattes dans chaque segment thoracique. Dans le céphalon et l'abdomen, des cellules semblables, mais plus petites, sont disséminées çà et là sur les côtés du corps.

En outre, dans les $2^{\mathrm{e}}$ et $3^{\mathrm{e}}$ segments thoraciques, sur les côtés des oviductes gonflés d'œufs, existent deux longues bandes cellulaires étroites $(\boldsymbol{n}\rangle$ de coloration grisàtre, qui constituent une autre catégorie de corps graisseux. Dans ces bandes, les cellules sont très grosses et contiennent un protoplasme plus finement granuleux que celui des ovules en voie de maturation, avec un noyau arrondi très petit. Leur constitution est d'ailleurs assez semblable à celle des cellules groupées à la 
base des pattes; mais leur forme est ellipsoïdale, et non point irréguliérement polygonale. La présence d'un corps graisseux ainsi localisé dans divers points de la cavité du corps a été constatée déjà pour d'autres espèces de Copépodes par Claus (55), Heider (107), Giesbrecht (90) et List (139).

Chez la femelle, l'appareil génital n'a pas été figuré avec exactitude par Della VALLE. Il comprend (PI. XVIII, fig. 2 et I 2 ) un ovaire germigène de forme conique, placé dorsalement à l'intestin dans les $1^{\mathrm{er}}, 2^{\mathrm{e}}$ et $3^{\mathrm{e}}$ segments thoraciques; cet ovaire est transparent. les cellules ovulaires y sont toujours petites. A droite et à gauche de son extrémité antérieure, se détachent deux larges oviductes germigènes, à paroi mince et extensible, qui se recourbent sur les côtés de l'intestin, traversent les $2^{\mathrm{e}}, 3^{\mathrm{e}}$ et $4^{\mathrm{e}}$ segments, au-delà desquels ils sont continués par des canaux plus étroits, à paroi cellulaire épaisse et servant de glande collétérique pour la constitution des sacs ovigères durant la ponte. Mais il n'existe point, dans Enterocola, de glande collétérique séparée en forme de gouttière, comme le signale SCHIMKEviTSCH (188) dans Enteropsis dubius SchimkevitsCH. Les oviductes se terminent aux deux orifices génitaux saillants $(\circ g)$ placés sur les côtés de la limite antérieure du premier segment abdominal, à l'angle formé par l'insertion des cinquièmes pattes thoraciques sur le tronc. Dans l'atrium génital précédant chaque ouverture des oviductes, viennent déboucher, vers l'intérieur, les canalicules séminifères minces et cylindriques $(c s)$, qui divergent à droite et à gauche du large réceptacle dans lequel le pore de fécondation ( $p o$ ) permet l'introduction des spermatozoïdes après l'accouplement. Le pore de fécondation, unique et ventral, est une large fente transversale placée au milieu du premier segment abdominal de la femelle.

Le système nerveux de la femelle se compose, sur la face ventrale et au-dessous de l'intestin, d'un amas cellulaire d'aspect granuleux (Pl. XVIII, fig. 2, sn), au contour légèrement ondulé, effilé à son extrémité postérieure et qui s'étend depuis la paroi œsophagienne jusqu'au milieu du $3^{\mathrm{e}}$ (et non du $2^{\mathrm{e}}$, ainsi que l'a figuré DelLA VALLE : loc. cit., fig. 4, n) segment thoracique. Il détache à droite et à gauche les rameaux nerveux des appendices et se continue au-delà de son extrémité postérieure par un cordon nerveux abdominal ( $n a b d$ ) assez épais et simple, que j'ai pu suivre jusqu'au niveau des pattes de la quatrième paire, sans le voir se bifurquer. Cette disposition du système nerveux ventral rappelle beaucoup ce qu'a décrit List (139) au sujet de Gastrodelphys.

De mème que chez Agnathaner, les replis pleuraux du céphalon, dans le màle d'Ent. fulgens, sont perforés d'une innombrable quantité d'orifices punctiformes, où s'attachent des poils sensoriels (P1. XVIII, fig. 4 et 5) particulièrement allongés et touffus vers l'angle latéro-postérieur. La femelle est d'ailleurs entièrement 
dépourvue d'organes de ce genre, et l'on peut y voir une divergence sexuelle de nature à augmenter les aptitudes sensorielles du mâle.

Le genre Enterocola est, en ce qui concerne les femelles, un véritable parasite des Synascidies, particulièrement des Aplidiens. Dans les cormus qu'il infeste, on le trouve enfoncé dans l'estomac des ascidiozoïdes et c'est seulement à la suite de contractions accidentelles qu'il passe dans la cavité branchiale du Tunicier. Après la ponte, l'extrémité postérieure du Copépode fait saillie dans la branchie, et les oufs s'y trouvent baignés d'un continuel courant d'eau; leur situation, relativement moins abritée dans cette cavité à parois délicates, explique la fréquente séparation des sacs ovigères durant la dissection des cormus parasités.

La comparaison des formes décrites par HESSE (110) d'une façon souvent bizarre, comme parasites des Ascidies composées de la rade de Brest, avec les espéces méthodiquement étudiées par des observateurs plus exercés, n'est point sans présenter d'énormes difficultés. Après avoir observé un certain nombre de Copépodes parasites des Synascidies, je ne puis pourtant m'associer à l'opinion si légèrement introduite par Della Valle (76, page 243, note 1), ni considérer les divers genres ascidicoles: Andranesius Hesse, Polycliniophilus H., Biocryptus H., Lygephilus H., Cryptopodus H., Hypnodes H., Mychephilus H., Narcodes H., Polyoon H. et Pachynesthes H., comme établis d’après une connaissance erronée sur le seul Enterocola fulgens vaN BEN. Certains genres établis par HeSSE s'attachent à des Copépodes bien différents d'Enterocola, mais ils n'en restent pas moins très problématiques par suite de l'incompétence du fondateur. C'est ainsi que Cryptopodus s'écarte d'Enterocola pour se rapprocher d'Enteropsis Aurivillius et d'Aplostoma Canu. Au contraire, Biocryptus semble bien se confondre avec le genre de VAN BENEDEN. Mais des conclusions plus rigoureuses - d'après de grossières ressemblances et sans le secours d'indications morphologiques ni éthologiques précises - seraient bien éloignées de la certitude scientifique! Il me semble plus rationnel, dans de semblables conditions, de laisser les formes considérees dans la foule des espèces incertaines sans tenter prématurément des homologations hasardées. Une étude nouvelle et complète des types observés par HesSE pourrait seule, si elle était possible, fixer notre opinion d’une manière suffisante.

\section{Enterocola fulgens, VAN BenEDEN.}

1861. Eutcrocola fulgens, VAN BENEDEN, 12, pages 151-160, avec 1 pl.

8883. ? Enterocola fulgens, V. Ben.; Della VAlLE, 76, pages 245-247, fig. 3-10.

1891. Entirocola fulgens, V. Ben,; Canu; 34 , pages 468,469 et 474 .

Planche XVIII, Fig. I-I2; Planche XIX, Fig. 12 el 13 
Taille: $q: 0,9-1,4 \mathrm{~m} / \mathrm{m} ; \sigma^{7}: 0,65-0,90^{\mathrm{m}} / \mathrm{m}$.

१. Forme générale large et ramassée (fig. 1), abdomen relativement court, à peine égal au quart du céphalothorax. Pièces furcales en forme de lamelles droites et légèrement aplaties, plus courtes que le dernier segment. Extrémité antérieure du céphalon arrondie; prolongement frontal court et large (fig. 2). - - . Pièces furcales larges, plus longues que le dernier segment, avec 4 grandes soies barbelées terminales (fig. 8) et de petites soies dorsale et marginale. Rostre frontal saillant et pointu, légèrement fendu à șon extrémité distale.

Appendices céphaliques sans caractères spécifiques. Dans le $\sigma$, les antennules 8-articulées ont les 2 articles basilaires plus gros et plus longs, avec de longues soies glabres, et l'article terminal pourvu à son bord distal antérieur d'un long filament sensoriel réfringent accolé à une longue soie glabre.

Pattes thoraciques des 4 premières paires larges et courtes chez la $q$, avec une base 2-articulée et deux rames simples: l'exopodite étant plus résistant que l'endopodite et renflé à sa base, avec l'extrémité amincie en épine légèrement courbée vers l'extérieur. Epine terminale de l'exopodite à peine plus longue et plus solide dans la $3^{\mathrm{e}}$ paire (fig. 11) que dans les autres (fig. 10). - Exopodite de la $\mathrm{I}^{\text {re }}$ patte du. J (fig. 6) 2-articulé, avec des épines marginales externes ou terminales et des soies barbelées internes.

Pattes de la $5^{\mathrm{B}}$ paire larges, courtes (fig. 9) et terminées par deux petites soies semblables, à peine visibles.

Tégument chitineux très mince, mais conservant encore l'apparence très nette de la segmentation du corps ; avec des traces de replis pleuraux dans les segments céphalique et thoraciques, où on peut encore les distinguer aisément par l'action dissolvante de la potasse caustique à froid. Sur la chitine de la face ventrale et des appendices thoraciques, on constate aussi la présence d'un grand nombre de petits poils disposés en séries régulières (Pl. XIX, Fig. 11).

Sacs ovigères gros et arrondis à leur extrémité distale, contenant un petit nombre d'œufs, mais atteignant jusqu'à la moitié de la taille de la femelle adulte. Cufs mûrs et pondus de couleur rouge.

Le mâle est semi-parasite et fréquent au printemps et en septembre; la femelle est parasite et commune en toutes saisons dans le canal digestif de Polyclinum luteum GIARD, Synascidie très répandue sur tous les rochers de la côte dans la zone des Laminaires. VAN BENEDEN dit avoir trouvé la femelle à Ostende, dans Aplidium ficus SAV. et ficoides V. BEN., Ascidies probablement identiques à celle du Boulonnais qui abrite le mème parasite; il n'existe à mon avis aucun doute sur l'identité des Copépodes étudiés par ce naturaliste et par moi. Grace à l'imperfection des descriptions de Della Valle, il peut rester quelque hésitation au sujet de l'espèce de Naples, surtout en ce qui concerne les caractères fournis par les antennes et les pattes thoraciques; la forme générale et la furca ressemblent à ceiles d'Ent. fulgens type. Les Tuniciers qui abritent à Naples cet Enterocola sont 
divers Aplidiens (Aplidium cristallinum D. V et $A$. gibbulosum D. V) et Didemniens.

Distribution: Mer du Nord, Manche, Méditerranée (?).

\section{Enterocola betencourti, CANU.}

1875. (?) Entcrocola, sp.; CLAuS, 55, pages 351 et 352, Taf. XXIV, fig. 31.

1891. Enterocola hetencourti; CANU, 34, pages 474 et 475.

Planche XVII, Fig. 13 et 14 ; Planche XX, Fig. $1-4$.

б. Taille: $1,2-1,4 \mathrm{~m} / \mathrm{m}$.

Forme étroite et allongée (fig. 1); abdomen égalant en longueur le tiers du céphalothorax. Extrémité antérieure du céphalon légèrement effilée, avec le rostre frontal long et étroit (tig. 13 et 14).

Antennules, antennes et pièces buccales comme dans E. fulgens. Pattes thoraciques des quatre premières paires très allongées, spécialement dans l'endopodite (fig. 2 et 3); exopodite large à la base, rétréci vers l'extrémité en forme d'épine, avec sa région distale rigide et particulièrement allongée dans la $3^{\mathrm{e}}$ paire (fig. 3 ).

Pattes thoraciques de la $5^{\mathrm{e}}$ paire en forme de cuillerons assez développés et pourvus a leur bord distal d'une épine uncinée et d'une petite soie grêle (fig. 4).

Pièces furcales (fig. 4) plus longues que le segment précédent, légèrement recourbées vers l'extérieur, avec la forme de lames irrégulières trois fois et demie ou quatre fois plus longues que larges.

Sacs ovigères relativement grêles, légèrement effilés vers leur extrémité distale, avec un petit nombre d'œufs rouges.

Cette espèce est bien distincte d'E. fulgens, dans son aspect général et dans l'organisation de diverses parties du corps. Par l'allongement des appendices, spécialement des pattes de la $3^{\mathrm{e}}$ paire, l'espèce observée par Claus se rapproche plutòt d' $E$. betencourti; toutefois la forme des pièces furcales est un peu différente.

Enterocola betencourti est commun dans Aplidium ficus Savigny, spécialement dans la variété foncée, verdâtre, qu'on trouve parfois aux Roches Bernard et dans les fonds côtiers du Boulonnais. Je l'ai recueilli également en Bretagne, dans les cormus d'Aplidium zostericola GIARD, qui abondent à Concarneau et aux Iles Glénans.

Distribution: Manche, Atlantique, Méditerranée (?).

\section{Enteropsis, Aurivillius.}

1868. Enterocola, V. Ben.; NORMAN, 157, page 300.

1878. Entirocola, V. Ben.; Brady, 20, vol. 1, page 147.

1885. Enleropsis; Aurivilulus, 4, pages 237 et 238.

1885. Haligryps; Auriviluius, 4 , pages 242 et 243. 
1886. Enteropsis, Aur.; CANu, 27, pages 365 et suiv.

1889. Enteropsis, Aur.; Sснімкеvitsch, 188, pages 75 et suiv.

1891. Enteropsis, Aur.; Canu, 34, page 475.

Ascidicolidés nageurs dans le sexe $\varnothing$ et rampeurs dans le sexe $\{$, avec:

$\mathrm{I}^{0}$ Le corps allongé et vermitorme dans la femelle, cyclopoïde dans le mâle comme dans

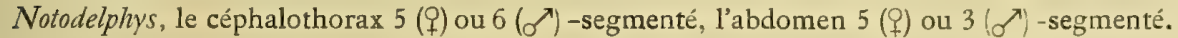

$2^{0}$ Les œufs réunis après la ponte en deux sacs ovigères externes, non abrités dans une cavité incubatrice.

$3^{0}$ Les antennules pluriarticulées et sétifères dans le mâle, 2-articulées avec quelques courtes épines dans la femelle.

$4^{0}$ Les antennes 2-articulées et épaisses dans la femelle, 3-articulées dans le mâle; préhensiles et terminées par deux épines recourbées dans les deux sexes.

$5^{\circ}$ Les mandibules longues et non masticatrices dans les deux sexes, biramées et terminées par de longues griffes plus ou moins effilées dans chaque rame.

$6^{0}$ Les premières maxilles totalement disparues à l'état adulte dans les deux sexes.

$7^{0}$ Les secondes maxilles sans portions distinctes interne ni externe, en forme de griffe recourbée, articulée, avec une petite soie appendiculée à l'extrémité distale du second article.

$8^{0}$ Les premières pattes thoraciques biramées dans les deux sexes, avec les rames courtes et simples dans la femelle, où l'exopodite est inerme et l'endopodite armé de trois ou quatre crochets uncinés peu développés; avec les rames longues, 2- ou 3-articulées dans le mâle où elles portent des épines denticulées et des soies plumeuses sur leurs bords latéraux et terminal.

$9^{0}$ Les $5^{\text {es }}$ pattes thoraciques très réduites dans les deux sexes; constizuées chez la femelle par deux bourrelets peu saillants non articulés, presque confondus avec le tronc et portant deux courtes soies.

$10^{0}$ Les pièces furcales courtes dans les deux sexes, avec des soies plumeuses dans le $\sigma^{\prime}$, sans soies plumeuses, avec un crochet terminal ou totalement inermes dans la femelle.

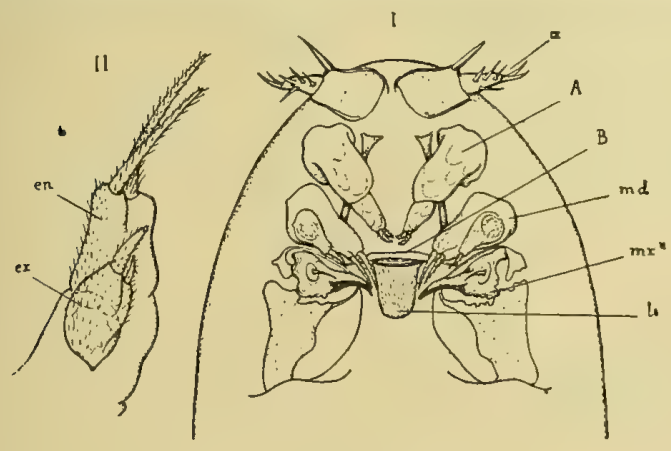

Fig. 17. - Enteropsis pilosus. CANU; femelle. I Céphalon vu par la face ventrale: $m d$, mandibule. II Mandibule plus fortement grossie.

Les particularités intéressantes du genre Enteropsis, déjà signalées plus haut au point de vue de leur morphologie, résident principalement dans le dimorphisme sexuel et dans la constitution des appendices buccaux. Sans insister longuement encore, je signalerai cependant pour son intérêt au point de vue systématique, la constitution des mandibules (voir ci-contre : fig. $17 ;$ md) avec les deux rames saillantes et bien dévelop- 
pées. Il convient d'observer que, dans ces appendices, l'exopodite se présente toujours plus court que l'endopodite, indiquant par ce fait une tendance rétrograde que nous verrons franchement affirmée dans un genre voisin, Aplostoma Canu.

Le genre Enteropsis AURIVILlius comporte actuellement un petit nombre d'espèces, parasites dans le sexe $q$, semi-parasites dans le sexe $\sigma$, et qui vivent dans les Ascidies simples ou sociales. Il n'a point de représentant connu (I) jusqu'à ce jour dans les Synascidies.

\section{Enteropsis pilosus, CanU.}

1886. Enteropsis pulosus; CANU, 27, pages ;65-372, PI. III, fig. 1-5.

1. Taille: $2,5^{\mathrm{u}} \% \mathrm{~m}$.

Corps allongé, presque aussi large dans la région abdominale que dans la région thoracique. Pièces furcales inermes.

Epines des antennules longues et rigides, effilées à leur extrémité.

Antennes, mandibules et tégument ventral postérieur à la bouche, garnis de poils courts et raides, disposés sans ordre ou bien arrangés régulièrement suivant plusieurs courbes espacées.

Second article de la seconde maxille long et mince, fonctionnant comme une griffe préhensile puissante.

Pattes thoraciques peu allongées.

Eufs mûrs et récemment pondus de couleur pâle, rosée. Sacs ovigères longs et cylindriques, avec un grand nombre d'œufs.

J'ai observé un seul exemplaire femelle de ce curieux Copépode, recueilli dans le sac branchial d'une Ascidie sociale: Diazona hebridica FORBES draguée en 1886 , au large du récif de la Jument, près des Iles Glénans, dans le voisinage de Concarneau. Par sa taille et l'ornementation du tégument sur les appendices buccaux, il se distingue nettement des espèces voisines: Enteropsis sphinx Auriviluius et Ent. dubius Schimkevitsch, atteignant jusqu'à 7 et $8 \mathrm{~m} / \mathrm{m}$ de longueur. Quant à Enteropsis eruca Norman, sa description est tellement incomplète, qu'elle ne peut ètre comparée utilement aux précédentes.

Distribution: Océan Atlantique.

\section{Aplostoma, C. $\mathrm{Anv}$.}

1886. Aplostoma; CanU, 27, pages 313 et suivantes.

1891. Aplostoma; Canu, 34, pages 474 et 475.

(1) A moins que le genre Cryptopodus Hesse ne se confonde avec lui, ce qui reste très douteux. 
Ascidicolidés nageurs dans le sexe $\sigma$, rampants dans le sexe $q$, avec:

$I^{0}$ Le corps complétement segmenté, cyclopoïde chez le mâle, presque vermiforme chez la femelle 8-segmentée.

$2^{0}$ Les œufs pondus en deux sacs ovigères externes, non abrités dans une cavité incubatrice.

$3^{\prime 3}$ Les antennules courtes : 4-articulées, sétifères et sensorielles chez le mále ; plus réduites et vaguement 5-articulées chez la femelle.

$4^{0}$ Les antennes 2-articulées, rendues adhésives par l'existence de quelques épines plus ( $\sigma$ ) ou moins ( $($ ) longues sur le bord du second article.

$5^{0} \mathrm{La}$ bouche située au sommet d'un cône buccal et disposée pour la succion, avec les mandibules réduites, simples et non masticatrices, placées sur les côtés de l'éminence buccale.

$6^{0}$ La première maxille totalement disparue.

$7^{0} \mathrm{La}$ seconde maxille simple et réduite à un appendice préhensile puissant, 3- ou 4-articulé, avec les $3^{\circ}$ et $4^{*}$ articles plus grêles, surmontés d'une épine recourbée et formant pince contre le $2^{\mathrm{e}}$ article renflé.

$8^{0}$ Les quatre premières pattes thoraciques biramées dans les deux sexes; avec les rames articulées et natatoires chez le mâle; plus courtes et non natatoires chez la femelle, où elles comprennent, comme chez Enteropsis, un exopodite lamellaire inerme et un endopodite incurvé avec trois épines marginales ou distales.

$9^{0}$ Les $5^{\text {es }}$ pattes thoraciques vaguement 2-articulées chez le mâle, simples et non articulées chez la femelle; , où elles forment deux petites saillies reportées vers la face dorsale, sur les côtés du dernier segment thoracique, et où elles sont terminées par deux petites soies lisses.

$10^{0}$ Les pièces furcales courtes, terminées par de longues soies hyalines chez le mâle, par une épine uncinée et quelques poils chitineux très courts chez la femelle, où elles sont utilisées dans la reptation.

Le genre Aplostoma Canu est en réalité très voisin du genre Enteropsis Aur.; comme je l'indiquais dès 1886, il peut être opposé au genre Enterocola V. BeN. par toute une série de caractères dont les plus importants consistent encore dans la structure différente des appendices, structure morphologiquement étudiée en détail dans la première partie de ce travail. Le dimorphisme sexuel très accentué, la disparition de la première maxille, relient étroitement Aplostoma et Enteropsis; dans le premier, l'exopodite mandibulaire est presque entièrement disparu et à peine distinct, la mandibule entière a subi une réduction considérable: tandis que dans le second, les deux rames mandibulaires demeurent bien reconnaissables. l'externe étant néanmoins plus faible que l'interne. 
Très commun dans certains points des côtes de France, le genre Aplostoma peut ètre très répandu sans avoir toutefois attire l'attention des zoologistes, en raison de sa taille très réduite. Dans les nombreux genres observés par HESSE, un seul semble s'en rapprocher; c'est le genre Cryptopodus, établi en 1865 (loc. cit., $5^{e}$ série, tome IV, page 237 , Pl. VII, fig. 2 et 3 ). Mais la description de cette forme laisse subsister de sérieux doutes sur la synonymie, spécialement en ce qui concerne le nombre des paires d’appendices existant dans la région céphalique. Hesse décrit, dans Cryptopodus flawus : une paire d'anternes depourvues de soies et de piquants, que nous accepterons comme les antennules; une premiere paire de pattes-máchoires avec une ou plusieurs griffes terminales, qui sont peut-être les antennes; une deuxième paire de pattes-màchoires large à sa base, comprimée latéralement et terminée par une griffe; et une troisième patte de méme conformation. Ceci nous donne à penser que Cryptopodus est réellement pourvu de deux paires d'appendices buccaux, bien développés, tels que HESSE ait pu les distinguer sans l'emploi des méthodes modernes et des microscopes puissants. Par suite, ce genre se rapproche d'Enteropsis plutòt que d'Aplostoma; c'est au premier chef un genre incerta sedis, et je ne me crois nullement autorisé

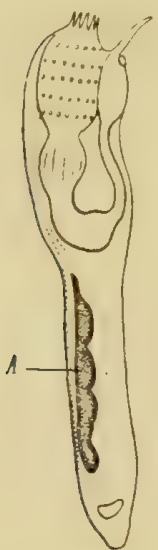

Fig. 18. - Figure schéinatique indiquant la place occupée dans l'ascidiozoide par une femelle d'Aplostoma (d) logée dans l'épicarde. à le faire rentrer, même avec doute, dans la synonymie d'Aplosloma.

A l'encontre d'Enteropsis Auriv., le genre Aplostoma est jusquà présent un parasite exclusif des Synascidies, particulièrement des genres Morchellium et Circinalium. Son mode de vie est lui-mème des plus dignes d'intérêt: au lieu d'habiter le tube digestif et la cavité respiratoire, comme tous les autres Ascidicolidés vermiformes, la femelle d'Aplostoma leur est extérieure. Elle se meut, à la façon d'un ver parasite, dans un espace voisin de la branchie, formant une cavité où elle peut se contourner dans tous les sens. De plus, lorsque cette femelle a pondu ses œufs, on peut constater fréquemment sa présence dans l'abdomen de l'ascidiozoïde, au-delà de l'anse intestinale (PI. XX, fig. 7). Elle est ainsi logée dans une dépendance de la cavité branchiale qui descend vers l'ovaire, formant une sorte de diverticule dénommé par ED. van BENEDEN et JuLIN l'épicarde. Aplostoma femelle distend considérablement les parois de cette cavité, comme le représente la figure schématique ci-dessus.

C'est avec les plus grandes difficultés qu'on arrive à disséquer les cormus de son hôte sans produire une rupture accidentelle des ascidiozoïdes dans la région qu'il 
comprime, et sans expulser le Copépode de sa retraite. A la suite d'une dissection malheureuse, Aplostoma semble vivre en liberté dans la cavité de la tunique commune du Tunicier ; mais c'est là une erreur contre laquelle je désire prémunir les naturalistes.

\section{Aplostoma brevicauda, CANU.}

1886. Aplostoma brevicauda; Canu ; 27, pages $313-320$; PI. II, fig. 1 à 4 .

Planche XX, Fig. 5-I8; Planche XXI, Fig. 13-15.

Taille : $q .1$ à $1,4 \mathrm{~m} / \mathrm{m} ; \sigma, 0,9 \mathrm{~m} / \mathrm{m}$.

Dans le sexe $q$. le corps est plus ou moins allongé suivant l’état d'extension assez variable.

La forme du céphalothorax est presque cylindrique, un peu rétrécie vers le céphalon et élargie vers le $4^{e}$ segment formé par l'union des $4^{\mathrm{e}}$ et $5^{\mathrm{e}}$ somites thoraciques; mais l'élargissement de la région céphalothoracique est dû principalement au développement des œufs en voie de maturation. L'abdomen (fig. 5) comprend seulement trois segments et les pièces furcales; il a la forme d'un tronc de cône, étant aminci vers l'extrémité postérieure; le $1^{\text {er }}$ et le $2^{\mathrm{e}}$ segments ne sont même plus très nettement séparés sur les échantillons dont la musculature a été disjointe par l'action de la potasse caustique (fig. 10). Le $\mathbf{1}^{\mathrm{er}}$ segment, plus, large, porte dorsalement les deux orifices externes $\log$ ) des oviductes, aux bords chitinisés et dentés; une plaque chitineuse épaissie, donnant appui aux muscles dilatateurs des orifices, les sépare sur lá face dorsale (fig. 5 et 10 ). Les $2^{\mathrm{e}}$ et $3^{\mathrm{p}}$ segments abdominaux sont moins longs que larges; le dernier porte sur sa face dorsale l'ouverture anale. Les pièces furcales sont un peu plus longues que le dernier segment, en forme de griffe amincie et recourbée vers l'extrémité, et armées d'une épine et d'une soie termininales avec une petite soie marginale insérée vers leur milieu. - Dans le sexe $\nearrow$, le corps rappelle, par sa forme générale, les genres Notodelphys et Enterocola. L'abdomen est très allongé et semble compter 6 segments et la furca. Le segment anal est très allongé, les pièces furcales plus courtes, avec deux longues soies terminales assez particulières, lisses, cylindriques et hyalines (fig. 18).

Les antennules sont, chez la femelle (fig. $8, a$ ), des appendices courts et épais, pourvus aux bords antérieur et distal d'épines peu résistantes ou de soies grêles. Chez le mâle (fig. 13 et 14), elles sont bien articulées, avec les deux premiers articles renfés et portant : le $1^{\text {er }}$, sur la face interne et postérieure, un groupe serré de longs filaments sensoriels $(y)$ hyalins, au contenu finement granuleux parsemé de vésicules plus claires, avec leur base plus étroite et un étranglement incomplet de la paroi vers le tiers distal de la longueur : le $2^{\mathrm{e}}$, a son bord antérieur, un certain nombre de grosses soies chitineuses insérées sur des saillies tuberculaires de la paroi. Les $3^{n}$ et $4^{\mathrm{e}}$ articles sont pourvus chacun d'un filament sensoriel plus gros, renflé en massue et étranglé dans le milieu de sa portion large et cylindrique.

Antennes assez semblables dans la femelle (fig. $8, A$ ) et dans le mâle (fig. 15), plus longues dans ce dernier, où les épines du second article sont plus grandes mais moins pointues.

Mandibules très réduites dans la femelle (fig. 8, md, fig. 9), allongées et 3-articulées dans le mâle (fig. $16, m d$ ). 
Seconde maxille assez semblable dans les deux sexes; 4-articulée et plus longue, spécialement dans sa région distale chez le mâle (fig. $16, m x^{2}$ ).

Pattes thoraciques des quatre premières paires très différentes dans les deux sexes; semblables entre elles chez la femelle (fig. 12) où l'exopodite porte seulement 3 ou 4 courtes épines en grifte; - dissemblables chez le $\sigma^{\circ}$ (PI. XXI), où la ${ }^{\text {re }}$ paire (fig. 13 ) se distingue des autres (fig. 15) par son endopodite (fig. 14) 2-articulé, ayant l'extrémité armée de deux fortes griffes uncinées qui correspondent peut-être au $3^{e}$ article des autres paires.

Cinquiènes pattes thoraciques réduites dans la femelle (Pl. XX, fig. 5) à un lobe saillant de forme irrégulière dans sa région distale armée de deux petites soies à peine visibles; formées dans le mâle (fig. 17) par deux articles très courts, le premier soudé au tronc et armé d'une soie externe, le second court et étroit avec deux soies grêles terminales.

Lacoloration est : dans les mâles, jaune brunâtre, avec de grands intervalles clairs et transparents sur les côtés du corps : - dans les femelles, uniformément composée de blanc rosé, absolument opaque à l'état vivant, la cavité du corps étant presque entièrement obstruée par les cellules graisseuses du tissu conjonctif.

Ovules mùrs et œufs pondus de couleur rosée; sacs ovigères (fig. 6) aussi longs que le corps, de forme cylindrique et comprenant un grand nombre d'œufs.

Aplostoma brevicauda est peu abondant dans diverses Synascidies des bancs rocheux du littoral du Boulonnais; je l'ai recueilli en tous points, dans Circinalium concrescens GIARD et Morchellium argus M.-EDw.; à la Pointe aux Oies et aux Roches Bernard dans Amaræcium nordmanni M. Edw., Amaræcium sp. voisin de A. punctum, et Polyclinum luteum GIARD.

Distribution: Manche.

\section{II. - COPEPODA DIPORODELPHYA.}

Copépodes ayant le corps plus ou moins nettement segmenté ou même sans divisions reconnaissables, et dont la femelle présente deux orifices sexuels pour la pénétration des spermatozoïdes.

Comme l'indique le tableau phylogénétique établi précédemment, les Copépodes diporodelphes comprennent les différentes familles de parasites et semi-parasites réunies par Claus (Traité de Zoologie dans le second groupe, celui des Parasites ou Siphonostomes, qu'il oppose au premier groupe des Nageurs ou Gnathostomes. 
Dans les Caligidae (52), les Lernaeidae (54, 211, 32), les Lernacopodidae (208), les Dichelestiida, les Ascomyzontida, dans les Coricaida, les Ergasilida, les Chondracanthida, les Lichomolgide et Sapphirinida, ainsi que dans les Nereicolidae (126), les Hersiliidce et dans leurs voisins très probables les Choniostomatidce (87), les pores de fécondation sont pairs et plus ou moins rapprochés des orifices externes ventraux, latéraux ou dorsaux des oviductes.

Le seul genre Pachysoma Claus, rangé jusqu'à présent parmi les Coricaida, semble faire exception, puisqu'il est monoporodelphe. La disposition de l'appareil sexuel déjà connue par une courte description de Claus ( $\mathbf{I}$ ) est confirmée par le $\mathrm{D}^{r}$ GiesBReChT (2) qui a pu réétudier ce Copépode et observer qu'à l'encontre des Coricéidés typiques, il présente un seul pore ventral sur le segment génital de la femelle. Il me paraît très douteux que Pachysoma soit un véritable Coricéidé, et de plus amples renseignements sont encore nécessaires pour fixer avec certitude la position systématique de ce genre.

\section{Monochila.}

Copépodes diporodelphes ayant:

$I^{0}$ Des habitudes parasitaires passagères ou persistantes, et la forme du corps très variable.

$2^{0}$ Les antennules formées d'un petit nombre d'articles, semblables dans les deux sexes, et les antennes simples, préhensiles et souvent plus puissantes chez le mâle.

$3^{0} \mathrm{La}$ lèvre supérieure convexe, recouvrant l'orifice buccal sans traces de paragnathes.

$4^{0}$ Les mandibules constituées par une lame aplatie (coxopodite), tranchante ou denticulée à son bord interne.

$5^{0}$ Les premières maxilles réduites à un moignon simple et très court, avec quelques petites soies à leur extrémité.

$6^{0}$ Les secondes maxilles parfois simples et masticatrices, et parfois doubles avec leur portion interne préhensile plus puissante chez le mâle.

$7^{0}$ Les pattes thoraciques des quatre premières paires biramées et natatoires ou bien

(1) " Bei Pachysoma (Tafel IX, fig. 8)..... warscheinlich wird das Sperma durch einen medianen Porus in " zwei enge Canäle aufgenommen und nach den Seitenflügeln der Drüse unterhalb der Geschlechtsöffinungen " geführt " (51, p. 66).

(2) Communication manuscrite. 
préhensiles et adhésives, très variables dans leur forme, parfois en partie absentes dans les deux sexes ou dans l'un d'eux.

$8^{0}$ Les pattes de la cinquième paire semblables dans les deux sexes, quand elles existent.

$9^{\circ} \mathrm{La}$ circulation lacunaire.

$10^{0}$ Les œufs pondus portés par la femelle en deux sacs extérieurs appendus à l'orifice des oviductes.

Ce sont les Coricceide de Claus (51), avec les diverses familles de parasites qui s'y rattachent indubitablement: les Lichomolgida, les Ergasilida, les Chondracanthida, et très probablement aussi les Philichthyida (I).

La disparition totale des paragnathes limite chez eux l'armature buccale à la lèvre supérieure parfois très développée, et la constitution spéciale de leurs appendices buccaux, déjà signalée plus haut, les distingue assez nettement de tous les autres Copépodes (voir ci-dessus, p. 56-58).

A ce titre, les Monochila semblent d'origine phylogénétique très précoce, ainsi que viennent encore en témoigner les nombreuses variations éthologiques et morphologiques dont ils sont susceptibles. A còté de parasites très étroitement adaptés, comme les Chondracanthidés et les Ergasilidés vivant sur les Poissons, viennent les Lichomolgidés, formes essentiellement semi-parasites, très éclectiques au point de vue de leur habitat puisqu'elles réunissent ċans un même genre des espèces parasites des Actiniaires aussi bien que des Mollusques et des Tuniciers. La fréquence des Lichomolgidés dans les pèches destinées à recueillir des Copépodes libres $(20$, vol. $3 ; \mathbf{6 6})$ indique suffisamment la faculté remarquable qu'ils possèdent de vivre longtemps à l'état de liberté (2).

Les Ergasilidés sont bien, comme l'ont fait remarquer plusieurs auteurs, reliés d'une manière très étroite aux Lichomolgidés, dont ils dérivent peut-être phylogénétiquement. De même, les Chondracanthidés se rattachent aux Ergasilidés.

Mais les Coricaida vrais ne me semblent guère, en raison de leur adaptation exagérée à l'existence pélagique, plus rapprochés de la souche que les autres Monochila (209, p. 97-99). Le véritable lien unissant les Copépodes primitifs aux Monochila n'est, en réalité, connu d'aucune manière, ainsi que je l'ai déjà fait observer précédemment.

(1) Et peut-être même les Bomolochida, dont on n'a pu fixer encore d'une manière à peu près certaine la position systématique $(52,55)$. Les curieux parasites cavicoles des Mollusques nudibranches (p. ex. Splanchnotrophus HANC, et NoRM., 104, 35) se placent certainement dans les Monrochila.

(2) Les mêmes habituđes se retrouvent dans les Sapphirinidés, qui ne sont d'ailleurs que des Lichomolgidés devenus pélagiques au même titre que leurs hôtes (Salpes) et adaptés comme eux à ce mode d'existence. 


\section{I C HOMOLGI D A E.}

Copépodes monochilés ayant :

$1^{0}$ Des habitudes semi-parasitaires très accusées.

$2^{0}$ La forme cyclopoïde la plus caractérisée.

$3^{0}$ Les antennules assez longues, 7 -articulées, semblables dans les deux sexes au point de vue de la disposition des articles et ne différant que par les longs filaments sensoriels hyalins, plus nombreux chez le mâle.

$4^{0}$ Les antennes 4 -articulées, terminées par des soies simples ou en crochet et par des griffes chitineuses.

$5^{0}$ Les secondes maxilles divisées en deux paires d'appendices dans les deux sexes ( 1 ), et dont l'interne est hypertrophiée en un puissant organe préhensile chez le mâle.

$6^{\circ}$ Les pattes thoraciques des quatre premières paires biramées et natatoires, de conformation normale.

$7^{0}$ Les pattes de la cinquième paire 2-articulées, de développement moyen.

En outre des propriétés éthologiques (2) citées plus haut, les Lichomolgidés se signalent parmi les Monochilés par diverses particularités que Claus (66) a récemment indiquées d'une manière satisfaisante (3) en redressant les erreurs commises par d'autres naturalistes (Kossmann, Della Valle....).

\section{Lichomolgus, THORELL.}

1860. Lichomolgus; THORELL. 204, P. 64-69.

1877. Stellicola; Kossmann. 119, p. 11.

1880. Lichomolgus, Th., pars; Brady, 20, vol. 3, p. 40.

1880. Lichomolgus, Th., pars; Della Valle, 75, p. 108-I IO.

1883. Lichomolgus, Th.; Aurivillius, 4, p. 38, 94, 96 et 106.

1885. Lichomolgus, Th., pars; Raffaele è Monticelli. 167, p. 43.

1889. Lichomolgus, Th.; Claus, 66, p. 11 et 12 .

1891. Lichomolgus, Th.; Canu, 34, p. 478.

(1) Exception faite de Myicola q, d'après Wright.

(2) Semi-parasitisme sur les animaux les plus divers du fond de la mer: Actinies, Echinodermes, Annelides, Mollusques, Tuniciers.

(3) Je ne renouvelle point cette discussion qui allongerait sans profit la description des espèces que j'ai recueillies. Les dissections minutieuses et difficiles que $\mathrm{j}^{\prime}$ ai faites pour étudier assez complètement les appendices de dix espèces de Lichomolgidés m'ont entièrement convaincu de l'exactitude des conclusions émises par CLaus sur l'organisation de ces animaux. Je ferai d'ailleurs observer qu'aucun zoologiste n'a fait jusqu'à ce jour l'étude personnelle d'un aussi grand nombre de ces semi-parasites, puisque THorell n'en posséda que 4 espèces, Claus 7, Kossmann 9, Della Valle 8 et Brady 6. 
Lichomolgidés ayant :

I" Le corps cyclopoïde plus ou moins élargi.

$2^{0}$ Le premier somite thoracique séparé (1) du segment céphalique.

$3^{0}$ Les antennes préhensiles, avec le $3^{\mathrm{e}}$ article très court porteur de courtes soies ou épines à son bord intérieur, avec l'article terminal armé d'une première épine en crochet plus longue, d'une deuxième plus courte et de quelques soies grèles recourbées (Pl. XXII, fig. 3 et 16 ; Pl. XXIII, fig. 7 .

$4^{0}$ Les mandibules falciformes effilées à leur extrémité, dépourvues de soie appendiculaire à leur bord antérieur concave (Pl. XXII, fig. 5 et 18 ; PI. XXIII, fig. 8).

$5^{\circ}$ Les secondes maxilles externes nettement 2 -articulées, avec le second article portant en avant de sa face intérieure : une petite épine triangulaire proximale et une longue soie denticulée plus éloignée de la base, et prolongé à son extrémité distale par une longue soie falciforme denticulée vers l'intérieur (PI. XXII, fig. 7 et fig. 19 ; PI. XXIII, fig. $8 m x^{2} e$ et fig. 9).

$6^{0}$ L'endopodite des quatrièmes pattes thoraciques 2-articulé, avec une soie barbelée au bord distal interne du premier article et deux épines terminales sur le second article (PI. XXII, fig. 10 et $11 ; \mathrm{Pl}$. XXIII, fig. 11 ).

$7^{0}$ Le second article des cinquièmes pattes thoraciques presque cylindrique et portant seulement une soie et une épine aux angles de son extrémité distale.

\section{Lichomolgus agilis, LEYDIG.}

1853. Doridicola agilis; LeYdIG, 135, p. $377-382$, Taf. XIV.

1875. Lichomolgus doridicola, Leydig; Claus, 55 , p. 348 et 349, Taf. XXIV, fig. 29 et 29 '.

1883. Lichomolgus chromodoridis; Della VAlLE, 75, p. 120 et 121 , fig. 43-48.

1889. Lichomolgus doridicola, Leydig; Chaus, 66, p. 12 et 13, Taf, II, fig. I-12.

1891. Lichomolgus doridicola, Leydig; Canu, 34, p. 478.

\section{Planche XXII, FIG. $1-14$.}

Taille : $f$, de 0,9 à $1,2 \mathrm{~m} / \mathrm{m} ; \sigma$, de 0,7 à $0,8 \mathrm{~m} / \mathrm{m}$.

Espèce bien caractérisée par sa forme élargie, spécialement dans le céphalothorax et le premier segment abdominal (fig. 1 et 2), par la brièveté des pièces furcales égales au segment qui les porte (fig. 1, 2 et 12) ou même plus courtes que lui ; par la taille des soies furcales dont la terminale interne dépasse de beaucoup la longueur de l'abdomen (fig. 1 ét 2).

(1) Au moins dans les trois espèces que j'ai étudiées (Pl. XXII, fig. 1 et 2 ; Pl. XXIII, fig. I, 2, 4 et 5). Claus Pindique comme soudé au céphalon dans Lich. agilis LeYdig et Lich. anemonice Cls, et Della Valle fait de même pouk Lich. agilis (二 chromodoridis D. V.),... etc. Dans Lich. agilis, la limite de ces segments est une ligne parfaitement visible à un grossissement de 120 diamètres, sur les exemplaires vivants comme sur les échantillons conservés que l'on éclaircit par l'action de la potasse caustique à froid. 
Parmi les appendices les plus caractéristiques sont:

Les antennules grêles et courtes (fig. 4).

Les antennes (fig. 3) épaisses et solides, avec le $3^{\mathrm{e}}$ article très court armé de petites soies raides à son bord distal interne, avec le $4^{\mathrm{e}}$ article terminé par deux crochets presque égaux qu'il dépasse en longueur, et par quelques soies très petites.

Les mandibules (fig. 5) larges à leur base et possédant sur leur bord interne et postérieur un lobe très saillant armé de denticules forts et pointus.

Les secondes maxilles externes (fig. 7) renflées, avec le prolongement terminal plus long que la soie distale interne et pourvu comme elle de longues épines effilées en petit nombre.

Les secondes maxilles internes de la femelle (fig. 3 et 8) 3-articulées, assez lungues, avec le $1_{1}^{\text {er }}$ article plus long que le $2^{\theta}$, celui-ci armé de deux soies grêles et barbelées sur sa face interne dépourvue de denticules, avec le $3^{e}$ article court, arrondi, terminé par deux épines denticulées courtes et épaisses.

Les secondes maxilles internes du màle (fig. 9) plus développées, transformées en pince préhensile par l'allongement du $3^{\mathrm{e}}$ article sous forme d'une lame recourbée portant à sa base élargie une longue soie accessoire et par le renflement du second article avec une longue soie rigide et une série ondulée de denticules larges et triangulaires sur sa face interne convexe.

Les cinquièmes pattes thoraciques (fig. 1, 2 et 11 ) avec le $1^{\text {er }}$ article soudé au tronc, mais encore très saillant sur la face dorsale du cinquième segment thoracique, où il porte une longue soie flexible, avec le second article très allongé, à bord interne irrégulier, et terminé par deux soies raides et minces.

Coloration blanchâtre ou orangée, variant avec la teinte de l'hôte.

Les œufs sont également blanchâtres, petits et très nombreux, disposés en huit ou dix rangées longitudinales dans chaque sac ovigère. Après l'éclosion, le nauplius (fig. 13 et 14) présente une forme assez allongée, avec des appendices bien développés. Son organisation intérieure (œil, cerveau secondaire, tube digestif divisé en trois renflements d'importance subégale dont le médian est plus glandulaire, soies furcales très allongées et très divergentes) est établie sur un type commun à tous les Lichomolgidés et même à d'autres Monochila (Splanchnotrophus HaNC. ET NoRM, par exemple). Dans les figures 13 et 14 relatives à cet embryon, se trouvent figurés avec plus d'exactitude ces divers caractères, représentés antérieurement d'une manière insuffisante par quelques auteurs.

Ce Copépode est abondant sur les Mollusques nudibranches du Boulonnais; ; 'ai constaté sa présence fréquente sur les branchies des grandes espèces de Doris: D. tuberculata Cuvier, D. millegrana Alder Et HanCock, D. johnstoni Alder ET HancoCK el sur les papilles dorsales de Eolis coronata FoRbes, Antiopa cristata Delle Chiaje, $A$. hyalina Alder et Hancock. Dans la baie de Concarneau, je l'ai recueilli communément sur Doris tuberculata et D. johnstoni. Leydig, Claus, Della Valle et Aurivillius le signalent sur diverses espèces de Doris. Sa présence que j'ai constatée sur les Eolis peut laisser supposer qu'il soit aussi le L. eolidicola 
de SarS (184), ce qui justifie l'abandon de la dénomination spécifique : doridicola, antérieurement acceptée.

Distribution: Mer du Nord, Manche, Atlantique, Méditerranée.

\section{Lichomolgus albens, THORELL.}

1860. Lichomolgus albens; THorell, 202, p. 69-71, Tab. X, XI et XII, fig. 15.

1875. Lichomolgus albens, Thor. ; Mceius, 151, p. 270 et suiv.

1877. ? Lichomolgus furcillatus, Thor. ; Kossmann, 119, p. 19, Taf. IV, fig. 1.

1885. Lichomolgus albens, Thor.; Auriviluus, 4, p. 94-96, Taf. Vil, fig. 9 et 10.

1891. Lichomolgus albens, Thor.; Canu, 34, p. 478.

Planche XXII, Fig. 15 et 23 ; Planche XXIII, Fig. I-4.

Taille : + , de 1 à $1,2 \mathrm{~m} / \mathrm{m} ;$ t, de 0,7 à $0,8 \mathrm{~m} / \mathrm{m}$.

La forme du corps est large, mais peu ramassée; l'abdomen relativement allongé a son dernier segment un peu plus long que celui qui le précède. Les pièces furcales sont deux fois plus longues que le dernier segment; les soies marginales y sont implantées vers le milieu de la longueur et les soies terminales ont une structure particulière jusqu’à présent méconnue: dans leur moitié proximale, la paroi chitineuse de ces soies est épaisse et porte des barbules serrées; dans leur moitié distale la paroi est hyaline et beaucoup moins réfringente que a portion axiale.

L'antennule (fig. 15) est bien développée; ainsi que l'a fait remarquer Møвius, les sept articles y sont nettement séparés, et le $3^{\mathrm{e}}$ article très court, égal en longueur au $7^{\mathrm{e}}$, est bien distinct du $4^{\theta}$ plus allongé. Cette séparation a d'ailleurs été vue par Thorell, quil'a figurée (loc. cit., Tab. XI, fig, I5 A') en continuation directe avec la base d'une soie attachée au bord distal antérieur, de sorte qu'il a pu considérer par erreur l'antennule comme 6-articulée. Dans la femelle, cet appendice porte des filaments sensoriels que l'on retrouve un peu plus longs et plus nombreux chez le mâle où ils sont au nombre de 5 , et non de 3 comme le dit MøвiUs.

L'antenne (fig. 16) a le $3^{\mathrm{e}}$ article court et armé de deux petites soies grêles, le $4^{\mathrm{e}}$ article pourvu de deux fortes épines plus longues que lui et accompagnées de petites soies.

La mandibule (fig. 18) longue et effilée à son extrémité, porte plusieurs séries de poils courts au bord postéro-intérieur, et une seule rangée marginale au bord antéro-extérieur.

La première maxille (fig. 18 ) est réduite à un moignon avec deux soies recoürbées.

La seconde maxille externe (fig. 19), renflée et trapue, a son $3^{\theta}$ article pourvu d'une épine proximale interne petite, d'une soie distale interne longue et garnie d'épines, d'un prolongement falciforme très long armé d'épines grêles dans toute sa longueur, avec une épine en crochet plus grosse près de sa base.

La seconde maxille interne est très différente dans le mâle (fig. 3 ) et dans la femelle (fig. 20). Dans le mâle, les deux derniers articles formant pince sont très allongés; le $3^{\circ}$ est mince et recourbé, de forme irrégulière; le $2^{\mathrm{e}}$ pourvu d'une convexité saillante à sa face interne, porte une petite rangée de fins poils chitineux et deux soies accessoires. Dans la femelle, l'appendice encore composé de trois articles, est particulièrement court et large. 
Les pattes natatoires sont, comme dans tout Lichomolgus, larges et pourvues de fortes épines et de longues soies plumeuses. L'endopodite de la $4^{8}$ paire est assez allongé, avec les deux épines denticulées terminales très développées.

La $5^{\circledR}$ patte a une forme assez caractéristique (fig. 22). Le premier article soudé au tronc est peu saillant; il porte à l'angle dorsal externe une très petite soic. Le second article est court, épais, élargi dans sa région distale par la présence d'une saillie interne légèrement incurvée ; il se termine par une épine denticulée courte, de la même longueur que lui, avec une soie barbelée double de sa longueur. C'est même en raison de la forme de cet appendice, ainsi que de la forme de l'abdomen dans l'animal figuré par Kossmann (loc. cit., Tat. IV, fig. 1), que je crois devoir considérer $L$. furcillatus THor., var. mediterranea Kossm., comme identique à Lich. albens THORELL.

Dans Sabelliphilus Sarsi ơ. Della Valle (75) a décrit les canaux déférents comme formés par deux organes symétriques, d'un blanc mat, en forme de massue très régulière, placés sur les côtés du tube digestif dans les segments thoraciques et aboutissant par leur région amincie aux sacs à spermatophores; mais il n'a pu voir la glande testiculaire qu'il suppose impaire, à l'encontre de WiERzejsk1, lequel donne (211), chez Lich. sepicola Crads, les canaux déférents comme des testicules pairs.

Avec quelque peine, j'ai pu suivre (PI. XXIII, fig. .4), en avant de chacun de ces renflements glandulaires des canaux déférents, la marche de canalicules étroits, à parois glanduleuses et plissées, qui remontent jusqu'auprès de l'extrémité antérieure de l'estomac, se recourbent dorsalement pour revenir en arrière $(e . f$.$) et rejoindre les angles antérieurs d'une$ glande testiculaire impaire $(T)$, placée à la limite du céphalon et du thorax. La supposition toute gratuite avancée par Della ValLe sur l'organisation de l'appareil génital mâle des Lichomolgides se trouve donc justifiée.

Lich. albens est de teinte blanchâtre, avec un œil rouge vif. Les œufs sont blancs, petits et nombreux, pondus dans deux sacs ovigères cylindriques.

Cette espèce, recueillie par un certain nombre de naturalistes, semble très répandue et relativement commune, si l'on tient compte de la difficulté que présente sa recherche. Dans le Boulonnais, je l'ai trouvée habitant la cavité péribranchiale et le cloaque de diverses Ascidies: Ciona intestinalis MUeller, Molgula socialis AldER, Cynthia lurida THORELl. Une variété naine $(0,6 \mathrm{~m} / \mathrm{m}$ de long) vit également dans Perophora listeri M. Don. - Thorell l'a obtenue dans Corella parallelogramma Alder, Phallusia mentula Mueller et Ciona canina Mueller; MœBIUs dans Molgula sp.; Della Valle ( 75 , p I08) dans Ciona intestinalis; et Aurivilluus dans Phallusia patula Alder, Ph. virginea Mueller, Corella parallelogramma ALDER.

Distribution : Mer du Nord, Manche, Méditerranée.

$$
\text { Lichomolgus poucbeti, CANU. }
$$

1891. Lichomolgus poucheti; CANU; 34, pages 478 et 479.

$$
\text { Planche XXIII, Fig. 5-12. }
$$


․ - Taille: 1 à $1,2 \mathrm{~m} / \mathrm{m}$.

Ce Copśpode se rapproche par quelques caractères généraux d'une espèce rare, décrite par Thorell et rétudiée par Aurrvilutus (4, pages 98-101) : Lich. furcillatus, J'indiquerai brièvement les points qui séparent ces espèces.

La forme du corps (fig. 5) est, dans Lich. poucheti, assez étroite et allongée (1) ; le céphalothorax est presque elliptique, le dernier segment abdominal (fig. 5 et 12) est deux fois et demie plus long que le précédent; les pièces buccales sont de même longueur que le dernier segment et les soies furcales assez courtes n'atteignent guère qu'à la moitié de la taille de l'abdomen.

L'antenne (fig. 7) est longue et forte, avec trois longues épines et deux soies recourbées à l'extrémité du $4^{\mathrm{e}}$ article, avec une épine recourbée et deux petites soies au bord distal interne du $3^{\circ}$. Cette armature rappelle Sabelliphilus, plutôt que les autres espèces de Lichomolgus.

La mandibule ( fig. 8, md ) est une lame falciforme très longue, avec la courbure très marquée, et ne présente nullement au bord postéro-interne la mêne armature que chez $L$. albens. Au contraire, à côté de petits poils raides disposés en série marginale, ce bord présente une lame proéminente membraneuse, finement striée dans le sens de sa largeur.

La première maxille (fig. $8, m x^{4}$ ) est longue et armée d'une seule grosse soie recourbée.

La seconde maxille externe (fig. $8, m x^{2} e$; fig. 9) diffère notablement de celles de $L$. albens et de $L$. furcillatus, que Thorell rapproche l'une de l'autre et dit pourvues d'une seule grosse épine à la base du prolongement falciforme. Dans Lich. poucheti, il existe en ce point cinq grandes épines solides, suivies de petites dents semblables à celles qui garnissent le bord de la longue soie interne distale.

La seconde maxille interne (fig. 10) est grêle, avec le $3^{\circ}$ article recourbé en griffe et très court.

L'endopodite des $4^{\text {es }}$ pattes natatoires (fig. 11) est assez court, avec les épines terminales relativement longues et grêles.

Couleur jaune-orangée; sacs ovigères cylindriques.

Malgré ces caractères, parmi les Lichomolgus vivant dans les Tuniciers, Lich. pouchetı se place auprès de Lich. furcillatus par la disposition analogue des segments abdominaux, par la forme auriculaire des orifices externes des oviductes, ainsi que par la brièveté des $5^{{ }^{e s}}$ pattes thoraciques et des pièces furcales.

J'ai trouvé cette espèce assez rare, dans la baie de Concarneau, vivant en semi-parasite à la surface des gros cormus de Morchellium argus M.-EDw. et de Fragarium areolatum GIARD; mais je ne l'ai point obtenue encore dans le Boulonnais.

\section{Distribution : Atlantique.}

(1) Dans Lich. furcillatus, THORELL (204, page 74) dit le corps plus élargi que dans les autres espèces, notamment dans $L$. albens. C'est l'inverse pour Lich. poucheti, comme on peut s'en convaincre en comparant les figures I et 5 de la planche XXIII. 


\section{Sabellipbilus, SARS.}

1862. Sabelliphilus; M. SARS; 185, page 139.

1870. Sabelliphilus, Sars; Claparède; 39 , page 6.

1875. Sabelliphilus, Sars; Claus; 55, page 341.

1876. Sabelliphilus, Sars, Claus; 56, page 161.

1877. Sabelliphilus, Sars; Kossmann ; 119, page 6 .

1880. Lichomolgus, Thorell, pars ; Della VAlLE; 75, pages 108-110.

1885. Sabelliphilus, Sars, pars; RafFaele È Monticelli; 167, page 16.

1889. Sabelliphilus, Sars; Claus; 66, page 14.

\section{Lichomolgide ayant :}

$I^{0}$ Le corps cyclopoïde relativement allongé.

$2^{0}$ Le premier somite thoracique séparé du céphalon et les deux premiers somites abdominaux soudés chez la femelle en un segment génital.

$3^{0}$ Les antennules très élargies et renflées dans les deux premiers articles.

$4^{0}$ Les antennes préhensiles 4 -articulées, épaisses, puissantes et pourvues : à la face interne du $2^{\theta}$ article, d'une crête chitineuse garnie de plusieurs denticules triangulaires ; au bord distal du $3^{\mathrm{e}}$ article, d'une épine en crochet et de deux soies; à l'extrémité du $4^{\mathrm{e}}$, de trois épines recourbées puissantes et de petites soies.

$5^{0}$ Les mandibules falciformes et effilées, sans soie antérieure.

$6^{0}$ Les secondes maxilles externes également prolongées en faucille, avec quelques grosses dents triangulaires au bord intérieur de ce prolongement, relativement court.

$7^{0}$ L'endopodite des $4^{\text {eg }}$ pattes thoraciques 3-articulé, avec deux soies barbelées à son bord interne et deux soies lisses effilées à son extrémité distale.

$8^{0}$ Le second article des $5^{\text {es }}$ pattes thoraciques presque cylindrique et portant seulement deux soies ou épines.

Le genre Sabelliphilus, bien caractérisé par M. SARS, doit être maintenu.

Il présente avec Lichomolgus THORELL des différences très nettes, et la discussion de la valeur générique ou sub-générique de ces caractères, soutenue par Della Valle et par Raffaele è Monticelli (loc. cit., page 4) n'a aucune raison d'être. Entre Lichomolgus THORELl et Sabelliphilus SARS, il existe autant de dissemblances qu'entre Lichomolgus Thorell et Anthessius Della Valle; cette simple constatation (167, page 4; 66, page 6) réduit à néant les critiques de Della VAlle (75, page 108).

Comprendre dans le genre Sabelliphilus tous les Lichomolgidés - distincts de 
Anthessius - ayant l'endopodite des $4^{\text {es }}$ pattes 3 -articulé (I), serait encore une. erreur grave, tant au point de vue bibliographique (2) qu'au point de vue de la critique systématique. Raffaele ̀̀ Monticell, défenseurs de cette opinion, méconnaissent d'ailleurs les relations systématiques des divers genres à endopodite 3-articulé dans la $4^{e}$ patte, puisque leur Lichomolgus spinosus, qu'ils rapportent à Sabelliphilus, montre tous les caractères d'Anthessius:

$\alpha$. Dans la présence d'une soie accessoire $(s p)$ sur le bord concave de la mandibule (167, page 5, fig. 8, $m d$ ).

F. Dans la forme irrégulièrement quadrangulaire de la première maxille (loc. cit., page 5 , fig. $8, m s)$.

$\gamma$. Dans l'endopodite des $4^{\text {es }}$ pattes thoraciques, garni à son extrémité de soies ou épines en nombre supérieur à deux (loc. cit., page 6, fig. 6).

6. Dans le second article des $5^{\mathrm{es}}$ pattes thoraciques, élargi et portant 4 (i) ou 5 ( $\left.\sigma^{7}\right)$ soies et épines ( $10 c$. cit., pages 6 et 7 , fig. 7 et I 1 ).

ع. Dans sa taille relativement considérable, entre 2,5 et $2 \mathrm{~m} / \mathrm{m}(3)$.

\section{Sabellipbilus sarsi, CLAPARÈdE.}

1870. Sabelliphilus Sarsii; ClAPARÈde ; 39, page 6, PI. VII.

1876. Sabolliphilus Sarsii, Clap.; Claus; 56, pages I6ı-164, Taf. X.

1877. Sabclliphilus Sarsii, Clap. ; Kossmann; 119, pages tó et 17.

1877. Sabelliphilus Leuckarti; Kossmann; 119, pages 17 et 18 , Taf. 11 , fig. 2 et j.

1880. Lichomolgus Sarsii, Clap.; Della VAlle; 75, pages $110-118$, fig. 1-26.

1887. Lichomolgus sabellae; I.-C. Thompson; 195, pages 68 et 69, Plate II.

1889. Sabelliphilus Sarsii. Clap.; Claus; 66, pages 14 et 15, Taf. 1, fig. 8-10.

1889. Sabelliphilus Sarsii, Clap., var. massiliensis; Gourrer ; Arch. de Biologie, Tome IX, pages 475-479, P1. XXXIV.

1889. Subelliphilus Sarsi, Clap. ; Canu ; 35, page 437.

Il est possible que cette forme se confonde avec le type décrit par M. SARS; toutefois, c'est la seule espèce du genre suffisamment caractérisée. Il ne peut être question de maintenir les espèces et variétés récemment établies à tort par quelques

(1) Comme le veulent Raffaele È Monticelli (loc, cit.): ".... subdividersi l'unico genere Lichomolgus in 3 sottogruppi di egual valore: Lichomolgus prop. detto, nel senso del fondatore del genere THorell; Sabelliphilus che comprenderebbe quei Lichomolgidi che hanno il ramo interno del $4^{\circ}$ paio di piedi tri-articulatõ ; è Anthessius, che a questo carattere aggiunge quello delle parti boccali \%. .

(2) En effet, M. SARS a indiqué dans la diagnose de Sabelliphilus certains caractères des antennules, antennes, etc, qui manquent dans les genres Paranthessius Claus, Modiolicola Aurivilulus, Herrmannella Canu, .... etc.

(3) Claus (66, page 15) dit d'Anthessius que ce genre paraît contenir en particulier les formes grandes et allongées qui vivent sur les Mollusques. 
naturalistes ignorants des descriptions précises et complètes fournies par leurs prédécesseurs au sujet de Sab. sarsi. En effet, quiconque prendra le soin de comparer les descriptions de Claus, Della Valle, etc., avec celles de Kossmann (I) pour Sab. Leuckarti, de THOMPSON pour Lich. Sabella, et de GourReT pour Sab. Sarsii, var. massiliensis, pourra aisément rectifier les erreurs d'observation contenues dans ces dernières et se convaincre de l'unité spécifique et sub-spécifique du parasite de Spirographis et de Sabella.

Copépode assez rare, aux Roches Bernard, sur les branchies de Sabella pavonina SAvigny, recueilli dans la zone des Laminaires. A Naples et Marseille, l'espèce habite sur Spirographis spallanzanii; à Liverpool, sur les Sabelles. L'hôte indiqué par SARS est également une Sabelle: Sabella sarsi KR $\emptyset$ YER.

Distribution : Mer du Nord?, Manche, Atlantique, Méditerranée.

\section{Herrmannella, CANU.}

1891. Hermannella; Canu; 34, pages 479 et 480.

Lichomolgide ayant :

$I^{0}$ Le corps cyclopoïde de forme assez ramassée.

$2^{0}$ Le $_{1}{ }^{\text {er }}$ somite thoracique séparé du céphalon; le $1^{\text {er }}$ somite abdominal libre et très élargi chez le mâle, soudé avec le $2^{\mathrm{e}}$ chez la femelle.

$3^{0}$ Les deux premiers articles des antennules cylindriques et nullement renflés.

$4^{0}$ Les antennes 4-articulées, avec une grande épine recourbée et quelques soies grêles à 'l'extrémité distale.

$5^{\circ}$ Les mandibules falciformes, très effilées, sans soie antérieure.

$6^{0}$ Les secondes maxilles externes prolongées en faucille, avec le prolongement richement denticulé et deux soies internes sur le $2^{\circ}$ article : l'une longue et denticulée, l'autre courte et lisse.

$7^{0}$ L'endopodite des $4^{\text {es }}$ pattes 3-articulé, avec deux soies barbelées à son bord interne et deux épines à son extrémité.

$8^{0}$ Le second article des $5^{\text {es }}$ pattes thoraciques presque cylindrique avec seulement deux soies ou épines.

Ce genre est assez nettement séparé des Lichomolgides aux endopodites

(1) Auquel il faut bien tenir compte des mauvaises conditions de confort dans lesquelles furent conduites ses observations sur les animaux de la Mer Rouge, surtout lorsqu'il s'agit de détails d'organisation si difficiles à étudier. 
3-articulés dans la $4^{\mathrm{e}}$ paire, pour qu'il me suffise de rappeler ici les divergences présentées:

a. - Dans Sabelliphilus SARS, par l'antennule, l'antenne et les pièces buccales.

b. - Dans Anthessius D. V., par la mandibule, la seconde maxille et les $4^{\mathrm{e}}$ et $5^{\mathrm{e}}$ pattes.

c. - Dans Modiolicola Aur., par le $\mathrm{I}^{\mathrm{er}}$ segment abdominal des deux sexes, par l'antenne, la mandibule et la seconde maxil'e externe.

d. - Dans Paranthessius CLs., far l'antenne, la seconde maxille externe et les $4^{\text {es }}$ pattes.

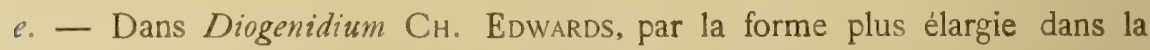
région intermédiaire du thorax et de l'abdomen, ainsi que par les pièces buccales.

f. - Dans Myicola Wright q, par la seconde maxille en partie absente (?) et par les mandibule et maxille.

Et pourtant, dans l'état vraiment précaire de nos connaissances actuelles, il ne peut ètre question de conclure en toute certitude à l'indépendance systématique des genres Myicola et Diogenidium. L'absence de la seconde maxille interne chez la femelle, indiquée par WRIGHT chez Myicola, n'ayant été constatée dans aucun autre Lichomolgide voisin d'Anthessius, il persiste quelques doutes sur l'exactitude de la diagnose de ce parasite de Mya arenaria. Les conditions particulières (Kystes libres dans la cavité générale d'une Holothuric) dans lesquelles ont été recueillis les exemplaires de Diogenidium étudiés par $\mathrm{CH}$. EDWARDs, et les quelques divergences séparant les organes buccaux, laissent supposer que Diogenidium et Ilerrmannella sont deux genres voisins mais pourtant distinçts, entre lesquels existent à peu près les mêmes rapports qu'entre les genres Anthessius D. V. et Paranthessius Cis.

\section{Herrmannella rostrata, CANU.}

1891. Herrmannella rostrata; CANU, 34, page 480 .

1891. Herrmannella rostrata; CANU, 35.

Planche XXIV, Fig. $1-13$.

Taille: $q 1,2^{\mathrm{m}} / \mathrm{m} ; \sigma, 0,85^{\mathrm{m}} / \mathrm{m}$.

Céphalothorax (fig. 1 et 2) épais et élargi, de forme ovalaire, avec le $5^{\circ}$ segment thoracique très étroit, le prolongement frontal recourbé ventralement et constituant une épine chitineuse solide. Abdomen (fig. 1, 2 et 13) 4-i) ou 5-( $\left.0^{7}\right)$ segmenté, avec le $1^{\text {er }}$ segment large et très renflé dans les deux sexes, et les autres segments à peu près aussi longs que larges. Pièces furcales larges et cylindriques, plus que doubles du segment précédent, avec les soies 
terminales bien développées, la médiane interne plus longue et égale aux deux tiers de l'abdomen, avec la soie marginale insérée vers le milieu de leur longueur.

Antennule (fig. 1,2 et 13 ) avec le $2^{\mathrm{e}}$ article très allongé, les $1^{\mathrm{er}}, 4^{\mathrm{e}}, 5^{\circ}, 6^{\mathrm{e}}$ et $7^{\circ}$ moindres à peu près égaux entre eux, et le $3^{e}$ plus court ; portant chez le mâle sept grands filaments sensoriels et chez la femelle trois filaments plus courts.

Antenne épaisse, à parois résistantes, avec le $2^{\mathrm{e}}$ article plus long, le $3^{\circ}$ très court et portant à son bord distal interne trois soies chitineuses recourbées, le $4^{\mathrm{e}}$ un peu plus long que large et armé d'une épine recourbée très grosse et très longue et de quelques soies rigides.

Mandibule (fig. 5) allongée, avec de petits denticules assez nombreux sur les bords antérieur et postérieur.

Première maxille (fig. 6) courte et cylindrique, terminée par deux courtes soies grêles.

Seconde maxille externe (fig. 7) large et convexe dans sa région basilaire; le second article assez allongé, cylindrique, portant une soie proximale interne plus longue que lui, une soie distale interne et un prolongement falciforme égaux en longueur et armés d'une douzaine d'épines semblables assez courtes.

Seconde maxille interne longue et forte dans les deux sexes. Chez la femelle (fig. 8) le $1^{\text {er }}$ article est égal à la moitié du $2^{\mathrm{e}}$; celui-ci porte vers son extrémité deux-soies accessoires internes mais ne présente pas de denticules; le $3^{\mathrm{B}}$ article est une griffe allongée, égale au $1^{\text {er }}$ article et sans courbure sensible. Chez le mâle (fig. 9 et 10 ), le $2^{e}$ article est plus élargi et porte, avec les deux épines accessoires internes plus longues, une série de fins denticules; le $3^{\mathrm{e}}$ est une longue griffe formant pince, avec une petite pointe recourbée à son extrémité distale et portant dans sa région proximale une soie accessoire rigide relativement très longue.

Pattes natatoires 3-articulées, avec l'endopodite bien développé et assez élargi dans la $4^{0}$ paire (fig. 11). Dans la ${ }_{1}^{\text {re }}$ paire, le basipodite porte (fig. $3, p^{1}$ ) à son bord interne une très longue épine recourbée vers l'extérieur et une série de denticules triangulaires.

Cinquièmes pattes (fig. 12 et 13 ) avec l'article basilaire très intimement soudé au tronc et pourvu d'une petite soie distale et dorsale; avec le second article presque cylindrique, garni d'une soie barbelée et d'une courte épine denticulée.

Ouvertures externes des oviductes placées (fig. 13) des deux côtés de la face dorsale, dans le milieu du ${ }^{\text {er }}$ segment abdominal de la femelle, et dirigés parallèlement au grand axe de l'animal, avec une forte épine chitineuse sur la lèvre supérieure devenue externe.

Sacs à spermatophores très développés et de forme assez particulière (fig. 2).

Sacs ovigères longs, de forme ovalaire ou même sub-cylindrique, avec les œufs colorés en blanc rosé, de même que les ovules en voie de maturation dans les oviductes.

Ce Copépode est assez répandu en semi-parasite dans la cavité palléale et sur les branchies de divers Mollusques lamellibranches du Boulonnais. Je l'ai observé dans Mactra stultorum LinNÉ, provenant des sables de la Pointe aux Oies et du Port en eau profonde de Boulogne, dans Cardium edule LinNé de la baie d'Authie et du Port en eau profonde; dans Pecten opercularis Linne dragué au large d'Étaples.

Distribution: Manche. 


\section{Modiolicola, Auriviluius.}

1883. Modiolicola; Auriviluus, 4, pages 9 et to.

1885. Lichomolgus, Th., pars; RAFFAeLE È MONTICELli, 167, pages 302 et 303.

1886. Modiolicola, Aur.; Auriviluuus, 7.

1891. Motiolicola, Aur.; Canu, 34, page 482.

1891. Modiolicola, Aur.; Canu; 35, page 436.

Lichomolgide ayant :

$I^{0}$ Le corps cyclopoïde de forme remarquablement allongée.

$2^{\mathrm{n}}$ Le $1^{\mathrm{er}}$ somite thoracique séparé du céphalon, et le $1^{\mathrm{er}}$ somite abdominal séparé du $2^{\mathrm{e}}$ dans les deux sexes.

$3^{0}$ Les deux premiers articles des antennules cylindriques et non renflés.

$4^{\circ}$ Les antennes 4 -articulées, solides, avec une épine recourbée plus forte, d'autres plus faibles et quelques soies.

$5^{\circ}$ Les mandibules falciformes effilées et sans soie antérieure.

$6^{0}$ Les secondes maxilles externes prolongées en faucille, avec des dents sur ce prolongement, et portant une seule soie interne lisse sur le second article.

$7^{0}$ L'endopodite des $4^{\text {es }}$ pattes 3-articulé, avec deux soies barbelées à son bord interne et deux épines à son extrémité.

$8^{0} \mathrm{Le}$ second article des $5^{\text {es }}$ pattes thoraciques presque cylindrique avec une longue épine et une soie à son extrémité.

Comme l'a primitivement indiqué (4) et ultérieurement soutenu ( $\boldsymbol{\nabla}$ ) Auriviluius, le genre Modiolicola est très nettement séparé des autres Lichomolgidés, et il peut paraltre étrange qu'il n'ait point attiré l'attention de Claus dans sa récente rérision de cette famille de Copépodes, où ne sont point mentionnés les mémoires d'Aurivillius et de Raffaele è Monticelli.

Par les deux espèces actuellement connues, et récoltées par moi dans le Boulonnais, ce genre semble exclusivement semi-parasite dans les Mollusques lameilibranches.

\section{Modiolicola insignis, Auriviluuus.}

1883. Modiolicola insignis; Auriviluus, 4, pages I0-14 et 39-44, Taf. II, fig. 1-10 et Taf. IV. fig. I-8.

1885. Lichomolgus insignis, Aur.; RafFaele È MonTICELL1, 167, pages 302 et gin, fig. 13-16.

1891. Modiolicola insignis, Aur.; Canv, 34, page 482.

\section{Planche XXIV, Fig. 14-20.}

Taille: $\left\{, 1,2^{\mathrm{m}} / \mathrm{m} ; \sigma^{7}, 0,9^{\mathrm{m}} / \mathrm{m}\right.$.

Céphalothorax de forme assez régulière, elliptique allongée dans les deux sexes (fig. 14 
et 15$)$. Abdomen presque cylindrique avec le $1^{\text {er }}$ segment portant les orifices génitaux et assez peu renflé dans les deux sexes. Face ventrale des $2^{\theta}, 3^{\theta}$ et $4^{e}$ segments abdomizaux garnie, vers son bord postérieur, d'une petite crête transversale avec 12 ou 15 épines triangulaires (fig. 25 et 26). Pièces furcales courtes ou cylindriques, à peu près égales au segment qui les porte (fig. 14,15 et 25 ), avec quatre soies terminales bien développées dont la médiane interne plus longue est égale aux deux tiers de l'abdomen, avec une soie marginale insérée vers le milieu de leur longueur.

Antennule (fig. 16) 7 -articulée, avec l'article basilaire court, le $2^{\mathrm{e}}$ article cylindrique et allongé, le $3^{\mathrm{e}}$ et le $7^{\mathrm{e}}$ plus courts que les $4^{\mathrm{e}}, 5^{\mathrm{e}}$ et $6^{\mathrm{e}}(1)$. Dans le mâle, les filaments sensoriels sont au nombre de six, tandis que dans la femelle on n'en trouve que trois.

Antenne (fig. ${ }^{17}$ ) très allongée, avec le $1^{\mathrm{er}}$ article plus court que le $3^{8}$, celui-ci entièrement dépourvu de soies et de crochet à son bord distal interne; les trois épines solides qui la terminent très recourbées en hameçon.

Mandibule (fig. 19 et 20) en lame falciforme tres effilée, avec la portion basilaire élargie, pourvues de petits denticules sur le bord interne et postérieur et de poils rigides plus grêles sur le bord antérieur.

Première maxille (fig. 18, 19 et 20) composée d'un court moignon presque cylindrique terminé par trois soies (2) assez raides et légèrement incurvées.

Seconde maxille externe bien différente dans les deux sexes. Chez la femelle (fig. 18 et 22), c'est un appendice assez court, 3-articulé, avec le $1^{\mathrm{er}}$ article renflé et court, le $2^{\mathrm{e}}$ plus long que le $1^{\text {er }}$ et garni à sa face interne d'une petite crête denticulée sans poils accessoires (fig. 18), le $3^{\mathrm{e}}$ réduit à un petit tubercule (3) sans soie accessoire et avec quelques denticules peu apparents. Chez le mâle, cet appendice, beaucoup plus développé (fig. 23), recouvre les autres pièces buccales : le $1^{\mathrm{er}}$ article est plus allongé; le $2^{\mathrm{e}}$ porte deux soies accessoires et deux rangées longitudinales de fins denticules sur sa face interne convexe; le $3^{\circ}$ constitue une longue lame recourbée formant pince, avec une grande soie accessoire sur le côté de sa portion basilaire élargie.

Pattes thoraciques natatoires avec l'endopodite différant dans la 4 e paire (4).

Pattes de la $5^{\circ}$ paire (fig. 14, 15, 25 et 26) courtes, recourbées postérieurement sur les côtés de l'abdomen, avec l'épine terminale du second article très forte et particulièrement longue, puisqu'elle atteint le milieu du $1^{\mathrm{er}}$ segment abdominal dans les deux sexes.

Orifices externes des oviductes (fig. 14 et 26) placés à l'angle latéro-dorsal et postérieur du $1^{\text {er }}$ segment abdominal et fortement inclinés sur l'axe longitudinal.

(1) La forme des antennules est mal figurée par Auriviluius (loc. cit.; Taf. II, fig. 1 et 2 ; Taf. IV, fig. 1).

(2) Et non par quatre soies (Aurivillius) ni par deux épines (RafF. ¿̇ Mont.).

(3) Légèrement prolongé à son bord distal par son petit bouton saillant qui est le vestige de l'épine terıninale du mâle ou des deux sexes dans les autres espèces. Cette disposition est légèrement indiquée par Raffaele È Monticelli (l.c.; fig. 16, pmp) mais totalement méconnue par Aurivillius ( $l$.c.; Taf. II, fig. 6).

(4) La patte figurée par Raffaele è Monticelli (l.c., fig. 15) comme appartenant à la $4^{0}$ paire, ne convient nullement à cet appendice, à cause de l'organisation de l'endopodite. Par la brièveté ct la largeur des deux rames, elle conviendrait plutôt à la $1^{\mathrm{r}}$ paire. 
Orifices des sacs à spermatophores (fig. 25) ventraux, larges, avec la lèvre supérieure munie de deux soies courtes sans denticules. Spermatophores de forme très régulière et ressemblant à une larme (fig. 15).

Sacs ovigères longs, elliptiques, contenant un grand nombre d'œufs colorés en rougeorangé, de même que les œufs múrs contenus dans les oviductes avant la ponte.

Ce Copépode est très abondant sur les branchies de gros Modiola modiolus L. dragués entre les bancs du Varne et du Colbart, au milieu du détroit du Pas-deCalais. Aurivilluus l'a découvert dans les Modioles: M. vulgaris Flemming et aussi dans les Moules: $M$. edulis L. Raffaele E Monticelli l'ont obtenu dans Mytilus edulis L., var. gallo-provincialis LAMARCK.

Distribution: Mer du Nord, Manche, Méditerranée.

\section{Modiolicola inermis, CANU.}

1891. Modiolicola nov. sp. ; Canu, 35, page 436.

Espèce très voisine de Modiolicola insignis Aur. dans sa forme, sa taille et la plupart des caractères généraux.

Abdomen 5-segmenté, avec le bord distal des $2^{e}, 3^{e}$ et $4^{e}$ segments portant sur la face ventrale, au lieu de 12 à 15 épines triangulaires comme chez M. insignis, une série de fins poils chitineux très courts et très serrés, au nombre de cinquante environ, visibles à l'aide d'objectifs assez

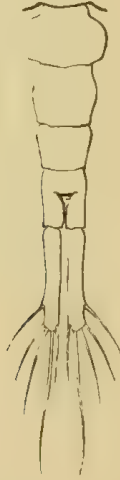

Fig. 19. - Modiolicola inermis CANu, Abdomen et pièces furcales du mâle jeune, vues par la face ventrale.

grande épine uncinée, une autre plus courte et des soies rigides. puissants.

Pièces furcales longues et cylindriques, doubles en longueur du dernier segment abdominal, avec quatre soies terminales bien développées et une soie marginale assez longue insérée auprès de l'extrémité postérieure, vers le quart distal de la longueur (voir fig. 19 dans le texte).

Antennule, mandibule, première maxille, seconde maxille externe, seconde maxille interne $\mathrm{du}$ mâle et appendices thoraciques semblables à ceux de $M$. insignis.

Antenne plus courte et beaucoup plus ramassée que dans $M$. insignis, se rapprochant un peu dans son aspect, de l'antenne de Sabelliphilus et de Herrmannella. Les quatre articles qui la composent sont de longueurs presque égales: le $3^{e}$ porte a son bord distal interne une épine chitineuse et de petites soies: le $4^{\mathrm{e}}$ se termine par une 
Ce Copépode habite également en semi-parasite la cavité palléale et la branchie d'un Mollusque lamellibranche, je l'ai recueilli en assez grande quantité dans Pecten opercularis LinNé, au large d'Etaples, en compagnie de Herrmannella rostrata CANU.

Distribution : Manche.

\section{Pseudanthessius, Claus.}

1880. Lichomolgus, Thorell, pars; Brady, 20, vol. 3, p. 40.

1889. Pseudanthessius; Claus, 66, p. 18.

1891. Pseudanthessius, Cls; Canu, 31, p. 480 et 481 .

Lichomolgide ayant :

$I^{0}$ Le corps cyclopoïde de forme très ramassée;

$2^{0}$ Le $1^{\mathrm{er}}$ somite thoracique soudé au céphalon, et le $1^{\mathrm{er}}$ somite abdominal soudé au $2^{\mathrm{e}}$ dans la femelle.

$3^{0}$ Les articles des antennules allongés et non renflés dans la région proximale.

$4^{0}$ Les antennes $4^{-a r t i c u l e ́ e s, ~ t r e ̀ s ~ a l l o n g e ́ e s, ~ a v e c ~ l e ~} 2^{\theta}$ article plus long et le $3^{\theta}$ plus court terminées par trois ou quatre épines sétiformes coudées à angle droit et plusieurs soies rigides et droites ; pourvues au bord distal du $3^{\mathrm{e}}$ article d'une épine coudée et de deux soies droites.

$5^{0}$ La lèvre supérieure profondément incisée sur la ligne médiane, avec deux lobes latéraux saillants recouvrant presque entièrement les mandibules courtes et épaisses, garnies de dents solides sur le bord postéro-interne et d'une courte épine courbée à son extrémité distale, sans prolongement falciforme grêle et flexible ni soie accessoire sur le bord antérieur concave.

$6^{0}$ Les secondes maxilles externes 2 -articulées, avec un prolongement falciforme garni de dents solides et espacées, et une soie distale interne denticulée.

$7^{0}$ L'endopodite des quatrièmes pattes thoraciques allongé et simple, avec deux soies épineuses terminales.

$8^{\circ}$ Les cinquièmes pattes thoraciques sans articulations, avec les deux articles habituels soudés en une lame adhérant au tronc et portant des soies ou une épine sur ses bords externe et distal.

Le genre Pseudanthessius, établi par Claus sur un unique exemplaire femelle recueilli à l'état de liberté, est des plus caractérisés parmi les Lichomolgidés. La forme du corps, la nature des épines antennaires préhensiles, grèles, sétiformes, coudées et articulées en leur point de courbure, la structure des mandibules garnies de quelques dents masticatrices particulièrement solides, l'endopodite simple des quatrièmes pattes et les cinquièmes pattes simples et entièrement soudées 
au segment qui les porte, sont autant de caractères morphologiques de grande valeur qui militent en faveur de la distinction générique de ces animaux. Par quelques-uns de ces caractères, deux espèces très incomplètement décrites par BRADY viennent s'adjoindre à celle qui fut établie par Claus. Ce sont : Lichomolgus thorelli BraDy (l. c.; p. 47-49, Pl. LXXXVIII, fig. I-9), une forme très voisine (I) de Pseudanthessius gracilis Claus; - et d'autre part Lich. liber BRADY ET Robertson (ibidem; p. 44-46, Pl. LXXXVI, fig. I-I3) qui se rapproche sûrement du genre Pseudanthessius par la nature des quatrième et cinquième paires de pattes. Malgré les divergences considérables que présentent entre elles (2) cette dernière espèce et une forme nouvelle que j'ai recueillie, je crois pouvoir les rapprocher l'une de l'autre à cause de quelques caractères communs (3). Peut-être même les divergenccs constatées ne sont-elles dues qu'à un mélange ou une confusion d'espèces de la part de BRADY, ce que font bien supposer les caractères trop aberrants que présentent certains organes de ces animaux. Toutefois diverses particularités saillantes, faciles à observer, non signalées par BradY, s'opposent absolument à la réunion spécifique des deux formes.

Une particularité intéressante du genre Pseudanthessius consiste dans la situation latéro-ventrale des orifices externes des oviductes dans la femelle, position primitive qui semble conservée dans ce seul genre de Lichomolgide. Cette situation ventrale des orifices femelles concorde avec la forme cylindrique plus parfaite de l'abdomen dans cet animal.

J'insisterai encore ici sur la présence de nombreuses cellules sensorielles dans les téguments du Pseudanthessius que j'ai pu étudier. Dans le céphalothorax, en particulier, sur les faces dorsale et latérale des segments, la chitine assez épaisse est perforée d'un grand nombre d'orifices punctiformes münis chacun d'un petit poil rigide ( $\mathrm{Pl}$. XXV, fig. 17). A ces orifices correspondent intérieuremeut de grosses cellules piriformes, au contenu finement granuleux parsemé de grosses vésicules claires et réfringentes, et pourvues d'un noyau nucléolé arrondi. Divers auteurs, et notamment Della VAlle dans Sabelliphilus sarsi, ont signalé ces cellules dans les Lichomolgidés et les Sapphirinidés.

(1) Si elle n'est point identique, comme le feraient supposer la forme de l'abdomen et des pièces furcales de la femelle (l. $c_{.}$, fig. 8$)$, celle de la seconde maxille interne de la femelle $(l . c .$, fig. 5$)$ et l'armature de son second article, l'armature du $4^{e}$ article de l'antenne $\left(l_{0} c\right.$. , fig. 2). Toutefois, les quatrièmes pattes thoraciques ont l'endopodite plus large et plus allongé (l. c., fig. 7 ) dans L. thorelli que dans Ps. gracilis.

(2) Notamment dans l'armature et la forme générale des antennules (20, vol. 3, Pl. LXXXVI, fig. 2), dans. l'armature des antennes (fig. 3 ), dans les mandibules (fig. 4), dans l'armature du $2^{\mathrm{e}}$ article des secondes maxilles internes (fig. 6 et 7 ).

(3) La forme de l'abdomen et des pièces furcales ( $l_{0} c_{0}$, fig. 1 et 12$)$, celle des quatrièrnes et cinquièmes pattes thoraciques (l. c., fig. 10 et $\$ 1$ ), l'aspect général de l'animal entier (l. c., fig. 1 ). 
 \\ Pseudanthessius sauvager, $\mathrm{C}_{\mathrm{AnU}}$.}

18a1. Pseudanthessius sauvagei; Canu, 34 p. 481.

Planche XXV, Fig. I-17.

Taille : $\sigma$ et $q$, de 1 aे $1,3 \mathrm{~m} / \mathrm{m}$.

Céphalothorax épais et fortement convexe sur la face dorsale (fig. 1 et 2 ); $5^{\mathrm{e}}$ segment thoracique réduit et très étroit (fig. 13 et 15 ).

Abdomen court et étroit, subcylindrique, avec les derniers segments presque égaux entre eux et plus courts que larges. Pièces furcales peu développées, épaisses, de forme cylindrique et dépassant en longueur le segment qui les porte; avec quatre grandes soies terminales barbelées, une petite soie dorsale et une sore marginale insérée aụ tiers distal de la longueur (fig. 1).

Antennule (fig. 1, 2 et 3 ) avec les articles presque cylindriques et allongés, spécialement le $2^{e}$, puis les $4^{e}, 5^{e}$ et $6^{\theta}$, et enfin les $1^{\text {en }}, 7^{e}$ et le $3^{e}$ plus court; avec des soies longues et flexibles sur tous les articles.

Antenne (fig. 3) avec des soies longues, et sur le $4^{\mathrm{e}}$ article quatre épines sétiformes en crochet.

Mandibule (fig. 4, 5 et 6 ) en forme de lame courte, élargie à sa base et vers son extrémité, et portant au bord interne cinq grosses dents et une série de denticules plus petits, avec une épine contournée en crochet à l'extrémité distale.

Première maxille (fig. 7) courte, avec trois soies terminales raides.

Seconde maxille externe (fig. $3, m x^{2} e$, fig. 8) avec l'article basilaire renflé et l'article terminal allongé, falciforme, portant à sa face interne, en outre d'une courte soie distale barbelée, une petite soie proximale délicatement denticulée.

Seconde maxille interne considérablement plus développée dans le sexe $\sigma$. - Chez la femelle (fig. 3 et 9), le $2^{\mathrm{e}}$ article est long, légèrement infléchi vers l'intérieur, sans revêtement denticulaire et pourvu sur la face interne d'une longue soie barbelée, épaisse et égale en longueur à l'appendice entier, et aussi d'une petite épine triangulaire; le $3^{\mathrm{e}}$ article est court, en griffe effilée, avec deux petites soies accessoires._Chez le mâle (fig.2 et 10), l'appendice recouvre toutes les pièces buccales: les $2^{e}$ et $3^{e}$ articles forment une pièce puissante; la face interne $\mathrm{du} 2^{\mathrm{e}}$, irrégulièrement convexe, porte deux groupes importants de denticules triangulaires avec deux épines triangulaires et pointues; le $3^{e}$ est une longue lame recourbée, arrondie $e_{i}$ spatule à son extrémité et flanquée de deux soies accessoires allongées (1).

Pattes natatoires bien développées. Endopodite de la" quatrième paire allongé, à bords presque parallèles et finement garnis de poils courts, sans lobe cilié saillant au bord externe du tiers proximal (2).

(1) Les secondes maxilles du male et de la femelle chez Pseudanthessius liber Brady ET RoBertson sont, d'après BRADY, armées de façon toute différente. Les caractères inđiqués ici pour Ps. sanvagei n’auraient pu, à cause de leur netteté et de leur importance, échapper à l'auteur anglais.

(2) Ce qui le rapproche de Ps. liber BR. Er RoBo, pour l'éloigner de Ps. gracilis Cls et Ps. thorelli Br. 
Cinquième patte thoracique (fig. 12, 13, 14 et 15) sessile et très peu saillante (1) avec une soie marginale dorsale correspondant à la soie du $1^{\mathrm{er}}$ article dans les autres Lichomolgidés, et, de plus, une soie marginale latérale, une forte épine dentelée et recourbée postérieurement, qui correspondent à l'armature habituelle du second article libre dans les autres genres.

Sacs ovigères assez courts et piritormes, contenant un grand nombre d'œufs dont la couleur est d'un blanc mat.

Lèvre supérieure des orifices externes des sacs à spermatophores en forme d'un bourrelet épais et saillant (fig. 15 et 16 ) avec deux longues soies grêles à l'angle latéro-postérieur. Spermatophores allongés et presque piriformes.

Pseudanthessius sauvagei est une espèce curieuse par l'existence semi-parasitaire qu'elle mène en compagnie de Echinocardium cordatum Pennant, oursin irrégulier si abondant dans les sables de la côte boulonnaise; je l'ai trouvée en assez grande abondance sortant des piquants de son hôte recueilili avec soin dans le but de découvrir ce parasite. L'indication des habitudes de cette espèce permettra sans doute de déterminer l'hôte des autres espèces du genre.

Distribution : Manche.

\section{E R GASI L I D A E.}

Copépodes monochilés ayant:

$1^{0}$ Des habitudes parasitaires ou semi-parasitaires.

$2^{0} \mathrm{La}$ forme assez irrégulière et tourmentée, voisine encore de celle des Cyclops par la distinction nette d'un céphalothorax segmenté et d'un abdomen également segmenté.

$3^{\circ}$ Les antennules courtes, peu (6-) articulées, avec des soies plus ou moins longues, semblables dàns les deux sexes.

$4^{0}$ Les antennes 3 -articulées, transformées en pinces exclusivement préhensiles, plus longues chez le màle.

$5^{0}$ Les secondes maxilles divisées seulement dans le sexe $\sigma^{\nearrow}$ en deux portions dont l'interne est préhensile, simples dans le sexe ?.

$6^{0}$ Les pattes thoraciques des quatre premières paires biramées et natatoires, de conformation presque normale.

$7^{0}$ Les pattes de la $5^{\theta}$ paire réduites à un simple article assez court, avec deux soies terminales.

(1) Ce qui rappelle encore $P_{s}$. liber BR. ET R., et non point $P_{s}$ gracilis et thorelli. 


\section{Thersites, Pagenstecher.}

1860. Thersites; PAgensteCher. 158 , page 118.

1863. Ergasilus, Von Nordmann, pars; Króyer, 125, pages 227 et 228.

1875. Ergasilus, Von Nordm., pars; Claus, 55, pages 339 et 340.

1891. Thersites, Pag.; Canu, 34, page 482.

Ergasilidés ayant :

$\mathrm{I}^{0} \mathrm{La}$ forme du corps particulièrement trapue par un renflement sphérique de la face dorsale des premiers segments céphalothoraciques et par la brièveté des autres segments thoraciques et abdominaux.

$2^{0}$ Les antennes courtes et épaisses, terminées par deux épines inégales, solides et recourbées, portées par le $3^{\mathrm{e}}$ article.

\section{Thersites gasterostei, Pagenstecher.}

1861. Thersites gasterostei; Pagenstecher, 158, pages $118-125$. Taf. VI, fig. $1-9$.

1863. Ergasilus gasterostei; KR $\phi$ YER, 125, pages $233-237$ et 239 , Taf XIl, fig $2 a-h$

1891. Thersites gasterostei, Pag.; Canu, 34 , page 482.

\section{Planche XXIII, Fig. I3-I8.}

․ - Taille : $0,5 \mathrm{~m} / \mathrm{m}$ de longueur et $0,34 \mathrm{~m} / \mathrm{m}$ de plus grande largeur.

Malgré les observations émises par Claus contre cette opinion, je considère cette forme comme parfaitement caractérisée en qualité de genre (ou de sous-genre?). La forme des antennes et celle du céphalothorax sont deux caractères concordants qui la séparent nettement des véritables Ergasilus.

Bien étudié par PAGenstecher, el moins complètement par KroYer, qui donne toutefois quelques renseignements sur le sexe $\sigma^{\nearrow}, T h$. gasterostei présente néanmoins quelques particularités d'organisation pour lesquelles il convient de rectifier ou de compléter les descriptions de ces auteurs.

La lèvre supérieure (fig. 14,ls) forme une sorte de capuchon bilobé recouvrant en grande partie les pièces buccales; il n'y a point de véritable lèvre inférieure reconnaissable.

Les mandibules ( fig. 14, 15 et $16 \mathrm{md}$ ), considérées par Pagenstecher comme premières pattes mâchoires (1), sont des lames aplaties et recourbées, plus étroites dans leur région distale denticulée vers l'intérieur, plus larges dans leur région proximale où le bord postérointérieur porte six grosses dents triangulaires.

Les premières maxilles ( fig. 14 et $16, m x^{1}$ ) sont de courts moignons cylindriques terminés par deux courtes soies et abrités presque entièrement sous les mandibules.

Les secondes maxilles ( fig. 14, 15 et $16, m x^{2}$ ) sont des appendices plus forts, composés d'un $1^{\mathrm{er}}$ article large et cylindrique, supportant une région distale plus grêle et recourbée vers

(1) A ce sujet, voir Claus, 55 , page 340 
la bouche. Cette dernière, incomprise de Pagenstecher et de Krjyer (1), n'est point simple comme Claus l'a décrite dans Ergasilus sieboldi Von Nordmann. Elle comprend au contraire trois articles successifs : le $1^{\text {er }}$, un peu plus large, porte à son bord externe une petite soie; le $2^{0}$ est plus allongé et légèrement courbé; le $3^{e}$, effilé et recourbé en lame de serpe, porte deux séries de poils denticulés sur ses bords et une épine conique à soz extrémité (2).

L'abdomen est court (fig, 17), avec le ${ }^{\text {er }}$ segment à peu près aussi long que large et garni de plusieurs séries ondulées de petits poils fins sur sa face ventrale; avec les $2^{\mathrm{e}}$ et $3^{\mathrm{e}}$ segments beaucoup plus courts et bordés de poils fins à leur limite postérieure; avec le $4^{\text {e }}$ segment profondément incisé en deux lobes donnant appui aux pièces furcales courtes et munies d'une longue soie en épine et de petits poils terminaux.

L'animal est d'un blanc assez éclatant dans la plus grande partie du corps, ce qui est dû en partie au développement du corps graisseux et des oviductes chargés d'œufs. Les ovules sont de la même couleur. Seuls font exception l'œil tripartite rouge-vif et le tube digestif, antérieurement cruciforme, d'une teinte bleu-indigo uniforme.

Les deux sacs ovigères cylindriques, avec un grand nombre d'œufs disposés assez régulièrement en 6 ou 8 rangées longitudinales, atteignent à peu près la longueur de l'animal.

A mesure que les œufs pondus avancent dans leur développement, apparaissent dans l'embryon nauplien deux séries de taches pigmentaires, l'une rouge en forme d'x pour l'œil (3); les trois autres circulaires pour l'intestin. De celles-ci, les deux extrêmes sont bleues pour l'intestin antérieur et postérieur, et la médiane jaune-grise pour l'intestin moyen glandulaire.

A l'éclosion, les nauplius, de forme trapue et ramassée, présentent encore ces mêmes colorations, d'abord l'œil rouge, puis le tube digestif partagé en trois régions arrondies : l'intérieure et la postéricure bleues, la médiane jaunâtre. Par divers caractères de sa forme générale, de son tube digestif et des soies furcales allongées, l'embryon libre de Thersites se rapproche beaucoup de celui des Lichomolgidés et appuie la parenté assez éloignée de ces animaux.

J'ai recueilli une dizaine d'exemplaires femelles de Thersites gasterostei sur les branchies, l'opercule et les nageoires pectorales de Gasterosteus aculeatus Linné, pêché dans l'eau saumâtre de l'estuaire du Wimereux. PAGENSTECHeR a recueilli cette espèce sur les branchies de $G$. aculeatus pris à Ostende dans l'eau saumâtre souvent mélangée d'eau salée et d'eau douce. KR $\varnothing$ YER trouva un assez grand nombre d'exemplaires de ce parasite dans les branchies ou les téguments de G. aculeatus, provenant des eaux saumâtres ou salées du Sund sur les côtes de

(1) Ce qu'expliquent suffisamment la petitesse des pièces buccales et les difficultés de leur étude.

(2) THORELl a figuré une structure analogue pour l'extrémité de la seconde maxille externe chez Ergasilus sieboldi (24, Taf. XI , fig. $16 \mathrm{M}^{2}$ ).

(3) Cet oil n'est nullement bleu, comme le dit PAGENSTECHER, qui le confond avec la région antérieure du tube digestif : «....durch die Anwesenheit eines blauen Augenfleckes die mehr fortgeschrittene Embryonalent wickelung gekennzeichnet. " Loc. cit, page 125. 
Danemark; il l'obtint également du Groenland et des Iles Feroë. En conséquence, ce parasite semble particulier aux Épinoches habituées aux milieux marins; on ne l'a point signalé dans des eaux absolument douces.

Distribution : Océan Arctique, Mer du Nord, Baltique, Manche.

\section{Auliostoma.}

Copépodes diporodelphes ayant :

$I^{0}$ Des habitudes parasitaires durables ou passagères sur les Invertébrés, et le corps de forme variable, cyclopoïde ou vermiforme.

$2^{0}$ Les antennules de constitution variable, généralement semblables dans les deux sexes, et les antennes préhensiles terminées par des soies ou des crochets.

$3^{0}$ La bouche située au fond d'une dépression aux bords saillants, disposés en bourrelets convexes ou en disque circulaire; abritée ainsi dans un appareil non articulé avec le tronc, recouvrant partiellement ou totalement les coxopodites mandibulaires sans aider à leur fonctionnement.

$4^{0}$ Les divers appendices plus ou moins développés et subissant fréquemment d'importantes réductions ou adaptations.

$5^{0} \mathrm{La}$ circulation lacunaire.

$6^{0}$.Les œufs pondus en sacs extérieurs ou abrités dans une cavité incubatrice.

Je n'insisterai point spécialement ici sur les relations de ce sous-groupe de Diporodelphes; elles ont été indiquées précédemment d'une manière suffisante.

La constitution de l'appareil buccal est le principal caractère qui réunisse ces étres encore trop peu connus et trop rarement observés pour qu'on puisse délimiter entièrement les rapports qui les unissent. Ces particularités de l'appareil buccal et mandibulaire se retrouvent à différents degrés de complication dans divers Copépodes parasites des Crustacés, des Annélides et des Echinodermes, qui sont distribués, dans l'état actuel de nos connaissances, en plusieurs petites familles des plus intéressantes.

\section{H E R S I L I I D A E.}

Copépodes auliostomes ayant :

$I^{0}$ Une existence plus ou moins parasite sur les Invertébrés (Crustacés, Annélides). 
$2^{0}$ Le corps complètement segmenté avec le premier somite thoracique soudé au céphalon.

$3^{\circ}$ Les antennules assez diversement articulées ( 7 à 10) et semblables dans les deux sexes.

$4^{\prime}$ Les antennes 4-articulées, coudées au niveau du troisième article qui sert de point d'appui pour l'adhésion, semblables dans les deux sexes.

$5^{\circ}$ L'atrium buccal plus ou moins clos et abritant des mandibules réduites au coxopodite, impropres à la mastication, mais disposées pour pincer les parois membraneuses.

$6^{\circ}$ Les premières maxilles bilobées, avec la partie basilaire interne armée d'épines comme un lobe masticateur, et la partie externe saillante, lamelleuse et pourvue d'un petit nombre de soies barbelées, ainsi qu'un palpe.

$7^{0}$ Les secondes maxilles nettement séparées : en secondes maxilles externes 2-articulées, préhensiles plutôt que masticatrices, et en secondes maxilles internes, pluriarticulées et préhensiles, modifiées et hypertrophiées dans le mâle.

$8^{0}$ Les pattes des quatre premières paires, natatoires, rarement adhésives.

91) Les pattes de la cinquième paire biarticulées, lamellaires.

$10^{0}$ La circulation lacunaire.

$17^{\circ}$ Les conduits génitaux femelles pairs et symétriques, s'ouvrant sur les côtés du corps, et les cuufs disposés en deux sacs après la ponte.

Ainsi délimitée, cette famille comprend actuellement les genres Clausidium Kossmann (1), Hersiliodes Canu, Giardella Canu et Nicothoe Aud. et M.-Edw.

L'étude de Nicothoe astaci Aud. et M.-EDw., que j'ai reprise sur un certain nombre d'echantillons bien conservés dans l'alcool $\left\{2, \mathrm{~m}^{\prime}\right.$ a convaincu de la parenté de ce Copépode avec les Hersiliens, ainsi que je l'avais entrevu en i 888 (3). Entre Nicothoe et Giardella, il existe des différences dans le degré de spécialisation des organes, mais point de véritables dissemblances morphologiques. Les antennules comptent onze articles (4) au lieu de sept, mais c'est là une divergence d'importance insignifiante. Les antennes 4-articulées sont conformées sur le mème type que dans Giardella et Hersiliodes; elles sont très courtes (Pl. XXX, fig. 3), mais coudées au niveau du troisième article, la concavité de l'appendice étant dirigée vers l'exterieur et un peu vers l'avant. Au coude ainsi formé se trouve la surface adhesive, comparable en tous points à celle que nous connaissons chez Hersiliodes

(1) Synonyme de Hersilia PHILIPPI.

2) J'ai obtenu ces échantillons, grâce à l'obligeance de mon ami M. le Dr V. Willem, assistant à l'Université de Gand.

(3) Voir 30 , page 426.

(4) Claus (41), confirmant l'indication de Rathke (172), en compte seulement dix, parce qu'il omet le premier article, caché par le second, cylindrique et allongé. 
(PI. XXX, fig. 4). Le troisième article se prolonge interieurement en une large saillie convexe vers l'intérieur et garnie de petits tubercules pour faciliter l'adhésion; il a été réuni par Claus (44) - qui donne l'antenne comme 3 -articulée avec le quatrième article plus court, lamellaire et porteur de truis épines terminales résistantes; la séparation de ces deux articles est d'ailleurs particulièrement nette, malgré la taille réduite de l'appendice.

La véritable différence consiste, chez $=$ Nicothoe, dans l'organisation de l'atrium buccal en un disque-suçoir.

La première maxille, assez bien décrite et figurée par Claus (4I), concorde dans sa disposition avec celle de Giardella ; la partie masticatrice interne est cependant réduite, ce que le genre de vie de l'animal rend parfaitement compréhensible.

La seconde maxille externe est également formée de trois parties: les deux articles basilaires ont la même forme que dans Giardella, mais sont dépourvus de soies internes; l'extrémité est exclusivement formée de deux épines recourbées en griffe, sans la troisième soie accessoire interne qui existe chez Giardella (P1. XXX, fig. 7). Dans la seconde maxille interne, l'extrémité préhensile, simple chez les autres Hersiliens, est formée chez Nicothoe d'une suite de trois articles courts, armés de faibles épines effilées, parfaitement distincts chez la femelle adulte.

Les pattes natatoires, avec leurs rames 3 -articulées, ont la même structure que chez Giardella, et présentent un allongement notable de leurs articles basilaires. La cinquième patte montre également la forme d'une lame aplatie.

En résumé, les caractères morphologiques de la femelle rattachent Nicothoe aux autres Hersiliens parasites de la Callianasse. La connaissance des caractères sexuels secondaires du sexe mâle serait désirable pour confirmer la parenté de ces Copépodes. Or, le mâle de Nicọthoe n'a jamais été rencontré avec les femelles sur les branchies du Homard.

L'échantillon unique - recueilli sur le Homard par LeUCKART - décrit par Claus (44) comme un mâle, n'est qu'une jeune femelle immature, avant sa déformation par le développement des ovaires. En effet, Claus n'a point observé les glandes sexuelles; et les arguments qu'il avanze pour appuyer son interprétation n'ont aucune valeur. Car l'abdomen de l'échantillon figuré est celui d'une jeune femelle; il porte au premier segment l'ébauche des orifices des oviductes. De plus, RatHKE (loc. cit., pages I I I et I15) n’a nullement suivi, dès les premières études du développement, l'apparition des ailes sacciformes produites sur la femelle adulte par l'expansion des oviductes et de l'estomac. D'autre part, VAn Beneden, $\mathbf{I}$ ) figure une jeune femelle de Nicothoe avec quatre paires de pattes natatoires et

(1) Mim. Acad. Sc. Belgique, tome XXIV. 
sans appendices dorsaux, ainsi que deux stades ultérieurs présentant ces appendices en voie de développement. Chez $N$ icothoe, comme dans la plupart des Copépodes parasites à l'extérieur des animaux, la déformation de la femelle a donc lieu après l'entière apparition de l'organisation fondamentale, ce qui réduit à néant l'interprétation avancée par Claus dans le cas qui nous occupe.

Pour la description détaillée des Hersiliens du Boulonnais, des genres Hersiliodes et Giardella, je renvoie le lecteur à l'étude que j'en ai publiée (31); j'insisterai sur quelques points nouveaux de leur histoire naturelle :

$I^{o}$ Leur qualité de Copépodes diporodelphes est indiscutable si l'on considère la disposition des ouver-

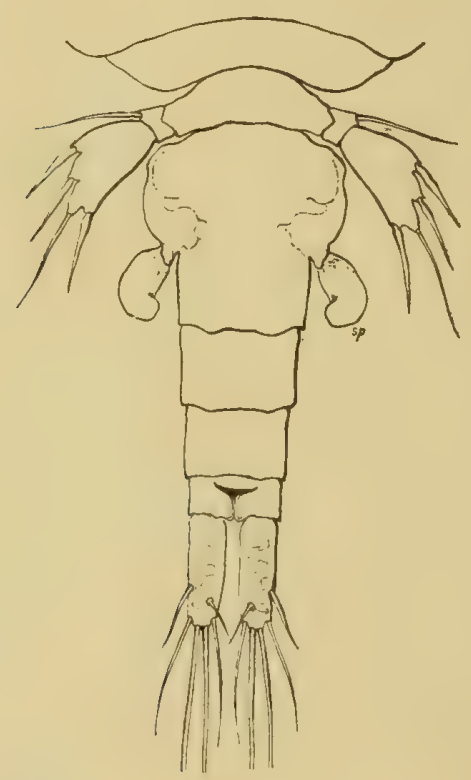

Fig. 20. - Hersiliodes thompsoni Canu ; femelle vue par la face dorsale, avec les deux spermatophores $(s p)$ fixés aux ouvertures sexuelles. tures sexuelles. Chez aucun d'entre eux, la face ventrale du premier segment abdominal ne présente, dans la femelle, l'orifice médian des monoporodelphes. La fig. 20 ci-contre montre les spermatophores d'Hersiliodes thompsoni fixés à l'angle postéro-inférieur de la dépression qui loge les ouvertures des oviductes. De chaque côté de l'abdomen, se trouve un pore de fécondation où sont accolés, après la copulation, les spermatophores brièvement pédiculés. De ces pores, partent les canaux séminifères qui les conduisent dans l'appareil génital femelle.

$2^{\circ}$ La disposition de l'appareil digestif présente dans cette famille un interêt tout spécial par suite des modifications considérables amenées par l'influence du mode de nutrition sur la région pharyngienne. Dans Giardella et Hersiliodes, la disposition du pharynx chitineux faisant suite à la cavité de l'atrium, ne diffère pas considérablement de celle que présentent la majorité des Copépodes libres ou 
semi-parasites. Le pharynx est alors un tube plus ou moins recourbé qui est soutenu dorsalement par de petits muscles élevateurs d'un développement normal, sous l'influence desquels il joue le rôle de tube aspirateur. Ce tube chitineux déborde légèrement, sous forme d'une collerette en entonnoir, à l'intérieur de la cavité stomacale. C'est ce qu'on voit dans bon nombre de Copépodes et mème dans beaucoup d'autres Entomostracés, comme l'ont décrit plusieurs naturalistes.

Dans Nicothoe astaci + , la disposition du pharynx est toute autre. Quand on observe par la face dorsale un exemplaire très légèrement éclairci dans une solution faible de potasse caustique ( $\mathrm{au} 4 / 100$ ), on aperçoit (P1. XXX, fig. 2), au-dessus des muscles en V $(n)$ qui rattachent la base du disque-suçoir aux faces latérodorsales de la carapace, deux faisceaux musculaires épais $(m)$ formant un angle presque droit ouvert antérieurement. A mesure que la préparation s'éclaircit, on suit le trajet des faisceaux $m$ unissant les parois latéro-dorsales du tégument céphalique à la face dorsale d'un tube chitineux assez vaste $(h p h)$ qui appartient à l'appareil digestif.

Le pharynx de Nicothoe'astaci montre un développement unique parmi les Copépodes, développement ignoré jusqu'à ce jour des naturalistes. Pour l'étudier, il est plus commode de débarrasser entièrement le segment céphalique des tissus qui gênent l'observation en le laissant longuement macérer dans une solution de potasse plus concentrée (à Io ou $15 \%$ ). Au travers du tégument dorsal, on aperçoit alors le pharynx ( $p h$, Pl. XXX, fig I) partant de la cavité du disque-suçoir, sous forme d'un tube étroit, presque cylindrique, et remontant vers la face dorsale. Bientôt, ce pharynx se recourbe et s'élargit; il débouche enfin dans un appareil chitineux très vaste $(h p h)$ ouvert à sa partie postérieure et qui atteint le niveau d'insertion des secondes maxilles internes. Cet appareil correspond sans aucun doute à l'hypopharynx des Crustacés malacostracés, en particulier des Amphipodes; il représente aussi, mais seulement dans sa partie postérieure, l'entonnoir saillant du pharynx chez les autres Copépodes (I). C'est un manchon chitineux, à parois minces et flexibles, qui présente sur la ligne médiane dorsale une carène saillante flanquée de deux faces latéro-dorsales. Sur ces faces concaves ou même repliées vers l'intérieur, s'insèrent les muscles latéro-dorsaux $m$. A l'état de relâchement, ils laissent l'hypopharynx étendu et rétréci dans l'effort exercé sur les extrémités. par le pharynx d'un côté, par l'estomac de l'autre. Lorsque les muscles se contractent, les faces latéro-dorsales sont amenées vers l'extérieur; l'hypopharynx est fortement gonflé, ce qui produit dans l'atrium buccal une énergique aspiration.

(1) Sur l'hypopharynx des Crustacés, consulter: Claus, Organismus der Phronimiden, Arb. Zool. Inst. Wien, Tom. II, 1879, qui compare cet organe dans les Phronimes, les Argules et d'autres Entomostracés. 
Par la contraction rhytmique des muscles s'établit un mouvement de succion capable de remplir un rôle important dans la prise de nourriture.

Aucune disposition semblable n'est connue chez les Copépodes, ni même chez les Entomostracés. Seul, l'hypopharynx des Amphipodes peut être comparé à ce que nous venons de décrire; mais il existe dans ces Malacostracés une complication bien plus grande de l'organe destiné à remplir une action masticatrice sur la nourriture ingérée. Parmi les Copépodes parasites des Annélides, LiST a signalé chez Gastrodelphys (139, pages 96-98, Taf. V, fig. 19 et 20) l'existence d'un pharynx aspirateur qui ne peut être non plus comparé, pour sa forme et sa musculature, à ce que j'ai étudié dans Nicothoe: l'œsophage de Gastrodelphys clausi GraEFFE est situé entre la bouche et le système nerveux, tandis que l'hypopharynx de Nicothoe astaci se trouve - comme celui des Amphipodes - au-delà de l'anneau péri-cesophagien.

\section{Hersiliodes, Canu.}

1888. Hersiliodes; CanU, 31 . pages 417 et 418.

1889. Hersiliodes, Canu; Thompson, 199, page 185.

Corps peu aplati. Mandibules terminées à l'extrémité distale par une griffe articulée, une plaque triangulaire denticulée et deux grosses soies barbelées ( $\mathrm{Pl} . \mathrm{XXX}$, fig. 5 ). Premières maxilles avec une grosse épine aplatie en cuilleron et dentelée, à l'extrémité de l'appendice.

\section{Hersiliodes pelseneeri, Canu.}

1888. Hersiliodes Polseneevi; Canu, 31, pages $418-422$, PI. XXIX.

Corps très étroit et allongé; abdomen un peu plus étroit que le céphalothorax. Pièces furcales longues, légèrement renflées à leur base, un peu plus minces vers leur extrémité.

Antennes garnies de courts tubercules chitineux sur la face interne du bord adhésif, dans les troisième et quatrième articles (Pl. XXX, fig 4).

Épine terminale de la seconde maxille externe solide, très large et terminée par cinq grosses dents légèrement recourbées. Seconde maxille interne très longue, avec les dẹx articles basilaires larges et l'épine terminale forte et plus longue que l'un de ces articles.

Cinquième patte thoracique formée d'une lame aplatie longue et étroite.

Tube digestif coloré en rouge vif, ainsi que les ovules.

Une jeune femelle immature de ce Copépode a été recueillie par moi dans le tube digestif d'un Clyménien abondant dans les sables de la Pointe-aux-Oies, près d'Ambleteuse. 
Les jeunes stades-copépodes de cette espèce, parfaitement reconnaissables aux caractères indiqués ci-dessus, vivent en pélagiques à la surface de l'eau; ils sont très fréquents parmi les Copépodes recueillis dans les pêches au filet fin aux environs de cette localité.

En I868, Grube a décrit et figuré ( $\mathbf{r}$ ) un Copépode vivant sur des Annélides clyméniens de Saint-Waast-la-Hougue et de Roscoff. Malgré quelques ressemblances qui existent entre Antaria latericia GruBE et Hersiliodes pelseneeri CANU (2), je crois devoir considérer ces deux formes comme distinctes. Une étude plus complète des caractères est indispensable pour permettre d'affirmer qu'il n'existe point - sur les Clymènes comme sur la Callianasse - plusieurs espèces d'un même genre. En effet, nous ne connaissons point l'ornementation des antennes (dites 3 -articulées!), pas plus que l'organisation, voire même le nombre exact (!), des pièces buccales. Dans ces conditions, comme caractères précis, il reste l'habitat, ou le contour général du corps figuré d'une manière bien insuffisante.

Si l'on considère que, parmi les Hersiliens eux-mêmes, trois espèces, réparties dans trois genres différents, vivent sur la Callianasse, et que deux d'entre elles sont recueillies côte à côte avec les mêmes hòtes et dans les mêmes bancs de sable, l'importance de ces caractères paraîtra - ce qu'elle est en réalité - tout à fait insignifiante. Dans ces conditions, il semble bien que Antaria latericia Grube vient augmenter encore le nombre déjà grand des formes incertaines ou problématiques, celles que certains naturalistes, comme le Prof. Claus, sont tout disposés à rayer des cadres systématiques.

\section{Hersiliodes thompsoni, $\mathrm{C}_{\mathrm{ANU}}$.}

1888. Hersiliodes thompsoni; CanU ; 31 , pages 422 et 423 , Pl. XXX, fig. $1-8$.

Taille de la $q$ adulte $: 2,3 \mathrm{~m} / \mathrm{m}$.

Corps élargi dans la région céphalothoracique, de forme cyclopoïde. Pièces furcales courtes et presque cylindriques.

Antennes garnies de petits poils raides au bord interne et adhésif des deuxième et troisième articles.

Épine terminale de la seconde maxille interne renflée, avec l'extrémité garnie de quatre dents légèrement divergentes.

Seconde maxille interne avec les deux articles basilaires allongés et l'épine terminale plus courte que chacun d'eux.

(1) Grube. Mittheilungen über St-Waast-la-Hougue, Abhandl. d. Schles. Ges. d. Naturto, i 868, Taf. Il, fig. 3. -Mittheilungen über Roscoff, ibidem, 1870, page 109.

(2) Tout récemment M. le Rév. A.-M. Norman a bien voulu attirer sur elles mon attention. 
Cinquièmes pattes thoraciques formées d'une lame aplatie, élargie en palette vers l'extrémité distale.

Ovaires et œufs pondus de couleur jaune soufre.

Espèce assez rare dans les bancs de sable de la Pointe-aux-Oies, où elle vit dans les galeries de la Callianasse, abritée parfois entre les pattes abdominales de ce Crustacé (I).

\section{Giardella, Canu.}

1888. Giardella; CANu ; 31, pages 409 et 410.

1889. Giardella, Canu; Thомpson; 199 , pages 184 et 185 .

Corps aplati, de forme cyclopoïde. Mandibules terminées à leur extrémité par une griffe articulée et deux larges soies barbelées. Premières maxilles terminées par une épine conique, légèrement entaillée sur le bord (PI. XXX, fig. 7).

\section{Giardella callianassae, CANU.}

1888. Giardella callianassa; ; Canu; 31, pages 410-417, Pl. XXVIII.

1889. Giardella callianassae, Canu; THOMPson ; 199 , pages 184 et 185.

La seule espèce du genre; bien reconnaissable à la forme aplatie de son corps, à la garniture de poils épineux très effilés que porte le troisième article des antennes, à la largeur de l'extrémité des cinquièmes pattes thoraciques, à la longueur des pièces furcales dépassant la moitié de l'abdomen, à la coloration rose des ovaires et des oufs chez la femelle, ainsi qu'à l'hypertrophie considérable des secondes maxilles internes, devenues préhensiles chez le mâle.

Elle vit communément à la Pointe-aux-Oies, dans les galeries de la Callianasse où l'on peut aisément recueillir les deux sexes, ainsi que les stades de développement pénultième et antépénultième. Les jeunes stades-cyclops sont pélagiques et abondent dans les pêches au filet fin. C'est d'ailleurs à l'état de jeunes pélagiques que cette espèce a été recueillie par THOMPSON dans la baie de Liverpool.

\section{CANCERILLA ET CALIGIDIUM.}

Ces deux curieux Copépodes doivent être, comme je l'ai déjà montré, séparés des autres familles pour diverses particularités communes, à savoir :

$I^{0}$ La constitution particulièrement robuste des antennes, simples, préhensiles et 4-articulées.

(1) L'espèce établie par Bourne (19, page 379, PI. XXV) comme Hersiliodes canuensis d'après de jeunes exemplaires pélagiques, est, selon toute vraisemblance, apparentée ou très voisine de thompsoni. 
$2^{0}$ L'organisation de l'atrium buccal en une saillie de forme tronc-conique, dirigée vers l'extrémité antérieure.

$3^{n}$ La réduction de la première maxille à ưn moignon simple, garni de trois soies.

$4^{0}$ La nature des appendices thoraciques bien développés dans les deux premiers somites, très réduits dans le troisième, disparus totalement dans le quatrième, et relativement normaux dans le cinquième.

$5^{0}$ Les caractères sexuels secondaires de la femelle, qui consistent dans une forte réduction des pattes natatoires des deux premiers somites et dans la transformation de ces organes en appareils préhenisiles par l'existence de crochets recourbés...., etc.

Le mâle de Caligidium est seul connu; il a été longuement décrit et figuré par Claus (66), d'après un unique exemplaire.

Les deux sexes de Cancerilla ont été étudiés avec beaucoup de détails par GIARD (85) dans une courte note sans figures. J'ai pu compléter et rectifier cette description d'après quelques échantillons quej'ai recueillis dans le Boulonnais.

\section{Cancerilla tubulata, DALYELL.}

1851. Cancerilla tubulata; Dalyell; The powers of the Creator....., vol. 1, page 233, P1. LXII, fig. 1-5. 1887. Cancerilla tubulata, Dalyell ; GIARD; 85 .

\section{Planche XXIX, Fig. 5-13}

Taille : $q$, longueur $1 \mathrm{~m} / \mathrm{m}$, largeur $1 \mathrm{~m} / \mathrm{m} ; \delta$, longueur $0,9 \mathrm{~m} / \mathrm{m}$, largeur $0,5 \mathrm{~m} / \mathrm{m}$.

La forme du corps diffère sensiblement dans les deux sexes.

La femelle adulte (fig. 5) a le premier segment céphalothoracique (céphalon $+1^{\mathrm{er}}$ somite) très large et convexe, bosselé par le développement des ovaires; les autres segments du thorax, très réduits en longueur, vont en diminuant rapidement de largeur, de sorte que le céphalothorax a une forme presque elliptique avec le grand axe transversal. L'abdomen est court et atteint à peine le tiers de la longueur du céphalothorax; le premier somite abdominal est très large, avec des expansions chitineuses aux angles latéro-postérieurs, où se trouvent les orifices de l'oviducte. Le deuxième segment abdominal est très court et partiellement recouvert par le bord postérieur du premier; le troisième est beaucoup plus long et porte deux pièces furcales presque cylindriques, de même longueur que lui, garnies d'une grosse soie rigide terminale, avec les autres soies terminales et marginales très petites.

Le mâle adulte a le céphalothorax plus allongé (fig. 6), entièrement semblable à celui de Caligidium Cuaus, et rappelant comme ce dernier la forme des Caliges. Le segment céphalique est à peu près aussi long que large, avec les angles latéro-postérieurs arrondis. Le second segment (deuxième somite thoracique) a la forme d'une lame arquée, dont les côtés sont très saillants et arrondis (fig. 7): le troisième, le quatrième et le cinquième sont presque cylin-

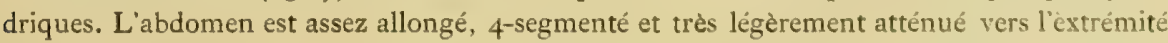


postérieure; le premier segment est un peu plus large que le cinquième somite thoracique; les deux suivants sont égaux et le dernier est un peu plus long. Les pièces furcales sont courtes et portent les mêmes soies que chez la femelle.

Prolongement frontal presque quadrangulaire (fig. 6).

Antennules (fig. 8) courtes, 6-articulées, portant des soies assez peu développées, semblables dans les deux sexes.

Antennes (fig. 9) préhensiles, 4-articulées, avec les trois premiers articles presque cylindriques et recouverts d'une chitine très épaisse, avec l'article terminal en forme d'ongle solide et résistant, à parois plus épaisses encore.

Mandibules (fig. 6 et 10), premières maxilles (fig. 6 et 11 ) et secondes maxilles (fig. 6) déjà décrites, réduites ou bien développées en organes préhensiles.

Pattes thoraciques de la première paire différentes dans les deux sexes. Chez le mâle (fig. 6, $\left.p^{3}\right)$, ce sont des pattes natatoires courtes, avec la base 2-articulée, l'endopodite 2-articulé et l'exopodite simple, ceux-ci porteurs d'un assez grand nombre de soies. Chez la femelle (fig. 12), ces appendices sont très réduits, avec la base 2-articulée, l'exopodite simple pourvu de soies, l'endopodite 2-articulé, mais très court et grêle, avec trois soies seulement.

Pattes de la deuxième paire également différentes. Chez le mâle (fig. $6, p^{2}$ ), elles sont plus longues que les premières, avec la base 2-articulée et les deux rames 2-articulées pourvues d'un grand nombre de soies. Chez la femelle (fig. 13), elles sont plus petites que dans la première paire et réduites à leur base 2-articulée portant à l'angle externe du deuxième article une épine rigide, avec une région terminale composée d'un seul moignon courtourné armé de six soies courtes ou épines.

Pattes de la troisième paire réduites à un simple repli tégumentaire, pourvu de deux ou trois soies très courtes ( fig. $6, p^{3}$ ) et situé dans l'angle postérieur ventral du segment.

Pattes de la quatrième paire faisant complètement défaut, si l'on néglige les faibles saillies du bord postérieur ventral du segment.

Pattes de la cinquième paire 2-articulées, avec l'article basilaire soudé au tronc et porteur d'une soie raide divergente, avec le second article ayant la forme d'une lame courte pourvue de quatre petites soies raides.

Ovaires et œufs verts; œufs pondus mesurant $0,2 \mathrm{~m} / \mathrm{m}$ de diamètre et réunis en deux sacs sphériques aussi volumineux que l'animal lui-même.

Les deux sexes de Cancerilla tubulata sont parasites à la face ventrale d'Amphiura squamata Delle-ChiAjE. Ils sont très rares dans le Boulonnais où trois exemplaires ont été recueillis à Wimereux et aux Roches-Bernard.

Caligidium vagabundum CLS se distingue nettement, dans le sexe male, de Cancerilla tubulata par l'allongement du corps et des appendices, par son antennule 9-articulée avec de gros filaments sensoriels réfringents, par la deuxième paire de pattes thoraciques dont les deux rames sont·3-articulées, par des aptitudes à la natation qui font entièrement défaut dans l'autre espèce.

Distribution : Côtes des Iles-Britanniques (localité inconnue), Manche (Boulonnais. Fécamp), (?) Océan Atlantique (Concarneau). 


\section{Rhynchostoma.}

Copépodes diporodelphes ayant :

$I^{0}$ Des habitudes parasitaires durables ou passagères sur les Vertébrés et les Invertébrés, et le corps de forme extrêmement variable, depuis l'aspect cyclopoïde jusqu'aux apparences vermiformes les plus bizarres.

$2^{0}$ Les antennules de constitution très variable, semblables ou non dans les deux sexes; les antennes préhensiles en forme de crochets plus ou moins articulés.

$3^{0} \mathrm{La}$ lèvre supérieure et la lèvre inférieure saillantes, soudées dans leur portion distale en un tube conique formant un appareil articulé avec le tronc et à l'ouverture duquel viennent souvent affleurer les coxopodites mandibulaires en stylets.

$4^{0}$ Les premières maxilles plus ou moins développées, présentant l'aspect d'un appendice divisé en lobe masticateur et lobe palpiforme séparés, sauf dans les cas où ce dernier n’existe qu'à l'état rudimentaire.

$5^{\circ}$ Les secondes maxilles variables, simples ou doubles, en forme de pince préhensile ou d'appareil adhésif.

$6^{0}$ Les pattes thoraciques normales ou subissant dimportantes modifications en vue d'un fonctionnement spécial, souvent en partie disparues.

$7^{0} \mathrm{La}$ circulation lacunaire, compliquée d'une circulation vasculaire spéciale dans quelques types.

$8^{\circ}$ Les œufs pondus rassemblés en sacs extérieurs portés par les femelles et disposés en une ou plusieurs séries longitudinales.

Les Copépodes qui composent ce sous-groupe sont, à vrai dire, les seuls Siphonostomes. Leur trompe prébuccale joue un rôle effectif dans le fonctionnement des organes buccaux. Tous sont parasites à divers degrés et vivent aux dépens d'autres animaux, en prenant à l'aide de leur siphon une nourriture toute superficielle (sécrétions, mucus). Ils pénètrent dans les tissus de leur hôte uniquement pour se fixer, et n'enfoncent point leur appareil buccal dans la peau des Poissons ni dans les tissus des Invertébrés qui les portent. Les femelles adultes de Lernéens, après la copulation, ne constituent elles-mèmes qu'une apparente exception à cette règle.

\section{ASCOMYZONTIDAE.}

Copépodes rhynchostomes ayant :

$I^{0}$ Des habitudes semi-parasitaires très accusées. 
$2^{0}$ La forme cyclopoïde la plus accentuée.

$3^{\circ}$ Les antennules longues ou courtes, généralement pourvues d'un très gros filament sensoriel, différentes dans les deux sexes par leur transformation en organes préhensiles des deux côtés chez le $\sigma$.

$4^{0}$ Les antennes 3- ou 4-articulées, préhensiles, avec un exopodite très rudimentaire réduit à un seul article portant 2 ou 3 soies.

$5^{11}$ Les mandibules en stylet composées d'un (mandibules sans palpe) ou de plusieurs (mandibules avec palpe) articles.

$6^{0}$ Les secondes maxilles divisées dans les deux sexes en deux portions formant des pinces puissantes, semblables dans le mâle et dans la femelle.

$7^{0}$ Les pattes thoraciques des quatre premières paires généralement biramées et natatoires, de conformation à peu près normale.

$8^{0}$ Les pattes de la $5^{\mathrm{e}}$ paire courtes et 2 -articulées, avec des épines et des soies marginales et terminales.

La famille des Ascomyzontida établie assez suffisamment par THORELL (204, pages 75-77) en 1859 et consolidée récemment par Claus (66, pages 20,2 I et 36 ), doit être maintenue à juste titre. Elle s'éloigne notablement de celle des Lichomolgide, ainsi que l'établissent une foule de caractères morphologiques ou éthologiques, et c'est à tort que. KOSSMANN $(118,199,120)$ a voulu rapprocher ces êtres cyclopiformes. Elle correspond à la famille des Artoirogida fondée par BRADY (20, vol. 3, page 52) en 1880.

En général, les Copépodes qui composent cette subdivision systématique, n'ont été recueillis qu'en nombre restreint, vivant en liberté sur le fond de la mer, au milieu des animaux invertébrés et des algues Un petit nombre d'espèces ont été trouvées sur les téguments ou dans les cavités de Tuniciers, d'Éponges, d'Échinodermes ou de Mollusques Nudibranches, animaux aux dépens desquels ils vivent. Leurs habitudes éthologiques restent donc très obscures, mais il résulte de nos connaissances actuelles qu'ils sont des semi-parasittes abandonnant facilement et pour longtemps leur hòte, afin de nager en liberté.

\section{Dermatomyzon, Claus.}

1880. Cyclopicera, pars; Brady, 20, vol. 3, page 53.

1889. Dermatomyzon; Claus, 66, pages 24 et 25.

1891. Dermatomyzon, Cls ; Canu, 34 , page 483. 
Ascomyzontide ayant :

$1^{0}$ Le céphalothorax 5 -segmenté dans les deux sexes; l'abdomen 4-segmenté chez la f́melle et 5-segmenté chez le mâle.

$2^{\circ}$ Les antennules 19 -articulées chez la femelle et 13 -articulées chez le mâle.

$3^{\circ}$ Le siphon court, large à sa base et rétréci vers son extrémité, ne dépassant point la base de la seconde maxille.

$4^{0}$ Les inandibules 2-articulées, avec le coxopodite styliforme, épais et solide, pourvu de denticules à son extrémité; avec le second article court et cylindrique, terminé par deux longues soies inégales.

$5^{\circ}$ Les premières maxilles avec les deux lobes presque égaux entre eux et dirigés vers la ligne médiane.

$6^{0}$ Le second article des $5^{\text {es }}$ pattes thoraciques large, foliacé, bordé de quatre épines et d'une soie

Ce genre, établi par Claus d'après un seul spécimen femelle, et dont j'ai pu étudier le mâle, serait, d'après son fondateur, séparé du genre Cyclopicera BraDY, en particulier de Cyclopicera nigripes BRADY ET ROBERTSON, uniquement par la segmentation plus complète de l'abdomen et par la forme de la première maxille.

En effet, BRADY décrit pour le genre Cyclopicera (loc. cit., page 53) et figure pour l'espèce $C$. nigripes (loc. cit., Pl. LXXXIX), l'abdomen de la femelle 3-segmenté (fig. I) et celui du malle 4-segmenté (fig. II). Mais, d'après la forme attribuée au premier segment abdominal dans cette figure I $\mathrm{I}, \mathrm{j}$ 'ai supposé(I) que BRADY y avait dessiné comme mâle une femelle montrant nettement la segmentation de l'abdomen, tandis que dans la figure I, attribuée à la femelle, il avait par erreur et d'après une préparation moins nette, réuni en un seul anneau les deux derniers segments précédant la furca.

Les proportions relatives des segments, dans les dessins reproduits par l'auteur anglais, appuient du reste cette supposition : c'est ainsi que dans la figure $\mathrm{I}$, le dernier segment est plus long que l'avant-dernier; tandis que dans la figure I 1 , c'est l'antépénultième qui est plus long que le dernier et que l'avant-dernier, et plus court que ceux-ci réunis. Il faut également observer que BRADY n'a point décrit ni figuré pour $C y c$. nigripes les attributs les plus caractéristiques du sexe $\sigma^{7}$ : les antennules préhensiles et les sacs à spermatophores avec leurs orifices exterieurs.

De ces remarques, je crois pouvoir conclure que BRADY n'a point observé le male de Cyclopicera nigripes BR. ET RoB., qu'il n'a étudié que des femelles et que

(1) Informé de cette opinion, M. le Prof. Brady a bien voulu me faire connaitre qu’il n'avait rien de spécial à objecter et que le fait était possible. 
sa donnée de l'abdomen 3-segmenté repose sur une erreur. En conséquence, Cyclopicera nigripes BR. ET RoB. est un véritable Dermatomyzon, voisin, sinon identique, de Derm. elegans Claus. D'après la description très incomplète que l'on en possède, le seul caractère permettant de séparer ces deux formes consiste dans la coloration foncée des pattes thoraciques et du corps (I) chez Dermaiomyzon nigripes $\mathrm{BR}$. ET RoB., ces parties ètant blanches ou transparentes chez $D$. elegans CLS.

\section{Dermatomy aon elegans, Ciuaus.}

1889. Dermatomyzon elegans; Claus, 66 , pages 25 et 26 , Taf. VI, fig. 5-10.

1891. Dermatomyzon clegans, Cls; Canu, 34 , page 483.

\section{Planche XXVI, Fig. I-IO.}

Taille : $+1,35 \mathrm{~m} / \mathrm{m}$ d'après Cuaus ; $\sigma, 0,9-1 \mathrm{~m} / \mathrm{m}$.

Corps de forme assez allongée, surtout dans le sexe $\sigma^{\nearrow}$. Céphalothorax ovalaire : replis pleuraux des derniers segments thoraciques bien accusés et $5^{\mathrm{e}}$ somite thoracique étroit et court. Prolongement frontal triangulaire, pointu et replié sur la face ventrale. Abdomen long et presque cylindrique; les segments génitaux des deux sexes un peu renflés portent: dans la femelle, les ouvertures des oviductes sur les faces latérales et vers leur milieu (2), dans le mâle (fig. 1 et 10), les ouvertures extérieures des sacs à spermatophores à l'angle postérieur saillant de la face ventrale. Dernier segment abdominal n'étant point plus long que celui qui précède. Pièces furcales courtes et larges (fig. 1), avec de longues soies terminales.

Antennules plus courtes que le segment céphalique (fig. 1); 19-articulées dans la femelle, d'après Craus, avec un filament sensoriel inséré sur le $17^{\circ}$ article. Dans le mâle, elles ne comptent que 13 articles, avec un filament sensoriel plus long et plus épais sur le $12^{\circ}$ article; la disposition de leurs articles (fig. 1) établit que les $11^{\mathrm{e}}, 12^{\mathrm{e}}$ et $13^{\mathrm{e}}$, et les $14^{\mathrm{e}}, 15^{\mathrm{e}}$, $16^{\text {e }}$ et $17^{\mathrm{e}}$ de la femelle ne forment respectivement qu'une seule pièce dans le mâle. Les deux premiers articles sont un peu plus longs et plus épais que ceux qui les suivent. et le $1^{\text {sr }}$ porte à son bord antérieur distal une longue soie rigide en forme d'épine.

Antennes (fig. 2, $A$, et 3) 4-articulées et coudées dans plusieurs directions aux points d'articulation, de manière à faciliter l'adhésion. Le $1^{\mathrm{er}}$ article est très court; le $2^{\mathrm{e}}$, très long, porte un exopodite réduit à un petit article garni de deux soies grêles avee un endopodite simple et solide, terminé par une longue épine légèrement courbée en griffe, d'une longueur égale au $\dot{2}^{\mathrm{e}}$ article.

Siphon buccal très court, élargi dans sa région basilaire (fig. 4 et 5) et prolongé dans sa région distale en un tube cylindrique à peine saillant. Le tube terminal, à parois plus

(1) Voir Brady, loc. cit., page 55: "Colour pale brown, the feet smaky black, a character which at once distinguishes the animal among others. "

(2) Ce qui est figuré par Brady ( $l, c_{0}$, fig. 11 ) dans l'abdomen 4 -articulé qu'il attribue à Cyclopicera nigripes of. 
minces, est fendu en sifflet à son extrémité et découvre ainsi le bout dentelé des coxopodites mandibulaires (fig. $5, m d$ ).

Dans chaque mandibule (fig. 6), sur une base large et courte, s'insèrent : d'abord le coxopodite styliforme, massif et trapu, partiellement abrité dans le siphon et rétréci en une lame distale tailladante et denticulée ̀̀ son extrémité; de plus, un palpe réduit à un seul article à peine égal en longueur au tiers du coxopodite et terminé par deux soies, l'externe très longue et l'interne beaucoup plus courte, garnies toutes deux de barbules courtes et espacées.

Premières maxilles (fig. $2 m x^{1}$, fig. 7) comprenant une base dont se détachent : un lobe basilaire interne armé de quatre soies courtes et raides à peine barbelées et un lobe distal externe armé de trois soies plus longues et lisses; ces deux lobes sont dirigés, ainsi que leurs soies, en travers de la ligne médiane du corps.

Secondes maxilles externes 2-articulées, avec l'article basilaire allongé, à contour subrectangulaire, avec la griffe terminale garnie de poils denticulaires sur son bord interne et pourvue d'un crochet terminal articulé (fig. $2, m x^{2} e$; fig. 8).

Secondes maxilles internes (fig $2, m x^{2} i$ ) 5-articulées, portant à leur extrémité une longue épine en griffe et un poil raide plus court.

Pattes natatoires avec les deux rames 3-articulées et semblables. Chez le mâle, dans la $1^{\text {re }}$ paire (fig. $2, p^{1}$ ), le $1^{\text {er }}$ article de l'exopodite porte à son bord distal externe une épine denticulée longue et recourbée vers l'intérieur, qui atteint le bord distal du $2^{\mathrm{e}}$ article. Les épines terminales des deux rames sont presque semblables, sétiformes et rigides.

Pattes de la $5^{\mathrm{e}}$ paire (fig. 9) avec leur article basilaire large et soudé au tronc, muni d'une courte soie au bord distal externe; avec leur second article armé à son extrémité distale d'une soie glabre très mince, - à son bord intérieur on médial, de deux épines à parois chitineuses épaissies et réfringentes, ayant leur hord externe limité par une mince membrane chitineuse hyaline (1), - à son bord extérieur, de deux grosses épines lancéolées garnies de poils courts rigides.

Les sacs ovigères n'ont pas été observés sur la femelle recueillie par Czaus.

Les sacs à spermatophores du mâle sont ovalaires ou piriformes.

La coloration est blanche dans les deux sexes, sans traces de cette teinte brunâtre signalée par Brady dans Cycl. nigripes.

Un certain nombre d'exemplaires mâles de Derm. elegans ont été recueillis par moi dans des produits de dragage effectués dans le détroit du Pas-de-Calais, par 35 mètres de profondeur, au milieu de Tuniciers, de Mollusques et d'Echinodermes, parmi lesquels il me fut impossible de distinguer l'hòte de ce semiparasite.

Distribution: Manche, Adriatique (Trieste).

(1) Disposition figurée par BradY dans Cyclopicera nigripes et nullement représentée par CLaus dans Derm. alegans ?. 


\section{Asterocheres, BOECK.}

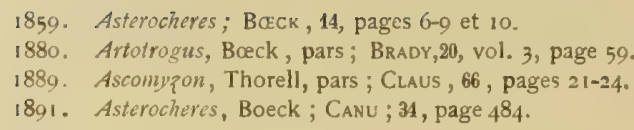

Ascomyzontide ayant :

$1^{0}$ Le céphalothorax 5-segmenté dans les deux sexes; l'abdomen 3-segmenté dans la femelle et 4 -segmenté dans le mâle.

$2^{0}$ Les antennules 19-articulées chez la femelle (1) et 17 -articulées chez le mâle, où le $12^{\circ}$ article est égal en longueur au $13^{\circ}$ et au $14^{\circ}$.

$3^{\prime \prime}$ Le siphon buccal court et ne dépassant pas la base des secondes maxilles, de forme conique très effilée et relativement peu élargie à la base, dans son aspect général plus court, plus large et plus fort que chez Artotrogus (2).

$4^{\prime \prime}$ Les mandibules 3-articulées avec le coxopodite styloïde sous forme d'une tige grêle et recourbée atteignant le bout du siphon par son extrémité tronquée et denticulée; avec les deux articles terminaux et cylindriques du palpe prolongés d'une longue soie et d'une petite soie barbelées.

$5^{0}$ Les premières maxilles bilogbées, avec le lobe basilaire ou masticateur plus allongé que l'externe palpiforme.

$6^{0}$ Le second article des $5^{\text {es }}$ pattes thoraciques lamellaire ou cylindrique, terminé par deux soies.

Le genre Asterocheres, établi par. BcEck d'après une seule espèce, ne doit nullefnent rentrer, comme l'ont pensé Brady et Claus, dans la synonymie des autres genres d'Ascomyzontides. J'ai pu comparer deux espèces de ce genre avec les descriptions et les dessins fournis par les différents auteurs sur les divers Copépodes dont Asterocheres fut ainsi rapproché, et cette étude m'a permis de rectifier les synonymies antérieurement introduites par les observateurs précités.

(i) $\mathrm{B} \propto \mathrm{CK}\left(l . c_{\text {. }}\right.$, pages 6-7 et page 10) les donne comme 18-articulées, mais d'après les dispositions des autres articles dans la figure 3 de sa planche 11 , je crois pouvoir admettre que la $18^{\circ}$ articulation a été omise et les $18^{\circ}$ et $19^{\circ}$ articles réunis en un seul.

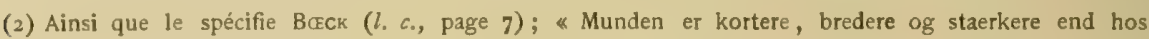
Artolrogus. . 
Le genre Asterocheres Bळeck, tel qu'il est ici diagnostiqué, se distingue nettement :

$\alpha$ - d'Artotrogus BєECK ( $\mathbf{r}$ ), par toute son organisation, notamment par ses antennules richement articulées et la forme de toutes les parties buccales.

ß-d'Ascomyzon Thorell (2), - non par les antennules de la femelle auxquelles ThORELl et Brady attribuent jusqu'à 20 articles, car il ressort des figures (3) publiées que leur $20^{\mathrm{e}}$ article n'est autre que l'extrémité réellement indivise du $19^{\mathrm{e}}-$; mais par l'organisation et le développement du siphon buccal très allongé, effilé, atteignant jusqu'au $5^{\mathrm{e}}$ somite thoracique ou jusqu'au $1^{\mathrm{er}}$ segment abdominal d'après les dessins (Tab. XIV, fig. 2 I $R$ et 2 I $A$ ) et le texte de Thorell, dépassant le céphalon d'après les dessins de Brady (PI. XCI, fig. 6,g).

$\gamma$ - de Cyclopicera BRADY (4), ou de son synonyme presque certain Echinocheres CLs, par les antennules 2 I-articulées de la femelle; par celles du mâle 17-articulées, avec le $\mathrm{I} 2^{\mathrm{e}}$ article renflé et beaucoup plus long que le $\mathrm{I} 3^{\mathrm{e}}$ et le $\mathrm{I} 4^{\mathrm{e}}(5)$; et aussi par la $I^{\mathrm{re}}$ maxille dont le lobe interne porte une longue soie plus forte et plus épaisse que toutes les autres, et dont le lobe externe est simple et réduit (6).

\section{Asterocheres renaudi, CAnU.}

1891. Asterocheres Renaudi; Canu, 34, page 485.

$$
\text { Planche XXVI, Fig. II-I9. }
$$

9. - Taille : $0,85 \mathrm{~m} / \mathrm{m}$.

Forme générale (fig. 1) assez allongée. Céphalothorax élargi avec les $4^{\mathrm{e}}$ et $5^{\mathrm{e}}$ somites thoraciques à peu près aussi larges l'un que l'autre; prolongement frontal étroit et pointu, replié sur la face ventrale (fig. 11 et 12). Abdomen (fig. 19) avec le $1^{\text {er }}$ segment plus large que long, sans cils sur ses bords et portant latéro-dorsalement les orifices extérieurs des oviductes sous forme de deux incisions assez profondes ; $2^{\mathrm{e}}$ segment abdominal très court; $3^{\mathrm{e}}$ segment

(1) Auquel Brady le rapporte!

(2) Dont il serait synonyme d'après CLaus, et pour lequel je prends 'comne type Ascomyzon lilljeborgi THORELL (= Arlotrogus backi BRADY).

(3) Thorell : l. c.; Taf. XIV, fig. 2 I $A$. n Brady : l. c.; PI. XCl, fig. 1.

(4) Auquel le réunit en partie Claus; et pour lequel je prends comme type l'espèce primitivement décrite en 1871: Cyclopicera lata BRADY.

(5) Comparer : Brady, loc. cit., PI. XC, fig. 12 ; et Claus, loc. cit., Taf. V, fig. 5 et $5^{\prime}$, et Taf. VI, fig. 3.

(6) Le genre /sopodius, si mal décrit par Kritchaguine (121: pages 398-403; PI. XIV, fig. 6-17) qui le rapprochait des Peltidiens, n'est probablement qu'un Cyclopicera, comme le font supposer les antennules 2l-articulées chez la femelle, dissemblables chez le mâle; l'abdomen 3 -segmenté dans la femelle et 4 -segmenté daus te mâle (fig. 7 et 8 ). 
abdominal plus que double du $2^{\ominus}$, avec ses limites postérieures finement ciliées vers la face ventrale. Pièces furcales courtes et larges, avec quatre soics terminales bien développées.

Antennules assez grêles (fig. 11 et 12 ), avec le $1^{\text {er }}$ article épais et allongé, les articles 2-9 courts et décroissant vers l'extrémité, tandis que les articles $10-19$, cylindriques et plus étroits, vont en s'allongeant de façon assez régulière. Le $17^{\sharp}$ article porte le filament sensoriel, assez grêle et égal en longueur aux articles 11-19 réunis.

Antennes (fig. 12) assez épaisses et trapues, avec leur chitine ornementée de stries ondulées presque parallêles, avec l'épine terminale longue et pointue, l'exopodite réduit portant deux courtes soies raides et lisses.

Siphon et pièces buccales ornées de stries comme les antennes; le premier large et court, avec son extrémité légèrement fendue sagittalement entre la lèvre supérieure étroite et effilée à son extrémité incurvée et garnie de poils (fig. 13 et 14,75 ), et la lèvre inférieure chitinisée et arrondie (fig. 14, li).

Mandibule avec le coxopodite long et recourbé, avec le palpe très court, aux deux articles subégaux (fig. 12, md; fig. 15).

Premières maxilles (fig $12, m x^{1}$; fig. 16) avec le lobe interne garni de quatre soies raides dirigées parallèlement au siphon, et le palpe réduit, terminé par trois soies faisant saillie en travers et au-dessus du siphon.

Secondes maxilles internes et externes trapues, solides, avec les griffes terminales relativement courtes et très recourbées, garnies de poils à leur face intérieure.

Cinquièmes pattes thoraciques saillantes sur les faces latéro-ventrales du $5^{\circ}$ somite, et composées de deux articles : le $1^{\text {er }}$ soudé au tronc avec une petite soie externe; le second court ct large, terminé par deux petites soies rigides et barbelées.

Cette espèce ne m'est connue que par un exemplaire femelle recueilli à la surface d'une grosse Éponge gluante ( Renieria sp?) draguée dans le détroit du Pas-deCalais, à l'accore $\mathrm{E}$ du banc du Colbart. Elle est particulièrement distincte en raison de la brièveté du palpe mandibulaire, des faibles dimensions de la $5^{\mathbf{e}}$ patte thoracique et de l'ornementation de la cuticule dans les appendices céphaliques.

Distribution: Manche.

\section{Asterocheres lilljeborgi, Boeck.}

1859. Asterocheres Lilljehorgi; $\mathrm{B} \Subset \mathrm{cK}, 11$, pages 6-9; PI II, fig. 1-11.

1891. Asterocheres Lilljehorgi, Bœck; Canu, 34, pages 484 et 485.

\section{Planche XXVII, Fig. I-6.}

Je n'ai étudié qu'un seul exemplaire mutilé et dépourvu de son abdomen; il appartient au sexe $\sigma^{7}$; le sexe $q$ était seul connu.

Taille : Céphalothorax atteignant $0,6 \mathrm{~m} / \mathrm{m}$ de longueur; par conséquent plus petit et aussi plus étroit (fig. 1) que celui de la femelle qui, d'après le dessin de Bøak, mesure $0,7 \mathrm{~m} / \mathrm{m}$.

Le $5^{\theta}$ somite thoracique est très étroit, dépourvu des replis pleuraux très développés dans les autres segments. Le prolongement frontal (fig. 3) est de forme pentagonale et pointu. 
L'antennule, sans le filament sensoriel, est presque aussi longue que le céphalon, et avec ce filament, plus longue que le $1^{\text {er }}$ segment. Son $1^{\text {er }}$ article est modérément allongé, les $2^{0}-9^{e}$ sont plus courts que larges, disposés de manière à recourber l'extrémité de l'antennule vers l'avant. Au-delà, les articles vont en croissant vers l'extrémité; le $15^{\circ}$ est le plus long et il est relié au $16^{\mathrm{e}}$ par une articulation géniculée; le $16^{\mathrm{e}}$ porte le filament sensoriel'et le $17^{\circ}$ est un peu plus court, plus grêle, garni de plusieurs soies raides et lisses.

L'antenne (fig. 3) est assez allongée, avec une griffe terminale fortement incurvée et un exopodite réduit portant deux petites soies.

Le siphon (fig. 3) est très effilé et presque tétraédrique vers son extrémité légèrement évasée (fig. 4).

La mandibule (fig. $4, m d$ ) a le coxopodite mince et effilé, le palpe très allongé avec le ${ }_{1}{ }^{\mathrm{er}}$ article deux fois plus long que le second $(1)$ et les soies barbelées très longues.

La ${ }^{\mathrm{re}}$ maxille (fig. $4, m x^{1}$ ) a le lobe interne pourvu de 3 soies allongées et le lobe palpiforme terminé par 3 soies grêles, avec une autre renflée et barbelée sur le bord externe.

Les secondes maxilles (fig. $3, m x^{2} e$ et $m x^{2} i$; fig. 5 ) sont bien développées, avec leurs griffes terminales longues, pointues et recourbées (2).

Les pattes thoraciques de la $1^{\mathrm{re}}$ paire $\left(\mathrm{fig} .3, p^{1}\right)$ ont l'épine marginale externe du $1^{\text {er }}$ article de l'exopodite très longue et recourbée, ce qui peut être un caractère sexuel secondaire, étant donné que la femelle présente en ce point une épine normale (3), et que le mâle de Dermatomyzon elegans possède aussi cette disposition.

Les $5^{\text {es }}$ pattes (fig. 7 ) sont larges, avec une soie rigide au bord externe du $1^{\text {er }}$ article soudé au tronc, et deux soies semblables sur le bord distal du second article large et aplati, bordé, non point de quatre épines marginales internes, comme le figure Bøck, mais de poils courts, plus fins à l'extérieur et plus gros vers l'intérieur.

Les canaux déférents (fig. 1) sont fortement recourbés et contournés; ils rappellent vaguement la disposition que nous avons constatée chez Lichomolgus albens.

L'exemplaire $\sigma^{7}$ ici décrit provient des produits, conservés dans l'alcool, d'un dragage effectué à bord de l'Ajax par M. le Dr SAuvaGe ; en vue du cap Gris-Nez. Dans ce dragage abondaient Molgula socialis ALDER, ainsi que Psammechinus miliaris LESKE; dans ces conditions, il ne fut pas possible de distinguer quel hôte habite ce Copépode, dont la femelle fut trouvée par BaEcK sur Echinaster sanguinolentus.

\section{Distribution : Mer du Nord, Manche.}

( 1 ) C'est ce palpe qui est représenté par Bøck (loc. cil.) dans la fig. 7 de sa P1. II, et dont il dit également que " sa base èst 2-articulée, avec le $1^{\text {or }}$ article deux fois aussi long et aussi épais que le $2^{6} \mathrm{n}$.

(2) Comme Bøck les a figurées dans la femelle : loc. cit.; fig. 5 et 6.

(3) Bøack (loc. cit., page 8) signale la présence de cette épine, sans insister sur sa longueur, ce qui peut indiquer qu'elle ne présente point un développement remarquable, comme c'est le cas dans le mâle. 


\section{Artotrogus, Bовск.}

1859. Artotrogus; BCECK ; 14, pages $2-5$ et 10.

1880. Artotrogus, Bœeck, pars; BradY, 20, vol. 3, page 59.

1889. Artotrogus, Bæeck; Claus, 66, pages 34 et 35.

Ascomyzontide ayant :

$I^{0}$ Le céphalothorax 5 -segmenté et l'abdomen 4-segmenté dans le mâle (1) et dans la femelle.

$2^{\circ}$ Les antennules courtes et 9-articulées dans les deux sexes.

$3^{0}$ Le siphon très allongé, renflé et ovalaire dans sa région proximale, effilé et tubiforme dans sa région distale; celle-ci fendue sagittalement vers son extrémité, pour constituer une sorte de bec laissant poindre les mandibules.

$4^{0}$ Les mandibules simples, sans palpe, et réduites au coxopodite styliforme dentelé en scie à son extrémité.

$5^{\circ}$ Les $1^{\text {res }}$ maxilles peu développées, avec deux lobes saillants pourvus d'épines denticulées ou d'une petite soie.

$6^{0}$ Les pattes thoraciques des quatre premières paires biramées; celles de la $5^{e}$ paire 2-articulées, avec des soies et des épines.

Le genre Artotrogus fut parfaitement délimité par BEcK, et les particularités des antennules, des mandibules sans palpe et de la longueur du siphon, auraient pu dispenser BraDY de le considérer comme synonyme d'autres genres bien distincts.

Dans la diagnose établie ci-dessus, sont corrigées diverses omissions ou erreurs des auteurs précédents, d'après l'étude d'un Copépode femelle appartenant indubitablement à ce genre; je citerai notamment les antennules de la femelle chez Art. magniceps, d'après Brady, les $5^{\mathrm{es}}$ pattes chez Art. Normani, d'après Brady; je ferai observer encore que l'exopodite antennaire subsiste dans ce genre, sous le mème état rudimentaire que chez les formes voisines.

\section{Artotrogus normani, Brady \& RoBertson.}

1875. Dyspontius striatus. BRADY ET ROBERTSON,

1880. Artotrogus Normani, B. et R; Brady, 20, vol. 3, pages 63 et 64 , PI. XCl, fig. 12-15, Pl. XCIl, fig. 14 , PI. XCIII, fig. 10.

1891. Artotrogus Normani, B. et R.; Canu, 34, pages 495-486.

Planche XXVII, fig. 7-17.

(1) D'après Brady (loc. cit., PI. XCIII, fig. 10), mais les caractères distinctifs du sexe $\nearrow$ ne sont pas indiqués. 
f. - Taille $: 1,6 \mathrm{~m} / \mathrm{m}$.

Forme allongée ( fig. 7), très différente des deux autres espèces du genre: $A$. orbicularis Borck et $A$. magniceps Brady, "par le rétrécissement du céphalothorax dans la région antérieure, par la séparation considérable entre les pleura des $2^{e}, 3^{e}$ et $t^{e}$ segments, par l'allongement de l'abdomen plus grêle. Prolongement frontal (fig. 8) triangulaire, à bords chitineux très épais. Pièces furcales (fig. 7) un peu plus longues que le segment précédent, spatuliformes, avec de grandes soies terminales plumeuses.

Tégument perforé d'une quantité considérable de petits orifices arrondis, garnis chacun d'un poil rigide et transparent, et qui donnent à la carapace l'aspect finement granulé caractéristique.

Antennules (flg. 8, a) avec les deux premiers articles cylindriques et allongés (1), avec le $9^{\mathrm{e}}$ article plus long que les six précédents et donnant attache dans son tiers distal, au filament sensoriel épais et cylindrique.

Antennes (fig. 8, A) très courtes, 4-articulées, avec l'exopodite distinct garni de deux soies, avec une épine terminale denticulée, accompagnée de deux soies terminales et d'une soie latérale intêrne sur le $4^{\circ}$ article.

Siphon allongé et grêle (fig. 9), dépassant quelque peu la limite postérieure du i ${ }^{\text {er }}$ segment céphalothoracique, avec deux longues et étroites échancrures basilaires latérales donnant passage aux coxopodites mandibulaires, avec le bec terminal fendu jusqu'au quart de la longueur totale (fig. 10).

Extrémité saillante des mandibules avec cinq dents mousses sur le côté interne (fig. 11 ).

Premières maxilles (fig. 12 et 13 ) avec la base large supportant: vers l'intérieur un lobe saillant aminci vers son extrémité garnie d'une petite soie, et cilié sur son bord externe; d'autre part, vers l'extérieur, un lobe palpiforme cilié, avec deux épines barbelées et une petite soie terminales.

Secondes maxilles externes (fig. 13) 3-articulées, avec le $2^{\mathrm{e}}$ article long et renflé, avec le $3^{\mathrm{e}}$ article sous forme d'une griffe allongée, rigide, fortement recourbée en crochet à son extrémité distale articulée.

Secondes maxilles internes (fig. 14) longues et solides, présentant bien les caractères indiqués par Brady, notamment : l'épine insérée sur le $3^{\mathrm{e}}$ article, vers le milieu de la face interne et qui semble manquer dans les autres espèces, l'épine terminale du $6^{\mathrm{e}}$ article très pointue à son extrémité libre.

Les pattes natatoires ont 'dans les trois premières paires (fig. 15) l'exopodite et l'endopodite de même longueur, armés d'épines semblables à leur extrémité distale. Dans la $4^{\circ}$ paire (fig. 16), l'endopodite réduit égale la moitié de l'exopodite; il porte un nombre restreint de soies plus courtes. Ces appendices vont en augmentant graduellement en longueur depuis la $1^{\text {re }}$ paire jusqu'à la $3^{\theta}$, la $4^{\theta}$ paire est plus courte que la précédente. Leurs coxopodites et leurs basipodites sont assez larges, avec les parois fortement chitinisées et les contours presque quadrangulaires. Dans la $1^{\text {re }}$ paire, le basipodite porte à son bord interne distal une grande épine barbelée.

(1) La subdivision du $2^{0}$ article de l'antennule, indiquée par Brady (l. C., Pl. XCIII, fig. 2) pour Art. magniceps, repose certainement sur une erreur; les antennules de cette espèce se rattachent trop complètement au type ici décrit, pour présenter une telle divergence. 
Cinquièmes pattes (fig. 17 ) nettement 2 -articulées, placées à la face ventrale du $5^{\mathrm{e}}$ somite thoracique, avec le ${ }^{\text {er }}$ article très large, lamellaire et pourvu d'une soie externe; avec le second article court et aplati, terriné par une épine droite et rigide, flanquée de deux soies (1).

Tandis que les deux autres espèces du genre sont très voisines l'une de l'autre, si mème elles ne sont pas identiques. Artotrogus normani est bien distinct par sa forme et son ornementation. La présence de ce Copépode sur les côtes du Boulonnais est démontrée par un seul exemplaire femelle que j'ai recueilli à Wimereux, près de la Tour de Croï, parmi les Hermelles, les Synascidies et les Éponges de la zone des Laminaires. Brady a dragué quelques spécimens sur les côtes de Durham, à la profondeur de 27 brasses; I.-C. Thompson le signale aux Iles Canaries et à Madère.

Distribution : Mer du Nord, Manche, Atlantique.

\section{Dyspontius, ThORELL.}

1860. Dyspontils; THORELL, 294 , pages 80 et 81 .

1866 ? Dyspontius, Th.; Hesse, 110, page 69.

1880. Dyspontius, Th.; Bradr, 20, vol. 3 , page 65.

Ascomyzontide ayant :

$I^{0}$ Le céphalothorax 5-segmenté; l'abdomen 5-segmenté dans le mâle et 4-segmenté dans la femelle.

$2^{0}$ Les antennules courtes, 9-(Brady) ou 10-(ThorelL) articulées dans la fernelle, 11articulées dans le mâle.

$3^{0}$ Le siphon allongé, comme dans Artotrogus, et s'étendant jusqu'au milieu du thorax , avec l'extrémité fendue sagittalement en un bec laissant poindre les mandibules.

$4^{0}$ Les mandibules simples, sans palpe, réduites au coxopodite styliforme dentelé en scie à son extrémité.

$5^{\circ}$ Les premières maxilles peu développées, bilobées comme dans Artotrogus.

$6^{0}$ Les pattes natatoires des 3 premières paires biramées, celles de la $4^{\mathrm{e}}$ paire réduites à l'exopodite 3 -articulé; les pattes de la $5^{\mathrm{e}}$ paire très réduites, mais formées encore de deux articles sétifères distincts (2).

(1) BRADY n'a point constaté la présence de cet appendice, probablement parce qu'il est dissimulé à la face ventrale de cet animal.

(2) Brady et Thorell disent les $5^{\text {es }}$ pattes disparues ou absentes; Thorell figure néanmoins (boc.,

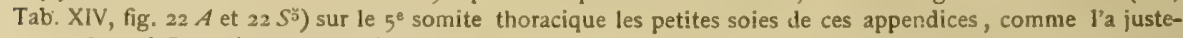
ment observé Claus (66, page 35 ). 
Ce genre est très voisin d'Artotrogus $\mathrm{B}$ ckK; il s'en distingue néanmoins avec la plus parfaite certitude, grâce à l'organisation de la $4^{\mathrm{e}}$ paire de pattes thoraciques, dans laquelle l'endopodite, déjà très réduit chez Artotrogus, manque complètement pour laisser seul un exopodite 3 -articulé de conformation normale, avec les soies et les épines marginales habituelles. L'étude que j'ai pu faire d'un jeune exemplaire de Dyspontius encore asexué, m'a démontré que l'endopodite disparaît à une époque très précoce dans cette $4^{\mathrm{e}}$ paire de pattes, puisque dans le $3^{\mathrm{e}}$ ou $4^{\mathrm{e}}$ stade cyclopoïde ( Pl. XXVIII, fig. I4), l'appendice comporte déjà une base 2-articulée formée par les coxopodite et basipodite, avec un exopodite 2-articulé, sans aucune trace d'endopodite.

\section{Dyspontius striatus, THORELl.}

1860. Dyspontius striatus; THorell, 204, pages 81 et 82, Tab. XIV, fig. 22.

1880. Dispontius striatus, Th.; BRADY, 20, vol. 3, pages 66-68, Pl. XCIl, fig. 1-13.

1891. Dyspontius striatus, Th.; CANu ; 34 , page 486.

Planche XXVIII, fig. I-I 4 .

J. - Taille : $1,1 \mathrm{~m} / \mathrm{m}$.

Corps aplati et transparent (fig. 1), avec le céphalothorax elliptique et allongé, pourvu dans les $2^{\mathrm{e}}, 3^{\mathrm{e}}$ et $4^{\mathrm{e}}$ segments de replis pleuraux aliformes considérables, avec l'abdomen assez large, ayant le $1^{\text {er }}$ segment très développé et les autres plus courts que larges. Le $5^{\circ}$ segment abdominal est plus long que les $2^{\mathrm{e}}, 3^{\mathrm{e}}$ et $4^{\mathrm{e}}$, et de même longueur que les pièces furcales aplaties, lamellaires, avec de longues soies terminales barbelées (fig. 1 et 12).

Antennules (fig. 2) 11-articulées, géniculées entre les $9^{\mathrm{e}}$ et $10^{\mathrm{e}}$ articles, garnies à leur bord antérieur d'un petit nombre de soies relativement courtes et portant au bord antérieur distal du $10^{\circ}$ article un long et gros filament sensoriel cylindrique. Les deux premiers articles sont plus longs, les $3^{\mathrm{e}}, 4^{\mathrm{e}}, 5^{\mathrm{e}}, 6^{\mathrm{e}}$ et $7^{\mathrm{o}}$ plus courts; le $8^{\mathrm{e}}$ est légèrement renflé pour loger le muscle fléchisseur et porte à son bord antérieur une crête ornée de deux épines recourbées; le $11^{\mathrm{e}}$ est plus court, réduit à un tubercule arrondi, avec une longue soie rigide terminale et une épine recourbée à son bord antérieur (fig. 3). Une particularité qui a échappé à Brady, le seul observateur qui ait étudié le mâle de $D$. striatus, consiste dans l'existence à la face ventrale de l'antennule (fig. 1), d'un grand nombre de filaments sensoriels cylindriques, plus longs et plus grêles que le filament terminal, et qui manquent dans le jeune asexué.

Antennes (fig. $4 \%$, avec l'exopodite réduit et deux articulations bien nettes, la $3^{\mathrm{e}}$ étant à peine visible entre les $1^{\text {er }}$ et $2^{\mathrm{e}}$ articles.

Pièces buccales et rostre assez semblables à ce qui existe dans Artotrogus. Le rostre est moins renflé dans sa région basilaire, plus épais et plus aplati dans sa région distale (fig. 5 et 6). La mandibule diffère un peu dans l'ornementation de son extrémité libre (fig. 6, md) constituée par une saillie dentelée en soie vers l'intérieur, et une grosse pointe saillante vers l'extérieur. Les soies terminales de la $1^{\text {re }}$ maxille sont plus longues et plus flexibles (fig. $5 \mathrm{mx}^{\mathrm{s}}$ fig. 7). 
Pattes natatoires allongées, terminées dans les deux rames par une épine distale, avec de courtes épines marginales externes et des soies barbelées marginales internes. - N'ayant observé qu'un seul individu mâle, je ne puis décider si la conformation présentée par sa $2^{\circ}$ patte thoracique gauche (fig. 10) correspond à un caractère sexuel secondaire ou à une malformation exceptionnelle.

Quatrième patte thoracique (fig. 11) avec les deux articles basilaires allongés, avec les trois articles de l'exopodite bien développés, garnis : vers l'extérieur, de courtes épines denticulées (1), vers l'intérieur, de soies barbelées, terminés par une grande épine denticulée sur le bord externe. Cette rame unique de la $4^{e}$ paire est plus large et moins longue que l'exopodite des paires précédentes (fig. 11 et fig. 9).

Cinquième patte thoracique (fig. 12) aux deux articles à peine saillants, tuberculiformes, avec deux longues soies grêles à l'extrémité du second et une soie analogue au bord externe distal du premier.

Soie furcale médiane interne presque aussi longue que le céphalothorax, et double de l'abdomen.

Ouvertures externes des sacs à spermatophores (fig. 12) large et saillante, avec une épine et deux soies sur la lèvre supérieure; spermatophores irrégulièrement piriformes, très renflés.

Tégument chitineux ornementé de stries réfringentes irrẻgulières et enchevêtrées (fig. 13). avec des orifices punctiformes espacés.

J'ai recueilli deux spécimens de cette espèce, sortis des Ascidies simples de petite taille, dans la zone des Laminaires, auprès de la Tour de Croï, à Wimereux. L'un était un mâle adulte, particulièrement remarquable par sa transparence, par le développement de l'ouil tripartite et par l'extension dans le céphalothorax des lobes latéraux cruciformes du tube digestif. L'autre est une jeune forme encore asexuée, aux rames natatoires des pattes thoraciques 2-articulées. Dyspontius striatus est une forme rare, toujours recueillie en nombre très restreint dans une localité déterminée; elle semble très répandue, mais ses habitudes restent néanmoins des plus problématiques.

Distribution: Merdu Nord, Manche, Atlantique.

\section{Acontiophorus, BRADY:}

1880. Acontiophorus; BradY, 20, vol. 3 , page 69.

1889. Acontiophorws, Br.; Cl.Aus, 66 , pages 31 et 32.

(1) Contre l'assertion de Bradx : o Fourth pair of feet (fig. 9) elongated, 1-branched, destitute of marginal spines... $x$ 
Ascomyzontide ayant :

$I^{0}$ Le céphalothorax 5-segmenté, l'abdomen 3-segmenté chez la femelle et 4-segmenté chez le mâle.

$2^{0}$ Les antennules variables au point de vue du nombre (q : 11 ou 16$)$ des articles.

$3^{0}$ Le siphon très allongé, étendu jusqu'au $4^{\circ}$ segment thoracique ou jusqu'aux pièces furcales, capillaire dans sa portion distale extrêmement grèle, avec la portion proximale conique et renflée abritant seule les mandibules.

$4^{0}$ Les mandibules styliformes, avec le coxopodite terminé par une soie grêle et flexible ne pénétrant point dans la région capillaire du siphon, avec la région distale palpiforme transformée en une grande et longue soie plumeuse.

$5^{0}$ Les premières maxilles bilobées, aux deux lobes presque équivalents, armés de quatre Iongues soies lisses ou barbelées.

$6^{0}$ Le second article des $5^{\text {es }}$ pattes thoraciques large et foliacé, avec cinq soies.

Le genre Acontiophorus, établi avec raison par BraDY et maintenu par Claus, est très suffisamment caractérisé par les importantes transformations morphologiques que subissent chez lui le siphon buccal et les mandibules. Le tube terminant l'appareil buccal joue un ròle indiscutable dans la préhension de la nourriture, rôle auquel ne peuvent évidemment participer les mandibules. Claus eut facilement compris ce fait s'il avait reconnu les relations et la forme exactes des coxopodites mandibulaires. En réalité ces organes (Pl. XXIX, fig. 3, md) conservent avec le siphon les mèmes rapports que dans les genres précédents, mais ils sont plus réduits et manquent de la rigidité nécessaire pour fonctionner utilement contre les matières nutritives. Le tube capillaire terminal n'étant que le prolongement de la cavité conique menagée en avant de la bouche, entre les lèvres supérieure et inférieure, on ne peut dire que les mandibules ne sont point en rapport avec la cavité de ce tube.

\section{Acontiophorus scutatus, Brady \& Robertson.}

1873. Solenostoma scutatum; BRADY ET ROBERTSON.

1880. Acontiophorus scutatus, B. et R.; BRADY, 20, vol. 3, pages 69-7 I, PI. XC, fig. 1-10.

1889. Acontiophorus scutatus, B. et R.; Claus, 66, page 33, Taf. VII, fig. 1-5.

1891. Acontiophorus scutatus, B. et R.; Canu, 34, page 486.

Planche XXIX, Fig. I-4.

t. - Taille : 0,9 à $1 \mathrm{~m} / \mathrm{m}$.

Forme très allongée. Derniers segments abdominaux parfaitement cylindriques, sans saillies anguleuses à leur limite postéro-latérale (fig. 1 et 4 ). Pièces furcales plus longues que larges, avec de grandes soies terminales plumeuses (fig. 1). 
Antennules très courtes (fig. 2), 11-articulées, avec des soies barbelées ou lisses, longues et raides, et un long filament sensoriel cylindrique sur le $8^{\mathrm{e}}$ article.

Siphon buccal replié sur la face ventrale et dépassant l'extrémité postérieure des pièces furcales (fig. 1).

Pattes thoraciques des quatre premières paires biramées et natatoires.

Pattes de la $5^{\mathrm{e}}$ paire (fig. 4), avec le $1^{\mathrm{er}}$ article élargi et soudé a la face ventrale dư segment, pourvu à son bord distal d'une longue soie glabre dépassant la limite postérieure du $1^{\text {er }}$ segment abdominal ; avec le second article long et large, pourvu à son bord externe de deux grosses soies plumeuses, à son extrémité distale d'une, et à son bord interne de deux longues soies glabres peu flexibles.

Ouvertures génitales femelles placées au milieu des faces latérales du premier segment abdominal.

Sacs ovigères petits, élargis dans le sens dorso-ventral, comprenant seulement quatre ou cinq gros œufs sphériques.

Coloration blanche du corps et des œufs.

Rare parmi les Éponges et les Ascidies, dans la zone des Laminaires et sur les fonds rocheux du large. J'ai recueilli un petit nombre d'exemplaires femelles à marée basse, près de la Tour de Croï, à Wimereux, et dans le produit des dragages de l'Ajax, dans le détroit du Pas-de-Calais.

Distribution: Mer du Nord, Manche, Atlantique, Adriatique.

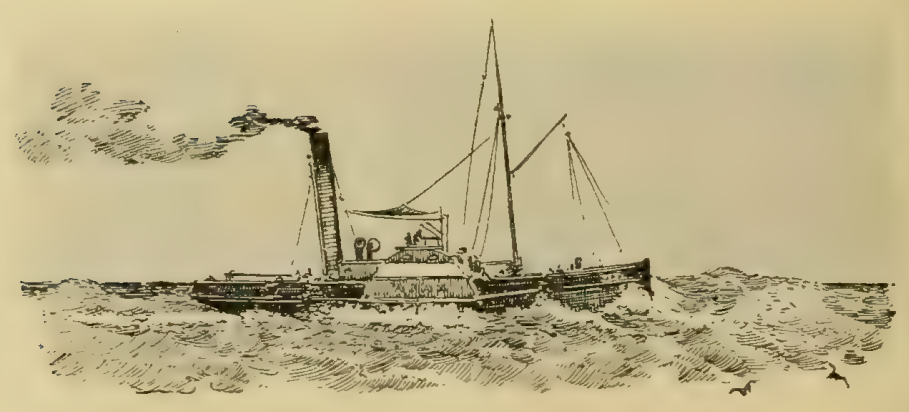




\section{INDEX ANALYTIQUE.}

\section{ABDOMEN}

Morphologie chez l'adulte.

3o-36

Nature et formation du somite furcal.

\section{APPAREIL DIGESTIF.}

Développement dans l'embryon chez les Ascidicolidés. $67-91$

Disposition dans le nauplius chez Lichomolgus................. $\quad 229$

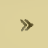

$\gg$ Thersites.

246

$\gg$

dans l'adulte chez Botryllophilus.

205

$\gg$

$\gg$ Enterocola.

215

Extension des lobes hépatiques chez Dyspontius

270

Organisation de l'hypopharynx chez Nicothoe.

251

Variations de la région pharyngienne chez les Hersiliidés.

$40-4^{3}$

$\gg$

$\gg$

$\gg$

$\gg$

$\gg$

$» \quad$ Auliostomés

$43-44$

" Ascomyzontidés...... $44-46$

$\gg \quad$ Ascidicolidés .......... $\quad 46-54$

\ Lichomolgidés........ 54

Ergasilidés ........... 55

\section{APPAREIL GÉNITAL FEMELLE.}

État rudimentaire dans l'embryon chez Doropygus.............. 77

État définitif chez les Ascidicolidés....................... $\sigma_{+}$

» $\quad$ Botryllophilus............................. 206

» $\quad$ Enterocola............................. 215

Disposition des pores de fécondation ........................ 127

chez Hersiliodes.............. 250 
Ovogenèse chez Notopterophorus .......................... $\quad 65$

» . Doroixys..................................... 65

\$ Botryllophilus ............................... 66

$\gg \quad$ Enterocola..................................... 66

APPAREIL GÉNITAL MALE.

État adulte chez Lichomolgus albens .......................... 231

\section{APPENDICES.}

Disparition de la mandibule chez Enterocola..................... $\quad 48$

première maxille chez Aplostoma.............. 51

Divergences dans les deux sexes............................ 111

» $\quad$ chez Asterocheres et Dermatomyzon.. $\quad 265$

Mode de formation embryonnaire .......................... 31

Morphologie chez l'adulte................................ 36

Transformations embryonnaires chez les Ascidicolidés............ 67

\section{CÉPHALOTHORAX.}

Morphologie chez l'adulte ............................... $\quad 26$

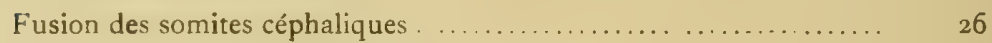

Soudure de divers somites thoraciques $\ldots \ldots \ldots \ldots \ldots \ldots \ldots \ldots \ldots \ldots . \ldots \ldots, \quad 27$

Divergences dans les deux sexes.......................... 2$\}, 111$

\section{CORPS GRAISSEUX.}

Apparition dans l'embryon chez Doropygus.................. $\quad 77$

Distribution dans l'adulte $\gg$ Doropygus.................... ${ }_{195}$

》 $\quad$ Enterocola ..................... 214

* $\quad \gg$ Aplostoma ...................... 224

FORME GÉNÉRALE DU CORPS.

Morphologie chez l'adulte.............................. 24

Mode d'apparition chez l'embryon......................... $\quad 26$ 


$$
-275-
$$

Transformations dans l'embryon chez les Ascidicolidés........... $67-90$

Divergences dans les deux sexes............................. $\quad 111$

Variations au point de vue de la systématique $\ldots \ldots \ldots \ldots \ldots \ldots \ldots . \quad 138-272$

\section{GLANDE ANTENNALE.}

Dans le nauplius chez Doropygus et les Ascidicolidés............. $\quad 70$

$» \quad$ Cyclops xquoreus....................... ${ }_{185}$

\section{GLANDE DU TEST.}

Dans les Copépodes en général.

Dans l'embryon cyclopoïde chez Doropygus

73,75

$\gg$

» Paryphes

78

Dans l'adulte chez Tačlnidius

155

\section{GLANDES HYPODERMIQUES.}

Dans l'abdomen chez Cyclops lubbocki.... 183

Dans le céphalothorax chez Pseudanthessius..................... $\quad 242$

Glande frontale du nauplius chez les Ascidicolidés.............. $\quad 69$

\section{MÉSODERME.}

Dans le nauplius avant l'éclosion chez Doroixys................. 84

» après „ 》 $\gg$ Doropygus................ 70

Mésoderme secondaire chez Doropygus....................... $7_{11}$

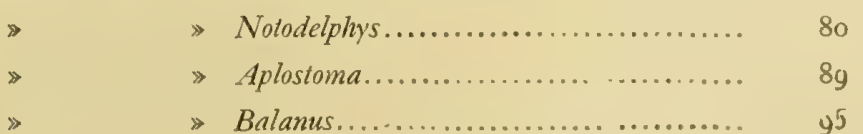

(EIL.

Variations chez les Peltidiens........................... 147

$\gg \quad$ Pontelliens............................... ${ }_{176}$ 


\section{ORGANE NUCHAL.}

Chez les Copépodes en général......................... $\quad 125$

\$ Tachidius ........................................... ${ }_{15} 155$

\$Laophonte ... ................................. ${ }_{164}$

\section{SYSTEME NERVEUX.}

Cerveau, dans l'embryon nauplien, chez Doropygus............. 69

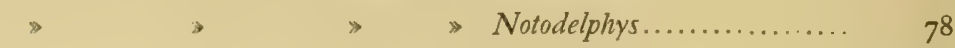

\$ $\quad \gg \quad$ Doroixys................ 83

\$ métanauplien chez Doropygus........... $\quad 73$

\$ $\quad$ cyclopoïde chez Doropygus............. $\quad 76$

Chaîne ventrale dans l'embryon cyclopoïde chez Doropygus......... $\quad 75$

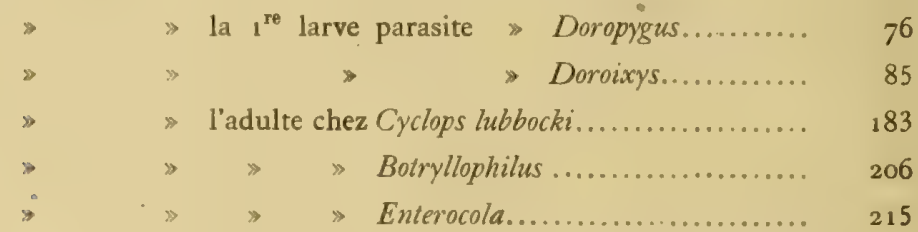




\section{LISTE SYNOPTIQUE}

\section{DES ESPÈCES ÉTUDIÉES,}

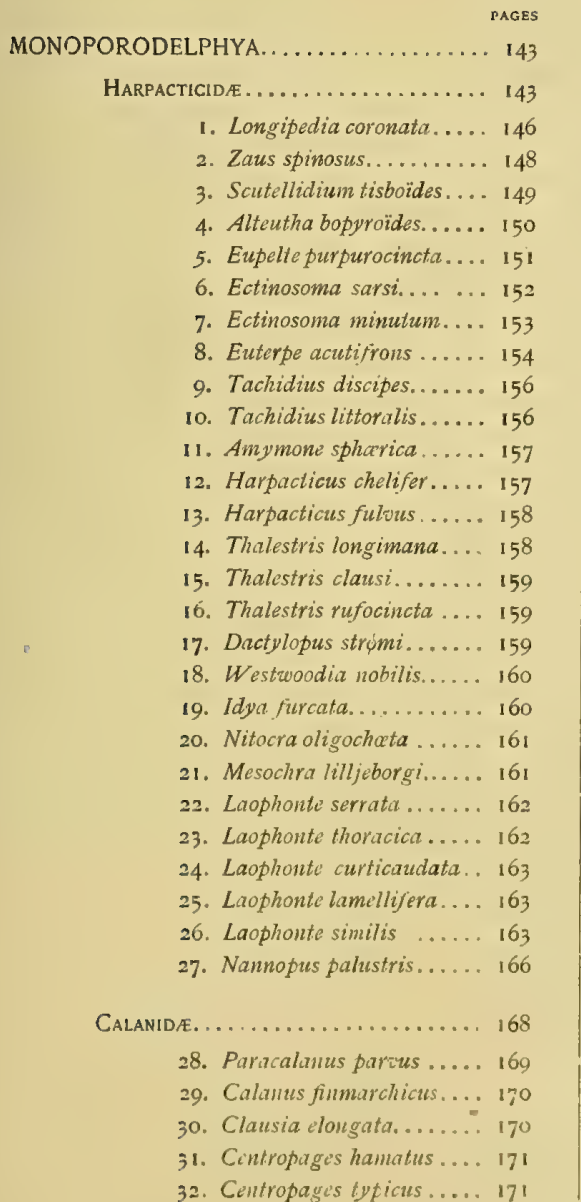

\begin{tabular}{|c|c|}
\hline \multicolumn{2}{|r|}{ PAGES } \\
\hline & Isias clavipes......... 172 \\
\hline & Temora longicornis...... I72 \\
\hline & Temorella affinis.. \\
\hline & Acartia clausi......... 174 \\
\hline & $\begin{array}{lll}\text { Acartia disc.zudala ..... } & 174 \\
\text { Parapontella brevicornis.. } & 175\end{array}$ \\
\hline & Labidocera wollastoni ... 176 \\
\hline & Pontclla lobiancoi....... $\quad{ }_{17} 8$ \\
\hline & Anomalocera patersol \\
\hline
\end{tabular}

Cyclopide.................... 179

42. Oithona helgolandica.... 180

43. Cyciopina gracilis ..... 18,

44. Thorellia brunnea...... 18

45. Cyclops lubbocki....... 182

46. Cyclops cequoreus ....... 184

AsCIDICOLIDE................ 186

47. Notodelphys agilis ....... 188

48. Notodelphys allmuni.... 188

49. Notodilplys elegans,... 189

50. Nolopterophorus elongatus 190

51. Notopterophorus gibber... 191

52. Doropygus psyllus ...... 194

53. Doropygus pulex........ 195

54. Bonnierilla longipes..... 197

55. Gunenotopliorus globularis 200

56. Doroixys uncinata ..... 202

57. Botryllophilus macropus. . 200

58. Ascidicola rosed ........ 209

59. Agnathwer typicus...... 211

60. Agnathaner minutus.... 212

61. Enterocola fulgens...... 216

62. Enterocola helencourti... 218

63. Enteropsis pilosus...... 220

64. Aplosloma brcoicauda ... 223 


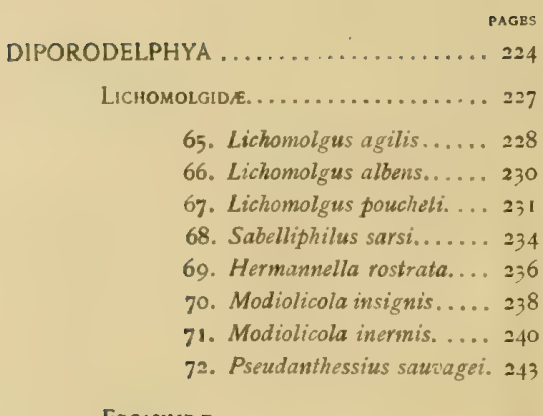

Ergasilidat................... 244

73. Thersites gasterostei.... 245
PACES

Hersilitof . . . . . . . . . . . . . 247

74. Hersiliudes pilseneiri... 252

75. Hersiliodes thompsoni.... 253

76. Giardilla vallianassa ... 254

77. Nicothoe astaci....... 248

78. Cancerilla tubulata..... 255

AscomYZONTIDE................. 257

79. Dermatomj zon elegans... 260

80. Asterocheres renaudi..... 263

81. Asterocheres lilljeborgi... 264

82. Artotrogus normani..... 266

83. Dyspontius striatus. ..... 269

84. Acontiophorus scutatus .. 27 


\title{
TABLE DES FIGURES
}

\author{
INTERCALEES DANS LE TEXTE.
}

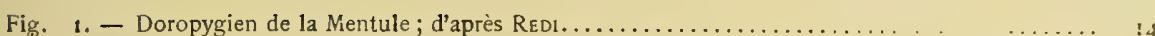

Fig. 2. - Première maxille et paragnathes d'Euphausia; d'après Claus ................... 4 i

Fig. 3. - Région buccale de Caligidium vagabundum Cıs; d'après Claus ................... 44

Fig. 4. - Enteropsis pilosus Canu. Fenrelle; céphalon et mandibule . . . . . . . . . . . . . . 52

Fig. 5. - Mâle adulte d'Enteropsis sphinx Aur.; d'après Auriviluius .................... 53

Fig. 6. - Jeune mâle d'Enteropsis sphinx Aur.; d'après Auriviluius.................... 53

Fig. 7. - Première maxille de Longipedia coronata Cls.; d'après GiesBrecht. ................. 57

Fig. $8 .-$ Première maxille de Zaus spinosus Claus ............................. 58

Fig. 9. - Nauplius de Calanus (Cetochilus) à l'éclosion; d'après Grobren.................... 94

Fig. 10. - Région postérieure d'un nauplius de Balanus balanö̈des Limné, peu de temps avant l'éclosion...................................................... 95

Fig. II. - Bandes mésodermiques secondaires du nauplius de Sacculina sp.; d'après GrobBen......... 9 ;

Fig. 12. - Forme jeune d'Apoblema appendiculatum Diesing, dans Clausia elongata BoECK, d'après

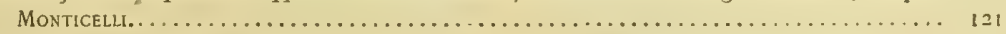

Fig. 13. - Région antérieure du céphalon de Labidocera wollastoni Luввоск, avec l'œil tripartite..... 176

Fig. 14. - Coupe d'une partie de l'œil chez Pontella sp.; d'après G. H. Parker............... I77

Fig. 15. - Région antérieure du céphalon chez Branchipres; d'après Claus................... 177

Fig. 16. - Femelle de Botryllophilus ruber Hesse; d'après Hesse.......... . . . . . . . . . . . . 205

Fig. 17. - Enteropsis pilosus Canu. Femelle : céphalon et mandibule................... 219

Fig. 18. - Figure schématique indiquant la place occupée dans l'ascidiozoïde par une femelle d'Aplostoma logée dans l'épicarde.................................. 222

Fig. 19. - Modiolicola inermis Canu. Abdomen et pièces furcales du mâle jeune............... 240

Fig. 20. - Hersiliodes thompsoni C CANU. Abdomen de la femelle avec deux spermatophores......... 250 



\section{LITTÉRATURE.}

1. Allman, Description of a new genus and species of Entomostraca; Ann. Mag. Nat. Hist., Sér. 1, vol. XX, 1847 .

2. - On the development of Notodelphys Allmann, a new genus of Entomostraca; Rep. Brit. Assoc. for 1847.

3. Audouin et Milne-Edwards, Mémoire sur la Nicothoe, animal singulier qui suce le sang des homards; Ann. d. sci. nat., Zoologic, sẻr. 1, vol. IX, 1826.

4. Aurivilutus, C. W. S., Bidrag till Kännedomen om Krustaceer, som lefva hos Mollusker och Tunikater; Akademisk Afhandling, Stockholm, 1883.

5. - Krustaceer hos arktiska Tunikater; Vega Expeditionens vetenskapliger Jakttagelser. Bd. IV, Stockholm, 1885.

6. - Hafsevertebrater från Nordligaste Trımsö amt och Vestfinmarken; Bihang till $K$. Svenska Vetensk. Akad. Handl., Bd XI, N ${ }^{\circ}$, Stockholm, 1886.

7. - Bemerkungen zu einem Aufsatze : « Descrizione di un nuovo Lichomolgus parassita del Mytilus galloprovincialis », Memoria dei signori Raffaele è Monticelli; Zool. Anzeiger, Jahrgang IX, N 215,8 février 1886.

8. Aurivilliưs, P. O. C., On a new genus and species of Harpacticida; Bihnng till $K$. Svenska Vetensk. Akad. Handl., $\mathrm{Bd} \mathrm{V}, \mathrm{N}^{0} 18$.

9. Baird, Natural history of British Entomostraca : Ray Society, London, 1850.

10. Blankaart, Stephan, Schon-burg der Rupsen, Wormen, Maden, etc...; Amsterdam, 1688.

11. Beneden, P. J. van, Organisation et développement des Nicothoes; Nouv. Mém. Acad. Belg., t. XXIV, 1848 (d ${ }^{0} \ldots$, Ann. d. sci. nat., Zoologie, sér. 3, t. XIII, 1850.

12. - Sur un nouveau genre de Crustacé Lernéen; Bull. Acad. Belg., sér. 2, t. IX, 1860.

13. - Recherches sur les Crustacés du littoral de Belgique, Nouv. Mém. Acad. Belg., t. XXXIII : 1861 .

14. Bовск, A., Tvende nye parasitiske Krebsdyr, Forhandl i Vidensk Selskah. i. Christiania, 1859 . 
15. - Oversigt over de ved Norges Kyster jaggtagne Copepoder henhorende til Calanidernes, Cyclopidernes og Harpactidernes Familier; Forhandl. i Vidensk. Selskab. $i$ Christionia, 1864.

16. - Om Sildeaat; Tidskr. for Fiskeri. Bd. I, 1867 (traduit en allemand dans : Arch. f. Naturg, Jahrgàng XXXIV, 1868).

17. - Nye Slægter og Arter of Saltvands Copepoder; Forhandl. i Vidensk. Selskab. i Christiania. 1872.

18. Bourne, G. C., Report on the pelagic Copepoda collected at Plymouth in 1888-89, Journ. Mar. biol. Assoc, new ser., vol. I, N’2 2, octobre 1889 .

19. - Report on the surface collections made by M. W. J. Grenfell in the North sea and West of Scotland, ibidem, new. ser., vol. I, $\mathrm{N}^{0} 4$, Novembre 1890.

20. Brady, Monograph of the free and semiparasitic Copepoda of the British Islanids, Ray Society, London, vol. I, 1878; vol. II, 1880 ; vol. III, 1880.

21. - Report on the Copepoda, Zool. Challenger. Expedit. Part XXIII (vol.'VIII), 1883.

22. - Notes on the Entomostraca, Fifth annual Report of the fishery Board for Scotland 1887 ; app. F, n ${ }^{r} \mathrm{XI}$.

23. Bruzelius, Lamippe rubra; Ofvers. Vetensk. Akad. Forhandl., Stockholm, 1858 (traduit en allemand dans Arch. f. Naturg. Jahrgang XXV, 1859).

24. Вuснноцz, Beiträge zur Kenntniss der innerhalb der Ascidien lebenden parasitischen Crustaceen des Mittelmeeres; Zeitschr. f. wiss. Zool. Bd. XIX, 1869.

25. - Die zweite deutsche Nordpolarfahrt in den Jahren 1869 und 1870 , Crustacea, in Bd. II.

26. Burmeister, Beschreibung einiger neuen oder wenig bekannter Schmarotzerkrebse, nebst allgemeinen Betrachtungen über die Gruppe, welche sie angehören ; Nova Acta Acad. Leopold.-Carol., Halle a. Saale, Bd. XVII, 1835.

27. Canu, Description de deux Copépodes nouveaux parasites des Synascidies; Bull. scientif., t. XVII, 1886

28. - Les Copépodes marins du Boulonnais, I, Calanidæ; Bull. scientif., t. XIX, 1888.

29. - Les Copépodes marins du Boulonnais, II, Description d'Isias Bonnieri ; $\imath$ bid.. t. XIX, 1888.

30. - Sur les Hersiliidæ, famille nouvelle de Copépodes commensaux, Comptes rendus Acad. sc. Paris, t. CVII, 12 novembre 1888.

31. - Les Copépodes marins du Boulonnais, III, Les Hersiliidæ; Bull. scientif., t. XIX, 1888.

32. - Copépodes, in GIARD, Le laboratoire de Wimereux en 1888 ; ibid., t. XIX, 1888. 
33. - Les Copépodes marins du Boulonnais, IV, Les Calanides pélagiques; ibid., t. XXII, 1890 .

34. - Les Copépodes marins du Boulonnais, V, Les Semi-parasites; ibid., t. XXIII, 1891 (extrait des Comptes rendus Acad. Sc. Paris, 189o).

35. - Sur quelques Copépodes semi-parasites du Boulonnais; Comptes rendus Acad. Sc. Paris, t. CXIII, 5 octobre 1891 .

36. Car, Lazar, Ein Beitrag zur Copepoden-Fauna des Adriatischen Meeres; Arch. $f$. Naturg., Jahrgang L, 188 .

3r. - Ein Beitrag zur Kenntniss der Copepoden von Trieste; Societas historico-naturalis croatica, Agram, Bd. V, 1890.

38. Claparède, Sur un Crustacé parasite de la Lobularia digitata, Delle Chiaje (Lamippe proteus, n. sp.); Ann. des sci. nat., Zoologie, sér. 5, t. VIII, 1867.

39. - Sur les Copépodes parasites des Annélides; ibid., sér. 5, t. XIII, 1870.

40. Claus, Zur Anatomie und Entwickelungsgeschichte der Copepoden ; Arch. f. Naturg., Jahrgang XXIV, 1858.

41. - Ueber den Bau und die Entwickelung parasitischer Crustaceen, Cassel, 1858.

42. - Ueber das Auge der Sapphirinen und Pontella; Arch. f. Anat. u. Phys., 1859.

43. - Beiträge zur Kenntniss der Entomostraken, Mäburg, 1860.

44. - Zur Morphologie der Crustaceen; Wurzb. naturwiss. Zeitschr., Bd. I, 1860 (traduit en anglais dans Quart. Journ. Micr. Sci., vol. I, 1861).

45. - Ueber den Bau von Notodelphys ascidicola; ibid., Bd. I, 1860.

46. - Ueber die blassen Kolben und Cylinder an der Antennen der Copepoden und Ostracoden ; ibid., Bd. I, 1860.

47. - Ueber die Familie der Lernæen; ibid., Bd. II, 1861.

48. - Ueber den Bau und die Entwickelung von Achteres percarum, Zeitsch. f. wiss. Zool, Bd. XI, 1861 .

49. - Untersuchungen über die Organisation und Verwandtschaft der Copepoden; Wurzb. naturwiss. Zeitschr., Bd. III, 1862.

50. - Ueber die morphologischen Beziehungen der Copepoden zu der werwandte Crustaceen-gruppen ; ibid., Bd. III, 1862.

51. - Die freilebenden Copepoden, mit besonderer Berücksichtigung der Fauna Deutschlands, der Nordsee und des Mittelmeeres. Leipzig, 1863.

52. - Beiträge zur Kenntniss der Schmarotzerkrebse; Zeitschr. f. wiss. Zool., Bd. XIV, 1864 . 
53. - Die Copepodenfauna von Nizza, Ein Beitrag zur Characteristik der Formen und deren Abänderungen in Sinne Darwin's; Schrift. d. Gesellsch. Beförd. der gesammt. Naturwiss., 1866.

54. - Beobachtungen über Lernæocera, Peniculus und Lernæa. Ein Beitrag zur Naturgeschichte der Lernæen, Marburg, 1868.

55. - Neue Beiträge zur Kenntniss parasitischer Copepoden nebst Bemerkungen über das System derselben; Zeitschr. f. wiss. Zool., Bd. XXV, 1875.

56. - Ueber Sabelliphilus Sarsii und das Männchen derselben; Zeitschr. f. wiss. Zool., Bd. XXVI, 1876 .

5\%. - Untersuchungen zur Erforschung der genealogischen Grundlage des Crustaceensystems. Ein Beitrag zur Descendenz-theorie, Wien; 1876.

58. - Die Schalendrüse der Copepoden, Sitzungsber. d.k. k. Akad. d. Wiss. Wien, Bd. LXXIV.

59. - Uecer die Gattungen Temora und Temorella nebst der zugehörigen Arten ; ibid., Bd. LXXXIII, 1881.

60. - Neue Beiträge zur Kenntniss der Copepoden unter besonderer Berücksichtigung der Triester Fauna; Arb. Zool. Inst. Wien, t. III, 1881.

61. - Neue Beiträge zur Morphologie der Crustaceen ; i.jid., t. VI, 1885.

62. - Untersuchungen über die Organisation und Entwickelung von Branchipus und Artemia, nebst vergleichenden Bemerkungen über ändere Phyllopoden; ibid.,t. VI. 1886.

63. - Ueber Lernæascus nematoxys Cus., und die Familie der Philichthyiden; ibid., t. VII, 1887 .

64. - Ueber den Organismus der Nebaliden und die systematische Stellung der Leptostraken; ibid., t. VIII, 1888.

65. - Copepoden Studien. - Heft I, Peltidien, Wien, 1889.

66. - Ueber neue oder wenig bekannte halbparasitische Copepoden, insbesonderc der Lichomolgiden-und Ascomyzontiden-gruppen; Arb. Zool. Inst. Wien, t. VIII, $188 \mathrm{~g}$.

67. - Ueber Goniopelte gracilis, eine neue Peltidie; ibid., t. IX, 1890.

68. - Ueber den feineren Bau des Medianauges der Crustaceen; Akad. Anzeiger Wiin, $\mathrm{N}^{r}$ XII (Sitz. d. math.-nat. Cl. von 14 mai 1891).

69. - Bemerkungen über secundäre Sexualcharactere an den zwischen Vorderantennen und den fünften Fusspaare gelegenen Gliedmassen der Copepoden, und die Prätensionen des $\mathrm{D}^{\text {rs }}$ Giesbrecht; Zoolog. Anzeiger, Jahrgang XIV, N ${ }^{0} 378,30$ Nov. 1891 . 
7o. - Das Medianauge der Crustaceen. - Ueber die Gattung Miracia Dana mit besonderer Berücksichtigung ihres Augenbaues; Arb. Zoot. Inst. Wien, t. IX, 1891.

r1. Costa, O. G., Fauna del Regno di Napoli, Entomostraca (1840), 1852 ..

72. Cuvier, Tableau élémentaire de l'histoire naturelle des animaux. Paris, 1798.

73. DaNa, United States Exploring Expeditions during the years 1838-39, 18 +o, 1841, 1842, vol. XIII, Crustacea, Philadelphie, 1852. Atlas, 1855.

74. De GEER, Mémoires pour servir à l'histoire naturelle des Insectes, vol. III, Stockholm, 1778 .

75. Della Valle, Sui coriceidi parassiti e sull'anatomia dell' gen. Lichomolgus; Mem. R. Accad.d. Lincei, sér. 3, vol. V, Roma, 1880.

76. - Sui Copepodi che vivono nelle Ascidie composte del Golfo di Napoli; ibid., sér. 3, vol. XV, Roma, 1883.

7r. Desmarets, Considérations générales sur la classe des Crustacés, Paris, 1825.

r8. Edwards, Charles L., Beschreibung einiger neuen Copepoden und einer neuen Copepoden-änhlichen Krebses : Leuckartella paradoxa; Arch. $f$. Naturg., Jahrgang LXVII.

r9. Fabricius, J. C., Systema entomologiæ sistens Insectorum classes, ordines, genera, species. Leipzig, $177^{5}$.

80. Fischer, Beiträge zur Kenntnis der Entomostraken; Abhandl. K. Bayer. Akad. d. Wiss., Bd. VIII, 1860.

81. Fric, J. A., Note préliminaire sur l'ontogénie des Copépodes d'eau douce; Zool. Anzeiger, Jahrgang V, 1882.

82. Gegenbaur, Mittheilungen über die Organisation von Phyllosoma und Sapphirina; Arch. f. Anat. u. Physiol., 1858.

83. Gerstaecker, Bronn's Klassen und Ordnungen des Thierreichs, Bd. V, Arthropoda.

84. Giard, Histoire naturelle des Synascidies, Arch. de Zool. Expér., sér. 1, t. II, 1873.

85. - Sur un Copépode (Cancerilla tubulata, DalyELt) parasite de l'Amphiura squamata Delle Chiaje; Comptes rendus Acad. sc. Paris, 25 avril 1887.

86. - Le laboratoire de Wimereux en 1889 , Recherches fauniques, Bull. scientif., t. XXII, 189o. (Cancerilla tubulata, p. 85).

87. - et Bonnier, Note sur Aspidœecia normani et sur la famille des Choniostomatidæ: Bull. scientif., t. XX, 1889 .

88. Giesbrecht, Vorläufige Mittheilung aus einer Arbeit über die freilebenden Copepoden des Kieler Hafens; Zool. Anzeiger, Jahrgang IV, 1881.

89. - Die freilebenden Copepoden der Kieler Föhrde, VIItres Jahresbericht d. Commiss. f. wiss. Unters d. deutschen Meere, 1882. 
90. - Zur Kenntniss einiger Notodelphyiden, Mitth. Zool. Stat. Neapel, Bd. III, 1882.

91. - Elenco dei Copepodi pelagici raccolti dal tenente di vascello Gaetano Chierchia durante il viaggio della R. Corvetta « Vettor Pisani »negli anni $1882-1885$ e dal tenente di vascello Francesco Orsini nel Mare Rosso nel 1884. Rendiconti $R$. Accad. d. Lincei, vol. IV, V, VII, 1888, 1889, 1891 .

92. - Pelagische Copepoden, in Kükenthal's Beiträge zur Fauna Spitzbergens, Arch. $f$. Naturg., Jahrgang. LV, 1889.

93. - Ueber secundære Sexualcharactere bei Copepoden, Zool. Anzeiger, No 372, 1891.

94. Goonsir, The genus Cetochilus, belonging to the order Copepoda and the family Pontia of Milne Edwards, Edinb. New Philos. Journ., vol. XXXV, 1843.

95. - On severæ new species of Crustaceous allied to Sapphirina, Ann. Mag. Nat. Hist., vol. XVI, 1845 .

96. GrefFFe, Gastrodelphys; Boll. Soc. Adriat. di Sci, nat., Trieste, vol. VIII.

97. Grobben, Die Entwickelungsgeschichte der Moina rectirostris, zugleich ein Beitrag zur Anatomie der Phyllopoden; Arb. Zool. Instit. Wien, T. II, 1879.

98. - Die Antennendrüse der Crustaceen; Arb. Zool. Inst. Wien, T. III, 1880.

99. - Die Entwickelungsgeschichte der Cetochilus septentrionalis ; ibid, t. III, 1881.

100. Grube, Mittheilungen über St-Waast-la-Hougue, über St-Malo und Roscoff; $A b h$. d. Schles. Gesellsch. Naturwis, 1868 et 1870.

101 Gruber, Beiträge zur Kenntniss der Generationsorgane der freilebenden Copepoden; Zeitsch. f. Wiss. Zool, Bd. XXXII.

102. Gunner, Skrifter som udi der Kjobenhavnske Selskabe: Acta Havnia, Deel X, 1765.

103. Haller, Beschreibung einiger neuen Peltidien; Arch. f. Naturg., Jahrgang XLVI, 188 o.

104. Hancock and Norman, On Splanchnotropus, an undescribed genus of Crustacea, parasit in Nudibranchiate Mollusca; Trans. Linn. Soc. London, Zoology, vol. XXIV, 1863.

105. Hansen, Oversigt over de paa Dijmphna-Togtet indsamlede Krebsdyr ; DijmphnaTogtet Zool. Botan. Udbytte, Kjobenhavn, 1888.

106. Hartog, The Morphology of Cyclops and the relations of the Copepoda ; Trans. Linn. Soc. London, Zoology, vol. V, 1888.

107. Heider, Die Gattung Lernanthropus; Arb. Zool. Inst. Wien, t. II, 1879.

108. Heller, Reise der Novara, Bd. II, Abth III, Crustaceen, Wien, 1866.

109. - Carcinologische Beiträge zur Fauna des Adriatischen Meeres, Verhandl. Zool. Bot. Gesellsch. Wien, Bd. XVI, 1866. 
110. Hesse, Recherches sur les Crustacés rares ou nouveaux des côtes de France, $A n n$. a. Sci. nat. Zoologie, sér. 4, t. XVIII, 1862 ; sér. 5 , t. I, 1864 ; t. IV, 1865; t. VI, 1865 ; t. VII, 186 ; ; t. IX, 1868 ; t. XI, 186 q ; sér. 6 , t. VII, 1878.

111. Hoek, De vrylevende Zoetwater-Copepoda der Nederlandsche Fauna; Tijdsch. Ned. Dierk, Vereen., Bd. III, 1876 (Presqu'intégralement reproduit en allemand dans Nied. Arch.f. Zool., Bd. III, 1876).

112. - Carcinologische Aanteckeningen. Bydräge tot de Kennis der Noordzee-Fauna; ibid., Bd. III, 1876 .

113. - Zur Entwickelungsgeschichte der Entomostraken, II, Zur Embryologie der freilebenden Copepoden ; Nied. Arch. f. Zool., Bd. IV, 1877.

114. JoliET, Observations sur quelques Crustacés de la Méditerranée. - Sur une troisième espèce du genre Lamippe ; Arch. d. Zool. Expér., sér. 1, t. X. 1882.

115. Jurine père, Histoire des. Monocles qui se trouvent aux environs de Genève, 1820.

116. KefErstein, Ueber einen neuen Schmarotzerkrebs, Nereicola ovata; Zeitschr. f. wiss Zool., Bd. XII, 1863.

117. Kerschner, Ueber zwei neue Notodelphyiden; Denksch. d. k. k. Akad. Wiss. Wien, Bd. XLI, 1879 .

118. Kossmann, Ueber Clausidium testudo, einen neuen Copepoden, nebst Bemerkungen über das System der halbparasitischen Copepoden; Verh.d. Wurzb. phys. med. Gesellsch, N. F., Bd. VII, 1874 .

119. - Zoologische Ergebnisse einer Reise in die Küstengebiete des Rothen Meeres. Erstes Hälfte, IV, Entomostraca; 1877 .

120. - Ueber den classificatorischen Werth der Mundorgane der Crustaceen. Erwiederung an Herrn Della Valle; Zool. Anzeiger, Jahrgang IV, N6 95, 24 octobre 1881.

121. Kritchaguine, Matériaux pour la faune du rivage oriental de la Mer Noire (en russe); Public. Soc. Nat. Kiew, t. III, 1873 .

122. Kroyer, Om Snyltkrebsene, isaer med Hensyn til danske Fauna; Naturhist. Tidsskr. I et II, $1837^{-1838 .}$

123. - Karcinologiske Bidrag, Slaegter Calanus. Pontia, etc.; ibid.. Nye Raekke, 2 Bd, , 1848-49.

124. - Voyage de la Commission scientifique du Nord en Scandinavie, en Laponie, au Spitzberg et aux Féroé, pendant les années 1838-1840 sur la cởvette La Recherche. Atlas, Ctustacés, Paris, 1842.

125. - Bidrag til Kundskab om Snyltekrebsene ; Naturhist. Tidskr., III Rxkke, 2 Bd., 1863.

126. Kurz, Eunicicola Clausii, ein neuer Anneliden Parasit; Sitzungsber. Wien. Akad. $d$. Wiss., Bd. LXXV, 1877 . 
127. Latreille, Précis des caractères génériques des Insectes, Brives, 1796.

128. - Le Règne animal (Cuvier), nouv. édit., vol. VI, 1829.

129. LEACH, Edinburg Encyclopædia, article Crustaceology, 1813-1814.

130. - A tabular view of the External Characters of four classes of animals which Linné arranged under Insecta; Trans. Linn. Soc. London, 1815.

131. - Entomostracés, in Dictionnaire des Sciences naturelles rédigé par Fréd. Cuvier, vol. XIV, 1819 .

132. Leeuwenhoer, Opera omnia, t. III. - Continuatio arcanarum naturæ. Epistolæ ad societatem regiam angliam. Epist. 121, p. 138-145, 1669.

133. Leucrart, Notopterophorus. - Ueber die Gesichtswerkzeuge der Copepoden; Arch. f. Naturg., Jahrgang XXV, 1859.

134. Levinsen, Om nogle parasitiske Krebsdyr der snylte hos annelider, Vidensk. Meddelels. fra Naturhist. Forening, Kjobenhavn, 3 Række, Bd. X, 1878.

135. Leydig, Zoologische Notizen. Neuer Schmarotzer-Krebs auf einem Weichthiere; Zeitschr. f. wiss. Zool. Bd. IV, 1853.

136. Lilzjeborg, De Crustaceis ex ordinibus tribus: Cladocera, Ostracoda et Copepoda in Scania occurentibus. Lund, 1853.

137. - De under Svenske vetenskapliga Expeditionen til Spitzbergen 1872-73 derstädes samlade Hafs-Entomostraceer; CEfvers. af k. Vetensk. Akad. Förhandl., 1875.

138. - Collection of chiefly fresh-water Crustacea from Sweden. Great Fisheries Exhibition London, 1883.

139. List, Das Genus Gastrodelphys; Zeitschr. f. wiss. Zool., Bd. XLIX, 1889.

140. Lubbock, Amn. Mag. Nat. Hist. ser. 2, vol. XI, 1853 ; a new genus: Labidocera, two new subgenera, Iva, Ivella.

141. - On some Entomostraca collected by Dr Sutherland in the Atlantic Ocean; Trans. Entomol. Soc. London, 口. ser., vol. IV, part. 2, 1856.

142. - On arctic Calanidæ; Ann. Mag. Nat. Hist., ser. 2, vol. XVII, 1857.

143. - Description of eight new species of Entomostraca found at Weymouth; ibid., sér. 2, vol. XX, 1859 .

144. - On some Oceanic Entomostraca, collected by Captain Toynbee; Trans. Linn. Soc. London, vol. XXIII, 1860.

145. Mac INrosh, On a Crustacean parasite of Nereis cultrifera; Quart. Journ. Micr. Sci, vol. X, 1870 .

146. MetzGer, Ueber das Männchen und Weibchen der Gattung Lernæa vor der 
Eintritt der sogenannt rückgeschreitenden Metamorphose ; Nachrichten Gesellsch. Wiss. Univ. Göttingen, 1868 (également dans Archiv.f. Naturg., Jahrgang XXXIV, 1868.

147. Mrlne Edwards, Classification dẹ Crustacés; Ann. d. Sci. Nat., sér. 1, t. XX, 1830.

148. - Mémoire sur l'organisation de la bouche chez les Crustacés suceurs; Ann. d. Sc. nat., sér. 1, t. XXIX, 1833.

149. - Histoire naturelle des Crustacés, Suites à Buffon, 3 vol., Paris, 1834-1840.

150. Mœвius, Die wirbellosen Thiere der Ostsee, Jahresber. $d$. Comm. f. wiss. Unters. $d$. deutschen Meere: Pomerania Expedition 1871, Kiel 1873.

151. - Copepoda und Cladocera; ibid. für $1872-73$, Berlin 1875 .

152. Mueller, O. F., Entomostraca seu Insecta testacea quæ in aquis Daniæ et Norvegiæ reperit, descripsit et iconibus illustravit. Lipsiæ et Havniæ 1785.

153. W. Mueller, Longipedina paguri n. s.; Archiv. f. Naturg., Jahrgang L., 1884.

154. Nordmann, A. von, Mikrographische Beiträge zur Naturgeschichte der wirbellosen Thiere, Heft 2, Berlin, 1832.

155. - Neue Beiträge zur Kenntniss parasistischer Copepoden, I, Bull. Soc. imp. Natur. Moscou, t. XXXVII, 1864 .

156. NorDQvist, Die Calaniden Finlands ; Bidrag til Kannedomen of Finlands Natur och Folk, Heft 47, Helsingfors, 1888.

157. Norman, Last Report on Dredging among the Shetland Isles; Rep. Brit. Assoc., 1868.

158. Pagenstecher, Thersites gasterostei und Leptodera Nicothoæ, eine neue Gattung parasitischer Crustaceen und eine Nematoden; Arch.f. Naturg., Jahrgang XVII, 1861.

159. PhilippI, Einige zoologische Notizen. Beobachtungen über Copepoden des Mittelmeeres; Arch. $f$. Naturg., Jahrg. V, VI, IX, X, $-1839,1840,1843$ et 1844 .

160. Pickering and Dana, Description of Crustaceous animal, Caligus americanus; Amer. Journ. of Sci., t. XXXIV, 1838.

161. Poppe, Ueber eine neue Art der Gattung Temora, Baird; Abhandl. der Naturw. Vereins zu Bremen, Bd. VII, 188 o.

162. -- Ueber einen neuen Harpacticiden (Tachidius littoralis); ibid., Bd. VII, 1881.

163. - Ueber die von den Herren Dr Arthur und Aurel Krause im Nördlichen Stillen Ocean und Behringsmeer gesammelten freilebenden Copepoden; Arch.f. Naturg. Jahrgang L, 1884 .

164. - Die freilebenden Copepoden des Jadebusens; Abhandl. der Naturw. Vereins $z$ u Bremen, Bd. IX, 1885. 
165. - Beschreibung einiger neuer Entomostraken; Zeitschr. f. wiss. Zool., Bd. XLV, 1887 .

166. - Beitrag zur Kenntniss der Gattung Clytemnestra Dana. Zur Litteratur des Genus Monstrilla Dana; Abhandl. Bremen, 1891 .

167. Rayfaele È Monticrili, Descrizione di un nuovo Lichomolgus parassita del Mytilus galloprovincialis; Mem.d.R. Accad. d. Lincei, ser. 4, vol. I, 1885.

168. Ramdohr, Beiträge zur Naturgeschichte einiger deutschen Monoculusarten, Halle a. S. 1805 .

169. Rathbun, List of Marine Invertebrata; Proc. U. S. Nat. Mus., vol. IV, 1881.

170. - Annotated list of described species of parasitic Copepoda; ibid., vol. VII, 1884.

1'y1. RathKE, Bemerkungen ueber den Bau des Dichelestium sturionis und der Lernæopoda stellata; Nove acta Acad. Leopold. Carol. Halle a. S. Bd., XIX, 1839.

172. - Beiträge zur Fauna Norvegens; ibid., Bd. XX, 1843.

173. REDI, Osservazioni intorno agli animali videnti che si trovano negli animali viventi, Firenze, 1684 .

174. Richard, Sur la glande du test des Copépodes d'eau douce; Bull. Soc. Zool. France, t. XV, 18 go.

175. - Recherches sur le système glandulaire et sur le système nerveux des Copépodes libres d'eau douce, suivies d'une révision des espèces de ce groupe qui vivent en France; Am.d. Sci.nat., Zoologie, sér. 7, t. XII, 1891.

176. Richiardi, Catalogo sistematico dei Crostacei che vivono sul corpo degli animali acquatici in Italia, Pisa, 1880.

17r. Rudley, On a new Copepod of the genus Doridicola; Ann. Mag. Nat. Hist., ser. 5, vol. IV, 1879 .

178. Rossor.., Ueber zwei neue an Echinodermen lebende parasitische Copepoden: Ascomyzon comatulæ und Astericola Clausii; Sitzungsber. K. Akad. Wiss. Wien, Bd. XCII, 1888.

179. Roussel de Vauzème, Description du Cetochilus australis, nouveau genre de Crustacé branchiopode; Ann.d. Sci, nat., Zoologie, sér. 2, t. I, 1834 .

180. Salensky, Sphæronella Leuckarti, ein neuer Schmarotzerkrebs ; Arch. f. Naturg., Jahrgang XXXIV, 1868.

181. Sars, G. O., Oversigt af irdenlandske Ferskvands-Copepoder, Forhandl. Vid. Selsk., Christiania, 1862 .

182. - Den norske Nordhavs Expedition, Zoologi, Crustacea, 1885, 1886.

183. SARs. M.; Forhandlingar ved de Skandin. Naturforskeres Mode i Christiania, 1856. 
184. - Beskrivelse med Afbildninger af fire nye parasitisk Copepoder; Forhandl. Vid. Selsk. i Christiania, Aar 1861, 1862.

185. - Beretning om et nyt lernæalignende Krebsdyr, Sabellacheres gracilis; ibid. ibid.

186. - VII Bidrag til Kundskab om Christianiafjordens, Crustacea. Beskrivelse af nye, pad Annelidar snyltende Copepodeformer. Beskrivelse of Anteacheres Duebenii, SARS; Nyt. Mag.f. Naturv., Bd. XIII, 1870.

18\%. Siebold, von, Ueber das Begattungsgeschäft des Cyclops Castor; Neueste Schr. $d$. Naturf. Gesellsch.in Danzig, Bd. III, 1839 (traduit dans Ann. d. Sci.nat.,Zoologie, sér. 2, t. XIV).

188. Schimkevitsch, Embryogénie des Copépodes parasites (Enteropsis dubius, Tracheliastes, Lernæopoda) (en russe). Trav. Soc. des Natural. de St-Peter bourg, section de Zool. et Phys., t. XX, livraison 2, 1889 .

189. Schmankevirsch, Quelques Crustacés des eaux salées et douces et leurs rapports avec l'élément environnant; (en russe), Public. Soc. natural. N Nhe Russie, Odessa t. III, 1875 .

190. - Zur Kenntniss des Einflusses der äusseren Lebendsbedingungen auf die Organisátion der Thiere; Zeitsch. f. wiss. Zool., Bd. XXIX, 1877.

191. SchmeIL, Beiträge zur Kenntniss der Süsswasser-Copepöden Deutschland's ; Jnaug. Diss., Leipzig, 1891.

192. Sparre-Schneidèr, Undersögelse of dyrelwe ti de arktiske fjörde. II. Kvænangsfjördens Crustaceer og Pygnogonider; Aarshefte Museum Tromsö, Bd. VII, 1884.

193. Ström, Skrifter, som udi det Kjöbenhavnske selskab. (Acta Havnice). Deel IX.

194. Thompson, I. C., On some Copepoda, new to Britain, found in Liverpool Bay; Pruc. Biol. Soc. Liverpool, vol. I, 1887.

195. - Second Report on the Copepoda of Liverpool Bay ; ibid. vol. II, 1887.

196. - Copepoda of Madeira and the Canary Islands, with Description of new genera and species; Journ. Linn. Soc., Zoology, vol. XX, 1887.

197. - Report on the Copepoda collected in Maltese seas by David Bruce; Proc. Binl. Soc. Liverpool, vol. III, 1888.

198. - Tow-net gatherings made by Riley on West-Coast of Norway up the North Cape; ibid., vol. III, $188 \mathrm{~g}$.

199. - Third Report on the Copepoda of Liverpool Bay, ibid. vol. III, 1889.

200. - Appendix to Third Report, ibid., ibid.

201. - Monstrilla and Cymbasomatidæ, ibid. vol. IV, 1890.

202. Thompson, G. M., NewZealand Copepoda; Trans. N. Zeal. Inst., vol. XV, 1883. 
203. - New Zealand Copepoda, II, N. Zeal. Journ. of Sci., vol. I, 1883.

204. Thorell, Bidrag til Kännedomen om Krustaceer som lefva i Arter Slägtet Ascidia ; K. Svensk. Vetensk. Akad. Handl. Stockholm, Bd. III, 1860. (Extrait en allemand dans Zeitschr. $f$. d. Gesam. Naturw., Jahrgang 186o, Bd. XV.

205. Urbanowicz, Zur Entwickelungsgeschichte der Cyclopiden (Vorläufige Mittheilung); Zool. Anzeiger, Jahrgang VII, 1884 .

206. - Przyczynck do embryologii rakow widlonogich (Copepoda) - Zur Embryologie der Copepoden); Kosmos, 1885.

207. VAlle, A., Supra una specie nuova del genere Stellicola Ksm。 - Crostacei parasiti dei Pesci di mare Adriatico; Boll. d. Soc. Adriat. d. Sci.nat. Trieste, vol. VI, 1881.

208. Vejdovsky, Untersuchungen über die Anatomie und Metamorphose von Tracheliastes polycolpus NordM。; Zeitschr. f. wiss. Zool., Bd. XXIX, 1877.

209. Vogt, C., Ocean und Mittelmeer. Reisebriefe, II, 1848.

210. - Recherches côtières faites à Roscoff. Crustacés parasites des poissons; Mém. Inst. nat. genevois, t. XIII. 1879.

211. Wierzejski, Ueber Schmarotzerkrebse von Cephalopoden; Zeitschr. f. wiss. Zool. Bd. XXIX, 1877 .

212. Witl, Ueber Staurosoma, einen in den Actinien lebenden Schmarotzer; Arch. $f$. Naturg., Jahrgang X, 1848.

213. Wright, Ramsay, On a parasit Copepod of the Clam (Myicola metisiensis); Amer. Naturalist, vol. XIX, 1885.

214. ZENKER, Anatomische-Systematische Studien über die Krebsthiere; Arch.f. Naturg., Jahrgang XX, 1854 . 
PLANCHES. 


\section{LETTRES COMMUNES}

\section{AUX FIGURES DE TOUTES LES PLANCHES.}

$a$, antennule.

$A$, antenne.

$a^{4}, a^{2}, a^{3}, a^{4}, a^{5}$, les cinq somites abdominaux.

$b$, bouche.

bsp, basipodite.

$c$, céphalon.

$c d$, canal déférent.

cer, cerveau.

cers, cerveau secondaire.

cpm, cellules mésodermiques polaires.

$c s$, canalicules seminifères.

cxp, coxopodite.

end, endoderme.

enp, endopodite.

exd, exoderme.

exp, exopodite.

$f$, somite terminal ou furca.

$f r$, prolongement frontal.

glfr, glande frontale.

$i$, intestin.

$l i$, lèvre inférieure.

$l s$, lèvre supérieure. md, mandibule.

$m s^{2}$, mésoderme secondaire.

$m x^{\downarrow}$, première maxille.

$m x^{2}$, seconde maxille.

$m x^{2} e$, seconde maxille externe.

$m x^{2} i$, seconde maxille interne.

$o e$, cil.

oes, œesophage.

og, orifice génital.

ov, ovaire.

ovd, oviducte.

$p^{1}, p^{2}, p^{3}, p^{4}, p^{5}$, les cinq appendices thoraciques.

po, pore de fécondation.

pr, paragnathe.

prp, protopodite.

$p s$, vésicule séminale.

$s n$, système nerveux.

$T$, testicule.

$t^{1}, t^{2}, t^{3}, t^{4}, t^{5}$, les cinq somites thoraciques.

$t d$, tube digestif.

$\nu$, repli intermédiaire des paires d'appendices. 


\section{- PLANCHE 1.}

Fig. I-I 2, Paracalanus parvus Cläus

Fig. I3-I9, Calamus finmarcbicus Gunner. 


\section{PLANCHE I.}

\section{Paracalanus parvus.}

Fig. I. Mâle adulte, vu par la face dorsale: $\frac{50}{1}$.

Fig. 2. Mâle jeune, à l'avant-dernier stade: $\frac{50}{1}$.

Fig. 3. Femelle adulte, vue par la face dorsale : $\frac{50}{1}$.

Fig. 4. Extrémité antérieure de la tête, vue ventrale : $\frac{55}{\mathrm{I}}$.

Fig. 5. Derniers segments thoraciques et premiers segments abdominaux du jeune mâle, vue ventrale : $\frac{160}{1}$.

Fig. 6. Cinquième patte thoracique de la femelle adulte: $\frac{160}{\mathrm{I}}$.

Fig. 7. Seconde maxille interne du mâle adulte : $\frac{160}{1}$.

Fig. 8. Seconde maxille interne de la femelle adulte : $\frac{160}{\mathrm{I}}$.

Fig. 9. Première patte natatoire de la femelle : $\frac{160}{1}$.

Fig. IO. Troisième patte natatoire de la femelle : $\frac{160}{1}$.

Fig. 11. Cinquième patte thoracique du mâle adulte : $\frac{160}{\mathrm{I}}$.

Firg. 12. Céphalothorax d'une femelle adulte, avec les organes génitaux : $\frac{120}{1}$. $m$, œuffs mûrs.

\section{Calanus finmarcbicus.}

Fig. 13. Mâle adulte vu par la face dorsale: $\frac{25}{1}$.

Fig. 14. Femelle adulte vue par la face dorsale : $\frac{25}{1}$.

Fig. I 5. Abdomen du mâle adulte: vue dorsale: $\frac{55}{1}$.

Fig. 16. Seconde maxille interne du mâle: $\frac{120}{1}$.

Fig. 17. Seconde maxille interne de la femelle: $\frac{55}{1}$

Fig. 18. Première patte thoracique: $\frac{55}{1}$

Fig. 19. Cinquième patte thoracique: 55 . 
Tian du Lab. de Wimereux. TomeVI.

1.1.1.1.\% I.

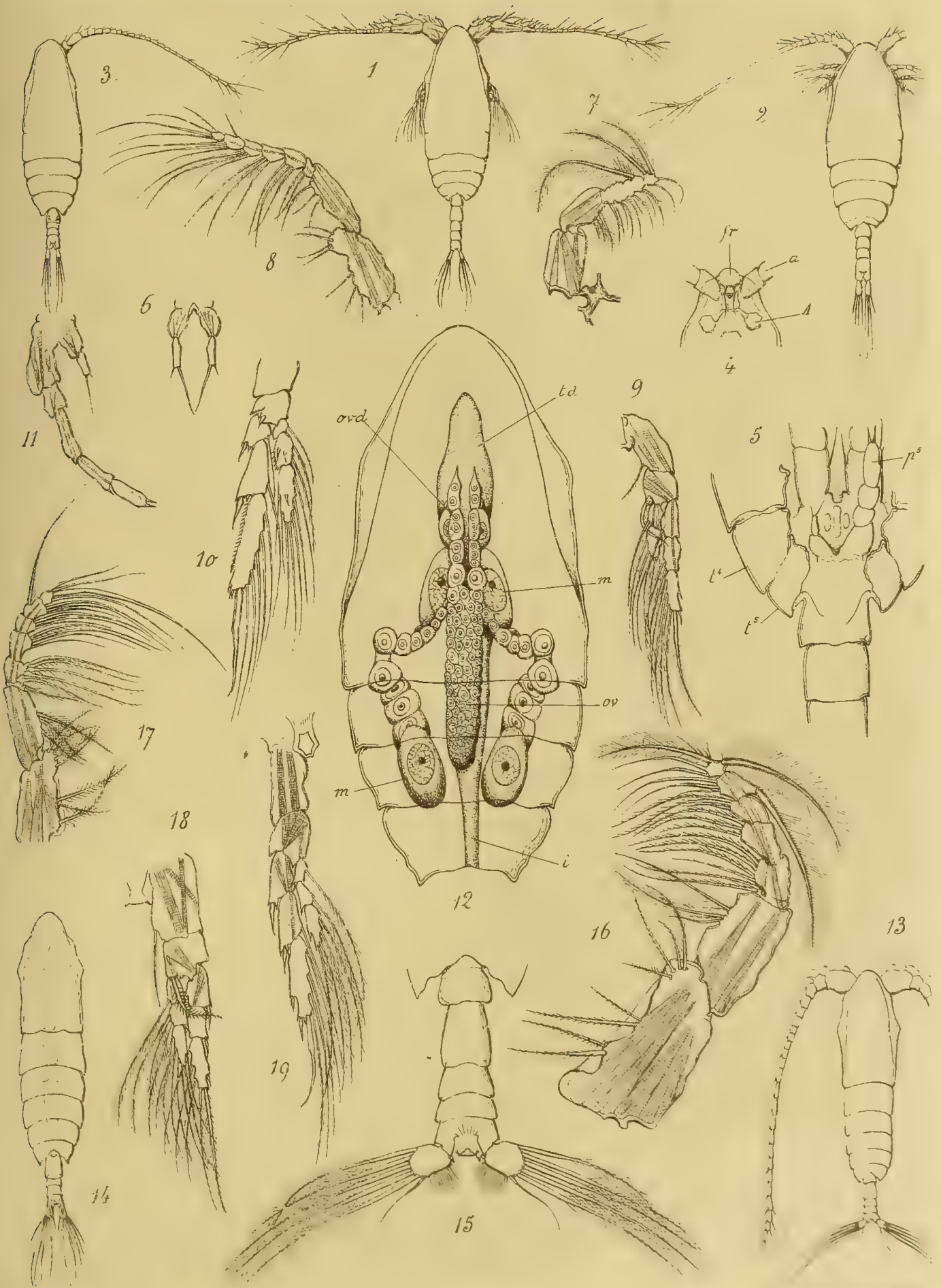





\section{PLANCHE II.}

Fig. I-I 3, Clausia elongata Bоеск.

Fig. I4-I5, Labidocera wollastoni LubBock.

Fig. I6-I7, Acartia discaudata Giesbrecht.

Fig. I8-ı, Acartia clausi Giesbrecht.

Fig. 2O-2I, Centropages bamatus Lilljeborg. 


\section{PLANCHE II.}

\section{Clausia elongata.}

Fig. I. Femelle adulte, vue de profil : $\frac{55}{1}$.

Fiğ. 2. Mâle adulte, vu de profil : $\frac{50}{1}$.

Firg. 3. Cinquième patte thoracique du mâle adulte: $\frac{130}{1}$.

Firs. 4. Femelle jeune, à l'avant-dernier stade: $\frac{50}{1}$.

Fig. 5. Mâle jeune, à l'avant-dernier stade $: \frac{50}{1}$.

Fig. 6. Cinquième patte thoracique de ce jeune mâle: $\frac{160}{1}$.

Fìrs. 7. Mâle jeune, au stade antépénultième : $\frac{50}{1}$.

Fìn. 8. Cinquième patte thoracique de ce jeune mâle: $\frac{160}{1}$.

Figr. 9. Mandibule de la femelle adulte $: \frac{160}{1}$.

Fìs. IO. Mandibule du mâle adulte: $\frac{350}{1}$.

Fin. I I. Première maxille de la femelle : $\frac{160}{1}$.

$\varepsilon p$, lobe épipodial du protopodite.

Fin. I2. Seconde maxille externe de la femelle: $\frac{160}{1}$.

Fing. 13. Seconde maxille externe du mâle: $\frac{350}{1}$.

\section{Labidocera wollastoni.}

Firg. 14. Céphalon du mâle, vue par la face dorsale: $\frac{55}{1}$.

Figr. 15. Céphalon de la femelle, vue par la face dorsale: $\frac{55}{1}$.

\section{Acartia discaudata.}

Firg. I6. Mâle, vu dorsalement, avec sa musculature: $\frac{80}{1}$.

Fìng. 17. Femelle, vue de même: $\frac{80}{1}$.

\section{Acartia clausi.}

Fìgr. 18. Mâle, vu dorsalement, avec sa musculature $: \frac{80}{1}$.

Fìn. 19. Femelle, de même: $\frac{55}{1}$.

\section{Centropages bamatus.}

Fig. 20. Deuxième patte thoracique du mâle, avec la musculature : $\frac{120}{1}$.

Fig. 2 I. Deuxième patte thoracique de la femelle, avec la musculature : $\frac{120}{I}$. $m$, muscle de la rame interne. 
Trandu Lab. de Himerenx. TomelT.

Plunche II.

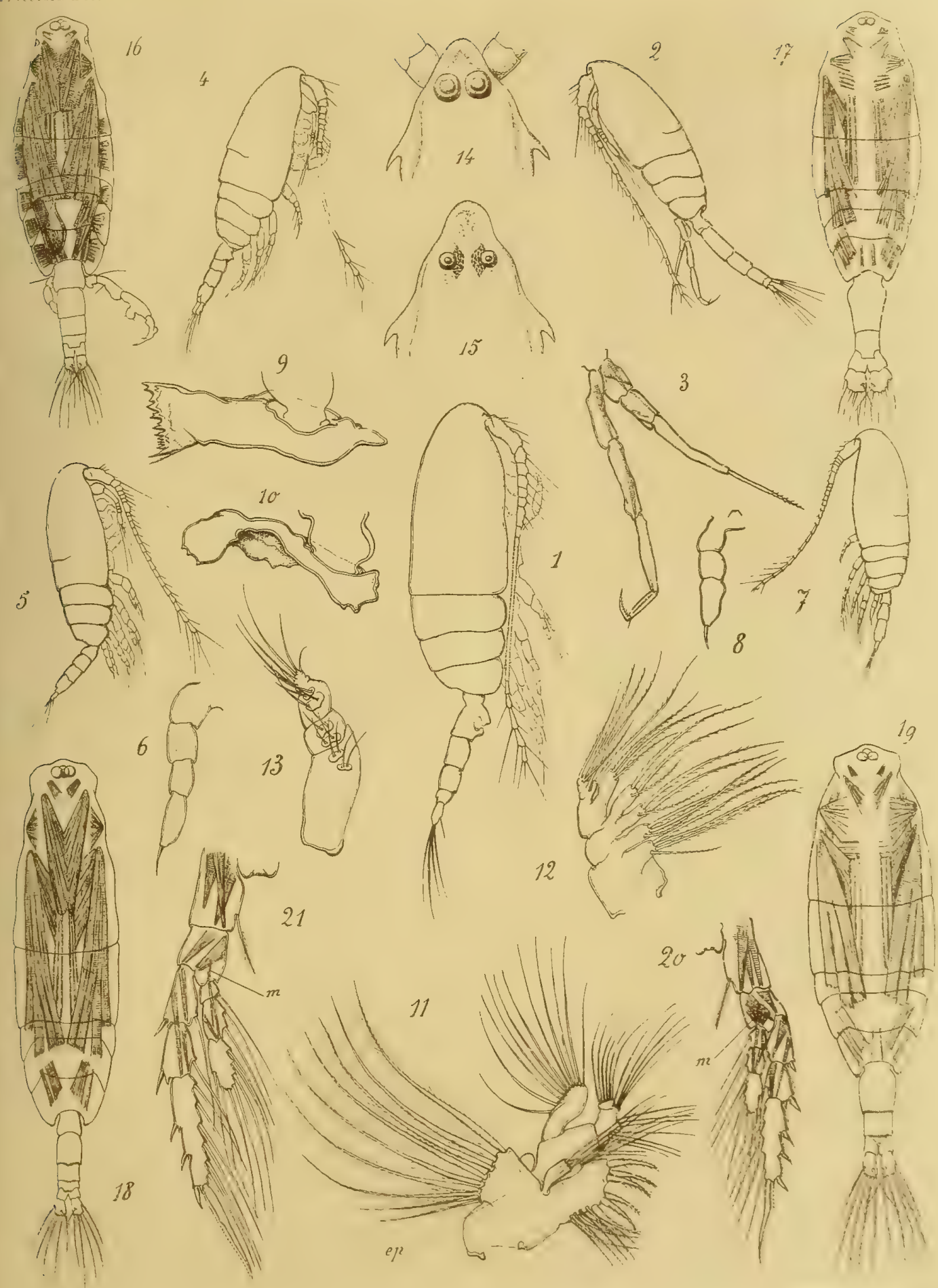





\section{PLANCHE III.}

Fig. I-IO, Cyclops aquoreus Fischer.

Fig. I I-I8, Cyclops lubbocki Brady. 


\section{PLANCHE III.}

\section{Cyclops aquoreus.}

Fig. 1. Femelle, vue par la face dorsale : $\frac{120}{1}$.

Fig. 2. Antenne de la femelle: $\frac{350}{1}$.

Fig. 3. Mandibule et première maxille de la femelle: $\frac{350}{1}$.

Fig. 4. Lobe masticateur de la mandibule: $\frac{670}{1}$.

Fig. 5. Seconde maxille de la femelle: $\frac{350}{1}$.

Fig. 6. Bord denté de la lèvre supérieure: $\frac{350}{1}$.

Fig. 7. Cinquième patte thoracique de la femelle: $\frac{340}{1}$.

Figr. 8. Antennule du mâle: $\frac{160}{1}$.

Fig. 9. Cinquième patte thoracique et premier segmentabdominal du mâle : $\frac{160}{I}$

Fig. IO. Nauplius vu par la face dorsale: $\frac{350}{1}$.

\section{Cyclops lubbocki.}

Fig. I 1. Antennule de la femelle: $\frac{160}{1}$.

Figg. I2. Antennule du mâle: $\frac{160}{\mathrm{I}}$.

Fig. 13. Première maxille de la femelle, vue par la face interne : $\frac{350}{1}$.

Fig. 14. Portion distale (palpe) de la première maxille: $\frac{350}{1}$.

Fig. 15. Seconde maxille de la femelle: $\frac{350}{1}$.

Fig. 16. Prolongement frontal et lèvre supérieure de la mème: $\frac{350}{1}$

Fig. 17. Abdomen de la femelle, vu par la face ventrale : $\frac{160}{I}$.

$n p^{5}$, nerf de la $5^{\circ}$ patte thoracique ; $n a$, cordons nerveux abdominaux; $g l$, glandes réfringentes.

Fig. 18. Extrémité terminale d'une pièce furcale, avec ses glandes réfringentes : $\frac{350}{1}$ ₹, cellules effilées en relation avec les glandes. 
Trm: du Lab.de Wimereux,Tome VT.

Plunche III.

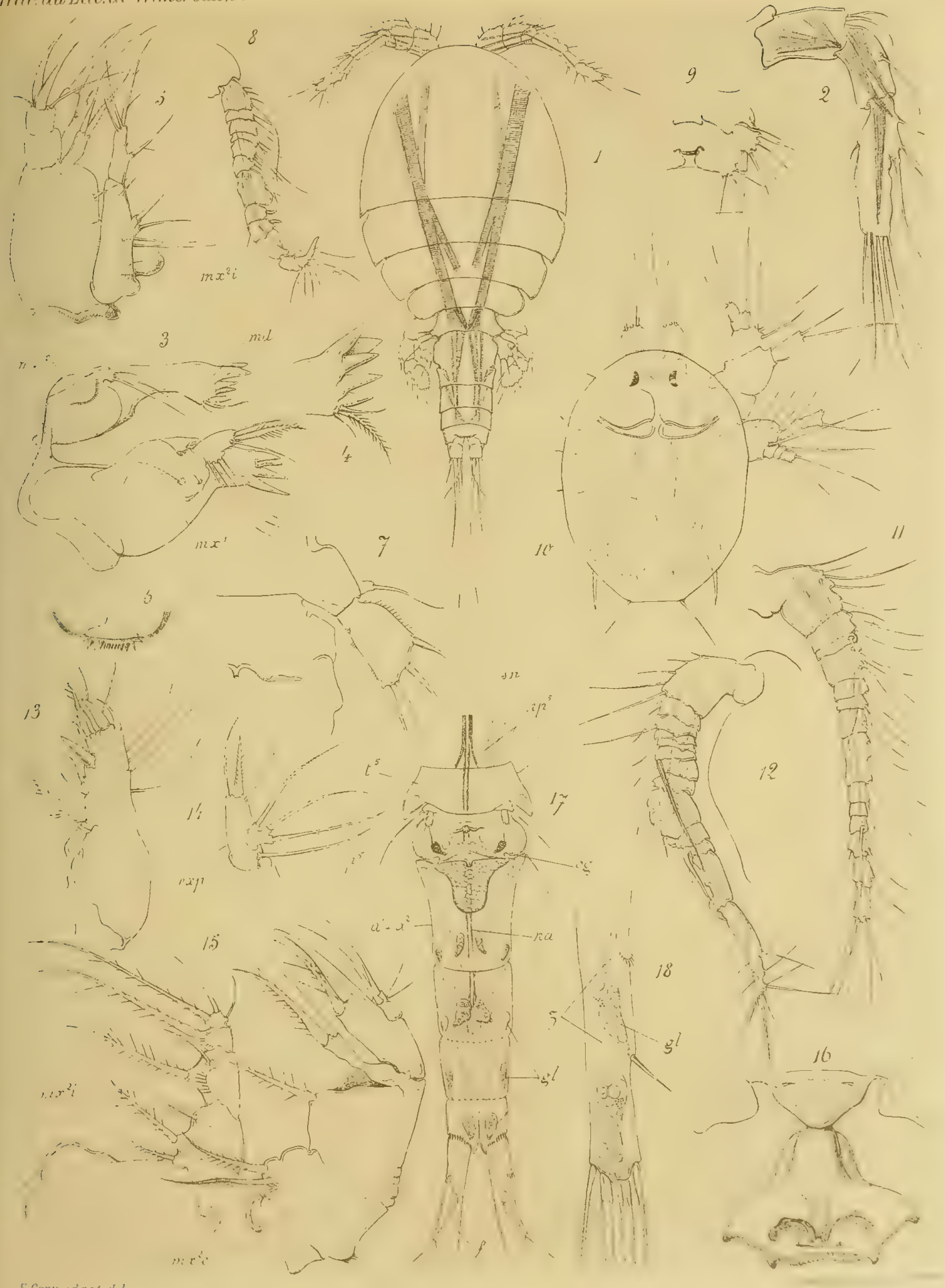





\section{PLANCHE IV.}

Fig. I-5, Cyclops lubbocki Brady.

Fig. 6-2I, Namnopus, palustris BRADY. 


\section{PLANCHE IV.}

\section{Cyclops lubbocki.}

Fig. I. Femelle portant ses œufs, vue dorsalement : $\frac{50}{1}$.

Fig. 2. Mâle, vu par la face dorsale : $\frac{50}{1}$.

Fig. 3. Antenne et rostre de la femelle, vus de profil : $\frac{160}{1}$

Fig. 4. Mandibule de la femelle: $\frac{350}{\mathrm{t}}$. Les soies du palpe ne sont pas représentées entièrement.

Fig. 5. Cinquième patte thoracique et premier segment abdominaladu mále : $\frac{350}{1}$

\section{Nannopus palustris.}

Fig. 6. Femelle vue par la face dorsale: $\frac{55}{1}$.

Fig. 7. Femelle vue de profil : $\frac{55}{1}$.

Fig. 8. Antennule de la femelle: $\frac{350}{8}$

Fig. 9. Antenne de la femelle : $\frac{350}{1}$.

Fig. IO. Mandibule : $\frac{350}{1}$.

Fig. II. Première maxille: $\frac{350}{1}$.

Fig. 12. Portion terminale de la première maxille: $\frac{350}{5}$.

Fig. 13. Seconde maxille interne: $\frac{350}{1}$.

Fig. 14. Seconde maxille externe: $\frac{350}{1}$.

Fig. 15. Première patte thoracique: $\frac{350}{1}$.

Fig. 16. Deuxième patte thoracique: $\frac{350}{8}$.

Fig. 17. Troisième patte thoracique: $\frac{350}{1}$.

Fig. 18. Quatrième patte thoracique: $\frac{350}{1}$.

Fig. 19. Cinquième patte thoracique : $\frac{350}{1}$.

Fig. 20. Abdomen de la femeile, vu ventralement: $\frac{160}{I}$.

Fig. 21. Orifices génitaux de la femelle: $\frac{550}{1}$. 

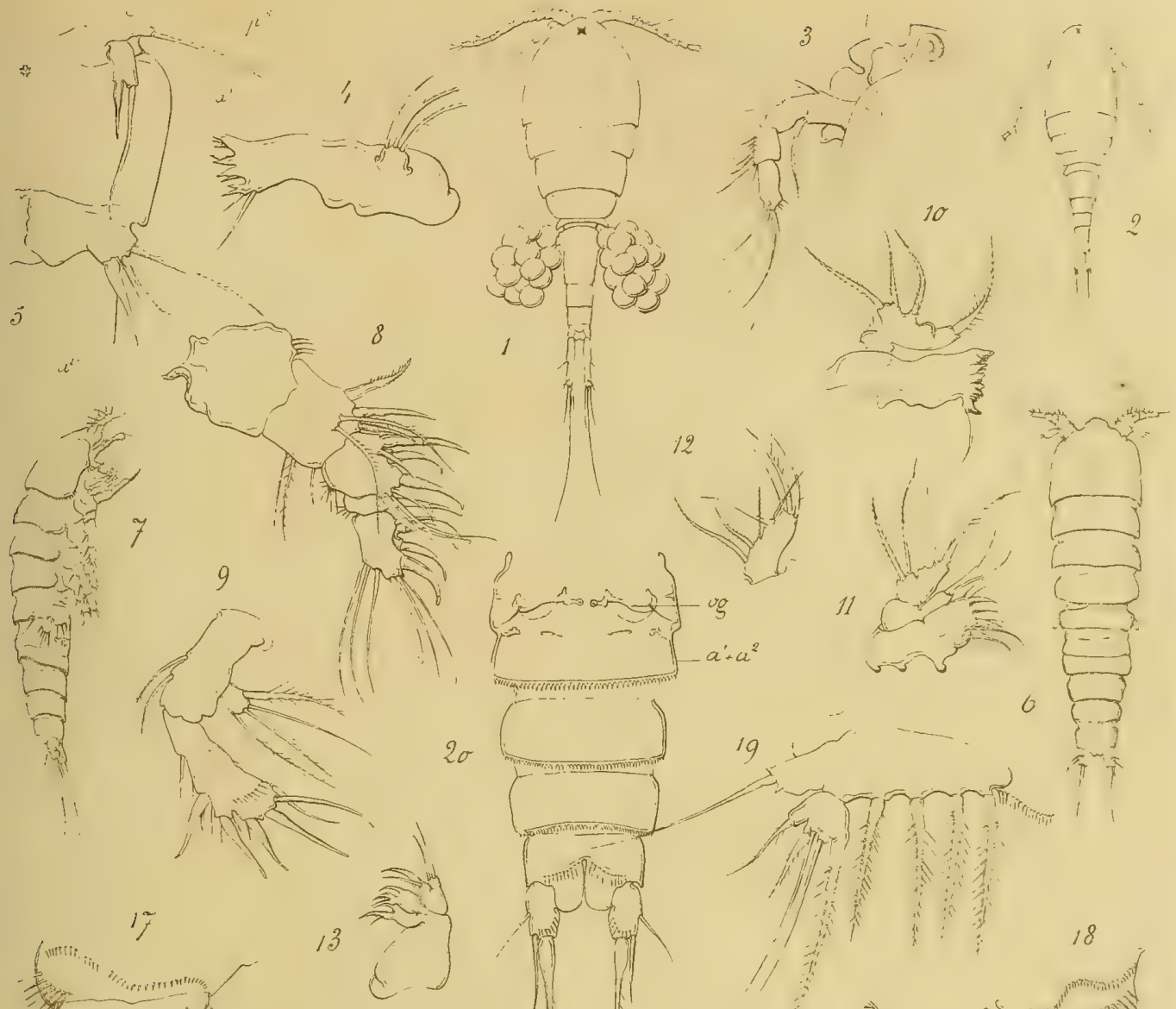

-

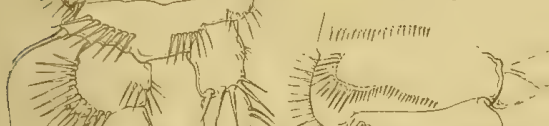

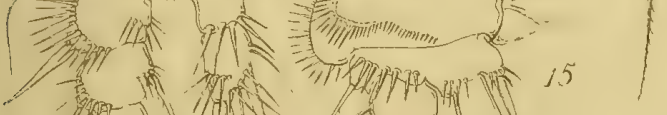
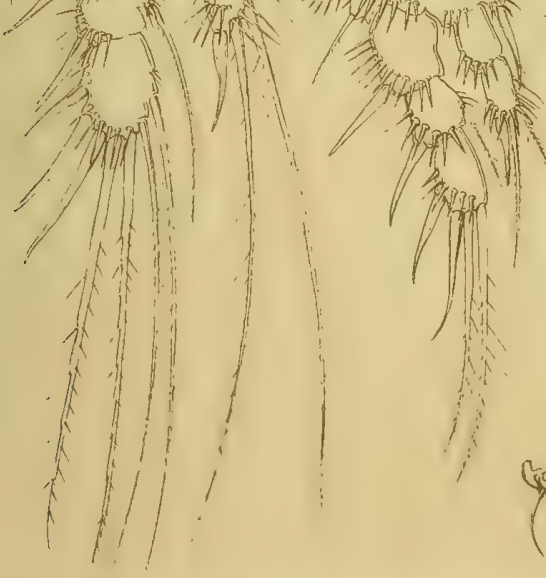

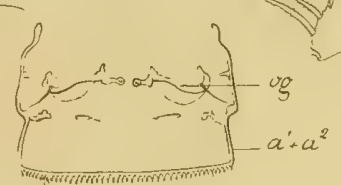
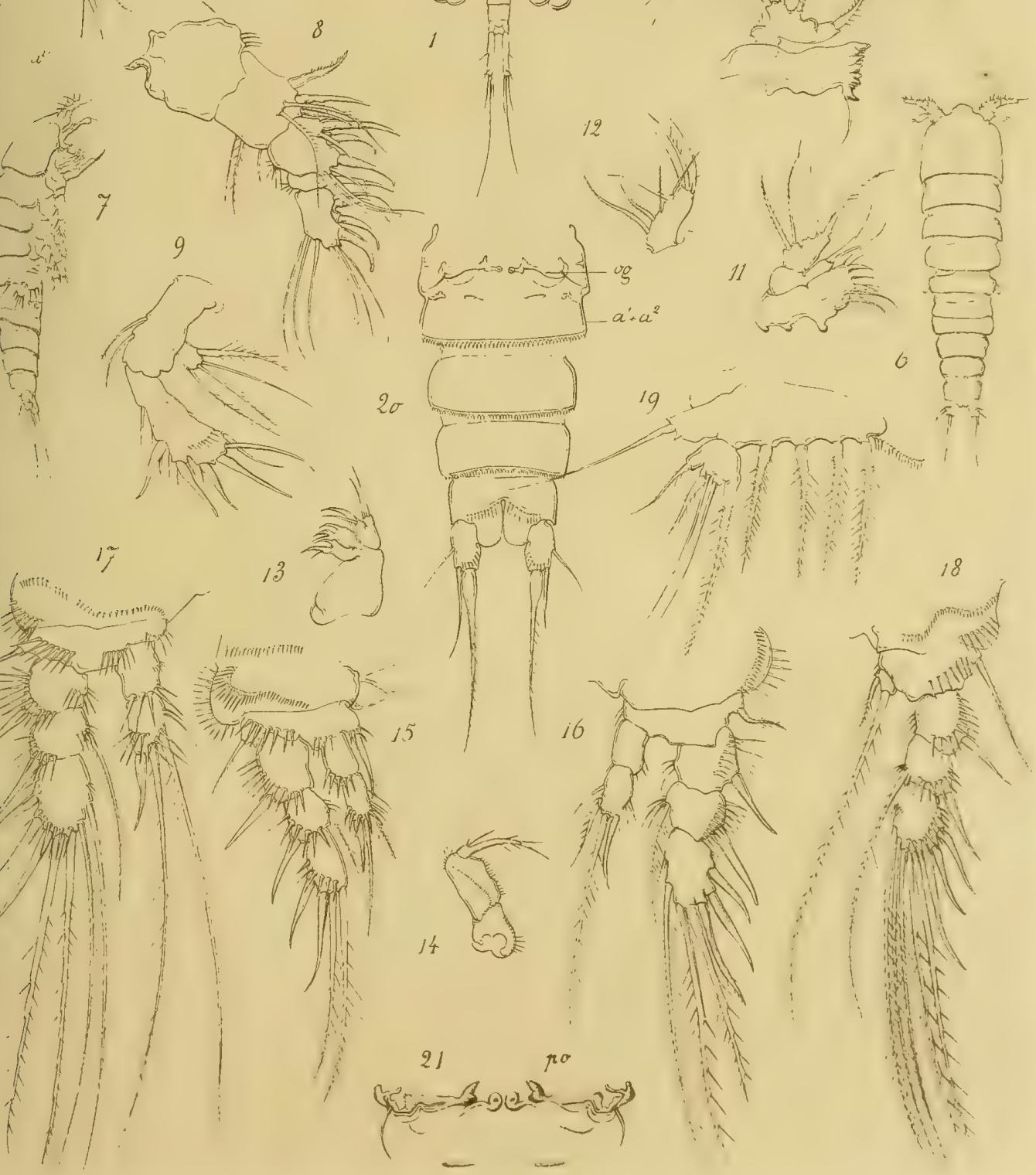

E Cares ad nat del 



\section{PLANCHE V.}

Fig. I-24, Laopbonte similis Claus.

Fig. 25-3 I, Doropygus gibber Thorel.L. 


\section{PLANCHE V.}

\section{Laophonte similis.}

Fig. 1. Mâle vu de profil: $\frac{55}{1}$.

Fig. 2. Femelle vue de profil : $-\frac{55}{1}$.

Fig. 3. Tête de la femelle, vue par la face dorsale. $\frac{\mathbf{2} 20}{\mathrm{I}}$.

Fig. 4. Antennule du mâle : $\frac{350}{1}$.

Fig. 5. La même, vue du côté opposé : $\frac{350}{1}$.

Fig. 6. Antennule et prolongement frontal de la femelle, vus par la face dorsale: $\frac{360}{1}$.

Fig. 7. Antenne de la femelle: $\frac{350}{1}$.

Fig. 8. Face ventrale de la région buccale montrant les cadres d'insertion des appendices: ${ }^{350}$.

Fig. 9. Mandibule de la femelle: $\frac{350}{1}$.

Fig. 10. Lame masticatrice de la mandibule: $\frac{350}{1}$.

Figr. I I. Première maxille de la femelle: $\frac{350}{1}$.

Fig. 12. La mème: $\frac{350}{1}$.

Fig. 13. Seconde maxille externe: $\frac{350}{1}$.

Fig. I4. Secondes maxilles internes: $\frac{350}{1}$.

Fig. 15. Première patte thoracique de la femelle : $\frac{350}{1}$.

Fig. I6. Deuxième patte thoracique de la femelle: $\frac{350}{1}$.

Fig. 17. Troisième patte thoracique de la femelle : $\frac{350}{1}$.

Fig. 18. Quatrième patte thoracique de la femelle : $\frac{350}{1}$.

Fig. 19. Endopodite de la deuxième patte thoracique du mâle: $\frac{356}{1}$.

Fig. 20. Endopodite de la troisième patte thoracique du mâle: $\frac{350}{I}$.

Fig. 2 1. Cinquième somite thoracique et premier somite abdominal du mâle : $\frac{350}{1}$

Fig. 22. Cinquième patte thoracique de la femelle: $\frac{350}{1}$.

Fig. 23. Ouvertures génitales de la femelle: $\frac{350}{1}$.

Fig. 24. Furca de la femelle: $\frac{350}{1}$.

\section{Doropygus gibber.}

Fig. 25. Antennule de la femelle : $\frac{120}{1}$.

Fig. 26. Antennule du mâle : $\frac{160}{1}$.

Fig. 27. Antenne de la femelle: $\frac{120}{1}$.

Fig. 28. Mandibule du mâle: $\frac{160}{1}$.

Fig. 29. Deuxième patte thoracique du mâle : $\frac{160}{1}$.

Fig. 30. Quatrième patte thoracique du mâle: $\frac{160}{1}$.

Fig. 3I. Cinquième patte thoracique et pore de fécondation de la femelle: $\frac{55}{1}$. 


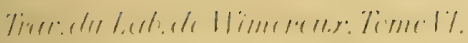

I1/udeler.
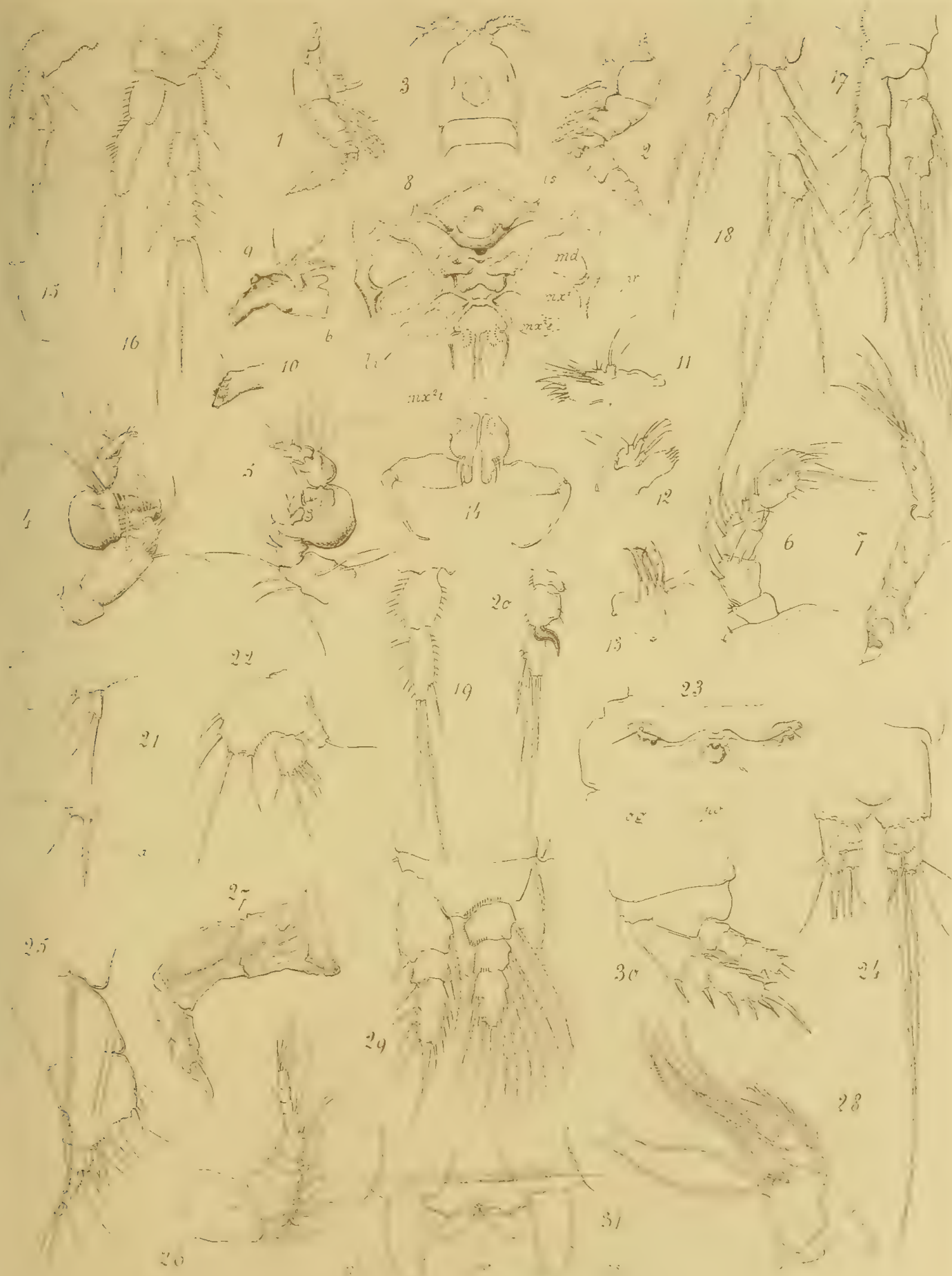

PLANCHE VI.

Fig. I-8, Doropygus gibber Thorell. 


\section{PLANCHE VI.}

\section{Doropygus gibber.}

Fig I. Femelles, de grandeur naturelle.

Fig. 2. Male, vu de profil: $\frac{55}{1}$.

Fig. 3. Femelle jeune, dans l'avant-dernier stade, vue de profil : $\frac{50}{1}$.

Fig. 4. Femelle eune, dans le dernier stade, vue de profil : $\frac{50}{1}$.

Fig. 5. Mandibule de la femelle : $\frac{120}{1}$.

Fig. 6. Première maxille de la femelle: $\frac{120}{1}$.

Fig. 7. Seconde maxille externe de la femelle : $\frac{160}{1}$. $\iota p$, soie en forme d'épine; $\mathrm{S}$, grosse soie barbelée.

Fig. 8. Seconde maxille interne de la femelle: $\frac{120}{1}$. 


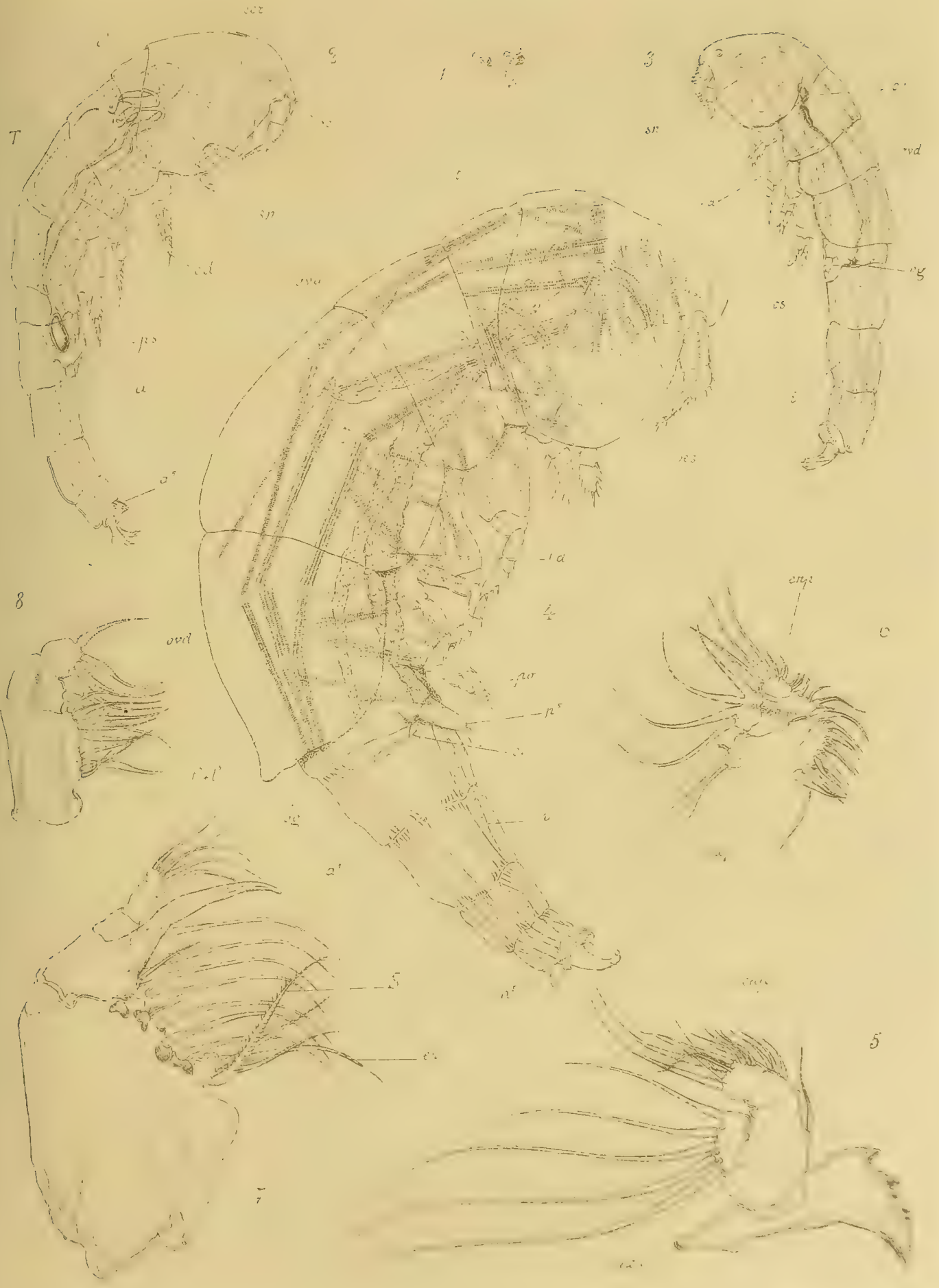





\section{PLANCHE VII.}

Fig. I-Io, Doropygus gibber Thorell.

Métamorphose. 


\section{PLANCHE VII.}

\section{Doropygus gibber.}

Fig. I. Nauplius à l'éclosion, vu dorsalement : $\frac{160}{\mathrm{I}}$.

Fìg. 2. Extrémité postérieure d'un nauplius plus âgé, vu dorsalement: $\frac{350}{1}$.

Fìg. 3. Métanauplius, vu ventrálement et montrant les divers appendices de l'embryon cyclopoïde : $\frac{160}{1}$.

Fig. 4. Métanauplius vu dorsalement et montrant les divers somites post-céphaliques $(1-5)$ de l'embryon cyclopoïde : $\frac{160}{1}$.

Fig. 5. Embryon au premier stade cyclopoïde, vu ventralement : $\frac{160}{1}$.

Fig. 6. Embryon au deuxième stade cyclopoïde, vu ventralement : $\frac{160}{1}$.

Fig. 7. Embryon plus âgé, vu dorsalement : $\frac{160}{1}$. $r g$, rudiment de la glande génitale.

Fig. 8. Embryon du même âge, vu de profil, présentant déjà l'apparence d'un parasite : $\frac{120}{1}$.

Fig. 9. Le céphalon et le premier somite thoracique du même embryon, vus de profil: $\frac{350}{1}$.

rg, rudiment de la glande génitale; $\mathrm{cm}$, grandes cellules du tissu conjonctif.

Fig. Io. Rudiment de la quatrième patte natatoire: $\frac{350}{1}$. 


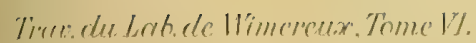

Planche VII.
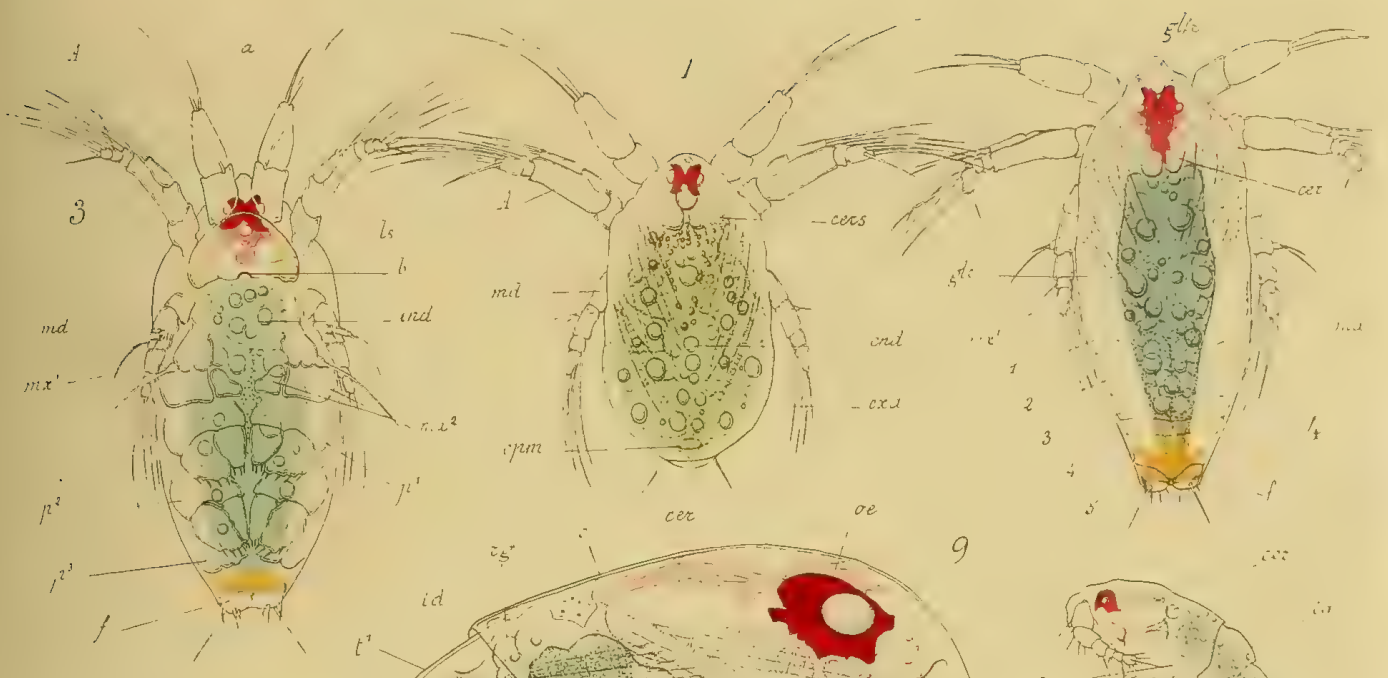

9

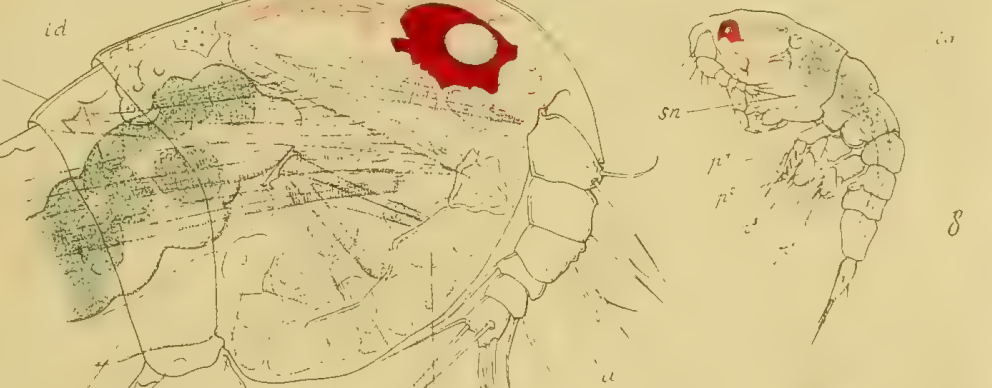

exd

m

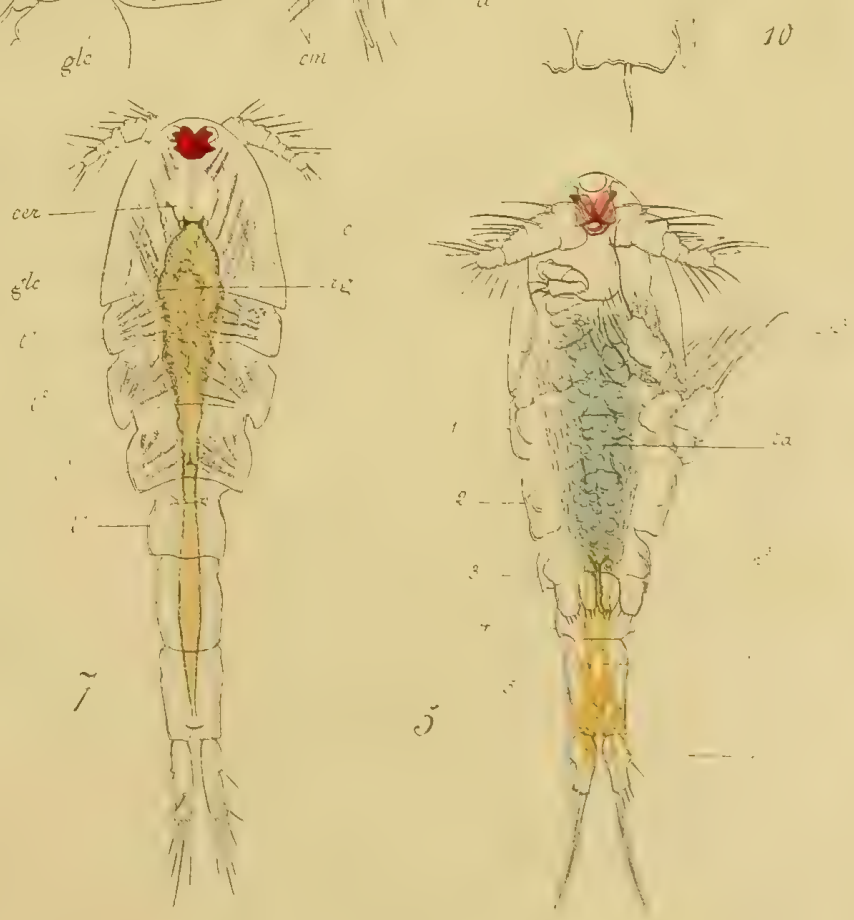





\section{PLANCHE VIII.}

Fig. I-II, Doropygus psyllus Thorell.

Fig. I2-22, Doropygus pulex Thorell. 


\section{PLANCHE VIII.}

\section{Doropygus psyllus o.}

Fig. I. L'animal vu de profil : $\frac{25}{1}$.

Fig. 2. Le mème, de grandeur naturelle.

Fig. 3. Antennule : $\frac{120}{1}$.

Fig. 4. Antenne: $\frac{120}{\mathrm{I}}$.

Fig. 5. Mandibule: $\frac{160}{1}$.

Fig. 6. Première maxille: $\frac{160}{1}$

Fig. 7. Seconde maxille externe: $\frac{160}{1}$.

Fig. 8. Seconde maxille interne: $\frac{160}{i}$.

Fig. 9. Première patte thoracique, sans l'endopodite : $\frac{160}{1}$

Fig. IO. Deuxième patte thoracique : $\frac{120}{1}$.

Fig. I I. Cinquième patte thoracique : $\frac{160}{1}$.

\section{Doropygus pulex.}

Fig. 12. Femelle, vue de profil: $\frac{160}{1}$.

Fig. I 3. Mâle, vu de profil : $\frac{55}{1}$. $c m$, grandes cellules du tissu conjonctif (corps graisseux).

Fig. 14. Antennule de la femelle : $\frac{120}{1}$.

Fig. I5. Antennule du mâle: $\frac{120}{1}$.

Fig. 16. Antenne du mâle: $\frac{120}{1}$.

Fig. I7. Coxopodite (lame masticatrice) de la mandibule: $\frac{160}{1}$.

Fig. 18. Basipodite, endopodite et exopodite de la mandibule: $\frac{160}{1}$.

Fig. I9. Première maxille de la femelle, vue par la face externe : $\frac{160}{1}$.

Fig. 20. Le mème appendice, vu par la face interne: $\frac{160}{\mathrm{t}}$.

Fig. 2 I. Patte thoracique de la deuxième paire du mâle : $\frac{120}{\mathrm{I}}$.

Fig. 22. Patte thoracique de la cinquième paire de la femelle: $\frac{55}{1}$. 


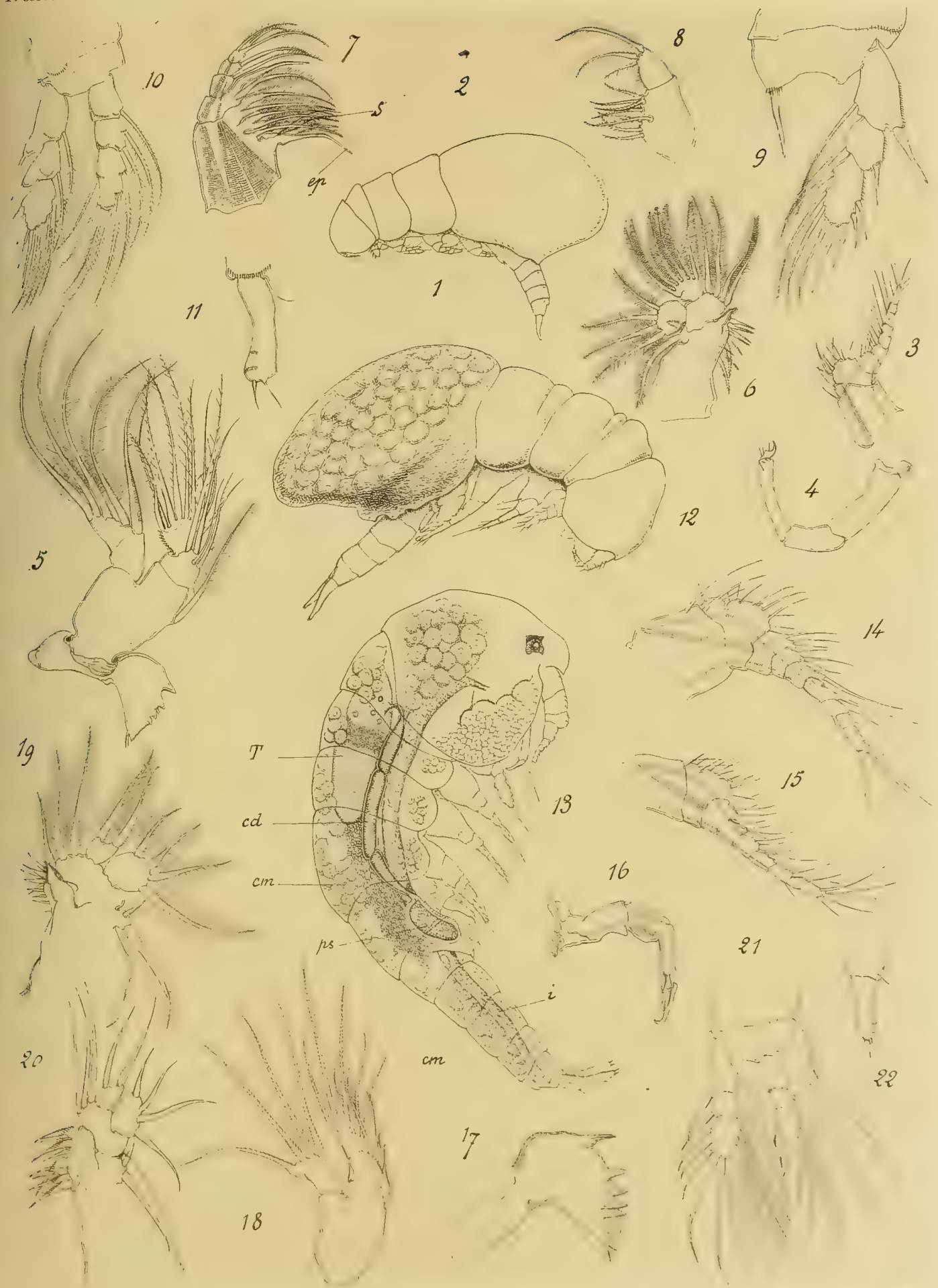





\section{PLANCHE IX.}

Fig. I-3, Doropygus pulex Thorell.

Fig. 4-I3, Bonnierilla longipes Kerschner. 


\section{PLANCHE IX.}

\section{Doropygus pulex.}

Fig. I. Seconde maxille externe de la femelle, vue par la face antérieure: $\frac{160}{I}$. $e p$, soie en forme d'épine; $S$, grosse soie hérissée de barbules.

Fig. 2. Seconde maxille interne de la femelle, vue par la face postérieure : $\frac{160}{1}$.

Fig. 3. Première patte thoracique du mâle: $\frac{160}{1}$.

\section{Bomierilla longipes ?.}

Fig. 4. Antennule et prolongement frontal: $\frac{160}{1}$.

Fig. 5. Antenne: $\frac{120}{1}$.

Fig. 6. Mandibule $: \frac{120}{1}$.

Fig. 7. Première maxille, vue par la face interne : $\frac{160}{1}$.

Fig. 8. Seconde maxille externe: $\frac{160}{1}$.

$e p$, soie en forme d'épine; $S$, grosse soie hérissée de barbules.

Fig. 9. Seconde maxille interne: $\frac{160}{1}$.

Fig. 10. Première patte thoracique : $\frac{120}{1}$.

Fig. I I. Troisième patte thoracique: $\frac{120}{2}$.

Fig. 12. Endopodite d'une quatrième patte thoracique : $\frac{55}{1}$.

Fig. I3. Cinquième patte thoracique: $\frac{120}{1}$. 
Trav.duLab. de Wimereux,TomeV.

Planche IX.

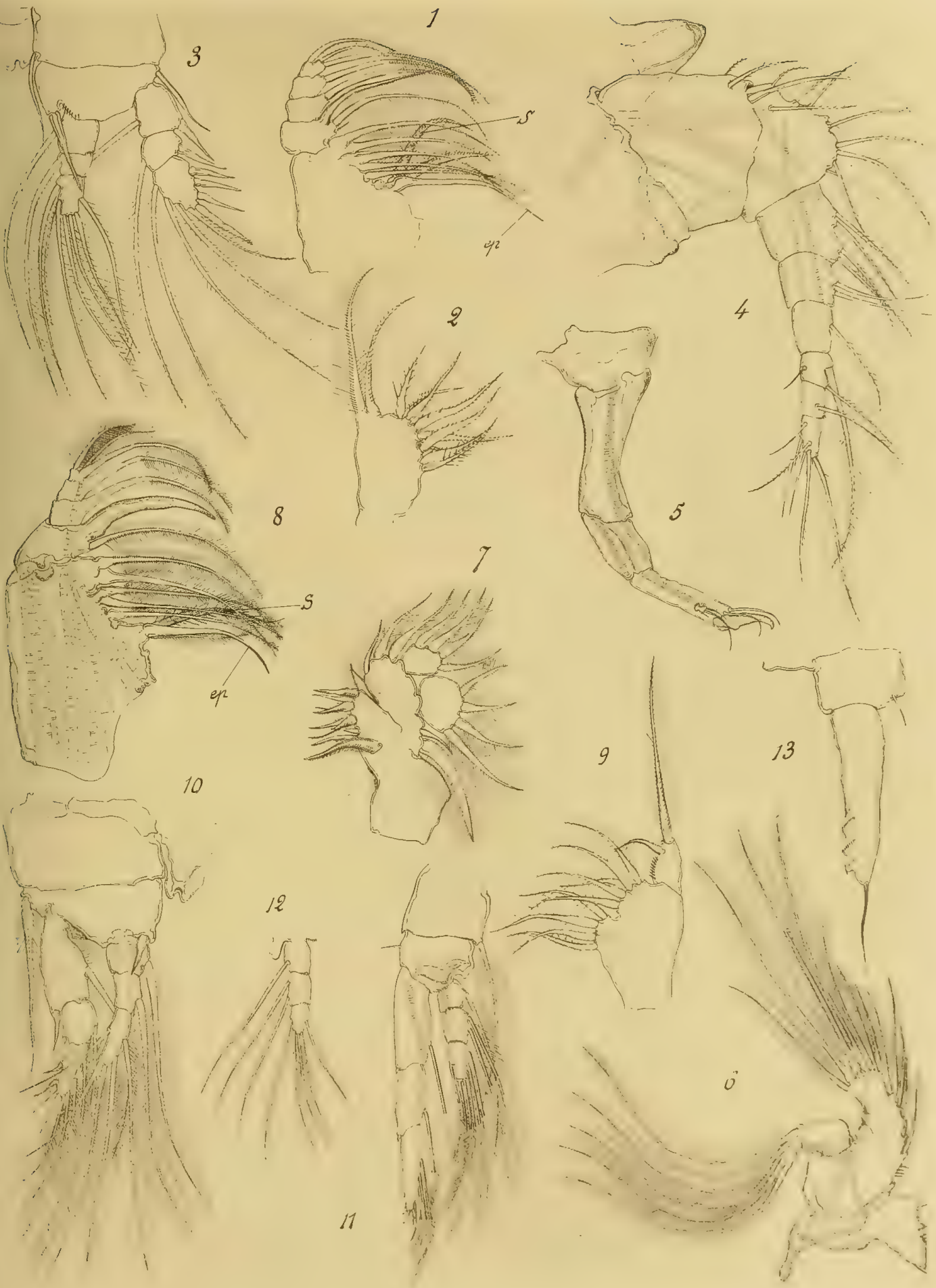





\section{PLANCHE X.}

Fig. I-8, Bonnierilla longipes Kerschner.

Métamorphose. 


\section{PLANCHE X.}

\section{Bomnierilla longipes.}

Fig. I. Femelle adulte portant des embryons sur le point d'éclore: $\frac{50}{1}$. $m$, cavité incubatrice remplie d'embryons.

Figr. I $a$. La mème, de grandeur naturelle, vue de profil.

Fig. I $b$. La même, de grandeur naturelle, vue dorsalement.

Fig. 2. Nauplius à l'éclosion, vu ventralement et montrant les appendices de l'embryon cyclopoïde. $\frac{160}{1}$.

Fig. 3. Métanauplius, vu par la face ventrale : $\frac{160}{1}$.

Fig. 4. Métanauplius, vu par la face dorsale et montrant l'indication du premier somite thoracique: $\frac{160}{1}$.

Fig. 5. Métanauplius, vu par la face dorsale, peu de temps avant la mue délivrant l'embryon cyclopoïde: $\frac{160}{\mathrm{I}}$.

$1,2,3,4,5$, les diverses somites de l'embryon cyclopoïde.

Fig. 6. Embryon au premier stade cyclopoïde, vu par la face ventrale $: \frac{160}{1}$.

Fig. 7. Le même embryon, vu par la face dorsale : $\frac{160}{1}$.

Fig. 8. Embryon au deuxième stade cyclopoïde, vu par la face dorsale : $\frac{160}{1}$. 
Tran del Lah de Himereux, Tome WT.

Planche X.
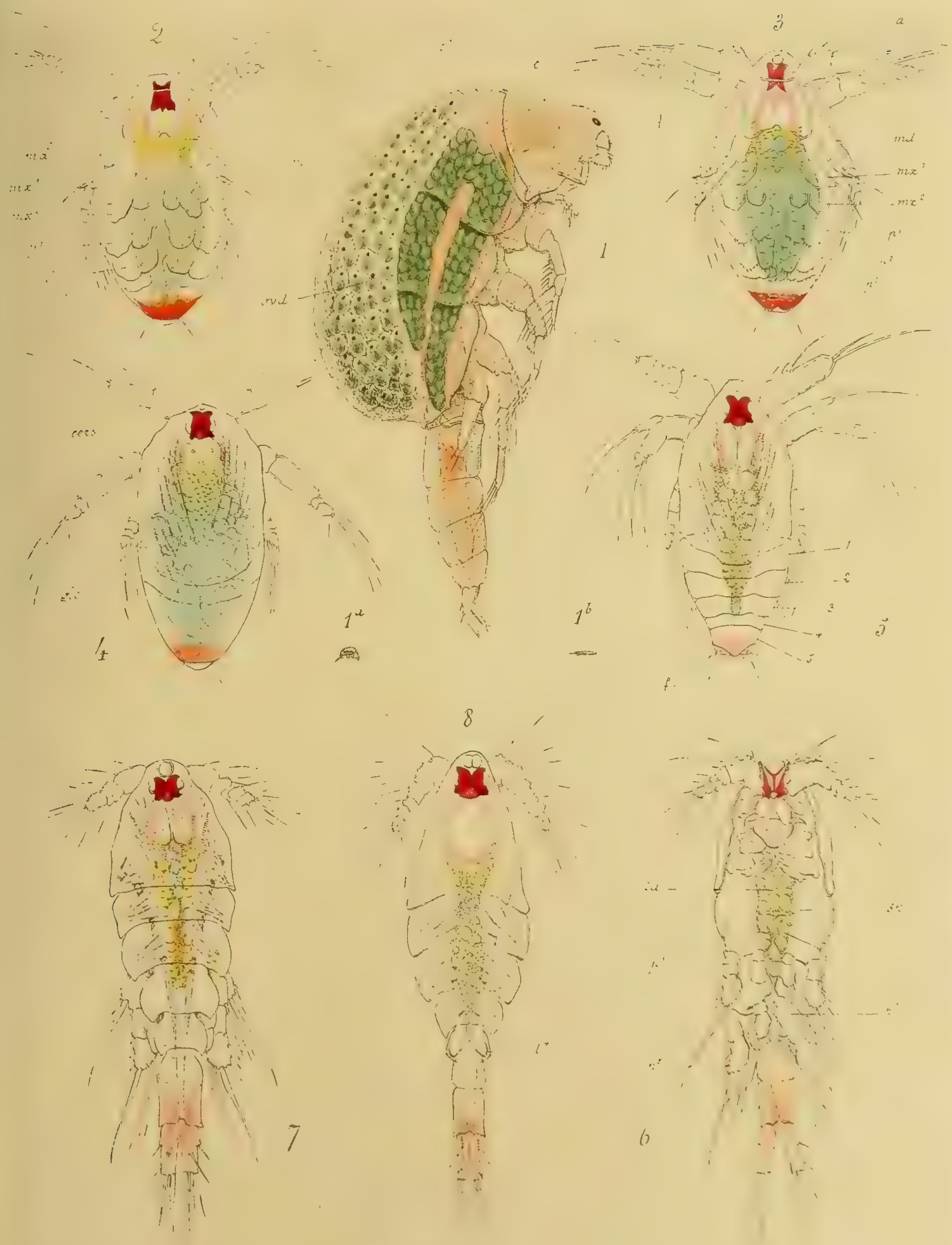



\section{PLANCHE XI.}

Fig. I-I2, Gunenotophorus globularis Costa.

Fig. I3 et I4, Doroixys uncinata Kerschner. 


\section{PLANCHE XI.}

\section{Gunenotophorus globularis ․}

Fig. I. Antennule et prolongement trontal, vus par la face dorsale: $\frac{160}{1}$.

Fig. 2. Antenne: $\frac{160}{1}$.

Fin. 3. Coxopodite de la mandibule: $\frac{350}{1}$.

Figr. 4. Basipodite, endopodite et exopodite de la mandibule: $\frac{160}{1}$.

Fï. 5. Première maxille vue par la face externe: $\frac{350}{1}$.

Fig. 6. Seconde maxille externe, vue par la face postérieure : $\frac{160}{1}$.

$e p$, soie en forme d'épine; $g$, grosse soie hérissée de barbules.

Fig. 7. Seconde maxille interne: $\frac{350}{2}$.

Fig. 8. Patte thoracique de la première paire: $\frac{130}{1}$.

Fig. 9. Patte de la troisième paire: $\frac{55}{1}$.

Fig. 10. Patte de la quatrième paire, endopodite: $\frac{160}{1}$

Fig. II. Patte de la cinquième paire: $\frac{350}{1}$.

Fig. I2. Abdomen vu par la face dorsale : $\frac{25}{1}$.

\section{Doroixys uncinata.}

Fig. I 3. Ovaire et portion terminale de l'oviducte, avec des œufs en voie de formation : $\frac{160}{1}$.

cov, plusieurs files d'ovules, issues de l'ovaire.

Fig. I4. Partie posterieure de l'ovaire et portion proximale de l'oviducte avec des œufs en voie de formation.

$\operatorname{cov}$, files linéaires d'ovules, issues de l'ovaire; $m$, ovules en voie de maturation. 

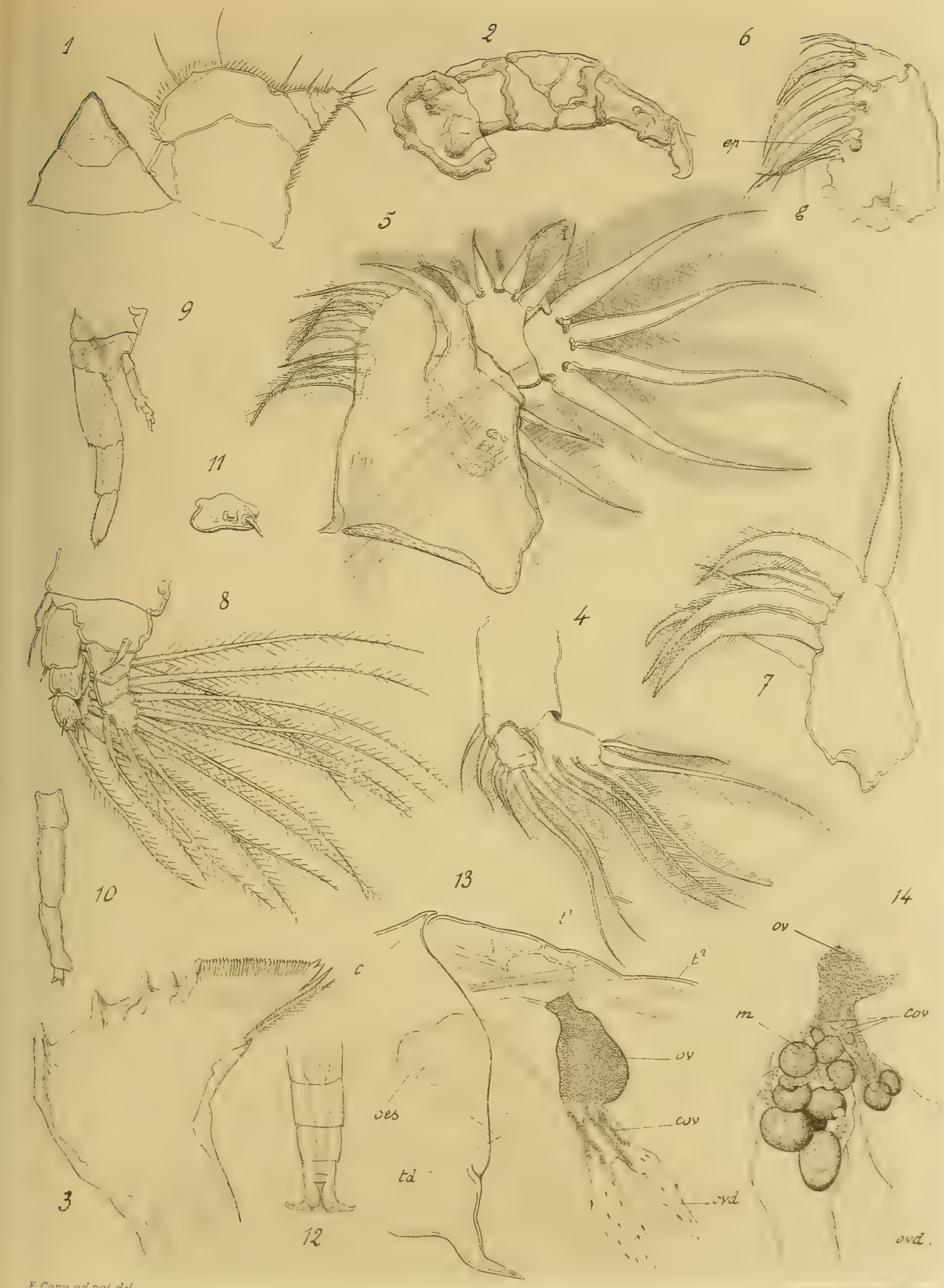

5

$+\infty$ (s) 



\section{PLANCHE XII.}

Fig. I-8, Notodelphys agilis THorell.

Métamorphose. 


\section{PLANCHE XII.}

\section{Notodelphys agilis.}

Fig. 1. Nauplius à l'éclosion, vu dorsalement: $\frac{160}{1}$.

Fig. 2. Extrémité antérieure du même, montrant la structure de l'œil nauplien : $\frac{350}{1}$.

Fig. 3. Métanauplius, vu par la face ventrale: $\frac{160}{1}$.

Fig. 4. Métanauplius plus âgé, vu par la face ventrale et montrant les appendices de l'embryon cyclopoïde : $\frac{160}{1}$.

Fig. 5. Le mème ménatauplius, vu ventralement et montrant les somites $(1-5)$ de l'embryon cyclopoïde: $\frac{160}{1}$.

Fig. 6. Métanauplius, vu par la face ventrale et montrant les appendices de l'embryon cyclopoïde dans un état de développement plus avancé : $\frac{160}{1}$.

Fig. 7. Embryon au premier stade cyclopoïde, vu par la face dorsale $\frac{160}{1}$.

Fig. 8. Embryon au deuxième stade cyclopoïde, vu par la face dorsale: $\frac{160}{\mathrm{I}}$. 
Tran: du Lab. de Wimereux. TomeVIT.

Planche XII.

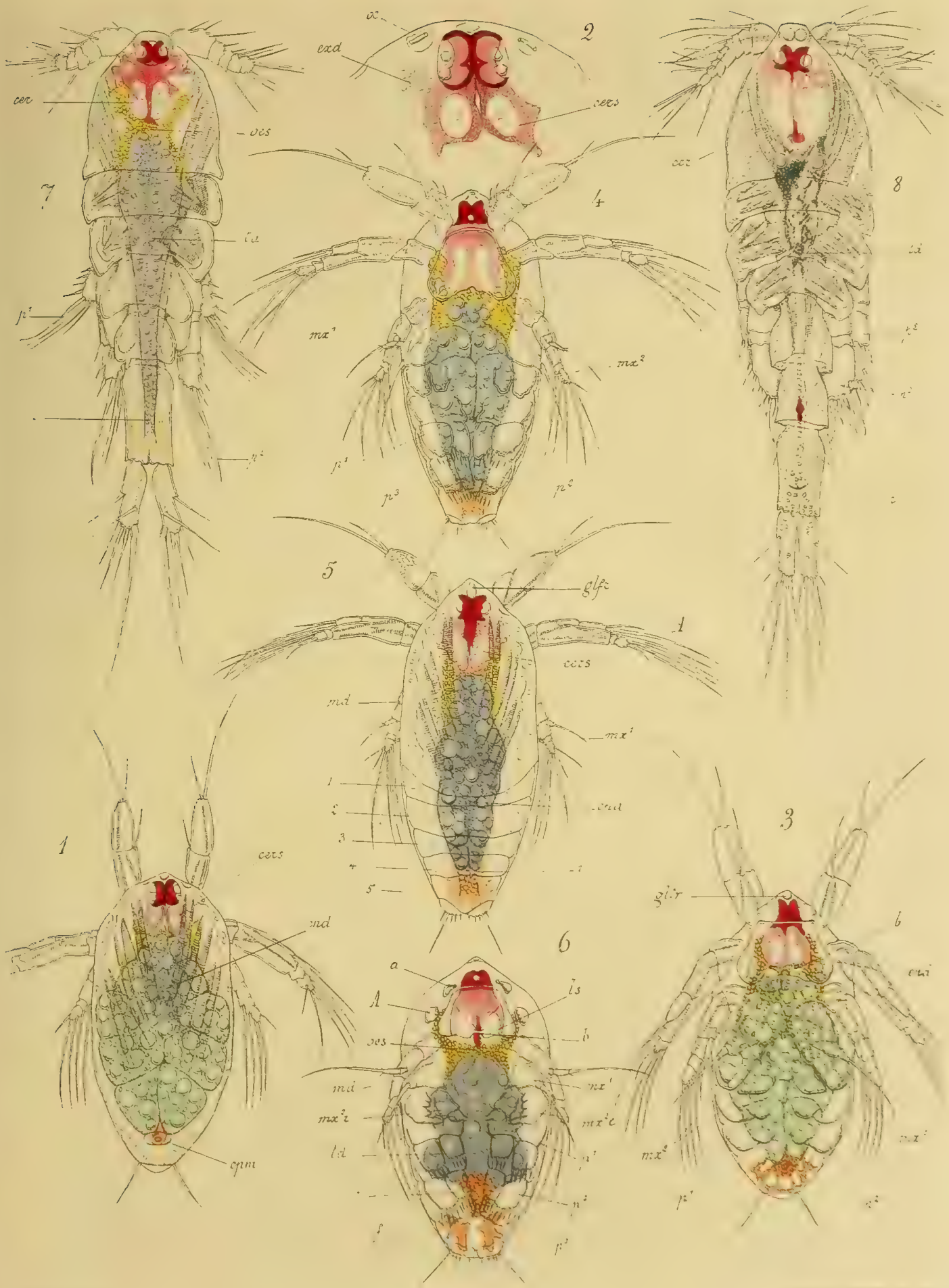





\section{PLANCHE XIII.}

Fig. I-6', Notodelphys allmani THorell.

Métamorphose. 


\section{PLANCHE XIII.}

\section{Notodelphys allman.}

Fig. I. Nauplius à l'éclosion, vu par la face dorsale : $\frac{160}{1}$.

$d$, amas pigmentaire du $3^{\text {e }}$ somite $; e$, tache pigmentaire des somites postérieurs $; g$, groupe de sphérules pigmentaires de l'extrémité postérieure.

Fig. 2. Nauplius plus àgé, vu par la face ventrale et montrant les premières indications des appendices postérieurs: $\frac{160}{\mathrm{I}}$.

Fig. 3. Métanauplius, vu par la face ventrale et montrant les appendices de l'embryon cyclopoïde : $\frac{160}{3}$.

Mêmes lettres que dans la fig. 1 .

Fig. 4. Métanauplius, vu par la face dorsale: $\frac{160}{1}$.

Mêmes lettres que dans la fig. $1 ; e^{\prime}, e^{\prime \prime}, e^{\prime \prime \prime}$, taches pigmentaires des somites postérieurs qui s'échelonnent dorsalement à mesure que les somites se constituent.

Fig. 5. Embryon au premier stade cyclopoïde, vu dorsalement : $\frac{i 60}{1}$.

Fig. 6. Antenne du même embryon: $\frac{350}{\mathrm{I}}$.

Les taches pigmentaires $e^{\prime \prime}, e^{\prime \prime}, e^{\prime \prime \prime}$, ont accompli leur migration et se trouvent disposées régulièrement dans les somites postérieurs.

Fig. 6'. Embryon au deuxième stade cyclopoïde, vu par la face ventrale : $\frac{160}{1}$. 
Trov du Lab. de Wimerenx. TomeVI.

Planche XIII.

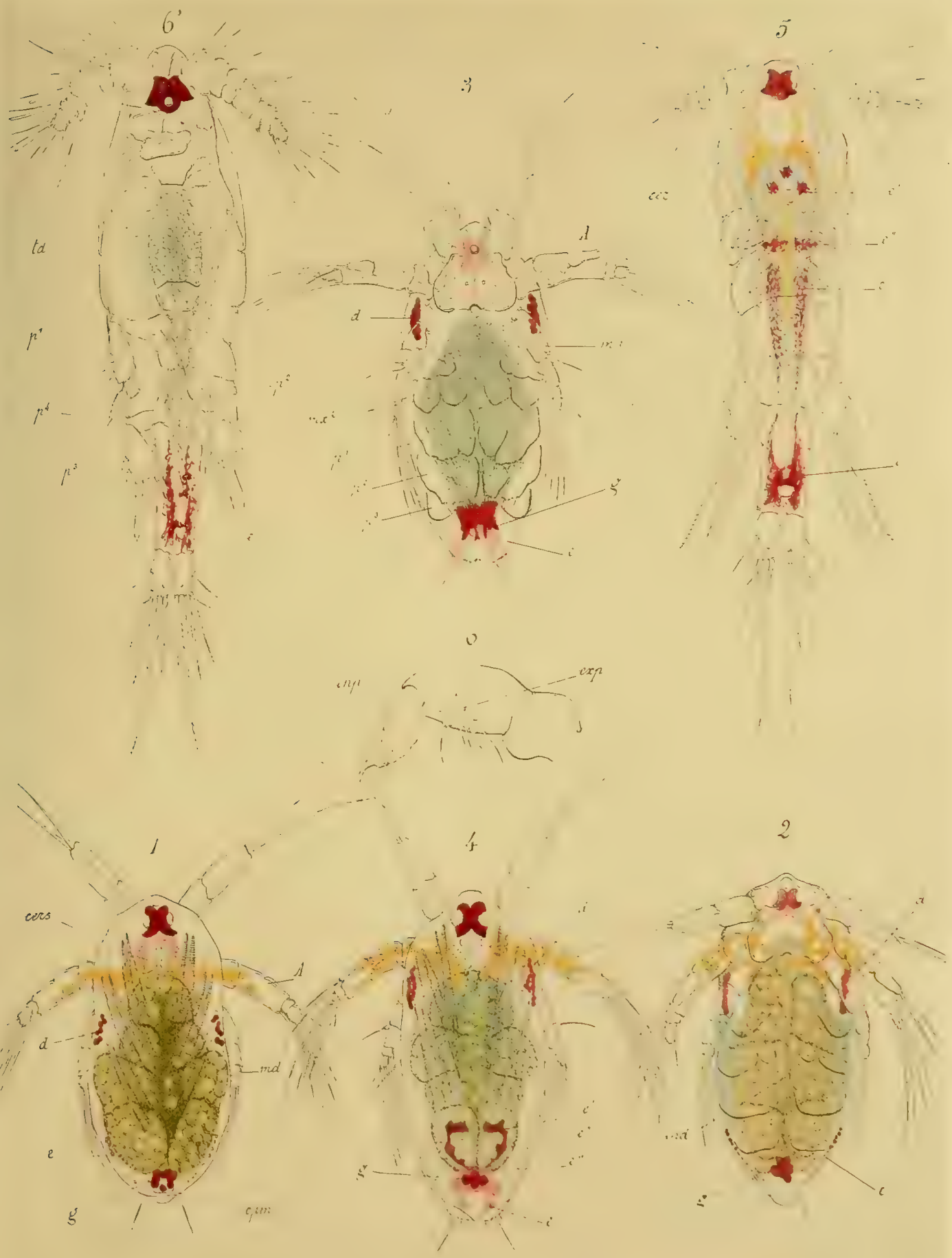





\section{PLANCHE XIV.}

Fig. I-I4, Doroixys uncinata Kerschner. 


\section{PLANCHE XIV.}

\section{Doroixys uncinata ․}

Fig. I. Adulte vu de profil: $\frac{25}{1}$.

Fig. 2. Antennule et prolongement frontal: $\frac{160}{1}$.

Fig. 3. Antenne: $\frac{160}{1}$.

Fig. 4. Mandibule: $\frac{350}{1}$.

Fig. 5. Première maxille: $\frac{350}{1}$.

Fig. 6. Seconde maxille externe: $\frac{350}{8}$.

$e p$, soie en forme d'épine; $S$, grosse soie hérissée de barbules.

Fig. 7. Paragnathes et secondes maxilles externes, avec la lèvre inférieure en place : $\frac{350}{1}$.

Fig. 8. Patte thoracique de la première paire: $\frac{160}{1}$.

Fig. 9. Patte de la deuxième paire $: \frac{160}{1}$.

Fig. 10. Patte de la troisième paire: $\frac{160}{1}$.

Fig. II. Patte de la quatrième paire: $\frac{160}{1}$.

Fig. I2. Patte de la quatrième paire, endopodite: $\frac{350}{1}$.

Fig. I3. Le mème, chez un autre individu: $\frac{350}{1}$.

Fig. 14. Abdomen et derniers somites thoraciques, vus de profil : $\frac{160}{1}$. 


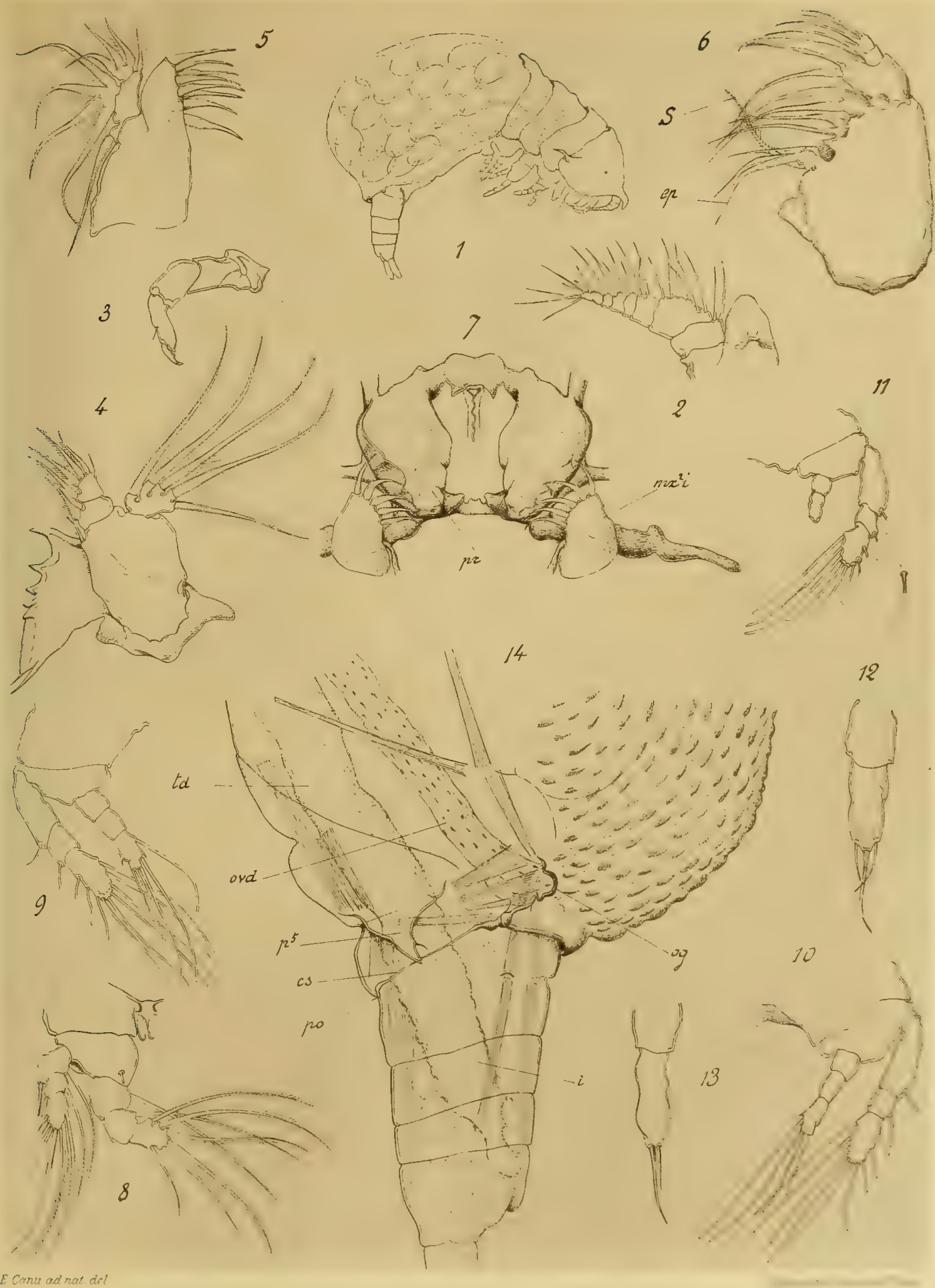





\section{PLANCHE XV.}

Fig. I-II, Doroixys uncinata Kerschner.

Métamorphose. 


\section{PLANCHE XV.}

\section{Doroixys uncinata.}

Fig. I. Nauplius dans l'œuf: $\frac{350}{1}$.

Fig. 2. Nauplius à l'éclosion: $\frac{160}{1}$.

Fig. 3. Métanauplius, vu ventralement et montrant les appendices de l'embryon cyclopoïde : $\frac{160}{2}$.

Fig. 4. Métanauplius, vu dorsalement et montrant les somites de l'embryon cyclopoïde : $\frac{110}{1}$.

Fig. 5. Embryon au premier stade cyclopoïde, vu par la face ventrale : $\frac{160}{1}$.

Fig. 6. Embryon au deuxième stade cyclopoïde, vu par la face dorsale : $\frac{160}{1}$.

Fig. 7. Deuxième patte natatoire du même embryon: $\frac{350}{\mathrm{I}}$.

Fig. 8. Troisième patte natatoire du même embryon: $\frac{350}{1}$.

Fig. 9. Embryon au premier stade parasite, vu par la face ventrale; $\frac{160}{1}$.

Fig. 10. Système nerveux ventral du même embryon: $\frac{200}{1}$.

Fiy. II. Jeune femelle parasite, vue ventralement: $\frac{160}{1}$.

ep. l'épine caractéristique sur le bord du céphalon. 
Trav. du Lab. de Wimereux, TomeVI.

Planche $\mathrm{XI}$.
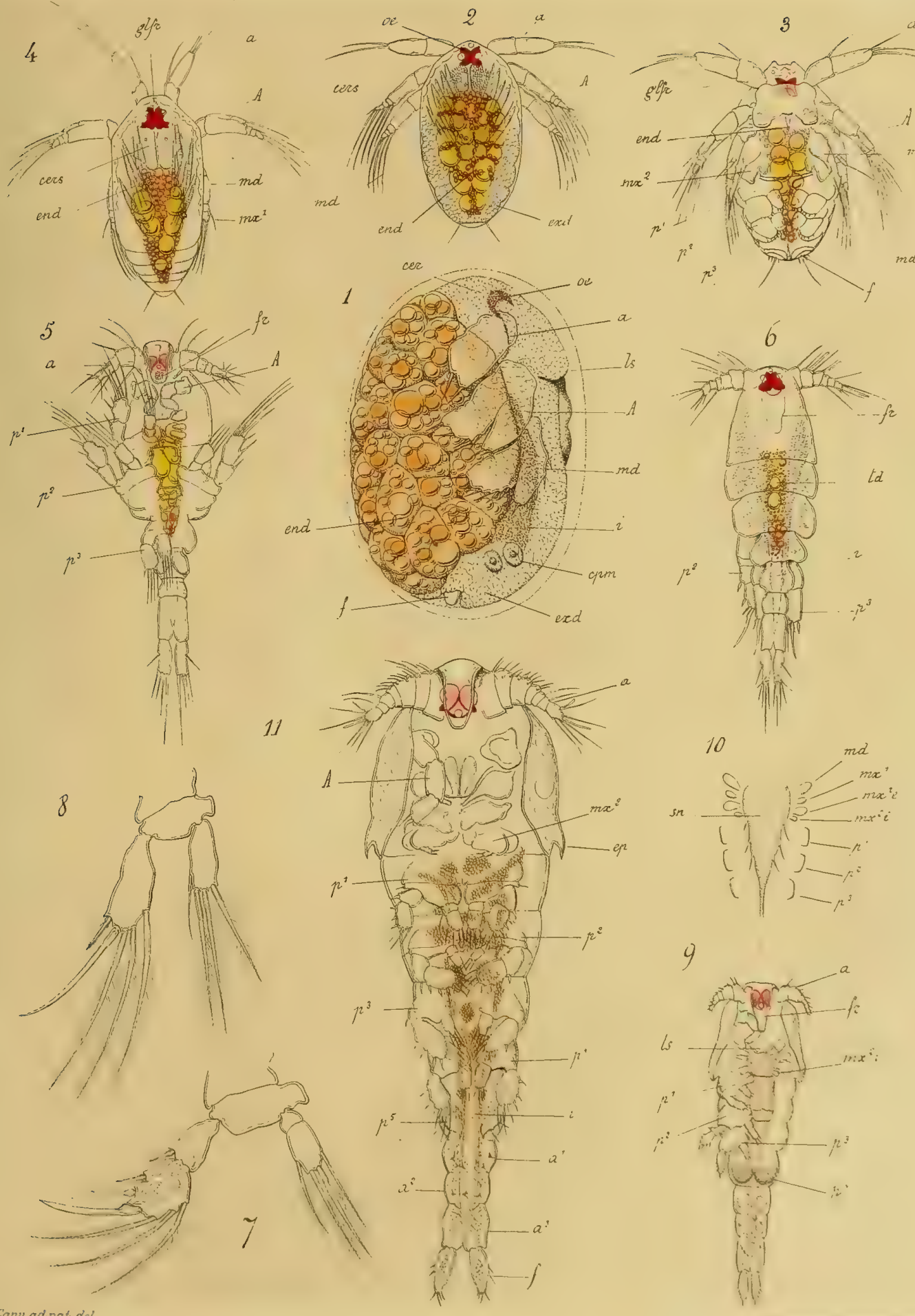



\section{PLANCHE XVI.}

Fig. I-I4, Botryllopbilus macropus CanU. 


\section{PLANCHE XVI.}

\section{Botryllopbilus macropus ?.}

Fig. 1. Femelle adulte, après la ponte, vue de profil : $\frac{50}{1}$.

Fig. 2. Femelle, peu de temps après la dernière mue, vue de profil : $\frac{50}{1}$. $n p^{2}, n p^{3}, n p^{4}$, nerfs des pattes thoraciques; nabd, nerf abdominal.

Fig. 3. Jeune mâle (?) recueilli dans Polyclinum luteum, vu par la face dorsale : $\frac{120}{1}$.

Fig. 4. Abdomen du précédent, vu de profil : $\frac{160}{1}$.

Fig. 5. Antennule de la femelle adulte: $\frac{160}{1}$.

Fig. 6. Antenne de la femelle adulte; $\frac{175}{1}$.

Fig. 7. Coxopodite mandibulaire, vu par la face externe et postérieure: $\frac{350}{1}$.

Fig. 8. Basipodite, endopodite et exopodite de la mandibule : $\frac{175}{I}$.

Fig. 9. Première maxille, vue par la face externe: $\frac{350}{1}$.

Fig. 10. Seconde maxille externe: $\frac{350}{1}$.

Fig. II. Seconde maxille interne: $\frac{160}{1}$.

Fig. 12. Patte thoracique gauche de la quatrième paire: $\frac{160}{1}$.

Fig. 13. Patte thoracique de la cinquième paire, dans une jeune femelle avant la dernière mue : $\frac{160}{1}$.

Fig. I4. Anneau génital d'une femelle adulte après la fonte, vu par la face dorsale. os, canalicules séminifères. 
Trav du Lab. de Wimereux, Tome VI.

Planche XI\%.
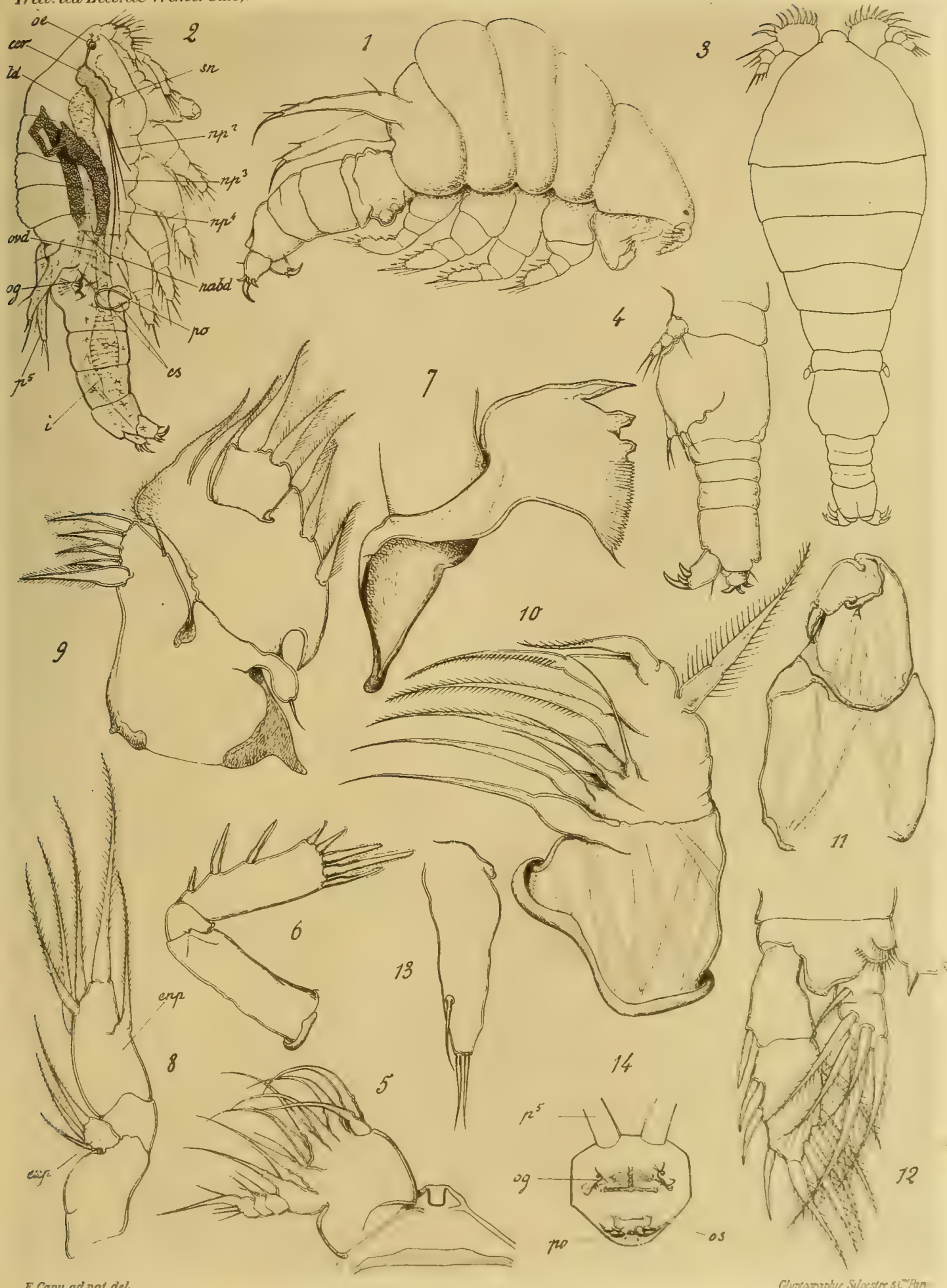



\section{PLANCHE XVII.}

Fig. I-Io, Agnathaner typicus CanU.

Fig. II-16, Agnathaner minutus CAnU. 


\section{PLANCHE XVII.}

\section{Agnatbaner typicus or.}

Fig. 1. L'animal vu dorsalement: $\frac{55}{1}$.

Fig. 2. Antennule et prolongement frontal: $\frac{160}{1}$.

Fig. 3. Antenne, vue par la face externe: $-\frac{350}{\mathrm{I}}$.

Fig. 4. Cône buccal et coxopodite mandibulaire, vus de face: $\frac{350}{1}$.

Fig. 5. Les mêmes, vus de profil : $\frac{350}{1}$.

Fig. 6. Mandibule: $\frac{350}{1}$.

Fig. 7. Première maxille: $\frac{350}{1}$.

Fig. 8. Seconde maxille externe: $\frac{350}{1}$.

Fig. 9. Seconde maxille interne $: \frac{350}{1}$.

Fig. 10. Patte thoracique de la cinquième paire: $\frac{350}{1}$.

\section{Agnatbaner minutus ơ.}

Fig. II. L'animal vu dorsalement: $\frac{55}{1}$.

Fig. 12. Céphalon, antennule et antenne, vus par la face ventrale: $\frac{160}{1}$.

Fig. 13. Cône et appendices buccaux vus ventralement: $\frac{160}{1}$.

Fig. 14. Patte de la première paire: $\frac{350}{1}$.

Fig. 15. Cinquième somite thoracique et premier somite abdominal, vus veniralement : $\frac{160}{1}$.

Fig. I6. Segments fostérieurs de l'abdomen, vus par la face ventrale : $\frac{160}{1}$. 
Tran: du Lab. de Wimereux. TomeVI.

Planche XIII.
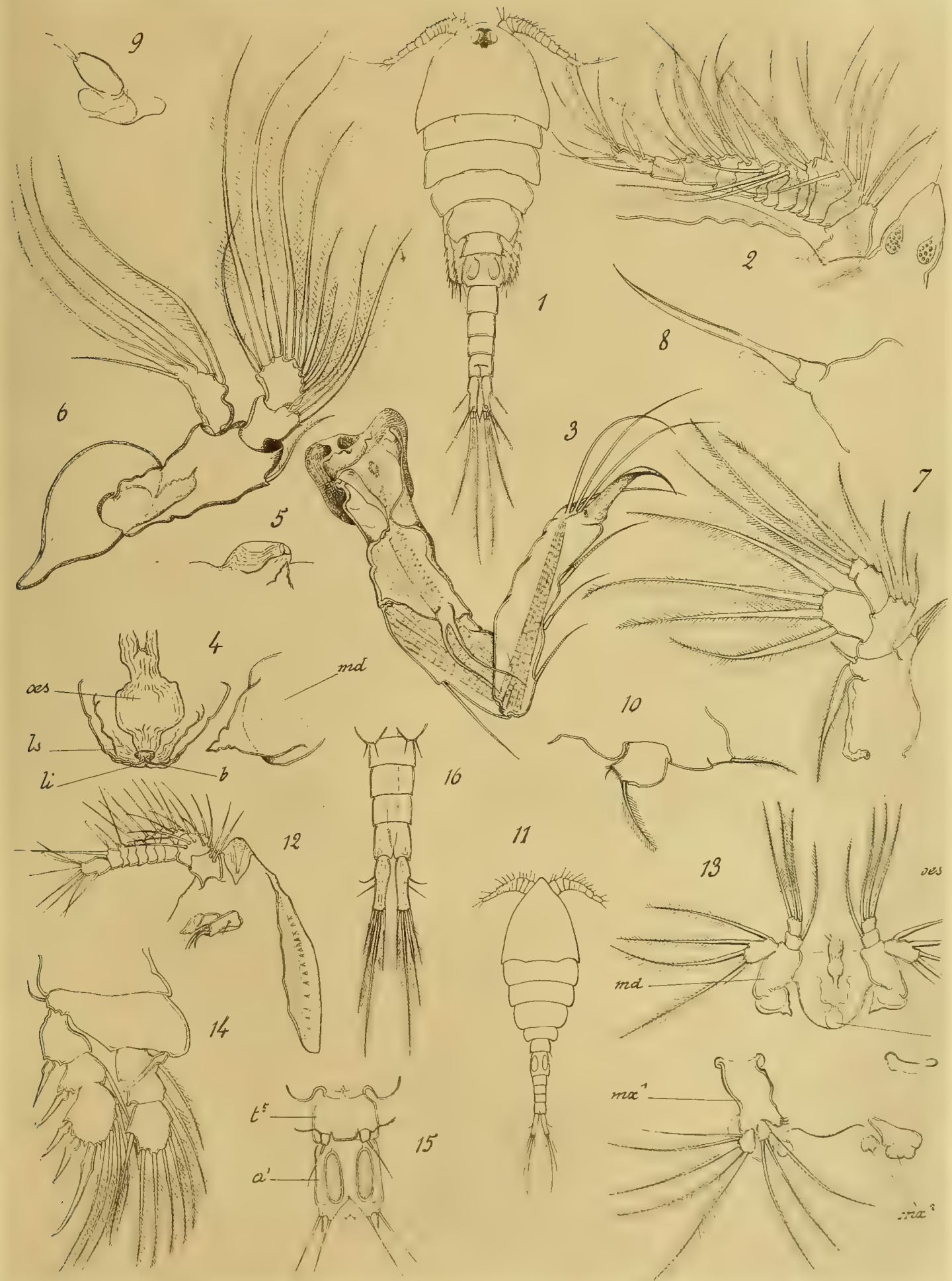

10

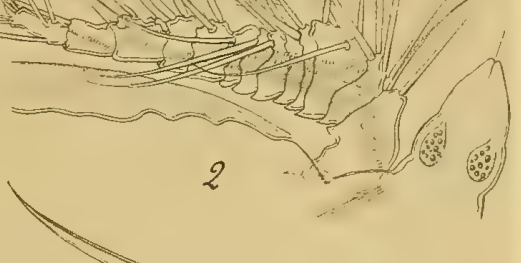

E. Canu ad nat. del.
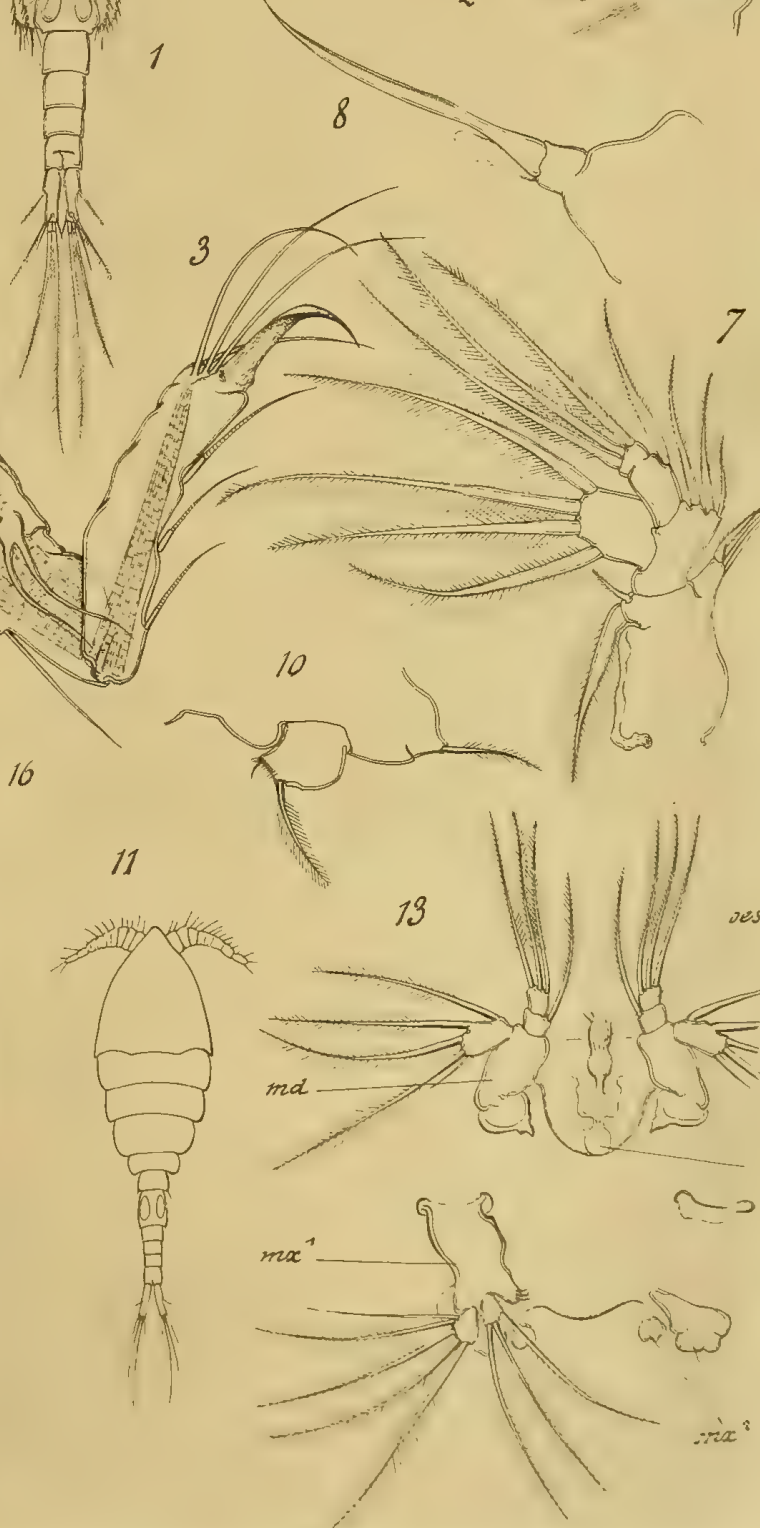



\section{PLANCHE XVIII.}

Fig. I-I2, Enterocola fulgens VAN Beneden.

Fig. I3 et I4, Enterocola betencourti Canu. 


\section{PLANCHE XVIII.}

\section{Enterocola fulgens.}

Fig. I. Femelle portant deux paquets d'œufs, vue par la face dorsale : $\frac{25}{1}$.

Fig. 2. Femelle, vue ventralement et montrant la disposition des organes intérieurs : $\frac{55}{1}$.

$n p^{2}, n p^{4}$, nerfs des pattes thoraciques; nabd, nerf abdominal; $c m$, grandes cellules du tissu conjonctif groupées à la base des pattes thoraciques; $m$, aufs en voie de maturation; $n$, les grosses cellules du tissu conjonctif disposées en deux groupes latéraux.

Fig. 3. Mâle vu de profil: $\frac{160}{1}$.

Fig. 4. Céphalon du mâle, avec ses appendices, vus ventralement : $\frac{160}{1}$.

Fig. 5. Les mémes, vus de profil : $\frac{160}{1}$.

Fig. 6. Première patte thoracique du mâle: $\frac{350}{1}$.

Fig. 7. Cinquième somite thoracique et premier somite abdominal du mâle, vus par la face ventrale: $\frac{160}{1}$.

Fig. 8. Extrémité de l'abdomen du mâle, vue ventralement: $\frac{160}{1}$.

Fig. 9. Abdomen de la femelle, vu ventralement : $\frac{120}{1}$.

Fig. 10. Deuxième patte thoracique de la femelle: $\frac{160}{I}$.

Fig. I1. Troisième patte thoracique de la femelle : $\frac{160}{1}$.

Fig. 12. Appareil génital de la femelle: $\frac{55}{1}$.

$m$, ceufs en voie de maturation.

\section{Enterocola betencourti $q$.}

Fig. 13. Le céphalon, vu par la face dorsale, pour montrer les cadres d'insertion des appendices et les crètes articulaires des deux paires de pièces buccales : $\frac{160}{1}$.

Fig. 14. Appendices céphaliques en place, vus par la face ventrale: $\frac{100}{1}$. 
Trav. du Lab. de Wimereux, TomeVI.

Planche XVIII.

a

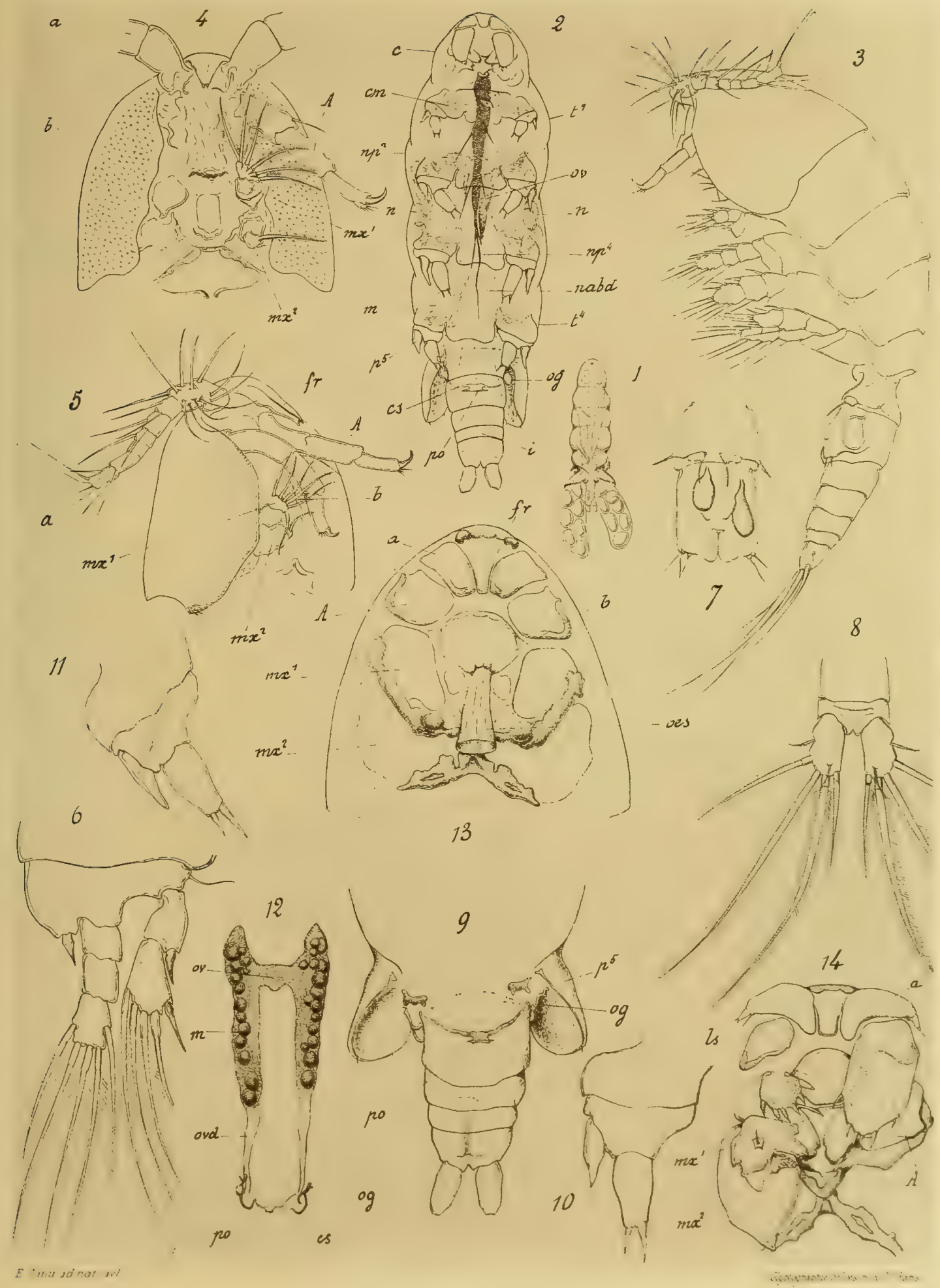





\section{PLANCHE XIX}

Fig. I-I3, Enterocola fulgens VAN BENEDEN.

Métamorphose. 


\section{PLANCHE XIX.}

\section{Enterocola fulgens.}

Fig. I. Nauplius, vu par la face ventrale et montrant les appendices de l'embryon cyclopoïde : $\frac{160}{1}$.

Fig. 2. Nauplius vu par la face dorsale, avec l'indication des somites thoraciques: $\frac{160}{\mathrm{I}}$.

Fig 3. Embryon au premier stade cyclopoïde, vu dorsalement : $\frac{160}{1}$.

Fig. 4. Le même, vu de profil : $\frac{160}{1}$.

Fig. 5. Appendices buccaux du même embryon: $\frac{350}{1}$.

Fig. 6. Première patte natatoire du même : $\frac{350}{1}$.

Fig. 7. Embryon au deuxième stade cyclopoïde, vu ventralement et montrant les divers appendices: $\frac{160}{x}$.

Fig. 8. Embryon femelle au premier stade parasite, vu de profil : $\frac{160}{1}$.

Fig. 9. Céphalon du même embryon, vu par la face ventrale : $\frac{340}{1}$.

Fig. IO. Céphalon du même, vu de profil: $\frac{350}{\mathrm{I}}$.

Fig. I I. Premier somite thoracique du même embryon, vu ventralement : $\frac{340}{1}$.

Fig. 12. Femelle adulte, vue dorsalement: $\frac{50}{1}$.

Fig. 13. Mâle adulte, vu dorsalement: $\frac{55}{1}$. 
Trav du Lab. de Wimereux, Tome VI.

Planche XIX.
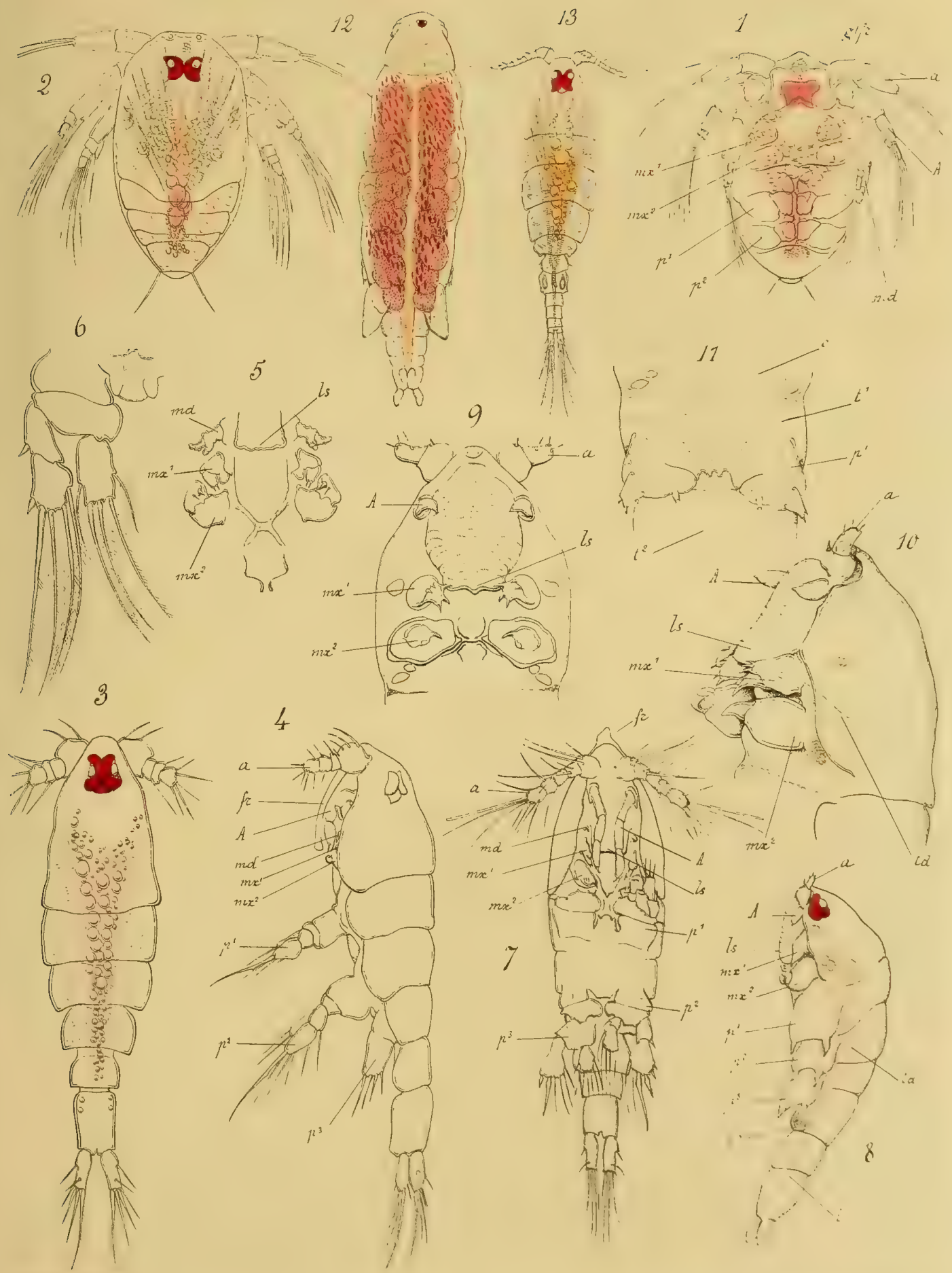



\section{PLANCHE XX.}

Fig. I-4, Enterocola betencourti CANU.

Fig. 5-I8, Aplostoma brevicauda $\mathrm{C}_{\mathrm{ANU}}$. 


\section{PLANCHE XX.}

\section{Enterocola betencourti.}

Fig. I. Femelle adulte, avec deux paquets d'œufs, vue dorsalement: $\frac{25}{1}$.

Fig. 2. Deuxième patte thoracique: $\frac{160}{1}$.

Fig. 3. Troisième patte thoracique: $\frac{160}{1}$.

Fig. 4. Abdomen vu par la face ventrale: $\frac{120}{1}$.

\section{Aplostoma brevicauda.}

Fig. 5. Femelle vue dorsalement: $\frac{50}{1}$.

Fig. 6. Plusieurs femelles, avec paquets d'œufs, en grandeur naturelle.

Fig. 7. Ascidiozoïde d'Amaræcium, avec son parasite: $\frac{25}{\mathrm{I}}$.

Fig. 8. Tête de la femelle, vue ventralement: $\frac{160}{x}$.

Fig. 9. Mandibule : $\frac{350}{1}$.

Fig. IO. Abdomen, vu dorsalement: $\frac{120}{1}$.

Fig. I I. Premier segment abdominal, vu ventralement et montrant le pore de fécondation et les canalicules séminaux : $\frac{160}{1}$.

Fig. 12. Une patte thoracique: $\frac{160}{\mathrm{r}}$.

Fig. 13. Aplostoma mâle, vu de profil : $\frac{120}{1}$.

Fig. I4. Antennule du même : $\frac{160}{1}$.

$x$, bâtonnet sensoriel de l'article basilaire: $\frac{670}{1}$

$y$, bâtonnet sensoriel des articles terminaux : $\frac{670}{i}$

Fig. 15. Antenne: $\frac{160}{1}$

Fig. I6. Pièces buccales du côté gauche, vues par la face ventrale: $\frac{350}{1}$.

Fig. 17. Patte thoracique de la cinquième paire: $\frac{350}{1}$.

Fig. I8. Extrémité de l'abdomen, vue ventralement: $\frac{160}{!}$. 


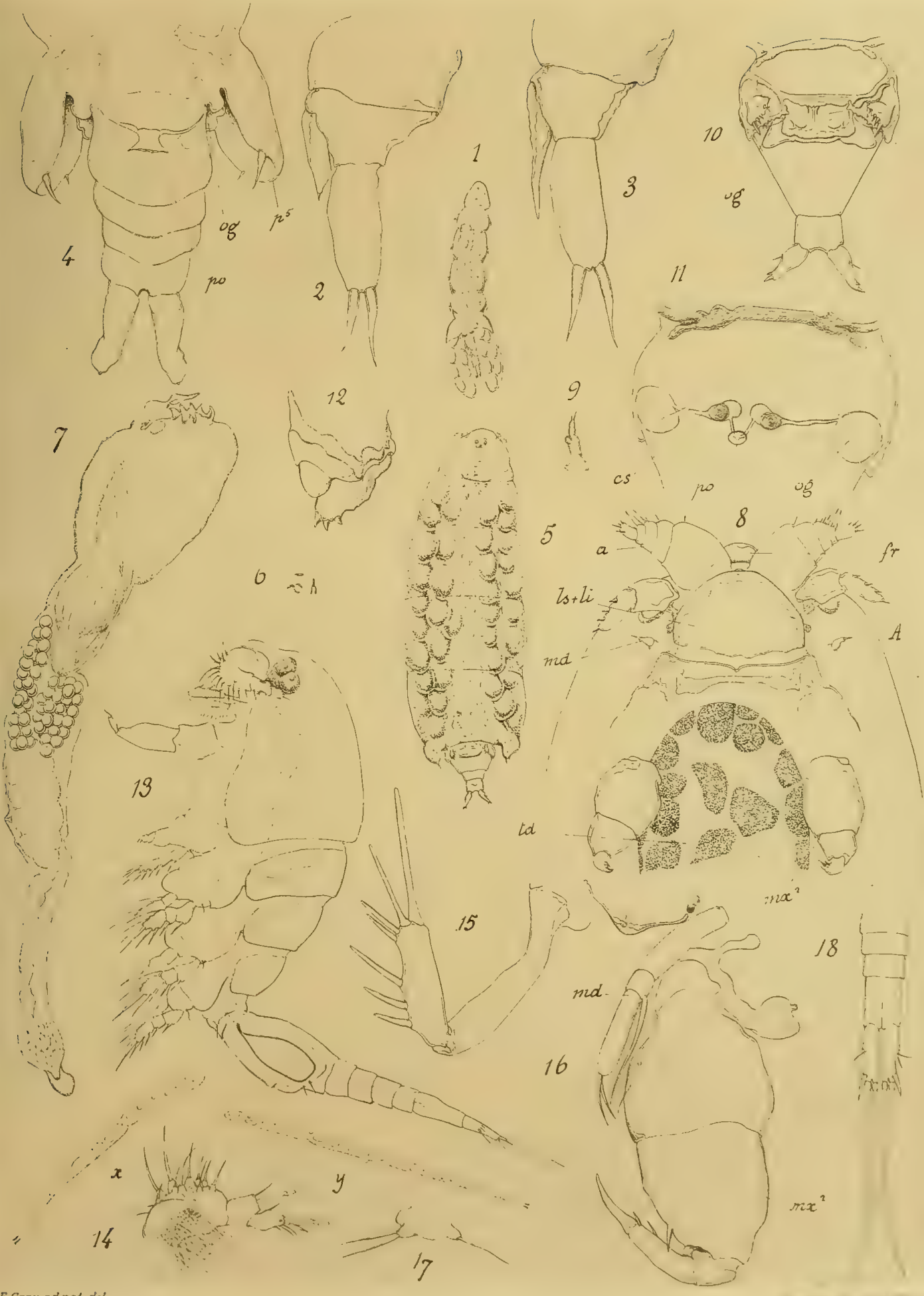

E. Canu ad nal del. 



\section{PLANCHE XXI.}

Fig. I-I2, Aplostoma brevicauda Canu.

Métamorphose.

Fig. I 3-I 5, Aplostoma sp., mâle. 


\section{PLANCHE XXI.}

\section{Aplostoma brevicauda.}

Fig. 1. Nauplius à l'éclosion, vu ventralemeut $\frac{160}{1}$.

Fig. 2. Le mème, vu dorsalement: $\frac{160}{1}$.

-Fig. 3. Nauplius plus âgé, vú dorsalement: $\frac{160}{1}$.

Fig. 4. Le même, vu ventralement : $\frac{160}{1}$.

Fig. 5. Nauplius encore plus àgé, vu dorsalement : $\frac{160}{1}$.

Fig. 6. Le même, vu ventralement et montrant les appendices de l'embryon cyclopoïde : $\frac{160}{1}$.

Fig. 7. Embryon au premier stade cyclopoïde, vu dorsalement : $\frac{160}{1}$.

Fig. 8. Céphalon et antennule du même: $\frac{350}{1}$.

$s, s^{\prime}$, soies sensorielles de l'antennule.

Fig. 9. Céphalon et premier somite thoracique du même: $\frac{350}{1}$.

Fig. Io. Cône buccal et appendices buccaux du même, vus de profil : $\frac{350}{1}$. ep, épine terminale, saillante, du cône buccal.

Fig. 11. Mandibule du même: $\frac{350}{1}$.

Fig. 12. Les trois derniers somites du même, vus ventralement: $\frac{350}{1}$.

\section{Aplostoma or}

Fig. I 3. Pattes thoraciques de la première paire: $\frac{160}{1}$.

Fig. 14. Endopodite des pattes de la première paire: $\frac{670}{1}$.

Fig. 15. Pattes thoraciques de la deuxième paire: $\frac{169}{1}$. 


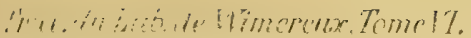

I'lunche XXI.
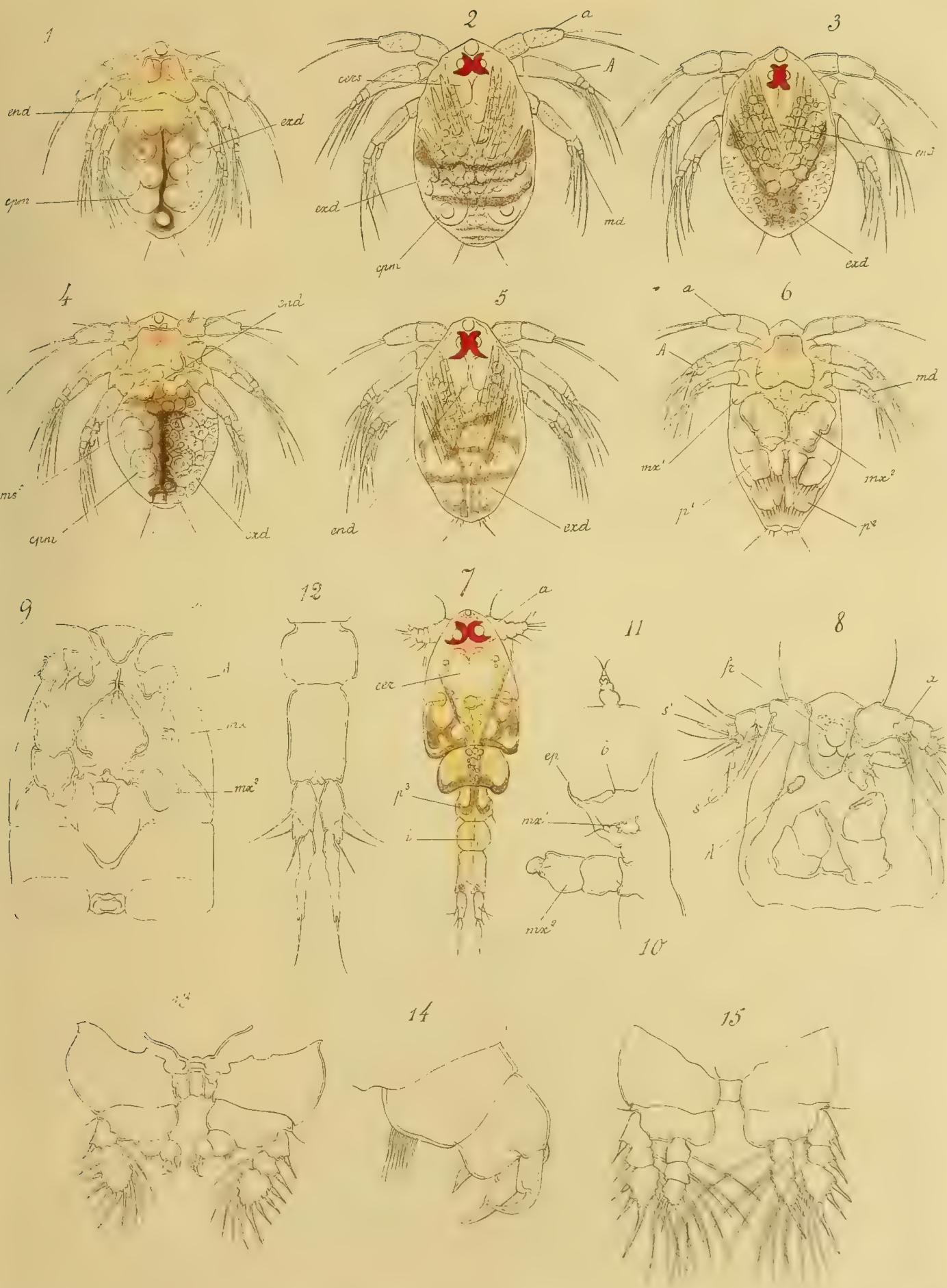



\section{PLANCHE XXII.}

Fig. I-I4, Lichomolgus doridicola LEYDIG.

Fig. I5-23 Lichomolgus albens Thorell. 


\section{PLANCHE XXII.}

\section{Licbomolgus diordicola.}

Fig. I. Femelle, vue dorsalement: $\frac{55}{1}$.

Fig. 2. Mâle, vu dorsalement: $\frac{55}{2}$

Fig. 3. Céphalon de la femelle, vu ventralement : $\frac{120}{8}$.

Fig. 4. Antennule de la femelle: $\frac{160}{1}$.

Fig. 5. Mandibule: $\frac{350}{\mathrm{I}}$.

Fig. 6. Première maxille: $\frac{350}{1}$.

Fig. 7. Seconde maxille externe: $\frac{350}{1}$.

Fig. 8. Seconde maxille interne, chez la femelle: $\frac{350}{1}$.

Fig. 9. Seconde maxille interne, chez le mâle: $\frac{350}{1}$.

Fig. 10. Quatrième patte thoracique : $\frac{160}{1}$.

Fig. I I. Cinquième patte thoracique de la femelle : $\frac{160}{\mathrm{x}}$.

Fig. 12. Extrémité de l'abdomen, vue dorsalement: $\frac{160}{1}$.

Fig. 13. Nauplius vu dorsalement: $\frac{350}{1}$.

Fig. 14. Face ventrale de la partie postérieure du nauplius: $\frac{350}{1}$.

\section{Lichomolgus albens.}

Fig. 15. Antennule: $\frac{350}{1}$.

Fig. 16. Antenne: $\frac{160}{1}$.

Fig. 17. Lèvre supérieure recouvrant la mandibule et la seconde maxille externe, avec le pharynx: $\frac{350}{1}$.

Fig. 18. Mandibule et première maxille $: \frac{350}{1}$.

Fig. 19. Seconde maxille externe: $\frac{350}{1}$.

Fig. 20. Seconde maxille interne, chez la femelle: $\frac{350}{1}$.

Fig. 2 I. Endopodite de la quatrième patte thoracique: $\frac{350}{1}$.

Fig. 22. Cinquièmé patte thoracique, vue dorsalement: $\frac{160}{1}$.

Fig. 23. Abdomen du mâle, vu ventralement: $\frac{1 \text { óo }}{1}$. $x$, la grande soie furcale : $\frac{350}{1}$. 
Trav du Lab. de Wimereux, TomeVI.

Planche XXII.

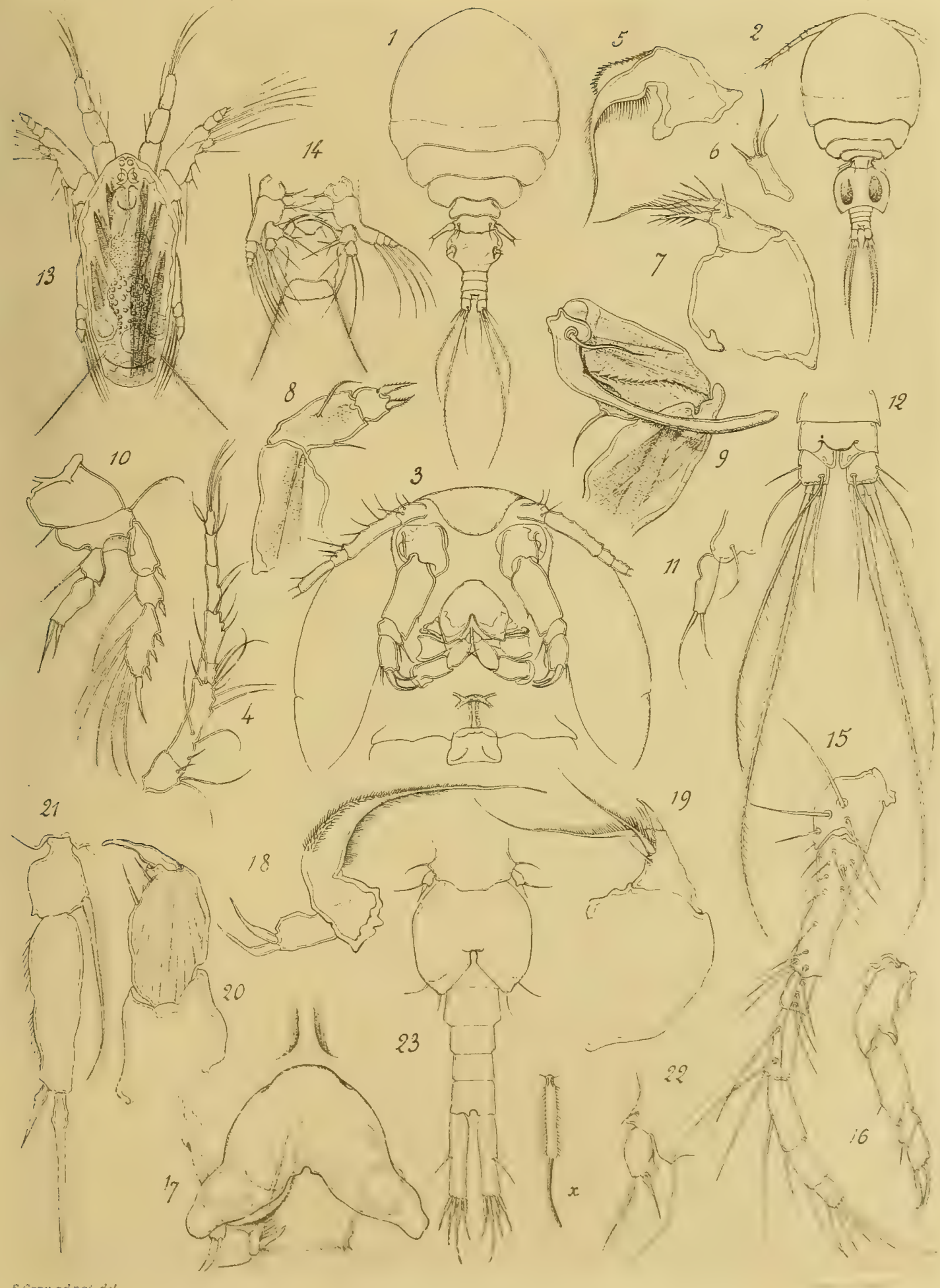





\section{PLANCHE XXIII.}

Fig. I-4, Lichomolgus albens Thorell.

Fig. 5-I2, Lichomolgus poucheti CANU.

Fig. I3-I8, Thersites gasterostei. Pagenstecher. 


\section{PLANCHE XXIII.}

\section{Lichomolgus albens.}

Fig. I. Femelle, vue dorsalement : $\frac{55}{1}$.

Figg. 2. Mâle, vu dorsalement: $\frac{55}{1}$.

Fig. 3. Seconde maxille interne du mâle: $\frac{350}{1}$.

Fig. 4. Appareil génital mâle, vu de profil : $\frac{160}{1}$.

cf, portion antérieure étroite et contournée du canal déférent.

\section{Lichomolgus poucbeti.}

Fig. 5. Femelle, vue dorsalement : $\frac{55}{1}$.

Fig. 6. Antennule et prolongement frontal de la même : $\frac{340}{1}$.

Firr. 7. Antenne et lèvre supérieure de la même: $\frac{34^{\circ}}{1}$.

Fí̌. 8. Appendices buccaux de la même, vus de profil: $\frac{350}{1}$.

Figr. 9. Seconde maxille externe de la mème: $\frac{350}{1}$.

Fig. 10. Seconde maxille interne de la même: $\frac{160}{1}$

Fig. I I. Quatrième patte natatoire de la même : $\frac{160}{1}$.

Fig. 12. Cinquième somite thoracique et abdomen de la même, vus par la face dorsale: $\frac{160}{1}$.

\section{Thersites gasterostei.}

Fig. 13. Antenne de la femelle: $\frac{350}{1}$.

Fig. 14. Appendices buccaux de la même, vus de profil : $\frac{350}{1}$.

Fig. 15. Les mêmes, vus de face: $\frac{350}{1}$.

Fig. I6. Mandibule et première maxille, vues par la face interne: $\frac{350}{1}$.

Fig. 17. Abdomen de la femelle, vu dorsalement: $\frac{160}{t}$.

Fig. 18. Nauplius, vu dorsalement : $\frac{160}{1}$. 
Trav du Lab. de Wimereux, Tome VI.

Planche XXIII.

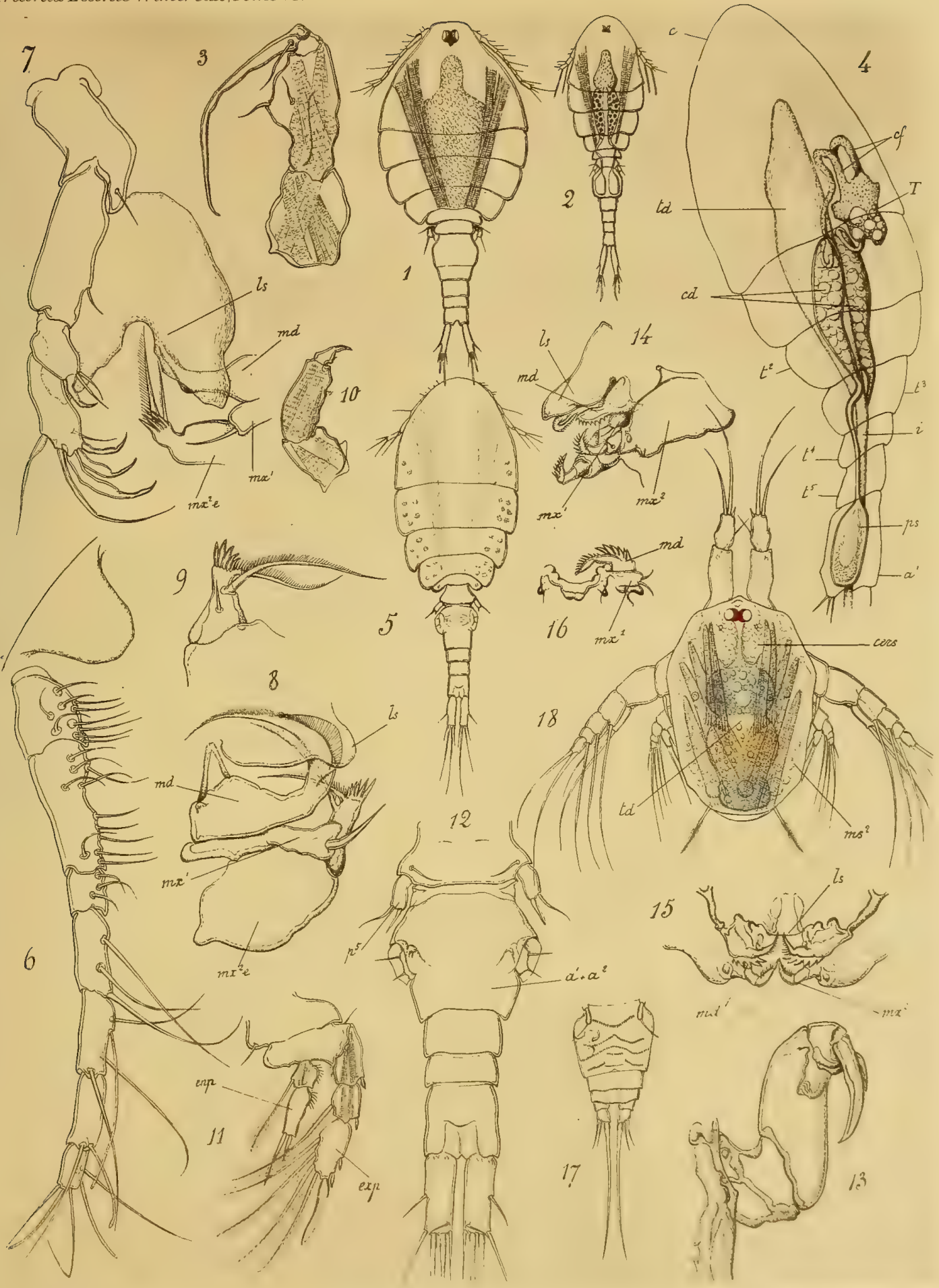





\section{PLANCHE XXIV.}

Fig. I-I3, Herrmamella rostrata Canu.

Fig. I4-26, Modiolicola insignis Aurivillius. 


\section{PLANCHE XXIV.}

\section{Herrmannella rostrata.}

Fig. I. Femelle aduite, vue dorsalement : $\frac{53}{1}$.

Fig. 2. Mâle, vu dorsalement: $\frac{55}{1}$.

Fig. 3. Céphalon et premier somite thoracique du mâle, vus ventralement: $\frac{160}{1}$.

Fig. 4. Antenne du mâle : $\frac{160}{x}$.

Fig. 5. Mandibule de la femelle: $\frac{350}{1}$.

Fig. 6. Première maxille: $\frac{350}{1}$.

Fig. 7. Seconde maxille externe: $\frac{350}{1}$.

Fig. 8. Seconde maxille interne, chez la femelle: $\frac{350}{1}$.

Fig. 9. La mème, chez le mâle, vue par sa face externe: $\frac{350}{\mathrm{I}}$.

Fig. Io. La même, chez le mâle, vue par sa face interne: $\frac{160}{1}$.

Figr. II Endopodite de la quatrième patte thoracique: $\frac{160}{1}$.

Fig. I 2 , Cinquieme somite thoracique avec ses appendices, chez le mâle : $\frac{160}{\mathrm{I}}$.

Figr. 13. Abdomen de la femelle et cinquième somite thoracique, vus dorsalement : $\frac{160}{\mathrm{I}}$.

\section{Modiolicola insignis.}

Fig. I4. Femelle, vue dorsalement: $\frac{50}{1}$.

Figg. I 5. Mâle, vu dorsalement: $\frac{50}{1}$.

Fig. I6. Antennule du mâle : $\frac{100}{1}$.

Fig. 17. Antenne du mâle: $\frac{160}{1}$.

Fig. 18. Région buccale de la femelle: $\frac{100}{1}$.

Fig. I9. Mandibule et première maxille, vues par la face externe: $\frac{350}{1}$.

Fig. 20. Les mèmes, vues par la face interne: $\frac{160}{1}$.

Fig. 21 . Seconde maxille externe: $\frac{350}{\mathrm{I}}$.

Fig. 22. Seconde maxille interne, chez la femelle: $\frac{160}{1}$.

Fig. 23. Seconde maxille interne, chez le mâle: $\frac{160}{1}$.

Fig. 24. Quatrième patte thoracique : $\frac{120}{1}$.

Fig. 25. Asbdomen du mâle, vu ventralement: $\frac{120}{1}$.

Fig. 26. Cinquieme somite thoracique et premiers somites abdominaux de la femelle, vus de profil : $\frac{160}{1}$. 
Trur: du iat, de Winerewar, Tome V.

I'turechedrys.

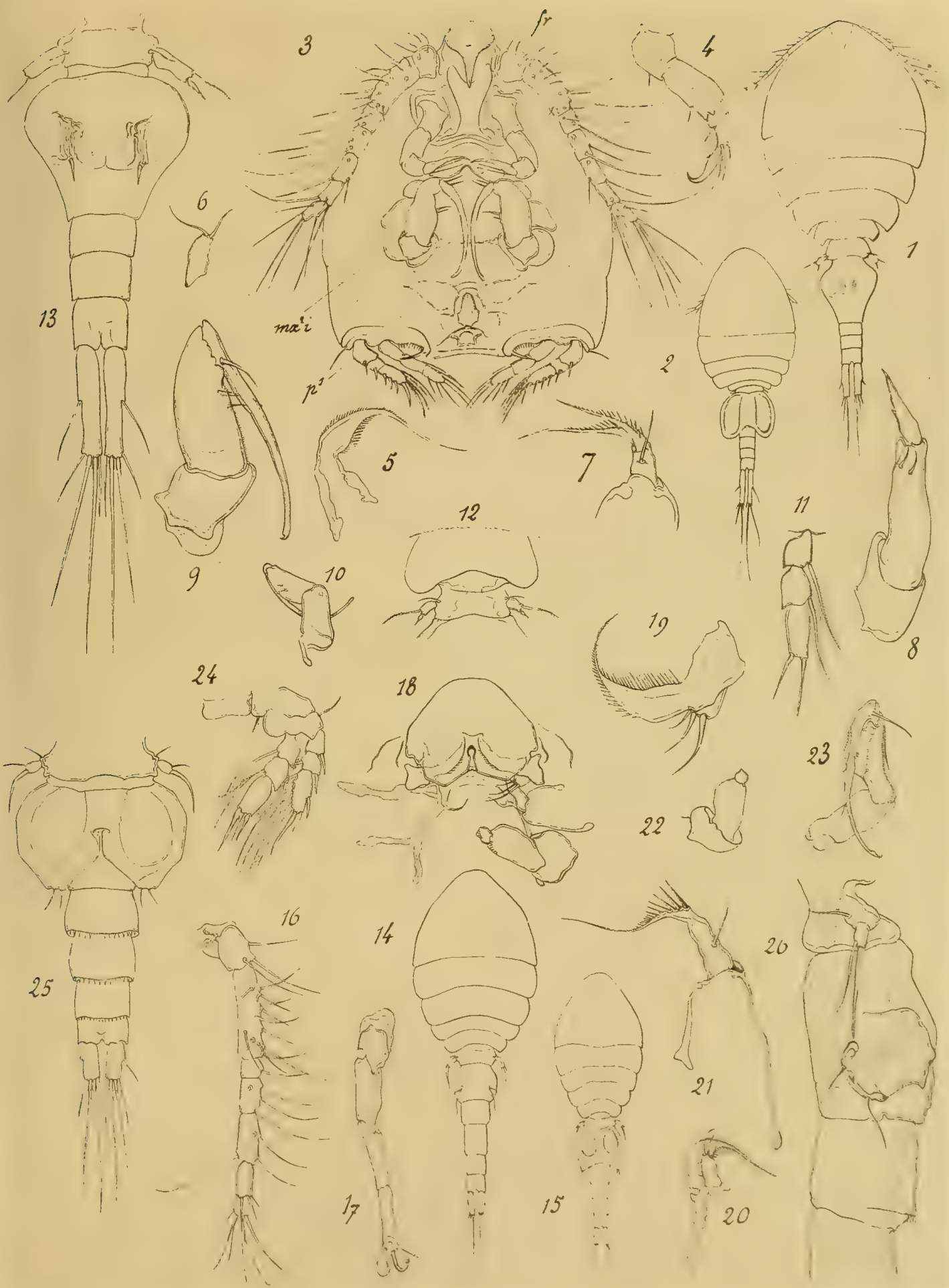





\section{PLANCHE XXV.}

Fig. I-I7, Pseudanthessius sauvagei $\mathrm{C} A \mathrm{ANU}$ 


\section{PLANCHE XXV.}

\section{Pseudanthessius sauvagei.}

Fig. I. Femelle, vue de profil : $\frac{55}{1}$.

Fig. 2. Male, vu de profil: $\frac{55}{1}$.

Fig. 3. Céphaion de la femelle, vu ventralement: $\frac{160}{1}$.

Fig. 4. Mandibule, vue par la face interne: $\frac{670}{1}$.

Fig. 5. Mandibule, vue de profil par le bord extérieur: $\frac{350}{1}$

Fig. 6 Mandibule, vue par la face externe: $\frac{350}{1}$.

Fig. 7. Première maxille: $\frac{670}{1}$.

Fig. 8. Seconde maxille externe: $\frac{350}{1}$.

Fig. 9. Seconde maxille interne, chez la femelle: $\frac{160}{1 .}$.

Fig. Io. Seconde maxille externe, chez le mâle: $\frac{350}{1}$.

Fig. I1. Quatrième patte thoracique: $\frac{160}{1}$.

Fig. 12. Cinquième patte thoracique du mâle: $\frac{350}{1}$.

Fig. 13. Abdomen de la femelle, vu dorsalement: $\frac{160}{\mathrm{I}}$.

Fig. 14. Cinquième somite thoracique et premier anneau abdominal de la femelle, vus de profil : $\frac{350}{1}$.

Fig. 15. Abdomen du mâle, vu ventralement: $\frac{160}{1}$.

Fig. I6. Bord postérieur du premier somite abdominal du mâle: $\frac{350}{1}$.

Fig. 17. Tégument dorsal du céphalon chez le mâle, pour montrer les cellules sensorielles glanduliformes: $\frac{350}{1}$. 


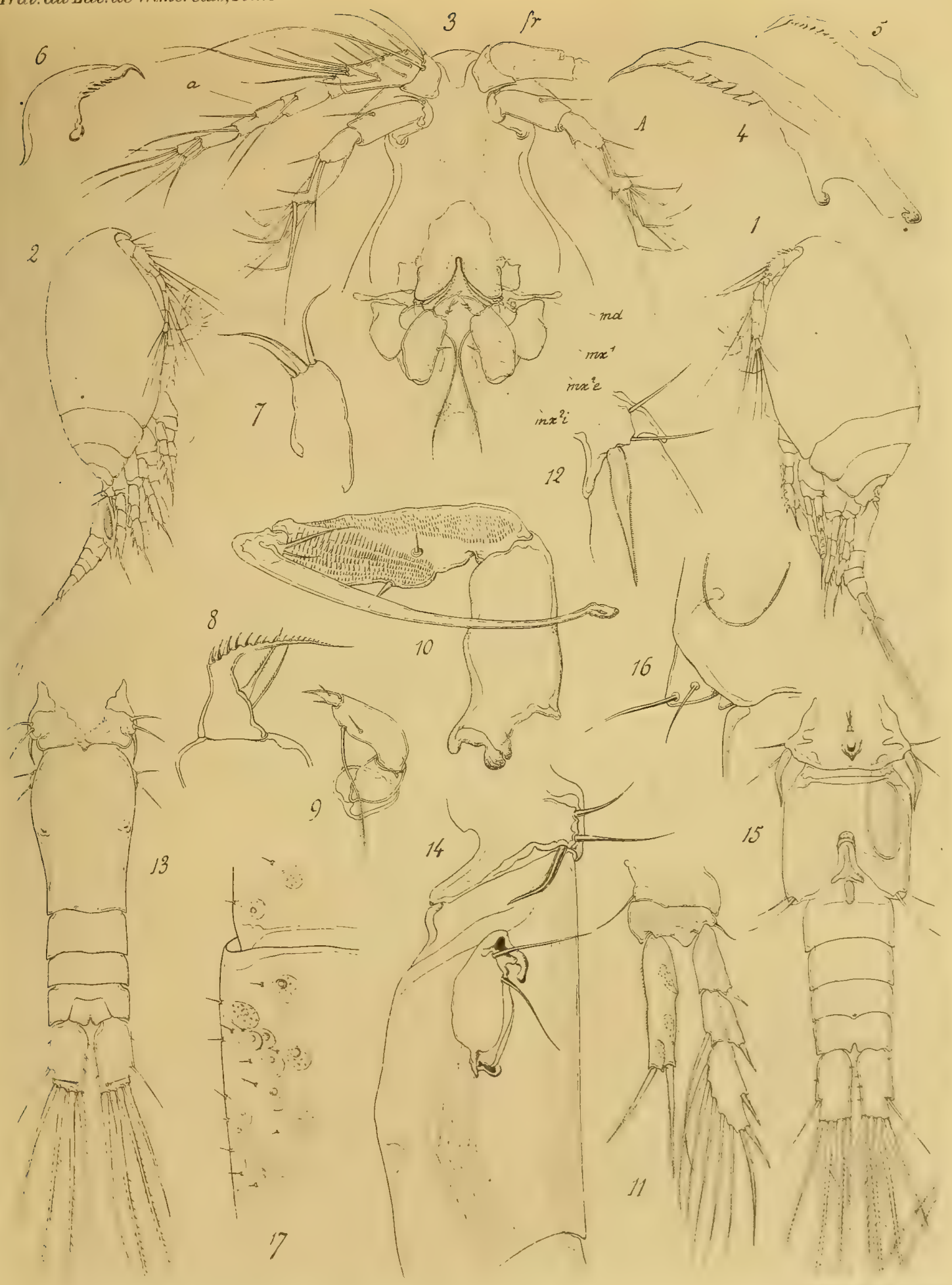





\section{PLANCHE XXVI.}

Fig. I-Io, Dermatomyzon elegans Claus.

Fig. I I-I9, Asterocheres renaudi Canu. 


\section{PLANCHE XXVI.}

\section{Dermatomyzon elegans $\sigma^{7}$.}

Figg. 1. Mâle, vu dorsalement: $\frac{55}{1}$.

Fig. 2. Céphalon et premier somite th oracique, vus par la face ventrale: $\frac{160}{1}$.

Fig. 3. Antenne: $\frac{350}{1}$.

Fig. 4. Trompe-siphon, vue par la face interne: $\frac{160}{1}$.

Fig. 5. Trompe-siphon, vue de profil: $\frac{350}{8}$.

Figr. 6. Mandibule : $\frac{350}{1}$.

Fig. 7. Première maxille: $\frac{350}{1}$.

Fig. 8. Seconde maxille externe: $\frac{350}{1}$.

Fig. 9. Cinquième patte thoracique: $\frac{100}{\mathrm{I}}$.

Fig. IO. Les deux premiers somites abdominaux, vus de profil : $\frac{350}{1}$.

\section{Asterocheres renaudi $\$$}

Fig. I I. Animal, vu dorsalement: $\frac{55}{1}$.

Fig. 12. Partie antérieure du céphalon, vue ventralement: $\frac{340}{1}$.

Fig. 13. Extrémité de la lèvre supérieure: $\frac{350}{1}$.

Fig. 14. Trompe-siphon, vue de profil: $\frac{350}{1}$.

Fig. 15. Mandibule: $\frac{350}{1}$.

Fig. I6. Première maxille: $\frac{350}{1}$.

Fig. 17. Seconde maxille externe: $\frac{340}{1}$.

Fig. 18. Seconde maxille interne: $\frac{340}{1}$.

Fig. I9. Cinquième somite thoracique et abdomen, vus par la face ventrale: $\frac{160}{1}$. 
Trav. du Lab. de Wimereux, Tome VI.

Planche XII']

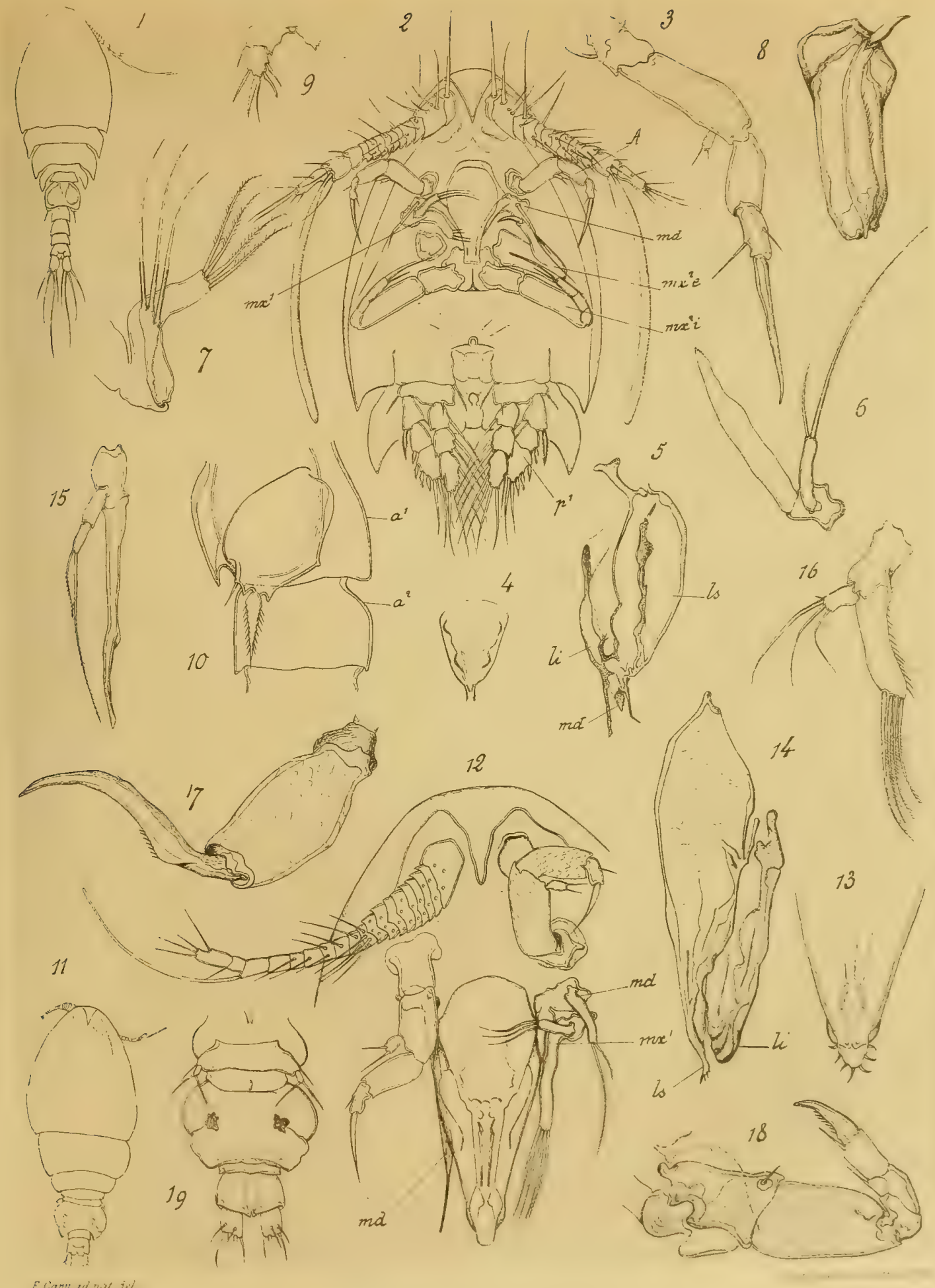





\section{PLANCHE XXVII.}

Fig. I-6, Asterocheres lilljeborgi Вовск.

Fig. 7-I7, Artotrogus normani Brady et Robertson. 


\section{PLANCHE XXVII.}

\section{Asterocheres lilljeborgi o'.}

Fig. I. Céphalothorax du mâle, vu dorsalement : $\frac{55}{1}$.

Fig. 2. Antennule: $\frac{350}{1}$.

Fig. 3. Région antérieure du corps, vue ventralement: $\frac{160}{1}$.

Fig. 4. Extrémité de la trompe-siphon, avec la mandibule et la maxille en place: $\frac{350}{1}$.

Fig. 5. Portion distale de la seconde maxille externe: $\frac{350}{1}$.

Fig. 6. Cinquième somite thoracique, vu dorsalement: $\frac{350}{1}$.

\section{Artotrogus normani.}

Fig. 7. Femelle, vue dorsalement: $\frac{50}{1}$.

Fig. 8. Antennule, antenne et prolongement frontal: $\frac{160}{1}$.

Fig. 9. Trompe-siphon, vue par la face externe: $\frac{55}{1}$.

Fig. Io. Extremité distale de la trompe-siphon: $\frac{350}{1}$

Fig. I1. Extrémité distale de la mandibule: $\frac{350}{1}$.

Fig. 12. Première maxille.

Fig. 13. Première maxille et seconde maxille externe: $\frac{55}{1}$.

Fig. 14. Seconde maxille interne: $\frac{55}{1}$.

Fig. 15. Troisième patte thoracique: $\frac{55}{1}$.

Fig. 16. Quatrième patte thoracique : $\frac{160}{1}$.

Fig. 17. Cinquième patte thoracique : $\frac{160}{3}$. 
Trav. du Lah. de Wimereux, TomeVI.

Planche XXVII.

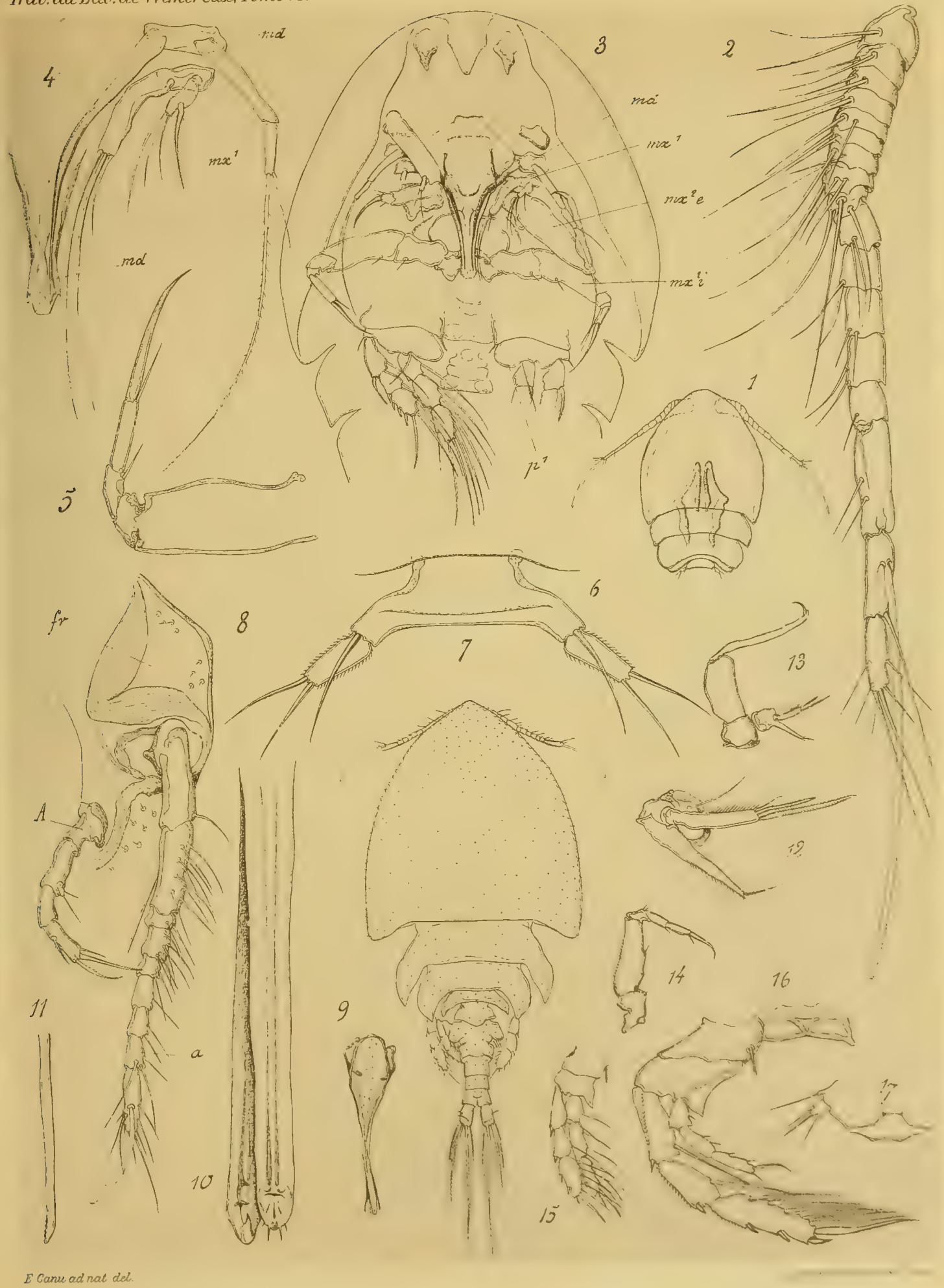





\section{PLANCHE XXVIII.}

Fig. I-I4, Dyspontius striatus Thorell. 


\section{PLANCHE XXVIII.}

\section{Drspontius striatus.}

Fig. 1. Mâle adulte, vu dorsalement: $\frac{55}{1}$.

Fig. 2. Antennule: $\frac{160}{1}$.

Fig. 3. Extrémité distale de l'antennule: $\frac{160}{1}$.

Fig. 4. Antenne: $\frac{350}{1}$.

Fig. 5. Base de la trompe-siphon, avec la mandibule et la première maxille, vues de profil : $\frac{350}{1}$.

Fig. 6. Extrémités de la trompe-siphon et de la mandibule : $\frac{350}{1}$.

Fig. 7. Première maxille, vue par la face externe $: \frac{350}{4}$.

Fig. 8. Seconde maxille : $\frac{160}{1}$.

Fig. 9. Troisième patte thoracique : $\frac{160}{1}$.

Fig. 10. Deuxième patte thoracique gauche: $\frac{160}{1}$

Fig. 11. Quatrième patte thoracique: $\frac{160}{1}$.

Fig. I2. Abdomen et cinquième somite thoracique, vus par la face ventrale: $\frac{160}{1}$.

Fig. 13. Ornementation de la cuticule: $\frac{160}{1}$.

Fig. 14. Jeune asexué, vu ventralement: $\frac{120}{1}$. 
Trov. du Lab. de Wimereux, TomeVI.

Planche XXVIII.

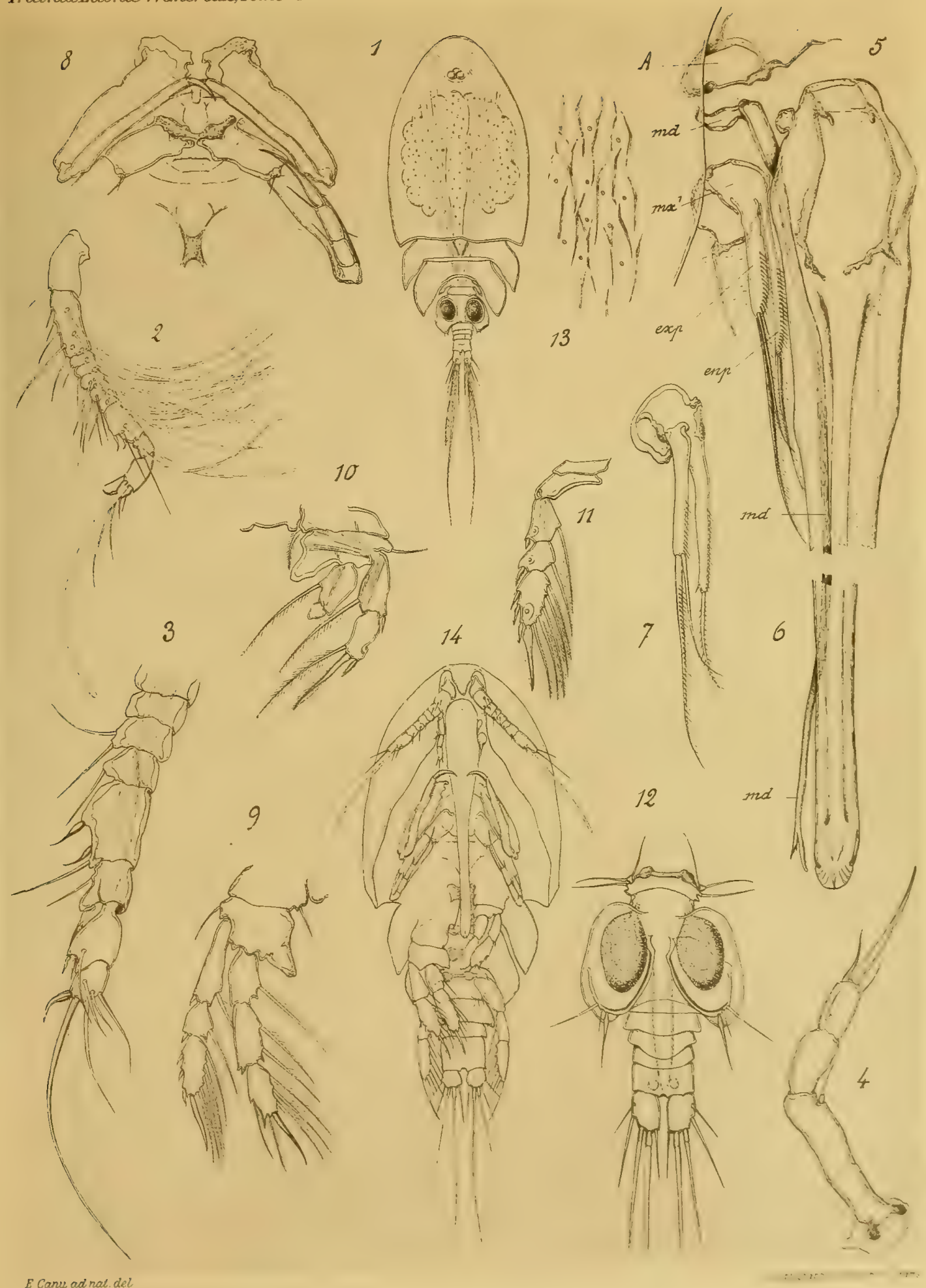





\section{PLANCHE XXIX.}

Fig. I-4, Acontiophorus scutatus Brady et Robertson.

Fig. 5-I3, Cancerilla tubulata DaLyell. 


\section{PLANCHE XXIX.}

\section{Acontiophorus scutatus o.}

Fig. I. Animal avec des œufs pondus, vu dorsalement: $\frac{55}{1}$.

L'extrémité distale de la trompe-siphon est visible entre les deux pièces de la furca.

Fig. 2. Antennule : $\frac{160}{1}$.

Fig. 3. Base de la trompe et appendices buccaux : $\frac{350}{1}$.

Fig. 4. Cinquième somite thoracique et premiers segments abdominaux, vus ventralement : $\frac{160}{1}$.

\section{Cancerilla tubulata.}

Fig. 5. Femelle, vue dorsalement: $\frac{55}{1}$.

Fig. 6. Mâle, vu ventralement: $\frac{55}{1}$.

Fig. 7. Derniers somites thoraciques et abdomen du mâle, vus dorsalement: $\frac{55}{1}$.

Fig. 8. Antennule de la femelle: $\frac{160}{1}$.

Fig. 9. Antenne de la femelle: $\frac{160}{1}$.

Fig. IO. Mandibule: $\frac{350}{1}$.

Fig. I I. Première maxille: $\frac{350}{i}$.

Fig. I2. Première patte thoracique de la femelle: $\frac{350}{1}$.

Fig. 13. Deuxième patte thoracique de la femelle: $\frac{350}{1}$ 


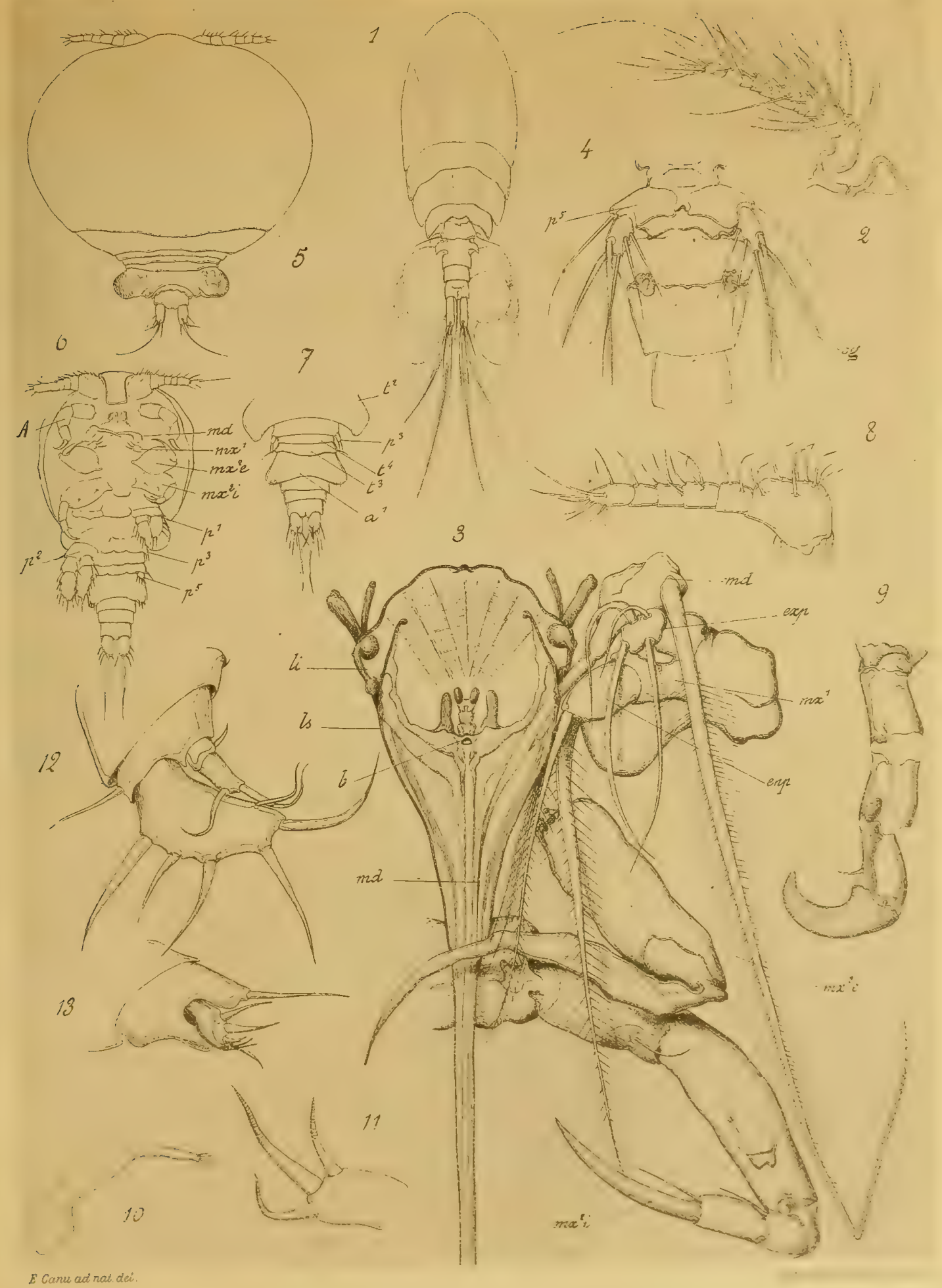





\section{PLANCHE XXX.}

Fig. I-3, Nicothoe astaci Audouin et Milne-Edwards.

Fig. 4-5 et io, Hersiliodes pelseneeri Canu.

Fig. 6-9, Grardella callianassa CANU. 


\section{PLANCHE XXX.}

\section{Nicotboe astaci .}

Fig. I. Segment céphalique, vu dorsalement et ne montrant que les parties chitineuses : $\frac{160}{1}$.

$p h$, pharynx débouchant dans le disque-suçoir ; hph, hypopharynx avec les replis de sa paroi ; $x, y$, crêtes chitineuses pour l'articulation de la seconde maxille.

Fig. 2. Céphalon et premiers somites thoraciques, vus par la face dorsale $: \frac{120}{1}$. Avec les muscles ( $m$ ) dorsaux-latéraux de l'hypopharynx et ceux ( $n$ ) du disque-suçoir.

Fig. 3. Antenne, vue par la face interne: $\frac{350}{1}$.

$r$, le lobe dentelé du $3^{\circ}$ article.

\section{Hersiliodes pelseneeri o.}

Fig. 4. Antenne: $\frac{160}{1}$.

Fig. 5. Mandibule, vue par la face interne: $\frac{160}{1}$.

\section{Giardella callianassa.}

Fig. 6. Première maxille: $\frac{340}{1}$.

Fig. 7. Seconde maxille externe: $\frac{350}{1}$.

Fig. 8. Seconde maxille interne: $\frac{160}{1}$.

Fig. 9. Région buccale: $\frac{350}{1}$.

\section{Hersiliodes pelseneeri.}

Fig. ro. Région buccale d'un embryon cyclopoïde: $\frac{350}{1}$. 


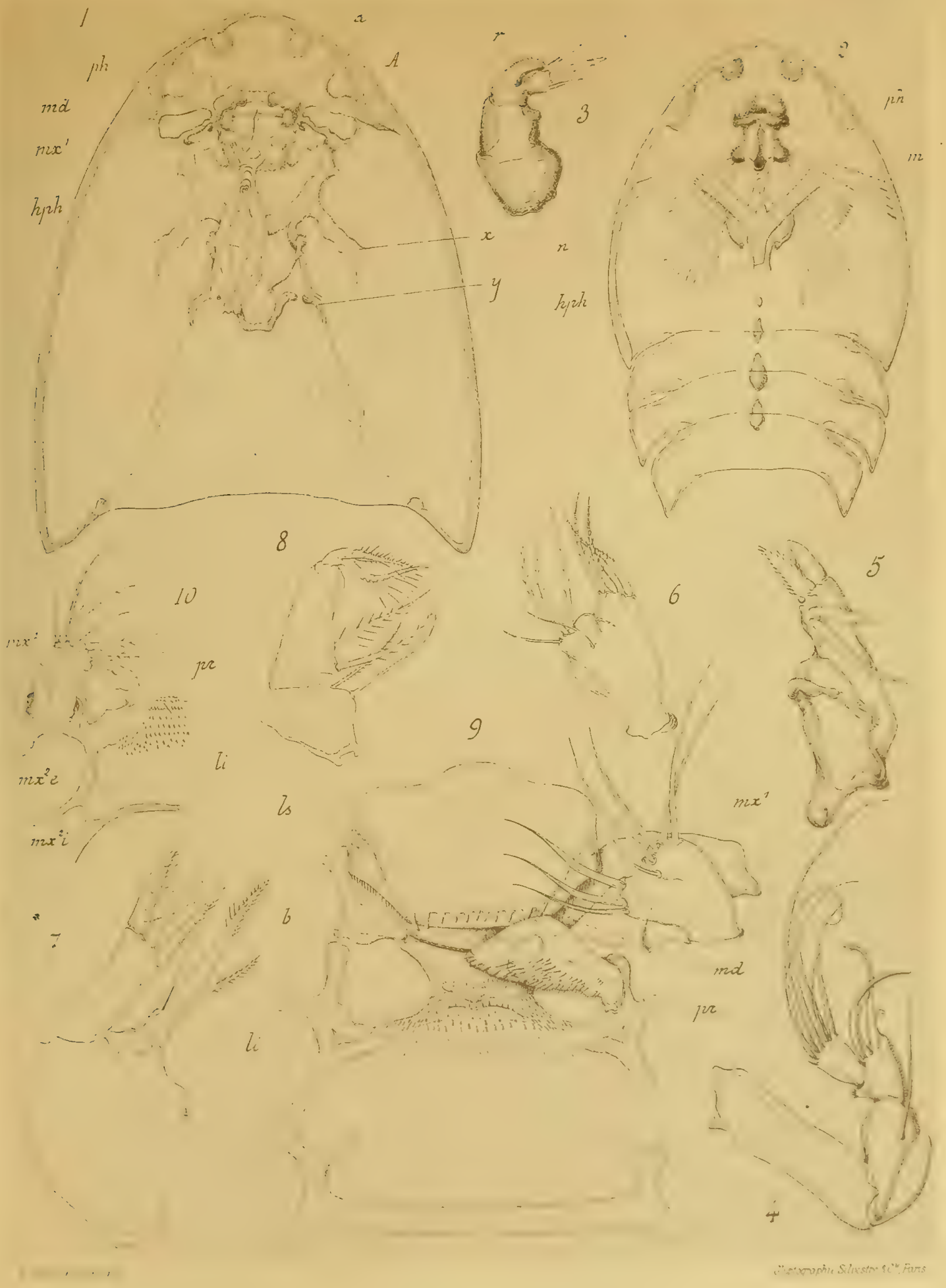



WILSON COPEPOD LIRRARY Smithsenian Institution

Invertebrate Zoology

(Crustacea)

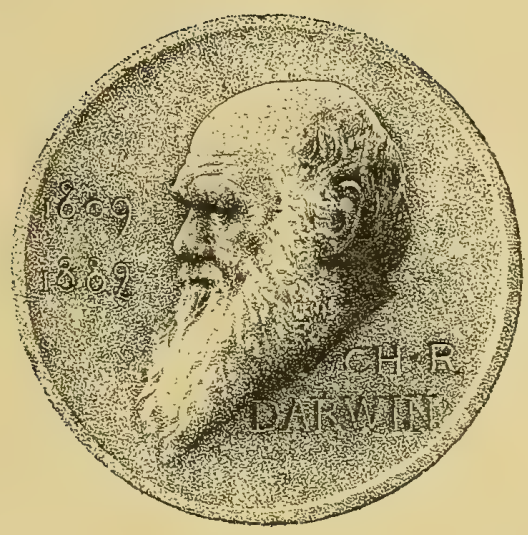



Publications de La station zOOLOGIQUe de WIMEREUX-AMbleteuse.

SOUS LA DIRECTION DE

ALFRED GIARD,

PROFESSEUR A LA SORBONNE.

II.

\section{TRAVAUX DU LABORATOIRE.}

1. ULes BARROIS, Recherches sur l'embryologie des Bryozoaires, in-4, 305 pages, 16 planches coloriées el noires $(1877) \ldots \ldots \ldots \ldots \ldots \ldots \ldots \ldots \ldots \ldots \ldots \ldots \ldots$ (épuisé)

1. Paul HALLEZ, Contributions à l'histoire naturelle des Turbellariés, in-4 213 pages, II plainches $(1879) \ldots \ldots \ldots \ldots \ldots \ldots \ldots \ldots \ldots \ldots \ldots \ldots \ldots \ldots \ldots \ldots \ldots \ldots \ldots$ (épuisé)

11. Roman MONIEZ, Essai monographique sur les Cysticerques, in-40, rgo pages,

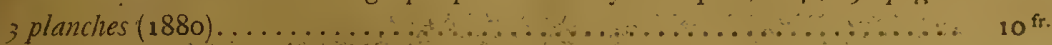

- Romarn MONIEZ, Mémoires sưr les Cestodes, in-4, 238 pages, 12 planches

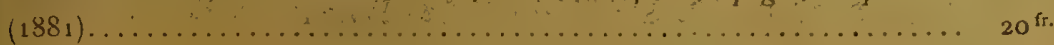

V. A. GIARD et J. BONNIER, Contributions à l'étude des Bopyriens, in-4", $27^{2}$ pages, 10 planches dont 6 colorices, et 26 ,ig. dans le texte $(1887) \ldots \ldots$. .

$40 \mathrm{fr}$.

VI. Eugène CANU, Les Copépodes du Boulonnais, in $-4^{\circ}, 354$ pages, 30 planches

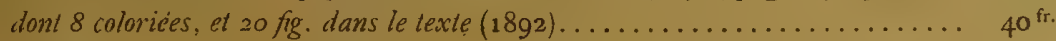

Dípositaires des Pubucations dǘ Laboratoire`de Wimereư-Ambleteusk:

Paris, Georges CARRE, 58, rue Saint-André-des-Arts ;

- PaUl KLINCKSIECK, 52, rue des Écoles;

Berlin, FRIEDLÄNDER \& SOHN, N.-W., 11, Carlstrasse ;

Londres, DULAU \& C , 37, Soho-Square. 


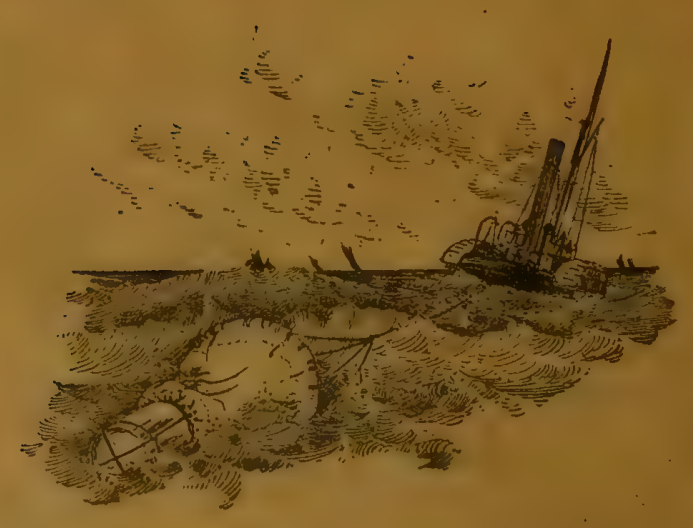

i 




SMITHSONIAN INSTITUTION LIBRARIES 